Prepared in cooperation with the

Colorado Department of Public Health and Environment

\title{
Effect of Drought on Streamflow and Stream-Water Quality in Colorado, July through September 2002
}

Scientific Investigations Report 2006-5322 


\section{Effect of Drought on Streamflow and Stream-Water Quality in Colorado, July through September 2002}

By Daniel T. Chafin and A. Douglas Druliner

Prepared in cooperation with the

Colorado Department of Public Health and Environment

Scientific Investigations Report 2006-5322 


\section{U.S. Department of the Interior DIRK KEMPTHORNE, Secretary}

\section{U.S. Geological Survey \\ Mark D. Myers, Director}

\section{U.S. Geological Survey, Reston, Virginia: 2007}

For product and ordering information:

World Wide Web: http://www.usgs.gov/pubprod

Telephone: 1-888-ASK-USGS

For more information on the USGS--the Federal source for science about the Earth, its natural and living resources, natural hazards, and the environment:

World Wide Web: http://www.usgs.gov

Telephone: 1-888-ASK-USGS

Any use of trade, product, or firm names is for descriptive purposes only and does not imply endorsement by the U.S. Government.

Although this report is in the public domain, permission must be secured from the individual copyright owners to reproduce any copyrighted materials contained within this report.

Suggested citation:

Chafin, D.T., and Druliner, A.D., 2007, Effect of drought on streamflow and stream-water quality in Colorado, July through September 2002: U.S. Geological Survey Scientific Investigations Report 2006-5322, 134 p. 


\section{Contents}

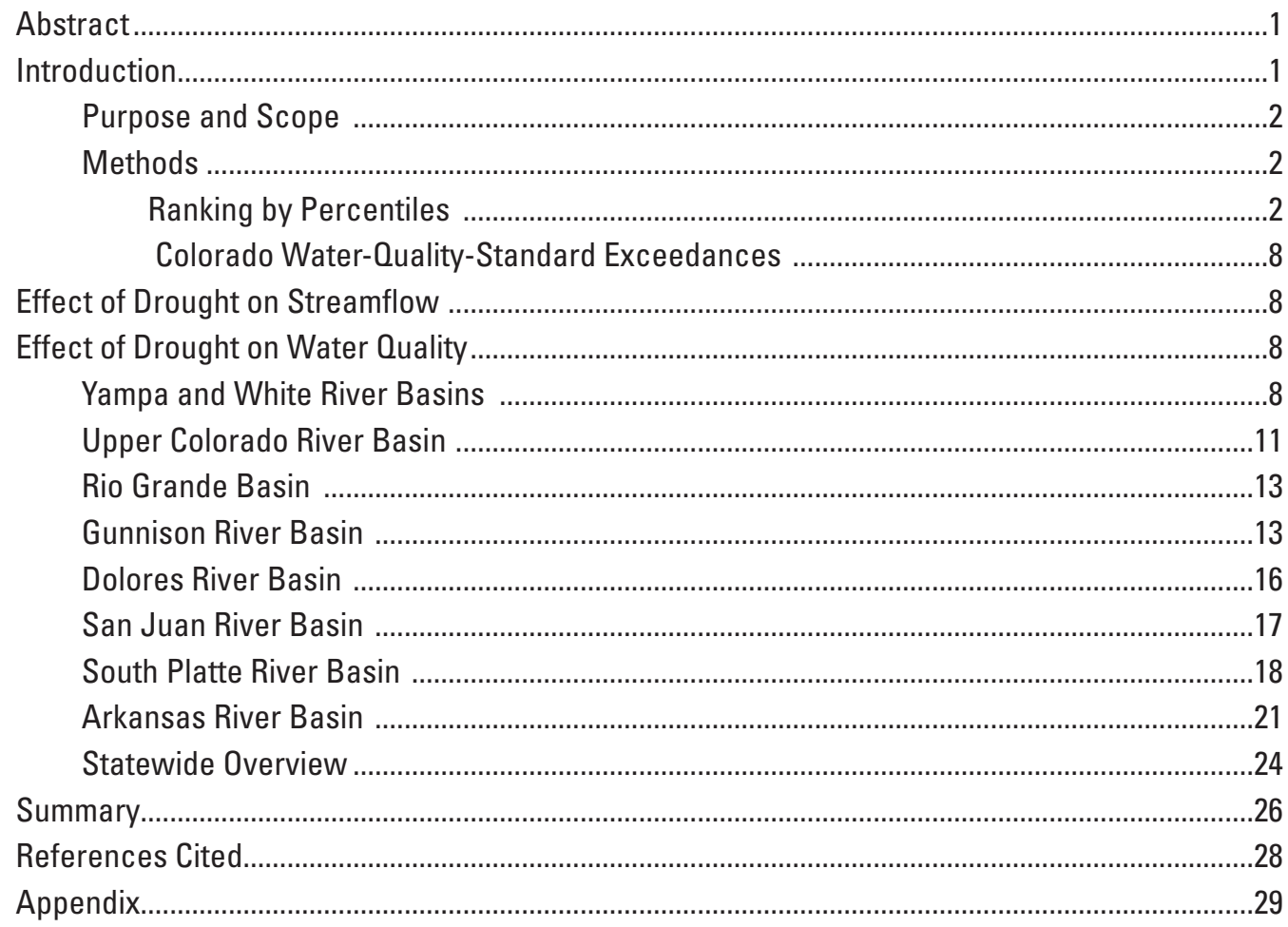

\section{Figures}

1-7. Maps showing:

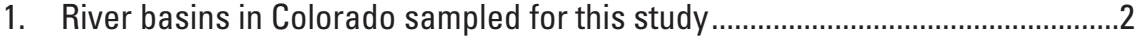

2. Percentiles of mean annual flow in Colorado for water year 2002 relative to mean annual flow during 1978-2002 ...................................................

3. Percentiles of mean summer (July-September) flow in Colorado for 2002 relative to mean summer flow during 1978-2002 .................................10

4. Sampling sites in the Yampa, White, and Upper Colorado River Basins ..........12

5. Sampling sites in the Rio Grande, Gunnison, Dolores, and San Juan

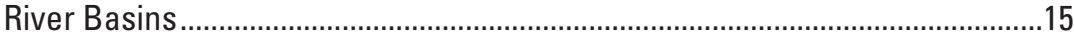

6. Sampling sites in the South Platte River Basin .................................................19

7. Sampling sites in the Arkansas River Basin ......................................................22 


\section{Tables}

1. Colorado stream sites where water-quality samples were collected during July through September 2002

2. Average percentiles and results for summer 2002 drought water-quality data from sites in the Yampa and White River Basins, relative to historical data from July through September, 1978-2002.

3. Average percentiles and results for summer 2002 drought water-quality data from sites in the Upper Colorado River Basin, relative to historical data from July through September, 1978-2002.

4. Average percentiles and results for summer 2002 drought water-quality data from sites in the Rio Grande Basin, relative to historical data from July through September, 1978-2002.

5. Average percentiles and results for summer 2002 drought water-quality data from sites in the Gunnison River Basin relative to historical data from July through September, 1978-2002

6. Average percentiles and results for summer 2002 drought water-quality data from sites in the Dolores River Basin relative to historical data from July through September, 1978-2002.

7. Average percentiles and results for summer 2002 drought water-quality data from sites in the San Juan River Basin, relative to historical data from July through September, 1978-2002.

8. Average percentiles and results for summer 2002 drought water-quality data from sites in the South Platte River Basin relative to historical data from July through September, 1978-2002.

9. Average percentiles and results for summer 2002 drought water-quality data from sites in the Arkansas River Basin relative to historical data from July through September, 1978-2002

10. Summary of characterization of percentiles for properties and constituents in drought samples collected from sites in Colorado during summer 2002 compared to 1978-2002.

11. Water-quality data for the Yampa and White River Basins, showing 2002 drought values, Colorado water-quality exceedances, and percentiles relative to historical data for selected properties and constituents from July through September, 1978-2002.

12. Water-quality data for the Upper Colorado River Basin, showing 2002 drought values, Colorado water-quality exceedances, and percentiles relative to historical data for selected properties and constituents from July through September, 1978-2002.

13. Water-quality data for the Rio Grande Basin showing 2002 drought values, Colorado water-quality exceedances, and percentiles relative to historical data for selected properties and constituents from July through September, 1978-2002.

14. Water-quality data for the Gunnison River Basin showing 2002 drought values, Colorado water-quality exceedances, and percentiles relative to historical data for selected properties and constituents from July through September, 1978-2002. 
15. Water-quality data for the Dolores River Basin showing 2002 drought values, Colorado water-quality exceedances, and percentiles relative to historical data for selected properties and constituents from July through September, 1978-2002.

16. Water-quality data for the San Juan River Basin showing 2002 drought values, Colorado water-quality exceedances, and percentiles relative to historical data for selected properties and constituents from July through September, 1978-2002

17. Water-quality data for the South Platte River Basin showing 2002 drought values, Colorado water-quality exceedances, and percentiles relative to historical data for selected properties and constituents from July through September, 1978-2002.

18. Water-Quality data for the Arkansas River Basin showing 2002 drought values, Colorado water-quality exceedances, and percentiles relative to historical data for selected properties and constituents from July through September, 1978-2002.

\section{Conversion Factors and Datums}

\begin{tabular}{|c|c|c|}
\hline Multiply & By & To obtain \\
\hline & Volume & \\
\hline \multirow[t]{2}{*}{ liter (L) } & 0.2642 & gallon (gal) \\
\hline & Mass & \\
\hline \multirow{2}{*}{ 1. $-5+5$} & 0.03527 & ounce, avoirdupois (oz) \\
\hline & Streamflow & \\
\hline cubic meters per second $\left(\mathrm{m}^{3} / \mathrm{s}\right)$ & 35.31 & cubic feet per second $\left(\mathrm{ft}^{3} / \mathrm{s}\right)$ \\
\hline
\end{tabular}

Temperature in degrees Celsius $\left({ }^{\circ} \mathrm{C}\right)$ may be converted to degrees Fahrenheit $\left({ }^{\circ} \mathrm{F}\right)$ as follows:

$$
{ }^{\circ} \mathrm{F}=\left(1.8 \times{ }^{\circ} \mathrm{C}\right)+32
$$

Vertical coordinate information is referenced to the North American Vertical Datum of 1988 (NAVD 88).

Horizontal coordinate information is referenced to the North American Datum of 1927 (NAD 27).

\section{Abbreviations or Terms}

$\begin{array}{ll}\text { as } \mathrm{N} & \text { as quantified, as measured nitrogen } \\ \text { as } \mathrm{P} & \text { as quantified, as measured phosphorus } \\ \mathrm{col} / 100 \mathrm{~mL} & \text { colonies per } 100 \text { milliliters } \\ \mu \mathrm{g} / \mathrm{L} & \text { micrograms per liter } \\ \mu \mathrm{S} / \mathrm{cm} & \text { microsiemens per centimeter at } 25 \text { degrees Celsius } \\ \mathrm{mg} / \mathrm{L} & \text { milligrams per liter } \\ \mathrm{Q} \% & \text { percentile } \\ \mathrm{SU} & \text { standard units }(\mathrm{pH})\end{array}$





\title{
Effect of Drought on Streamflow and Stream-Water Quality in Colorado, July through September 2002
}

\author{
By Daniel T. Chafin and A. Douglas Druliner
}

\section{Abstract}

During 2002, Colorado experienced the State's worst drought since 1977. In 2003, the U.S. Geological Survey entered into cooperative agreement with the Colorado Department of Public Health and Environment to evaluate the general effects of drought on the water quality of streams in Colorado during summer 2002 by analyzing a water-quality data set obtained during summer 2002 in cooperation with a variety of State and local governments. Water samples were collected at 148 stream sites in Colorado and were measured or analyzed for field properties, major ions, nutrients, organic carbon, bacteria, and dissolved and total recoverable metals.

Mean annual streamflow was analyzed at 134 sites in Colorado, and mean summer (July-September) streamflow for 2002 was determined for 146 sites for water years 1978-2002. Mean annual streamflow for 2002 had an average percentile of 29.4 and mean summer streamflow for 2002 had an average percentile of 7.6 relative to 1978-2002. These results indicate that streamflow in Colorado was substantially less than median streamflow for the period and that the effect of drought on streamflow was greater during summer 2002 than during water year 2002 (October 1, 2001, through September 30, 2002).

Few measured constituent concentrations or values were elevated or depressed on a widespread basis during summer 2002. Specific conductance was elevated (in the upper quartile relative to historical data) in five of the seven basins that had sufficient data for characterization, indicating that specific conductance likely was affected by drought in those basins. Chloride concentrations were elevated in three of five basins with sufficient data and indicate that chloride concentration generally was affected by drought in those basins. Sulfate concentration was elevated in four of six basins with sufficient data. The widespread elevation of specific conductance and concentrations of chloride and sulfate indicates that salinity generally was affected by drought in Colorado streams during July-September 2002, likely because streamflow at most sites was dominated by base flow of ground water, which usually has substantially greater salinity compared to runoff from precipitation. Total-recoverable iron and manganese concentrations were depressed (in the lower quartile of historical data) in the Arkansas River Basin, which likely was due to reduced land-surface washoff of sediment containing oxyhydroxides of these metals.

Of the 246 water samples collected at 148 sites during the summer of 2002, constituents in 115 exceeded Colorado water-quality standards. Constituents that exceeded waterquality standards were $\mathrm{pH}$ (all 9.0 standard unit exceedances; 9 samples), chloride (1 sample), sulfate (9 samples), dissolved ammonia (10 samples), dissolved nitrite nitrogen (3 samples), E. coli (Escherichia coli) bacteria (34 samples, 20 in Arkansas River Basin), fecal-coliform bacteria (18 samples, all in Arkansas River Basin), dissolved copper (1 sample), dissolved iron (3 samples), total-recoverable iron (3 samples), dissolved manganese (13 samples), dissolved selenium (10 samples), and dissolved zinc ( 1 sample). Of these 115 exceedances, historical data were sufficient to conclude that 21 probably were affected by drought, that 39 probably were not affected by drought, and that 55 were of indeterminate nature.

Specific conductance indicates that the San Juan River Basin (average percentile 95.2) experienced the greatest effects of drought on water quality during summer 2002 compared to other basins in Colorado, followed by the Upper Colorado (90.0) and Dolores River (85.7) Basins. The South Platte River Basin (70.9) experienced the least effect of drought, and the Yampa and White River Basin group (73.7) had the second smallest effect. The Gunnison River (82.1) and Arkansas River (81.2) Basins had intermediate drought effects. The Rio Grande had insufficient data to rank the relative effect of drought on salinity.

\section{Introduction}

During 2002, Colorado experienced the State's worst drought since 1977 (Kuhn, 2005). To determine the effects of the 2002 drought on streamflow and stream-water quality in the State during the summer months, a variety of State and local governments cooperated with the U.S. Geological Survey (USGS) to collect water-quality samples at 148 stream sites in the nine major river basins in Colorado (fig. 1). In 2003, the U.S. Geological Survey, in cooperation with the Colorado Department of Public Health and Environment, began 


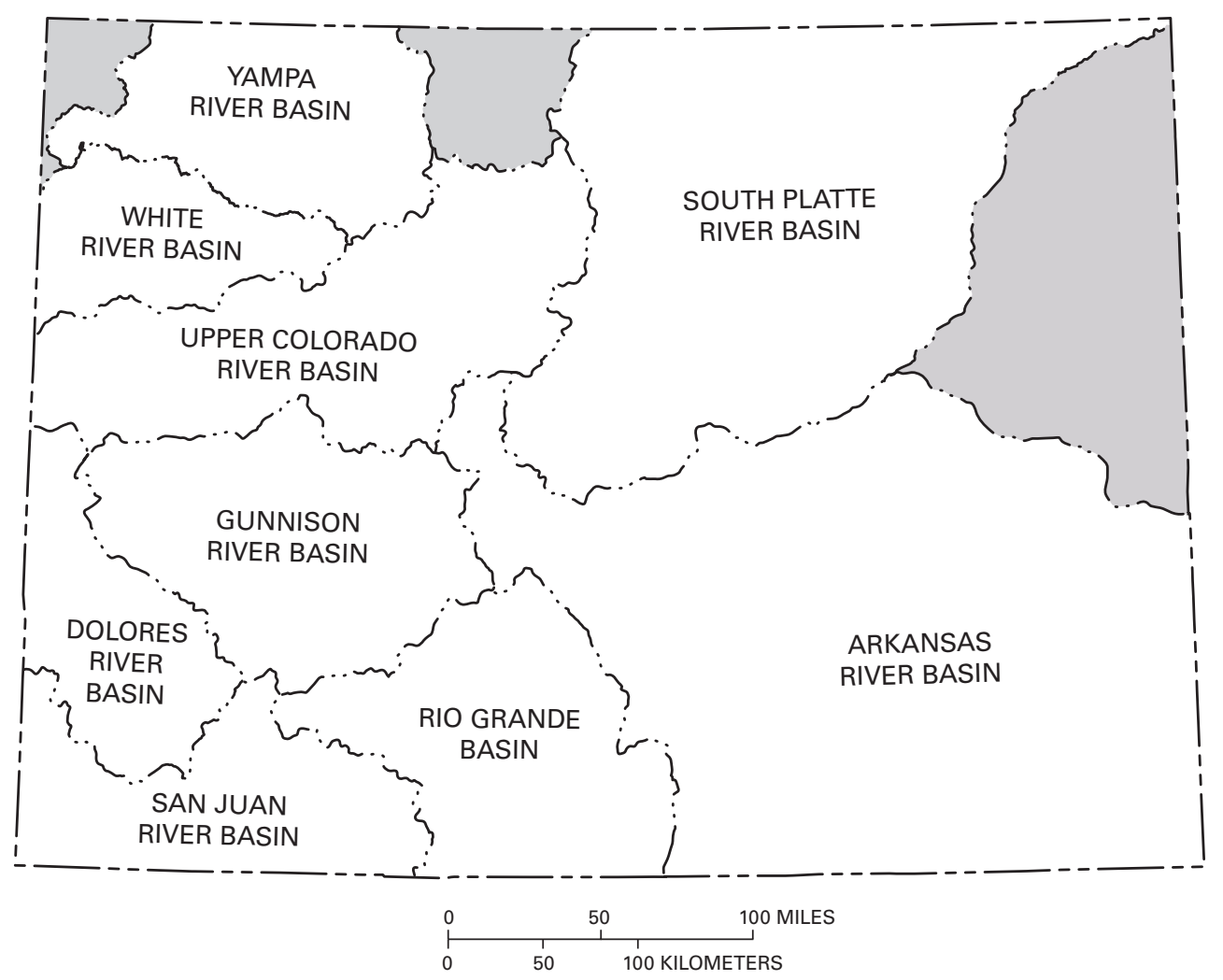

Figure 1. River basins in Colorado sampled for this study.

to examine these data. Water samples were analyzed for field properties, major ions, nutrients, organic carbon, bacteria, and dissolved metals at all sites, although individual constituents in these categories varied among basins. Results of the analyses were reported in Crowfoot, Payne, and O'Neill (2003) and in Crowfoot, Boulger, and O'Neill (2003).

\section{Purpose and Scope}

This report presents water-quality data collected at 148 sites on rivers and creeks in Colorado during drought conditions for the period July-September 2002. The report summarizes water-quality data for 2002 relative to data from 1978 through 2001 and percentiles of constituent concentrations during summer 2002 relative to historical data for each site for the months of July through September during 1978 through 2001. In addition, the report presents exceedances of Colorado water-quality standards for constituents sampled during summer 2002.

\section{Methods}

Between July 11, 2002, and September 30, 2002, 148 stream sites (table 1) were sampled by the equal-widthincrement method (U.S. Geological Survey, 1998) to obtain representative water-quality samples. Water samples were collected twice at 98 of these sites for a total of 246 samples.
Water samples collected at these sites are hereinafter referred to as "drought samples." Individual drought samples were measured for field properties (dissolved oxygen, $\mathrm{pH}$, specific conductance, and water temperature) and collected for laboratory analysis and were processed, preserved, and shipped according to standard guidelines (U.S. Geological Survey, 1998). All samples were shipped to the USGS National Water-Quality Laboratory in Lakewood, Colorado, for laboratory analyses. Data are reported by basin in tables 11-18 in the Appendix at the back of this report.

\section{Ranking by Percentiles}

The primary tool used to evaluate concentrations of constituents in water-quality samples collected during summer 2002 was ranking by percentile. Percentiles were calculated for each water-quality constituent for each site sampled during the 2002 drought by using the population of all samples for each site from July through September for 1978 through 2002 that had 20 or more samples (including the samples collected during the drought). Percentiles calculated for fewer than 20 samples were not considered to be statistically reliable. Percentiles were calculated only for the months of July-September to maintain a common basis for comparison among years so that the effects of drought during July-September 2002 could be assessed. Data collected prior to 1978 were not included, primarily to preclude comparison 
Table 1. Colorado stream sites where water-quality samples were collected during July through September 2002.

\begin{tabular}{|c|c|c|}
\hline $\begin{array}{c}\text { Site } \\
\text { number in } \\
\text { figures } 4-7\end{array}$ & $\begin{array}{l}\text { U.S. Geological } \\
\text { Survey } \\
\text { station number }\end{array}$ & U.S. Geological Survey station name \\
\hline 1 & 09238900 & Fish Creek at upper station near Steamboat Springs \\
\hline 2 & 09239500 & Yampa River at Steamboat Springs \\
\hline 4 & 09246400 & Elkhead Creek below Maynard Gulch near Craig \\
\hline 5 & 09247600 & Yampa River below Craig \\
\hline 6 & 09249750 & Williams Fork at mouth \\
\hline 7 & 09251000 & Yampa River near Maybell \\
\hline \multicolumn{3}{|r|}{ White River Basin (figure 4) } \\
\hline 10 & 09304000 & South Fork White River at Buford \\
\hline 11 & 09304200 & White River above Coal Creek near Meeker \\
\hline 12 & 09304800 & White River below Meeker \\
\hline 13 & 09306200 & Piceance Creek below Ryan Gulch near Rio Blanco \\
\hline 14 & 09306222 & Piceance Creek at White River \\
\hline 15 & 09306242 & Corral Gulch near Rangely \\
\hline 16 & 09306255 & Yellow Creek near White \\
\hline 17 & 09306290 & White River below Boise Creek near Rangely \\
\hline 23 & 09025010 & Fraser River below Vasquez Creek at Winter Park \\
\hline 24 & 09027100 & Fraser River at Tabernash \\
\hline 25 & 09033100 & Ranch Creek below Meadow Creek near Tabernash \\
\hline 26 & 09033300 & Fraser River below Crooked Creek at Tabernash \\
\hline 27 & 09034250 & Colorado River at Windy Gap near Granby \\
\hline 28 & 09038500 & Williams Fork below Williams Fork Reservoir \\
\hline 29 & 09040500 & Troublesome Creek near Troublesome \\
\hline 30 & 09041090 & Muddy Creek above Antelope Creek near Kremmling \\
\hline 31 & 09041400 & Muddy Creek below Wolford Mountain Reservoir near Kremmling \\
\hline 32 & 09057700 & Blue River at mouth near Kremmling \\
\hline 33 & 09058000 & Colorado River near Kremmling \\
\hline 34 & 09063000 & Eagle River at Red Cliff \\
\hline 35 & 09066510 & Gore Creek at mouth near Minturn \\
\hline 36 & 09067005 & Eagle River at Avon \\
\hline 37 & 09069000 & Eagle River at Gypsum \\
\hline
\end{tabular}


Table 1. Colorado stream sites where water-quality samples were collected during July through September 2002.—Continued

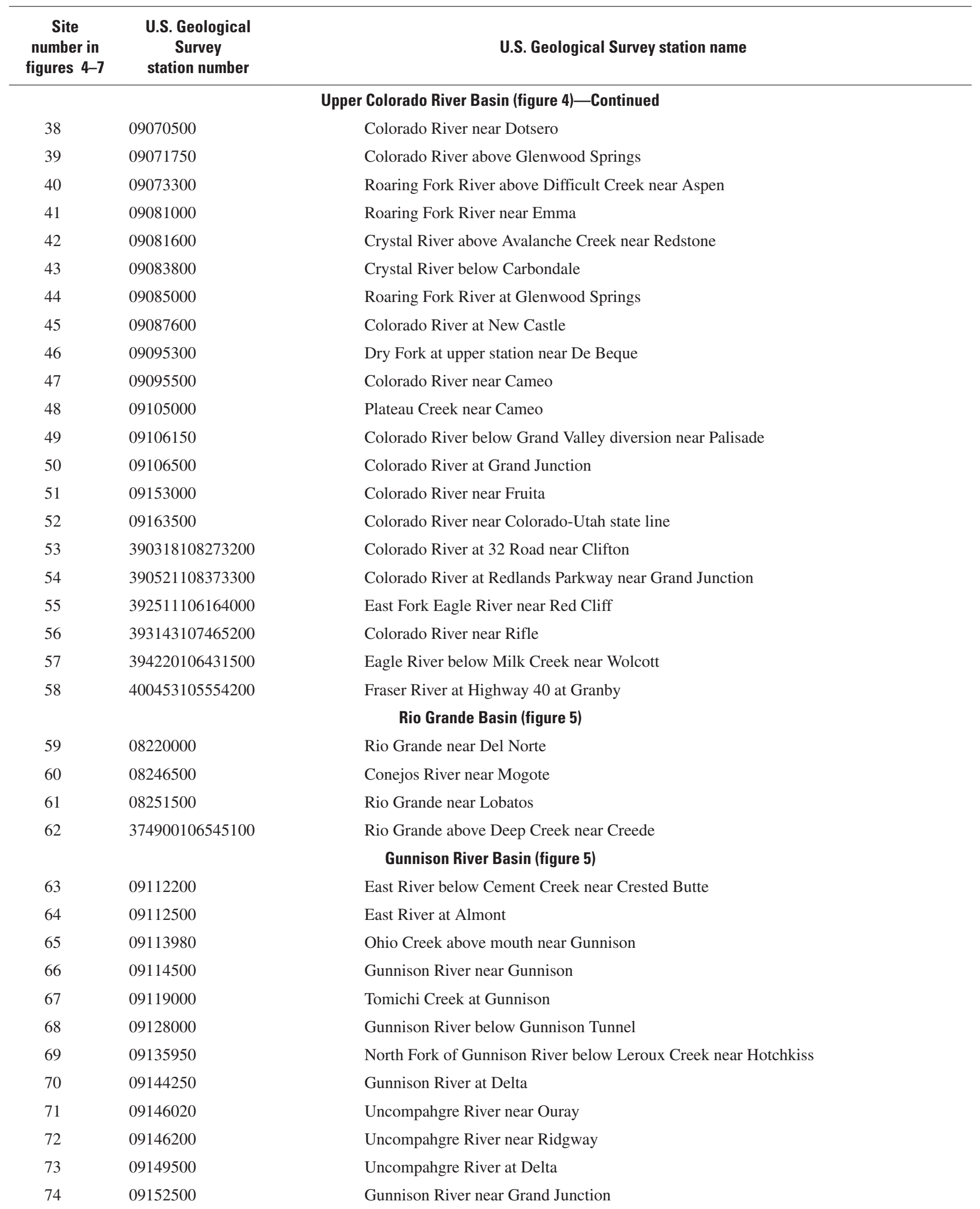


Table 1. Colorado stream sites where water-quality samples were collected during July through September 2002. —Continued

\begin{tabular}{|c|c|c|}
\hline $\begin{array}{c}\text { Site } \\
\text { number in } \\
\text { figures } 4-7\end{array}$ & $\begin{array}{c}\text { U.S. Geological } \\
\text { Survey } \\
\text { station number }\end{array}$ & U.S. Geological Survey station name \\
\hline \multicolumn{3}{|r|}{ Gunnison River Basin (figure 5)—Continued } \\
\hline 75 & 383103106594200 & Gunnison River at County Road 32 below Gunnison \\
\hline 76 & 383126106475600 & Tomichi Creek below Chetopa Creek near Parlin \\
\hline 77 & 383604106312400 & Quartz Creek below Pitkin \\
\hline 78 & 384624107570701 & Gunnison River at 2200 Road bridge at Austin \\
\hline 79 & 384950106544200 & East River above Slate River near Crested Butte \\
\hline 80 & 385224106590100 & Coal Creek above mouth at Crested Butte \\
\hline 81 & 385240106583600 & Slate River above Coal Creek near Crested Butte \\
\hline 82 & 385325106581200 & Washington Gulch below Woods Creek at Mount Crested Butte \\
\hline 83 & 385408106543600 & East River above Crested Butte \\
\hline \multicolumn{3}{|r|}{ Dolores River Basin (figure 5) } \\
\hline 84 & 09166500 & Dolores River at Dolores \\
\hline 85 & 09169500 & Dolores River at Bedrock \\
\hline 86 & 09171100 & Dolores River near Bedrock \\
\hline 87 & 09172500 & San Miguel River near Placerville \\
\hline 88 & 09177000 & San Miguel River at Uravan \\
\hline \multicolumn{3}{|r|}{ San Juan River Basin (figure 5) } \\
\hline 89 & 09349800 & Piedra River near Arboles \\
\hline 90 & 09352900 & Vallecito Creek near Bayfield \\
\hline 91 & 09354500 & Los Pinos River at La Boca \\
\hline 92 & 09359020 & Animas River below Silverton \\
\hline 93 & 09361500 & Animas River at Durango \\
\hline 94 & 09363200 & Florida River at mouth at Bondad \\
\hline 95 & 09366500 & La Plata River at Colorado-New Mexico state line \\
\hline 96 & 09370000 & Mancos River near Mancos \\
\hline 97 & 09371492 & Mud Creek at State Highway 32 near Cortez \\
\hline 98 & 09371520 & McElmo Creek above Trail Canyon near Cortez \\
\hline 99 & 09372000 & McElmo Creek near Colorado-Utah state line \\
\hline 100 & 370907107530401 & Animas River at Weaselskin Bridge near La Posta \\
\hline 101 & 371319107515001 & Animas River near Carbon Junction \\
\hline 102 & 371500107004601 & San Juan River below Pagosa Springs \\
\hline \multicolumn{3}{|r|}{ South Platte River Basin (figure 6) } \\
\hline 103 & 06713500 & Cherry Creek at Denver \\
\hline 104 & 06714000 & South Platte River at Denver \\
\hline 105 & 06719505 & Clear Creek at Golden \\
\hline 106 & 06720500 & South Platte River at Henderson \\
\hline 107 & 06733000 & Big Thompson River at Estes Park \\
\hline 108 & 06734900 & Olympus tunnel at Lake Estes \\
\hline 109 & 06735500 & Big Thompson River near Estes Park \\
\hline 110 & 06736000 & North Fork Big Thompson River at Drake \\
\hline
\end{tabular}


Table 1. Colorado stream sites where water-quality samples were collected during July through September 2002.—Continued

\begin{tabular}{|c|c|c|}
\hline $\begin{array}{c}\text { Site } \\
\text { number in } \\
\text { figures } 4-7\end{array}$ & $\begin{array}{l}\text { U.S. Geological } \\
\text { Survey } \\
\text { station number }\end{array}$ & U.S. Geological Survey station name \\
\hline \multicolumn{3}{|r|}{ South Platte River Basin (figure 6)—Continued } \\
\hline 111 & 06741510 & Big Thompson River at Loveland \\
\hline 112 & 06741530 & Big Thompson River at Interstate 25 near Loveland \\
\hline 114 & 06752280 & Cache la Poudre River above Boxelder Canyon near Timnath \\
\hline 115 & 06753990 & Lonetree Creek near Greeley \\
\hline 116 & 06754000 & South Platte River near Kersey \\
\hline 117 & 06758500 & South Platte River near Weldona \\
\hline 121 & 402114105350101 & Big Thompson River below Moraine Park near Estes Park \\
\hline 122 & 402533105124300 & Big Thompson River below Sulzer Gulch near Loveland \\
\hline 123 & 402535105105700 & Buckhorn Creek at County Road 24 near Loveland \\
\hline 124 & 402554105202100 & Big Thompson River above North Fork of Big Thompson at Drake \\
\hline 125 & 403048105042701 & Fossil Creek at College Avenue at Fort Collins \\
\hline \multicolumn{3}{|r|}{ Arkansas River Basin (figure 7) } \\
\hline 126 & 07086000 & Arkansas River at Granite \\
\hline 127 & 07096000 & Arkansas River at Canon City \\
\hline 134 & 07103970 & Monument Creek above Woodmen Road at Colorado Springs \\
\hline 135 & 07104905 & Monument Creek at Bijou Street at Colorado Springs \\
\hline 136 & 07105500 & Fountain Creek at Colorado Springs \\
\hline 137 & 07105530 & Fountain Creek below Janitell Road below Colorado Springs \\
\hline 138 & 07105800 & Fountain Creek at Security \\
\hline 139 & 07106000 & Fountain Creek near Fountain \\
\hline 140 & 07106300 & Fountain Creek near Pinon \\
\hline 141 & 07109500 & Arkansas River near Avondale \\
\hline 142 & 07119700 & Arkansas River at Catlin Dam near Fowler \\
\hline 143 & 07120500 & Arkansas River near Rocky Ford \\
\hline 144 & 07123000 & Arkansas River at La Junta \\
\hline 145 & 07124000 & Arkansas River at Las Animas \\
\hline 146 & 07124200 & Purgatoire River at Madrid \\
\hline 147 & 07126500 & Purgatoire River at Ninemile Dam near Higbee \\
\hline 148 & 07133000 & Arkansas River at Lamar \\
\hline
\end{tabular}


with data that were collected during earlier droughts, including the last one, which ended in 1977. This restriction also minimized comparison of 2002 data with data that were collected before construction of major reservoirs in the 1960s and 1970s. For major ions, percentiles were calculated only for chloride and sulfate because they are the only major ions for which Colorado water-quality standards have been established. Percentiles also were calculated for mean annual and mean summer streamflow during 2002 relative to 1978-2002 to evaluate the effect of drought on streamflow.

The percentile for a specific observation in a data set is defined as the percentage of the number of observations in the data set that have values less than or equal to that of the specific observation (Helsel and Hirsch, 1992). For example, if a $\mathrm{pH}$ value of $7.4 \mathrm{in}$ a data set is ranked at the 30th percentile, then about 30 percent of the ranked $\mathrm{pH}$ values are less than or equal to 7.4. Percentiles were calculated using the Weibull formula as described by (Helsel and Hirsch, 1992):

$$
Q \%=100 \times R /(N+1)
$$

where

$$
\begin{gathered}
Q \% \quad \text { is the value of the percentile for a given } \\
\text { observation, } \\
R \quad \begin{array}{c}
\text { is the whole-number, high rank of the } \\
\text { observation (ascending order), }
\end{array}
\end{gathered}
$$

and

$$
\begin{gathered}
N \quad \text { is the number of observations in the ranked } \\
\text { data set }
\end{gathered}
$$

Calculation of percentiles for censored data sets-those having some observations reported as "less than" $(<)$ a threshold concentration-required recensoring to a common basis when the threshold concentration varied among censored observations or when uncensored concentrations were less than one or more censored concentrations. Recensoring was required because of the inability to distinguish ranks given the varying thresholds for censored data. For example, dissolved cadmium concentrations of $<0.1,<1$, and $<50 \mu \mathrm{g} / \mathrm{L}$ (micrograms per liter) cannot be distinguished by rank because the actual concentrations reported as $<1 \mu \mathrm{g} / \mathrm{L}$ and $<50 \mu \mathrm{g} / \mathrm{L}$ might be smaller than the actual concentration reported as $<0.1 \mu \mathrm{g} / \mathrm{L}$. Therefore, the smaller two of these concentrations would require recensoring to $<50 \mu \mathrm{g} / \mathrm{L}$, as would any uncensored concentration less than $50 \mu \mathrm{g} / \mathrm{L}$ (Helsel, 1990). In this example, if only one or two observations were reported as $<50 \mu \mathrm{g} / \mathrm{L}$, the drought sample was censored at a smaller threshold concentration, and the $<50 \mu \mathrm{g} / \mathrm{L}$ observations was discarded from the data set (assuming at least 20 observations remained) to allow better resolution of the percentile for the drought sample; then recensoring would be done to the largest censored concentration that remained. Recensoring was not necessary when the drought sample had a concentration that exceeded the largest censored concentration or when the censored drought sample was unambiguously the smallest concentration.
Assigning ranks to censored or tied values involves some ambiguity. This ambiguity is illustrated in the following generic example with eight observations, which shows how "low ranks" and "high ranks" may be assigned to such values. The low and high ranks bound the possible range of ranks that might be associated with censored values:

\begin{tabular}{ccc}
\hline Value & High rank & Low rank \\
\hline$<1$ & 3 & 1 \\
$<1$ & 3 & 1 \\
$<1$ & 3 & 1 \\
1 & 4 & 4 \\
2 & 5 & 5 \\
3 & 7 & 6 \\
3 & 7 & 6 \\
4 & 8 & 8 \\
\hline
\end{tabular}

Using high ranking as previously described, ties (censored, recensored, or uncensored) in some data sets resulted in drought-sample percentiles that assumed the maximum possible rank for the tied data. For example, if a hypothetical drought sample and 94 historical samples for a site had a dissolved-iron concentration of $<10 \mu \mathrm{g} / \mathrm{L}$ and only 4 historical samples exceeded this concentration, the resulting droughtsample percentile of 95 percent would spuriously indicate an elevated drought-sample concentration when, as far as the data can indicate, the true drought-sample concentration could fall anywhere in the lowest 95 percent of the data. Similarly, in some cases, ties of uncensored observations spuriously indicate elevated drought-sample values or concentrations. For example, if a drought sample and 10 historical samples for a site had a $\mathrm{pH}$ of 8.1,82 historical samples had lower $\mathrm{pH}$, and 6 historical samples had higher $\mathrm{pH}$, then the calculated drought-sample percentile would be 93 percent when, in fact, the true drought-sample $\mathrm{pH}$ (which hypothetically could be indicated by values reported with greater precision) might rank anywhere between a percentile of 83 and 93 percent (inclusive). In cases where tied ranks caused uncertainty in calculated percentiles, the possible percentile range was calculated by using both low and high ranks, and the possible percentile range was reported and flagged by footnotes indicating the type of ties in tables 11-18 (at the back of this report).

To determine the effect of drought on the water quality of individual basins, a mean percentile was calculated for each constituent with a calculated percentile for all sites in the basin. For sites with more than one drought sample, only the most recent sample was used to compute the average percentile for that constituent for that basin. Also, the mean percentile was used in place of percentile ranges for individual samples when calculating basin constituent percentiles. The constituent was concluded to be "elevated" in concentration or value when the mean percentile was equal to or greater than 75 (that is, was in the upper quartile of the data). The 
constituent was concluded to be "depressed" when the mean percentile was less than 25 (that is, was in the lower quartile of the data). The constituent was considered to be "medial" when the mean percentile was between 25 and 74.9 (that is, was in the interquartile range of the data). Basins with fewer than three sites with calculated constituent percentiles were not rated. In cases where tied observations caused a range in possible percentile for one or more sites, the mean (midpoint) of the range(s) was used to calculate mean percentile for the basin. In cases where the conclusion of average effect was an "elevated" or "depressed" value or concentration and the uncertainty associated with the percentile range(s) indiciated the possibility of another conclusion, the conclusion was designated as "tentative" and identified as such in tables 2-9 in the "Effect of Drought on Water Quality" section and in table 10 in the "Statewide Overview" section.

\section{Colorado Water-Quality-Standard Exceedances}

Water-quality data collected during July through September 2002 were compared to regulatory standards promulgated by the State of Colorado (Colorado Department of Public Health and Environment, 2005) to determine which constituents had concentrations or values that exceeded regulatory standards. Because prolonged exceedances are required before there is a legal violation of the water-quality standard, the designation of exceedances in this report are not be considered as actual legal violations of water-quality standards.

Most dissolved-metal concentrations in samples were compared to standards based on water hardness (a function of dissolved calcium and magnesium concentrations). The standard for un-ionized ammonia was applied by calculating the fraction of un-ionized ammonia in each sample, using total dissolved-ammonia concentration, water temperature, and $\mathrm{pH}$. Water-quality exceedances for drought samples at each site were compared to historical exceedances for each site for July-September 1978 through 2001 to determine whether the drought-sample exceedances might have been affected by drought. Exceedances for samples collected during the drought are assumed to be affected by the drought: (1) if there is a total of 6 or more historical samples at the same site as the drought sample, all of which show no exceedances and the maximum historical value is one-half or less of the value of the lowest drought exceedance; or (2) if there are 10 or more historical samples at the same site with no more than 10 percent exceeding the standard.

\section{Effect of Drought on Streamflow}

To determine the effect of the 2002 drought on streamflow in Colorado for the 25-water-year period 1978 through 2002, percentiles were calculated for mean annual streamflow and mean summer (defined as July 1 through September 30) streamflow, using equation 1. (Mean annual streamflow is based on water year, which begins on October 1 and ends on September 30 of the following year.)

Mean annual streamflow was analyzed at 134 sites in Colorado for years 1978-2002 (fig. 2). Forty-one of these sites also were sampled during the 2002 drought. Mean annual streamflow for 2002 had an average percentile of 29.4 percent during this period, indicating that overall streamflow in Colorado was substantially less than median percentile streamflow for the period. Twenty sites (15 percent) had the smallest mean annual streamflow during 2002, and only 16 sites (12 percent) had percentiles for 2002 that exceeded median streamflow during 1978-2002. These data indicate substantial drought conditions during water year 2002.

Mean summer (July-September) streamflow for 2002 (fig. 3) was determined for 146 sites in Colorado, and percentiles averaged 7.6 percent when compared to summer streamflow for 1978-2002 for 134 sites across Colorado. Fifty-two of these sites were sampled during the 2002 drought. During 2002, 116 sites (79 percent) had the smallest mean summer streamflow for 1978-2002, and only 3 sites ( 2 percent) had percentiles for 2002 that exceeded median streamflow for the period. Furthermore, because mean summer streamflow for 2002 had a substantially smaller average percentile (7.6 percent) than did mean annual streamflow for 2002 (29.4 percent), one can conclude that the effect of drought on streamflow was greater during summer 2002 than during water year 2002.

\section{Effect of Drought on Water Quality}

Results of water-quality sampling during 2002, calculated percentiles (Q\%), number (N) of 1978-2002 samples (including only drought samples for 2002), and Colorado water-quality exceedances are shown by river basin in tables 11-18 in the Appendix at the back of this report. Percentiles are not reported for major ions except chloride and sulfate or for any property or constituent that did not have at least 20 samples at a site. Columns showing percentile and number of samples were omitted for properties or constituents with no reported percentile in each table. The following sections discuss results for the following river basins (fig. 1): (1) Yampa and White (combined); (2) Upper Colorado; (3) Rio Grande; (4) Gunnison; (5) Dolores; (6) San Juan; (7) South Platte; and (8) Arkansas. Finally, a statewide overview section discusses general results for all of these basins, including properties or constituents for which results were too infrequent for discussion by basin.

\section{Yampa and White River Basins}

Drought samples were collected at 8 sites in the Yampa River Basin and at 11 sites in the White River Basin (table 11, fig. 4). Because both basins are adjacent, independent tributaries of the Green River, samples collected in these basins 


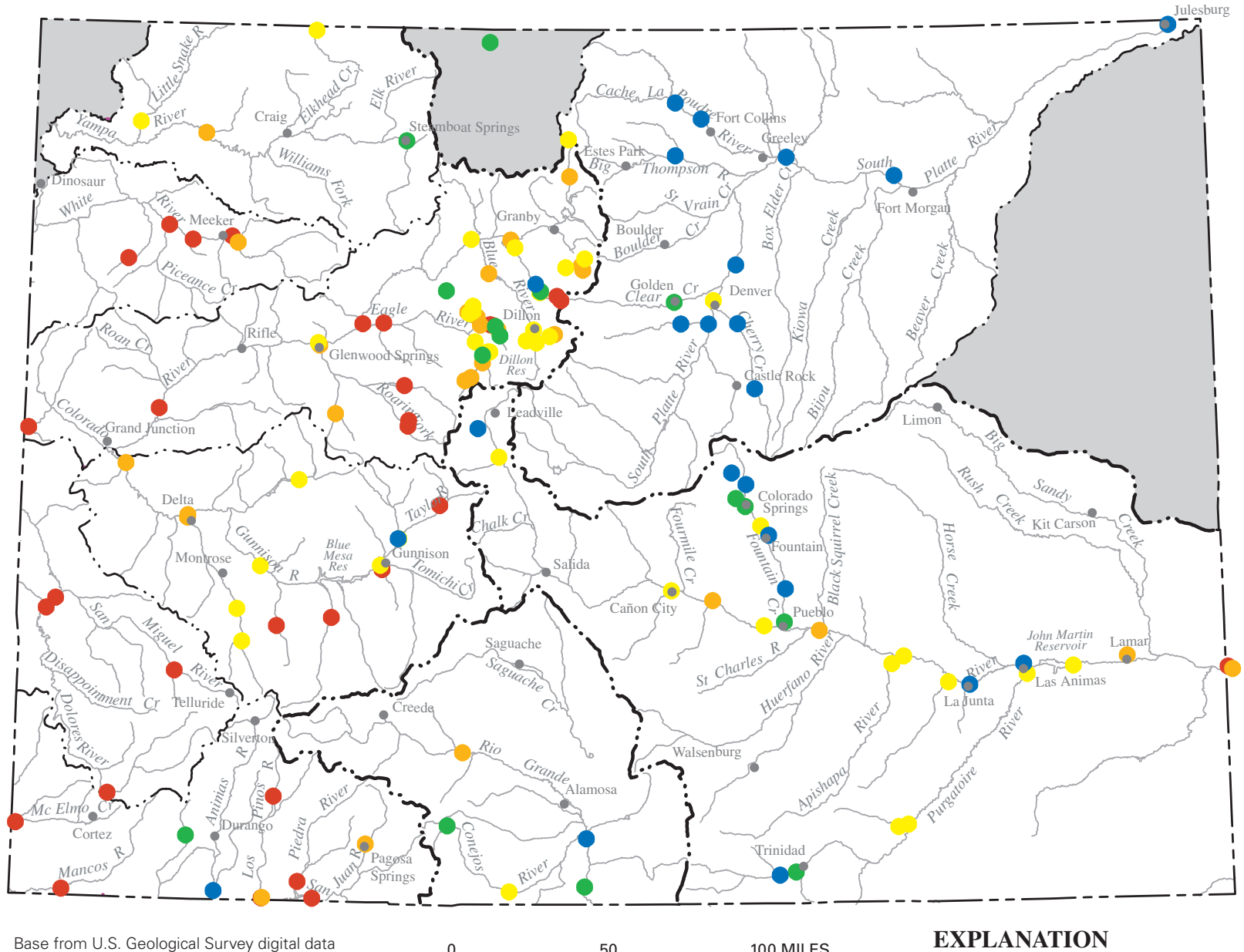

1:100,000 Lambert Conformal Conic projection Standard parallels $33^{\circ} \mathrm{N}$ and $45^{\circ} \mathrm{N}$

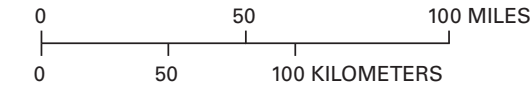

EXPLANATION

Percentiles of surface-water stations

- 3.8 to 7.7

11.5 to 23.1

26.9 to 38.5

- 42.3 to 49.9

- 50.0 to 84.6

Figure 2. Percentiles of mean annual flow in Colorado for water year 2002 relative to mean annual flow during 1978-2002. 


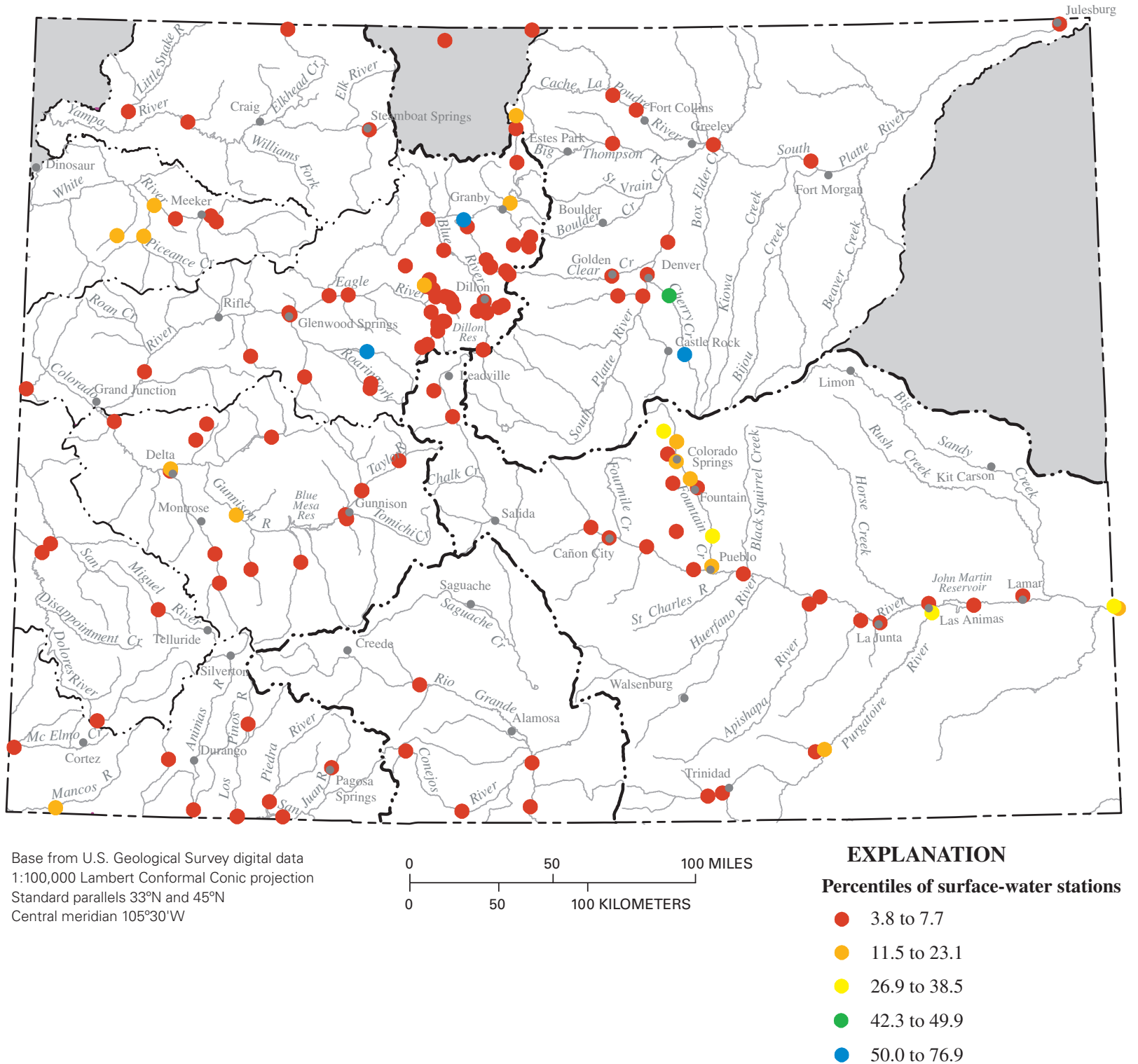

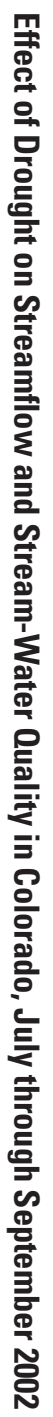

Figure 3. Percentiles of mean summer (July-September) flow in Colorado for 2002 relative to mean summer flow during 1978-2002. 
were jointly interpreted. Average percentile for properties and constituents (table 2) indicate medial values or concentrations for all properties or constituents with sufficient data-including field properties, dissolved major and minor constituents (chloride, fluoride, silica, and sulfate), most dissolved and total-recoverable nutrients, and dissolved boron and iron. Thus, it appears that drought conditions did not have a substantial influence on concentrations of these constituents. Dissolved organic carbon and dissolved metals (manganese, selenium, and zinc) had fewer than three useful percentiles and could not be characterized. Total-recoverable metals were not sampled in these two basins.

Only two drought samples exceeded Colorado waterquality standards (table 11). On July 29, 2002, the $\mathrm{pH}$ at site 2 was $9.3 \mathrm{SU}$, exceeding the upper standard for aquatic life, recreational use, and domestic water supply of 9.0 standard units. Eleven historical samples had a maximum pH of 8.9.
Because of the potential diurnal fluctuation of $\mathrm{pH}$ at these sites and variable measurement time (Chafin, 2002), it is uncertain if this exceedance was affected by drought. On September 4, 2002 , the dissolved manganese concentration at site 8 was $65 \mu \mathrm{g} / \mathrm{L}$, exceeding the drinking-water standard of $50 \mu \mathrm{g} / \mathrm{L}$; five historical samples showed a maximum concentration of $7.2 \mu \mathrm{g} / \mathrm{L}$. In summary, only the dissolved manganese exceedance at site 8 was drought affected.

\section{Upper Colorado River Basin}

Drought samples were collected at 39 sites in the Colorado River Basin (table 12 in Appendix, fig. 4). Average percentile for properties and constituents (table 3 ) indicate elevated specific conductance and elevated concentrations of chloride, fluoride (tentative), and sulfate relative to nondrought

Table 2. Average percentiles and results for summer 2002 drought water-quality data from sites in the Yampa and White River Basins, relative to historical data from July through September, 1978-2002.

[Q\%, percentile; Medial, average Q\% between 25 and 74.9; ID, insufficient number of sites (fewer than 3) to determine result]

\begin{tabular}{lccc}
\hline \multicolumn{1}{c}{ Property or constituent } & Average $\mathbf{0}$ \% & Number of sites & Result \\
\hline Oxygen, dissolved & 44.0 & 12 & Medial \\
pH & 50.4 & 12 & Medial \\
Specific conductance & 73.7 & 17 & Medial \\
Temperature, water & 54.1 & 17 & Medial \\
Chloride, dissolved & 70.2 & 9 & Medial \\
Fluoride, dissolved & 69.2 & 7 & Medial \\
Silica, dissolved & 42.0 & 6 & Medial \\
Sulfate, dissolved & 69.4 & 9 & Medial \\
Nitrogen, ammonia, dissolved & 38.7 & 8 & Medial \\
Nitrogen, organic plus ammonia, dissolved & 55.0 & 6 & Medial \\
Nitrogen, organic plus ammonia, total recoverable & 71.5 & 3 & Medial \\
Nitrogen, nitrite plus nitrate, dissolved & 35.8 & 10 & Medial \\
Nitrogen, nitrite, dissolved & 48.9 & 7 & Medial \\
Phosphorus, dissolved & 58.9 & 7 & Medial \\
Phosphorus, total recoverable & 72.1 & 3 & Medial \\
Phosphorus, orthophosphate, dissolved & 49.0 & Medial \\
Carbon, organic, dissolved & 60.0 & 8 & ID \\
Boron, dissolved & 48.5 & 1 & Medial \\
Iron, dissolved & 42.3 & Medial \\
Manganese, dissolved & 41.3 & ID \\
Selenium, dissolved & 81.0 & 4 & ID \\
Zinc, dissolved & 40.4 & 2 & 2 \\
\hline
\end{tabular}




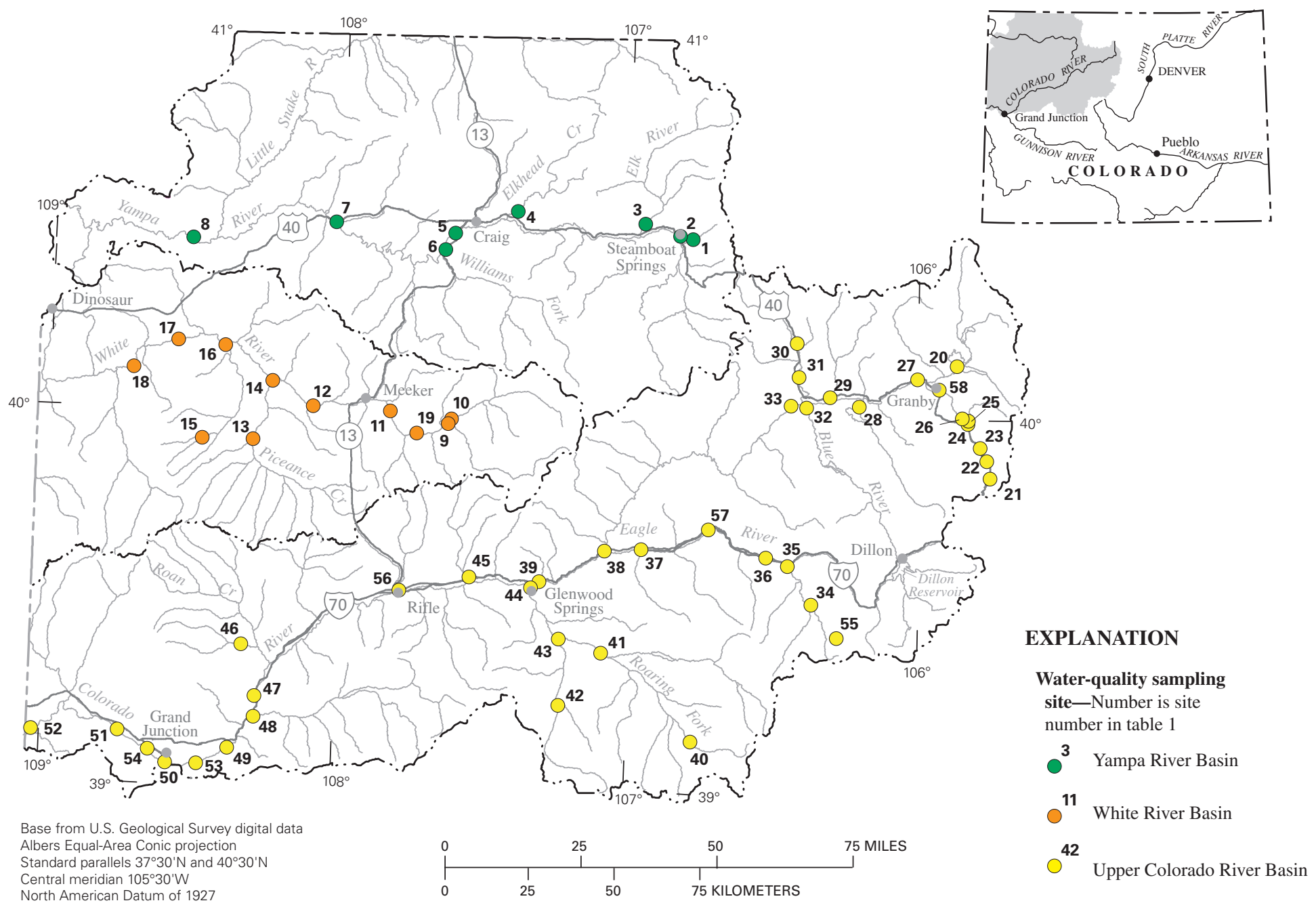

Figure 4. Sampling sites in the Yampa, White, and Upper Colorado River Basins. 
conditions. All other constituents with sufficient data (field properties, silica, dissolved and total-recoverable nutrients, dissolved iron, and dissolved manganese) indicate medial values or concentrations. Other dissolved metals and the only total-recoverable metals sampled (iron and manganese) had too few sites and associated percentiles for characterization.

Drought samples collected in the basin had water-quality exceedances for $\mathrm{pH}$ and concentrations of chloride, sulfate, nitrite nitrogen, dissolved iron, dissolved manganese, and dissolved selenium (table 12). $\mathrm{pH}$ exceeded the upper standard of 9.0 at sites 24, 25, 26, and 27. Out of 85 historical $\mathrm{pH}$ measurements at site 24, 28 showed $\mathrm{pH}$ exceedances; no historical exceedances were measured at site 25 (13 samples), site 26 ( 8 samples), or site 27 (6 samples); therefore, drought probably did not cause the exceedance at site 24 , but the data are insufficient to assess drought effect for sites 25-27 because of the potential diurnal fluctuation of $\mathrm{pH}$ at these sites and variable measurement time. The chloride standard of $250 \mathrm{mg} / \mathrm{L}$ was exceeded only at site 57 on September 4, 2002 (292 mg/L); six historical samples showed a maximum concentration of $120 \mathrm{mg} / \mathrm{L}$. Thus, historical samples and elevated average percentile for chloride in this basin indicate that drought may have caused the exceedance at site 57.

The sulfate water-supply standard of $250 \mathrm{mg} / \mathrm{L}$ was exceeded at sites 37 (256 and $310 \mathrm{mg} / \mathrm{L}), 46$ (1,910 mg/L), 50 (338 mg/L), and 53 (259 mg/L). Sites 51, 52, and 54 also had sulfate concentrations in excess of $250 \mathrm{mg} / \mathrm{L}$, but no sulfate standards have been established for these reaches. Historical samples showed sulfate exceedances for 2 out of 29 samples at site 37 (maximum $260 \mathrm{mg} / \mathrm{L}$ ); 15 out of 15 samples at site 46 (maximum 1,860 mg/L); zero out of 6 samples at site 50 (maximum $240 \mathrm{mg} / \mathrm{L}$ ); and zero out of 3 samples at site 53 (all in 1994; maximum $180 \mathrm{mg} / \mathrm{L}$ ). Historical samples and elevated sulfate concentration in this basin indicate that the greater exceedance at site 37 and the exceedance at site 50 were likely affected by drought; the exceedance at site 53 appears to have been affected by drought, but the data are insufficient to allow a more definitive conclusion.

Other drought samples collected in the basin for which samples exceeded water-quality standards were nitrite nitrogen, dissolved iron, dissolved manganese, and dissolved selenium. Nitrite nitrogen exceeded the chronic standard of $0.05 \mathrm{mg} / \mathrm{L}$ at site 57 (0.081 and $0.117 \mathrm{mg} / \mathrm{L})$. Six historical samples from 2000 through 2001 showed no exceedances; however, because the 2002 concentrations at this site exceeded the historical samples by 5 to 8 times, these exceedances likely are affected by drought.

Dissolved iron exceeded the chronic standard of $300 \mu \mathrm{g} / \mathrm{L}$ at site 26 (339 and $383 \mu \mathrm{g} / \mathrm{L})$; one of seven historical samples exceeded this standard (maximum $354 \mu \mathrm{g} / \mathrm{L}$ ). Medial concentrations of iron in this basin and these historical data strongly indicate that this exceedance is not affected by drought. Dissolved manganese exceeded the hardness-based chronic standard of $50 \mu \mathrm{g} / \mathrm{L}$ at sites $30(60$ and $115 \mu \mathrm{g} / \mathrm{L})$ and 31 ( $250 \mu \mathrm{g} / \mathrm{L}) ; 2$ of 26 historical samples from site 30 showed exceedances ( 68 and $83 \mu \mathrm{g} / \mathrm{L}$ ), and 3 of 19 samples from site
31 showed exceedances (maximum $342 \mu \mathrm{g} / \mathrm{L}$ ). Therefore, the exceedances at site 30 probably were affected by drought, but the exceedance at site 31 probably was not. Dissolved selenium exceeded the chronic standard of $4.6 \mu \mathrm{g} / \mathrm{L}$ at sites 51 $(6.3 \mu \mathrm{g} / \mathrm{L}), 52(8.8$ and $11 \mu \mathrm{g} / \mathrm{L})$, and $54(5.8 \mu \mathrm{g} / \mathrm{L})$; this standard was exceeded by 3 of 6 historical samples from site 51 (maximum $6 \mu \mathrm{g} / \mathrm{L}$ ), by 27 of 45 samples from site 52 (maximum $16 \mu \mathrm{g} / \mathrm{L}$ ), and by 3 of 6 samples from site 54 (maximum $6 \mu \mathrm{g} / \mathrm{L}$ ), indicating that the dissolved selenium exceedances at these sites were not affected by drought. In summary, the data indicate drought-affected exceedances only for chloride at site 57, sulfate at sites 37 and 50, nitrite at site 57, and dissolved manganese (chronic standard) at site 30 .

\section{Rio Grande Basin}

Drought samples were collected at four sites in the Rio Grande Basin (table 13 in Appendix, fig. 5). Average percentiles for properties and constituents (table 4) only indicate a medial percentile for water temperature. All other properties and constituents had too few data to calculate percentiles for basinwide characterization.

The only water-quality exceedance for this basin was for dissolved zinc at site $59(54 \mu \mathrm{g} / \mathrm{L})$ (table 13), which exceeded the acute and chronic standards of $49.3 \mu \mathrm{g} / \mathrm{L}$ and $49.7 \mu \mathrm{g} / \mathrm{L}$. Only two historical samples (1984) were analyzed for dissolved zinc, and they did not exceed these hardness-based standards. Therefore, these data are insufficient to indicate whether this dissolved zinc exceedance was affected by drought.

\section{Gunnison River Basin}

Drought samples were collected at 21 sites in the Gunnison River Basin (table 14 in Appendix, fig. 5). Average percentiles for properties and constituents (table 5) indicate elevated specific conductance and elevated concentrations of dissolved chloride, dissolved silica, and dissolved sulfate. All other constituents with sufficient data-including other field properties and most dissolved and total-recoverable nutrients-indicated medial values or concentrations. Fluoride, total-recoverable organic plus ammonia nitrogen, and dissolved metals had too few percentiles for calculation. Totalrecoverable metals were not sampled in this basin before 2002; and, therefore, no percentiles could be calculated.

Drought samples collected in the basin for which samples exceeded water-quality standards were sulfate, E. coli bacteria, total-recoverable iron, dissolved manganese, and dissolved selenium (table 14). Sulfate exceeded the water-supply standard of $480 \mathrm{mg} / \mathrm{L}$ at site 74 on September 24, 2002, and 12 of 55 historical samples (maximum $670 \mathrm{mg} / \mathrm{L}$ ) exceeded this standard. No sulfate standards have been established for sites 69 and 73, which showed concentrations from 608 to $743 \mathrm{mg} / \mathrm{L}$. E. coli bacteria exceeded the standard of $126 \mathrm{col} / 100 \mathrm{~mL}$ (colonies per 100 milliliters) at sites 69 


\section{Effect of Drought on Streamflow and Stream-Water Quality in Colorado, July through September 2002}

Table 3. Average percentiles and results for summer 2002 drought water-quality data from sites in the Upper Colorado River Basin, relative to historical data from July through September, 1978-2002.

[Q\%, percentile; Medial, average Q\% between 25 and 74.9; elevated, average Q\% greater than or equal to 75; ID, insufficient number of sites (fewer than 3) to determine result; text in bold type highlights elevated average percentiles]

\begin{tabular}{|c|c|c|c|}
\hline Property or constituent & Average $0 \%$ & Number of sites & Result \\
\hline Oxygen, dissolved & 33.3 & 13 & Medial \\
\hline $\mathrm{pH}$ & 63.6 & 15 & Medial \\
\hline Specific conductance & 90.0 & 24 & Elevated \\
\hline Temperature, water & 66.1 & 23 & Medial \\
\hline Chloride, dissolved & 95.0 & 14 & Elevated \\
\hline Fluoride, dissolved & 80.1 & 5 & Elevated $^{1}$ \\
\hline Silica, dissolved & 32.7 & 10 & Medial \\
\hline Sulfate, dissolved & 81.6 & 11 & Elevated \\
\hline Nitrogen, ammonia, dissolved & 37.1 & 10 & Medial \\
\hline Nitrogen, organic plus ammonia, dissolved & 68.0 & 5 & Medial \\
\hline Nitrogen, organic plus ammonia, total recoverable & 71.2 & 5 & Medial \\
\hline Nitrogen, nitrite plus nitrate, dissolved & 55.8 & 11 & Medial \\
\hline Nitrogen, nitrite, dissolved & 48.9 & 10 & Medial \\
\hline Phosphorus, dissolved & 56.7 & 10 & Medial \\
\hline Phosphorus, total recoverable & 63.5 & 6 & Medial \\
\hline Phosphorus, orthophosphate, dissolved & 48.1 & 10 & Medial \\
\hline Carbon, organic, dissolved & 87.5 & 1 & ID \\
\hline Cadmium, dissolved & 44.5 & 2 & ID \\
\hline Chromium, dissolved & 50.0 & 1 & ID \\
\hline Copper, dissolved & 66.4 & 2 & ID \\
\hline Iron, dissolved & 51.6 & 5 & Medial \\
\hline Iron, total recoverable & 70.0 & 2 & ID \\
\hline Lead, dissolved & 47.7 & 2 & ID \\
\hline Manganese, dissolved & 73.9 & 5 & Medial \\
\hline Manganese, total recoverable & 93.7 & 2 & ID \\
\hline Molybdenum, dissolved & 63.6 & 1 & ID \\
\hline Nickel, dissolved & 47.7 & 1 & ID \\
\hline Selenium, dissolved & 95.8 & 1 & ID \\
\hline Silver, dissolved & 46.7 & 2 & ID \\
\hline Zinc, dissolved & 30.4 & 2 & ID \\
\hline
\end{tabular}

${ }^{1}$ Tentative result because of possible range of Q\% for two sites (table 3); minimum values in ranges result in average Q\% of 71.7 (medial result). 


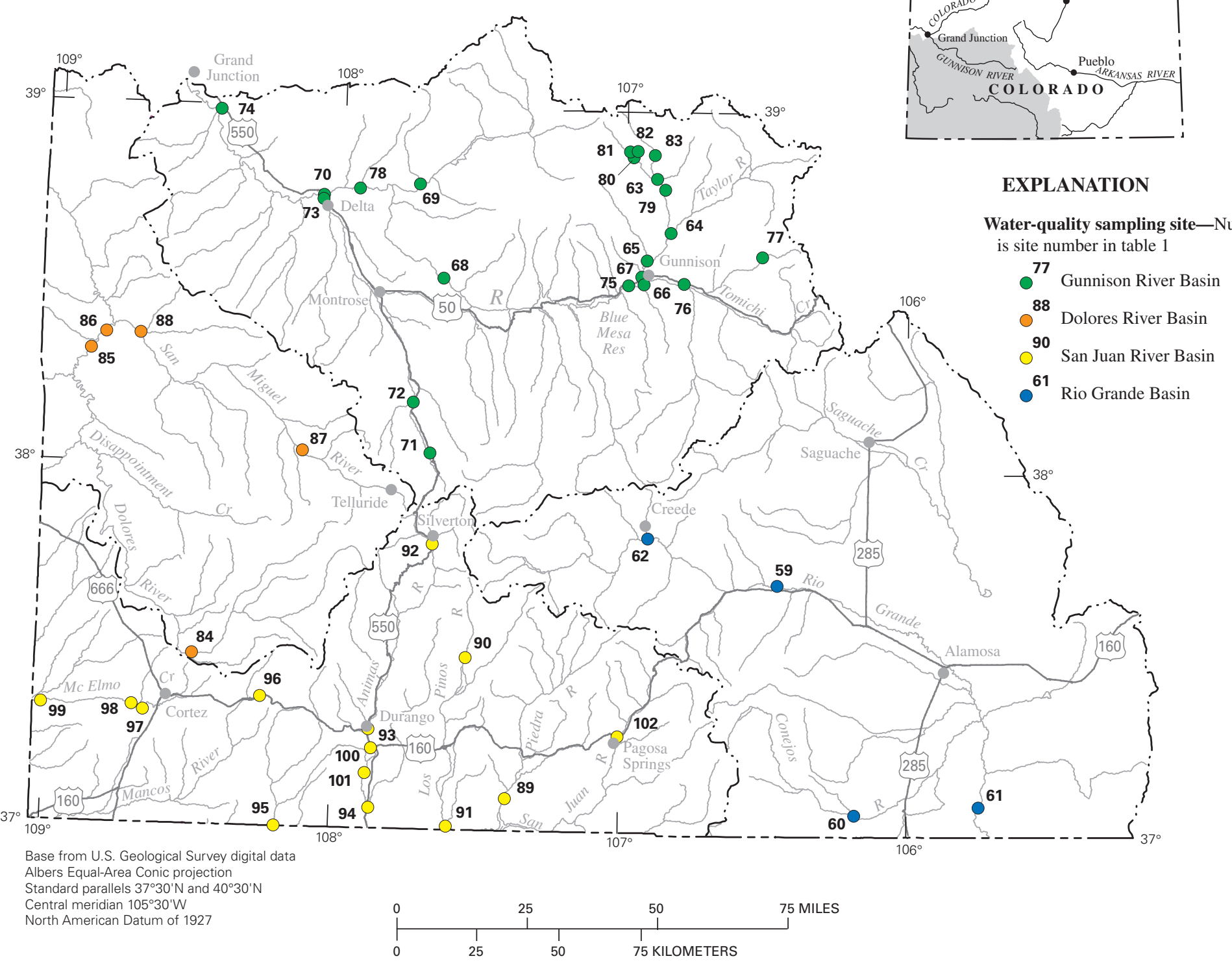

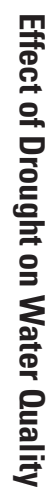

Figure 5. Sampling sites in the Rio Grande, Gunnison, Dolores, and San Juan River Basins. 
Table 4. Average percentiles and results for summer 2002 drought water-quality data from sites in the Rio Grande Basin, relative to historical data from July through September, 1978-2002.

[Q\%, percentile; medial, average Q\% between 25 and 74.9; elevated, average Q\% greater than or equal to 75; depressed, average Q\% less than 25; ID, insufficient number of sites (fewer than 3 ) to determine result]

\begin{tabular}{llll}
\hline & Average $\mathbf{0} \%$ & Number of sites & Result \\
\hline Oxygen, dissolved & 38.2 & 1 & ID \\
pH & 94.6 & 1 & ID \\
Specific conductance & 62.9 & 1 & ID \\
Temperature, water & 26.6 & 3 & Medial \\
Chloride, dissolved & 97.1 & 1 & ID \\
Fluoride, dissolved & 97.1 & 1 & ID \\
Silica, dissolved & 2.9 & 1 & ID \\
Sulfate dissolved & 68.6 & 1 & ID \\
Nitrogen, ammonia, dissolved & 40.3 & 1 & ID \\
Nitrogen, organic plus ammonia, dissolved & 65.4 & 1 & ID \\
Nitrogen, organic plus ammonia, total recoverable & 56.8 & 1 & ID \\
Nitrogen, nitrite plus nitrate, dissolved & 45.5 & 1 & ID \\
Nitrogen, nitrite, dissolved & 50.0 & 1 & ID \\
Phosphorus, dissolved & 10.8 & 1 & ID \\
Phosphorus, total recoverable & 4.1 & 1 & ID \\
Phosphorus, orthophosphate, dissolved & 10.0 & 1 & ID \\
Bacteria, fecal coliform & 40.9 & 1 & ID \\
Iron, dissolved & 29.6 & 1 & ID \\
Manganese, dissolved & 86.0 & 1 & ID \\
\hline
\end{tabular}

(580 and $210 \mathrm{col} / 100 \mathrm{~mL}), 72(210 \mathrm{col} / 100 \mathrm{~mL}), 73(280$ and $210 \mathrm{col} / 100 \mathrm{~mL})$, and $82(800 \mathrm{col} / 100 \mathrm{~mL})$; however, the effect of drought cannot be determined for these sites because of insufficient historical data. The chronic standard of $1,500 \mu \mathrm{g} / \mathrm{L}$ for total-recoverable iron was exceeded at sites $71(4,050 \mu \mathrm{g} / \mathrm{L})$ and $72(2,640 \mu \mathrm{g} / \mathrm{L})$; two of two 2001 historical samples for site 71 exceeded this standard (maximum 6,520 $\mu \mathrm{g} / \mathrm{L}$ ), and two of three historical samples for site 72 exceeded this standard (maximum 19,900 $\mu \mathrm{g} / \mathrm{L}$ ). The hardness-based, chronic standard for dissolved manganese was exceeded at site 67 on August 7, 2002 (57 $\mu \mathrm{g} / \mathrm{L})$; zero of nine historical samples exceeded this standard (maximum $35 \mu \mathrm{g} / \mathrm{L}$ ). The hardness-based chronic dissolved selenium standard of $4.6 \mu \mathrm{g} / \mathrm{L}$ was exceeded at site 70 on August 8 $(5.0 \mu \mathrm{g} / \mathrm{L})$ and September 11, $2002(7 \mu \mathrm{g} / \mathrm{L})$. The fixed chronic standard of $6.0 \mu \mathrm{g} / \mathrm{L}$ was exceeded at site 74 on September 24, $2002(8 \mu \mathrm{g} / \mathrm{L})$. For site 70, 6 of 13 historical samples for the seasonal period of record and 31 of 50 samples for site 74 exceeded these standards (maximum 8 and $25 \mu \mathrm{g} / \mathrm{L}$, respectively). In summary, the only exceedance that was affected by drought was dissolved manganese (chronic standard) at site 67. The data indicate that the exceedances for sulfate, total iron, and dissolved selenium were not affected by drought.

\section{Dolores River Basin}

Drought samples were collected at five sites in the Dolores River Basin (table 15 in Appendix, fig. 5). Average percentiles (table 6) indicate that specific conductance was elevated and that water temperature had medial values. $\mathrm{pH}$, chloride, fluoride, silica, and sulfate had too few sites for basin characterization, although all had high average percentiles, indicating elevated levels for the available data. 
Table 5. Average percentiles and results for summer 2002 drought water-quality data from sites in the Gunnison River Basin relative to historical data from July through September, 1978-2002.

[Q\%, percentile; Medial, average Q\% between 25 and 74.9; elevated, average Q\% greater than or equal to 75; ID, insufficient number of sites (fewer than 3) to determine result; text in bold type highlights elevated average percentiles]

\begin{tabular}{|c|c|c|c|}
\hline Property or constituent & Average $\mathbf{0} \%$ & Number of sites & Result \\
\hline Oxygen, dissolved & 35.5 & 4 & Medial \\
\hline $\mathrm{pH}$ & 70.8 & 5 & Medial \\
\hline Specific conductance & 82.1 & 9 & Elevated \\
\hline Temperature, water & 47.5 & 9 & Medial \\
\hline Chloride, dissolved & 79.0 & 3 & Elevated \\
\hline Fluoride, dissolved & 77.8 & 1 & ID \\
\hline Silica, dissolved & 75.9 & 3 & Elevated $^{1}$ \\
\hline Sulfate, dissolved & 84.4 & 3 & Elevated \\
\hline Nitrogen, ammonia, dissolved & 35.8 & 3 & Medial \\
\hline Nitrogen, organic plus ammonia, dissolved & 48.6 & 3 & Medial \\
\hline Nitrogen, organic plus ammonia, total recoverable & 50.0 & 1 & ID \\
\hline Nitrogen, nitrite plus nitrate, dissolved & 44.0 & 3 & Medial \\
\hline Nitrogen, nitrite, dissolved & 41.9 & 3 & Medial \\
\hline Phosphorus, dissolved & 49.9 & 3 & Medial \\
\hline Phosphorus, total recoverable & 64.3 & 3 & Medial \\
\hline Phosphorus, orthophosphate, dissolved & 36.8 & 3 & Medial \\
\hline Cadmium, dissolved & 48.1 & 1 & ID \\
\hline Copper, dissolved & 76.9 & 1 & ID \\
\hline Iron, dissolved & 27.9 & 2 & ID \\
\hline Lead, dissolved & 46.2 & 1 & ID \\
\hline Manganese, dissolved & 25.6 & 1 & ID \\
\hline Selenium, dissolved & 68.3 & 2 & ID \\
\hline Silver, dissolved & 93.3 & 1 & ID \\
\hline Zinc, dissolved & 42.3 & 1 & ID \\
\hline
\end{tabular}

${ }^{1}$ Tentative result because of possible range of Q\% for one site (table 5); minimum value in range results in average Q\% of 74.7 (medial result).

E. coli bacteria was the only constituent showing a waterquality exceedance in this basin: site 87 exceeded the standard of $126 \mathrm{col} / 100 \mathrm{~mL}$ on August 6, $2002(210 \mathrm{col} / 100 \mathrm{~mL})$. However, because there are no historical samples for this constituent at this site, no conclusion can be made about the effect of drought on this exceedance.

\section{San Juan River Basin}

Drought samples were collected at 14 sites in the San Juan River Basin (table 16 in Appendix, fig. 5). Average percentiles (table 7) indicate that specific conductance and concentrations of chloride, fluoride, and sulfate were elevated and likely were affected by drought. $\mathrm{pH}$ and water temperature values and silica concentrations indicated medial values. Dissolved and total-recoverable nutrients had too few sites for characterization. Metals were sampled too infrequently to allow calculation of reliable percentiles.

Drought samples exceeded water-quality standards for sulfate, E. coli bacteria, and dissolved iron (table 16). The sulfate standard of $250 \mathrm{mg} / \mathrm{L}$ was exceeded at site 102 on September 6, 2002 (419 mg/L); no historical samples exist for this constituent at this site. The standard of $126 \mathrm{col} / 100 \mathrm{~mL}$ for E. coli bacteria was exceeded at site 94 on August 2, 2002 (140 col/100 mL), at site 95 on September 4, 2002 
Table 6. Average percentiles and results for summer 2002 drought water-quality data from sites in the Dolores River Basin relative to historical data from July through September, 1978-2002.

[Q\%, percentile; medial, average Q\% between 25 and 74.9; elevated, average $\mathrm{Q} \%$ greater than or equal to 75; ID, insufficient number of sites (fewer than 3) to determine result; text in bold type highlights elevated average percentiles]

\begin{tabular}{lccl}
\hline \multicolumn{1}{c}{ Property or constituent } & Average Q\% & $\begin{array}{c}\text { Number of } \\
\text { sites }\end{array}$ & Result \\
\hline $\mathrm{pH}$ & 88.8 & 2 & ID \\
Specific conductance & 85.7 & 5 & Elevated \\
Temperature, water & 36.8 & 5 & Medial \\
Chloride, dissolved & 95.0 & 2 & ID \\
Fluoride, dissolved & 82.0 & 2 & ID \\
Silica, dissolved & 95.0 & 2 & ID \\
Sulfate dissolved & 86.5 & 2 & ID \\
\hline
\end{tabular}

Table 7. Average percentiles and results for summer 2002 drought water-quality data from sites in the San Juan River Basin, relative to historical data from July through September, 1978-2002.

[Q\%, percentile; medial, average Q\% between 25 and 50; elevated, average $\mathrm{Q} \%$ greater than or equal to 75; ID, insufficient number of sites (fewer than 3) to determine result; text in bold type highlights elevated average percentiles]

\begin{tabular}{lccl}
\hline Property or constituent & Average Q\% & $\begin{array}{c}\text { Number of } \\
\text { sites }\end{array}$ & Result \\
\hline $\mathrm{pH}$ & 43.8 & 4 & Medial \\
Specific conductance & 95.2 & 6 & Elevated \\
Temperature, water & 53.3 & 7 & Medial \\
$\begin{array}{l}\text { Chloride, dissolved } \\
\text { Fluoride, dissolved }\end{array}$ & 86.0 & 4 & Elevated \\
$\begin{array}{l}\text { Silica, dissolved } \\
\text { Sulfate, dissolved }\end{array}$ & 92.9 & 3 & Elevated \\
$\begin{array}{l}\text { Nitrogen, ammonia, } \\
\text { dissolved }\end{array}$ & 88.2 & 6 & Medial \\
$\begin{array}{l}\text { Nitrogen, organic plus } \\
\text { ammonia, total } \\
\text { recoverable }\end{array}$ & 30.6 & 1 & Elevated \\
$\begin{array}{l}\text { Nitrogen, nitrite plus } \\
\text { nitrate, dissolved }\end{array}$ & 94.7 & 1 & ID \\
$\begin{array}{l}\text { Phosphorus, dissolved } \\
\text { Phosphorus, total } \\
\text { recoverable }\end{array}$ & 34.8 & 1 & ID \\
\hline & 26.1 & 1 & ID \\
\hline
\end{tabular}

(380 col/100 mL), at site 97 (220 and $260 \mathrm{col} / 100 \mathrm{~mL}$ ) August 7 and September 18, 2002, at site 98 on September 10, 2002 (540 col/100 mL), and at site 99 on August 7 and September 10, 2002 (220 and 1,100 col/100 mL); no historical data exist for this constituent at any of these sites, so the effect of drought cannot be assessed. The monthly dependent, chronic standard of 1,286 $\mu \mathrm{g} / \mathrm{L}$ for dissolved iron at site 92 was exceeded on July 31, 2002, with a concentration of $1,900 \mu \mathrm{g} / \mathrm{L}$. Seven historical samples show zero exceedances for dissolved iron (maximum 1,200 $\mu \mathrm{g} / \mathrm{L}$ ), although the maximum historical value is more than half of the drought exceedance. In summary, it is unknown if any of the standard exceedances were affected by drought.

\section{South Platte River Basin}

Drought samples were collected at 23 sites in the South Platte River Basin (table 17 in Appendix, fig. 6). Average percentiles (table 8) indicated medial values and concentrations for field properties, most dissolved and total-recoverable nutrients, dissolved organic carbon, and some dissolved trace metals (copper, iron, manganese, and silver). Too few sites had reliable percentiles to allow characterization of dissolved organic plus ammonia nitrogen, total-recoverable organic carbon, total-recoverable iron, dissolved lead, and dissolved nickel.

Drought samples exceeded water-quality standards for $\mathrm{pH}$, dissolved ammonia, dissolved nitrite, dissolved copper, and dissolved manganese (table 17). The upper standard of 9.0 SU for $\mathrm{pH}$ was exceeded at sites 108 (9.1) and 109 (9.1) on September 9, 2002, and at site 119 (9.3) on September 10, 2002; historical samples for site 108 (maximum $\mathrm{pH}$ of 8.2) show 1 out of $47 \mathrm{pH}$ measurements was above the acceptable range, but that measurement was below the 6.5 standard; zero of 6 historical samples for site 109 exceeded the $\mathrm{pH}$ standard (maximum 7.8); 2 of 58 historical samples for site 119 (maximum 8.5 ) had $\mathrm{pH}$ less than the 6.5 standard, but none exceeded the upper standard. Therefore, although all three of the upper-standard $\mathrm{pH}$ exceedances appear to be affected by drought, the problem of diurnal variation in $\mathrm{pH}$ and variable measurement times (Chafin, 2002) preclude a valid basis for comparing drought and historical measurements, so no causative conclusion can be made.

The reach-specific chronic standard for dissolved ammonia was exceeded in drought samples at sites 104, 106, 108, 109, 116, and 122 (table 17). Sites 104, 106, 108, and 122 also exceeded their reach-specific chronic standards for ammonia more than two-thirds of the time during the seasonal period of record, and site 116 exceeded the standard about one-half of the time. The effect of drought on site 109 is uncertain because one ammonia concentration in six exceeded the standard during the seasonal period of record. These results indicate that the chronic ammonia exceedances in the South Platte River Basin during 2002 were not directly affected by drought. 


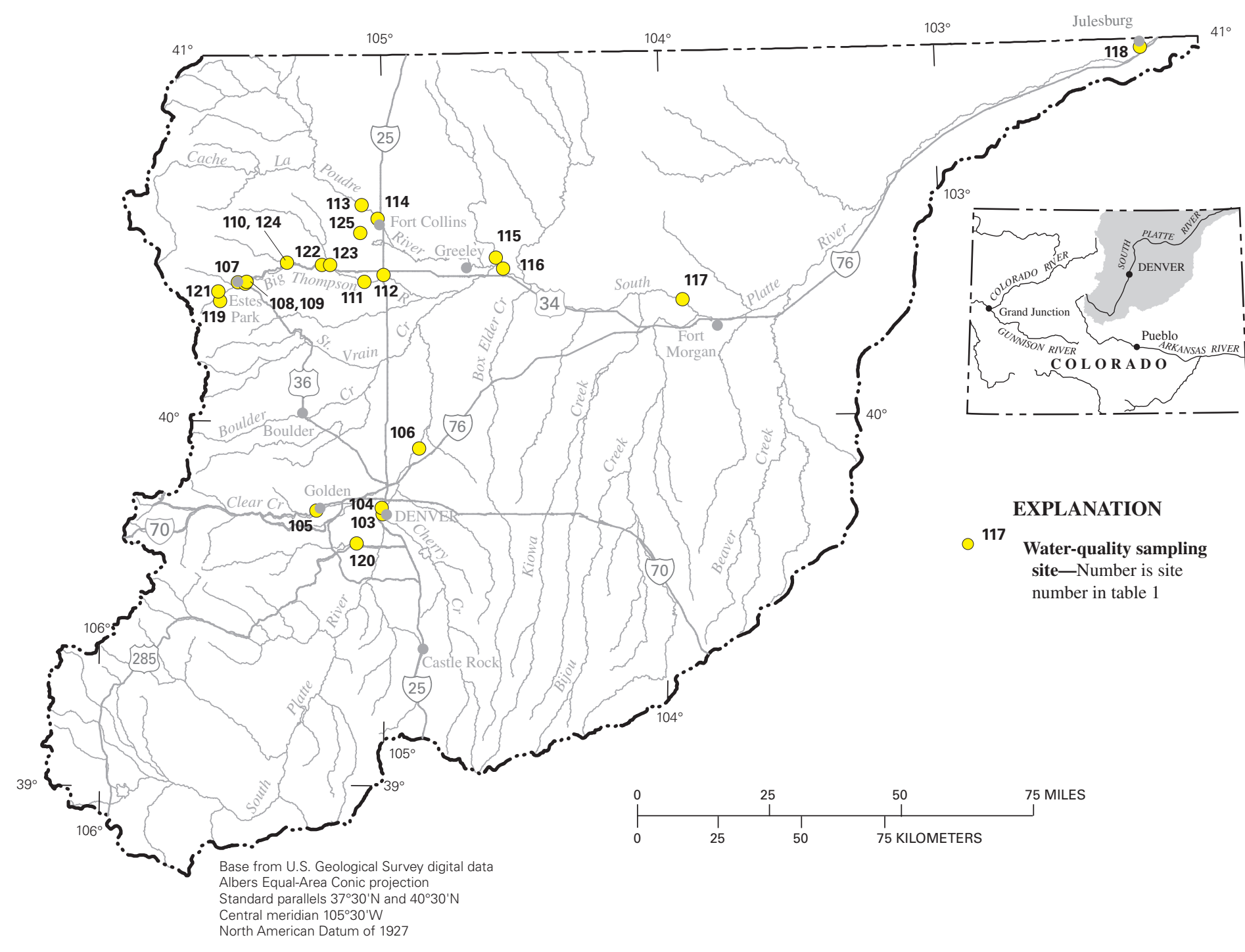

Figure 6. Sampling sites in the South Platte River Basin. 
Table 8. Average percentiles and results for summer 2002 drought water-quality data from sites in the South Platte River Basin relative to historical data from July through September, 1978-2002.

[Q\%, percentile; Medial, average Q\% between 25 and 74.9; elevated, average Q\% greater than or equal to 75; ID, insufficient number of sites (fewer than 3) to determine result]

\begin{tabular}{|c|c|c|c|}
\hline Property or constituent & Average $0 \%$ & Number of sites & Result \\
\hline Oxygen, dissolved & 43.7 & 12 & Medial \\
\hline $\mathrm{pH}$ & 53.0 & 12 & Medial \\
\hline Specific conductance & 70.9 & 13 & Medial \\
\hline Temperature, water & 56.3 & 15 & Medial \\
\hline Chloride, dissolved & 74.9 & 9 & Medial \\
\hline Silica, dissolved & 66.7 & 7 & Medial \\
\hline Sulfate, dissolved & 59.3 & 9 & Medial \\
\hline Nitrogen, ammonia, dissolved & 43.7 & 11 & Medial \\
\hline Nitrogen, organic plus ammonia, dissolved & 83.9 & 1 & ID \\
\hline Nitrogen, organic plus ammonia, total recoverable & 35.2 & 6 & Medial \\
\hline Nitrogen, nitrite plus nitrate, dissolved & 68.2 & 12 & Medial \\
\hline Nitrogen, nitrite, dissolved & 60.2 & 7 & Medial \\
\hline Phosphorus, dissolved & 46.2 & 8 & Medial \\
\hline Phosphorus, total recoverable & 46.0 & 9 & Medial \\
\hline Phosphorus, orthophosphate, dissolved & 53.4 & 10 & Medial \\
\hline Carbon, organic, dissolved & 58.8 & 3 & Medial \\
\hline Carbon, organic, total recoverable & 25.9 & 1 & ID \\
\hline Copper, dissolved & 62.3 & 5 & Medial \\
\hline Iron, dissolved & 33.4 & 10 & Medial \\
\hline Iron, total recoverable & 42.0 & 2 & ID \\
\hline Lead, dissolved & 48.5 & 1 & ID \\
\hline Manganese, dissolved & 63.5 & 6 & Medial \\
\hline Nickel, dissolved & 50.0 & 1 & ID \\
\hline Silver, dissolved & 45.9 & 4 & Medial \\
\hline
\end{tabular}


Dissolved nitrite nitrogen exceeded the reach-specific standard of 1.0 at site 106 on August 13, 2002 (1.21 mg/L); one of 22 historical samples exceeded the standard (maximum $1.1 \mathrm{mg} / \mathrm{L})$, making it likely that the drought-sample exceedance was affected by drought. Dissolved copper exceeded the hardness-based acute and chronic standards at site 110 on August 6, 2002 (1.9 $\mu \mathrm{g} / \mathrm{L})$; six historical samples showed no exceedances, but four of these samples had reported concentrations of less than $2 \mu \mathrm{g} / \mathrm{L}$; therefore, no conclusion can be reached about the effect of drought on the dissolved copper exceedances at site 110. The drinking-water standard for dissolved manganese was exceeded at site 120 on August 22 $(69 \mu \mathrm{g} / \mathrm{L})$ and September 16, $2002(63 \mu \mathrm{g} / \mathrm{L})$, but there are no historical data for manganese for this site, so the effect of drought cannot be determined.

In summary, the 2002 drought appears to have caused an exceedance for nitrite at one site when compared to the seasonal period of record for all sites.

\section{Arkansas River Basin}

Drought samples were collected at 23 sites in the Arkansas River Basin (table 18 in Appendix, fig. 7). Percentiles for specific conductance (table 9) indicated elevated values for specific conductance and sulfate concentration; concentrations of total-recoverable iron and manganese (tentative) were depressed. Dissolved oxygen, $\mathrm{pH}$, most dissolved and totalrecoverable nutrients, fecal-coliform bacteria, dissolved cadmium, iron, lead, manganese, and zinc, and total-recoverable cadmium, chromium, and zinc indicated medial values and concentrations. Too few sites had reliable percentiles for totalrecoverable organic plus ammonia nitrogen; dissolved arsenic, chromium, copper, nickel, selenium, and silver; and totalrecoverable copper, mercury, and nickel.

Drought samples indicate water-quality exceedances for sulfate, E. coli bacteria, fecal-coliform bacteria, total-recoverable iron, dissolved manganese, and dissolved selenium (table 18). Sulfate exceeded the reach-specific standard of $1,078 \mu \mathrm{g} / \mathrm{L}$ at sites $144(1,080 \mu \mathrm{g} / \mathrm{L})$ and 145 $(1,590 \mu \mathrm{g} / \mathrm{L})$; historical samples showed no exceedances in 5 samples at site 144 (maximum $940 \mu \mathrm{g} / \mathrm{L}$ ) and 1 exceedance in 10 samples at site 145 (maximum 1,440 $\mu \mathrm{g} / \mathrm{L}$ ). Historicalsample exceedances are inconclusive indicators of drought effect on sulfate exceedance at site 144; however, elevated average percentile for sulfate in this basin indicates that drought could have caused some exceedances of water-quality standards and probably did so for the drought samples collected at sites 144 and 145 .

Most sites (16 of 23) had samples with E. coli colonies that exceeded the standard of $126 \mathrm{col} / 100 \mathrm{~mL}$ (maximum 3,900 colonies per $100 \mathrm{~mL}$ ); however, all historical samples were collected in 2001 and no more than three samples were collected at each site. Therefore, no conclusion can be made about the effect of drought on these E. coli exceedances. Most sites (14 of 23) also had drought samples with fecal-coliform colonies that exceeded the general standard of $200 \mathrm{col} / 100 \mathrm{~mL}$. More than one-half of the samples collected at these sites historically had exceedances that generally were substantially larger than drought samples (except for site 143, which had no historical data, and for site 131, which had one exceedance in seven historical samples and for which drought-sample colonies substantially exceeded the maximum historical count by 3 to 25 times). Therefore, exceedances of water-quality standards for fecal coliform for 14 of 18 samples collected in this basin probably were not affected by drought conditions.

The reach-specific chronic standard of 2,690 $\mu \mathrm{g} / \mathrm{L}$ for total-recoverable iron was exceeded at site $140(3,840 \mu \mathrm{g} / \mathrm{L})$; historical data for the seasonal period of record show 11 exceedances in 18 samples (maximum 54,100 $\mu \mathrm{g} / \mathrm{L}$ ), indicating that the drought-sample exceedance was not affected by drought conditions - a conclusion supported by the depressed average percentile for total-recoverable iron.

The chronic dissolved manganese standard of $50 \mu \mathrm{g} / \mathrm{L}$ was exceeded at sites $130(162 \mu \mathrm{g} / \mathrm{L}), 131$ (141 $\mu \mathrm{g} / \mathrm{L}), 133$ (52 $\mu \mathrm{g} / \mathrm{L}), 141$ (71 $\mu \mathrm{g} / \mathrm{L}), 143$ (115 $\mu \mathrm{g} / \mathrm{L})$, and 145 (133 $\mu \mathrm{g} / \mathrm{L})$; historical samples show one exceedance out of 14 samples at site 130 (maximum $80 \mu \mathrm{g} / \mathrm{L})$, zero out of 12 at site 131 (maximum $30 \mu \mathrm{g} / \mathrm{L}$ ); 23 out of 45 at site 133 (maximum $150 \mu \mathrm{g} / \mathrm{L})$; zero out of 9 at site 141 ; and zero out of 10 at site 145 (maximum $30 \mu \mathrm{g} / \mathrm{L}$ ). There are no historical data for site 143. These data indicate that the drought-sample exceedance at site 133 was not affected by drought conditions, whereas those at sites $130,131,141$, and 145 probably were, and those at site 143 are indeterminate.

The acute and chronic standards for dissolved selenium were exceeded at site $131(28 \mu \mathrm{g} / \mathrm{L})$; the variable chronic standard also was exceeded at sites $135(15 \mu \mathrm{g} / \mathrm{L})$ and 136 $(14 \mu \mathrm{g} / \mathrm{L})$. Historical data indicate no exceedances for site 131 (11 samples; maximum $6 \mu \mathrm{g} / \mathrm{L}$ ) and 135 (4 samples; maximum $9 \mu \mathrm{g} / \mathrm{L})$; site 136 had one exceedance out of 25 samples (maximum $7 \mu \mathrm{g} / \mathrm{L}$ ). These data indicate that exceedances at sites 131 and 136 probably were affected by drought, but the effect at site 135 is uncertain.

In summary, drought conditions probably contributed to exceedances of water-quality standards for sulfate at site 144 and site 145 . Fourteen of 18 exceedances in fecal-coliform colonies probably were not affected by drought, but two exceedances (site 131) possibly were affected by drought and two (site 143) could not be assessed for cause. The chronic standard for total-recoverable iron at site 140 probably was not affected by drought. The chronic dissolved manganese exceedance at site 133 probably was not affected by drought conditions, whereas those at sites 130, 131, 141, and 145 probably were, and the exceedance at site 143 cannot be assessed for cause. Exceedances in dissolved selenium at sites 131 (acute and chronic) and 136 (chronic) probably were affected by drought, but the chronic exceedance at site 135 could not be characterized. 


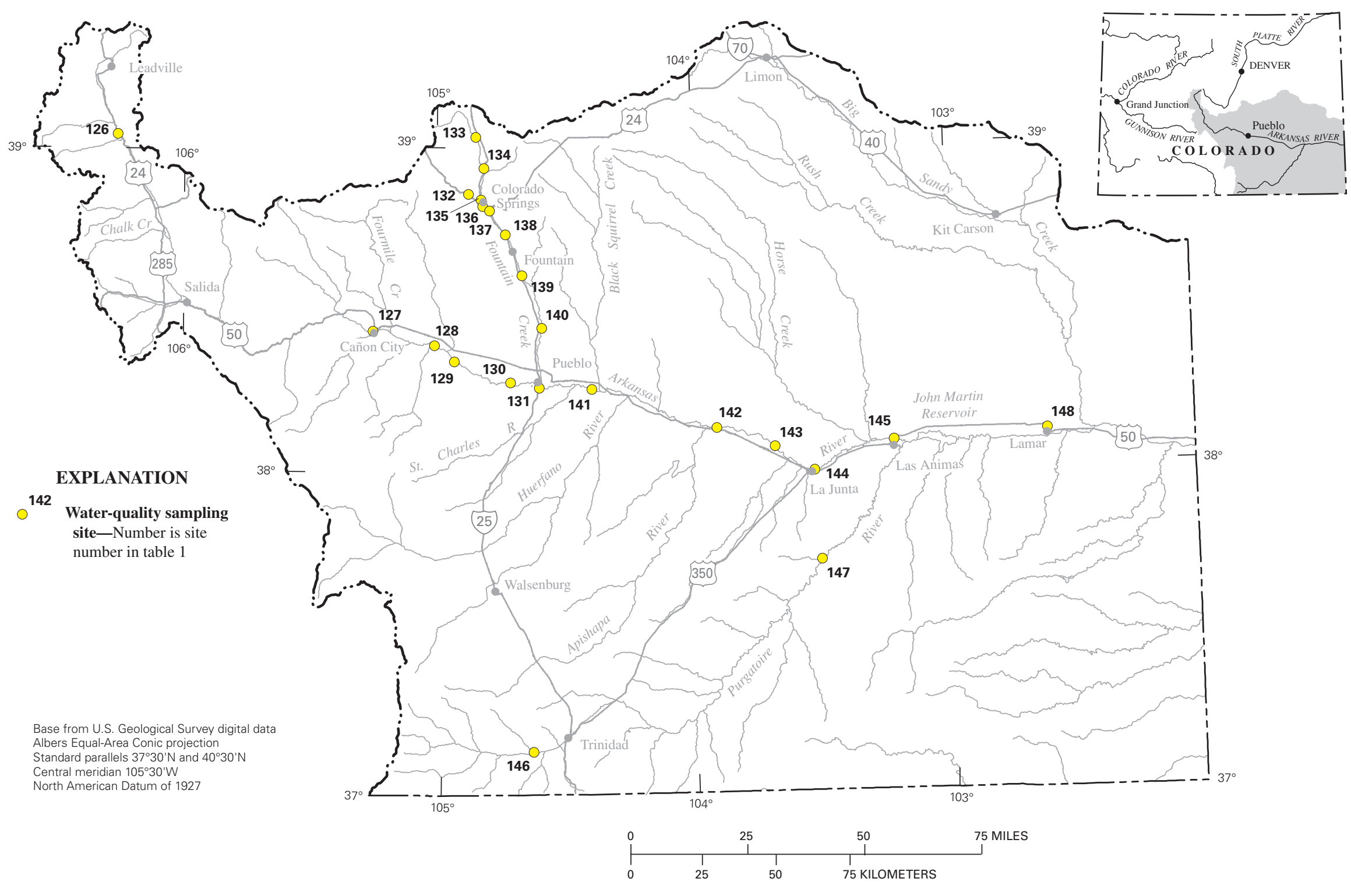

Figure 7. Sampling sites in the Arkansas River Basin. 
Table 9. Average percentiles and results for summer 2002 drought water-quality data from sites in the Arkansas River Basin relative to historical data from July through September, 1978-2002.

[Q\%, percentile; medial, average Q\% between 25 and 74.9; elevated, average Q\% greater than or equal to 75; depressed, average Q\% less than 25; ID, insufficient number of sites (fewer than 3) to determine result; text in bold type highlights depressed or elevated average percentiles]

\begin{tabular}{|c|c|c|c|}
\hline Property or constituent & Average $0 \%$ & Number of sites & Result \\
\hline Oxygen, dissolved & 55.5 & 10 & Medial \\
\hline $\mathrm{pH}$ & 64.9 & 11 & Medial \\
\hline Specific conductance & 81.2 & 20 & Elevated \\
\hline Temperature, water & 63.8 & 19 & Medial \\
\hline Fluoride, dissolved & 49.6 & 6 & Medial \\
\hline Sulfate dissolved & 86.7 & 6 & Elevated \\
\hline Nitrogen, ammonia, dissolved & 25.1 & 5 & Medial \\
\hline Nitrogen, organic plus ammonia, total recoverable & 46.9 & 1 & ID \\
\hline Nitrogen, nitrite plus nitrate, dissolved & 54.7 & 6 & Medial \\
\hline Phosphorus, total recoverable & 47.9 & 4 & Medial \\
\hline Phosphorus, orthophosphate, dissolved & 51.4 & 4 & Medial \\
\hline Bacteria, fecal coliform & 48.7 & 6 & Medial \\
\hline Arsenic, dissolved & 31.0 & 1 & ID \\
\hline Cadmium, dissolved & 48.2 & 4 & Medial \\
\hline Cadmium, total recoverable & 47.6 & 4 & Medial \\
\hline Chromium, dissolved & 95.7 & 1 & ID \\
\hline Chromium, total recoverable & 51.8 & 5 & Medial \\
\hline Copper, dissolved & 46.4 & 1 & ID \\
\hline Copper, total recoverable & 62.8 & 1 & ID \\
\hline Iron, dissolved & 25.6 & 7 & Medial \\
\hline Iron, total recoverable & 20.4 & 6 & Depressed \\
\hline Lead, dissolved & 46.8 & 5 & Medial \\
\hline Manganese, dissolved & 42.6 & 5 & Medial \\
\hline Manganese, total recoverable & 24.4 & 6 & Depressed $^{1}$ \\
\hline Mercury, total recoverable & 47.6 & 1 & ID \\
\hline Nickel, dissolved & 79.3 & 1 & ID \\
\hline Nickel, total recoverable & 89.3 & 1 & ID \\
\hline Selenium, dissolved & 87.7 & 2 & ID \\
\hline Silver, dissolved & 48.3 & 1 & ID \\
\hline Zinc, dissolved & 59.6 & 6 & Medial \\
\hline Zinc, total recoverable & 28.3 & 6 & Medial \\
\hline
\end{tabular}

\footnotetext{
${ }^{1}$ Tentative result because of possible range of Q\% for four sites (table 18 in Appendix); maximum values in ranges result in average Q\% of 25.6 (medial result).
} 


\section{Statewide Overview}

A summary of characterization of percentiles for all drought samples collected in Colorado during summer 2002 (table 10) indicates that few measured properties or constituents were elevated or depressed in concentration or value on a widespread basis. Specific conductance was elevated in five of the seven basins (or basin groups) that had sufficient data for characterization (that is, those not showing "ID" or "--" in table 10); although specific conductance was medial in the Yampa and White and the South Platte River Basins, large average percentiles of 73.7 (table 2) and 70.9 (table 8 ) indicate that specific conductance likely was affected by drought in those basins. For the 95 sites for which a specific-conductance percentile could be calculated, the average percentile of 81.8 indicates substantial elevation on a statewide basis. Similarly, chloride concentrations were elevated in three of five basins with sufficient data; although chloride concentration was medial in the Yampa and White and the South Platte River Basins, above-median average percentiles of 70.2 (table 2) and 74.9 (table 8) indicate that chloride concentration generally was affected by drought in those basins, too (average percentile of 83.7 for 43 sites). Sulfate concentration was elevated in four of six basins with sufficient data; the Yampa and White and the South Platte River Basins had medial concentrations; an above-median average percentile of 69.4 (table 2) in the Yampa and White River Basin group indicates some increase in sulfate concentration there; sulfate concentration was slightly increased in the South Platte River Basin (average percentile equals 59.3; table 8). All basins that exhibited elevated concentrations of chloride and sulfate also exhibited elevated specific conductance. The widespread elevation of specific conductance and concentrations of chloride and sulfate indicates that salinity generally was affected by drought in Colorado streams during July through September 2002, undoubtedly because streamflow at most sites was dominated by base flow of ground water, which usually has considerable salinity compared to runoff from precipitation (Winter and others, 1999).

Fluoride concentrations were elevated in two (tentative in Upper Colorado; San Juan) of four basins with sufficient data to characterize, and two of the other basins (Yampa/White and Arkansas) had medial concentrations. Both basins with elevated concentrations of fluoride had elevated specific conductance and concentrations of chloride and sulfate, which is consistent with a ground-water source for fluoride; however, the Arkansas River Basin had medial concentrations of fluoride despite having elevated specific conductance and sulfate concentrations. The reasons for this apparent contradiction cannot be readily determined but may be the result of basin geology, an artifact of sampling-site distribution, or both.

Dissolved and total-recoverable nutrients (nitrogen and phosphorus) showed medial (and near medial) concentrations in the five basins with sufficient data to characterize. However, dissolved ammonia concentrations were below median concentrations (average percentile less than 50) in the five basins with sufficient data to characterize; the Arkansas River Basin nearly had depressed average percentile (25.1; table 9). The widespread below-median concentration of dissolved ammonia indicates that drought affected the ammonia concentrations. The overall pronounced consistency of medial concentrations of nutrients probably is the result of nutrient uptake by aquatic plants upstream from most sites that were sampled-which tends to keep nutrient concentrations low during summer, low-flow conditions (Sprague and others, 2002). This mechanism would explain why reduced dilution of nutrients did not cause elevated nutrient concentration at some sites that receive sewage-wastewater effluent.

Dissolved organic carbon could be characterized only for the South Platte River Basin, which had medial concentrations. E. coli bacteria could not be characterized for any basin. Fecal-coliform bacteria could be characterized only for the Arkansas River Basin, which had medial percentiles.

Dissolved and total-recoverable concentrations of metals - except those for dissolved iron and manganesecould only be characterized for one constituent each in four separate basins: the Arkansas River Basin had medial concentrations of cadmium (dissolved and total-recoverable), totalrecoverable chromium, dissolved lead, and zinc (dissolved and total-recoverable); the Yampa and White River Basin had medial concentrations of dissolved boron; and the South Platte River Basin had medial concentrations of dissolved copper and silver. Dissolved iron showed medial concentrations in the Yampa and White, Upper Colorado, South Platte, and Arkansas Basins. Dissolved manganese showed medial concentrations in the Upper Colorado, South Platte, and Arkansas Basins. Total-recoverable iron and manganese could only be characterized for the Arkansas River Basin, which had depressed concentrations of both constituents (tentative for manganese).

Medial concentrations of all of these metals except for copper, iron, and manganese result from the fact that most historical and drought samples had censored, recensored, or low (near-censored) concentrations, probably due to limitations of source and solubility. Medial concentrations of dissolved copper, iron, and manganese possibly resulted from the fact that these metals are abundant enough in drainage basins (from natural and anthropogenic sources) to dissolve into the runoff component of streamflow in concentrations similar to those found in ground water; if so, the greater dominance of ground-water base flow during drought conditions would not be expected to shift concentrations substantially compared to historical summer periods when runoff contributed a larger fraction of streamflow.

The depressed concentrations of total-recoverable iron and manganese in the Arkansas River Basin during drought conditions of 2002 probably were the result of reduced landsurface washoff of sediment containing oxyhydroxides of these metals; the relatively low average percentile of 28.3 for total-recoverable zinc indicates the same effect, although censored and recensored concentrations of total-recoverable zinc for many drought and historical samples could preclude 
Table 10. Summary of characterization of percentiles for properties and constituents in drought samples collected from sites in Colorado during summer 2002 compared to 1978-2002.

[Medial, average basin percentile between 25 and 74.9; elevated, average basin percentile greater than or equal to 75; depressed, average basin percentile less than 25; ID, insufficient number (less than 3) of sites to calculate average percentile; --, constituent not measured in drought samples or had insufficient number (fewer than 20) of historical and drought samples at any site to calculate percentile. Constituents for which there were too few or no drought samples for all study river bains are not included in this table; text in bold highlights depressed or elevated average percentiles]

\begin{tabular}{|c|c|c|c|c|c|c|c|c|}
\hline \multirow[b]{2}{*}{ Property or constituent } & \multicolumn{8}{|c|}{ River basin } \\
\hline & $\begin{array}{c}\text { Yampa } \\
\text { and } \\
\text { White } \\
\text { (table 2) }\end{array}$ & $\begin{array}{c}\text { Upper } \\
\text { Colorado } \\
\text { (table 3) }\end{array}$ & $\begin{array}{c}\text { Rio } \\
\text { Grande } \\
\text { (table 4) }\end{array}$ & $\begin{array}{c}\text { Gunnison } \\
\text { (table 5) }\end{array}$ & $\begin{array}{l}\text { Dolores } \\
\text { (table 6) }\end{array}$ & $\begin{array}{c}\text { San } \\
\text { Juan } \\
\text { (table 7) }\end{array}$ & $\begin{array}{c}\text { South } \\
\text { Platte } \\
\text { (table 8) }\end{array}$ & $\begin{array}{c}\text { Arkansas } \\
\text { (table 9) }\end{array}$ \\
\hline Oxygen, dissolved & Medial & Medial & ID & Medial & -- & -- & Medial & Medial \\
\hline $\mathrm{pH}$ & Medial & Medial & ID & Medial & ID & Medial & Medial & Medial \\
\hline Specific conductance & Medial & Elevated & ID & Elevated & Elevated & Elevated & Medial & Elevated \\
\hline Temperature, water & Medial & Medial & Medial & Medial & Medial & Medial & Medial & Medial \\
\hline Chloride, dissolved & Medial & Elevated & ID & Elevated & ID & Elevated & Medial & -- \\
\hline Fluoride, dissolved & Medial & Elevated $^{1}$ & ID & ID & ID & Elevated & -- & Medial \\
\hline Silica, dissolved & Medial & Medial & ID & Elevated $^{1}$ & ID & Medial & Medial & -- \\
\hline Sulfate, dissolved & Medial & Elevated & ID & Elevated & ID & Elevated & Medial & Elevated \\
\hline Nitrogen, ammonia, dissolved & Medial & Medial & ID & Medial & -- & ID & Medial & Medial \\
\hline Nitrogen, organic plus ammonia, dissolved & Medial & Medial & ID & Medial & -- & -- & ID & -- \\
\hline $\begin{array}{l}\text { Nitrogen, organic plus ammonia, } \\
\text { total recoverable }\end{array}$ & Medial & Medial & ID & ID & -- & ID & Medial & ID \\
\hline Nitrogen, nitrite plus nitrate, dissolved & Medial & Medial & ID & Medial & -- & ID & Medial & Medial \\
\hline Nitrogen, nitrite, dissolved & Medial & Medial & ID & Medial & -- & -- & Medial & -- \\
\hline Phosphorus, dissolved & Medial & Medial & ID & Medial & -- & ID & Medial & -- \\
\hline Phosphorus, total recoverable & Medial & Medial & ID & Medial & -- & ID & Medial & Medial \\
\hline Phosphorus, orthophosphate, dissolved & Medial & Medial & ID & Medial & -- & -- & Medial & Medial \\
\hline Carbon, organic, dissolved & ID & ID & -- & -- & -- & -- & Medial & -- \\
\hline Bacteria, fecal coliform & -- & -- & ID & -- & -- & -- & -- & Medial \\
\hline Boron, dissolved & Medial & -- & -- & -- & -- & -- & -- & -- \\
\hline Cadmium, dissolved & -- & ID & -- & ID & -- & -- & -- & Medial \\
\hline Cadmium, total recoverable & -- & -- & -- & -- & -- & -- & -- & Medial \\
\hline Chromium, total recoverable & -- & -- & -- & -- & -- & -- & -- & Medial \\
\hline Copper, dissolved & -- & ID & -- & ID & -- & -- & Medial & ID \\
\hline Iron, dissolved & Medial & Medial & ID & ID & -- & -- & Medial & Medial \\
\hline Iron, total recoverable & -- & ID & -- & -- & -- & -- & ID & Depressed \\
\hline Lead, dissolved & -- & ID & -- & ID & -- & -- & ID & Medial \\
\hline Manganese, dissolved & ID & Medial & ID & ID & -- & -- & Medial & Medial \\
\hline Manganese, total recoverable & -- & ID & -- & -- & -- & -- & -- & Depressed $^{1}$ \\
\hline Nickel, dissolved & -- & ID & -- & -- & -- & -- & ID & ID \\
\hline Silver, dissolved & -- & ID & -- & ID & -- & -- & Medial & ID \\
\hline Zinc, dissolved & ID & ID & -- & ID & -- & -- & -- & Medial \\
\hline Zinc, total recoverable & -- & -- & -- & -- & -- & -- & -- & Medial \\
\hline
\end{tabular}

\footnotetext{
${ }^{1}$ Tentative (borderline) result; see table listed in column heading for details.
} 
the ability of average percentile to indicate depressed concentration.

In total, 115 exceedances of 2002 Colorado water-quality standards were identified in the 246 drought samples collected at 148 sites. Constituents showing exceedances of water quality-standards were $\mathrm{pH}$ (all 9.0 exceedances; 9 samples), chloride (1 sample), sulfate (9 samples), dissolved ammonia (10 samples), dissolved nitrite nitrogen (3 samples), E. coli bacteria (34 samples, 20 in the Arkansas River Basin), fecalcoliform bacteria (18 samples, all in the Arkansas River Basin), dissolved copper (1 sample), dissolved iron (3 samples), total-recoverable iron (3 samples), dissolved manganese (13 samples), dissolved selenium (10 samples), and dissolved zinc (1 sample). Of these 115 exceedances, historical data were sufficient to conclude that 21 probably were affected by drought, 39 probably were not affected by drought, and 55 were of indeterminate nature (that is, were considered to be possibly or apparently affected by drought, or data were insufficient for judging). Excluding all of the exceedances of E. coli and fecal-coliform bacteria (52) because of their overrepresentation in the Arkansas River Basin, 19 of the total 63 exceedances probably were affected by drought, 25 probably were not, and 19 were of indeterminate nature. Therefore, based on the first two categories, about 38 percent of the exceedances probably would have occurred under nondrought conditions. However, eight dissolved manganese exceedances were affected by drought compared to two that were considered not to be affected by drought, and sulfate showed five exceedances that were affected by drought and two that were not affected by drought. Overall, the data indicate that most water-quality exceedances probably were not drought related but that drought affected concentrations of some constituents (especially dissolved manganese and sulfate) were enough to cause local exceedances of water-quality standards.

Specific-conductance values indicate that the San Juan River Basin (average percentile 95.2; table 7) experienced the greatest effects of drought on water quality during summer 2002 compared to other basins in Colorado, followed by the Upper Colorado (90.0; table 3) and Dolores River (85.7; table 6) Basins. The South Platte River Basin (70.9; table 8) experienced the least effect of drought, and the Yampa and White River Basin group (73.7; table 2) had the second smallest drought effect. The Gunnison River (82.1; table 5) and Arkansas River (81.2; table 9) Basins showed an intermediate drought effect. The Rio Grande Basin had insufficient data to determine the relative effect of drought on water quality.

\section{Summary}

During 2002, Colorado experienced the State's worst drought since 1977. To determine the effects of this drought on the water quality of streams in the State during the summer of 2002, a variety of State and local governments cooperated with the U.S. Geological Survey to collect water-quality samples at 148 stream sites in all major river basins in Colorado. Water samples were analyzed for field properties, major ions, nutrients, organic carbon, bacteria, and dissolved metals at all sites, although individual constituents in these categories varied among basins. At some sites, water samples also were analyzed for total-recoverable metals. In 2003, the U.S. Geological Survey, in cooperation with the Colorado Department of Public Health and Environment, analyzed these data to determine the general effects of drought on the water quality of streams in Colorado during summer 2002. This report summarizes streamflow for 2002 relative to the 1978 through 2001 period and presents percentiles of concentrations of water-quality constituents from the summer of 2002 relative to historical data for each site for the months of July through September during 1978 through 2001. Exceedances of Colorado water-quality standards for constituents sampled during summer 2002 are included.

Mean annual streamflow was analyzed at 134 sites in Colorado for water years 1978-2002. Mean annual streamflow for 2002 had an average percentile of 29.4 percent during this period, indicating that streamflow in Colorado during 2002 was substantially less than median percentile streamflow for 1978-2002. Mean summer (July-September) streamflow for 2002 was analyzed for 146 sites in Colorado, and percentiles averaged 7.6 percent when compared to summer streamflow for 1978-2002 for 134 sites across Colorado. Because mean summer streamflow for 2002 had a substantially smaller average percentile ( 7.6 percent) than did mean annual streamflow for 2002 (29.4 percent), the effect of drought on streamflow was greater during summer 2002 than during water year 2002.

Drought samples were collected at 8 sites in the Yampa River Basin and at 11 sites in the White River Basin. Average percentiles (for July-September 1978-2002) for constituents collected in both basins indicate medial (in interquartile range of 25-75) values or concentrations for all constituents with sufficient data-including field properties, chloride, fluoride, silica, and sulfate, most dissolved and total-recoverable nutrients, and dissolved boron and iron. The only exceedances of 2002 Colorado water-quality standards were for $\mathrm{pH}$ (upper standard of $9.0 \mathrm{SU}$ ) at one site and for dissolved manganese at another.

Drought samples were collected at 39 sites in the Upper Colorado River Basin. Average percentiles for properties and constituents indicate elevated (in upper quartile range of 75 or greater) specific conductance and elevated concentrations of chloride, fluoride (tentative), and sulfate. All other constituents with sufficient data (field properties, silica, dissolved and total-recoverable nutrients, dissolved iron and manganese) indicate medial values or concentrations.

Drought samples from the Upper Colorado River Basin had water-quality exceedances for $\mathrm{pH}$ and concentrations of chloride, sulfate, nitrite nitrogen, dissolved iron, dissolved manganese, and dissolved selenium. $\mathrm{pH}$ exceeded the upper standard of 9.0 SU five times at four sites; the effect of the drought on these exceedances is unknown. The chloride standard of $250 \mathrm{mg} / \mathrm{L}$ was exceeded once at one site, and the 
exceedance was likely a result of the drought. The sulfate standard of $250 \mathrm{mg} / \mathrm{L}$ was exceeded five times at four sites, and three of the exceedances were likely an effect of the drought. Nitrite nitrogen exceeded the chronic standard of $0.05 \mathrm{mg} / \mathrm{L}$ twice at one site, and the exceedances were likely affected by drought. Dissolved iron exceeded the chronic standard of 300 $\mu \mathrm{g} / \mathrm{L}$ twice at one site and the exceedances were not affected by drought. Dissolved manganese exceeded the hardnessbased chronic standard of $50 \mu \mathrm{g} / \mathrm{L}$ three times at two sites; two exceedances at one site were affected by drought. Dissolved selenium exceeded the chronic standard of $4.6 \mu \mathrm{g} / \mathrm{L}$ four times at three sites; historical data indicate that these exceedances were not affected by drought.

Drought samples were collected at four sites in the Rio Grande Basin. Average percentiles for properties and constituents only indicate result for medial water temperature, but all other properties and constituents had too few percentiles for characterization. The only water-quality exceedance for this basin was for dissolved zinc in one sample at one site; it is unknown if this sample was affected by drought.

Drought samples were collected at 21 sites in the Gunnison River Basin. Average percentiles for properties and constituents indicate elevated specific conductance and elevated concentrations of dissolved chloride, dissolved silica, and dissolved sulfate. All other constituents with sufficient data-including other field properties and most dissolved and total-recoverable nutrients - indicated medial values or concentrations. Drought samples collected in the basin had water-quality exceedances for sulfate (one sample, one site), E. coli bacteria (four samples, three sites), total-recoverable iron (two samples, two sites), dissolved manganese (one sample, one site), and dissolved selenium (three samples, two sites). The only exceedance that likely was affected by drought was dissolved manganese.

Drought samples were collected at five sites in the Dolores River Basin. Average percentiles indicate that specific conductance was elevated and that temperature was medial. $\mathrm{pH}$, chloride, fluoride, silica, and sulfate had too few sites for basin characterization, although all had high average percentiles, indicating elevated levels. $E$. coli bacteria was the only constituent showing a water-quality exceedance (one sample, one site) and it is unknown if the exceedance was affected by drought.

Drought samples were collected at 14 sites in the San Juan River Basin. Average percentiles indicate that specific conductance and concentrations of chloride, fluoride, and sulfate were elevated, whereas $\mathrm{pH}$ and temperature values and silica concentrations had medial values. Dissolved and total-recoverable nutrients had too few sites for characterization. Metals were sampled too infrequently to allow calculation of reliable percentiles. Drought samples had water-quality exceedances for sulfate (one sample, one site), E. coli bacteria (seven samples, five sites), and dissolved iron (one sample, one site). It is unknown if any of the exceedances were affected by drought.
Drought samples were collected at 23 sites in the South Platte River Basin. Average percentiles indicated medial values and concentrations for field properties, most dissolved and total-recoverable nutrients, dissolved organic carbon, and some dissolved trace metals (copper, iron, manganese, and silver). Too few sites had reliable percentiles to allow characterization of dissolved organic plus ammonia nitrogen, totalrecoverable organic carbon, total-recoverable iron, dissolved lead, and dissolved nickel.

Drought samples exceeded water-quality standards for pH (9.0 standard units; 3 samples, three sites), dissolved ammonia (10 samples, six sites), dissolved nitrite (1 sample, one site), dissolved copper (1 sample, one site), and dissolved manganese ( 2 samples, one site). Historical data indicate that the single nitrite exceedance likely was affected by drought.

Drought samples were collected at 23 sites in the Arkansas River Basin. Average percentiles indicate elevated specific conductance values and elevated sulfate concentration; concentrations of total-recoverable iron and manganese (tentative) were depressed. Dissolved oxygen, $\mathrm{pH}$, most dissolved and total-recoverable nutrients, fecal-coliform bacteria, some dissolved metals (cadmium, iron, lead, manganese, and zinc), and some total-recoverable metals (cadmium, chromium, and zinc) indicated medial values and concentrations. Too few sites had reliable percentiles for total-recoverable organic plus ammonia nitrogen, some dissolved metals (arsenic, chromium, copper, nickel, selenium, and silver), and some total-recoverable metals (copper, mercury, and nickel). Drought samples indicate water-quality exceedances for sulfate (2 samples, 2 sites), E. coli bacteria (20 samples, 16 sites), fecal-coliform bacteria (18 samples, 14 sites), total-recoverable iron (1 sample, 1 site), dissolved manganese (6 samples, 6 sites), and dissolved selenium (3 samples, 3 sites). Drought conditions probably caused exceedances of water-quality standards for sulfate at two sites, fecal coliform at one site, manganese at four sites, and selenium at two sites.

Percentiles for all drought samples collected in Colorado during summer 2002 indicate that few measured constituents were elevated or depressed in concentration or value on a widespread basis. Specific conductance was elevated at five of the seven basins (or basin groups) that had sufficient data for characterization. Similarly, chloride concentrations were elevated in three of five basins with sufficient data, indicating that chloride concentration generally was affected by drought in those basins. Sulfate concentration was elevated in four of six basins with sufficient data. All basins that exhibited elevated concentrations of chloride and sulfate also exhibited elevated specific conductance. The widespread elevation of specific conductance and concentrations of chloride and sulfate indicates that salinity generally was affected by drought in Colorado streams during July through September 2002undoubtedly because streamflow at most sites was dominated by base flow of ground water, which usually has considerable salinity compared to runoff from precipitation.

Fluoride showed elevated concentrations in two of four basins with sufficient data to characterize (Upper Colorado 
(tentative); San Juan). Both basins with elevated concentrations of fluoride exhibited elevated specific conductance and elevated concentrations of chloride and sulfate, which is consistent with a ground-water source for fluoride. Dissolved and total-recoverable nutrients (nitrogen and phosphorus) showed medial (and near-medial) concentrations in all basins with sufficient data to characterize. Dissolved and total-recoverable concentrations of metals were mostly medial and could for the most part only be characterized for individual basins. Percentiles for concentrations of total recoverable iron and total recoverable manganese in the Arkansas Basin were depressed.

In total, 115 of the 246 water samples collected at 148 sites in the summer of 2002 exceeded Colorado waterquality standards. Constituents that exceeded water-quality standards were $\mathrm{pH}$ (all exceeding 9.0; 9 samples), chloride (1 sample), sulfate (9 samples), dissolved ammonia (10 samples), dissolved nitrite nitrogen (3 samples), E. coli bacteria (34 samples, 20 in Arkansas River Basin), fecal-coliform bacteria (18 samples, all in Arkansas River Basin), dissolved copper (1 sample), dissolved iron (3 samples), total-recoverable iron (3 samples), dissolved manganese (13 samples), dissolved selenium (10 samples), and dissolved zinc (1 sample). For these 115 exceedances, historical data were sufficient to conclude that 21 probably were affected by drought, 39 probably were not affected by drought, and 55 were of indeterminate nature. Overall, the data indicate that some water-quality exceedances probably would have occurred during July through September 2002 without drought but that drought probably affected concentrations of some constituents (especially dissolved manganese and sulfate) enough to locally cause exceedances of water-quality standards.

Specific-conductance values indicate the San Juan River Basin (average percentile 95.2) experienced the greatest effects of drought on water quality during summer 2002 compared to other basins in Colorado, followed by the Upper Colorado (90.0) and Dolores River (85.7) Basins. The South Platte River Basin (70.9) experienced the least effect of drought, and the Yampa and White River Basin group (73.7) had the second smallest effect. The Gunnison River (82.1) and Arkansas River (81.2) Basins had intermediate drought effects. The Rio Grande Basin had insufficient data to rank the relative effect of drought on salinity.

\section{References Cited}

Chafin, D. T., 2002, Evaluation of trends in $\mathrm{pH}$ in the Yampa River, northwestern Colorado, 1950-2000: U.S. Geological Survey Water-Resources Investigations Report 2002-4038, $41 \mathrm{p}$.

Colorado Department of Public Health and Environment, 2005, Water quality regulations: Web site accessed October 2005 at http://www.cdphe.state.co.us/regulations/wqccregs/ index.html

Crowfoot, R.M., Boulger, R.W., and O'Neill, G.B., 2003, Water resources data, Colorado, water year 2002Volume 2. Colorado River Basin: U.S. Geological Survey Water-Data Report CO-02-2, 623 p.

Crowfoot, R.M., Payne, W.F., and O’Neill, G.B., 2003, Water resources data, Colorado, water year 2002_-Volume 1. Missouri River Basin, Arkansas River Basin, and Rio Grande Basin: U.S. Geological Survey Water-Data Report CO-02-1, 609 p.

Helsel, D. R., 1990, Less than obvious-Statistical treatment of data below the detection limit: Environmental Science and Technology, v. 24, no. 12, p. 1766-1774

Helsel, D.R., and Hirsch, R.M., 1992, Statistical methods in water resources: New York, Elsevier, 529 p.

Kuhn, Gerhard, 2005, Historic perspective of statewide streamflows during the 2002 and 1977 droughts in Colorado: U.S. Geological Survey Scientific Investigations Report 2005-5174, 84 p.

Sprague, L.A., Kimbrough, R.A., and Ranalli, A.J., 2002, What happens to nutrients in offstream reservoirs in the lower South Platte River Basin?: U.S. Geological Survey Fact Sheet 044-02, 6 p.

U.S. Geological Survey, 1998, National field manual for the collection of water-quality data: U.S. Geological Survey Techniques of Water-Resources Investigations, book 9, chap. A4, variously paginated.

Winter, T.C., Harvey, J.W., Franke, O.L., and Alley., W.M., 1999, Ground water and surface water-A single resource: U.S. Geological Survey Circular 1139, 79 p. 


\section{Appendix}

This appendix contains data tables in support of the findings for this study. 
Table 11. Water-quality data for the Yampa and White River Basins, showing 2002 drought values, Colorado water-quality exceedances, and percentiles relative to historical data for selected properties and constituents from July through September, 1978-2002.

$\left[\mathrm{ft}^{3} / \mathrm{s}\right.$, cubic feet per second; Conc, concentration; Q\%, percentile (percentage of data less than or equal to reported value or concentration) of droughtsample constituent; $\mathrm{N}$, total number of historical and drought samples; $\mathrm{mg} / \mathrm{L}$, milligrams per liter; $\mu \mathrm{S} / \mathrm{cm}$, microsiemens per centimeter at $25^{\circ} \mathrm{Celsius}$; ${ }^{\circ} \mathrm{C}$, degrees Celsius; E. coli, Escherichia coli; $\mathrm{mL}$, milliliter; $\mu \mathrm{g} / \mathrm{L}$, micrograms per liter; $\mathrm{CaCO}_{3}$, calcium carbonate; $\mathrm{SiO}_{2}$, silica; $\mathrm{SO}_{4}$, sulfate; ID, insufficient number of samples (less than 20) to calculate percentile; <, less than; --, no drought-sample measurement; values or concentrations in bold type indicate 2002 Colorado water-quality exceedances; c, chronic water-quality exceedance]

\begin{tabular}{|c|c|c|c|c|c|c|c|c|c|}
\hline \multirow{2}{*}{$\begin{array}{c}\text { Site number } \\
\text { in table } 1 \\
\text { and fig. } 4\end{array}$} & \multirow{2}{*}{$\begin{array}{l}\text { Sampling } \\
\text { date }\end{array}$} & \multirow{2}{*}{$\begin{array}{l}\text { Sampling } \\
\text { time }\end{array}$} & \multirow{2}{*}{$\begin{array}{c}\text { Discharge, } \\
\text { in } \mathrm{ft}^{3} / \mathrm{s}\end{array}$} & \multicolumn{3}{|c|}{ 0xygen, dissolved, in $\mathrm{mg} / \mathrm{L}$} & \multicolumn{3}{|c|}{$\mathrm{pH}$, in standard units } \\
\hline & & & & Conc & $0 \%$ & $\mathrm{~N}$ & Value & $0 \%$ & $\mathrm{~N}$ \\
\hline \multicolumn{10}{|c|}{ Yampa River Basin } \\
\hline \multirow[t]{2}{*}{1} & $7-29-2002$ & 1415 & 3.0 & 6.2 & ID & 5 & 7.5 & ID & 8 \\
\hline & $9-3-2002$ & 1130 & 2.4 & 7.9 & ID & 5 & 7.6 & ID & 8 \\
\hline 2 & $7-29-2002$ & 1540 & 65.0 & 8.2 & ID & 9 & 9.3 & ID & 12 \\
\hline \multirow[t]{2}{*}{3} & $7-30-2002$ & 850 & 54.0 & 8.3 & ID & 11 & 8.1 & ID & 11 \\
\hline & $9-3-2002$ & 1400 & 8.3 & 7.8 & ID & 11 & 8.2 & ID & 11 \\
\hline 4 & $7-30-2002$ & 1100 & 1.9 & 7.3 & ID & 19 & 8.8 & ID & 19 \\
\hline 5 & $7-30-2002$ & 1520 & 57.0 & 9.8 & ${ }^{1} 66.7-71.4$ & 20 & 8.8 & ${ }^{1} 73.9-91.3$ & 22 \\
\hline \multirow[t]{2}{*}{6} & $7-30-2002$ & 1400 & 20.0 & 6.9 & ${ }^{1} 14.3-19.0$ & 20 & 8.4 & ${ }^{1} 59.1-63.6$ & 21 \\
\hline & $9-3-2002$ & 1655 & 2.9 & 7.6 & 42.9 & 20 & 8.0 & 9.1 & 21 \\
\hline \multirow[t]{2}{*}{7} & $7-31-2002$ & 850 & 48.0 & 6.6 & 2.2 & 45 & 8.4 & ${ }^{1} 39.1-54.3$ & 45 \\
\hline & $9-4-2002$ & 910 & 4.0 & 8.4 & 60.9 & 45 & 8.2 & ${ }^{1} 15.2-28.3$ & 45 \\
\hline \multirow[t]{2}{*}{8} & $7-31-2002$ & 1020 & 70.0 & 7.0 & ID & 7 & 8.4 & ID & 8 \\
\hline & $9-4-2002$ & 1130 & 4.2 & 8.6 & ID & 7 & 8.4 & ID & 8 \\
\hline \multicolumn{10}{|c|}{ White River Basin } \\
\hline \multirow[t]{2}{*}{9} & $7-29-2002$ & 1230 & 140.0 & 8.2 & ${ }^{1} 26.1-30.4$ & 22 & 8.6 & 96.0 & 24 \\
\hline & $9-9-2002$ & 1400 & 119.0 & 8.1 & ${ }^{1} 17.4-21.7$ & 22 & 8.5 & ${ }^{1} 76.0-92.0$ & 24 \\
\hline \multirow[t]{2}{*}{10} & $7-29-2002$ & 1425 & 119.0 & 8.2 & ${ }^{1} 45.5-50.0$ & 21 & 8.6 & ${ }^{1} 72.0-84.0$ & 24 \\
\hline & $9-9-2002$ & 1555 & 122.0 & 8.2 & ${ }^{1} 45.5-50.0$ & 21 & 8.6 & ${ }^{1} 72.0-84.0$ & 24 \\
\hline \multirow[t]{2}{*}{11} & $7-30-2002$ & 1250 & 24.0 & 7.3 & 13.0 & 22 & 8.6 & ${ }^{1} 82.5-92.2$ & 102 \\
\hline & $9-10-2002$ & 1035 & 12.0 & 8.2 & ${ }^{1} 43.5-47.8$ & 22 & 8.3 & ${ }^{1} 33.0-49.5$ & 102 \\
\hline \multirow[t]{2}{*}{12} & $7-30-2002$ & 1505 & 205.0 & 7.7 & 5.6 & 35 & 8.6 & ${ }^{1} 83.3-92.2$ & 101 \\
\hline & $9-10-2002$ & 1400 & 180.0 & 7.9 & ${ }^{1} 13.9-16.7$ & 35 & 8.4 & ${ }^{1} 59.8-74.5$ & 101 \\
\hline 13 & $9-11-2002$ & 1155 & 8.6 & 9.0 & ${ }^{1} 54.3-56.5$ & 45 & 8.3 & ${ }^{1} 38.8-71.4$ & 48 \\
\hline 14 & $7-31-2002$ & 900 & 3.0 & 8.9 & 64.1 & 38 & 8.8 & ${ }^{1} 78.6-95.2$ & 41 \\
\hline 15 & $9-11-2002$ & 1049 & 0.4 & 9.0 & 89.3 & 27 & 7.9 & ${ }^{1} 32.1-60.7$ & 27 \\
\hline 16 & $7-31-2002$ & 1107 & 1.6 & 8.4 & 27.6 & 28 & 8.5 & ${ }^{1} 10.3-20.7$ & 28 \\
\hline \multirow[t]{2}{*}{17} & $7-31-2002$ & 1400 & 176.0 & 7.8 & 27.5 & 39 & 8.5 & ${ }^{1} 53.2-72.3$ & 46 \\
\hline & $9-10-2002$ & 1715 & 176.0 & 7.7 & 17.5 & 39 & 8.3 & ${ }^{1} 6.4-27.7$ & 46 \\
\hline \multirow[t]{2}{*}{18} & $7-31-2002$ & 1600 & 180.0 & 7.2 & ID & 16 & 8.5 & ID & 16 \\
\hline & $9-10-2002$ & 1944 & 176.0 & 7.8 & ID & 16 & 8.4 & ID & 16 \\
\hline \multirow[t]{2}{*}{19} & $7-30-2002$ & 1030 & 266.0 & 8.1 & ID & 9 & 8.6 & ID & 9 \\
\hline & $9-9-2002$ & 1750 & 290.0 & 8.1 & ID & 9 & 8.6 & ID & 9 \\
\hline
\end{tabular}


Table 11. Water-quality data for the Yampa and White River Basins, showing 2002 drought values, Colorado water-quality exceedances, and percentiles relative to historical data for selected properties and constituents from July through September, 1978-2002.-Continued

[ft $\mathrm{ft}^{3} / \mathrm{s}$, cubic feet per second; Conc, concentration; $\mathrm{Q} \%$, percentile (percentage of data less than or equal to reported value or concentration) of droughtsample constituent; $\mathrm{N}$, total number of historical and drought samples; $\mathrm{mg} / \mathrm{L}$, milligrams per liter; $\mu \mathrm{S} / \mathrm{cm}$, microsiemens per centimeter at $25^{\circ} \mathrm{Celsius;}$ ${ }^{\circ} \mathrm{C}$, degrees Celsius; E. coli, Escherichia coli; $\mathrm{mL}$, milliliter; $\mu \mathrm{g} / \mathrm{L}$, micrograms per liter; $\mathrm{CaCO}_{3}$, calcium carbonate; $\mathrm{SiO}_{2}$, silica; $\mathrm{SO}_{4}$, sulfate; $\mathrm{ID}_{\text {, insuf- }}$ ficient number of samples (less than 20) to calculate percentile; <, less than; --, no drought-sample measurement; values or concentrations in bold type indicate 2002 Colorado water-quality exceedances; c, chronic water-quality exceedance]

\begin{tabular}{|c|c|c|c|c|c|c|c|c|c|c|}
\hline \multirow{2}{*}{$\begin{array}{c}\text { Site number } \\
\text { in table } 1 \\
\text { and fig. } 4\end{array}$} & \multirow{2}{*}{$\begin{array}{l}\text { Sampling } \\
\text { date }\end{array}$} & \multicolumn{3}{|c|}{ Specific conductance, in $\boldsymbol{\mu} S / \mathrm{cm}$} & \multicolumn{3}{|c|}{ Temperature, water, in ${ }^{\circ} \mathrm{C}$} & \multirow{2}{*}{$\begin{array}{c}\text { Hardness, } \\
\text { total, } \\
\text { in } \mathrm{mg} / \mathrm{L} \\
\text { as } \mathrm{CaCO}_{3}\end{array}$} & \multirow{2}{*}{$\begin{array}{l}\text { Calcium, } \\
\text { dissolved, } \\
\text { in } \mathrm{mg} / \mathrm{L}\end{array}$} & \multirow{2}{*}{$\begin{array}{c}\text { Magnesium, } \\
\text { dissolved, } \\
\text { in } \mathrm{mg} / \mathrm{L}\end{array}$} \\
\hline & & Value & $0 \%$ & $\mathrm{~N}$ & Value & $0 \%$ & $\mathrm{~N}$ & & & \\
\hline & & \multicolumn{9}{|c|}{ Yampa River Basin—Continued } \\
\hline \multirow[t]{2}{*}{1} & $7-29-2002$ & 25 & ${ }^{1} 30.8-41$ & 38 & 16.5 & 89.5 & 37 & 9 & 2.7 & 0.5 \\
\hline & $9-3-2002$ & 24 & 28.2 & 38 & 11.5 & ${ }^{1} 23.7-26.3$ & 37 & 8 & 2.5 & 0.5 \\
\hline 2 & $7-29-2002$ & 199 & 31.3 & 63 & 24.0 & ${ }^{1} 96.8-98.4$ & 62 & 86 & 22.7 & 7.2 \\
\hline \multirow[t]{2}{*}{3} & $7-30-2002$ & 147 & 90.7 & 42 & 16.0 & ${ }^{1} 46.5-53.5$ & 42 & 64 & 19.2 & 4.0 \\
\hline & 9-3-2002 & 229 & 97.7 & 42 & 16.6 & 55.8 & 42 & 98 & 28.3 & 6.6 \\
\hline 4 & $7-30-2002$ & 343 & 57.9 & 26 & 22.5 & 59.3 & 27 & 130 & 30.7 & 12.5 \\
\hline 5 & $7-30-2002$ & 373 & ${ }^{1} 60.2-61.2$ & 102 & 25.0 & 96.1 & 102 & 120 & 29.7 & 11.4 \\
\hline \multirow[t]{2}{*}{6} & $7-30-2002$ & 684 & ${ }^{1} 83.2-85.1$ & 100 & 24.0 & ${ }^{1} 90.3-94.2$ & 102 & 240 & 48.9 & 29.0 \\
\hline & $9-3-2002$ & 1,040 & 99.0 & 100 & 19.9 & ${ }^{1} 62.1-63.1$ & 102 & 240 & 44.1 & 32.1 \\
\hline \multirow[t]{2}{*}{7} & $7-31-2002$ & 866 & 93.9 & 65 & 18.5 & ${ }^{1} 25.6-31.1$ & 89 & 250 & 49.0 & 30.2 \\
\hline & $9-4-2002$ & 1,350 & 98.5 & 65 & 13.9 & 6.7 & 89 & 350 & 78.9 & 37.0 \\
\hline \multirow[t]{3}{*}{8} & $7-31-2002$ & 956 & 93.9 & 48 & 22.5 & ${ }^{1} 63.5-65.4$ & 51 & 260 & 59.9 & 27.3 \\
\hline & $9-4-2002$ & 972 & 98.0 & 48 & 16.1 & 1.9 & 51 & 290 & 62.6 & 31.8 \\
\hline & & \multicolumn{9}{|c|}{ White River Basin—Continued } \\
\hline \multirow[t]{2}{*}{9} & $7-29-2002$ & 374 & 96.0 & 74 & 16.5 & 90.8 & 75 & 190 & 59.9 & 10.7 \\
\hline & $9-9-2002$ & 397 & 98.7 & 74 & 15.5 & ${ }^{1} 85.5-86.8$ & 75 & -- & -- & -- \\
\hline \multirow[t]{2}{*}{10} & $7-29-2002$ & 336 & ${ }^{1} 96.9-98.4$ & 63 & 17.3 & 87.5 & 63 & 180 & 52.5 & 10.8 \\
\hline & $9-9-2002$ & 287 & 50.0 & 63 & 16.8 & 84.4 & 63 & -- & -- & -- \\
\hline \multirow[t]{2}{*}{11} & $7-30-2002$ & 570 & 98.6 & 145 & 22.5 & 98.6 & 143 & 290 & 87.9 & 16.4 \\
\hline & $9-10-2002$ & 576 & 99.3 & 145 & 15.1 & ${ }^{1} 61.8-62.5$ & 143 & -- & -- & -- \\
\hline \multirow[t]{2}{*}{12} & $7-30-2002$ & 785 & 94.8 & 133 & 22.7 & 98.6 & 138 & 380 & 102.0 & 31.9 \\
\hline & $9-10-2002$ & 891 & 99.3 & 133 & 19.2 & ${ }^{1} 82.7-83.5$ & 138 & 420 & 107.0 & 37.5 \\
\hline 13 & $9-11-2002$ & 1,620 & 39.4 & 65 & 13.7 & 18.6 & 85 & 480 & 62.1 & 77.7 \\
\hline 14 & $7-31-2002$ & 4,330 & 94.4 & 70 & 15.0 & ${ }^{1} 17.8-23.8$ & 100 & 400 & 13.6 & 88.9 \\
\hline 15 & $9-11-2002$ & 1,390 & 22.4 & 48 & 12.2 & ${ }^{1} 13.7-15.1$ & 72 & 550 & 100.0 & 71.2 \\
\hline 16 & $7-31-2002$ & 3,720 & 80.0 & 54 & 21.5 & ${ }^{1} 70.6-71.8$ & 84 & 690 & 22.7 & 152.0 \\
\hline \multirow[t]{2}{*}{17} & $7-31-2002$ & 843 & 90.0 & 79 & 23.7 & 93.9 & 130 & 350 & 83.7 & 35.2 \\
\hline & $9-10-2002$ & 1,030 & 98.8 & 79 & 21.7 & 74.0 & 130 & -- & -- & -- \\
\hline \multirow[t]{2}{*}{18} & $7-31-2002$ & 930 & ID & 16 & 23.9 & ID & 16 & 350 & 77.8 & 36.9 \\
\hline & $9-10-2002$ & 966 & ID & 16 & 20.2 & ID & 16 & -- & -- & -- \\
\hline \multirow[t]{2}{*}{19} & $7-30-2002$ & 418 & ID & 9 & 15.0 & ID & 9 & 220 & 66.8 & 12.0 \\
\hline & $9-9-2002$ & 400 & ID & 9 & 16.0 & ID & 9 & -- & -- & -- \\
\hline
\end{tabular}


Table 11. Water-quality data for the Yampa and White River Basins, showing 2002 drought values, Colorado water-quality exceedances, and percentiles relative to historical data for selected properties and constituents from July through September, 1978-2002.-Continued

[ft $3 / \mathrm{s}$, cubic feet per second; Conc, concentration; Q\%, percentile (percentage of data less than or equal to reported value or concentration) of droughtsample constituent; $\mathrm{N}$, total number of historical and drought samples; $\mathrm{mg} / \mathrm{L}$, milligrams per liter; $\mu \mathrm{S} / \mathrm{cm}$, microsiemens per centimeter at $25^{\circ} \mathrm{Celsius}$; ${ }^{\circ} \mathrm{C}$, degrees Celsius; E. coli, Escherichia coli; $\mathrm{mL}$, milliliter; $\mu \mathrm{g} / \mathrm{L}$, micrograms per liter; $\mathrm{CaCO}_{3}$, calcium carbonate; $\mathrm{SiO}_{2}$, silica; $\mathrm{SO}_{4}$, sulfate; ID, insufficient number of samples (less than 20) to calculate percentile; <, less than; --, no drought-sample measurement; values or concentrations in bold type indicate 2002 Colorado water-quality exceedances; c, chronic water-quality exceedance]

\begin{tabular}{|c|c|c|c|c|c|c|c|c|c|c|}
\hline \multirow{2}{*}{$\begin{array}{c}\text { Site number } \\
\text { in table } 1 \\
\text { and fig. } 4\end{array}$} & \multirow{2}{*}{$\begin{array}{l}\text { Sampling } \\
\text { date }\end{array}$} & \multirow{2}{*}{$\begin{array}{c}\text { Potassium, } \\
\text { dissolved, } \\
\text { in } \mathrm{mg} / \mathrm{L}\end{array}$} & \multirow{2}{*}{$\begin{array}{c}\text { Sodium, } \\
\text { dissolved, } \\
\text { in } \mathrm{mg} / \mathrm{L}\end{array}$} & \multirow{2}{*}{$\begin{array}{c}\text { Alkalinity, } \\
\text { laboratory, } \\
\text { in } \mathrm{mg} / \mathrm{L} \\
\text { as } \mathrm{CaCO}_{3}\end{array}$} & \multicolumn{3}{|c|}{ Chloride, dissolved, in $\mathrm{mg} / \mathrm{L}$} & \multicolumn{3}{|c|}{ Fluoride, dissolved, in $\mathrm{mg} / \mathrm{L}$} \\
\hline & & & & & Conc & $0 \%$ & $\mathrm{~N}$ & Conc & $0 \%$ & $\mathrm{~N}$ \\
\hline & & \multicolumn{9}{|c|}{ Yampa River Basin—Continued } \\
\hline 1 & $7-29-2002$ & 0.6 & 1.2 & 11 & 0.2 & ID & 8 & -- & -- & -- \\
\hline & $9-3-2002$ & 0.6 & 1.2 & 10 & 0.3 & ID & 8 & -- & -- & -- \\
\hline 2 & $7-29-2002$ & 1.4 & 7.7 & -- & -- & -- & -- & -- & -- & -- \\
\hline \multirow[t]{2}{*}{3} & $7-30-2002$ & 1.6 & 4.3 & 62 & 1.7 & ID & 11 & -- & -- & -- \\
\hline & $9-3-2002$ & 1.8 & 7.5 & 85 & 3.9 & ID & 11 & -- & -- & -- \\
\hline 4 & $7-30-2002$ & 1.6 & 20.5 & 107 & 4.4 & ID & 19 & 0.2 & ID & 19 \\
\hline 5 & $7-30-2002$ & 2.8 & 29.9 & 116 & 12.4 & 81.0 & 20 & 0.3 & ${ }^{1} 66.7-95.2$ & 20 \\
\hline \multirow[t]{2}{*}{6} & $7-30-2002$ & 3.0 & 54.7 & 266 & 6.3 & ID & 18 & -- & -- & -- \\
\hline & $9-3-2002$ & 3.7 & 146.0 & 417 & 11.6 & ID & 18 & -- & -- & -- \\
\hline \multirow[t]{2}{*}{7} & $7-31-2002$ & 4.7 & 95.1 & 219 & 30.6 & 90.9 & 43 & -- & -- & -- \\
\hline & $9-4-2002$ & 6.2 & 154.0 & 232 & 126.0 & 97.7 & 43 & 0.5 & ${ }^{1} 95.3-97.7$ & 42 \\
\hline \multirow[t]{2}{*}{8} & $7-31-2002$ & 4.8 & 102.0 & 187 & 75.3 & ID & 9 & 0.4 & ID & 8 \\
\hline & 9-4-2002 & 4.9 & 95.6 & 197 & 62.9 & ID & 9 & -- & -- & -- \\
\hline & & \multicolumn{9}{|c|}{ White River Basin—Continued } \\
\hline \multirow[t]{2}{*}{9} & $7-29-2002$ & 1.0 & 2.8 & 98 & 0.4 & ID & 11 & -- & -- & -- \\
\hline & $9-9-2002$ & -- & -- & -- & -- & -- & -- & -- & -- & -- \\
\hline \multirow[t]{2}{*}{10} & $7-29-2002$ & 1.0 & 2.2 & 130 & 0.5 & ID & 10 & -- & -- & -- \\
\hline & $9-9-2002$ & -- & -- & -- & -- & -- & -- & -- & -- & -- \\
\hline \multirow[t]{2}{*}{11} & $7-30-2002$ & 1.7 & 11.3 & 197 & 5.8 & 95.8 & 94 & -- & -- & -- \\
\hline & $9-10-2002$ & -- & -- & -- & -- & -- & -- & -- & -- & -- \\
\hline \multirow[t]{2}{*}{12} & $7-30-2002$ & 2.0 & 28.1 & 231 & 10.7 & 38.9 & 94 & 0.3 & ${ }^{1} 61.5-92.3$ & 25 \\
\hline & $9-10-2002$ & 2.8 & 38.8 & 245 & 13.0 & ${ }^{1} 48.4-49.5$ & 94 & 0.4 & 96.2 & 25 \\
\hline 13 & $9-11-2002$ & 2.7 & 194.0 & 471 & 16.9 & 45.0 & 39 & 0.7 & ${ }^{1} 25.0-40.0$ & 39 \\
\hline 14 & $7-31-2002$ & 5.1 & 989.0 & 1,970 & 169.0 & 91.7 & 35 & 3.4 & 94.4 & 35 \\
\hline 15 & $9-11-2002$ & 1.8 & 109.0 & 398 & 12.7 & 46.4 & 27 & 0.3 & 28.6 & 27 \\
\hline 16 & $7-31-2002$ & 3.8 & 746.0 & 1,290 & 109.0 & 48.3 & 28 & 1.9 & 55.2 & 28 \\
\hline \multirow[t]{2}{*}{17} & $7-31-2002$ & 2.4 & 49.0 & 217 & 14.7 & 76.7 & 29 & -- & -- & -- \\
\hline & $9-10-2002$ & -- & -- & -- & -- & -- & -- & -- & -- & -- \\
\hline \multirow[t]{2}{*}{18} & $7-31-2002$ & 2.8 & 74.2 & 237 & 22.7 & ID & 5 & -- & -- & -- \\
\hline & $9-10-2002$ & -- & -- & -- & -- & -- & -- & -- & -- & -- \\
\hline \multirow[t]{2}{*}{19} & $7-30-2002$ & 1.0 & 2.9 & 122 & 0.6 & ID & 1 & -- & -- & -- \\
\hline & $9-9-2002$ & -- & -- & -- & -- & -- & -- & -- & -- & -- \\
\hline
\end{tabular}


Table 11. Water-quality data for the Yampa and White River Basins, showing 2002 drought values, Colorado water-quality exceedances, and percentiles relative to historical data for selected properties and constituents from July through September, 1978-2002.-Continued

[ $\mathrm{ft}^{3} / \mathrm{s}$, cubic feet per second; Conc, concentration; Q\%, percentile (percentage of data less than or equal to reported value or concentration) of droughtsample constituent; $\mathrm{N}$, total number of historical and drought samples; $\mathrm{mg} / \mathrm{L}$, milligrams per liter; $\mu \mathrm{S} / \mathrm{cm}$, microsiemens per centimeter at $25^{\circ} \mathrm{Celsius;}$ ${ }^{\circ} \mathrm{C}$, degrees Celsius; E. coli, Escherichia coli; $\mathrm{mL}$, milliliter; $\mu \mathrm{g} / \mathrm{L}$, micrograms per liter; $\mathrm{CaCO}_{3}$, calcium carbonate; $\mathrm{SiO}_{2}$, silica; $\mathrm{SO}_{4}$, sulfate; ID, insufficient number of samples (less than 20) to calculate percentile; <, less than; --, no drought-sample measurement; values or concentrations in bold type indicate 2002 Colorado water-quality exceedances; c, chronic water-quality exceedance]

\begin{tabular}{|c|c|c|c|c|c|c|c|c|c|c|c|}
\hline \multirow{2}{*}{$\begin{array}{l}\text { Site number } \\
\text { in table } 1 \\
\text { and fig. } 4\end{array}$} & \multirow{2}{*}{$\begin{array}{l}\text { Sampling } \\
\text { date }\end{array}$} & \multicolumn{3}{|c|}{ Silica, dissolved, in $\mathrm{mg} / \mathrm{L}$ as $\mathrm{SiO}_{2}$} & \multicolumn{3}{|c|}{ Sulfate, dissolved, in $\mathrm{mg} / \mathrm{L}$ as $\mathrm{SO}_{4}$} & \multirow{2}{*}{$\begin{array}{l}\text { Solids, dissolved, } \\
\text { residue on } \\
\text { evaporation at } \\
180^{\circ} \mathrm{C} \text {, in } \mathrm{mg} / \mathrm{L}\end{array}$} & \multicolumn{3}{|c|}{$\begin{array}{c}\text { Nitrogen, ammonia, dissolved, in } \\
\mathrm{mg} / \mathrm{L} \text { as } \mathrm{N}\end{array}$} \\
\hline & & Conc & $0 \%$ & $\mathrm{~N}$ & Conc & $0 \%$ & $\mathrm{~N}$ & & Conc & $0 \%$ & $\mathrm{~N}$ \\
\hline & & \multicolumn{10}{|c|}{ Yampa River Basin-Continued } \\
\hline \multirow[t]{2}{*}{1} & $7-29-2002$ & 5.8 & ID & 8 & 1.5 & ID & 8 & -- & $<0.015$ & ID & 7 \\
\hline & $9-3-2002$ & 5.3 & ID & 8 & 1.3 & ID & 8 & -- & 0.01 & ID & 7 \\
\hline 2 & $7-29-2002$ & -- & -- & -- & -- & -- & -- & -- & 0.01 & ID & 7 \\
\hline \multirow[t]{2}{*}{3} & $7-30-2002$ & 3.3 & ID & 11 & 10.1 & ID & 11 & -- & $<0.015$ & ID & 7 \\
\hline & $9-3-2002$ & 2.3 & ID & 11 & 24.0 & ID & 11 & -- & $<0.015$ & ID & 7 \\
\hline 4 & $7-30-2002$ & 6.6 & ID & 19 & 61.3 & ID & 19 & -- & $<0.04$ & ID & 19 \\
\hline 5 & $7-30-2002$ & 1.5 & ID & 19 & 51.6 & 57.1 & 20 & -- & $<0.04$ & ID & 8 \\
\hline \multirow[t]{2}{*}{6} & $7-30-2002$ & 14.4 & ID & 18 & 94.0 & ID & 18 & -- & $<0.04$ & ID & 11 \\
\hline & $9-3-2002$ & 12.9 & ID & 18 & 135.0 & ID & 18 & -- & 0.06 & ID & 11 \\
\hline \multirow[t]{2}{*}{7} & $7-31-2002$ & 5.8 & 72.7 & 43 & 195.0 & 93.2 & 43 & 565 & $<0.04$ & $24.2-62.5$ & 23 \\
\hline & $9-4-2002$ & 6.9 & ${ }^{1} 84.1-86.4$ & 43 & 276.0 & 97.7 & 43 & 859 & $<0.04$ & $24.2-62.5$ & 23 \\
\hline \multirow[t]{3}{*}{8} & $7-31-2002$ & 13.3 & ID & 9 & 193.0 & ID & 9 & -- & $<0.04$ & ID & 7 \\
\hline & 9-4-2002 & 11.4 & ID & 9 & 209.0 & ID & 9 & -- & $<0.04$ & ID & 7 \\
\hline & & \multicolumn{10}{|c|}{ White River Basin—Continued } \\
\hline \multirow[t]{2}{*}{9} & $7-29-2002$ & 18.4 & ID & 11 & 90.4 & ID & 11 & -- & $<0.02$ & $24.3-56.5$ & 22 \\
\hline & 9-9-2002 & -- & -- & -- & -- & -- & -- & -- & $<0.02$ & $24.3-56.5$ & 22 \\
\hline \multirow[t]{2}{*}{10} & $7-29-2002$ & 14.9 & ID & 10 & 48.6 & ID & 10 & -- & $<0.02$ & $24.5-63.6$ & 21 \\
\hline & 9-9-2002 & -- & -- & -- & -- & -- & -- & -- & $<0.02$ & $24.5-63.6$ & 21 \\
\hline \multirow[t]{2}{*}{11} & $7-30-2002$ & 17.3 & ID & 11 & 106.0 & 97.8 & 89 & -- & $<0.04$ & $24.5-90.9$ & 21 \\
\hline & $9-10-2002$ & -- & -- & -- & -- & -- & -- & -- & $<0.04$ & $24.5-90.9$ & 21 \\
\hline \multirow[t]{2}{*}{12} & $7-30-2002$ & 14.0 & ${ }^{1} 7.7-23.1$ & 25 & 192.0 & 96.6 & 88 & -- & $<0.04$ & $24.0-80.0$ & 24 \\
\hline & $9-10-2002$ & 16.6 & 80.8 & 25 & 224.0 & 98.9 & 88 & -- & $<0.04$ & $24.0-80.0$ & 24 \\
\hline 13 & $9-11-2002$ & 11.8 & 7.5 & 39 & 425.0 & 50.0 & 39 & 1,120 & $<0.04$ & $23.2-74.2$ & 30 \\
\hline 14 & $7-31-2002$ & -- & -- & -- & 473.0 & 52.8 & 35 & 2,980 & $<0.04$ & $24.2--70.8$ & 23 \\
\hline 15 & $9-11-2002$ & 21.2 & ${ }^{1} 37.0-40.7$ & 26 & 363.0 & 21.4 & 27 & -- & $<0.04$ & ID & 17 \\
\hline 16 & $7-31-2002$ & 7.9 & ${ }^{1} 31.0-34.5$ & 28 & 839.0 & 62.1 & 28 & -- & $<0.04$ & ID & 19 \\
\hline \multirow[t]{2}{*}{17} & $7-31-2002$ & 10.5 & 6.9 & 28 & 220.0 & 86.7 & 29 & -- & $<0.04$ & $22.9-91.2$ & 33 \\
\hline & $9-10-2002$ & -- & -- & -- & -- & -- & -- & -- & $<0.04$ & 22.9-91.2 & 33 \\
\hline \multirow[t]{2}{*}{18} & $7-31-2002$ & 9.9 & ID & 4 & 234.0 & ID & 5 & -- & 0.02 & ID & 16 \\
\hline & $9-10-2002$ & -- & -- & -- & -- & -- & -- & -- & $<0.04$ & ID & 16 \\
\hline \multirow[t]{2}{*}{19} & $7-30-2002$ & 16.3 & ID & 1 & 93.9 & ID & 1 & -- & $<0.015$ & ID & 9 \\
\hline & $9-9-2002$ & -- & -- & -- & -- & -- & -- & -- & $<0.015$ & ID & 9 \\
\hline
\end{tabular}


Table 11. Water-quality data for the Yampa and White River Basins, showing 2002 drought values, Colorado water-quality exceedances, and percentiles relative to historical data for selected properties and constituents from July through September, 1978-2002.-Continued

[ft $3 / \mathrm{s}$, cubic feet per second; Conc, concentration; $\mathrm{Q} \%$, percentile (percentage of data less than or equal to reported value or concentration) of droughtsample constituent; $\mathrm{N}$, total number of historical and drought samples; $\mathrm{mg} / \mathrm{L}$, milligrams per liter; $\mu \mathrm{S} / \mathrm{cm}$, microsiemens per centimeter at $25^{\circ} \mathrm{Celsius}$; ${ }^{\circ} \mathrm{C}$, degrees Celsius; E. coli, Escherichia coli; $\mathrm{mL}$, milliliter; $\mu \mathrm{g} / \mathrm{L}$, micrograms per liter; $\mathrm{CaCO}_{3}$, calcium carbonate; $\mathrm{SiO}_{2}$, silica; $\mathrm{SO}_{4}$, sulfate; ID, insufficient number of samples (less than 20) to calculate percentile; <, less than; --, no drought-sample measurement; values or concentrations in bold type indicate 2002 Colorado water-quality exceedances; c, chronic water-quality exceedance]

\begin{tabular}{|c|c|c|c|c|c|c|c|c|c|c|}
\hline \multirow{2}{*}{$\begin{array}{l}\text { Site number } \\
\text { in table } 1 \\
\text { and fig. } 4\end{array}$} & \multirow{2}{*}{$\begin{array}{l}\text { Sampling } \\
\text { date }\end{array}$} & \multicolumn{3}{|c|}{$\begin{array}{l}\text { Nitrogen, organic plus ammonia, } \\
\text { dissolved, in } \mathrm{mg} / \mathrm{L} \text { as } \mathrm{N}\end{array}$} & \multicolumn{3}{|c|}{$\begin{array}{l}\text { Nitrogen, organic plus ammonia, } \\
\text { total recoverable, in } \mathrm{mg} / \mathrm{L} \text { as } \mathrm{N}\end{array}$} & \multicolumn{3}{|c|}{$\begin{array}{l}\text { Nitrogen, nitrite plus nitrate, dissolved, } \\
\text { in } \mathrm{mg} / \mathrm{L} \text { as } \mathrm{N}\end{array}$} \\
\hline & & Conc & $0 \%$ & $\mathrm{~N}$ & Conc & $0 \%$ & $\mathrm{~N}$ & Conc & $0 \%$ & $\mathrm{~N}$ \\
\hline & & \multicolumn{9}{|c|}{ Yampa River Basin_-Continued } \\
\hline \multirow[t]{2}{*}{1} & $7-29-2002$ & 0.13 & ID & 7 & -- & -- & -- & 0.072 & ID & 7 \\
\hline & $9-3-2002$ & 0.23 & ID & 7 & -- & -- & -- & 0.090 & ID & 7 \\
\hline 2 & $7-29-2002$ & 0.42 & ID & 7 & 0.73 & ID & 8 & $<0.013$ & ID & 9 \\
\hline \multirow[t]{2}{*}{3} & $7-30-2002$ & 0.29 & ID & 7 & -- & -- & -- & $<0.013$ & ID & 7 \\
\hline & 9-3-2002 & 0.23 & ID & 7 & -- & -- & -- & $<0.013$ & ID & 7 \\
\hline 4 & $7-30-2002$ & -- & -- & -- & 0.55 & ID & 19 & 0.030 & ID & 19 \\
\hline 5 & $7-30-2002$ & -- & -- & -- & 0.54 & ID & 16 & $<0.05$ & ID & 8 \\
\hline \multirow[t]{2}{*}{6} & $7-30-2002$ & 0.33 & ID & 11 & -- & -- & -- & $<0.05$ & ID & 11 \\
\hline & $9-3-2002$ & 0.33 & ID & 11 & -- & -- & -- & $<0.013$ & ID & 11 \\
\hline \multirow[t]{2}{*}{7} & $7-31-2002$ & 0.46 & ID & 12 & -- & -- & -- & $<0.1$ & ${ }^{2} 3.8-92.3$ & 25 \\
\hline & $9-4-2002$ & -- & -- & -- & 0.59 & 44.0 & 24 & $<0.1$ & ${ }^{2} 3.8-92.3$ & 25 \\
\hline \multirow[t]{3}{*}{8} & $7-31-2002$ & -- & -- & -- & 0.39 & ID & 6 & $<0.05$ & ID & 9 \\
\hline & $9-4-2002$ & 0.28 & ID & 6 & -- & -- & -- & $<0.05$ & ID & 9 \\
\hline & & \multicolumn{9}{|c|}{ White River Basin—Continued } \\
\hline \multirow[t]{2}{*}{9} & $7-29-2002$ & 0.11 & ${ }^{2} 4.5-68.2$ & 21 & -- & -- & -- & $<0.1$ & ${ }^{2} 4.3-91.3$ & 22 \\
\hline & $9-9-2002$ & -- & -- & -- & 0.13 & ID & 16 & $<0.1$ & ${ }^{2} 4.3-91.3$ & 22 \\
\hline \multirow[t]{2}{*}{10} & $7-29-2002$ & 0.11 & ${ }^{2} 4.8-76.2$ & 20 & -- & -- & -- & $<0.1$ & ${ }^{2} 4.5-90.9$ & 21 \\
\hline & $9-9-2002$ & -- & -- & -- & 0.11 & ID & 16 & $<0.1$ & ${ }^{2} 4.5-90.9$ & 21 \\
\hline \multirow[t]{2}{*}{11} & $7-30-2002$ & 0.18 & 81.0 & 20 & -- & -- & -- & $<0.1$ & ${ }^{2} 4.5-90.9$ & 21 \\
\hline & $9-10-2002$ & -- & -- & -- & 0.20 & ID & 17 & $<0.1$ & ${ }^{2} 4.5-90.9$ & 21 \\
\hline \multirow[t]{2}{*}{12} & $7-30-2002$ & -- & -- & -- & 0.46 & ${ }^{1} 70.0-73.3$ & 29 & $<0.1$ & ${ }^{2} 4.0-92.0$ & 24 \\
\hline & $9-10-2002$ & -- & -- & -- & 0.52 & 83.3 & 29 & $<0.1$ & ${ }^{2} 4.0-92.0$ & 24 \\
\hline 13 & $9-11-2002$ & 0.35 & 28.6 & 27 & -- & -- & -- & $<0.1$ & ${ }^{2} 2.5-42.5$ & 39 \\
\hline 14 & $7-31-2002$ & 0.69 & 61.9 & 20 & -- & -- & -- & $<0.05$ & ${ }^{2} 3.1-34.4$ & 31 \\
\hline 15 & $9-11-2002$ & 0.29 & ID & 17 & -- & -- & -- & 0.280 & 42.9 & 20 \\
\hline 16 & $7-31-2002$ & 0.66 & ID & 19 & -- & -- & -- & $<0.05$ & 4.8 & 20 \\
\hline \multirow[t]{2}{*}{17} & $7-31-2002$ & 0.37 & 81.8 & 32 & -- & -- & -- & $<0.05$ & ${ }^{2} 3.3-56.7$ & 29 \\
\hline & $9-10-2002$ & -- & -- & -- & 0.88 & ${ }^{1} 85.2-88.9$ & 26 & $<0.05$ & ${ }^{2} 3.3-56.7$ & 29 \\
\hline \multirow[t]{2}{*}{18} & $7-31-2002$ & 0.46 & ID & 15 & -- & -- & -- & 0.030 & ID & 16 \\
\hline & $9-10-2002$ & -- & -- & -- & 0.53 & ID & 15 & $<0.05$ & ID & 16 \\
\hline \multirow[t]{2}{*}{19} & $7-30-2002$ & 0.12 & ID & 8 & -- & -- & -- & $<0.013$ & ID & 9 \\
\hline & $9-9-2002$ & -- & -- & -- & 0.18 & ID & 8 & $<0.013$ & ID & 9 \\
\hline
\end{tabular}


Table 11. Water-quality data for the Yampa and White River Basins, showing 2002 drought values, Colorado water-quality exceedances, and percentiles relative to historical data for selected properties and constituents from July through September, 1978-2002.-Continued

[ft $\mathrm{ft}^{3} / \mathrm{s}$, cubic feet per second; Conc, concentration; Q\%, percentile (percentage of data less than or equal to reported value or concentration) of droughtsample constituent; $\mathrm{N}$, total number of historical and drought samples; $\mathrm{mg} / \mathrm{L}$, milligrams per liter; $\mu \mathrm{S} / \mathrm{cm}$, microsiemens per centimeter at $25^{\circ} \mathrm{Celsius;}$ ${ }^{\circ} \mathrm{C}$, degrees Celsius; E. coli, Escherichia coli; $\mathrm{mL}$, milliliter; $\mu \mathrm{g} / \mathrm{L}$, micrograms per liter; $\mathrm{CaCO}_{3}$, calcium carbonate; $\mathrm{SiO}_{2}$, silica; $\mathrm{SO}_{4}$, sulfate; ID, insufficient number of samples (less than 20) to calculate percentile; <, less than; --, no drought-sample measurement; values or concentrations in bold type indicate 2002 Colorado water-quality exceedances; c, chronic water-quality exceedance]

\begin{tabular}{|c|c|c|c|c|c|c|c|c|c|c|}
\hline \multirow{2}{*}{$\begin{array}{c}\text { Site number } \\
\text { in table } 1 \\
\text { and fig. } 4\end{array}$} & \multirow{2}{*}{$\begin{array}{l}\text { Sampling } \\
\text { date }\end{array}$} & \multicolumn{3}{|c|}{$\begin{array}{c}\text { Nitrogen, nitrite, dissolved, in } \\
\mathrm{mg} / \mathrm{L} \text { as } \mathrm{N}\end{array}$} & \multicolumn{3}{|c|}{ Phosphorus, dissolved, in $\mathrm{mg} / \mathrm{L}$ as $\mathrm{P}$} & \multicolumn{3}{|c|}{$\begin{array}{c}\text { Phosphorus, total recoverable, } \\
\text { in } \mathrm{mg} / \mathrm{L} \text { as } \mathrm{P}\end{array}$} \\
\hline & & Conc & $0 \%$ & $\mathrm{~N}$ & Conc & $0 \%$ & $\mathrm{~N}$ & Conc & $0 \%$ & $\mathrm{~N}$ \\
\hline & & \multicolumn{9}{|c|}{ Yampa River Basin—Continued } \\
\hline \multirow[t]{2}{*}{1} & $7-29-2002$ & $<0.002$ & ID & 7 & 0.002 & ID & 7 & 0.006 & ID & 2 \\
\hline & 9-3-2002 & $<0.002$ & ID & 7 & 0.005 & ID & 7 & 0.008 & ID & 2 \\
\hline 2 & $7-29-2002$ & 0.002 & ID & 7 & 0.077 & ID & 7 & 0.121 & ID & 8 \\
\hline \multirow[t]{2}{*}{3} & $7-30-2002$ & $<0.002$ & ID & 7 & 0.008 & ID & 7 & 0.019 & ID & 6 \\
\hline & $9-3-2002$ & $<0.002$ & ID & 7 & 0.004 & ID & 7 & 0.009 & ID & 6 \\
\hline 4 & $7-30-2002$ & $<0.008$ & ID & 19 & -- & -- & -- & 0.032 & ID & 19 \\
\hline 5 & $7-30-2002$ & $<0.008$ & ID & 8 & -- & -- & -- & 0.063 & ID & 16 \\
\hline \multirow[t]{2}{*}{6} & $7-30-2002$ & $<0.008$ & ID & 11 & $<0.06$ & ID & 11 & $<0.06$ & ID & 11 \\
\hline & 9-3-2002 & $<0.002$ & ID & 11 & 0.005 & ID & 11 & 0.033 & ID & 11 \\
\hline \multirow[t]{2}{*}{7} & $7-31-2002$ & $<0.008$ & ID & 13 & $<0.06$ & ${ }^{2} 3.6-89.3$ & 27 & 0.040 & ${ }^{1} 57.7-61.5$ & 25 \\
\hline & 9-4-2002 & $<0.008$ & ID & 13 & -- & -- & -- & 0.044 & 65.4 & 25 \\
\hline \multirow[t]{3}{*}{8} & $7-31-2002$ & $<0.008$ & ID & 7 & -- & -- & -- & 0.035 & ID & 7 \\
\hline & 9-4-2002 & $<0.008$ & ID & 7 & $<0.06$ & ID & 6 & 0.040 & ID & 7 \\
\hline & & \multicolumn{9}{|c|}{ White River Basin—Continued } \\
\hline \multirow[t]{2}{*}{9} & $7-29-2002$ & $<0.01$ & ${ }^{2} 4.3-87.0$ & 22 & -- & -- & -- & 0.022 & ID & 17 \\
\hline & 9-9-2002 & $<0.01$ & ${ }^{2} 4.3-87.0$ & 22 & 0.017 & 63.6 & 21 & 0.023 & ID & 17 \\
\hline \multirow[t]{2}{*}{10} & $7-29-2002$ & $<0.01$ & ${ }^{2} 4.5-90.9$ & 21 & 0.013 & 81.0 & 20 & 0.016 & ID & 17 \\
\hline & $9-9-2002$ & $<0.01$ & ${ }^{2} 4.5-90.9$ & 21 & -- & -- & -- & 0.011 & ID & 17 \\
\hline \multirow[t]{2}{*}{11} & $7-30-2002$ & $<0.01$ & ${ }^{2} 4.5-90.9$ & 21 & 0.058 & 90.5 & 20 & 0.056 & ID & 18 \\
\hline & $9-10-2002$ & $<0.01$ & ${ }^{2} 4.5-90.9$ & 21 & -- & -- & -- & 0.024 & ID & 18 \\
\hline \multirow[t]{2}{*}{12} & $7-30-2002$ & $<0.01$ & ${ }^{2} 4.2-91.7$ & 23 & -- & -- & -- & 0.053 & ${ }^{1} 70.0-73.3$ & 29 \\
\hline & $9-10-2002$ & $<0.01$ & ${ }^{2} 4.2--91.7$ & 23 & -- & -- & -- & 0.053 & ${ }^{1} 70.0-73.3$ & 29 \\
\hline 13 & $9-11-2002$ & $<0.008$ & 67.7 & 30 & 0.030 & 50.0 & 27 & 0.060 & ID & 10 \\
\hline 14 & $7-31-2002$ & $<0.02$ & ${ }^{2} 4.2-79.2$ & 23 & $<0.05$ & ${ }^{2} 4.8-57.1$ & 20 & 0.058 & ID & 10 \\
\hline 15 & $9-11-2002$ & 0.005 & ID & 15 & $<0.06$ & ID & 15 & 0.198 & ID & 2 \\
\hline 16 & $7-31-2002$ & $<0.008$ & ID & 15 & $<0.06$ & ID & 16 & 0.024 & ID & 4 \\
\hline \multirow[t]{2}{*}{17} & $7-31-2002$ & $<0.01$ & ${ }^{2} 2.9-85.3$ & 33 & $<0.06$ & ${ }^{2} 2.9-97.1$ & 33 & 0.030 & 31.0 & 28 \\
\hline & $9-10-2002$ & $<0.01$ & ${ }^{2} 2.9-85.3$ & 33 & -- & -- & -- & 0.166 & 79.3 & 28 \\
\hline \multirow[t]{2}{*}{18} & $7-31-2002$ & 0.007 & ID & 16 & $<0.06$ & ID & 15 & $<0.06$ & ID & 16 \\
\hline & $9-10-2002$ & $<0.008$ & ID & 16 & -- & -- & -- & 0.026 & ID & 16 \\
\hline \multirow[t]{2}{*}{19} & $7-30-2002$ & $<0.002$ & ID & 9 & 0.014 & ID & 8 & 0.035 & ID & 9 \\
\hline & $9-9-2002$ & $<0.002$ & ID & 9 & -- & -- & -- & 0.023 & ID & 9 \\
\hline
\end{tabular}


Table 11. Water-quality data for the Yampa and White River Basins, showing 2002 drought values, Colorado water-quality exceedances, and percentiles relative to historical data for selected properties and constituents from July through September, 1978-2002.-Continued

[ft $\mathrm{t}^{3} / \mathrm{s}$, cubic feet per second; Conc, concentration; Q\%, percentile (percentage of data less than or equal to reported value or concentration) of droughtsample constituent; $\mathrm{N}$, total number of historical and drought samples; $\mathrm{mg} / \mathrm{L}$, milligrams per liter; $\mu \mathrm{S} / \mathrm{cm}$, microsiemens per centimeter at $25^{\circ} \mathrm{Celsius}$; ${ }^{\circ} \mathrm{C}$, degrees Celsius; E. coli, Escherichia coli; $\mathrm{mL}$, milliliter; $\mu \mathrm{g} / \mathrm{L}$, micrograms per liter; $\mathrm{CaCO}_{3}$, calcium carbonate; $\mathrm{SiO}_{2}$, silica; $\mathrm{SO}_{4}$, sulfate; $\mathrm{ID}$, insufficient number of samples (less than 20) to calculate percentile; <, less than; --, no drought-sample measurement; values or concentrations in bold type indicate 2002 Colorado water-quality exceedances; c, chronic water-quality exceedance]

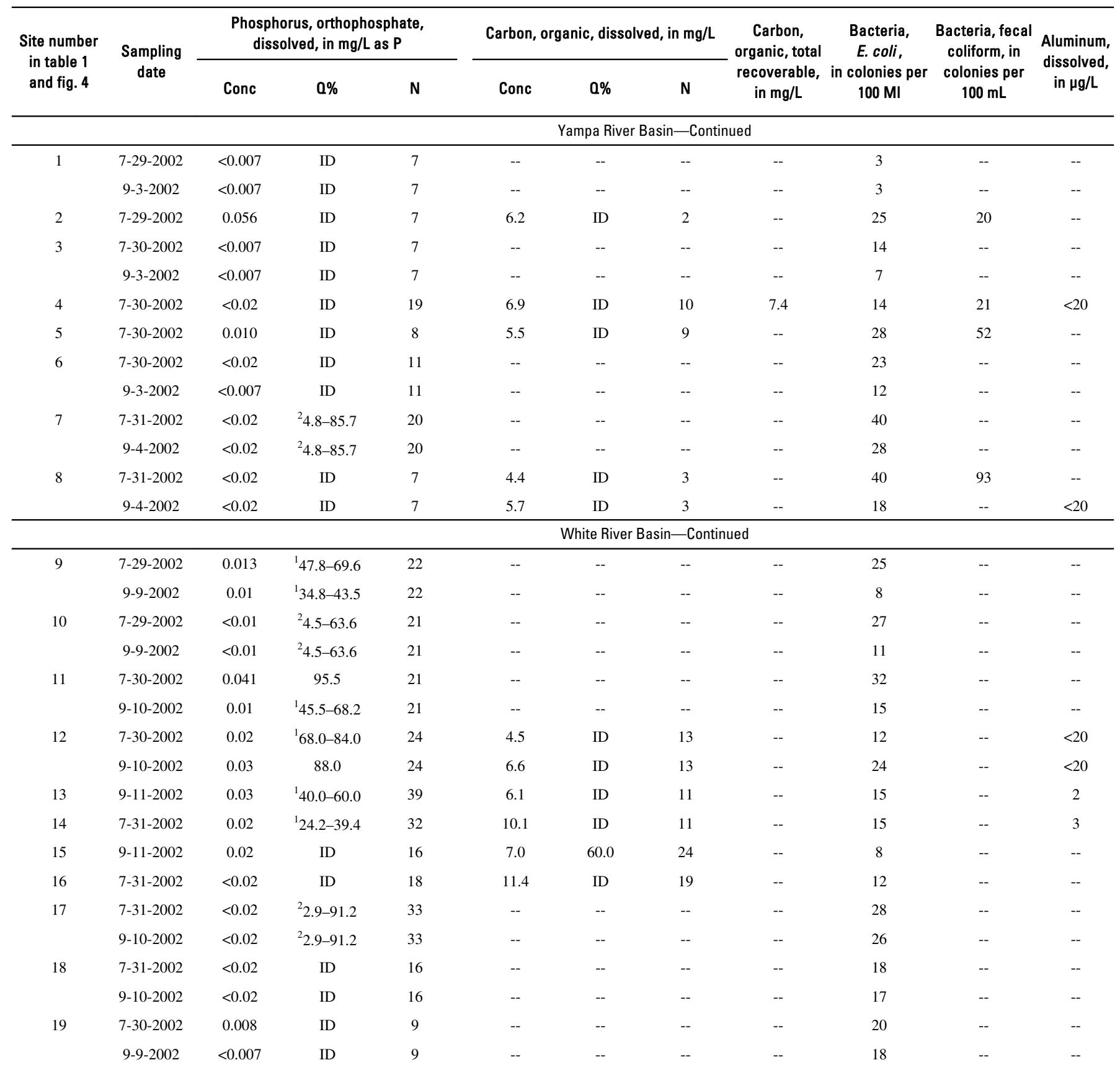


Table 11. Water-quality data for the Yampa and White River Basins, showing 2002 drought values, Colorado water-quality exceedances, and percentiles relative to historical data for selected properties and constituents from July through September, 1978-2002.-Continued

$[\mathrm{ft} / \mathrm{s}$, cubic feet per second; Conc, concentration; $\mathrm{Q} \%$, percentile (percentage of data less than or equal to reported value or concentration) of droughtsample constituent; $\mathrm{N}$, total number of historical and drought samples; $\mathrm{mg} / \mathrm{L}$, milligrams per liter; $\mu \mathrm{S} / \mathrm{cm}$, microsiemens per centimeter at $25^{\circ} \mathrm{Celsius;}$ ${ }^{\circ} \mathrm{C}$, degrees Celsius; E. coli, Escherichia coli; $\mathrm{mL}$, milliliter; $\mu \mathrm{g} / \mathrm{L}$, micrograms per liter; $\mathrm{CaCO}_{3}$, calcium carbonate; $\mathrm{SiO}_{2}$, silica; $\mathrm{SO}{ }_{4}, \mathrm{sulfate} ; \mathrm{ID}$, insufficient number of samples (less than 20) to calculate percentile; <, less than; --, no drought-sample measurement; values or concentrations in bold type indicate 2002 Colorado water-quality exceedances; c, chronic water-quality exceedance]

\begin{tabular}{|c|c|c|c|c|c|c|c|c|c|c|c|c|}
\hline \multirow{2}{*}{$\begin{array}{c}\text { Site number } \\
\text { in table } 1 \\
\text { and fig. } 4\end{array}$} & \multirow{2}{*}{$\begin{array}{c}\text { Sampling } \\
\text { date }\end{array}$} & \multirow{2}{*}{$\begin{array}{c}\text { Arsenic, } \\
\text { dissolved, } \\
\text { in } \mu \mathrm{g} / \mathrm{L}\end{array}$} & \multicolumn{3}{|c|}{ Boron, dissolved, in $\mu \mathrm{g} / \mathrm{L}$} & \multirow{2}{*}{$\begin{array}{l}\text { Cadmium, } \\
\text { dissolved, } \\
\text { in } \mu \mathrm{g} / \mathrm{L}\end{array}$} & \multirow{2}{*}{$\begin{array}{c}\text { Chromium, } \\
\text { dissolved, } \\
\text { in } \mu \mathrm{g} / \mathrm{L}\end{array}$} & \multirow{2}{*}{$\begin{array}{c}\text { Copper, } \\
\text { dissolved, } \\
\text { in } \mu \mathrm{g} / \mathrm{L}\end{array}$} & \multicolumn{3}{|c|}{ Iron, dissolved, in $\mu \mathrm{g} / \mathrm{L}$} & \multirow{2}{*}{$\begin{array}{l}\text { Iron, total } \\
\text { recoverable, } \\
\text { in } \mu \mathrm{g} / \mathrm{L}\end{array}$} \\
\hline & & & Conc & $0 \%$ & $\mathrm{~N}$ & & & & Conc & $0 \%$ & $\mathrm{~N}$ & \\
\hline & & \multicolumn{11}{|c|}{ Yampa River Basin-Continued } \\
\hline \multirow[t]{2}{*}{1} & $7-29-2002$ & -- & -- & -- & -- & -- & -- & -- & 74 & ID & 3 & -- \\
\hline & $9-3-2002$ & -- & -- & -- & -- & -- & -- & -- & 221 & ID & 3 & -- \\
\hline 2 & $7-29-2002$ & -- & -- & -- & -- & $<0.1$ & -- & 1.4 & -- & -- & -- & 370 \\
\hline \multirow[t]{2}{*}{3} & $7-30-2002$ & -- & -- & -- & -- & -- & -- & -- & 69 & ID & 3 & -- \\
\hline & $9-3-2002$ & -- & -- & -- & -- & -- & -- & -- & 74 & ID & 3 & -- \\
\hline 4 & $7-30-2002$ & -- & -- & -- & -- & $<0.04$ & -- & 1.7 & 14 & ID & -- & -- \\
\hline 5 & $7-30-2002$ & -- & -- & -- & -- & $<0.2$ & -- & 0.6 & -- & -- & -- & 190 \\
\hline \multirow[t]{2}{*}{6} & $7-30-2002$ & -- & -- & -- & -- & -- & -- & -- & 10 & ID & 10 & -- \\
\hline & $9-3-2002$ & -- & -- & -- & -- & -- & -- & -- & 8 & ID & 10 & -- \\
\hline \multirow[t]{2}{*}{7} & $7-31-2002$ & -- & -- & -- & -- & -- & -- & -- & $<10$ & ${ }^{2} 4.5-27.3$ & 21 & -- \\
\hline & $9-4-2002$ & -- & -- & -- & -- & -- & -- & -- & -- & -- & -- & -- \\
\hline \multirow[t]{2}{*}{8} & $7-31-2002$ & -- & -- & -- & -- & $<0.2$ & -- & 0.7 & -- & -- & -- & 330 \\
\hline & $9-4-2002$ & -- & -- & -- & -- & $<0.04$ & -- & 1.7 & $<10$ & ID & 1 & -- \\
\hline & & \multicolumn{11}{|c|}{ White River Basin—Continued } \\
\hline \multirow[t]{2}{*}{9} & $7-29-2002$ & -- & -- & -- & -- & -- & -- & -- & -- & -- & -- & -- \\
\hline & $9-9-2002$ & -- & -- & -- & -- & -- & -- & -- & 6 & ID & 5 & -- \\
\hline \multirow[t]{2}{*}{10} & $7-29-2002$ & -- & -- & -- & -- & -- & -- & -- & 9 & ID & 4 & -- \\
\hline & $9-9-2002$ & -- & -- & -- & -- & -- & -- & -- & -- & -- & -- & -- \\
\hline \multirow[t]{2}{*}{11} & $7-30-2002$ & -- & -- & -- & -- & -- & -- & -- & 18 & ID & 5 & -- \\
\hline & $9-10-2002$ & -- & -- & -- & -- & -- & -- & -- & -- & -- & -- & -- \\
\hline \multirow[t]{2}{*}{12} & $7-30-2002$ & -- & -- & -- & -- & 0.20 & -- & 0.7 & 15 & ID & 17 & -- \\
\hline & $9-10-2002$ & -- & -- & -- & -- & $<0.04$ & -- & 1.8 & 23 & ID & 17 & -- \\
\hline 13 & $9-11-2002$ & 2 & 210 & ${ }^{1} 35-37.5$ & 39 & 0.13 & $<0.8$ & 2.6 & 14 & 50.0 & 23 & -- \\
\hline 14 & $7-31-2002$ & 7 & 700 & 83.3 & 35 & 0.05 & $<0.8$ & 3.1 & $<30$ & ${ }^{2} 4.3-60.9$ & 22 & -- \\
\hline 15 & $9-11-2002$ & -- & 100 & ${ }^{1} 3.6-17.9$ & 27 & -- & -- & -- & -- & -- & -- & -- \\
\hline 16 & $7-31-2002$ & 7 & 650 & ${ }^{1} 62.1-65.5$ & 28 & -- & -- & -- & 11 & ID & 14 & -- \\
\hline \multirow[t]{2}{*}{17} & $7-31-2002$ & -- & -- & -- & -- & -- & -- & -- & 17 & 70.8 & 23 & -- \\
\hline & $9-10-2002$ & -- & -- & -- & -- & -- & -- & -- & -- & -- & -- & -- \\
\hline \multirow[t]{2}{*}{18} & $7-31-2002$ & -- & -- & -- & -- & -- & -- & -- & $<10$ & ID & 3 & -- \\
\hline & $9-10-2002$ & -- & -- & -- & -- & -- & -- & -- & -- & -- & -- & -- \\
\hline \multirow[t]{2}{*}{19} & $7-30-2002$ & -- & -- & -- & -- & -- & -- & -- & 6 & ID & 1 & -- \\
\hline & $9-9-2002$ & -- & -- & -- & -- & -- & -- & -- & -- & -- & -- & -- \\
\hline
\end{tabular}


Table 11. Water-quality data for the Yampa and White River Basins, showing 2002 drought values, Colorado water-quality exceedances, and percentiles relative to historical data for selected properties and constituents from July through September, 1978-2002.-Continued

[ft $3 / \mathrm{s}$, cubic feet per second; Conc, concentration; Q\%, percentile (percentage of data less than or equal to reported value or concentration) of droughtsample constituent; $\mathrm{N}$, total number of historical and drought samples; $\mathrm{mg} / \mathrm{L}$, milligrams per liter; $\mu \mathrm{S} / \mathrm{cm}$, microsiemens per centimeter at $25^{\circ} \mathrm{Celsius}$; ${ }^{\circ} \mathrm{C}$, degrees Celsius; E. coli, Escherichia coli; $\mathrm{mL}$, milliliter; $\mu \mathrm{g} / \mathrm{L}$, micrograms per liter; $\mathrm{CaCO}_{3}$, calcium carbonate; $\mathrm{SiO}_{2}, \mathrm{silica}_{3} \mathrm{SO}_{4}$, sulfate; ID, insufficient number of samples (less than 20) to calculate percentile; <, less than; --, no drought-sample measurement; values or concentrations in bold type indicate 2002 Colorado water-quality exceedances; c, chronic water-quality exceedance]

\begin{tabular}{|c|c|c|c|c|c|c|c|c|c|}
\hline \multirow{2}{*}{$\begin{array}{l}\text { Site number } \\
\text { in table } 1 \\
\text { and fig. } 4\end{array}$} & \multirow{2}{*}{$\begin{array}{c}\text { Sampling } \\
\text { date }\end{array}$} & \multirow{2}{*}{$\begin{array}{l}\text { Lead, } \\
\text { dissolved, } \\
\text { in } \mu \mathrm{g} / \mathrm{L}\end{array}$} & \multicolumn{3}{|c|}{ Manganese, dissolved, in $\mu \mathrm{g} / \mathrm{L}$} & \multirow{2}{*}{$\begin{array}{l}\text { Manganese, } \\
\text { total } \\
\text { recoverable, } \\
\text { in } \mu \mathrm{g} / \mathrm{L}\end{array}$} & \multirow{2}{*}{$\begin{array}{l}\text { Mercury, } \\
\text { dissolved, } \\
\text { in } \mu \mathrm{g} / \mathrm{L}\end{array}$} & \multirow{2}{*}{$\begin{array}{l}\text { Molybdenum, } \\
\text { dissolved, } \\
\text { in } \mu \mathrm{g} / \mathrm{L}\end{array}$} & \multirow{2}{*}{$\begin{array}{c}\text { Nickel, } \\
\text { dissolved, } \\
\text { in } \mu \mathrm{g} / \mathrm{L}\end{array}$} \\
\hline & & & Conc & $0 \%$ & $\mathrm{~N}$ & & & & \\
\hline & & \multicolumn{8}{|c|}{ Yampa River Basin—Continued } \\
\hline \multirow[t]{2}{*}{1} & $7-29-2002$ & -- & -- & -- & -- & -- & -- & -- & -- \\
\hline & $9-3-2002$ & -- & -- & -- & -- & -- & -- & -- & -- \\
\hline 2 & $7-29-2002$ & $<1$ & 26.6 & ID & 9 & 88.1 & $<0.01$ & -- & -- \\
\hline \multirow[t]{2}{*}{3} & $7-30-2002$ & -- & -- & -- & -- & -- & -- & -- & -- \\
\hline & $9-3-2002$ & -- & -- & -- & -- & -- & -- & -- & -- \\
\hline 4 & $7-30-2002$ & 0.1 & 23.7 & ID & 7 & -- & -- & -- & -- \\
\hline 5 & $7-30-2002$ & $<1$ & 15.4 & ID & 15 & 71.3 & $<0.01$ & -- & -- \\
\hline \multirow[t]{2}{*}{6} & $7-30-2002$ & -- & -- & -- & -- & -- & -- & -- & -- \\
\hline & $9-3-2002$ & -- & -- & -- & -- & -- & -- & -- & -- \\
\hline \multirow[t]{2}{*}{7} & $7-31-2002$ & -- & -- & -- & -- & -- & -- & -- & -- \\
\hline & $9-4-2002$ & -- & -- & -- & -- & -- & -- & -- & -- \\
\hline \multirow[t]{3}{*}{8} & $7-31-2002$ & $<1$ & 4.6 & ID & 7 & 50.4 & $<0.01$ & -- & -- \\
\hline & 9-4-2002 & $<0.08$ & $65 \mathrm{c}$ & ID & 7 & -- & -- & -- & -- \\
\hline & & \multicolumn{8}{|c|}{ White River Basin—Continued } \\
\hline \multirow[t]{2}{*}{9} & $7-29-2002$ & -- & -- & -- & -- & -- & -- & -- & -- \\
\hline & $9-9-2002$ & -- & -- & -- & -- & -- & -- & -- & -- \\
\hline \multirow[t]{2}{*}{10} & $7-29-2002$ & -- & -- & -- & -- & -- & -- & -- & -- \\
\hline & $9-9-2002$ & -- & -- & -- & -- & -- & -- & -- & -- \\
\hline \multirow[t]{2}{*}{11} & $7-30-2002$ & -- & -- & -- & -- & -- & -- & -- & -- \\
\hline & $9-10-2002$ & -- & -- & -- & -- & -- & -- & -- & -- \\
\hline \multirow[t]{2}{*}{12} & $7-30-2002$ & $<1$ & 32.1 & ID & 10 & -- & -- & -- & -- \\
\hline & $9-10-2002$ & 0.1 & 21.9 & ID & 10 & -- & -- & -- & -- \\
\hline 13 & $9-11-2002$ & 0.1 & 47.8 & 47.8 & 22 & -- & 0.01 & 7.6 & 5.2 \\
\hline 14 & $7-31-2002$ & $<0.2$ & 1.3 & ${ }^{2} 4.3-65.2$ & 22 & -- & $<0.06$ & 10.3 & 1.2 \\
\hline 15 & $9-11-2002$ & -- & -- & -- & -- & -- & -- & -- & -- \\
\hline 16 & $7-31-2002$ & -- & 1.5 & ID & 14 & -- & -- & 27.3 & 1.0 \\
\hline \multirow[t]{2}{*}{17} & $7-31-2002$ & -- & -- & -- & -- & -- & -- & -- & -- \\
\hline & $9-10-2002$ & -- & -- & -- & -- & -- & -- & -- & -- \\
\hline \multirow[t]{2}{*}{18} & $7-31-2002$ & -- & -- & -- & -- & -- & -- & -- & -- \\
\hline & $9-10-2002$ & -- & -- & -- & -- & -- & -- & -- & -- \\
\hline \multirow[t]{2}{*}{19} & $7-30-2002$ & -- & -- & -- & -- & -- & -- & -- & -- \\
\hline & $9-9-2002$ & -- & -- & -- & -- & -- & -- & -- & -- \\
\hline
\end{tabular}


Table 11. Water-quality data for the Yampa and White River Basins, showing 2002 drought values, Colorado water-quality exceedances, and percentiles relative to historical data for selected properties and constituents from July through September, 1978-2002.-Continued

[ $\left[\mathrm{ft}^{3} / \mathrm{s}\right.$, cubic feet per second; Conc, concentration; $\mathrm{Q} \%$, percentile (percentage of data less than or equal to reported value or concentration) of droughtsample constituent; $\mathrm{N}$, total number of historical and drought samples; $\mathrm{mg} / \mathrm{L}$, milligrams per liter; $\mu \mathrm{S} / \mathrm{cm}$, microsiemens per centimeter at $25^{\circ} \mathrm{Celsius;}$ ${ }^{\circ} \mathrm{C}$, degrees Celsius; E. coli, Escherichia coli; $\mathrm{mL}$, milliliter; $\mu \mathrm{g} / \mathrm{L}$, micrograms per liter; $\mathrm{CaCO}_{3}$, calcium carbonate; $\mathrm{SiO}_{2}, \mathrm{silica}_{\mathrm{S}} \mathrm{SO}_{4}$, sulfate; ID, insufficient number of samples (less than 20) to calculate percentile; <, less than; --, no drought-sample measurement; values or concentrations in bold type indicate 2002 Colorado water-quality exceedances; c, chronic water-quality exceedance]

\begin{tabular}{|c|c|c|c|c|c|c|c|c|}
\hline \multirow{2}{*}{$\begin{array}{c}\text { Site number } \\
\text { in table } 1 \\
\text { and fig. } 4\end{array}$} & \multirow{2}{*}{$\begin{array}{l}\text { Sampling } \\
\text { date }\end{array}$} & \multicolumn{3}{|c|}{ Selenium, dissolved, in $\mu \mathrm{g} / \mathrm{L}$} & \multirow{2}{*}{$\begin{array}{c}\text { Silver, } \\
\text { dissolved, } \\
\text { in } \mu \mathrm{g} / \mathrm{L}\end{array}$} & \multicolumn{3}{|c|}{ Zinc, dissolved, in $\mu \mathrm{g} / \mathrm{L}$} \\
\hline & & Conc & $0 \%$ & $\mathrm{~N}$ & & Conc & $0 \%$ & $\mathrm{~N}$ \\
\hline & & \multicolumn{7}{|c|}{ Yampa River Basin—Continued } \\
\hline \multirow[t]{2}{*}{1} & $7-29-2002$ & -- & -- & -- & -- & -- & -- & -- \\
\hline & 9-3-2002 & -- & -- & -- & -- & -- & -- & -- \\
\hline 2 & $7-29-2002$ & $<2$ & ID & 10 & $<0.1$ & $<24$ & ID & 9 \\
\hline \multirow[t]{2}{*}{3} & $7-30-2002$ & -- & -- & -- & -- & -- & -- & -- \\
\hline & 9-3-2002 & -- & -- & -- & -- & -- & -- & -- \\
\hline 4 & $7-30-2002$ & -- & -- & -- & -- & $<1$ & ID & 5 \\
\hline 5 & $7-30-2002$ & $<2$ & ID & 17 & $<0.2$ & $<24$ & ID & 13 \\
\hline \multirow[t]{2}{*}{6} & $7-30-2002$ & -- & -- & -- & -- & -- & -- & -- \\
\hline & $9-3-2002$ & -- & -- & -- & -- & -- & -- & -- \\
\hline \multirow[t]{2}{*}{7} & $7-31-2002$ & 0.7 & ${ }^{1} 69.0-72.4$ & 28 & -- & -- & -- & -- \\
\hline & $9-4-2002$ & 1.0 & ${ }^{1} 75.9-86.2$ & 28 & -- & -- & -- & -- \\
\hline \multirow[t]{3}{*}{8} & $7-31-2002$ & $<2$ & ID & 7 & $<0.2$ & $<24$ & ID & 7 \\
\hline & $9-4-2002$ & -- & -- & -- & -- & 1.0 & ID & 7 \\
\hline & & \multicolumn{7}{|c|}{ White River Basin-Continued } \\
\hline \multirow[t]{2}{*}{9} & $7-29-2002$ & -- & -- & -- & -- & -- & -- & -- \\
\hline & $9-9-2002$ & -- & -- & -- & -- & -- & -- & -- \\
\hline \multirow[t]{2}{*}{10} & $7-29-2002$ & -- & -- & -- & -- & -- & -- & -- \\
\hline & $9-9-2002$ & -- & -- & -- & -- & -- & -- & -- \\
\hline \multirow[t]{2}{*}{11} & $7-30-2002$ & -- & -- & -- & -- & -- & -- & -- \\
\hline & $9-10-2002$ & -- & -- & -- & -- & -- & -- & -- \\
\hline \multirow[t]{2}{*}{12} & $7-30-2002$ & -- & -- & -- & -- & $<24$ & ID & 6 \\
\hline & $9-10-2002$ & -- & -- & -- & -- & 1.0 & ID & 6 \\
\hline 13 & $9-11-2002$ & $<2$ & ID & 14 & $<1$ & $<20$ & ${ }^{2} 4.2-83.3$ & 23 \\
\hline 14 & $7-31-2002$ & $<2$ & ID & 17 & $<2$ & $<20$ & ${ }^{2} 4.3-69.6$ & 22 \\
\hline 15 & $9-11-2002$ & -- & -- & -- & -- & -- & -- & -- \\
\hline 16 & $7-31-2002$ & -- & -- & -- & -- & $<24$ & ID & 14 \\
\hline \multirow[t]{2}{*}{17} & $7-31-2002$ & -- & -- & -- & -- & -- & -- & -- \\
\hline & $9-10-2002$ & -- & -- & -- & -- & -- & -- & -- \\
\hline \multirow[t]{2}{*}{18} & $7-31-2002$ & -- & -- & -- & -- & -- & -- & -- \\
\hline & $9-10-2002$ & -- & -- & -- & -- & -- & -- & -- \\
\hline \multirow[t]{2}{*}{19} & $7-30-2002$ & -- & -- & -- & -- & -- & -- & -- \\
\hline & 9-9-2002 & -- & -- & -- & -- & -- & -- & -- \\
\hline
\end{tabular}

${ }^{1}$ Possible percentile range because of uncensored ties in data.

${ }^{2}$ Possible percentile range because of censored or recensored ties in data. 
Table 12. Water-quality data for the Upper Colorado River Basin, showing 2002 drought values, Colorado water-quality exceedances, and percentiles relative to historical data for selected properties and constituents from July through September, 1978-2002.

$\left[\mathrm{ft}^{3} / \mathrm{s}\right.$, cubic feet per second; Conc, concentration; $\mathrm{Q} \%$, percentile (percentage of data less than or equal to reported value or concentration) of drought-sample constituent; $\mathrm{N}$, total number of historical and drought samples; $\mathrm{mg} / \mathrm{L}$, milligrams per liter; $\mu \mathrm{S} / \mathrm{cm}$, microsiemens per centimeter at $25^{\circ} \mathrm{Celsius;}{ }^{\circ} \mathrm{C}$, degrees Celsius; E. coli, Escherichia coli; $\mathrm{mL}$, milliliter; $\mu \mathrm{g} / \mathrm{L}$, micrograms per liter; $\mathrm{CaCO}_{3}$, calcium carbonate; $\mathrm{SiO}_{2}$, silica; $\mathrm{SO}_{4}$, sulfate; ID, insufficient number of samples (less than 20) to calculate percentile; <, less than; --, no drought-sample measurement; values or concentrations in bold type indicate 2002 Colorado water-quality exceedances; $c$, chronic water-quality exceedance]

\begin{tabular}{|c|c|c|c|c|c|c|}
\hline \multirow{2}{*}{$\begin{array}{l}\text { Site number } \\
\text { in table } 1 \\
\text { and fig. } 4\end{array}$} & \multirow{2}{*}{ Sampling date } & \multirow{2}{*}{$\begin{array}{l}\text { Sampling } \\
\text { time }\end{array}$} & \multirow{2}{*}{$\begin{array}{c}\text { Discharge, } \\
\text { in } \mathrm{ft}^{3} / \mathrm{s}\end{array}$} & \multicolumn{3}{|c|}{ Oxygen, dissolved, in $\mathrm{mg} / \mathrm{L}$} \\
\hline & & & & Conc & $0 \%$ & $\mathrm{~N}$ \\
\hline 20 & $8-1-2002$ & 1300 & 40 & 9.0 & ID & 2 \\
\hline \multirow[t]{2}{*}{21} & $7-15-2002$ & 1415 & 7.5 & 8.0 & 15.4 & 25 \\
\hline & $9-4-2002$ & 1030 & 2.7 & 7.3 & ${ }^{1} 3.8-7.7$ & 25 \\
\hline \multirow[t]{2}{*}{22} & $7-15-2002$ & 1330 & 14 & 7.9 & ${ }^{1} 1.3-3.8$ & 77 \\
\hline & $9-4-2002$ & 1115 & 5.7 & 7.9 & ${ }^{1} 1.3-3.8$ & 77 \\
\hline \multirow[t]{2}{*}{23} & $7-15-2002$ & 1230 & 16 & 7.4 & ${ }^{1} 2.4-3.7$ & 81 \\
\hline & $9-4-2002$ & 1415 & 11 & 8.3 & ${ }^{1} 20.7-28.0$ & 81 \\
\hline \multirow[t]{2}{*}{24} & $7-15-2002$ & 1015 & 11 & 8.9 & ${ }^{1} 42.7-46.3$ & 81 \\
\hline & $9-4-2002$ & 1230 & 11 & 9.0 & ${ }^{1} 47.6-52.4$ & 81 \\
\hline \multirow[t]{2}{*}{25} & $7-15-2002$ & 1115 & 3.8 & 8.6 & ID & 15 \\
\hline & $9-4-2002$ & 1315 & 3.7 & 10.4 & ID & 15 \\
\hline \multirow[t]{2}{*}{26} & $7-17-2002$ & 1115 & 23 & 9.5 & ID & 10 \\
\hline & $9-6-2002$ & 1130 & 17 & 10.8 & ID & 10 \\
\hline 27 & $8-1-2002$ & 1130 & 119 & 9.0 & ID & 9 \\
\hline \multirow[t]{2}{*}{28} & $8-8-2002$ & 1315 & 234 & 6.6 & ID & 10 \\
\hline & $9-11-2002$ & 1215 & 214 & 6.1 & ID & 10 \\
\hline \multirow[t]{2}{*}{29} & 8-8-2002 & 1215 & 9.1 & 9.9 & ID & 2 \\
\hline & $9-11-2002$ & 1300 & 7.9 & 7.8 & ID & 2 \\
\hline \multirow[t]{2}{*}{30} & $7-29-2002$ & 1200 & 3.0 & 8.5 & ${ }^{1} 84.8-89.1$ & 45 \\
\hline & $9-10-2002$ & 1300 & 0.8 & 6.4 & 4.3 & 45 \\
\hline \multirow[t]{2}{*}{31} & $7-29-2002$ & 1330 & 21 & 8.9 & ${ }^{1} 60.0-64.0$ & 24 \\
\hline & $9-10-2002$ & 1130 & 223 & 8.6 & 48 & 24 \\
\hline \multirow[t]{2}{*}{32} & 8-8-2002 & 1100 & 257 & 10.0 & ID & 5 \\
\hline & $9-11-2002$ & 1115 & 62 & 8.6 & ID & 5 \\
\hline 33 & $7-30-2002$ & 1115 & 919 & 7.0 & ${ }^{1} 13.0-17.4$ & 22 \\
\hline 34 & $7-30-2002$ & 1035 & 8.7 & 7.2 & ID & 9 \\
\hline \multirow[t]{2}{*}{35} & $7-30-2002$ & 1330 & 22 & 7.5 & 4.3 & 22 \\
\hline & $9-3-2002$ & 1215 & 12 & 8.2 & 17.4 & 22 \\
\hline \multirow[t]{2}{*}{36} & $7-30-2002$ & 1555 & 58 & 6.8 & ID & 18 \\
\hline & $9-3-2002$ & 1620 & 32 & 7.7 & ID & 18 \\
\hline \multirow[t]{2}{*}{37} & $7-31-2002$ & 1735 & 97 & 7.8 & ${ }^{1} 5.9-11.8$ & 33 \\
\hline & $9-4-2002$ & 1320 & 74 & 10.5 & 97.1 & 33 \\
\hline
\end{tabular}


Table 12. Water-quality data for the Upper Colorado River Basin, showing 2002 drought values, Colorado water-quality exceedances, and percentiles relative to historical data for selected properties and constituents from July through September, 1978-2002.—Continued

$\left[\mathrm{ft}^{3} / \mathrm{s}\right.$, cubic feet per second; Conc, concentration; Q\%, percentile (percentage of data less than or equal to reported value or concentration) of drought-sample constituent; N, total number of historical and drought samples; $\mathrm{mg} / \mathrm{L}$, milligrams per liter; $\mu \mathrm{S} / \mathrm{cm}$, microsiemens per centimeter at $25^{\circ} \mathrm{Celsius;}{ }^{\circ} \mathrm{C}$, degrees Celsius; E. coli, Escherichia coli; $\mathrm{mL}$, milliliter; $\mu \mathrm{g} / \mathrm{L}$, micrograms per liter; $\mathrm{CaCO}_{3}$, calcium carbonate; $\mathrm{SiO}_{2}$, silica; $\mathrm{SO}_{4}$, sulfate; ID, insufficient number of samples (less than 20) to calculate percentile; <, less than; --, no drought-sample measurement; values or concentrations in bold type indicate 2002 Colorado water-quality exceedances; c, chronic water-quality exceedance]

\begin{tabular}{|c|c|c|c|c|c|c|}
\hline \multirow{2}{*}{$\begin{array}{l}\text { Site number } \\
\text { in table } 1 \\
\text { and fig. } 4\end{array}$} & \multirow{2}{*}{ Sampling date } & \multirow{2}{*}{$\begin{array}{l}\text { Sampling } \\
\text { time }\end{array}$} & \multirow{2}{*}{$\begin{array}{c}\text { Discharge, } \\
\text { in } \mathrm{ft}^{3} / \mathrm{s}\end{array}$} & \multicolumn{3}{|c|}{ Oxygen, dissolved, in $\mathrm{mg} / \mathrm{L}$} \\
\hline & & & & Conc & $0 \%$ & $\mathrm{~N}$ \\
\hline \multirow[t]{2}{*}{38} & $8-1-2002$ & 0900 & 982 & 7.4 & ID & 14 \\
\hline & $9-5-2002$ & 1500 & 596 & 9.6 & ID & 14 \\
\hline \multirow[t]{2}{*}{39} & $8-1-2002$ & 1130 & 1,040 & 7.1 & 9.1 & 32 \\
\hline & $9-6-2002$ & 0910 & 746 & 8.4 & ${ }^{1} 54.5-63.6$ & 32 \\
\hline \multirow[t]{2}{*}{40} & $7-29-2002$ & 1350 & 24 & 8.2 & ID & 12 \\
\hline & $9-3-2002$ & 1700 & 13 & 7.8 & ID & 12 \\
\hline \multirow[t]{2}{*}{41} & $7-29-2002$ & 1610 & 237 & 8.1 & ID & 11 \\
\hline & $9-4-2002$ & 0910 & 308 & 9.4 & ID & 11 \\
\hline \multirow[t]{2}{*}{42} & $7-30-2002$ & 0905 & 70 & 8.7 & ID & 12 \\
\hline & $9-4-2002$ & 1205 & 44 & 8.8 & ID & 12 \\
\hline \multirow[t]{2}{*}{43} & $7-30-2002$ & 1035 & 45 & 9.4 & ID & 6 \\
\hline & $9-4-2002$ & 1455 & 30 & 9.3 & ID & 6 \\
\hline \multirow[t]{2}{*}{44} & $7-30-2002$ & 1320 & 392 & 11.0 & ID & 16 \\
\hline & $9-5-2002$ & 0945 & 445 & 10.6 & ID & 16 \\
\hline \multirow[t]{2}{*}{45} & $7-31-2002$ & 0915 & 1,460 & 8.0 & ID & 2 \\
\hline & $9-6-2002$ & 1035 & 1,180 & 8.4 & ID & 2 \\
\hline 46 & $8-2-2002$ & 1340 & $<0.01$ & 9.3 & ID & 16 \\
\hline \multirow[t]{2}{*}{47} & $8-1-2002$ & 1300 & 1,440 & 7.2 & ${ }^{1} 2.4-4.9$ & 40 \\
\hline & $9-3-2002$ & 1030 & 1,270 & 7.9 & ${ }^{1} 34.1-41.5$ & 40 \\
\hline \multirow[t]{2}{*}{48} & $8-1-2002$ & 1000 & 8.7 & 7.4 & 7.7 & 25 \\
\hline & $9-5-2002$ & 1030 & 16 & 7.8 & 30.8 & 25 \\
\hline 49 & $7-31-2002$ & 0930 & 75 & 7.1 & ID & 4 \\
\hline 50 & $7-31-2002$ & 1645 & 256 & -- & -- & -- \\
\hline 51 & 8-1-2002 & 1515 & 1,050 & 8.2 & ID & 1 \\
\hline \multirow[t]{2}{*}{52} & $8-1-2002$ & 0910 & 1,280 & 6.6 & ${ }^{1} 1.9-5.6$ & 53 \\
\hline & $9-17-2002$ & 0940 & 2,520 & 7.4 & ${ }^{1} 35.2-46.3$ & 53 \\
\hline 53 & $7-31-2002$ & 1145 & 216 & 10.2 & ID & 1 \\
\hline 54 & 8-1-2002 & 1145 & 992 & 7.4 & ID & 1 \\
\hline 55 & $7-30-2002$ & 0840 & 1.4 & 7.5 & ID & 7 \\
\hline \multirow{2}{*}{56} & $7-31-2002$ & 1200 & 1,460 & 9.1 & ID & 2 \\
\hline & $9-5-2002$ & 1325 & 1,190 & 9.5 & ID & 2 \\
\hline \multirow[t]{2}{*}{57} & $7-31-2002$ & 1515 & 72 & 7.1 & ID & 8 \\
\hline & $9-4-2002$ & 1015 & 57 & 10.4 & ID & 8 \\
\hline \multirow[t]{2}{*}{58} & $7-16-2002$ & 1315 & 14 & 8.0 & ID & 6 \\
\hline & $9-5-2002$ & 0915 & 9.4 & 8.5 & ID & 6 \\
\hline
\end{tabular}


Table 12. Water-quality data for the Upper Colorado River Basin, showing 2002 drought values, Colorado water-quality exceedances, and percentiles relative to historical data for selected properties and constituents from July through September, 1978-2002. - Continued

$\left[\mathrm{ft}^{3} / \mathrm{s}\right.$, cubic feet per second; Conc, concentration; Q\%, percentile (percentage of data less than or equal to reported value or concentration) of drought-sample constituent; N, total number of historical and drought samples; $\mathrm{mg} / \mathrm{L}$, milligrams per liter; $\mu \mathrm{S} / \mathrm{cm}$, microsiemens per centimeter at $25^{\circ} \mathrm{Celsius;}{ }^{\circ} \mathrm{C}$, degrees Celsius; E. coli, Escherichia coli; $\mathrm{mL}$, milliliter; $\mu \mathrm{g} / \mathrm{L}$, micrograms per liter; $\mathrm{CaCO}_{3}$, calcium carbonate; $\mathrm{SiO}_{2}$, silica; $\mathrm{SO}_{4}$, sulfate; ID, insufficient number of samples (less than 20) to calculate percentile; <, less than; --, no drought-sample measurement; values or concentrations in bold type indicate 2002 Colorado water-quality exceedances; $c$, chronic water-quality exceedance]

\begin{tabular}{|c|c|c|c|c|c|c|c|}
\hline \multirow{2}{*}{$\begin{array}{l}\text { Site number } \\
\text { in table } 1 \\
\text { and fig. } 4\end{array}$} & \multirow{2}{*}{ Sampling date } & \multicolumn{3}{|c|}{$\mathrm{pH}$, in standard units } & \multicolumn{3}{|c|}{ Specific conductance, in $\mu \mathrm{S} / \mathrm{cm}$} \\
\hline & & Value & $0 \%$ & $\mathrm{~N}$ & Value & $0 \%$ & $\mathrm{~N}$ \\
\hline 20 & $8-1-2002$ & 7.6 & ID & 2 & 61 & ID & 12 \\
\hline \multirow[t]{2}{*}{21} & $7-15-2002$ & 8.0 & ${ }^{1} 30.8-38.5$ & 25 & 74 & ${ }^{1} 65.6-71.9$ & 63 \\
\hline & $9-4-2002$ & 8.0 & ${ }^{1} 30.8-38.5$ & 25 & 108 & 98.4 & 63 \\
\hline \multirow[t]{2}{*}{22} & $7-15-2002$ & 8.4 & ${ }^{1} 94.0-96.4$ & 82 & 85 & ${ }^{1} 82.1-83.3$ & 83 \\
\hline & $9-4-2002$ & 8.1 & ${ }^{1} 62.7-80.7$ & 82 & 108 & 98.8 & 83 \\
\hline \multirow[t]{2}{*}{23} & $7-15-2002$ & 8.1 & ${ }^{1} 69.0-78.2$ & 86 & 75 & ${ }^{1} 84.1-86.4$ & 87 \\
\hline & $9-4-2002$ & 8.4 & ${ }^{1} 90.8-94.3$ & 86 & 96 & 98.9 & 87 \\
\hline \multirow[t]{2}{*}{24} & $7-15-2002$ & 8.7 & ${ }^{1} 48.9-56.8$ & 107 & 115 & 97.7 & 87 \\
\hline & $9-4-2002$ & 9.1 & ${ }^{1} 68.2-73.9$ & 107 & 133 & 98.9 & 87 \\
\hline \multirow[t]{2}{*}{25} & $7-15-2002$ & 8.7 & ID & 15 & 160 & 96.2 & 25 \\
\hline & $9-4-2002$ & 9.2 & ID & 15 & 154 & 92.3 & 25 \\
\hline \multirow[t]{2}{*}{26} & $7-17-2002$ & 9.1 & ID & 10 & 132 & ID & 11 \\
\hline & $9-6-2002$ & 9.1 & ID & 10 & 143 & ID & 11 \\
\hline 27 & $8-1-2002$ & 9.1 & ID & 7 & 120 & ${ }^{1} 23.6-27.3$ & 54 \\
\hline \multirow[t]{2}{*}{28} & 8-8-2002 & 7.5 & ID & 10 & 121 & ${ }^{1} 84.6-86.2$ & 64 \\
\hline & $9-11-2002$ & 8.1 & ID & 10 & 133 & 98.5 & 64 \\
\hline \multirow[t]{2}{*}{29} & 8-8-2002 & 8.3 & ID & 2 & 559 & ID & 2 \\
\hline & $9-11-2002$ & 8.5 & ID & 2 & 555 & ID & 2 \\
\hline \multirow[t]{2}{*}{30} & $7-29-2002$ & 8.4 & ${ }^{1} 40.4-66.0$ & 46 & 664 & 77.1 & 47 \\
\hline & $9-10-2002$ & 8.1 & ${ }^{1} 6.4-14.9$ & 46 & 589 & ${ }^{1} 66.7-68.8$ & 47 \\
\hline \multirow[t]{2}{*}{31} & $7-29-2002$ & 8.3 & ${ }^{1} 56.0-76.0$ & 24 & 719 & 92.0 & 24 \\
\hline & $9-10-2002$ & 8.0 & ${ }^{1} 12.0-16.0$ & 24 & 722 & 96.0 & 24 \\
\hline \multirow[t]{2}{*}{32} & $8-8-2002$ & 8.4 & ID & 5 & 232 & ID & 5 \\
\hline & $9-11-2002$ & 8.5 & ID & 5 & 255 & ID & 5 \\
\hline 33 & $7-30-2002$ & 8.4 & ${ }^{1} 91.7-95.8$ & 23 & 220 & ${ }^{1} 56.9-58.8$ & 50 \\
\hline 34 & $7-30-2002$ & 8.5 & ID & 9 & 247 & 94.2 & 51 \\
\hline \multirow[t]{2}{*}{35} & $7-30-2002$ & 9.0 & ${ }^{1} 87.5-91.7$ & 23 & 364 & ${ }^{1} 88.0-92.0$ & 24 \\
\hline & $9-3-2002$ & 8.9 & ${ }^{1} 79.2-83.3$ & 23 & 466 & 96.0 & 24 \\
\hline \multirow[t]{2}{*}{36} & $7-30-2002$ & 8.6 & ID & 18 & 297 & 92.1 & 37 \\
\hline & $9-3-2002$ & 8.6 & ID & 18 & 416 & 97.4 & 37 \\
\hline \multirow[t]{2}{*}{37} & $7-31-2002$ & 8.5 & ${ }^{1} 68.6-82.9$ & 34 & 1,050 & 91.2 & 33 \\
\hline & $9-4-2002$ & 8.5 & ${ }^{1} 68.6-82.9$ & 34 & 1,360 & 97.1 & 33 \\
\hline
\end{tabular}


Table 12. Water-quality data for the Upper Colorado River Basin, showing 2002 drought values, Colorado water-quality exceedances, and percentiles relative to historical data for selected properties and constituents from July through September, 1978-2002.—Continued

[ft $3 / \mathrm{s}$, cubic feet per second; Conc, concentration; Q\%, percentile (percentage of data less than or equal to reported value or concentration) of drought-sample constituent; N, total number of historical and drought samples; $\mathrm{mg} / \mathrm{L}$, milligrams per liter; $\mu \mathrm{S} / \mathrm{cm}$, microsiemens per centimeter at $25^{\circ} \mathrm{Celsius;}{ }^{\circ} \mathrm{C}$, degrees Celsius; E. coli, Escherichia coli; $\mathrm{mL}$, milliliter; $\mu \mathrm{g} / \mathrm{L}$, micrograms per liter; $\mathrm{CaCO}_{3}$, calcium carbonate; $\mathrm{SiO}_{2}$, silica; $\mathrm{SO}_{4}$, sulfate; ID, insufficient number of samples (less than 20) to calculate percentile; <, less than; --, no drought-sample measurement; values or concentrations in bold type indicate 2002 Colorado water-quality exceedances; c, chronic water-quality exceedance]

\begin{tabular}{|c|c|c|c|c|c|c|c|}
\hline \multirow{2}{*}{$\begin{array}{l}\text { Site number } \\
\text { in table } 1 \\
\text { and fig. } 4\end{array}$} & \multirow{2}{*}{ Sampling date } & \multicolumn{3}{|c|}{$\mathrm{pH}$, in standard units } & \multicolumn{3}{|c|}{ Specific conductance, in $\mu S / \mathrm{cm}$} \\
\hline & & Value & $0 \%$ & $\mathrm{~N}$ & Value & $0 \%$ & $\mathrm{~N}$ \\
\hline \multirow[t]{2}{*}{38} & $8-1-2002$ & 8.3 & ${ }^{1} 75.0-80.0$ & 39 & 420 & ${ }^{1} 47.7-50.5$ & 110 \\
\hline & $9-5-2002$ & 8.6 & 97.5 & 39 & 621 & 99.1 & 110 \\
\hline \multirow[t]{2}{*}{39} & $8-1-2002$ & 8.5 & ${ }^{1} 81.0-90.5$ & 41 & 618 & 62.8 & 42 \\
\hline & $9-6-2002$ & 8.4 & ${ }^{1} 59.5-78.6$ & 41 & 932 & 97.7 & 42 \\
\hline \multirow[t]{2}{*}{40} & 7-29-2002 & 7.9 & ID & 12 & 77 & 85.7 & 55 \\
\hline & $9-3-2002$ & 7.8 & ID & 12 & 90 & 94.6 & 55 \\
\hline \multirow[t]{2}{*}{41} & $7-29-2002$ & 8.3 & ID & 11 & 392 & ID & 16 \\
\hline & $9-4-2002$ & 8.1 & ID & 11 & 327 & ID & 16 \\
\hline \multirow[t]{2}{*}{42} & $7-30-2002$ & 7.8 & ID & 12 & 519 & 96.6 & 57 \\
\hline & $9-4-2002$ & 7.7 & ID & 12 & 655 & 98.3 & 57 \\
\hline \multirow[t]{2}{*}{43} & $7-30-2002$ & 8.4 & ID & 6 & 633 & ID & 10 \\
\hline & $9-4-2002$ & 8.4 & ID & 6 & 658 & ID & 10 \\
\hline \multirow[t]{2}{*}{44} & $7-30-2002$ & 8.6 & 93.0 & 42 & 702 & 91.4 & 115 \\
\hline & $9-5-2002$ & 8.5 & ${ }^{1} 83.7-90.7$ & 42 & 653 & 81.0 & 115 \\
\hline \multirow[t]{2}{*}{45} & $7-31-2002$ & 8.2 & ID & 2 & 1,060 & ID & 2 \\
\hline & $9-6-2002$ & 8.3 & ID & 2 & 1,330 & ID & 2 \\
\hline 46 & $8-2-2002$ & 9.0 & ID & 16 & 3,850 & ID & 16 \\
\hline \multirow[t]{2}{*}{47} & $8-1-2002$ & 8.5 & ${ }^{1} 64.8-80.3$ & 70 & 1,100 & 95.5 & 178 \\
\hline & $9-3-2002$ & 8.4 & ${ }^{1} 47.9-63.4$ & 70 & 1,210 & ${ }^{1} 98.3-98.9$ & 178 \\
\hline \multirow[t]{2}{*}{48} & $8-1-2002$ & 8.6 & ${ }^{1} 46.4-75.0$ & 27 & 769 & 86.6 & 66 \\
\hline & $9-5-2002$ & 8.6 & ${ }^{1} 46.4-75.0$ & 27 & 774 & ${ }^{1} 88.1-89.6$ & 66 \\
\hline 49 & $7-31-2002$ & 8.5 & ID & 7 & 1,070 & ${ }^{1} 91.7-93.8$ & 47 \\
\hline 50 & $7-31-2002$ & 8.3 & ID & 4 & 1,470 & 94.7 & 18 \\
\hline 51 & $8-1-2002$ & 8.4 & ID & 4 & 1,240 & ID & 7 \\
\hline \multirow[t]{2}{*}{52} & $8-1-2002$ & 8.3 & ${ }^{1} 51.6-67.2$ & 63 & 1,400 & ${ }^{1} 85.7-87.1$ & 69 \\
\hline & $9-17-2002$ & 8.2 & ${ }^{1} 26.6-50.0$ & 63 & 1,600 & ${ }^{1} 97.1-98.6$ & 69 \\
\hline 53 & $7-31-2002$ & 8.2 & ID & 1 & 1,350 & ID & 14 \\
\hline 54 & $8-1-2002$ & 8.3 & ID & 4 & 1,180 & ID & 7 \\
\hline 55 & $7-30-2002$ & 8.2 & ID & 8 & 164 & ID & 8 \\
\hline \multirow[t]{2}{*}{56} & $7-31-2002$ & 8.5 & ID & 2 & 1,070 & ID & 2 \\
\hline & $9-5-2002$ & 8.5 & ID & 2 & 1,290 & ID & 2 \\
\hline \multirow[t]{2}{*}{57} & $7-31-2002$ & 8.8 & ID & 8 & 1,090 & ID & 8 \\
\hline & $9-4-2002$ & 8.6 & ID & 8 & 1,500 & ID & 8 \\
\hline \multirow[t]{2}{*}{58} & $7-16-2002$ & 8.4 & ID & 6 & 150 & ID & 6 \\
\hline & $9-5-2002$ & 8.1 & ID & 6 & 168 & ID & 6 \\
\hline
\end{tabular}


Table 12. Water-quality data for the Upper Colorado River Basin, showing 2002 drought values, Colorado water-quality exceedances, and percentiles relative to historical data for selected properties and constituents from July through September, 1978-2002.—Continued

$\left[\mathrm{ft}^{3} / \mathrm{s}\right.$, cubic feet per second; Conc, concentration; Q\%, percentile (percentage of data less than or equal to reported value or concentration) of drought-sample constituent; $\mathrm{N}$, total number of historical and drought samples; $\mathrm{mg} / \mathrm{L}$, milligrams per liter; $\mu \mathrm{S} / \mathrm{cm}$, microsiemens per centimeter at $25^{\circ} \mathrm{Celsius;}{ }^{\circ} \mathrm{C}$, degrees Celsius; E. coli, Escherichia coli; $\mathrm{mL}$, milliliter; $\mu \mathrm{g} / \mathrm{L}$, micrograms per liter; $\mathrm{CaCO}_{3}$, calcium carbonate; $\mathrm{SiO}_{2}$, silica; $\mathrm{SO}_{4}$, sulfate; ID, insufficient number of samples (less than 20) to calculate percentile; <, less than; --, no drought-sample measurement; values or concentrations in bold type indicate 2002 Colorado water-quality exceedances; c, chronic water-quality exceedance]

\begin{tabular}{|c|c|c|c|c|c|c|c|}
\hline \multirow{2}{*}{$\begin{array}{l}\text { Site number } \\
\text { in table } 1 \\
\text { and fig. } 4\end{array}$} & \multirow{2}{*}{ Sampling date } & \multicolumn{3}{|c|}{ Temperature, water, in ${ }^{\circ} \mathrm{C}$} & \multirow{2}{*}{$\begin{array}{l}\text { Hardness, } \\
\text { total, in } \mathrm{mg} / \mathrm{L} \\
\text { as } \mathrm{CaCO}_{3}\end{array}$} & \multirow{2}{*}{$\begin{array}{l}\text { Calcium, } \\
\text { dissolved, in } \\
\text { mg/L }\end{array}$} & \multirow{2}{*}{$\begin{array}{c}\text { Magnesium, } \\
\text { dissolved, } \\
\text { in } \mathrm{mg} / \mathrm{L}\end{array}$} \\
\hline & & Value & $0 \%$ & $\mathrm{~N}$ & & & \\
\hline 20 & $8-1-2002$ & 14.5 & ID & 12 & 24 & 7.5 & 1.3 \\
\hline \multirow[t]{2}{*}{21} & $7-15-2002$ & 13.0 & 98.4 & 63 & 23 & 5.3 & 2.3 \\
\hline & $9-4-2002$ & 6.0 & ${ }^{1} 23.4-28.1$ & 63 & 33 & 7.4 & 3.7 \\
\hline \multirow[t]{2}{*}{22} & $7-15-2002$ & 14.0 & 98.8 & 83 & 26 & 6.4 & 2.5 \\
\hline & $9-4-2002$ & 10.0 & ${ }^{1} 83.3-88.1$ & 83 & 37 & 9.3 & 3.4 \\
\hline \multirow[t]{2}{*}{23} & $7-15-2002$ & 16.0 & 98.9 & 87 & 25 & 6.6 & 2.1 \\
\hline & $9-4-2002$ & 14.0 & ${ }^{1} 95.5-96.6$ & 87 & 33 & 8.3 & 3.0 \\
\hline \multirow[t]{2}{*}{24} & $7-15-2002$ & 16.0 & ${ }^{1} 76.1-78.4$ & 87 & 38 & 10.8 & 2.7 \\
\hline & $9-4-2002$ & 16.0 & ${ }^{1} 76.1-78.4$ & 87 & 48 & 13.9 & 3.3 \\
\hline \multirow[t]{2}{*}{25} & $7-15-2002$ & 17.0 & ${ }^{1} 61.5-65.4$ & 25 & -- & -- & -- \\
\hline & $9-4-2002$ & 18.0 & 76.9 & 25 & -- & -- & -- \\
\hline \multirow[t]{2}{*}{26} & $7-17-2002$ & 18.0 & ID & 11 & 52 & 16.0 & 2.9 \\
\hline & $9-6-2002$ & 15.0 & ID & 11 & 59 & 17.9 & 3.4 \\
\hline 27 & $8-1-2002$ & 19.5 & ${ }^{1} 92.7-94.5$ & 54 & 51 & 16.2 & 2.7 \\
\hline \multirow[t]{2}{*}{28} & 8-8-2002 & 10.0 & ${ }^{1} 70.8-83.1$ & 64 & 52 & 16.4 & 2.7 \\
\hline & $9-11-2002$ & 15.0 & 98.5 & 64 & 59 & 18.6 & 3.0 \\
\hline \multirow[t]{2}{*}{29} & $8-8-2002$ & 18.0 & ID & 2 & 190 & 63 & 7.5 \\
\hline & $9-11-2002$ & 16.0 & ID & 2 & 190 & 63 & 7.2 \\
\hline \multirow[t]{2}{*}{30} & $7-29-2002$ & 17.0 & ${ }^{1} 40.0-46.0$ & 49 & 320 & 85 & 24.8 \\
\hline & $9-10-2002$ & 17.0 & ${ }^{1} 40.0-46.0$ & 49 & 240 & 63 & 20.1 \\
\hline \multirow[t]{2}{*}{31} & $7-29-2002$ & 12.0 & ${ }^{1} 44.0-52.0$ & 24 & 330 & 85 & 29.1 \\
\hline & $9-10-2002$ & 14.0 & 76.0 & 24 & 320 & 81 & 29.0 \\
\hline \multirow[t]{2}{*}{32} & 8-8-2002 & 15.0 & ID & 5 & 98 & 31 & 5.0 \\
\hline & $9-11-2002$ & 17.0 & ID & 5 & 120 & 36 & 6.2 \\
\hline 33 & $7-30-2002$ & 16.0 & ${ }^{1} 78.8-80.8$ & 51 & 98 & 30 & 5.3 \\
\hline 34 & $7-30-2002$ & 14.5 & ${ }^{1} 77.4-81.1$ & 52 & 130 & 31 & 13.6 \\
\hline \multirow[t]{2}{*}{35} & $7-30-2002$ & 17.5 & 96.0 & 24 & 180 & 57 & 9.7 \\
\hline & $9-3-2002$ & 14.5 & 92.0 & 24 & 230 & 72 & 12.1 \\
\hline \multirow[t]{2}{*}{36} & $7-30-2002$ & 20.0 & 97.4 & 37 & 150 & 42 & 10.6 \\
\hline & $9-3-2002$ & 14.5 & 68.4 & 37 & 200 & 58 & 14.4 \\
\hline \multirow[t]{2}{*}{37} & $7-31-2002$ & 24.0 & 97.1 & 34 & 400 & 123 & 21.6 \\
\hline & $9-4-2002$ & 18.5 & ${ }^{1} 85.7-88.6$ & 34 & 490 & 152 & 25.4 \\
\hline
\end{tabular}


Table 12. Water-quality data for the Upper Colorado River Basin, showing 2002 drought values, Colorado water-quality exceedances, and percentiles relative to historical data for selected properties and constituents from July through September, 1978-2002.—Continued

$\left[\mathrm{ft}^{3} / \mathrm{s}\right.$, cubic feet per second; Conc, concentration; Q\% percentile (percentage of data less than or equal to reported value or concentration) of drought-sample constituent; N, total number of historical and drought samples; $\mathrm{mg} / \mathrm{L}$, milligrams per liter; $\mu \mathrm{S} / \mathrm{cm}$, microsiemens per centimeter at $25^{\circ} \mathrm{Celsius;}{ }^{\circ} \mathrm{C}$, degrees Celsius; E. coli, Escherichia coli; $\mathrm{mL}$, milliliter; $\mu \mathrm{g} / \mathrm{L}$, micrograms per liter; $\mathrm{CaCO}_{3}$, calcium carbonate; $\mathrm{SiO}_{2}$, silica; $\mathrm{SO}_{4}$, sulfate; ID, insufficient number of samples (less than 20) to calculate percentile; <, less than; --, no drought-sample measurement; values or concentrations in bold type indicate 2002 Colorado water-quality exceedances; c, chronic water-quality exceedance]

\begin{tabular}{|c|c|c|c|c|c|c|c|}
\hline \multirow{2}{*}{$\begin{array}{l}\text { Site number } \\
\text { in table } 1 \\
\text { and fig. } 4\end{array}$} & \multirow{2}{*}{ Sampling date } & \multicolumn{3}{|c|}{ Temperature, water, in ${ }^{\circ} \mathrm{C}$} & \multirow{2}{*}{$\begin{array}{l}\text { Hardness, } \\
\text { total, in } \mathrm{mg} / \mathrm{L} \\
\text { as } \mathrm{CaCO}_{3}\end{array}$} & \multirow{2}{*}{$\begin{array}{l}\text { Calcium, } \\
\text { dissolved, in } \\
\mathrm{mg} / \mathrm{L}\end{array}$} & \multirow{2}{*}{$\begin{array}{c}\text { Magnesium, } \\
\text { dissolved, } \\
\text { in } \mathrm{mg} / \mathrm{L}\end{array}$} \\
\hline & & Value & $0 \%$ & $\mathrm{~N}$ & & & \\
\hline \multirow[t]{2}{*}{38} & 8-1-2002 & 18.0 & ${ }^{1} 80.2-87.4$ & 110 & 150 & 45 & 8.5 \\
\hline & $9-5-2002$ & 18.0 & ${ }^{1} 80.2-87.4$ & 110 & 230 & 67 & 16.0 \\
\hline \multirow[t]{2}{*}{39} & $8-1-2002$ & 20.5 & 97.7 & 42 & 160 & 49 & 9.3 \\
\hline & $9-6-2002$ & 18.0 & ${ }^{1} 58.1-62.8$ & 42 & 250 & 71 & 16.7 \\
\hline \multirow[t]{2}{*}{40} & $7-29-2002$ & 14.7 & 96.5 & 56 & 30 & 9.5 & 1.6 \\
\hline & $9-3-2002$ & 11.6 & ${ }^{1} 63.2-64.9$ & 56 & -- & -- & -- \\
\hline \multirow[t]{2}{*}{41} & $7-29-2002$ & 17.0 & ID & 16 & 190 & 58 & 9.6 \\
\hline & $9-4-2002$ & 9.5 & ID & 16 & -- & -- & -- \\
\hline \multirow[t]{2}{*}{42} & $7-30-2002$ & 13.1 & ${ }^{1} 49.2-50.8$ & 58 & 220 & 75 & 8.6 \\
\hline & $9-4-2002$ & 13.8 & ${ }^{1} 54.2-55.9$ & 58 & -- & -- & -- \\
\hline \multirow[t]{2}{*}{43} & $7-30-2002$ & 14.5 & ID & 10 & 310 & 95 & 16.3 \\
\hline & $9-4-2002$ & 16.3 & ID & 10 & -- & -- & -- \\
\hline \multirow[t]{2}{*}{44} & $7-30-2002$ & 18.5 & 95.8 & 117 & 270 & 83 & 15.0 \\
\hline & $9-5-2002$ & 12.0 & ${ }^{1} 16.9-20.3$ & 117 & -- & -- & -- \\
\hline \multirow[t]{2}{*}{45} & $7-31-2002$ & 18.6 & ID & 2 & 210 & 64 & 12.0 \\
\hline & $9-6-2002$ & 15.2 & ID & 2 & 270 & 79 & 16.5 \\
\hline 46 & 8-2-2002 & 24.3 & ID & 16 & 1,100 & 46 & 228.0 \\
\hline \multirow[t]{2}{*}{47} & $8-1-2002$ & 22.0 & ${ }^{1} 96.0-98.0$ & 200 & 220 & 66 & 14.4 \\
\hline & $9-3-2002$ & 17.5 & ${ }^{1} 36.3-40.8$ & 200 & 250 & 73 & 16.7 \\
\hline \multirow[t]{2}{*}{48} & $8-1-2002$ & 20.5 & ${ }^{1} 71.6-74.6$ & 66 & 250 & 29 & 41.8 \\
\hline & $9-5-2002$ & 16.5 & ${ }^{1} 22.4-25.4$ & 66 & 270 & 40 & 41.6 \\
\hline 49 & $7-31-2002$ & 22.8 & ${ }^{1} 77.1-79.2$ & 47 & 230 & 67 & 15.1 \\
\hline 50 & $7-31-2002$ & -- & -- & -- & 430 & 116 & 34.9 \\
\hline 51 & $8-1-2002$ & 24.3 & ID & 7 & 450 & 121 & 34.9 \\
\hline \multirow[t]{2}{*}{52} & $8-1-2002$ & 23.5 & ${ }^{1} 88.7-93.0$ & 70 & 520 & 139 & 42.2 \\
\hline & $9-17-2002$ & 18.0 & ${ }^{1} 15.5-19.7$ & 70 & 600 & 171 & 42.4 \\
\hline 53 & $7-31-2002$ & 23.3 & ID & 14 & 380 & 106 & 27.4 \\
\hline 54 & $8-1-2002$ & 23.1 & ID & 7 & 440 & 119 & 33.3 \\
\hline 55 & $7-30-2002$ & 9.5 & ID & 8 & 88 & 21 & 8.8 \\
\hline \multirow[t]{2}{*}{56} & $7-31-2002$ & 20.2 & ID & 2 & 210 & 64 & 12.8 \\
\hline & $9-5-2002$ & 17.9 & ID & 2 & 260 & 77 & 16.7 \\
\hline \multirow[t]{2}{*}{57} & $7-31-2002$ & 23.0 & ID & 8 & 250 & 74 & 16.0 \\
\hline & $9-4-2002$ & 13.5 & ID & 8 & 320 & 93 & 20.1 \\
\hline \multirow[t]{2}{*}{58} & 7-16-2002 & 21.0 & ID & 6 & -- & -- & -- \\
\hline & $9-5-2002$ & 10.0 & ID & 6 & -- & -- & -- \\
\hline
\end{tabular}


Table 12. Water-quality data for the Upper Colorado River Basin, showing 2002 drought values, Colorado water-quality exceedances, and percentiles relative to historical data for selected properties and constituents from July through September, 1978-2002. - Continued

$\left[\mathrm{ft}^{3} / \mathrm{s}\right.$, cubic feet per second; Conc, concentration; Q\%, percentile (percentage of data less than or equal to reported value or concentration) of drought-sample constituent; $\mathrm{N}$, total number of historical and drought samples; $\mathrm{mg} / \mathrm{L}$, milligrams per liter; $\mu \mathrm{S} / \mathrm{cm}$, microsiemens per centimeter at $25^{\circ} \mathrm{Celsius;}{ }^{\circ} \mathrm{C}$, degrees Celsius; E. coli, Escherichia coli; $\mathrm{mL}$, milliliter; $\mu \mathrm{g} / \mathrm{L}$, micrograms per liter; $\mathrm{CaCO}_{3}$, calcium carbonate; $\mathrm{SiO}_{2}$, silica; $\mathrm{SO}_{4}$, sulfate; ID, insufficient number of samples (less than 20) to calculate percentile; <, less than; --, no drought-sample measurement; values or concentrations in bold type indicate 2002 Colorado water-quality exceedances; c, chronic water-quality exceedance]

\begin{tabular}{|c|c|c|c|c|c|c|}
\hline $\begin{array}{l}\text { Site number } \\
\text { in table } 1 \\
\text { and fig. } 4\end{array}$ & Sampling date & $\begin{array}{c}\text { Potassium, } \\
\text { dissolved, in } \\
\mathrm{mg} / \mathrm{L}\end{array}$ & $\begin{array}{l}\text { Sodium, } \\
\text { dissolved, in } \\
\mathrm{mg} / \mathrm{L}\end{array}$ & $\begin{array}{c}\text { Acid- } \\
\text { neutralizing } \\
\text { capacity, } \\
\text { in } \mathrm{mg} / \mathrm{L} \\
\text { as } \mathrm{CaCO}_{3}\end{array}$ & $\begin{array}{c}\text { Alkalinity, } \\
\text { laboratory, in } \\
\mathrm{mg} / \mathrm{L} \text { as } \mathrm{CaCO}_{3}\end{array}$ & $\begin{array}{c}\text { Alkalinity, } \\
\text { onsite, in } \\
\mathrm{mg} / \mathrm{L} \text { as } \\
\mathrm{CaCO}_{3}\end{array}$ \\
\hline 20 & $8-1-2002$ & 0.69 & 2.1 & -- & 26 & -- \\
\hline \multirow{2}{*}{21} & $7-15-2002$ & -- & -- & -- & -- & -- \\
\hline & $9-4-2002$ & -- & -- & -- & -- & -- \\
\hline \multirow[t]{2}{*}{22} & $7-15-2002$ & -- & -- & -- & -- & -- \\
\hline & $9-4-2002$ & -- & -- & -- & -- & -- \\
\hline \multirow[t]{2}{*}{23} & $7-15-2002$ & -- & -- & -- & -- & -- \\
\hline & $9-4-2002$ & -- & -- & -- & -- & -- \\
\hline \multirow[t]{2}{*}{24} & $7-15-2002$ & -- & -- & -- & -- & -- \\
\hline & $9-4-2002$ & -- & -- & -- & -- & -- \\
\hline \multirow[t]{2}{*}{25} & $7-15-2002$ & -- & -- & -- & -- & -- \\
\hline & $9-4-2002$ & -- & -- & -- & -- & -- \\
\hline \multirow[t]{2}{*}{26} & $7-17-2002$ & 1.4 & 6.1 & -- & 55 & -- \\
\hline & $9-6-2002$ & 2.0 & 7.5 & -- & 61 & -- \\
\hline 27 & $8-1-2002$ & 1.1 & 5.5 & 58 & -- & -- \\
\hline \multirow[t]{2}{*}{28} & 8-8-2002 & -- & 3.1 & -- & 57 & -- \\
\hline & $9-11-2002$ & 1.7 & 3.3 & -- & 62 & -- \\
\hline \multirow[t]{2}{*}{29} & 8-8-2002 & 5.6 & 45 & -- & 229 & -- \\
\hline & $9-11-2002$ & 5.5 & 45 & -- & 222 & -- \\
\hline \multirow[t]{2}{*}{30} & $7-29-2002$ & 2.9 & 33 & 246 & -- & -- \\
\hline & $9-10-2002$ & 2.4 & 33 & 195 & -- & -- \\
\hline \multirow[t]{2}{*}{31} & $7-29-2002$ & 2.5 & 33 & 132 & -- & -- \\
\hline & $9-10-2002$ & 2.4 & 33 & 139 & -- & -- \\
\hline \multirow[t]{2}{*}{32} & 8-8-2002 & 1.8 & 5.3 & -- & 73 & -- \\
\hline & $9-11-2002$ & 1.9 & 6.3 & -- & 82 & -- \\
\hline 33 & $7-30-2002$ & 1.9 & 6.2 & 71 & -- & -- \\
\hline 34 & $7-30-2002$ & 1.0 & 2.8 & -- & 130 & 107 \\
\hline \multirow[t]{2}{*}{35} & $7-30-2002$ & 1.5 & 8.8 & -- & 123 & 102 \\
\hline & $9-3-2002$ & 1.8 & 11.3 & -- & 141 & 122 \\
\hline \multirow[t]{2}{*}{36} & $7-30-2002$ & 1.1 & 5.1 & -- & 101 & 88 \\
\hline & $9-3-2002$ & 1.3 & 7.7 & -- & 120 & 99 \\
\hline \multirow[t]{2}{*}{37} & $7-31-2002$ & 3.9 & 84 & -- & 138 & 120 \\
\hline & $9-4-2002$ & 4.0 & 102 & -- & -- & 148 \\
\hline
\end{tabular}


Table 12. Water-quality data for the Upper Colorado River Basin, showing 2002 drought values, Colorado water-quality exceedances, and percentiles relative to historical data for selected properties and constituents from July through September, 1978-2002.-Continued

[ft $3 / \mathrm{s}$, cubic feet per second; Conc, concentration; Q\%, percentile (percentage of data less than or equal to reported value or concentration) of drought-sample constituent; N, total number of historical and drought samples; $\mathrm{mg} / \mathrm{L}$, milligrams per liter; $\mu \mathrm{S} / \mathrm{cm}$, microsiemens per centimeter at $25^{\circ} \mathrm{Celsius;}{ }^{\circ} \mathrm{C}$, degrees Celsius; E. coli, Escherichia coli; $\mathrm{mL}$, milliliter; $\mu \mathrm{g} / \mathrm{L}$, micrograms per liter; $\mathrm{CaCO}_{3}$, calcium carbonate; $\mathrm{SiO}_{2}$, silica; $\mathrm{SO}_{4}$, sulfate; ID, insufficient number of samples (less than 20) to calculate percentile; <, less than; --, no drought-sample measurement; values or concentrations in bold type indicate 2002 Colorado water-quality exceedances; c, chronic water-quality exceedance]

\begin{tabular}{|c|c|c|c|c|c|c|}
\hline $\begin{array}{l}\text { Site number } \\
\text { in table } 1 \\
\text { and fig. } 4\end{array}$ & Sampling date & $\begin{array}{l}\text { Potassium, } \\
\text { dissolved, in } \\
\mathrm{mg} / \mathrm{L}\end{array}$ & $\begin{array}{l}\text { Sodium, } \\
\text { dissolved, in } \\
\mathrm{mg} / \mathrm{L}\end{array}$ & $\begin{array}{c}\text { Acid- } \\
\text { neutralizing } \\
\text { capacity, } \\
\text { in } \mathrm{mg} / \mathrm{L} \\
\text { as } \mathrm{CaCO}_{3}\end{array}$ & $\begin{array}{c}\text { Alkalinity, } \\
\text { laboratory, in } \\
\mathrm{mg} / \mathrm{L} \text { as } \mathrm{CaCO}_{3}\end{array}$ & $\begin{array}{c}\text { Alkalinity, } \\
\text { onsite, in } \\
\mathrm{mg} / \mathrm{L} \text { as } \\
\mathrm{CaCO}_{3}\end{array}$ \\
\hline
\end{tabular}

\begin{tabular}{|c|c|c|c|c|c|c|}
\hline \multirow[t]{2}{*}{38} & 8-1-2002 & 2.2 & 25 & -- & 91 & -- \\
\hline & $9-5-2002$ & 2.4 & 41 & -- & 114 & -- \\
\hline \multirow[t]{2}{*}{39} & $8-1-2002$ & 2.5 & 63 & -- & 94 & -- \\
\hline & $9-6-2002$ & 3.1 & 97 & -- & 121 & -- \\
\hline \multirow[t]{2}{*}{40} & $7-29-2002$ & 0.37 & 1.8 & -- & 28 & -- \\
\hline & $9-3-2002$ & -- & -- & -- & -- & -- \\
\hline \multirow[t]{2}{*}{41} & $7-29-2002$ & 1.2 & 4.0 & -- & 109 & -- \\
\hline & $9-4-2002$ & -- & -- & -- & -- & -- \\
\hline \multirow[t]{2}{*}{42} & $7-30-2002$ & 1.5 & 16.4 & -- & 111 & -- \\
\hline & $9-4-2002$ & -- & -- & -- & -- & -- \\
\hline \multirow[t]{2}{*}{43} & $7-30-2002$ & 1.7 & 12.0 & -- & 197 & -- \\
\hline & $9-4-2002$ & -- & -- & -- & -- & -- \\
\hline \multirow[t]{2}{*}{44} & $7-30-2002$ & 1.7 & 40 & -- & 150 & -- \\
\hline & $9-5-2002$ & -- & -- & -- & -- & -- \\
\hline \multirow[t]{2}{*}{45} & $7-31-2002$ & 3.8 & 125 & -- & 120 & -- \\
\hline & $9-6-2002$ & 4.1 & 160 & -- & 137 & -- \\
\hline 46 & $8-2-2002$ & 3.6 & 581 & -- & 326 & 274 \\
\hline \multirow[t]{2}{*}{47} & $8-1-2002$ & 4.1 & 131 & -- & 130 & -- \\
\hline & $9-3-2002$ & 3.8 & 142 & -- & 133 & -- \\
\hline \multirow[t]{2}{*}{48} & $8-1-2002$ & 7.6 & 81 & -- & 325 & -- \\
\hline & $9-5-2002$ & 7.0 & 75 & -- & 320 & -- \\
\hline 49 & $7-31-2002$ & 4.0 & 125 & -- & 132 & -- \\
\hline 50 & $7-31-2002$ & 4.9 & 144 & -- & 135 & -- \\
\hline 51 & 8-1-2002 & 4.1 & 95 & -- & 153 & -- \\
\hline \multirow[t]{2}{*}{52} & $8-1-2002$ & 4.4 & 113 & -- & 161 & 138 \\
\hline & $9-17-2002$ & 5.3 & 132 & -- & 182 & 156 \\
\hline 53 & $7-31-2002$ & 4.4 & 135 & -- & 160 & -- \\
\hline 54 & 8-1-2002 & 3.8 & 84 & -- & 152 & -- \\
\hline 55 & $7-30-2002$ & 0.84 & 1.6 & -- & 86 & -- \\
\hline \multirow[t]{2}{*}{56} & $7-31-2002$ & 3.8 & 125 & -- & 126 & -- \\
\hline & $9-5-2002$ & 4.1 & 154 & -- & 138 & -- \\
\hline \multirow[t]{2}{*}{57} & $7-31-2002$ & 3.9 & 123 & -- & 126 & 108 \\
\hline & $9-4-2002$ & 4.6 & 182 & -- & 141 & 136 \\
\hline \multirow[t]{2}{*}{58} & $7-16-2002$ & -- & -- & -- & -- & -- \\
\hline & $9-5-2002$ & -- & -- & -- & -- & -- \\
\hline
\end{tabular}


Table 12. Water-quality data for the Upper Colorado River Basin, showing 2002 drought values, Colorado water-quality exceedances, and percentiles relative to historical data for selected properties and constituents from July through September, 1978-2002. - Continued

$\left[\mathrm{ft}^{3} / \mathrm{s}\right.$, cubic feet per second; Conc, concentration; Q\%, percentile (percentage of data less than or equal to reported value or concentration) of drought-sample constituent; N, total number of historical and drought samples; $\mathrm{mg} / \mathrm{L}$, milligrams per liter; $\mu \mathrm{S} / \mathrm{cm}$, microsiemens per centimeter at $25^{\circ} \mathrm{Celsius;}{ }^{\circ} \mathrm{C}$, degrees Celsius; E. coli, Escherichia coli; $\mathrm{mL}$, milliliter; $\mu \mathrm{g} / \mathrm{L}$, micrograms per liter; $\mathrm{CaCO}_{3}$, calcium carbonate; $\mathrm{SiO}_{2}$, silica; $\mathrm{SO}_{4}$, sulfate; ID, insufficient number of samples (less than 20) to calculate percentile; <, less than; --, no drought-sample measurement; values or concentrations in bold type indicate 2002 Colorado water-quality exceedances; c, chronic water-quality exceedance]

\begin{tabular}{|c|c|c|c|c|c|c|c|}
\hline \multirow{2}{*}{$\begin{array}{l}\text { Site number } \\
\text { in table } 1 \\
\text { and fig. } 4\end{array}$} & \multirow{2}{*}{ Sampling date } & \multicolumn{3}{|c|}{ Chloride, dissolved, in $\mathrm{mg} / \mathrm{L}$} & \multicolumn{3}{|c|}{ Fluoride, dissolved, in $\mathrm{mg} / \mathrm{L}$} \\
\hline & & Conc & $0 \%$ & $\mathrm{~N}$ & Conc & $0 \%$ & $\mathrm{~N}$ \\
\hline 20 & $8-1-2002$ & 0.47 & ID & 2 & -- & -- & -- \\
\hline \multirow[t]{2}{*}{21} & $7-15-2002$ & 9.1 & 89.3 & 27 & -- & -- & -- \\
\hline & $9-4-2002$ & 10.1 & 92.9 & 27 & -- & -- & -- \\
\hline \multirow[t]{2}{*}{22} & $7-15-2002$ & 9.3 & 88.9 & 26 & -- & -- & -- \\
\hline & $9-4-2002$ & 10.6 & 96.3 & 26 & -- & -- & -- \\
\hline \multirow[t]{2}{*}{23} & $7-15-2002$ & 5.9 & 79.2 & 23 & -- & -- & -- \\
\hline & $9-4-2002$ & 7.1 & 95.8 & 23 & -- & -- & -- \\
\hline \multirow[t]{2}{*}{24} & $7-15-2002$ & 7.9 & 92.0 & 24 & -- & -- & -- \\
\hline & $9-4-2002$ & 9.9 & 96.0 & 24 & -- & -- & -- \\
\hline \multirow[t]{2}{*}{25} & $7-15-2002$ & 2.5 & ID & 15 & -- & -- & -- \\
\hline & $9-4-2002$ & 2.0 & ID & 15 & -- & -- & -- \\
\hline \multirow[t]{2}{*}{26} & $7-17-2002$ & 6.5 & ID & 10 & 0.22 & ID & 9 \\
\hline & $9-6-2002$ & 7.9 & ID & 10 & 0.3 & ID & 9 \\
\hline 27 & $8-1-2002$ & 2.5 & ID & 7 & 0.18 & ID & 7 \\
\hline \multirow[t]{2}{*}{28} & $8-8-2002$ & 1.4 & ID & 6 & -- & -- & -- \\
\hline & $9-11-2002$ & 1.3 & ID & 6 & -- & -- & -- \\
\hline \multirow[t]{2}{*}{29} & $8-8-2002$ & 6.7 & ID & 2 & -- & -- & -- \\
\hline & $9-11-2002$ & 6.9 & ID & 2 & -- & -- & -- \\
\hline \multirow[t]{2}{*}{30} & $7-29-2002$ & 3.3 & 89.6 & 47 & 0.23 & 81.3 & 47 \\
\hline & $9-10-2002$ & 3.5 & 91.7 & 47 & 0.25 & 83.3 & 47 \\
\hline \multirow[t]{2}{*}{31} & $7-29-2002$ & 3.9 & 92.0 & 24 & 0.19 & 20.0 & 24 \\
\hline & $9-10-2002$ & 4.3 & 96.0 & 24 & 0.21 & 96.0 & 24 \\
\hline \multirow[t]{2}{*}{32} & $8-8-2002$ & 6.3 & ID & 2 & -- & -- & -- \\
\hline & $9-11-2002$ & 6.4 & ID & 2 & -- & -- & -- \\
\hline 33 & $7-30-2002$ & 5.4 & 95.8 & 23 & 0.31 & 75.0 & 23 \\
\hline 34 & $7-30-2002$ & 1.1 & ID & 6 & 0.1 & ID & 6 \\
\hline \multirow[t]{2}{*}{35} & $7-30-2002$ & 15.4 & 91.3 & 22 & 0.1 & ${ }^{1} 43.5-95.7$ & 22 \\
\hline & $9-3-2002$ & 20 & 95.7 & 22 & 0.1 & ${ }^{1} 43.5-95.7$ & 22 \\
\hline \multirow[t]{2}{*}{36} & $7-30-2002$ & 6.5 & ID & 12 & 0.1 & ID & 12 \\
\hline & $9-3-2002$ & 9.0 & ID & 12 & 0.1 & ID & 12 \\
\hline \multirow[t]{2}{*}{37} & $7-31-2002$ & 129 & 93.8 & 31 & 0.2 & ${ }^{1} 62.5-90.6$ & 31 \\
\hline & $9-4-2002$ & 165 & 96.9 & 31 & 0.2 & ${ }^{1} 62.5-90.6$ & 31 \\
\hline
\end{tabular}


Table 12. Water-quality data for the Upper Colorado River Basin, showing 2002 drought values, Colorado water-quality exceedances, and percentiles relative to historical data for selected properties and constituents from July through September, 1978-2002.—Continued

[ft $3 / \mathrm{s}$, cubic feet per second; Conc, concentration; Q\%, percentile (percentage of data less than or equal to reported value or concentration) of drought-sample constituent; N, total number of historical and drought samples; $\mathrm{mg} / \mathrm{L}$, milligrams per liter; $\mu \mathrm{S} / \mathrm{cm}$, microsiemens per centimeter at $25^{\circ} \mathrm{Celsius;}{ }^{\circ} \mathrm{C}$, degrees Celsius; E. coli, Escherichia coli; $\mathrm{mL}$, milliliter; $\mu \mathrm{g} / \mathrm{L}$, micrograms per liter; $\mathrm{CaCO}_{3}$, calcium carbonate; $\mathrm{SiO}_{2}$, silica; $\mathrm{SO}_{4}$, sulfate; ID, insufficient number of samples (less than 20) to calculate percentile; <, less than; --, no drought-sample measurement; values or concentrations in bold type indicate 2002 Colorado water-quality exceedances; c, chronic water-quality exceedance]

\begin{tabular}{|c|c|c|c|c|c|c|c|}
\hline \multirow{2}{*}{$\begin{array}{l}\text { Site number } \\
\text { in table } 1 \\
\text { and fig. } 4\end{array}$} & \multirow{2}{*}{ Sampling date } & \multicolumn{3}{|c|}{ Chloride, dissolved, in $\mathrm{mg} / \mathrm{L}$} & \multicolumn{3}{|c|}{ Fluoride, dissolved, in $\mathrm{mg} / \mathrm{L}$} \\
\hline & & Conc & $0 \%$ & $\mathrm{~N}$ & Conc & $0 \%$ & $\mathrm{~N}$ \\
\hline \multirow[t]{2}{*}{38} & $8-1-2002$ & 33 & 90.1 & 80 & -- & -- & -- \\
\hline & $9-5-2002$ & 46 & 98.8 & 80 & -- & -- & -- \\
\hline \multirow[t]{2}{*}{39} & $8-1-2002$ & 91 & 92.7 & 40 & -- & -- & -- \\
\hline & $9-6-2002$ & 135 & 97.6 & 40 & -- & -- & -- \\
\hline \multirow[t]{2}{*}{40} & $7-29-2002$ & 0.53 & ID & 6 & 0.4 & ID & 6 \\
\hline & $9-3-2002$ & -- & -- & -- & -- & -- & -- \\
\hline \multirow[t]{2}{*}{41} & $7-29-2002$ & 3.5 & ID & 6 & 0.2 & ID & 6 \\
\hline & $9-4-2002$ & -- & -- & -- & -- & -- & -- \\
\hline \multirow[t]{2}{*}{42} & $7-30-2002$ & 7.4 & ID & 6 & 0.3 & ID & 6 \\
\hline & $9-4-2002$ & -- & -- & -- & -- & -- & -- \\
\hline \multirow[t]{2}{*}{43} & $7-30-2002$ & 4.5 & ID & 3 & 0.2 & ID & 3 \\
\hline & $9-4-2002$ & -- & -- & -- & -- & -- & -- \\
\hline \multirow[t]{2}{*}{44} & $7-30-2002$ & 55 & 92.0 & 74 & 0.2 & ID & 7 \\
\hline & $9-5-2002$ & -- & -- & -- & -- & -- & -- \\
\hline \multirow[t]{2}{*}{45} & $7-31-2002$ & 188 & ID & 2 & -- & -- & -- \\
\hline & $9-6-2002$ & 237 & ID & 2 & -- & -- & -- \\
\hline 46 & $8-2-2002$ & 18.3 & ID & 16 & 0.3 & ID & 16 \\
\hline \multirow[t]{2}{*}{47} & 8-1-2002 & 184 & 96.9 & 64 & -- & -- & -- \\
\hline & $9-3-2002$ & 210 & 98.5 & 64 & -- & -- & -- \\
\hline \multirow[t]{2}{*}{48} & 8-1-2002 & 8.6 & 88.9 & 26 & -- & -- & -- \\
\hline & $9-5-2002$ & 7.9 & 85.2 & 26 & -- & -- & -- \\
\hline 49 & $7-31-2002$ & 179 & ID & 9 & -- & -- & -- \\
\hline 50 & $7-31-2002$ & 179 & ID & 7 & -- & -- & -- \\
\hline 51 & 8-1-2002 & 77 & ID & 7 & -- & -- & -- \\
\hline \multirow[t]{2}{*}{52} & $8-1-2002$ & 98 & ${ }^{1} 87.3-88.9$ & 62 & -- & -- & -- \\
\hline & $9-17-2002$ & 112 & 96.8 & 62 & -- & -- & -- \\
\hline 53 & $7-31-2002$ & 182 & ID & 4 & -- & -- & -- \\
\hline 54 & 8-1-2002 & 60 & ID & 7 & -- & -- & -- \\
\hline 55 & $7-30-2002$ & 0.41 & ID & 5 & 0.1 & ID & 5 \\
\hline \multirow[t]{2}{*}{56} & $7-31-2002$ & 188 & ID & 2 & -- & -- & -- \\
\hline & $9-5-2002$ & 227 & ID & 2 & -- & -- & -- \\
\hline \multirow[t]{2}{*}{57} & $7-31-2002$ & 198 & ID & 8 & 0.1 & ID & 8 \\
\hline & $9-4-2002$ & 292 & ID & 8 & 0.2 & ID & 8 \\
\hline \multirow[t]{2}{*}{58} & $7-16-2002$ & 5.8 & ID & 6 & -- & -- & -- \\
\hline & $9-5-2002$ & 6.9 & ID & 6 & -- & -- & -- \\
\hline
\end{tabular}


Table 12. Water-quality data for the Upper Colorado River Basin, showing 2002 drought values, Colorado water-quality exceedances, and percentiles relative to historical data for selected properties and constituents from July through September, 1978-2002.-Continued

$\left[\mathrm{ft}^{3} / \mathrm{s}\right.$, cubic feet per second; Conc, concentration; Q\%, percentile (percentage of data less than or equal to reported value or concentration) of drought-sample constituent; N, total number of historical and drought samples; $\mathrm{mg} / \mathrm{L}$, milligrams per liter; $\mu \mathrm{S} / \mathrm{cm}$, microsiemens per centimeter at $25^{\circ} \mathrm{Celsius;}{ }^{\circ} \mathrm{C}$, degrees Celsius; E. coli, Escherichia coli; $\mathrm{mL}$, milliliter; $\mu \mathrm{g} / \mathrm{L}$, micrograms per liter; $\mathrm{CaCO}_{3}$, calcium carbonate; $\mathrm{SiO}_{2}$, silica; $\mathrm{SO}_{4}$, sulfate; ID, insufficient number of samples (less than 20) to calculate percentile; <, less than; --, no drought-sample measurement; values or concentrations in bold type indicate 2002 Colorado water-quality exceedances; c, chronic water-quality exceedance]

\begin{tabular}{|c|c|c|c|c|c|c|c|c|}
\hline \multirow{2}{*}{$\begin{array}{l}\text { Site number } \\
\text { in table } 1 \\
\text { and fig. } 4\end{array}$} & \multirow{2}{*}{ Sampling date } & \multicolumn{3}{|c|}{ Silica, dissolved, in $\mathrm{mg} / \mathrm{L}$ as $\mathrm{SiO}_{2}$} & \multicolumn{3}{|c|}{ Sulfate, dissolved, in $\mathrm{mg} / \mathrm{L}$ as $\mathrm{SO}_{4}$} & \multirow{2}{*}{$\begin{array}{c}\text { Solids, } \\
\text { dissolved, } \\
\text { residue on } \\
\text { evaporation at } \\
180^{\circ} \mathrm{C} \text {, } \\
\text { in } \mathrm{mg} / \mathrm{L}\end{array}$} \\
\hline & & Conc & $0 \%$ & $\mathrm{~N}$ & Conc & $0 \%$ & $\mathrm{~N}$ & \\
\hline 20 & $8-1-2002$ & 4.5 & 33.3 & 2 & 3.2 & 66.7 & 2 & -- \\
\hline \multirow[t]{2}{*}{21} & $7-15-2002$ & -- & -- & -- & -- & -- & -- & -- \\
\hline & 9-4-2002 & -- & -- & -- & -- & -- & -- & -- \\
\hline \multirow[t]{2}{*}{22} & $7-15-2002$ & -- & -- & -- & -- & -- & -- & -- \\
\hline & $9-4-2002$ & -- & -- & -- & -- & -- & -- & -- \\
\hline \multirow[t]{2}{*}{23} & $7-15-2002$ & -- & -- & -- & -- & -- & -- & -- \\
\hline & $9-4-2002$ & -- & -- & -- & -- & -- & -- & -- \\
\hline \multirow[t]{2}{*}{24} & $7-15-2002$ & -- & -- & -- & -- & -- & -- & -- \\
\hline & $9-4-2002$ & -- & -- & -- & -- & -- & -- & -- \\
\hline \multirow[t]{2}{*}{25} & $7-15-2002$ & -- & -- & -- & -- & -- & -- & -- \\
\hline & $9-4-2002$ & -- & -- & -- & -- & -- & -- & -- \\
\hline \multirow[t]{2}{*}{26} & $7-17-2002$ & 13.3 & ID & 9 & 2.3 & ID & 9 & 94 \\
\hline & $9-6-2002$ & 12.9 & ID & 9 & 2.8 & ID & 9 & 100 \\
\hline 27 & 8-1-2002 & 8.7 & ID & 7 & 3.0 & ID & 7 & -- \\
\hline \multirow[t]{2}{*}{28} & $8-8-2002$ & 12.0 & ID & 6 & 5.5 & ID & 6 & -- \\
\hline & $9-11-2002$ & 12.8 & ID & 6 & 5.9 & ID & 6 & -- \\
\hline \multirow[t]{2}{*}{29} & 8-8-2002 & 29 & ID & 2 & 69 & ID & 2 & -- \\
\hline & $9-11-2002$ & 29 & ID & 2 & 70 & ID & 2 & -- \\
\hline \multirow[t]{2}{*}{30} & $7-29-2002$ & 10.6 & 83.3 & 47 & 116 & ${ }^{1} 66.7-68.8$ & 47 & 438 \\
\hline & $9-10-2002$ & 9.3 & 64.6 & 47 & 116 & ${ }^{1} 66.7-68.8$ & 47 & 391 \\
\hline \multirow[t]{2}{*}{31} & $7-29-2002$ & 8.2 & ${ }^{1} 40.0-48.0$ & 24 & 246 & 92.0 & 24 & 517 \\
\hline & $9-10-2002$ & 7.8 & 20.0 & 24 & 237 & ${ }^{1} 84.0-88.0$ & 24 & 524 \\
\hline \multirow[t]{2}{*}{32} & 8-8-2002 & 4.8 & ID & 2 & 35 & ID & 2 & -- \\
\hline & $9-11-2002$ & 4.9 & ID & 2 & 39 & ID & 2 & -- \\
\hline 33 & $7-30-2002$ & 7.1 & 16.7 & 23 & 32 & 20.8 & 23 & 126 \\
\hline 34 & $7-30-2002$ & 6.9 & ID & 6 & 11.2 & ID & 6 & -- \\
\hline \multirow[t]{2}{*}{35} & $7-30-2002$ & 3.9 & 17.4 & 22 & 53 & 91.3 & 22 & -- \\
\hline & $9-3-2002$ & 3.9 & 26.1 & 22 & 75 & 95.7 & 22 & -- \\
\hline \multirow[t]{2}{*}{36} & $7-30-2002$ & 5.1 & ID & 12 & 48 & ID & 12 & -- \\
\hline & $9-3-2002$ & 5.2 & ID & 12 & 83 & ID & 12 & -- \\
\hline \multirow[t]{2}{*}{37} & 7-31-2002 & 6.8 & 28.1 & 31 & 256 & 87.5 & 31 & -- \\
\hline & $9-4-2002$ & 4.4 & 6.3 & 31 & 310 & 96.9 & 31 & -- \\
\hline
\end{tabular}


Table 12. Water-quality data for the Upper Colorado River Basin, showing 2002 drought values, Colorado water-quality exceedances, and percentiles relative to historical data for selected properties and constituents from July through September, 1978-2002.—Continued

[ft $3 / \mathrm{s}$, cubic feet per second; Conc, concentration; Q\%, percentile (percentage of data less than or equal to reported value or concentration) of drought-sample constituent; N, total number of historical and drought samples; $\mathrm{mg} / \mathrm{L}$, milligrams per liter; $\mu \mathrm{S} / \mathrm{cm}$, microsiemens per centimeter at $25^{\circ} \mathrm{Celsius;}{ }^{\circ} \mathrm{C}$, degrees Celsius; E. coli, Escherichia coli; $\mathrm{mL}$, milliliter; $\mu \mathrm{g} / \mathrm{L}$, micrograms per liter; $\mathrm{CaCO}_{3}$, calcium carbonate; $\mathrm{SiO}_{2}$, silica; $\mathrm{SO}_{4}$, sulfate; ID, insufficient number of samples (less than 20) to calculate percentile; <, less than; --, no drought-sample measurement; values or concentrations in bold type indicate 2002 Colorado water-quality exceedances; c, chronic water-quality exceedance]

\begin{tabular}{|c|c|c|c|c|c|c|c|c|}
\hline \multirow{2}{*}{$\begin{array}{l}\text { Site number } \\
\text { in table } 1 \\
\text { and fig. } 4\end{array}$} & \multirow{2}{*}{ Sampling date } & \multicolumn{3}{|c|}{ Silica, dissolved, in $\mathrm{mg} / \mathrm{L}$ as $\mathrm{SiO}_{2}$} & \multicolumn{3}{|c|}{ Sulfate, dissolved, in $\mathrm{mg} / \mathrm{L}$ as $\mathrm{SO}_{4}$} & \multirow{2}{*}{$\begin{array}{c}\text { Solids, } \\
\text { dissolved, } \\
\text { residue on } \\
\text { evaporation at } \\
180^{\circ} \mathrm{C}, \\
\text { in } \mathrm{mg} / \mathrm{L}\end{array}$} \\
\hline & & Conc & $0 \%$ & $\mathrm{~N}$ & Conc & $0 \%$ & $\mathrm{~N}$ & \\
\hline \multirow[t]{2}{*}{38} & $8-1-2002$ & 6.1 & ID & 14 & 64 & 33.3 & 80 & -- \\
\hline & $9-5-2002$ & 8.1 & ID & 14 & 132 & 98.8 & 80 & -- \\
\hline \multirow[t]{2}{*}{39} & $8-1-2002$ & 6.3 & 2.4 & 40 & 68 & 24.4 & 40 & 358 \\
\hline & $9-6-2002$ & 7.8 & 31.7 & 40 & 137 & 97.6 & 40 & 549 \\
\hline \multirow[t]{2}{*}{40} & $7-29-2002$ & 5.2 & ID & 6 & 7.9 & ID & 6 & -- \\
\hline & $9-3-2002$ & -- & -- & -- & -- & -- & -- & -- \\
\hline \multirow[t]{2}{*}{41} & $7-29-2002$ & 7.6 & ID & 6 & 81 & ID & 6 & -- \\
\hline & $9-4-2002$ & -- & -- & -- & -- & -- & -- & -- \\
\hline \multirow[t]{2}{*}{42} & $7-30-2002$ & 8.5 & ID & 6 & 134 & ID & 6 & -- \\
\hline & $9-4-2002$ & -- & -- & -- & -- & -- & -- & -- \\
\hline \multirow[t]{2}{*}{43} & $7-30-2002$ & 12.9 & ID & 3 & 133 & ID & 3 & -- \\
\hline & $9-4-2002$ & -- & -- & -- & -- & -- & -- & -- \\
\hline \multirow[t]{2}{*}{44} & $7-30-2002$ & 10.3 & ID & 7 & 130 & 80.0 & 74 & -- \\
\hline & $9-5-2002$ & -- & -- & -- & -- & -- & -- & -- \\
\hline \multirow[t]{2}{*}{45} & $7-31-2002$ & 7.4 & ID & 2 & 99 & ID & 2 & -- \\
\hline & $9-6-2002$ & 7.5 & ID & 2 & 143 & ID & 2 & -- \\
\hline 46 & $8-2-2002$ & 0.30 & ID & 16 & 1,910 & ID & 16 & -- \\
\hline \multirow[t]{2}{*}{47} & $8-1-2002$ & 6.4 & 11.1 & 53 & 114 & 56.9 & 64 & -- \\
\hline & $9-3-2002$ & 5.6 & 1.9 & 53 & 144 & 89.2 & 64 & -- \\
\hline \multirow[t]{2}{*}{48} & $8-1-2002$ & 28 & 63.0 & 26 & 94 & 92.6 & 26 & -- \\
\hline & $9-5-2002$ & 26 & 33.3 & 26 & 91 & 85.2 & 26 & -- \\
\hline 49 & $7-31-2002$ & 7.6 & ID & 9 & 121 & ID & 9 & -- \\
\hline 50 & $7-31-2002$ & 7.5 & ID & 7 & 338 & ID & 7 & -- \\
\hline 51 & $8-1-2002$ & 8.1 & ID & 7 & 388 & ID & 7 & -- \\
\hline \multirow[t]{2}{*}{52} & $8-1-2002$ & 6.8 & 4.8 & 62 & 437 & 87.3 & 62 & -- \\
\hline & $9-17-2002$ & 12.9 & 93.7 & 62 & 496 & 93.7 & 62 & -- \\
\hline 53 & $7-31-2002$ & 7.8 & ID & 4 & 259 & ID & 4 & -- \\
\hline 54 & 8-1-2002 & 8.2 & ID & 7 & 370 & ID & 7 & -- \\
\hline 55 & $7-30-2002$ & 4.7 & ID & 5 & 5.9 & ID & 5 & -- \\
\hline \multirow[t]{2}{*}{56} & $7-31-2002$ & 7.2 & ID & 2 & 103 & ID & 2 & -- \\
\hline & $9-5-2002$ & 6.8 & ID & 2 & 140 & ID & 2 & -- \\
\hline \multirow[t]{2}{*}{57} & $7-31-2002$ & 3.9 & ID & 8 & 113 & ID & 8 & -- \\
\hline & $9-4-2002$ & 3.7 & ID & 8 & 165 & ID & 8 & -- \\
\hline \multirow[t]{2}{*}{58} & 7-16-2002 & -- & -- & -- & -- & -- & -- & -- \\
\hline & $9-5-2002$ & -- & -- & -- & -- & -- & -- & -- \\
\hline
\end{tabular}


Table 12. Water-quality data for the Upper Colorado River Basin, showing 2002 drought values, Colorado water-quality exceedances, and percentiles relative to historical data for selected properties and constituents from July through September, 1978-2002. - Continued

$[\mathrm{ft} / \mathrm{s}$, cubic feet per second; Conc, concentration; Q\%, percentile (percentage of data less than or equal to reported value or concentration) of drought-sample constituent; $\mathrm{N}$, total number of historical and drought samples; $\mathrm{mg} / \mathrm{L}$, milligrams per liter; $\mu \mathrm{S} / \mathrm{cm}$, microsiemens per centimeter at $25^{\circ} \mathrm{Celsius;}{ }^{\circ} \mathrm{C}$, degrees Celsius; E. coli, Escherichia coli; $\mathrm{mL}$, milliliter; $\mu \mathrm{g} / \mathrm{L}$, micrograms per liter; $\mathrm{CaCO}_{3}$, calcium carbonate; $\mathrm{SiO}_{2}$, silica; $\mathrm{SO}_{4}$, sulfate; ID, insufficient number of samples (less than 20) to calculate percentile; <, less than; --, no drought-sample measurement; values or concentrations in bold type indicate 2002 Colorado water-quality exceedances; c, chronic water-quality exceedance]

\begin{tabular}{|c|c|c|c|c|c|c|c|}
\hline \multirow{2}{*}{$\begin{array}{l}\text { Site number } \\
\text { in table } 1 \\
\text { and fig. } 4\end{array}$} & \multirow{2}{*}{ Sampling date } & \multicolumn{3}{|c|}{$\begin{array}{l}\text { Nitrogen, ammonia, dissolved, in } \\
\mathrm{mg} / \mathrm{L} \text { as } \mathrm{N}\end{array}$} & \multicolumn{3}{|c|}{$\begin{array}{c}\text { Nitrogen, organic plus ammonia } \\
\text { dissolved, } \\
\text { in } \mathrm{mg} / \mathrm{L} \text { as } \mathrm{N} \\
\end{array}$} \\
\hline & & Conc & $0 \%$ & $\mathrm{~N}$ & Conc & $0 \%$ & $\mathrm{~N}$ \\
\hline 20 & $8-1-2002$ & $<0.007$ & ID & 2 & -- & -- & -- \\
\hline \multirow[t]{2}{*}{21} & $7-15-2002$ & $<0.002$ & ${ }^{2} 4.2-54.2$ & 23 & -- & -- & -- \\
\hline & $9-4-2002$ & $<0.002$ & ${ }^{2} 4.2-54.2$ & 23 & -- & -- & -- \\
\hline \multirow[t]{2}{*}{22} & $7-15-2002$ & $<0.002$ & ${ }^{2} 3.3-56.7$ & 29 & -- & -- & -- \\
\hline & $9-4-2002$ & $<0.002$ & ${ }^{2} 3.3-56.7$ & 29 & -- & -- & -- \\
\hline \multirow[t]{2}{*}{23} & $7-15-2002$ & $<0.015$ & ${ }^{2} 2.9-88.2$ & 33 & -- & -- & -- \\
\hline & $9-4-2002$ & $<0.015$ & ${ }^{2} 2.9-88.2$ & 33 & -- & -- & -- \\
\hline \multirow[t]{2}{*}{24} & $7-15-2002$ & 0.011 & 41.2 & 33 & -- & -- & -- \\
\hline & $9-4-2002$ & 0.010 & ${ }^{1} 35.3-38.2$ & 33 & -- & -- & -- \\
\hline \multirow[t]{2}{*}{25} & $7-15-2002$ & $<0.015$ & ID & 15 & -- & -- & -- \\
\hline & $9-4-2002$ & $<0.015$ & ID & 15 & -- & -- & -- \\
\hline \multirow[t]{2}{*}{26} & $7-17-2002$ & 0.008 & ID & 10 & 0.30 & ID & 9 \\
\hline & $9-6-2002$ & $<0.015$ & ID & 10 & 0.28 & ID & 9 \\
\hline 27 & $8-1-2002$ & $<0.04$ & ID & 8 & 0.26 & ID & 8 \\
\hline \multirow[t]{2}{*}{28} & 8-8-2002 & $<0.015$ & ID & 5 & 0.15 & ID & 5 \\
\hline & $9-11-2002$ & 0.010 & ID & 5 & 0.16 & ID & 5 \\
\hline \multirow[t]{2}{*}{29} & 8-8-2002 & $<0.015$ & ID & 2 & 0.34 & ID & 2 \\
\hline & $9-11-2002$ & $<0.015$ & ID & 2 & 0.30 & ID & 2 \\
\hline \multirow[t]{2}{*}{30} & $7-29-2002$ & $<0.02$ & ${ }^{2} 2.6-56.4$ & 38 & 0.53 & 92.3 & 38 \\
\hline & $9-10-2002$ & $<0.02$ & ${ }^{2} 2.6-56.4$ & 38 & 0.37 & ${ }^{1} 61.5-64.1$ & 38 \\
\hline \multirow[t]{2}{*}{31} & $7-29-2002$ & 0.028 & 66.7 & 23 & 0.33 & ${ }^{1} 70.8-79.2$ & 23 \\
\hline & $9-10-2002$ & 0.081 & 87.5 & 23 & 0.41 & 87.5 & 23 \\
\hline \multirow[t]{2}{*}{32} & 8-8-2002 & $<0.015$ & ID & 2 & 0.12 & ID & 2 \\
\hline & $9-11-2002$ & 0.014 & ID & 2 & 0.18 & ID & 2 \\
\hline 33 & $7-30-2002$ & $<0.015$ & ${ }^{2} 4.3-39.1$ & 22 & -- & -- & -- \\
\hline 34 & $7-30-2002$ & $<0.015$ & ID & 9 & 0.07 & ID & 9 \\
\hline \multirow[t]{2}{*}{35} & $7-30-2002$ & 0.025 & 86.4 & 21 & 0.19 & ${ }^{2} 4.3-91.3$ & 22 \\
\hline & $9-3-2002$ & 0.010 & 40.9 & 21 & 0.17 & ${ }^{2} 4.3-91.3$ & 22 \\
\hline \multirow[t]{2}{*}{36} & $7-30-2002$ & 0.009 & ID & 18 & 0.12 & ID & 18 \\
\hline & $9-3-2002$ & 0.009 & ID & 18 & 0.12 & ID & 18 \\
\hline \multirow[t]{2}{*}{37} & $7-31-2002$ & 0.050 & ${ }^{1} 85.7-90.5$ & 20 & 0.22 & 64.5 & 30 \\
\hline & $9-4-2002$ & $<0.02$ & ${ }^{2} 4.8-28.6$ & 20 & 0.21 & 61.3 & 30 \\
\hline
\end{tabular}


Table 12. Water-quality data for the Upper Colorado River Basin, showing 2002 drought values, Colorado water-quality exceedances, and percentiles relative to historical data for selected properties and constituents from July through September, 1978-2002.—Continued

[ft $3 / \mathrm{s}$, cubic feet per second; Conc, concentration; Q\%, percentile (percentage of data less than or equal to reported value or concentration) of drought-sample constituent; N, total number of historical and drought samples; $\mathrm{mg} / \mathrm{L}$, milligrams per liter; $\mu \mathrm{S} / \mathrm{cm}$, microsiemens per centimeter at $25^{\circ} \mathrm{Celsius;}{ }^{\circ} \mathrm{C}$, degrees Celsius; E. coli, Escherichia coli; $\mathrm{mL}$, milliliter; $\mu \mathrm{g} / \mathrm{L}$, micrograms per liter; $\mathrm{CaCO}_{3}$, calcium carbonate; $\mathrm{SiO}_{2}$, silica; $\mathrm{SO}_{4}$, sulfate; ID, insufficient number of samples (less than 20) to calculate percentile; <, less than; --, no drought-sample measurement; values or concentrations in bold type indicate 2002 Colorado water-quality exceedances; c, chronic water-quality exceedance]

\begin{tabular}{|c|c|c|c|c|c|c|c|}
\hline \multirow{2}{*}{$\begin{array}{l}\text { Site number } \\
\text { in table } 1 \\
\text { and fig. } 4\end{array}$} & \multirow{2}{*}{ Sampling date } & \multicolumn{3}{|c|}{$\begin{array}{l}\text { Nitrogen, ammonia, dissolved, in } \\
\mathrm{mg} / \mathrm{L} \text { as N }\end{array}$} & \multicolumn{3}{|c|}{$\begin{array}{c}\text { Nitrogen, organic plus ammonia } \\
\text { dissolved, } \\
\text { in } \mathrm{mg} / \mathrm{L} \text { as } \mathrm{N}\end{array}$} \\
\hline & & Conc & $0 \%$ & $\mathrm{~N}$ & Conc & $0 \%$ & $\mathrm{~N}$ \\
\hline \multirow[t]{2}{*}{38} & $8-1-2002$ & $<0.015$ & ID & 12 & 0.18 & ID & 12 \\
\hline & $9-5-2002$ & $<0.015$ & ID & 12 & 0.20 & ID & 12 \\
\hline \multirow[t]{2}{*}{39} & $8-1-2002$ & 0.013 & ID & 2 & 0.16 & ID & 2 \\
\hline & $9-6-2002$ & $<0.015$ & ID & 2 & 0.20 & ID & 2 \\
\hline \multirow[t]{2}{*}{40} & $7-29-2002$ & $<0.015$ & ID & 12 & 0.05 & ID & 12 \\
\hline & $9-3-2002$ & $<0.015$ & ID & 12 & 0.09 & ID & 12 \\
\hline 41 & $\begin{array}{l}7-29-2002 \\
9-4-2002\end{array}$ & $\begin{array}{c}0.016 \\
<0.015\end{array}$ & $\begin{array}{l}\text { ID } \\
\text { ID }\end{array}$ & $\begin{array}{l}11 \\
11\end{array}$ & $\begin{array}{l}0.13 \\
0.11\end{array}$ & $\begin{array}{l}\text { ID } \\
\text { ID }\end{array}$ & $\begin{array}{l}11 \\
11\end{array}$ \\
\hline \multirow[t]{2}{*}{42} & $7-30-2002$ & 0.017 & ID & 12 & $<0.1$ & ID & 12 \\
\hline & $9-4-2002$ & 0.008 & ID & 12 & 0.05 & ID & 12 \\
\hline \multirow[t]{2}{*}{43} & $7-30-2002$ & 0.034 & ID & 6 & 0.09 & ID & 6 \\
\hline & $9-4-2002$ & 0.013 & ID & 6 & 0.12 & ID & 6 \\
\hline \multirow[t]{2}{*}{44} & $7-30-2002$ & 0.021 & ID & 13 & 0.11 & ID & 13 \\
\hline & $9-5-2002$ & $<0.015$ & ID & 13 & 0.12 & ID & 13 \\
\hline \multirow[t]{2}{*}{45} & $7-31-2002$ & $<0.015$ & ID & 2 & 0.15 & ID & 2 \\
\hline & $9-6-2002$ & $<0.015$ & ID & 2 & 0.20 & ID & 2 \\
\hline 46 & $8-2-2002$ & $<0.04$ & ID & 16 & -- & -- & -- \\
\hline \multirow[t]{2}{*}{47} & $8-1-2002$ & $<0.04$ & ID & 17 & 0.15 & ID & 17 \\
\hline & $9-3-2002$ & $<0.04$ & ID & 17 & 0.16 & ID & 17 \\
\hline \multirow[t]{2}{*}{48} & $8-1-2002$ & $<0.04$ & ID & 3 & 0.43 & ID & 2 \\
\hline & $9-5-2002$ & $<0.04$ & ID & 3 & 0.36 & ID & 2 \\
\hline 49 & $7-31-2002$ & 0.023 & ID & 1 & 0.18 & ID & 1 \\
\hline 50 & $7-31-2002$ & 0.035 & ID & 1 & 0.26 & ID & 1 \\
\hline 51 & 8-1-2002 & 0.061 & ID & 1 & 0.35 & ID & 1 \\
\hline \multirow[t]{2}{*}{52} & $8-1-2002$ & $<0.04$ & ${ }^{1} 2.1-64.6$ & 47 & 0.29 & 67.7 & 30 \\
\hline & $9-17-2002$ & $<0.04$ & ${ }^{1} 2.1-64.6$ & 47 & 0.33 & 80.6 & 30 \\
\hline 53 & $7-31-2002$ & 0.092 & ID & 1 & 0.29 & ID & 1 \\
\hline 54 & $8-1-2002$ & 0.042 & ID & 1 & 0.27 & ID & 1 \\
\hline 55 & $7-30-2002$ & $<0.015$ & ID & 8 & 0.08 & ID & 8 \\
\hline \multirow[t]{2}{*}{56} & $7-31-2002$ & $<0.04$ & ID & 2 & 0.15 & ID & 2 \\
\hline & $9-5-2002$ & 0.030 & ID & 2 & 0.18 & ID & 2 \\
\hline \multirow[t]{2}{*}{57} & $7-31-2002$ & 0.026 & ID & 8 & 0.26 & ID & 8 \\
\hline & $9-4-2002$ & 0.014 & ID & 8 & 0.27 & ID & 8 \\
\hline \multirow[t]{2}{*}{58} & 7-16-2002 & 0.014 & ID & 6 & -- & -- & -- \\
\hline & $9-5-2002$ & $<0.015$ & ID & 6 & -- & -- & -- \\
\hline
\end{tabular}


Table 12. Water-quality data for the Upper Colorado River Basin, showing 2002 drought values, Colorado water-quality exceedances, and percentiles relative to historical data for selected properties and constituents from July through September, 1978-2002.—Continued

$\left[\mathrm{ft} \mathrm{t}^{3} / \mathrm{s}\right.$, cubic feet per second; Conc, concentration; Q\%, percentile (percentage of data less than or equal to reported value or concentration) of drought-sample constituent; N, total number of historical and drought samples; $\mathrm{mg} / \mathrm{L}$, milligrams per liter; $\mu \mathrm{S} / \mathrm{cm}$, microsiemens per centimeter at $25^{\circ} \mathrm{Celsius;}{ }^{\circ} \mathrm{C}$, degrees Celsius; E. coli, Escherichia coli; $\mathrm{mL}$, milliliter; $\mu \mathrm{g} / \mathrm{L}$, micrograms per liter; $\mathrm{CaCO}_{3}$, calcium carbonate; $\mathrm{SiO}_{2}$, silica; $\mathrm{SO}_{4}$, sulfate; ID, insufficient number of samples (less than 20) to calculate percentile; <, less than; --, no drought-sample measurement; values or concentrations in bold type indicate 2002 Colorado water-quality exceedances; $c$, chronic water-quality exceedance]

\begin{tabular}{|c|c|c|c|c|c|c|c|}
\hline \multirow{2}{*}{$\begin{array}{l}\text { Site number } \\
\text { in table } 1 \\
\text { and fig. } 4\end{array}$} & \multirow{2}{*}{ Sampling date } & \multicolumn{3}{|c|}{$\begin{array}{c}\text { Nitrogen, organic plus ammonia, } \\
\text { total recoverable, } \\
\text { in } \mathrm{mg} / \mathrm{L} \text { as } \mathrm{N}\end{array}$} & \multicolumn{3}{|c|}{$\begin{array}{l}\text { Nitrogen, nitrite plus nitrate, } \\
\text { dissolved, in } \mathrm{mg} / \mathrm{L} \text { as } \mathrm{N}\end{array}$} \\
\hline & & Conc & $0 \%$ & $\mathrm{~N}$ & Conc & $0 \%$ & $\mathrm{~N}$ \\
\hline 20 & $8-1-2002$ & 0.22 & ID & 2 & -- & -- & -- \\
\hline \multirow[t]{2}{*}{21} & $7-15-2002$ & -- & -- & -- & 0.055 & 58.3 & 23 \\
\hline & $9-4-2002$ & -- & -- & -- & 0.087 & 87.5 & 23 \\
\hline \multirow[t]{2}{*}{22} & $7-15-2002$ & -- & -- & -- & 0.040 & 43.3 & 29 \\
\hline & $9-4-2002$ & -- & -- & -- & 0.030 & 30.0 & 29 \\
\hline \multirow[t]{2}{*}{23} & $7-15-2002$ & -- & -- & -- & 0.078 & ${ }^{1} 61.8-64.7$ & 33 \\
\hline & $9-4-2002$ & -- & -- & -- & 0.039 & 29.4 & 33 \\
\hline \multirow[t]{2}{*}{24} & $7-15-2002$ & -- & -- & -- & 0.312 & 97.1 & 33 \\
\hline & $9-4-2002$ & -- & -- & -- & 0.108 & 76.5 & 33 \\
\hline \multirow[t]{2}{*}{25} & $7-15-2002$ & -- & -- & -- & $<0.013$ & ID & 15 \\
\hline & $9-4-2002$ & -- & -- & -- & $<0.013$ & ID & 15 \\
\hline \multirow[t]{2}{*}{26} & $7-17-2002$ & 0.42 & ID & 9 & 0.147 & ID & 10 \\
\hline & $9-6-2002$ & 0.35 & ID & 9 & 0.165 & ID & 10 \\
\hline 27 & $8-1-2002$ & 0.42 & ID & 8 & $<0.05$ & ID & 8 \\
\hline \multirow[t]{2}{*}{28} & $8-8-2002$ & -- & -- & -- & 0.126 & ID & 5 \\
\hline & $9-11-2002$ & -- & -- & -- & 0.046 & ID & 5 \\
\hline \multirow[t]{2}{*}{29} & $8-8-2002$ & -- & -- & -- & $<0.013$ & ID & 2 \\
\hline & $9-11-2002$ & -- & -- & -- & $<0.013$ & ID & 2 \\
\hline \multirow[t]{2}{*}{30} & $7-29-2002$ & 0.69 & 91.5 & 46 & $<0.013$ & ${ }^{2} 2.6-86.8$ & 37 \\
\hline & $9-10-2002$ & 0.43 & 63.8 & 46 & $<0.013$ & ${ }^{2} 2.6-86.8$ & 37 \\
\hline \multirow[t]{2}{*}{31} & $7-29-2002$ & 0.40 & 76.0 & 24 & 0.206 & 54.2 & 23 \\
\hline & $9-10-2002$ & 0.49 & 84.0 & 24 & 0.081 & 25.0 & 23 \\
\hline \multirow[t]{2}{*}{32} & $8-8-2002$ & -- & -- & -- & 0.150 & ID & 2 \\
\hline & $9-11-2002$ & -- & -- & -- & 0.059 & ID & 2 \\
\hline 33 & $7-30-2002$ & 0.22 & ${ }^{1} 50.0-58.3$ & 23 & 0.107 & 62.5 & 23 \\
\hline 34 & $7-30-2002$ & 0.06 & ID & 9 & 0.012 & ID & 9 \\
\hline \multirow[t]{2}{*}{35} & $7-30-2002$ & 0.42 & 95.7 & 22 & 0.771 & 91.3 & 22 \\
\hline & $9-3-2002$ & 0.27 & 91.3 & 22 & 1.12 & 95.7 & 22 \\
\hline \multirow[t]{2}{*}{36} & $7-30-2002$ & 0.17 & ID & 15 & 0.288 & ID & 18 \\
\hline & $9-3-2002$ & 0.15 & ID & 15 & 0.342 & ID & 18 \\
\hline \multirow[t]{2}{*}{37} & $7-31-2002$ & 0.37 & 74.2 & 30 & 0.276 & 57.1 & 34 \\
\hline & $9-4-2002$ & 0.26 & ${ }^{1} 61.3-64.5$ & 30 & 0.236 & 42.9 & 34 \\
\hline
\end{tabular}


Table 12. Water-quality data for the Upper Colorado River Basin, showing 2002 drought values, Colorado water-quality exceedances, and percentiles relative to historical data for selected properties and constituents from July through September, 1978-2002.-Continued

[ft $3 / \mathrm{s}$, cubic feet per second; Conc, concentration; Q\%, percentile (percentage of data less than or equal to reported value or concentration) of drought-sample constituent; N, total number of historical and drought samples; $\mathrm{mg} / \mathrm{L}$, milligrams per liter; $\mu \mathrm{S} / \mathrm{cm}$, microsiemens per centimeter at $25^{\circ} \mathrm{Celsius;}{ }^{\circ} \mathrm{C}$, degrees Celsius; E. coli, Escherichia coli; $\mathrm{mL}$, milliliter; $\mu \mathrm{g} / \mathrm{L}$, micrograms per liter; $\mathrm{CaCO}_{3}$, calcium carbonate; $\mathrm{SiO}_{2}$, silica; $\mathrm{SO}_{4}$, sulfate; ID, insufficient number of samples (less than 20) to calculate percentile; <, less than; --, no drought-sample measurement; values or concentrations in bold type indicate 2002 Colorado water-quality exceedances; c, chronic water-quality exceedance]

\begin{tabular}{|c|c|c|c|c|c|c|c|}
\hline \multirow{2}{*}{$\begin{array}{l}\text { Site number } \\
\text { in table } 1 \\
\text { and fig. } 4\end{array}$} & \multirow{2}{*}{ Sampling date } & \multicolumn{3}{|c|}{$\begin{array}{c}\text { Nitrogen, organic plus ammonia, } \\
\text { total recoverable, } \\
\text { in } \mathrm{mg} / \mathrm{L} \text { as } \mathrm{N} \\
\end{array}$} & \multicolumn{3}{|c|}{$\begin{array}{l}\text { Nitrogen, nitrite plus nitrate, } \\
\text { dissolved, in } \mathrm{mg} / \mathrm{L} \text { as } \mathrm{N}\end{array}$} \\
\hline & & Conc & $0 \%$ & $\mathrm{~N}$ & Conc & $0 \%$ & $\mathrm{~N}$ \\
\hline \multirow[t]{2}{*}{38} & $8-1-2002$ & -- & -- & -- & 0.016 & ID & 14 \\
\hline & $9-5-2002$ & -- & -- & -- & $<0.013$ & ID & 14 \\
\hline \multirow[t]{2}{*}{39} & $8-1-2002$ & -- & -- & -- & 0.026 & ID & 11 \\
\hline & $9-6-2002$ & -- & -- & -- & 0.030 & ID & 11 \\
\hline \multirow[t]{2}{*}{40} & $7-29-2002$ & 0.06 & ID & 12 & 0.012 & ID & 12 \\
\hline & $9-3-2002$ & 0.05 & ID & 12 & 0.010 & ID & 12 \\
\hline \multirow[t]{2}{*}{41} & $7-29-2002$ & 0.17 & ID & 11 & 0.103 & ID & 11 \\
\hline & $9-4-2002$ & 0.13 & ID & 11 & 0.091 & ID & 11 \\
\hline \multirow[t]{2}{*}{42} & $7-30-2002$ & 0.05 & ID & 12 & 0.031 & ID & 12 \\
\hline & $9-4-2002$ & 0.06 & ID & 12 & 0.026 & ID & 12 \\
\hline \multirow[t]{2}{*}{43} & $7-30-2002$ & 0.11 & ID & 6 & 0.245 & ID & 6 \\
\hline & $9-4-2002$ & 0.13 & ID & 6 & 0.200 & ID & 6 \\
\hline \multirow[t]{2}{*}{44} & $7-30-2002$ & 0.18 & ID & 13 & 0.042 & ID & 13 \\
\hline & $9-5-2002$ & 0.16 & ID & 13 & 0.047 & ID & 13 \\
\hline \multirow[t]{2}{*}{45} & $7-31-2002$ & -- & -- & -- & 0.031 & ID & 2 \\
\hline & $9-6-2002$ & -- & -- & -- & $<0.013$ & ID & 2 \\
\hline 46 & $8-2-2002$ & 0.65 & ID & 16 & $<0.05$ & ID & 16 \\
\hline \multirow[t]{2}{*}{47} & $8-1-2002$ & -- & -- & -- & $<0.05$ & ${ }^{2} 3.8-50$ & 25 \\
\hline & $9-3-2002$ & -- & -- & -- & $<0.05$ & ${ }^{2} 3.8-50$ & 25 \\
\hline \multirow[t]{2}{*}{48} & $8-1-2002$ & -- & -- & -- & $<0.05$ & ID & 6 \\
\hline & $9-5-2002$ & -- & -- & -- & $<0.05$ & ID & 6 \\
\hline 49 & $7-31-2002$ & -- & -- & -- & 0.023 & ID & 1 \\
\hline 50 & $7-31-2002$ & -- & -- & -- & 0.204 & ID & 1 \\
\hline 51 & $8-1-2002$ & -- & -- & -- & 0.974 & ID & 1 \\
\hline \multirow[t]{2}{*}{52} & $8-1-2002$ & -- & -- & -- & 0.870 & 50.9 & 54 \\
\hline & $9-17-2002$ & -- & -- & -- & 1.31 & 92.7 & 54 \\
\hline 53 & $7-31-2002$ & -- & -- & -- & 0.301 & ID & 1 \\
\hline 54 & $8-1-2002$ & -- & -- & -- & 0.752 & ID & 1 \\
\hline 55 & $7-30-2002$ & 0.08 & ID & 8 & 0.037 & ID & 8 \\
\hline \multirow[t]{2}{*}{56} & $7-31-2002$ & -- & -- & -- & $<0.05$ & ID & 2 \\
\hline & $9-5-2002$ & -- & -- & -- & $<0.05$ & ID & 2 \\
\hline \multirow[t]{2}{*}{57} & $7-31-2002$ & 0.40 & ID & 8 & 0.894 & ID & 8 \\
\hline & $9-4-2002$ & 0.34 & ID & 8 & 1.11 & ID & 8 \\
\hline \multirow[t]{2}{*}{58} & $7-16-2002$ & -- & -- & -- & 0.009 & ID & 6 \\
\hline & $9-5-2002$ & -- & -- & -- & $<0.013$ & ID & 6 \\
\hline
\end{tabular}


Table 12. Water-quality data for the Upper Colorado River Basin, showing 2002 drought values, Colorado water-quality exceedances, and percentiles relative to historical data for selected properties and constituents from July through September, 1978-2002. - Continued

$\left[\mathrm{ft}^{3} / \mathrm{s}\right.$, cubic feet per second; Conc, concentration; Q\%, percentile (percentage of data less than or equal to reported value or concentration) of drought-sample constituent; $\mathrm{N}$, total number of historical and drought samples; $\mathrm{mg} / \mathrm{L}$, milligrams per liter; $\mu \mathrm{S} / \mathrm{cm}$, microsiemens per centimeter at $25^{\circ} \mathrm{Celsius;}{ }^{\circ} \mathrm{C}$, degrees Celsius; E. coli, Escherichia coli; $\mathrm{mL}$, milliliter; $\mu \mathrm{g} / \mathrm{L}$, micrograms per liter; $\mathrm{CaCO}_{3}$, calcium carbonate; $\mathrm{SiO}_{2}$, silica; $\mathrm{SO}_{4}$, sulfate; ID, insufficient number of samples (less than 20) to calculate percentile; <, less than; --, no drought-sample measurement; values or concentrations in bold type indicate 2002 Colorado water-quality exceedances; c, chronic water-quality exceedance]

\begin{tabular}{|c|c|c|c|c|c|c|c|}
\hline \multirow{2}{*}{$\begin{array}{l}\text { Site number } \\
\text { in table } 1 \\
\text { and fig. } 4\end{array}$} & \multirow{2}{*}{ Sampling date } & \multicolumn{3}{|c|}{$\begin{array}{l}\text { Nitrogen, nitrite, dissolved, } \\
\text { in } \mathrm{mg} / \mathrm{L} \text { as } \mathrm{N}\end{array}$} & \multicolumn{3}{|c|}{$\begin{array}{l}\text { Phosphorus, dissolved, } \\
\text { in } \mathrm{mg} / \mathrm{L} \text { as } \mathrm{P}\end{array}$} \\
\hline & & Conc & $0 \%$ & $\mathrm{~N}$ & Conc & $0 \%$ & $\mathrm{~N}$ \\
\hline 20 & $8-1-2002$ & -- & -- & -- & -- & -- & -- \\
\hline \multirow[t]{2}{*}{21} & $7-15-2002$ & $<0.002$ & ${ }^{2} 4.2-83.3$ & 23 & 0.003 & ${ }^{1} 4.0-96.0$ & 24 \\
\hline & $9-4-2002$ & $<0.002$ & ${ }^{2} 4.2-83.3$ & 23 & $<0.004$ & ${ }^{1} 4.0-96.0$ & 24 \\
\hline \multirow[t]{2}{*}{22} & $7-15-2002$ & $<0.002$ & ${ }^{2} 3.3-83.3$ & 29 & 0.004 & ${ }^{1} 61.9-81.0$ & 20 \\
\hline & $9-4-2002$ & $<0.002$ & ${ }^{2} 3.3-83.3$ & 29 & 0.003 & ${ }^{2} 4.8-57.1$ & 20 \\
\hline \multirow[t]{2}{*}{23} & $7-15-2002$ & 0.002 & ${ }^{1} 45.5-69.7$ & 32 & 0.031 & 96.0 & 24 \\
\hline & $9-4-2002$ & $<0.002$ & ${ }^{2} 3-42.4$ & 32 & 0.022 & 92.0 & 24 \\
\hline \multirow[t]{2}{*}{24} & $7-15-2002$ & 0.021 & 97.1 & 33 & 0.113 & 92.0 & 24 \\
\hline & $9-4-2002$ & 0.006 & ${ }^{1} 44.1-50$ & 33 & 0.123 & 96.0 & 24 \\
\hline \multirow[t]{2}{*}{25} & $7-15-2002$ & $<0.002$ & ID & 15 & 0.022 & ID & 15 \\
\hline & $9-4-2002$ & $<0.002$ & ID & 15 & 0.021 & ID & 15 \\
\hline \multirow[t]{2}{*}{26} & $7-17-2002$ & 0.005 & ID & 10 & 0.082 & ID & 10 \\
\hline & $9-6-2002$ & 0.004 & ID & 10 & 0.08 & ID & 10 \\
\hline 27 & $8-1-2002$ & $<0.008$ & ID & 8 & 0.03 & ID & 8 \\
\hline \multirow[t]{2}{*}{28} & $8-8-2002$ & $<0.002$ & ID & 2 & 0.016 & ID & 5 \\
\hline & $9-11-2002$ & 0.003 & ID & 2 & 0.01 & ID & 5 \\
\hline \multirow[t]{2}{*}{29} & 8-8-2002 & $<0.002$ & ID & 2 & 0.099 & ID & 2 \\
\hline & $9-11-2002$ & $<0.002$ & ID & 2 & 0.099 & ID & 2 \\
\hline \multirow[t]{2}{*}{30} & $7-29-2002$ & $<0.002$ & ${ }^{2} 2.6-94.9$ & 38 & 0.008 & ${ }^{2} 2.6-97.4$ & 38 \\
\hline & $9-10-2002$ & $<0.002$ & ${ }^{2} 2.6-94.9$ & 38 & 0.006 & ${ }^{2} 2.6-97.4$ & 38 \\
\hline \multirow[t]{2}{*}{31} & $7-29-2002$ & 0.004 & ${ }^{2} 4.2-87.5$ & 23 & 0.004 & ${ }^{1} 29.2-79.2$ & 23 \\
\hline & $9-10-2002$ & 0.004 & ${ }^{2} 4.2-87.5$ & 23 & 0.009 & ${ }^{1} 29.2-79.2$ & 23 \\
\hline \multirow[t]{2}{*}{32} & $8-8-2002$ & 0.003 & ID & 2 & 0.004 & ID & 2 \\
\hline & $9-11-2002$ & 0.003 & ID & 2 & 0.004 & ID & 2 \\
\hline 33 & $7-30-2002$ & 0.002 & ${ }^{2} 4.2-87.5$ & 23 & 0.007 & 4.3 & 22 \\
\hline 34 & $7-30-2002$ & $<0.002$ & ID & 9 & 0.003 & ID & 9 \\
\hline \multirow[t]{2}{*}{35} & $7-30-2002$ & 0.016 & 95.7 & 22 & 0.174 & 91.3 & 22 \\
\hline & $9-3-2002$ & 0.014 & 91.3 & 22 & 0.25 & 95.7 & 22 \\
\hline \multirow[t]{2}{*}{36} & $7-30-2002$ & 0.005 & ID & 18 & 0.038 & ID & 18 \\
\hline & $9-3-2002$ & 0.004 & ID & 18 & 0.047 & ID & 18 \\
\hline \multirow[t]{2}{*}{37} & $7-31-2002$ & 0.009 & ${ }^{2} 4.8-71.4$ & 20 & 0.028 & 48.5 & 32 \\
\hline & $9-4-2002$ & 0.008 & ${ }^{2} 4.8-71.4$ & 20 & 0.01 & ${ }^{1} 15.2-24.2$ & 32 \\
\hline
\end{tabular}


Table 12. Water-quality data for the Upper Colorado River Basin, showing 2002 drought values, Colorado water-quality exceedances, and percentiles relative to historical data for selected properties and constituents from July through September, 1978-2002.—Continued

[ft $3 / \mathrm{s}$, cubic feet per second; Conc, concentration; Q\%, percentile (percentage of data less than or equal to reported value or concentration) of drought-sample constituent; N, total number of historical and drought samples; $\mathrm{mg} / \mathrm{L}$, milligrams per liter; $\mu \mathrm{S} / \mathrm{cm}$, microsiemens per centimeter at $25^{\circ} \mathrm{Celsius;}{ }^{\circ} \mathrm{C}$, degrees Celsius; E. coli, Escherichia coli; $\mathrm{mL}$, milliliter; $\mu \mathrm{g} / \mathrm{L}$, micrograms per liter; $\mathrm{CaCO}_{3}$, calcium carbonate; $\mathrm{SiO}_{2}$, silica; $\mathrm{SO}_{4}$, sulfate; ID, insufficient number of samples (less than 20) to calculate percentile; <, less than; --, no drought-sample measurement; values or concentrations in bold type indicate 2002 Colorado water-quality exceedances; c, chronic water-quality exceedance]

\begin{tabular}{|c|c|c|c|c|c|c|c|}
\hline \multirow{2}{*}{$\begin{array}{l}\text { Site number } \\
\text { in table } 1 \\
\text { and fig. } 4\end{array}$} & \multirow{2}{*}{ Sampling date } & \multicolumn{3}{|c|}{$\begin{array}{c}\text { Nitrogen, nitrite, dissolved, } \\
\text { in } \mathrm{mg} / \mathrm{L} \text { as } \mathrm{N}\end{array}$} & \multicolumn{3}{|c|}{$\begin{array}{l}\text { Phosphorus, dissolved, } \\
\text { in } \mathrm{mg} / \mathrm{L} \text { as } \mathrm{P}\end{array}$} \\
\hline & & Conc & $0 \%$ & $\mathrm{~N}$ & Conc & $0 \%$ & $\mathrm{~N}$ \\
\hline \multirow[t]{2}{*}{38} & $8-1-2002$ & $<0.002$ & ID & 12 & 0.007 & ID & 13 \\
\hline & $9-5-2002$ & $<0.002$ & ID & 12 & 0.004 & ID & 13 \\
\hline \multirow[t]{2}{*}{39} & $8-1-2002$ & 0.002 & ID & 2 & 0.009 & ID & 2 \\
\hline & $9-6-2002$ & $<0.002$ & ID & 2 & 0.004 & ID & 2 \\
\hline \multirow[t]{2}{*}{40} & $7-29-2002$ & $<0.002$ & ID & 12 & $<0.004$ & ID & 12 \\
\hline & $9-3-2002$ & $<0.002$ & ID & 12 & $<0.004$ & ID & 12 \\
\hline \multirow[t]{2}{*}{41} & $7-29-2002$ & 0.002 & ID & 11 & 0.017 & ID & 11 \\
\hline & $9-4-2002$ & 0.002 & ID & 11 & 0.01 & ID & 11 \\
\hline \multirow[t]{2}{*}{42} & $7-30-2002$ & $<0.002$ & ID & 12 & $<0.004$ & ID & 12 \\
\hline & $9-4-2002$ & $<0.002$ & ID & 12 & $<0.004$ & ID & 12 \\
\hline \multirow[t]{2}{*}{43} & $7-30-2002$ & 0.003 & ID & 6 & 0.004 & ID & 6 \\
\hline & $9-4-2002$ & 0.004 & ID & 6 & 0.002 & ID & 6 \\
\hline \multirow[t]{2}{*}{44} & $7-30-2002$ & 0.002 & ID & 13 & 0.008 & ID & 13 \\
\hline & $9-5-2002$ & 0.002 & ID & 13 & 0.003 & ID & 13 \\
\hline \multirow[t]{2}{*}{45} & $7-31-2002$ & 0.002 & ID & 2 & 0.009 & ID & 2 \\
\hline & $9-6-2002$ & $<0.002$ & ID & 2 & 0.005 & ID & 2 \\
\hline 46 & $8-2-2002$ & $<0.008$ & ID & 16 & -- & -- & -- \\
\hline \multirow[t]{2}{*}{47} & $8-1-2002$ & $<0.008$ & ID & 17 & $<0.06$ & ID & 19 \\
\hline & $9-3-2002$ & $<0.008$ & ID & 17 & $<0.06$ & ID & 19 \\
\hline \multirow[t]{2}{*}{48} & $8-1-2002$ & $<0.008$ & ID & 3 & $<0.06$ & ID & 3 \\
\hline & $9-5-2002$ & $<0.008$ & ID & 3 & $<0.06$ & ID & 3 \\
\hline 49 & $7-31-2002$ & 0.004 & ID & 1 & 0.02 & ID & 1 \\
\hline 50 & $7-31-2002$ & 0.007 & ID & 1 & 0.009 & ID & 1 \\
\hline 51 & $8-1-2002$ & 0.022 & ID & 1 & 0.034 & ID & 1 \\
\hline \multirow[t]{2}{*}{52} & $8-1-2002$ & 0.012 & 51.4 & 36 & $<0.06$ & ${ }^{2} 2.1-89.6$ & 47 \\
\hline & $9-17-2002$ & 0.014 & 62.2 & 36 & $<0.06$ & ${ }^{2} 2.1-89.6$ & 47 \\
\hline 53 & $7-31-2002$ & 0.005 & ID & 1 & 0.025 & ID & 1 \\
\hline 54 & $8-1-2002$ & 0.008 & ID & 1 & 0.007 & ID & 1 \\
\hline 55 & $7-30-2002$ & $<0.002$ & ID & 8 & 0.003 & ID & 8 \\
\hline \multirow[t]{2}{*}{56} & $7-31-2002$ & $<0.008$ & ID & 2 & $<0.06$ & ID & 2 \\
\hline & $9-5-2002$ & $<0.008$ & ID & 2 & $<0.06$ & ID & 2 \\
\hline \multirow[t]{2}{*}{57} & $7-31-2002$ & 0.081 & ID & 8 & 0.115 & ID & 8 \\
\hline & $9-4-2002$ & 0.117 & ID & 8 & 0.15 & ID & 8 \\
\hline \multirow[t]{2}{*}{58} & $7-16-2002$ & $<0.002$ & ID & 6 & 0.035 & ID & 6 \\
\hline & $9-5-2002$ & $<0.002$ & ID & 6 & 0.029 & ID & 6 \\
\hline
\end{tabular}


Table 12. Water-quality data for the Upper Colorado River Basin, showing 2002 drought values, Colorado water-quality exceedances, and percentiles relative to historical data for selected properties and constituents from July through September, 1978-2002. - Continued

$\left[\mathrm{ft}^{3} / \mathrm{s}\right.$, cubic feet per second; Conc, concentration; Q\%, percentile (percentage of data less than or equal to reported value or concentration) of drought-sample constituent; N, total number of historical and drought samples; $\mathrm{mg} / \mathrm{L}$, milligrams per liter; $\mu \mathrm{S} / \mathrm{cm}$, microsiemens per centimeter at $25^{\circ} \mathrm{Celsius;}{ }^{\circ} \mathrm{C}$, degrees Celsius; E. coli, Escherichia coli; $\mathrm{mL}$, milliliter; $\mu \mathrm{g} / \mathrm{L}$, micrograms per liter; $\mathrm{CaCO}_{3}$, calcium carbonate; $\mathrm{SiO}_{2}$, silica; $\mathrm{SO}_{4}$, sulfate; ID, insufficient number of samples (less than 20) to calculate percentile; <, less than; --, no drought-sample measurement; values or concentrations in bold type indicate 2002 Colorado water-quality exceedances; c, chronic water-quality exceedance]

\begin{tabular}{|c|c|c|c|c|c|c|c|}
\hline \multirow{2}{*}{$\begin{array}{l}\text { Site number } \\
\text { in table } 1 \\
\text { and fig. } 4\end{array}$} & \multirow{2}{*}{ Sampling date } & \multicolumn{3}{|c|}{$\begin{array}{c}\text { Phosphorus, total recoverable, in } \\
\mathrm{mg} / \mathrm{L} \text { as } \mathrm{P}\end{array}$} & \multicolumn{3}{|c|}{$\begin{array}{l}\text { Phosphorus, orthophosphate, } \\
\text { dissolved, in } \mathrm{mg} / \mathrm{L} \text { as } \mathrm{P}\end{array}$} \\
\hline & & Conc & $0 \%$ & $\mathrm{~N}$ & Conc & $0 \%$ & $\mathrm{~N}$ \\
\hline 20 & $8-1-2002$ & 0.013 & ID & 2 & $<0.007$ & ID & 2 \\
\hline \multirow[t]{2}{*}{21} & $7-15-2002$ & 0.005 & ID & 12 & $<0.007$ & ${ }^{2} 4.0-96.0$ & 24 \\
\hline & $9-4-2002$ & 0.004 & ID & 12 & $<0.007$ & ${ }^{2} 4.0-96.0$ & 24 \\
\hline \multirow[t]{2}{*}{22} & $7-15-2002$ & 0.011 & ID & 11 & $<0.007$ & ${ }^{2} 4.3-91.3$ & 22 \\
\hline & $9-4-2002$ & 0.01 & ID & 11 & $<0.007$ & ${ }^{2} 4.3-91.3$ & 22 \\
\hline \multirow[t]{2}{*}{23} & $7-15-2002$ & 0.046 & ID & 12 & 0.024 & 96.0 & 24 \\
\hline & $9-4-2002$ & 0.042 & ID & 12 & 0.015 & 88.0 & 24 \\
\hline \multirow[t]{2}{*}{24} & $7-15-2002$ & 0.175 & ID & 12 & 0.094 & 92.0 & 24 \\
\hline & $9-4-2002$ & 0.159 & ID & 12 & 0.101 & 96.0 & 24 \\
\hline \multirow[t]{2}{*}{25} & $7-15-2002$ & 0.036 & ID & 9 & 0.014 & ID & 15 \\
\hline & $9-4-2002$ & 0.032 & ID & 9 & 0.012 & ID & 15 \\
\hline \multirow[t]{2}{*}{26} & $7-17-2002$ & 0.118 & ID & 10 & 0.06 & ID & 10 \\
\hline & $9-6-2002$ & 0.114 & ID & 10 & 0.063 & ID & 10 \\
\hline 27 & $8-1-2002$ & 0.05 & ID & 8 & 0.03 & ID & 8 \\
\hline \multirow[t]{2}{*}{28} & $8-8-2002$ & 0.024 & ID & 6 & 0.011 & ID & 5 \\
\hline & $9-11-2002$ & 0.021 & ID & 6 & 0.005 & ID & 5 \\
\hline \multirow[t]{2}{*}{29} & 8-8-2002 & 0.114 & ID & 2 & 0.079 & ID & 2 \\
\hline & $9-11-2002$ & 0.113 & ID & 2 & 0.083 & ID & 2 \\
\hline \multirow[t]{2}{*}{30} & $7-29-2002$ & 0.026 & 76.2 & 41 & $<0.007$ & ${ }^{2} 2.6-76.9$ & 38 \\
\hline & $9-10-2002$ & 0.018 & 59.5 & 41 & $<0.007$ & ${ }^{2} 2.6-76.9$ & 38 \\
\hline \multirow[t]{2}{*}{31} & $7-29-2002$ & 0.015 & ${ }^{2} 4.0-88.0$ & 24 & $<0.007$ & ${ }^{2} 4.2-45.8$ & 23 \\
\hline & $9-10-2002$ & 0.03 & ${ }^{2} 4.0-88.0$ & 24 & 0.004 & ${ }^{2} 4.2-45.8$ & 23 \\
\hline \multirow[t]{2}{*}{32} & $8-8-2002$ & 0.013 & ID & 2 & $<0.007$ & ID & 2 \\
\hline & $9-11-2002$ & 0.008 & ID & 2 & $<0.007$ & ID & 2 \\
\hline 33 & $7-30-2002$ & 0.021 & 62.5 & 23 & $<0.007$ & 4.2 & 23 \\
\hline 34 & $7-30-2002$ & 0.006 & ID & 9 & $<0.007$ & ID & 9 \\
\hline \multirow[t]{2}{*}{35} & $7-30-2002$ & 0.18 & 91.3 & 22 & 0.148 & 91.3 & 22 \\
\hline & $9-3-2002$ & 0.25 & 95.7 & 22 & 0.218 & 95.7 & 22 \\
\hline \multirow[t]{2}{*}{36} & $7-30-2002$ & 0.046 & ID & 15 & 0.029 & ID & 18 \\
\hline & $9-3-2002$ & 0.055 & ID & 15 & 0.038 & ID & 18 \\
\hline \multirow[t]{2}{*}{37} & $7-31-2002$ & 0.068 & 83.9 & 30 & 0.017 & 27.3 & 21 \\
\hline & $9-4-2002$ & 0.022 & 19.4 & 30 & $<0.007$ & 4.5 & 21 \\
\hline
\end{tabular}


Table 12. Water-quality data for the Upper Colorado River Basin, showing 2002 drought values, Colorado water-quality exceedances, and percentiles relative to historical data for selected properties and constituents from July through September, 1978-2002.-Continued

[ft $3 / \mathrm{s}$, cubic feet per second; Conc, concentration; Q\%, percentile (percentage of data less than or equal to reported value or concentration) of drought-sample constituent; N, total number of historical and drought samples; $\mathrm{mg} / \mathrm{L}$, milligrams per liter; $\mu \mathrm{S} / \mathrm{cm}$, microsiemens per centimeter at $25^{\circ} \mathrm{Celsius;}{ }^{\circ} \mathrm{C}$, degrees Celsius; E. coli, Escherichia coli; $\mathrm{mL}$, milliliter; $\mu \mathrm{g} / \mathrm{L}$, micrograms per liter; $\mathrm{CaCO}_{3}$, calcium carbonate; $\mathrm{SiO}_{2}$, silica; $\mathrm{SO}_{4}$, sulfate; ID, insufficient number of samples (less than 20) to calculate percentile; <, less than; --, no drought-sample measurement; values or concentrations in bold type indicate 2002 Colorado water-quality exceedances; c, chronic water-quality exceedance]

\begin{tabular}{|c|c|c|c|c|c|c|c|}
\hline \multirow{2}{*}{$\begin{array}{l}\text { Site number } \\
\text { in table } 1 \\
\text { and fig. } 4\end{array}$} & \multirow{2}{*}{ Sampling date } & \multicolumn{3}{|c|}{$\begin{array}{c}\text { Phosphorus, total recoverable, in } \\
\mathrm{mg} / \mathrm{L} \text { as } \mathrm{P}\end{array}$} & \multicolumn{3}{|c|}{$\begin{array}{c}\text { Phosphorus, orthophosphate, } \\
\text { dissolved, in } \mathrm{mg} / \mathrm{L} \text { as } \mathrm{P}\end{array}$} \\
\hline & & Conc & $0 \%$ & $\mathrm{~N}$ & Conc & $0 \%$ & $\mathrm{~N}$ \\
\hline \multirow[t]{2}{*}{38} & $8-1-2002$ & 0.029 & ID & 12 & $<0.007$ & ID & 13 \\
\hline & $9-5-2002$ & 0.018 & ID & 12 & $<0.007$ & ID & 13 \\
\hline \multirow[t]{2}{*}{39} & $8-1-2002$ & 0.048 & ID & 2 & $<0.007$ & ID & 2 \\
\hline & $9-6-2002$ & 0.029 & ID & 2 & $<0.007$ & ID & 2 \\
\hline \multirow[t]{2}{*}{40} & $7-29-2002$ & 0.003 & ID & 12 & $<0.007$ & ID & 12 \\
\hline & $9-3-2002$ & $<0.004$ & ID & 12 & $<0.007$ & ID & 12 \\
\hline \multirow[t]{2}{*}{41} & $7-29-2002$ & 0.023 & ID & 11 & 0.012 & ID & 11 \\
\hline & $9-4-2002$ & 0.021 & ID & 11 & 0.006 & ID & 11 \\
\hline \multirow[t]{2}{*}{42} & $7-30-2002$ & 0.004 & ID & 12 & $<0.007$ & ID & 12 \\
\hline & $9-4-2002$ & 0.002 & ID & 12 & $<0.007$ & ID & 12 \\
\hline \multirow[t]{2}{*}{43} & $7-30-2002$ & 0.008 & ID & 6 & $<0.007$ & ID & 6 \\
\hline & $9-4-2002$ & 0.005 & ID & 6 & $<0.007$ & ID & 6 \\
\hline \multirow[t]{2}{*}{44} & $7-30-2002$ & 0.014 & ID & 13 & $<0.007$ & ID & 13 \\
\hline & $9-5-2002$ & 0.012 & ID & 13 & $<0.007$ & ID & 13 \\
\hline \multirow[t]{2}{*}{45} & $7-31-2002$ & 0.043 & ID & 2 & $<0.007$ & ID & 2 \\
\hline & $9-6-2002$ & 0.021 & ID & 2 & $<0.007$ & ID & 2 \\
\hline 46 & $8-2-2002$ & 0.023 & ID & 16 & $<0.02$ & ID & 16 \\
\hline \multirow[t]{2}{*}{47} & $8-1-2002$ & 0.06 & ID & 13 & $<0.02$ & ID & 19 \\
\hline & $9-3-2002$ & 0.04 & ID & 13 & $<0.02$ & ID & 19 \\
\hline \multirow[t]{2}{*}{48} & $8-1-2002$ & $<0.06$ & ID & 2 & $<0.02$ & ID & 4 \\
\hline & $9-5-2002$ & $<0.06$ & ID & 2 & $<0.02$ & ID & 4 \\
\hline 49 & $7-31-2002$ & 0.083 & ID & 1 & 0.012 & ID & 1 \\
\hline 50 & $7-31-2002$ & 0.042 & ID & 1 & $<0.007$ & ID & 1 \\
\hline 51 & 8-1-2002 & 0.094 & ID & 1 & 0.023 & ID & 1 \\
\hline \multirow[t]{2}{*}{52} & $8-1-2002$ & 0.11 & ${ }^{1} 32.6-39.1$ & 45 & 0.01 & ${ }^{2} 2.3-58.1$ & 42 \\
\hline & $9-17-2002$ & 1.95 & 97.8 & 45 & $<0.02$ & ${ }^{2} 2.3-58.1$ & 42 \\
\hline 53 & $7-31-2002$ & 0.07 & ID & 1 & 0.015 & ID & 1 \\
\hline 54 & 8-1-2002 & 0.051 & ID & 1 & $<0.007$ & ID & 1 \\
\hline 55 & $7-30-2002$ & 0.006 & ID & 8 & $<0.007$ & ID & 8 \\
\hline \multirow[t]{2}{*}{56} & $7-31-2002$ & 0.03 & ID & 2 & $<0.02$ & ID & 2 \\
\hline & $9-5-2002$ & $<0.06$ & ID & 2 & $<0.02$ & ID & 2 \\
\hline \multirow[t]{2}{*}{57} & $7-31-2002$ & 0.147 & ID & 8 & 0.094 & ID & 8 \\
\hline & $9-4-2002$ & 0.164 & ID & 8 & 0.124 & ID & 8 \\
\hline \multirow[t]{2}{*}{58} & $7-16-2002$ & 0.058 & ID & 6 & 0.024 & ID & 6 \\
\hline & $9-5-2002$ & 0.045 & ID & 6 & 0.016 & ID & 6 \\
\hline
\end{tabular}


Table 12. Water-quality data for the Upper Colorado River Basin, showing 2002 drought values, Colorado water-quality exceedances, and percentiles relative to historical data for selected properties and constituents from July through September, 1978-2002.-Continued

$\left[\mathrm{ft}^{3} / \mathrm{s}\right.$, cubic feet per second; Conc, concentration; Q\%, percentile (percentage of data less than or equal to reported value or concentration) of drought-sample constituent; N, total number of historical and drought samples; $\mathrm{mg} / \mathrm{L}$, milligrams per liter; $\mu \mathrm{S} / \mathrm{cm}$, microsiemens per centimeter at $25^{\circ} \mathrm{Celsius;}{ }^{\circ} \mathrm{C}$, degrees Celsius; E. coli, Escherichia coli; $\mathrm{mL}$, milliliter; $\mu \mathrm{g} / \mathrm{L}$, micrograms per liter; $\mathrm{CaCO}_{3}$, calcium carbonate; $\mathrm{SiO}_{2}$, silica; $\mathrm{SO}_{4}$, sulfate; ID, insufficient number of samples (less than 20) to calculate percentile; <, less than; --, no drought-sample measurement; values or concentrations in bold type indicate 2002 Colorado water-quality exceedances; c, chronic water-quality exceedance]

\begin{tabular}{|c|c|c|c|c|c|c|c|}
\hline \multirow{2}{*}{$\begin{array}{l}\text { Site number } \\
\text { in table } 1 \\
\text { and fig. } 4\end{array}$} & \multirow{2}{*}{ Sampling date } & \multicolumn{3}{|c|}{$\begin{array}{c}\text { Carbon, organic, dissolved, } \\
\text { in } \mathrm{mg} / \mathrm{L}\end{array}$} & \multirow{2}{*}{$\begin{array}{c}\text { Bacteria, } \\
\text { E. coli, } \\
\text { in colonies } \\
\text { per } 100 \mathrm{~mL}\end{array}$} & \multirow{2}{*}{$\begin{array}{l}\text { Bacteria, } \\
\text { fecal } \\
\text { coliform, in } \\
\text { colonies per } \\
100 \mathrm{~mL}\end{array}$} & \multirow{2}{*}{$\begin{array}{c}\text { Aluminum, } \\
\text { dissolved, } \\
\text { in } \mu \mathrm{g} / \mathrm{L}\end{array}$} \\
\hline & & Conc & $0 \%$ & $\mathrm{~N}$ & & & \\
\hline 20 & $8-1-2002$ & 2.6 & ID & 2 & -- & -- & -- \\
\hline \multirow[t]{2}{*}{21} & $7-15-2002$ & -- & -- & -- & -- & -- & -- \\
\hline & $9-4-2002$ & -- & -- & -- & -- & -- & -- \\
\hline \multirow[t]{2}{*}{22} & $7-15-2002$ & -- & -- & -- & -- & -- & -- \\
\hline & $9-4-2002$ & -- & -- & -- & -- & -- & -- \\
\hline \multirow[t]{2}{*}{23} & $7-15-2002$ & -- & -- & -- & -- & -- & -- \\
\hline & $9-4-2002$ & -- & -- & -- & -- & -- & -- \\
\hline \multirow[t]{2}{*}{24} & $7-15-2002$ & -- & -- & -- & -- & -- & -- \\
\hline & $9-4-2002$ & -- & -- & -- & -- & -- & -- \\
\hline \multirow[t]{2}{*}{25} & $7-15-2002$ & -- & -- & -- & 12 & -- & -- \\
\hline & $9-4-2002$ & -- & -- & -- & 1 & -- & -- \\
\hline \multirow[t]{2}{*}{26} & 7-17-2002 & 4.2 & ID & 9 & 30 & -- & -- \\
\hline & $9-6-2002$ & 4.3 & ID & 9 & 15 & -- & -- \\
\hline 27 & 8-1-2002 & -- & -- & -- & -- & -- & -- \\
\hline \multirow[t]{2}{*}{28} & $8-8-2002$ & 2.6 & ID & 3 & 2 & -- & $<20$ \\
\hline & $9-11-2002$ & 2.6 & ID & 3 & 1 & -- & $<20$ \\
\hline \multirow[t]{2}{*}{29} & 8-8-2002 & -- & -- & -- & 4 & -- & -- \\
\hline & $9-11-2002$ & -- & -- & -- & 33 & -- & -- \\
\hline \multirow[t]{2}{*}{30} & $7-29-2002$ & 8.9 & ${ }^{1} 90.9-95.5$ & 21 & 20 & -- & -- \\
\hline & $9-10-2002$ & -- & -- & -- & 3 & -- & -- \\
\hline \multirow[t]{2}{*}{31} & $7-29-2002$ & 6.7 & ID & 16 & 1 & -- & -- \\
\hline & $9-10-2002$ & -- & -- & -- & 1 & -- & -- \\
\hline \multirow[t]{2}{*}{32} & 8-8-2002 & 2 & ID & 2 & 1 & -- & $<20$ \\
\hline & $9-11-2002$ & 2.1 & ID & 2 & 4 & -- & $<20$ \\
\hline 33 & $7-30-2002$ & -- & -- & -- & 19 & -- & -- \\
\hline 34 & $7-30-2002$ & 1.5 & ID & 3 & 2 & 2 & -- \\
\hline \multirow[t]{2}{*}{35} & $7-30-2002$ & 1.7 & ID & 17 & $<1$ & 3 & -- \\
\hline & $9-3-2002$ & -- & -- & -- & 2 & -- & -- \\
\hline \multirow[t]{2}{*}{36} & $7-30-2002$ & 1.7 & ID & 4 & 9 & 12 & -- \\
\hline & $9-3-2002$ & -- & -- & -- & 14 & -- & -- \\
\hline \multirow[t]{2}{*}{37} & $7-31-2002$ & 1.8 & ID & 5 & 10 & 11 & -- \\
\hline & $9-4-2002$ & 2.3 & ID & 5 & 9 & -- & $<20$ \\
\hline
\end{tabular}


Table 12. Water-quality data for the Upper Colorado River Basin, showing 2002 drought values, Colorado water-quality exceedances, and percentiles relative to historical data for selected properties and constituents from July through September, 1978-2002.—Continued

[ft $3 / \mathrm{s}$, cubic feet per second; Conc, concentration; Q\%, percentile (percentage of data less than or equal to reported value or concentration) of drought-sample constituent; $\mathrm{N}$, total number of historical and drought samples; $\mathrm{mg} / \mathrm{L}$, milligrams per liter; $\mu \mathrm{S} / \mathrm{cm}$, microsiemens per centimeter at $25^{\circ} \mathrm{Celsius;}{ }^{\circ} \mathrm{C}$, degrees Celsius; E. coli, Escherichia coli; $\mathrm{mL}$, milliliter; $\mu \mathrm{g} / \mathrm{L}$, micrograms per liter; $\mathrm{CaCO}_{3}$, calcium carbonate; $\mathrm{SiO}_{2}$, silica; $\mathrm{SO}_{4}$, sulfate; ID, insufficient number of samples (less than 20) to calculate percentile; <, less than; --, no drought-sample measurement; values or concentrations in bold type indicate 2002 Colorado water-quality exceedances; c, chronic water-quality exceedance]

\begin{tabular}{|c|c|c|c|c|c|c|c|}
\hline \multirow{2}{*}{$\begin{array}{l}\text { Site number } \\
\text { in table } 1 \\
\text { and fig. } 4\end{array}$} & \multirow{2}{*}{ Sampling date } & \multicolumn{3}{|c|}{$\begin{array}{l}\text { Carbon, organic, dissolved, } \\
\text { in } \mathrm{mg} / \mathrm{L}\end{array}$} & \multirow{2}{*}{$\begin{array}{c}\text { Bacteria, } \\
\text { E. coli, } \\
\text { in colonies } \\
\text { per } 100 \mathrm{~mL}\end{array}$} & \multirow{2}{*}{$\begin{array}{l}\text { Bacteria, } \\
\text { fecal } \\
\text { coliform, in } \\
\text { colonies per } \\
100 \mathrm{~mL}\end{array}$} & \multirow{2}{*}{$\begin{array}{c}\text { Aluminum, } \\
\text { dissolved, } \\
\text { in } \mu \mathrm{g} / \mathrm{L}\end{array}$} \\
\hline & & Conc & $0 \%$ & $\mathrm{~N}$ & & & \\
\hline \multirow[t]{2}{*}{38} & $8-1-2002$ & -- & -- & -- & 11 & -- & -- \\
\hline & $9-5-2002$ & -- & -- & -- & 2 & -- & -- \\
\hline \multirow[t]{2}{*}{39} & $8-1-2002$ & 2.3 & ID & 2 & 4 & -- & -- \\
\hline & $9-6-2002$ & 3.6 & ID & 2 & 4 & -- & -- \\
\hline \multirow[t]{2}{*}{40} & 7-29-2002 & 1.1 & ID & 1 & 2 & 2 & -- \\
\hline & $9-3-2002$ & -- & -- & -- & 3 & 3 & -- \\
\hline \multirow[t]{2}{*}{41} & $7-29-2002$ & 1.8 & ID & 2 & 42 & 34 & -- \\
\hline & $9-4-2002$ & -- & -- & -- & 34 & 40 & -- \\
\hline \multirow[t]{2}{*}{42} & $7-30-2002$ & 0.6 & ID & 1 & 31 & 18 & -- \\
\hline & $9-4-2002$ & -- & -- & -- & 6 & 12 & -- \\
\hline \multirow[t]{2}{*}{43} & $7-30-2002$ & 1.2 & ID & 1 & 23 & 19 & -- \\
\hline & $9-4-2002$ & -- & -- & -- & 20 & 24 & -- \\
\hline \multirow[t]{2}{*}{44} & $7-30-2002$ & 1.6 & ID & 3 & 18 & 16 & -- \\
\hline & $9-5-2002$ & 1.8 & ID & 3 & 52 & 55 & $<20$ \\
\hline \multirow[t]{2}{*}{45} & $7-31-2002$ & 1.8 & ID & 2 & 40 & 23 & $<20$ \\
\hline & $9-6-2002$ & 2.9 & ID & 2 & 10 & -- & $<20$ \\
\hline 46 & $8-2-2002$ & -- & -- & -- & 100 & -- & -- \\
\hline \multirow[t]{2}{*}{47} & $8-1-2002$ & -- & -- & -- & 11 & -- & -- \\
\hline & $9-3-2002$ & -- & -- & -- & 24 & -- & -- \\
\hline \multirow[t]{2}{*}{48} & $8-1-2002$ & -- & -- & -- & 100 & -- & -- \\
\hline & $9-5-2002$ & -- & -- & -- & 56 & -- & -- \\
\hline 49 & $7-31-2002$ & -- & -- & -- & -- & -- & -- \\
\hline 50 & $7-31-2002$ & -- & -- & -- & -- & -- & -- \\
\hline 51 & $8-1-2002$ & -- & -- & -- & -- & -- & -- \\
\hline \multirow[t]{2}{*}{52} & 8-1-2002 & 3.7 & 79.2 & 23 & -- & -- & $<20$ \\
\hline & $9-17-2002$ & 4.3 & 87.5 & 23 & -- & -- & $<20$ \\
\hline 53 & $7-31-2002$ & -- & -- & -- & -- & -- & -- \\
\hline 54 & $8-1-2002$ & -- & -- & -- & -- & -- & -- \\
\hline 55 & $7-30-2002$ & 1.8 & ID & 2 & 17 & 10 & -- \\
\hline \multirow[t]{2}{*}{56} & $7-31-2002$ & -- & -- & -- & -- & -- & -- \\
\hline & $9-5-2002$ & -- & -- & -- & 20 & -- & -- \\
\hline \multirow[t]{2}{*}{57} & $7-31-2002$ & 2 & ID & 3 & 11 & 7 & -- \\
\hline & $9-4-2002$ & -- & -- & -- & 6 & -- & -- \\
\hline \multirow[t]{2}{*}{58} & $7-16-2002$ & -- & -- & -- & 7 & -- & -- \\
\hline & $9-5-2002$ & -- & -- & -- & 13 & -- & -- \\
\hline
\end{tabular}


Table 12. Water-quality data for the Upper Colorado River Basin, showing 2002 drought values, Colorado water-quality exceedances, and percentiles relative to historical data for selected properties and constituents from July through September, 1978-2002. - Continued

$\left[\mathrm{ft}^{3} / \mathrm{s}\right.$, cubic feet per second; Conc, concentration; Q\%, percentile (percentage of data less than or equal to reported value or concentration) of drought-sample constituent; N, total number of historical and drought samples; $\mathrm{mg} / \mathrm{L}$, milligrams per liter; $\mu \mathrm{S} / \mathrm{cm}$, microsiemens per centimeter at $25^{\circ} \mathrm{Celsius;}{ }^{\circ} \mathrm{C}$, degrees Celsius; E. coli, Escherichia coli; $\mathrm{mL}$, milliliter; $\mu \mathrm{g} / \mathrm{L}$, micrograms per liter; $\mathrm{CaCO}_{3}$, calcium carbonate; $\mathrm{SiO}_{2}$, silica; $\mathrm{SO}_{4}$, sulfate; ID, insufficient number of samples (less than 20) to calculate percentile; <, less than; --, no drought-sample measurement; values or concentrations in bold type indicate 2002 Colorado water-quality exceedances; c, chronic water-quality exceedance]

\begin{tabular}{|c|c|c|c|c|c|c|c|c|}
\hline \multirow{2}{*}{$\begin{array}{l}\text { Site number } \\
\text { in table } 1 \\
\text { and fig. } 4\end{array}$} & \multirow{2}{*}{ Sampling date } & \multirow{2}{*}{$\begin{array}{l}\text { Aluminum, } \\
\text { total } \\
\text { recoverable, } \\
\text { in } \mu \mathrm{g} / \mathrm{L}\end{array}$} & \multicolumn{3}{|c|}{ Arsenic, dissolved, in $\mu \mathrm{g} / \mathrm{L}$} & \multicolumn{3}{|c|}{$\begin{array}{l}\text { Arsenic, total recoverable, in } \\
\qquad \mu \mathrm{g} / \mathrm{L}\end{array}$} \\
\hline & & & Conc & $0 \%$ & $\mathrm{~N}$ & Conc & $0 \%$ & $\mathrm{~N}$ \\
\hline 20 & $8-1-2002$ & -- & -- & -- & -- & -- & -- & -- \\
\hline \multirow[t]{2}{*}{21} & $7-15-2002$ & -- & -- & -- & -- & -- & -- & -- \\
\hline & $9-4-2002$ & -- & -- & -- & -- & -- & -- & -- \\
\hline \multirow[t]{2}{*}{22} & $7-15-2002$ & -- & -- & -- & -- & -- & -- & -- \\
\hline & $9-4-2002$ & -- & -- & -- & -- & -- & -- & -- \\
\hline \multirow[t]{2}{*}{23} & $7-15-2002$ & -- & -- & -- & -- & -- & -- & -- \\
\hline & $9-4-2002$ & -- & -- & -- & -- & -- & -- & -- \\
\hline \multirow[t]{2}{*}{24} & $7-15-2002$ & -- & -- & -- & -- & -- & -- & -- \\
\hline & $9-4-2002$ & -- & -- & -- & -- & -- & -- & -- \\
\hline \multirow[t]{2}{*}{25} & $7-15-2002$ & -- & -- & -- & -- & -- & -- & -- \\
\hline & $9-4-2002$ & -- & -- & -- & -- & -- & -- & -- \\
\hline \multirow[t]{2}{*}{26} & $7-17-2002$ & -- & -- & -- & -- & -- & -- & -- \\
\hline & $9-6-2002$ & -- & -- & -- & -- & -- & -- & -- \\
\hline 27 & $8-1-2002$ & -- & $<2$ & ID & 8 & $<2$ & ID & 8 \\
\hline \multirow[t]{2}{*}{28} & 8-8-2002 & -- & -- & -- & -- & -- & -- & -- \\
\hline & $9-11-2002$ & -- & -- & -- & -- & -- & -- & -- \\
\hline \multirow[t]{2}{*}{29} & 8-8-2002 & -- & -- & -- & -- & -- & -- & -- \\
\hline & $9-11-2002$ & -- & -- & -- & -- & -- & -- & -- \\
\hline \multirow[t]{2}{*}{30} & $7-29-2002$ & 78 & 1 & ID & 14 & 1 & ID & 13 \\
\hline & $9-10-2002$ & -- & -- & -- & -- & -- & -- & -- \\
\hline \multirow[t]{2}{*}{31} & $7-29-2002$ & 29 & $<2$ & ID & 14 & 1 & ID & 12 \\
\hline & $9-10-2002$ & -- & -- & -- & -- & -- & -- & -- \\
\hline \multirow[t]{2}{*}{32} & 8-8-2002 & -- & -- & -- & -- & -- & -- & -- \\
\hline & $9-11-2002$ & -- & -- & -- & -- & -- & -- & -- \\
\hline 33 & $7-30-2002$ & -- & -- & -- & -- & -- & -- & -- \\
\hline 34 & $7-30-2002$ & -- & -- & -- & -- & -- & -- & -- \\
\hline \multirow[t]{2}{*}{35} & $7-30-2002$ & -- & -- & -- & -- & -- & -- & -- \\
\hline & $9-3-2002$ & -- & -- & -- & -- & -- & -- & -- \\
\hline \multirow[t]{2}{*}{36} & $7-30-2002$ & -- & -- & -- & -- & -- & -- & -- \\
\hline & $9-3-2002$ & -- & -- & -- & -- & -- & -- & -- \\
\hline \multirow[t]{2}{*}{37} & $7-31-2002$ & -- & $<2$ & ${ }^{2} 4.5-95.5$ & 21 & $<2$ & ${ }^{2} 4.8-90.5$ & 20 \\
\hline & $9-4-2002$ & -- & -- & -- & -- & -- & -- & -- \\
\hline
\end{tabular}


Table 12. Water-quality data for the Upper Colorado River Basin, showing 2002 drought values, Colorado water-quality exceedances, and percentiles relative to historical data for selected properties and constituents from July through September, 1978-2002.-Continued

[ft $\mathrm{f}^{3} / \mathrm{s}$, cubic feet per second; Conc, concentration; Q\%, percentile (percentage of data less than or equal to reported value or concentration) of drought-sample constituent; N, total number of historical and drought samples; $\mathrm{mg} / \mathrm{L}$, milligrams per liter; $\mu \mathrm{S} / \mathrm{cm}$, microsiemens per centimeter at $25^{\circ} \mathrm{Celsius;}{ }^{\circ} \mathrm{C}$, degrees Celsius; E. coli, Escherichia coli; $\mathrm{mL}$, milliliter; $\mu \mathrm{g} / \mathrm{L}$, micrograms per liter; $\mathrm{CaCO}_{3}$, calcium carbonate; $\mathrm{SiO}_{2}$, silica; $\mathrm{SO}_{4}$, sulfate; ID, insufficient number of samples (less than 20) to calculate percentile; <, less than; --, no drought-sample measurement; values or concentrations in bold type indicate 2002 Colorado water-quality exceedances; c, chronic water-quality exceedance]

\begin{tabular}{|c|c|c|c|c|c|c|c|c|}
\hline \multirow{2}{*}{$\begin{array}{l}\text { Site number } \\
\text { in table } 1 \\
\text { and fig. } 4\end{array}$} & \multirow{2}{*}{ Sampling date } & \multirow{2}{*}{$\begin{array}{l}\text { Aluminum, } \\
\text { total } \\
\text { recoverable, } \\
\text { in } \mu \mathrm{g} / \mathrm{L}\end{array}$} & \multicolumn{3}{|c|}{ Arsenic, dissolved, in $\mu \mathrm{g} / \mathrm{L}$} & \multicolumn{3}{|c|}{$\begin{array}{l}\text { Arsenic, total recoverable, in } \\
\qquad \mu \mathrm{g} / \mathrm{L}\end{array}$} \\
\hline & & & Conc & $0 \%$ & $\mathrm{~N}$ & Conc & $0 \%$ & $\mathrm{~N}$ \\
\hline \multirow[t]{2}{*}{38} & $8-1-2002$ & -- & -- & -- & -- & -- & -- & -- \\
\hline & $9-5-2002$ & -- & -- & -- & -- & -- & -- & -- \\
\hline \multirow[t]{2}{*}{39} & $8-1-2002$ & -- & -- & -- & -- & -- & -- & -- \\
\hline & $9-6-2002$ & -- & -- & -- & -- & -- & -- & -- \\
\hline \multirow[t]{2}{*}{40} & $7-29-2002$ & -- & -- & -- & -- & -- & -- & -- \\
\hline & $9-3-2002$ & -- & -- & -- & -- & -- & -- & -- \\
\hline \multirow[t]{2}{*}{41} & $7-29-2002$ & -- & -- & -- & -- & -- & -- & -- \\
\hline & $9-4-2002$ & -- & -- & -- & -- & -- & -- & -- \\
\hline \multirow[t]{2}{*}{42} & $7-30-2002$ & -- & -- & -- & -- & -- & -- & -- \\
\hline & $9-4-2002$ & -- & -- & -- & -- & -- & -- & -- \\
\hline \multirow[t]{2}{*}{43} & $7-30-2002$ & -- & -- & -- & -- & -- & -- & -- \\
\hline & $9-4-2002$ & -- & -- & -- & -- & -- & -- & -- \\
\hline \multirow[t]{2}{*}{44} & $7-30-2002$ & -- & -- & -- & -- & -- & -- & -- \\
\hline & $9-5-2002$ & -- & -- & -- & -- & -- & -- & -- \\
\hline \multirow[t]{2}{*}{45} & $7-31-2002$ & -- & -- & -- & -- & -- & -- & -- \\
\hline & $9-6-2002$ & -- & -- & -- & -- & -- & -- & -- \\
\hline 46 & $8-2-2002$ & -- & -- & -- & -- & -- & -- & -- \\
\hline \multirow[t]{2}{*}{47} & $8-1-2002$ & -- & -- & -- & -- & -- & -- & -- \\
\hline & $9-3-2002$ & -- & -- & -- & -- & -- & -- & -- \\
\hline \multirow[t]{2}{*}{48} & $8-1-2002$ & -- & -- & -- & -- & -- & -- & -- \\
\hline & $9-5-2002$ & -- & -- & -- & -- & -- & -- & -- \\
\hline 49 & $7-31-2002$ & -- & -- & -- & -- & -- & -- & -- \\
\hline 50 & $7-31-2002$ & -- & -- & -- & -- & -- & -- & -- \\
\hline 51 & 8-1-2002 & -- & -- & -- & -- & -- & -- & -- \\
\hline \multirow[t]{2}{*}{52} & 8-1-2002 & -- & -- & -- & -- & -- & -- & -- \\
\hline & $9-17-2002$ & -- & -- & -- & -- & -- & -- & -- \\
\hline 53 & $7-31-2002$ & -- & -- & -- & -- & -- & -- & -- \\
\hline 54 & $8-1-2002$ & -- & -- & -- & -- & -- & -- & -- \\
\hline 55 & $7-30-2002$ & -- & -- & -- & -- & -- & -- & -- \\
\hline \multirow[t]{2}{*}{56} & $7-31-2002$ & -- & -- & -- & -- & -- & -- & -- \\
\hline & $9-5-2002$ & -- & -- & -- & -- & -- & -- & -- \\
\hline \multirow[t]{2}{*}{57} & $7-31-2002$ & -- & -- & -- & -- & -- & -- & -- \\
\hline & $9-4-2002$ & -- & -- & -- & -- & -- & -- & -- \\
\hline \multirow[t]{2}{*}{58} & 7-16-2002 & -- & -- & -- & -- & -- & -- & -- \\
\hline & $9-5-2002$ & -- & -- & -- & -- & -- & -- & -- \\
\hline
\end{tabular}


Table 12. Water-quality data for the Upper Colorado River Basin, showing 2002 drought values, Colorado water-quality exceedances, and percentiles relative to historical data for selected properties and constituents from July through September, 1978-2002.-Continued

$\left[\mathrm{ft}^{3} / \mathrm{s}\right.$, cubic feet per second; Conc, concentration; Q\%, percentile (percentage of data less than or equal to reported value or concentration) of drought-sample constituent; N, total number of historical and drought samples; $\mathrm{mg} / \mathrm{L}$, milligrams per liter; $\mu \mathrm{S} / \mathrm{cm}$, microsiemens per centimeter at $25^{\circ} \mathrm{Celsius;}{ }^{\circ} \mathrm{C}$, degrees Celsius; E. coli, Escherichia coli; $\mathrm{mL}$, milliliter; $\mu \mathrm{g} / \mathrm{L}$, micrograms per liter; $\mathrm{CaCO}_{3}$, calcium carbonate; $\mathrm{SiO}_{2}$, silica; $\mathrm{SO}_{4}$, sulfate; ID, insufficient number of samples (less than 20) to calculate percentile; <, less than; --, no drought-sample measurement; values or concentrations in bold type indicate 2002 Colorado water-quality exceedances; c, chronic water-quality exceedance]

\begin{tabular}{|c|c|c|c|c|c|c|c|c|}
\hline \multirow{2}{*}{$\begin{array}{l}\text { Site number } \\
\text { in table } 1 \\
\text { and fig. } 4\end{array}$} & \multirow{2}{*}{ Sampling date } & \multirow{2}{*}{$\begin{array}{c}\text { Boron, } \\
\text { dissolved, in } \\
\mu \mathrm{g} / \mathrm{L}\end{array}$} & \multicolumn{3}{|c|}{ Cadmium, dissolved, in $\mu \mathrm{g} / \mathrm{L}$} & \multicolumn{3}{|c|}{$\begin{array}{c}\text { Cadmium, total recoverable, in } \\
\mu \mathrm{g} / \mathrm{L}\end{array}$} \\
\hline & & & Conc & $0 \%$ & $\mathrm{~N}$ & Conc & $0 \%$ & $\mathrm{~N}$ \\
\hline 20 & $8-1-2002$ & -- & -- & -- & -- & -- & -- & -- \\
\hline \multirow[t]{2}{*}{21} & $7-15-2002$ & -- & -- & -- & -- & -- & -- & -- \\
\hline & $9-4-2002$ & -- & -- & -- & -- & -- & -- & -- \\
\hline \multirow[t]{2}{*}{22} & $7-15-2002$ & -- & -- & -- & -- & -- & -- & -- \\
\hline & $9-4-2002$ & -- & -- & -- & -- & -- & -- & -- \\
\hline \multirow[t]{2}{*}{23} & $7-15-2002$ & -- & -- & -- & -- & -- & -- & -- \\
\hline & $9-4-2002$ & -- & -- & -- & -- & -- & -- & -- \\
\hline \multirow[t]{2}{*}{24} & $7-15-2002$ & -- & -- & -- & -- & -- & -- & -- \\
\hline & $9-4-2002$ & -- & -- & -- & -- & -- & -- & -- \\
\hline \multirow[t]{2}{*}{25} & $7-15-2002$ & -- & -- & -- & -- & -- & -- & -- \\
\hline & $9-4-2002$ & -- & -- & -- & -- & -- & -- & -- \\
\hline \multirow[t]{2}{*}{26} & $7-17-2002$ & -- & -- & -- & -- & -- & -- & -- \\
\hline & $9-6-2002$ & -- & -- & -- & -- & -- & -- & -- \\
\hline 27 & 8-1-2002 & -- & $<0.1$ & ID & 8 & $<0.1$ & ID & 8 \\
\hline \multirow[t]{2}{*}{28} & $8-8-2002$ & -- & 0.05 & ID & 2 & -- & -- & -- \\
\hline & $9-11-2002$ & -- & 0.02 & ID & 2 & -- & -- & -- \\
\hline \multirow[t]{2}{*}{29} & 8-8-2002 & -- & -- & -- & -- & -- & -- & -- \\
\hline & $9-11-2002$ & -- & -- & -- & -- & -- & -- & -- \\
\hline \multirow[t]{2}{*}{30} & $7-29-2002$ & 80 & $<0.1$ & ID & 14 & $<0.1$ & ID & 14 \\
\hline & $9-10-2002$ & -- & -- & -- & -- & -- & -- & -- \\
\hline \multirow[t]{2}{*}{31} & 7-29-2002 & 60 & $<0.1$ & ID & 14 & $<0.1$ & ID & 14 \\
\hline & $9-10-2002$ & -- & -- & -- & -- & -- & -- & -- \\
\hline \multirow[t]{2}{*}{32} & $8-8-2002$ & -- & 0.12 & ID & 2 & -- & -- & -- \\
\hline & $9-11-2002$ & -- & 0.11 & ID & 2 & -- & -- & -- \\
\hline 33 & $7-30-2002$ & -- & $<0.1$ & ${ }^{2} 4.5-86.4$ & 21 & -- & -- & -- \\
\hline 34 & $7-30-2002$ & -- & $<0.1$ & ID & 6 & -- & -- & -- \\
\hline \multirow[t]{2}{*}{35} & $7-30-2002$ & -- & 0.1 & ID & 5 & -- & -- & -- \\
\hline & $9-3-2002$ & -- & -- & -- & -- & -- & -- & -- \\
\hline \multirow[t]{2}{*}{36} & $7-30-2002$ & -- & 0.2 & ID & 9 & -- & -- & -- \\
\hline & $9-3-2002$ & -- & -- & -- & -- & -- & -- & -- \\
\hline \multirow[t]{2}{*}{37} & $7-31-2002$ & -- & 0.1 & ${ }^{2} 4.3-82.6$ & 22 & 0.1 & ${ }^{2} 4.5-86.4$ & 21 \\
\hline & $9-4-2002$ & -- & 0.04 & ${ }^{2} 4.3-82.6$ & 22 & -- & -- & -- \\
\hline
\end{tabular}


Table 12. Water-quality data for the Upper Colorado River Basin, showing 2002 drought values, Colorado water-quality exceedances, and percentiles relative to historical data for selected properties and constituents from July through September, 1978-2002.-Continued

[ft $3 / \mathrm{s}$, cubic feet per second; Conc, concentration; Q\%, percentile (percentage of data less than or equal to reported value or concentration) of drought-sample constituent; N, total number of historical and drought samples; $\mathrm{mg} / \mathrm{L}$, milligrams per liter; $\mu \mathrm{S} / \mathrm{cm}$, microsiemens per centimeter at $25^{\circ} \mathrm{Celsius;}{ }^{\circ} \mathrm{C}$, degrees Celsius; E. coli, Escherichia coli; $\mathrm{mL}$, milliliter; $\mu \mathrm{g} / \mathrm{L}$, micrograms per liter; $\mathrm{CaCO}_{3}$, calcium carbonate; $\mathrm{SiO}_{2}$, silica; $\mathrm{SO}_{4}$, sulfate; ID, insufficient number of samples (less than 20) to calculate percentile; <, less than; --, no drought-sample measurement; values or concentrations in bold type indicate 2002 Colorado water-quality exceedances; c, chronic water-quality exceedance]

\begin{tabular}{|c|c|c|c|c|c|c|c|c|}
\hline \multirow{2}{*}{$\begin{array}{l}\text { Site number } \\
\text { in table } 1 \\
\text { and fig. } 4\end{array}$} & \multirow{2}{*}{ Sampling date } & \multirow{2}{*}{$\begin{array}{c}\text { Boron, } \\
\text { dissolved, in } \\
\mu \mathrm{g} / \mathrm{L}\end{array}$} & \multicolumn{3}{|c|}{ Cadmium, dissolved, in $\mu \mathrm{g} / \mathrm{L}$} & \multicolumn{3}{|c|}{$\begin{array}{c}\text { Cadmium, total recoverable, in } \\
\mu \mathrm{g} / \mathrm{L}\end{array}$} \\
\hline & & & Conc & $0 \%$ & $\mathrm{~N}$ & Conc & $0 \%$ & $\mathrm{~N}$ \\
\hline \multirow[t]{2}{*}{38} & $8-1-2002$ & -- & -- & -- & -- & -- & -- & -- \\
\hline & $9-5-2002$ & -- & -- & -- & -- & -- & -- & -- \\
\hline \multirow[t]{2}{*}{39} & 8-1-2002 & -- & -- & -- & -- & -- & -- & -- \\
\hline & $9-6-2002$ & -- & -- & -- & -- & -- & -- & -- \\
\hline \multirow[t]{2}{*}{40} & $7-29-2002$ & -- & $<0.1$ & ID & 6 & -- & -- & -- \\
\hline & $9-3-2002$ & -- & -- & -- & -- & -- & -- & -- \\
\hline \multirow[t]{2}{*}{41} & $7-29-2002$ & -- & 0.1 & ID & 5 & -- & -- & -- \\
\hline & $9-4-2002$ & -- & -- & -- & -- & -- & -- & -- \\
\hline \multirow[t]{2}{*}{42} & $7-30-2002$ & -- & 0.2 & ID & 6 & -- & -- & -- \\
\hline & $9-4-2002$ & -- & -- & -- & -- & -- & -- & -- \\
\hline \multirow[t]{2}{*}{43} & $7-30-2002$ & -- & 0.1 & ID & 3 & -- & -- & -- \\
\hline & 9-4-2002 & -- & -- & -- & -- & -- & -- & -- \\
\hline \multirow[t]{2}{*}{44} & $7-30-2002$ & -- & $<0.1$ & ID & 10 & -- & -- & -- \\
\hline & $9-5-2002$ & -- & 0.02 & ID & 10 & -- & -- & -- \\
\hline \multirow[t]{2}{*}{45} & $7-31-2002$ & -- & 0.04 & ID & 2 & -- & -- & -- \\
\hline & $9-6-2002$ & -- & $<0.04$ & ID & 2 & -- & -- & -- \\
\hline 46 & $8-2-2002$ & -- & -- & -- & -- & -- & -- & -- \\
\hline \multirow[t]{2}{*}{47} & $8-1-2002$ & -- & -- & -- & -- & -- & -- & -- \\
\hline & $9-3-2002$ & -- & -- & -- & -- & -- & -- & -- \\
\hline \multirow[t]{2}{*}{48} & 8-1-2002 & -- & -- & -- & -- & -- & -- & -- \\
\hline & $9-5-2002$ & -- & -- & -- & -- & -- & -- & -- \\
\hline 49 & $7-31-2002$ & -- & -- & -- & -- & -- & -- & -- \\
\hline 50 & $7-31-2002$ & -- & -- & -- & -- & -- & -- & -- \\
\hline 51 & $8-1-2002$ & -- & -- & -- & -- & -- & -- & -- \\
\hline \multirow[t]{2}{*}{52} & 8-1-2002 & -- & 0.03 & ID & 19 & -- & -- & -- \\
\hline & $9-17-2002$ & -- & 0.03 & ID & 19 & -- & -- & -- \\
\hline 53 & $7-31-2002$ & -- & -- & -- & -- & -- & -- & -- \\
\hline 54 & 8-1-2002 & -- & -- & -- & -- & -- & -- & -- \\
\hline 55 & $7-30-2002$ & -- & $<0.1$ & ID & 5 & -- & -- & -- \\
\hline \multirow[t]{2}{*}{56} & $7-31-2002$ & -- & -- & -- & -- & -- & -- & -- \\
\hline & $9-5-2002$ & -- & -- & -- & -- & -- & -- & -- \\
\hline \multirow[t]{2}{*}{57} & 7-31-2002 & -- & 0.2 & ID & 3 & -- & -- & -- \\
\hline & $9-4-2002$ & -- & -- & -- & -- & -- & -- & -- \\
\hline \multirow[t]{2}{*}{58} & 7-16-2002 & -- & -- & -- & -- & -- & -- & -- \\
\hline & $9-5-2002$ & -- & -- & -- & -- & -- & -- & -- \\
\hline
\end{tabular}


Table 12. Water-quality data for the Upper Colorado River Basin, showing 2002 drought values, Colorado water-quality exceedances, and percentiles relative to historical data for selected properties and constituents from July through September, 1978-2002.-Continued

$[\mathrm{ft} / \mathrm{s}$, cubic feet per second; Conc, concentration; Q\%, percentile (percentage of data less than or equal to reported value or concentration) of drought-sample constituent; $\mathrm{N}$, total number of historical and drought samples; $\mathrm{mg} / \mathrm{L}$, milligrams per liter; $\mu \mathrm{S} / \mathrm{cm}$, microsiemens per centimeter at $25^{\circ} \mathrm{Celsius;}{ }^{\circ} \mathrm{C}$, degrees Celsius; E. coli, Escherichia coli; $\mathrm{mL}$, milliliter; $\mu \mathrm{g} / \mathrm{L}$, micrograms per liter; $\mathrm{CaCO}_{3}$, calcium carbonate; $\mathrm{SiO}_{2}$, silica; $\mathrm{SO}_{4}$, sulfate; ID, insufficient number of samples (less than 20) to calculate percentile; <, less than; --, no drought-sample measurement; values or concentrations in bold type indicate 2002 Colorado water-quality exceedances; c, chronic water-quality exceedance]

\begin{tabular}{|c|c|c|c|c|c|c|c|}
\hline \multirow{2}{*}{$\begin{array}{l}\text { Site number } \\
\text { in table } 1 \\
\text { and fig. } 4\end{array}$} & \multirow{2}{*}{ Sampling date } & \multicolumn{3}{|c|}{ Chromium, dissolved, in $\mu \mathrm{g} / \mathrm{L}$} & \multicolumn{3}{|c|}{$\begin{array}{c}\text { Chromium, total recoverable, in } \\
\mu \mathrm{g} / \mathrm{L}\end{array}$} \\
\hline & & Conc & $0 \%$ & $\mathrm{~N}$ & Conc & $0 \%$ & $\mathrm{~N}$ \\
\hline 20 & $8-1-2002$ & -- & -- & -- & -- & -- & -- \\
\hline \multirow[t]{2}{*}{21} & $7-15-2002$ & -- & -- & -- & -- & -- & -- \\
\hline & $9-4-2002$ & -- & -- & -- & -- & -- & -- \\
\hline \multirow[t]{2}{*}{22} & $7-15-2002$ & -- & -- & -- & -- & -- & -- \\
\hline & $9-4-2002$ & -- & -- & -- & -- & -- & -- \\
\hline \multirow[t]{2}{*}{23} & $7-15-2002$ & -- & -- & -- & -- & -- & -- \\
\hline & $9-4-2002$ & -- & -- & -- & -- & -- & -- \\
\hline \multirow[t]{2}{*}{24} & $7-15-2002$ & -- & -- & -- & -- & -- & -- \\
\hline & $9-4-2002$ & -- & -- & -- & -- & -- & -- \\
\hline \multirow[t]{2}{*}{25} & $7-15-2002$ & -- & -- & -- & -- & -- & -- \\
\hline & $9-4-2002$ & -- & -- & -- & -- & -- & -- \\
\hline \multirow[t]{2}{*}{26} & $7-17-2002$ & -- & -- & -- & -- & -- & -- \\
\hline & $9-6-2002$ & -- & -- & -- & -- & -- & -- \\
\hline 27 & $8-1-2002$ & $<0.8$ & ID & 8 & $<0.8$ & ID & 8 \\
\hline \multirow[t]{2}{*}{28} & 8-8-2002 & -- & -- & -- & -- & -- & -- \\
\hline & $9-11-2002$ & -- & -- & -- & -- & -- & -- \\
\hline \multirow[t]{2}{*}{29} & $8-8-2002$ & -- & -- & -- & -- & -- & -- \\
\hline & $9-11-2002$ & -- & -- & -- & -- & -- & -- \\
\hline \multirow[t]{2}{*}{30} & $7-29-2002$ & $<0.8$ & ID & 14 & $<0.8$ & ID & 14 \\
\hline & $9-10-2002$ & -- & -- & -- & -- & -- & -- \\
\hline \multirow[t]{2}{*}{31} & $7-29-2002$ & $<0.8$ & ID & 14 & $<0.8$ & ID & 14 \\
\hline & $9-10-2002$ & -- & -- & -- & -- & -- & -- \\
\hline \multirow[t]{2}{*}{32} & 8-8-2002 & -- & -- & -- & -- & -- & -- \\
\hline & $9-11-2002$ & -- & -- & -- & -- & -- & -- \\
\hline 33 & $7-30-2002$ & $<0.8$ & ${ }^{2} 4.5-95.5$ & 21 & -- & -- & -- \\
\hline 34 & $7-30-2002$ & -- & -- & -- & -- & -- & -- \\
\hline \multirow[t]{2}{*}{35} & $7-30-2002$ & $<0.8$ & ID & 5 & -- & -- & -- \\
\hline & $9-3-2002$ & -- & -- & -- & -- & -- & -- \\
\hline \multirow[t]{2}{*}{36} & $7-30-2002$ & -- & -- & -- & -- & -- & -- \\
\hline & $9-3-2002$ & -- & -- & -- & -- & -- & -- \\
\hline \multirow[t]{2}{*}{37} & $7-31-2002$ & $<0.8$ & ${ }^{2} 4.5-95.5$ & 21 & $<0.8$ & ${ }^{2} 4.5-63.6$ & 21 \\
\hline & $9-4-2002$ & -- & -- & -- & -- & -- & -- \\
\hline
\end{tabular}


Table 12. Water-quality data for the Upper Colorado River Basin, showing 2002 drought values, Colorado water-quality exceedances, and percentiles relative to historical data for selected properties and constituents from July through September, 1978-2002.-Continued

[ft $3 / \mathrm{s}$, cubic feet per second; Conc, concentration; Q\%, percentile (percentage of data less than or equal to reported value or concentration) of drought-sample constituent; N, total number of historical and drought samples; $\mathrm{mg} / \mathrm{L}$, milligrams per liter; $\mu \mathrm{S} / \mathrm{cm}$, microsiemens per centimeter at $25^{\circ} \mathrm{Celsius;}{ }^{\circ} \mathrm{C}$, degrees Celsius; E. coli, Escherichia coli; $\mathrm{mL}$, milliliter; $\mu \mathrm{g} / \mathrm{L}$, micrograms per liter; $\mathrm{CaCO}_{3}$, calcium carbonate; $\mathrm{SiO}_{2}$, silica; $\mathrm{SO}_{4}$, sulfate; $\mathrm{ID}$, insufficient number of samples (less than 20) to calculate percentile; <, less than; --, no drought-sample measurement; values or concentrations in bold type indicate 2002 Colorado water-quality exceedances; c, chronic water-quality exceedance]

\begin{tabular}{|c|c|c|c|c|c|c|c|}
\hline \multirow{2}{*}{$\begin{array}{l}\text { Site number } \\
\text { in table } 1 \\
\text { and fig. } 4\end{array}$} & \multirow{2}{*}{ Sampling date } & \multicolumn{3}{|c|}{ Chromium, dissolved, in $\mu \mathrm{g} / \mathrm{L}$} & \multicolumn{3}{|c|}{$\begin{array}{c}\text { Chromium, total recoverable, in } \\
\mu \mathrm{g} / \mathrm{L}\end{array}$} \\
\hline & & Conc & $0 \%$ & $\mathrm{~N}$ & Conc & $0 \%$ & $\mathrm{~N}$ \\
\hline \multirow[t]{2}{*}{38} & $8-1-2002$ & -- & -- & -- & -- & -- & -- \\
\hline & $9-5-2002$ & -- & -- & -- & -- & -- & -- \\
\hline \multirow[t]{2}{*}{39} & $8-1-2002$ & -- & -- & -- & -- & -- & -- \\
\hline & $9-6-2002$ & -- & -- & -- & -- & -- & -- \\
\hline \multirow[t]{2}{*}{40} & $7-29-2002$ & -- & -- & -- & -- & -- & -- \\
\hline & $9-3-2002$ & -- & -- & -- & -- & -- & -- \\
\hline \multirow[t]{2}{*}{41} & $7-29-2002$ & -- & -- & -- & -- & -- & -- \\
\hline & $9-4-2002$ & -- & -- & -- & -- & -- & -- \\
\hline \multirow[t]{2}{*}{42} & $7-30-2002$ & -- & -- & -- & -- & -- & -- \\
\hline & $9-4-2002$ & -- & -- & -- & -- & -- & -- \\
\hline \multirow[t]{2}{*}{43} & $7-30-2002$ & -- & -- & -- & -- & -- & -- \\
\hline & $9-4-2002$ & -- & -- & -- & -- & -- & -- \\
\hline \multirow[t]{2}{*}{44} & $7-30-2002$ & -- & -- & -- & -- & -- & -- \\
\hline & $9-5-2002$ & -- & -- & -- & -- & -- & -- \\
\hline \multirow[t]{2}{*}{45} & $7-31-2002$ & -- & -- & -- & -- & -- & -- \\
\hline & $9-6-2002$ & -- & -- & -- & -- & -- & -- \\
\hline 46 & $8-2-2002$ & -- & -- & -- & -- & -- & -- \\
\hline \multirow[t]{2}{*}{47} & 8-1-2002 & -- & -- & -- & -- & -- & -- \\
\hline & $9-3-2002$ & -- & -- & -- & -- & -- & -- \\
\hline \multirow[t]{2}{*}{48} & $8-1-2002$ & -- & -- & -- & -- & -- & -- \\
\hline & $9-5-2002$ & -- & -- & -- & -- & -- & -- \\
\hline 49 & $7-31-2002$ & -- & -- & -- & -- & -- & -- \\
\hline 50 & $7-31-2002$ & -- & -- & -- & -- & -- & -- \\
\hline 51 & $8-1-2002$ & -- & -- & -- & -- & -- & -- \\
\hline \multirow[t]{2}{*}{52} & $8-1-2002$ & -- & -- & -- & -- & -- & -- \\
\hline & $9-17-2002$ & -- & -- & -- & -- & -- & -- \\
\hline 53 & $7-31-2002$ & -- & -- & -- & -- & -- & -- \\
\hline 54 & $8-1-2002$ & -- & -- & -- & -- & -- & -- \\
\hline 55 & $7-30-2002$ & -- & -- & -- & -- & -- & -- \\
\hline \multirow[t]{2}{*}{56} & $7-31-2002$ & -- & -- & -- & -- & -- & -- \\
\hline & $9-5-2002$ & -- & -- & -- & -- & -- & -- \\
\hline \multirow[t]{2}{*}{57} & $7-31-2002$ & $<0.8$ & ID & 3 & -- & -- & -- \\
\hline & $9-4-2002$ & -- & -- & -- & -- & -- & -- \\
\hline \multirow[t]{2}{*}{58} & $7-16-2002$ & -- & -- & -- & -- & -- & -- \\
\hline & $9-5-2002$ & -- & -- & -- & -- & -- & -- \\
\hline
\end{tabular}


Table 12. Water-quality data for the Upper Colorado River Basin, showing 2002 drought values, Colorado water-quality exceedances, and percentiles relative to historical data for selected properties and constituents from July through September, 1978-2002.-Continued

$[\mathrm{ft} / \mathrm{s}$, cubic feet per second; Conc, concentration; Q\%, percentile (percentage of data less than or equal to reported value or concentration) of drought-sample constituent; N, total number of historical and drought samples; $\mathrm{mg} / \mathrm{L}$, milligrams per liter; $\mu \mathrm{S} / \mathrm{cm}$, microsiemens per centimeter at $25^{\circ} \mathrm{Celsius;}{ }^{\circ} \mathrm{C}$, degrees Celsius; E. coli, Escherichia coli; $\mathrm{mL}$, milliliter; $\mu \mathrm{g} / \mathrm{L}$, micrograms per liter; $\mathrm{CaCO}_{3}$, calcium carbonate; $\mathrm{SiO}_{2}$, silica; $\mathrm{SO}_{4}$, sulfate; ID, insufficient number of samples (less than 20) to calculate percentile; <, less than; --, no drought-sample measurement; values or concentrations in bold type indicate 2002 Colorado water-quality exceedances; c, chronic water-quality exceedance]

\begin{tabular}{|c|c|c|c|c|c|c|c|}
\hline \multirow{2}{*}{$\begin{array}{l}\text { Site number } \\
\text { in table } 1 \\
\text { and fig. } 4\end{array}$} & \multirow{2}{*}{ Sampling date } & \multicolumn{3}{|c|}{ Copper, dissolved, in $\mu \mathrm{g} / \mathrm{L}$} & \multicolumn{3}{|c|}{ Copper, total recoverable, in $\mu \mathrm{g} / \mathrm{L}$} \\
\hline & & Conc & $0 \%$ & $\mathrm{~N}$ & Conc & $0 \%$ & $\mathrm{~N}$ \\
\hline 20 & 8-1-2002 & -- & $\begin{array}{l}- \\
\end{array}$ & -- & -- & -- & -- \\
\hline \multirow[t]{2}{*}{21} & $7-15-2002$ & -- & -- & -- & -- & -- & -- \\
\hline & $9-4-2002$ & -- & -- & -- & -- & -- & -- \\
\hline \multirow[t]{2}{*}{22} & 7-15-2002 & -- & -- & -- & -- & -- & -- \\
\hline & $9-4-2002$ & -- & -- & -- & -- & -- & -- \\
\hline \multirow[t]{2}{*}{23} & $7-15-2002$ & -- & -- & -- & -- & -- & -- \\
\hline & $9-4-2002$ & -- & -- & -- & -- & -- & -- \\
\hline \multirow[t]{2}{*}{24} & $7-15-2002$ & -- & -- & -- & -- & -- & -- \\
\hline & $9-4-2002$ & -- & -- & -- & -- & -- & -- \\
\hline \multirow[t]{2}{*}{25} & $7-15-2002$ & -- & -- & -- & -- & -- & -- \\
\hline & $9-4-2002$ & -- & -- & -- & -- & -- & -- \\
\hline \multirow[t]{2}{*}{26} & 7-17-2002 & -- & -- & -- & -- & -- & -- \\
\hline & $9-6-2002$ & -- & -- & -- & -- & -- & -- \\
\hline 27 & 8-1-2002 & 0.8 & ID & 8 & 1 & ID & 8 \\
\hline \multirow[t]{2}{*}{28} & $8-8-2002$ & 0.9 & ID & 2 & -- & -- & -- \\
\hline & $9-11-2002$ & 0.7 & ID & 2 & -- & -- & -- \\
\hline \multirow[t]{2}{*}{29} & 8-8-2002 & -- & -- & -- & -- & -- & -- \\
\hline & $9-11-2002$ & -- & -- & -- & -- & -- & -- \\
\hline \multirow[t]{2}{*}{30} & 7-29-2002 & $<1$ & ID & 14 & 22 & ID & 14 \\
\hline & $9-10-2002$ & -- & -- & -- & -- & -- & -- \\
\hline \multirow[t]{2}{*}{31} & $7-29-2002$ & 0.8 & ID & 14 & 2.7 & ID & 14 \\
\hline & $9-10-2002$ & -- & -- & -- & -- & -- & -- \\
\hline \multirow[t]{2}{*}{32} & 8-8-2002 & 1 & ID & 2 & -- & -- & -- \\
\hline & 9-11-2002 & 1.1 & ID & 2 & -- & -- & -- \\
\hline 33 & $7-30-2002$ & 0.9 & ${ }^{2} 4.2-87.5$ & 23 & -- & -- & -- \\
\hline 34 & $7-30-2002$ & $<1$ & ID & 6 & -- & -- & -- \\
\hline \multirow[t]{2}{*}{35} & $7-30-2002$ & 1.6 & ID & 5 & -- & -- & -- \\
\hline & $9-3-2002$ & -- & -- & -- & -- & -- & -- \\
\hline \multirow[t]{2}{*}{36} & $7-30-2002$ & 0.9 & ID & 9 & -- & -- & -- \\
\hline & $9-3-2002$ & -- & -- & -- & -- & -- & -- \\
\hline \multirow[t]{2}{*}{37} & $7-31-2002$ & 0.9 & ${ }^{2} 4.3-39.1$ & 22 & 1.6 & ${ }^{1} 40.9-45.5$ & 21 \\
\hline & $9-4-2002$ & 2.6 & 87.0 & 22 & -- & -- & -- \\
\hline
\end{tabular}


Table 12. Water-quality data for the Upper Colorado River Basin, showing 2002 drought values, Colorado water-quality exceedances, and percentiles relative to historical data for selected properties and constituents from July through September, 1978-2002.-Continued

[ft $3 / \mathrm{s}$, cubic feet per second; Conc, concentration; Q\%, percentile (percentage of data less than or equal to reported value or concentration) of drought-sample constituent; N, total number of historical and drought samples; $\mathrm{mg} / \mathrm{L}$, milligrams per liter; $\mu \mathrm{S} / \mathrm{cm}$, microsiemens per centimeter at $25^{\circ} \mathrm{Celsius;}{ }^{\circ} \mathrm{C}$, degrees Celsius; E. coli, Escherichia coli; $\mathrm{mL}$, milliliter; $\mu \mathrm{g} / \mathrm{L}$, micrograms per liter; $\mathrm{CaCO}_{3}$, calcium carbonate; $\mathrm{SiO}_{2}$, silica; $\mathrm{SO}_{4}$, sulfate; ID, insufficient number of samples (less than 20) to calculate percentile; <, less than; --, no drought-sample measurement; values or concentrations in bold type indicate 2002 Colorado water-quality exceedances; c, chronic water-quality exceedance]

\begin{tabular}{|c|c|c|c|c|c|c|c|}
\hline \multirow{2}{*}{$\begin{array}{l}\text { Site number } \\
\text { in table } 1 \\
\text { and fig. } 4\end{array}$} & \multirow{2}{*}{ Sampling date } & \multicolumn{3}{|c|}{ Copper, dissolved, in $\mu \mathrm{g} / \mathrm{L}$} & \multicolumn{3}{|c|}{ Copper, total recoverable, in $\mu \mathrm{g} / \mathrm{L}$} \\
\hline & & Conc & $0 \%$ & $\mathrm{~N}$ & Conc & $0 \%$ & $\mathrm{~N}$ \\
\hline \multirow[t]{2}{*}{38} & $8-1-2002$ & -- & -- & -- & -- & -- & -- \\
\hline & $9-5-2002$ & -- & -- & -- & -- & -- & -- \\
\hline \multirow[t]{2}{*}{39} & $8-1-2002$ & -- & -- & -- & -- & -- & -- \\
\hline & $9-6-2002$ & -- & -- & -- & -- & -- & -- \\
\hline \multirow[t]{2}{*}{40} & $7-29-2002$ & 0.8 & ID & 6 & -- & -- & -- \\
\hline & $9-3-2002$ & -- & -- & -- & -- & -- & -- \\
\hline \multirow[t]{2}{*}{41} & $7-29-2002$ & $<1$ & ID & 5 & -- & -- & -- \\
\hline & $9-4-2002$ & -- & -- & -- & -- & -- & -- \\
\hline \multirow[t]{2}{*}{42} & $7-30-2002$ & $<1$ & ID & 6 & -- & -- & -- \\
\hline & $9-4-2002$ & -- & -- & -- & -- & -- & -- \\
\hline \multirow[t]{2}{*}{43} & $7-30-2002$ & $<1$ & ID & 3 & -- & -- & -- \\
\hline & $9-4-2002$ & -- & -- & -- & -- & -- & -- \\
\hline \multirow[t]{2}{*}{44} & $7-30-2002$ & 0.8 & ID & 10 & -- & -- & -- \\
\hline & $9-5-2002$ & 1.1 & ID & 10 & -- & -- & -- \\
\hline \multirow[t]{2}{*}{45} & $7-31-2002$ & 1.2 & ID & 2 & -- & -- & -- \\
\hline & $9-6-2002$ & 1.3 & ID & 2 & -- & -- & -- \\
\hline 46 & $8-2-2002$ & -- & -- & -- & -- & -- & -- \\
\hline \multirow[t]{2}{*}{47} & $8-1-2002$ & -- & -- & -- & -- & -- & -- \\
\hline & $9-3-2002$ & -- & -- & -- & -- & -- & -- \\
\hline \multirow[t]{2}{*}{48} & $8-1-2002$ & -- & -- & -- & -- & -- & -- \\
\hline & $9-5-2002$ & -- & -- & -- & -- & -- & -- \\
\hline 49 & $7-31-2002$ & -- & -- & -- & -- & -- & -- \\
\hline 50 & $7-31-2002$ & -- & -- & -- & -- & -- & -- \\
\hline 51 & $8-1-2002$ & -- & -- & -- & -- & -- & -- \\
\hline \multirow[t]{2}{*}{52} & $8-1-2002$ & 2.5 & ID & 19 & -- & -- & -- \\
\hline & $9-17-2002$ & 2.8 & ID & 19 & -- & -- & -- \\
\hline 53 & $7-31-2002$ & -- & -- & -- & -- & -- & -- \\
\hline 54 & 8-1-2002 & -- & -- & -- & -- & -- & -- \\
\hline 55 & $7-30-2002$ & 0.7 & ID & 5 & -- & -- & -- \\
\hline \multirow[t]{2}{*}{56} & $7-31-2002$ & -- & -- & -- & -- & -- & -- \\
\hline & $9-5-2002$ & -- & -- & -- & -- & -- & -- \\
\hline \multirow[t]{2}{*}{57} & $7-31-2002$ & 1.9 & ID & 3 & -- & -- & -- \\
\hline & 9-4-2002 & -- & -- & -- & -- & -- & -- \\
\hline \multirow[t]{2}{*}{58} & $7-16-2002$ & -- & -- & -- & -- & -- & -- \\
\hline & $9-5-2002$ & -- & -- & -- & -- & -- & -- \\
\hline
\end{tabular}


Table 12. Water-quality data for the Upper Colorado River Basin, showing 2002 drought values, Colorado water-quality exceedances, and percentiles relative to historical data for selected properties and constituents from July through September, 1978-2002. - Continued

$\left[\mathrm{ft}^{3} / \mathrm{s}\right.$, cubic feet per second; Conc, concentration; Q\%, percentile (percentage of data less than or equal to reported value or concentration) of drought-sample constituent; $\mathrm{N}$, total number of historical and drought samples; $\mathrm{mg} / \mathrm{L}$, milligrams per liter; $\mu \mathrm{S} / \mathrm{cm}$, microsiemens per centimeter at $25^{\circ} \mathrm{Celsius;}{ }^{\circ} \mathrm{C}$, degrees Celsius; E. coli, Escherichia coli; $\mathrm{mL}$, milliliter; $\mu \mathrm{g} / \mathrm{L}$, micrograms per liter; $\mathrm{CaCO}_{3}$, calcium carbonate; $\mathrm{SiO}_{2}$, silica; $\mathrm{SO}_{4}$, sulfate; ID, insufficient number of samples (less than 20) to calculate percentile; <, less than; --, no drought-sample measurement; values or concentrations in bold type indicate 2002 Colorado water-quality exceedances; c, chronic water-quality exceedance]

\begin{tabular}{|c|c|c|c|c|c|c|c|}
\hline \multirow{2}{*}{$\begin{array}{l}\text { Site number } \\
\text { in table } 1 \\
\text { and fig. } 4\end{array}$} & \multirow{2}{*}{ Sampling date } & \multicolumn{3}{|c|}{ Iron, dissolved, in $\mu \mathrm{g} / \mathrm{L}$} & \multicolumn{3}{|c|}{ Iron, total recoverable, in $\mu \mathrm{g} / \mathrm{L}$} \\
\hline & & Conc & $0 \%$ & $\mathrm{~N}$ & Conc & $0 \%$ & $\mathrm{~N}$ \\
\hline 20 & $8-1-2002$ & -- & -- & -- & -- & -- & -- \\
\hline \multirow[t]{2}{*}{21} & $7-15-2002$ & -- & -- & -- & -- & -- & -- \\
\hline & $9-4-2002$ & -- & -- & -- & -- & -- & -- \\
\hline \multirow[t]{2}{*}{22} & $7-15-2002$ & -- & -- & -- & -- & -- & -- \\
\hline & $9-4-2002$ & -- & -- & -- & -- & -- & -- \\
\hline \multirow[t]{2}{*}{23} & $7-15-2002$ & -- & -- & -- & -- & -- & -- \\
\hline & $9-4-2002$ & -- & -- & -- & -- & -- & -- \\
\hline \multirow[t]{2}{*}{24} & $7-15-2002$ & -- & -- & -- & -- & -- & -- \\
\hline & $9-4-2002$ & -- & -- & -- & -- & -- & -- \\
\hline \multirow[t]{2}{*}{25} & $7-15-2002$ & -- & -- & -- & -- & -- & -- \\
\hline & $9-4-2002$ & -- & -- & -- & -- & -- & -- \\
\hline \multirow[t]{2}{*}{26} & $7-17-2002$ & $339 c$ & ID & 9 & -- & -- & -- \\
\hline & $9-6-2002$ & $383 c$ & ID & 9 & -- & -- & -- \\
\hline 27 & $8-1-2002$ & 231 & ID & 7 & -- & -- & -- \\
\hline \multirow[t]{2}{*}{28} & 8-8-2002 & $<10$ & ID & 2 & -- & -- & -- \\
\hline & $9-11-2002$ & 9 & ID & 2 & -- & -- & -- \\
\hline \multirow[t]{2}{*}{29} & 8-8-2002 & 45 & ID & 2 & -- & -- & -- \\
\hline & $9-11-2002$ & 66 & ID & 2 & -- & -- & -- \\
\hline \multirow[t]{2}{*}{30} & $7-29-2002$ & 42 & 70.8 & 47 & 330 & ${ }^{1} 39.3-50.0$ & 27 \\
\hline & $9-10-2002$ & 32 & ${ }^{1} 58.3-60.4$ & 47 & 330 & ${ }^{1} 39.3-50.0$ & 27 \\
\hline \multirow[t]{2}{*}{31} & $7-29-2002$ & $<10$ & ${ }^{2} 4.0-48.0$ & 24 & 50 & ${ }^{1} 4.5-13.6$ & 21 \\
\hline & $9-10-2002$ & 55 & 88.0 & 24 & 260 & 95.5 & 21 \\
\hline \multirow[t]{2}{*}{32} & 8-8-2002 & 7 & ID & 2 & -- & -- & -- \\
\hline & $9-11-2002$ & 28 & ID & 2 & -- & -- & -- \\
\hline 33 & $7-30-2002$ & 33 & ${ }^{1} 45.8-50$ & 23 & -- & -- & -- \\
\hline 34 & $7-30-2002$ & -- & -- & -- & 90 & ID & 6 \\
\hline \multirow[t]{2}{*}{35} & $7-30-2002$ & $<10$ & ID & 17 & 10 & ID & 5 \\
\hline & $9-3-2002$ & -- & -- & -- & -- & -- & -- \\
\hline \multirow[t]{2}{*}{36} & $7-30-2002$ & -- & -- & -- & 180 & ID & 9 \\
\hline & $9-3-2002$ & -- & -- & -- & -- & -- & -- \\
\hline \multirow[t]{2}{*}{37} & $7-31-2002$ & 7 & 13.3 & 29 & -- & -- & -- \\
\hline & $9-4-2002$ & 13 & ${ }^{1} 26.7-33.3$ & 29 & -- & -- & -- \\
\hline
\end{tabular}


Table 12. Water-quality data for the Upper Colorado River Basin, showing 2002 drought values, Colorado water-quality exceedances, and percentiles relative to historical data for selected properties and constituents from July through September, 1978-2002.—Continued

[ft $3 / \mathrm{s}$, cubic feet per second; Conc, concentration; Q\%, percentile (percentage of data less than or equal to reported value or concentration) of drought-sample constituent; N, total number of historical and drought samples; $\mathrm{mg} / \mathrm{L}$, milligrams per liter; $\mu \mathrm{S} / \mathrm{cm}$, microsiemens per centimeter at $25^{\circ} \mathrm{Celsius;}{ }^{\circ} \mathrm{C}$, degrees Celsius; E. coli, Escherichia coli; $\mathrm{mL}$, milliliter; $\mu \mathrm{g} / \mathrm{L}$, micrograms per liter; $\mathrm{CaCO}_{3}$, calcium carbonate; $\mathrm{SiO}_{2}$, silica; $\mathrm{SO}_{4}$, sulfate; ID, insufficient number of samples (less than 20) to calculate percentile; <, less than; --, no drought-sample measurement; values or concentrations in bold type indicate 2002 Colorado water-quality exceedances; c, chronic water-quality exceedance]

\begin{tabular}{|c|c|c|c|c|c|c|c|}
\hline \multirow{2}{*}{$\begin{array}{l}\text { Site number } \\
\text { in table } 1 \\
\text { and fig. } 4\end{array}$} & \multirow{2}{*}{ Sampling date } & \multicolumn{3}{|c|}{ Iron, dissolved, in $\mu \mathrm{g} / \mathrm{L}$} & \multicolumn{3}{|c|}{ Iron, total recoverable, in $\mu \mathrm{g} / \mathrm{L}$} \\
\hline & & Conc & $0 \%$ & $\mathrm{~N}$ & Conc & $0 \%$ & $\mathrm{~N}$ \\
\hline \multirow[t]{2}{*}{38} & $8-1-2002$ & 23 & ID & 14 & -- & -- & -- \\
\hline & $9-5-2002$ & 15 & ID & 14 & -- & -- & -- \\
\hline \multirow[t]{2}{*}{39} & $8-1-2002$ & $<10$ & ID & 5 & -- & -- & -- \\
\hline & $9-6-2002$ & 17 & ID & 5 & -- & -- & -- \\
\hline \multirow[t]{2}{*}{40} & $7-29-2002$ & -- & -- & -- & 40 & ID & 6 \\
\hline & $9-3-2002$ & -- & -- & -- & -- & -- & -- \\
\hline \multirow[t]{2}{*}{41} & $7-29-2002$ & -- & -- & -- & 50 & ID & 5 \\
\hline & $9-4-2002$ & -- & -- & -- & -- & -- & -- \\
\hline \multirow[t]{2}{*}{42} & $7-30-2002$ & -- & -- & -- & 50 & ID & 6 \\
\hline & $9-4-2002$ & -- & -- & -- & -- & -- & -- \\
\hline \multirow[t]{2}{*}{43} & $7-30-2002$ & -- & -- & -- & 20 & ID & 3 \\
\hline & $9-4-2002$ & -- & -- & -- & -- & -- & -- \\
\hline \multirow[t]{2}{*}{44} & $7-30-2002$ & -- & -- & -- & 40 & ID & 9 \\
\hline & $9-5-2002$ & 5 & ID & 2 & -- & -- & -- \\
\hline \multirow[t]{2}{*}{45} & $7-31-2002$ & 12 & ID & 2 & -- & -- & -- \\
\hline & $9-6-2002$ & 7 & ID & 2 & -- & -- & -- \\
\hline 46 & $8-2-2002$ & -- & -- & -- & -- & -- & -- \\
\hline \multirow[t]{2}{*}{47} & $8-1-2002$ & 7 & ID & 19 & -- & -- & -- \\
\hline & $9-3-2002$ & 8 & ID & 19 & -- & -- & -- \\
\hline \multirow[t]{2}{*}{48} & $8-1-2002$ & $<10$ & ID & 5 & -- & -- & -- \\
\hline & $9-5-2002$ & 30 & ID & 5 & -- & -- & -- \\
\hline 49 & $7-31-2002$ & $<10$ & ID & 2 & -- & -- & -- \\
\hline 50 & $7-31-2002$ & 6 & ID & 1 & -- & -- & -- \\
\hline 51 & $8-1-2002$ & $<10$ & ID & 1 & -- & -- & -- \\
\hline \multirow[t]{2}{*}{52} & $8-1-2002$ & $<10$ & ${ }^{2} 2.2-63.0$ & 45 & -- & -- & -- \\
\hline & $9-17-2002$ & $<10$ & ${ }^{2} 2.2-63.0$ & 45 & -- & -- & -- \\
\hline 53 & $7-31-2002$ & 10 & ID & 1 & -- & -- & -- \\
\hline 54 & $8-1-2002$ & $<10$ & ID & 1 & -- & -- & -- \\
\hline 55 & $7-30-2002$ & -- & -- & -- & 510 & ID & 5 \\
\hline \multirow[t]{2}{*}{56} & $7-31-2002$ & 11 & ID & 2 & -- & -- & -- \\
\hline & $9-5-2002$ & 11 & ID & 2 & -- & -- & -- \\
\hline \multirow[t]{2}{*}{57} & $7-31-2002$ & 25 & ID & 3 & 280 & ID & 3 \\
\hline & $9-4-2002$ & -- & -- & -- & -- & -- & -- \\
\hline \multirow[t]{2}{*}{58} & $7-16-2002$ & -- & -- & -- & -- & -- & -- \\
\hline & $9-5-2002$ & -- & -- & -- & -- & -- & -- \\
\hline
\end{tabular}


Table 12. Water-quality data for the Upper Colorado River Basin, showing 2002 drought values, Colorado water-quality exceedances, and percentiles relative to historical data for selected properties and constituents from July through September, 1978-2002. - Continued

$\left[\mathrm{ft}^{3} / \mathrm{s}\right.$, cubic feet per second; Conc, concentration; Q\%, percentile (percentage of data less than or equal to reported value or concentration) of drought-sample constituent; $\mathrm{N}$, total number of historical and drought samples; $\mathrm{mg} / \mathrm{L}$, milligrams per liter; $\mu \mathrm{S} / \mathrm{cm}$, microsiemens per centimeter at $25^{\circ} \mathrm{Celsius;}{ }^{\circ} \mathrm{C}$, degrees Celsius; E. coli, Escherichia coli; $\mathrm{mL}$, milliliter; $\mu \mathrm{g} / \mathrm{L}$, micrograms per liter; $\mathrm{CaCO}_{3}$, calcium carbonate; $\mathrm{SiO}_{2}$, silica; $\mathrm{SO}_{4}$, sulfate; ID, insufficient number of samples (less than 20) to calculate percentile; <, less than; --, no drought-sample measurement; values or concentrations in bold type indicate 2002 Colorado water-quality exceedances; c, chronic water-quality exceedance]

\begin{tabular}{|c|c|c|c|c|c|c|c|}
\hline \multirow{2}{*}{$\begin{array}{l}\text { Site number } \\
\text { in table } 1 \\
\text { and fig. } 4\end{array}$} & \multirow{2}{*}{ Sampling date } & \multicolumn{3}{|c|}{ Lead, dissolved, in $\mu \mathrm{g} / \mathrm{L}$} & \multicolumn{3}{|c|}{ Manganese, dissolved, in $\mu \mathrm{g} / \mathrm{L}$} \\
\hline & & Conc & $0 \%$ & $\mathrm{~N}$ & Conc & $0 \%$ & $\mathrm{~N}$ \\
\hline 20 & $8-1-2002$ & $\begin{array}{ll}- \\
-\end{array}$ & -- & -- & -- & -- & -- \\
\hline \multirow[t]{2}{*}{21} & $7-15-2002$ & -- & -- & -- & -- & -- & -- \\
\hline & $9-4-2002$ & -- & -- & -- & -- & -- & -- \\
\hline \multirow[t]{2}{*}{22} & $7-15-2002$ & -- & -- & -- & -- & -- & -- \\
\hline & $9-4-2002$ & -- & -- & -- & -- & -- & -- \\
\hline \multirow[t]{2}{*}{23} & $7-15-2002$ & -- & -- & -- & -- & -- & -- \\
\hline & $9-4-2002$ & -- & -- & -- & -- & -- & -- \\
\hline \multirow[t]{2}{*}{24} & $7-15-2002$ & -- & -- & -- & -- & -- & -- \\
\hline & 9-4-2002 & -- & -- & -- & -- & -- & -- \\
\hline \multirow[t]{2}{*}{25} & $7-15-2002$ & -- & -- & -- & -- & -- & -- \\
\hline & $9-4-2002$ & -- & -- & -- & -- & -- & -- \\
\hline \multirow[t]{2}{*}{26} & $7-17-2002$ & -- & -- & -- & 20 & ID & 9 \\
\hline & $9-6-2002$ & -- & -- & -- & 19.1 & ID & 9 \\
\hline 27 & $8-1-2002$ & 0.09 & ID & 8 & 19.3 & ID & 7 \\
\hline \multirow[t]{2}{*}{28} & 8-8-2002 & $<0.08$ & ID & 2 & 6 & ID & 2 \\
\hline & $9-11-2002$ & $<0.08$ & ID & 2 & 1.6 & ID & 2 \\
\hline \multirow[t]{2}{*}{29} & 8-8-2002 & -- & -- & -- & -- & -- & -- \\
\hline & $9-11-2002$ & -- & -- & -- & -- & -- & -- \\
\hline \multirow[t]{2}{*}{30} & $7-29-2002$ & $<1$ & ID & 14 & $60 \mathrm{c}$ & 86.2 & 28 \\
\hline & $9-10-2002$ & -- & -- & -- & $115 c$ & 96.6 & 28 \\
\hline \multirow[t]{2}{*}{31} & $7-29-2002$ & $<1$ & ID & 14 & 10.3 & 59.1 & 21 \\
\hline & $9-10-2002$ & -- & -- & -- & 250c & 90.9 & 21 \\
\hline \multirow[t]{2}{*}{32} & 8-8-2002 & $<0.08$ & ID & 2 & 13.7 & ID & 2 \\
\hline & $9-11-2002$ & 0.13 & ID & 2 & 21 & ID & 2 \\
\hline 33 & $7-30-2002$ & $<0.08$ & ${ }^{2} 4.5-86.4$ & 21 & 21 & 37.5 & 23 \\
\hline 34 & $7-30-2002$ & $<1$ & ID & 6 & 9.3 & ID & 6 \\
\hline \multirow[t]{2}{*}{35} & $7-30-2002$ & 0.05 & ID & 5 & 2 & ID & 17 \\
\hline & $9-3-2002$ & -- & -- & -- & -- & -- & -- \\
\hline \multirow[t]{2}{*}{36} & $7-30-2002$ & $<1$ & ID & 9 & 15.5 & ID & 10 \\
\hline & $9-3-2002$ & -- & -- & -- & -- & -- & -- \\
\hline \multirow[t]{2}{*}{37} & $7-31-2002$ & $<1$ & ${ }^{2} 4.3-95.7$ & 22 & 21 & 26.7 & 29 \\
\hline & $9-4-2002$ & 0.07 & ${ }^{2} 4.3-95.7$ & 22 & 29 & 46.7 & 29 \\
\hline
\end{tabular}


Table 12. Water-quality data for the Upper Colorado River Basin, showing 2002 drought values, Colorado water-quality exceedances, and percentiles relative to historical data for selected properties and constituents from July through September, 1978-2002.-Continued

[ $\mathrm{ft}^{3} / \mathrm{s}$, cubic feet per second; Conc, concentration; Q\%, percentile (percentage of data less than or equal to reported value or concentration) of drought-sample constituent; $\mathrm{N}$, total number of historical and drought samples; $\mathrm{mg} / \mathrm{L}$, milligrams per liter; $\mu \mathrm{S} / \mathrm{cm}$, microsiemens per centimeter at $25^{\circ} \mathrm{Celsius;}{ }^{\circ} \mathrm{C}$, degrees Celsius; E. coli, Escherichia coli; $\mathrm{mL}$, milliliter; $\mu \mathrm{g} / \mathrm{L}$, micrograms per liter; $\mathrm{CaCO}_{3}$, calcium carbonate; $\mathrm{SiO}_{2}$, silica; $\mathrm{SO}_{4}$, sulfate; ID, insufficient number of samples (less than 20) to calculate percentile; <, less than; --, no drought-sample measurement; values or concentrations in bold type indicate 2002 Colorado water-quality exceedances; $c$, chronic water-quality exceedance]

\begin{tabular}{|c|c|c|c|c|c|c|c|}
\hline \multirow{2}{*}{$\begin{array}{l}\text { Site number } \\
\text { in table } 1 \\
\text { and fig. } 4\end{array}$} & \multirow{2}{*}{ Sampling date } & \multicolumn{3}{|c|}{ Lead, dissolved, in $\mu \mathrm{g} / \mathrm{L}$} & \multicolumn{3}{|c|}{ Manganese, dissolved, in $\mu \mathrm{g} / \mathrm{L}$} \\
\hline & & Conc & $0 \%$ & $\mathrm{~N}$ & Conc & $0 \%$ & $\mathrm{~N}$ \\
\hline \multirow[t]{2}{*}{38} & $8-1-2002$ & -- & -- & -- & -- & -- & -- \\
\hline & $9-5-2002$ & -- & -- & -- & -- & -- & -- \\
\hline \multirow[t]{2}{*}{39} & $8-1-2002$ & -- & -- & -- & -- & -- & -- \\
\hline & $9-6-2002$ & -- & -- & -- & -- & -- & -- \\
\hline \multirow[t]{2}{*}{40} & $7-29-2002$ & $<1$ & ID & 6 & 1.4 & ID & 6 \\
\hline & $9-3-2002$ & -- & -- & -- & -- & -- & -- \\
\hline \multirow[t]{2}{*}{41} & $7-29-2002$ & $<1$ & ID & 5 & 5.6 & ID & 6 \\
\hline & $9-4-2002$ & -- & -- & -- & -- & -- & -- \\
\hline \multirow[t]{2}{*}{42} & $7-30-2002$ & $<1$ & ID & 6 & 7.7 & ID & 6 \\
\hline & $9-4-2002$ & -- & -- & -- & -- & -- & -- \\
\hline \multirow[t]{2}{*}{43} & $7-30-2002$ & $<1$ & ID & 3 & 4.1 & ID & 3 \\
\hline & $9-4-2002$ & -- & -- & -- & -- & -- & -- \\
\hline \multirow[t]{2}{*}{44} & $7-30-2002$ & $<1$ & ID & 10 & 5 & ID & 11 \\
\hline & $9-5-2002$ & 0.14 & ID & 10 & 4.1 & ID & 11 \\
\hline \multirow[t]{2}{*}{45} & $7-31-2002$ & 0.07 & ID & 2 & 6.7 & ID & 2 \\
\hline & $9-6-2002$ & 0.09 & ID & 2 & 5.9 & ID & 2 \\
\hline 46 & $8-2-2002$ & -- & -- & -- & -- & -- & -- \\
\hline \multirow[t]{2}{*}{47} & $8-1-2002$ & -- & -- & -- & -- & -- & -- \\
\hline & $9-3-2002$ & -- & -- & -- & -- & -- & -- \\
\hline \multirow[t]{2}{*}{48} & $8-1-2002$ & -- & -- & -- & -- & -- & -- \\
\hline & $9-5-2002$ & -- & -- & -- & -- & -- & -- \\
\hline 49 & $7-31-2002$ & -- & -- & -- & -- & -- & -- \\
\hline 50 & $7-31-2002$ & -- & -- & -- & -- & -- & -- \\
\hline 51 & 8-1-2002 & -- & -- & -- & -- & -- & -- \\
\hline \multirow[t]{2}{*}{52} & $8-1-2002$ & 0.05 & ID & 19 & 5.4 & 93.5 & 45 \\
\hline & $9-17-2002$ & $<0.08$ & ID & 19 & 37 & 97.8 & 45 \\
\hline 53 & $7-31-2002$ & -- & -- & -- & -- & -- & -- \\
\hline 54 & $8-1-2002$ & -- & -- & -- & -- & -- & -- \\
\hline 55 & $7-30-2002$ & $<1$ & ID & 5 & 13.8 & ID & 5 \\
\hline \multirow[t]{2}{*}{56} & $7-31-2002$ & -- & -- & -- & -- & -- & -- \\
\hline & $9-5-2002$ & -- & -- & -- & -- & -- & -- \\
\hline \multirow[t]{2}{*}{57} & $7-31-2002$ & 0.12 & ID & 3 & 17.8 & ID & 3 \\
\hline & $9-4-2002$ & -- & -- & -- & -- & -- & -- \\
\hline \multirow[t]{2}{*}{58} & $7-16-2002$ & -- & -- & -- & -- & -- & -- \\
\hline & $9-5-2002$ & -- & -- & -- & -- & -- & -- \\
\hline
\end{tabular}


Table 12. Water-quality data for the Upper Colorado River Basin, showing 2002 drought values, Colorado water-quality exceedances, and percentiles relative to historical data for selected properties and constituents from July through September, 1978-2002.—Continued

$\left[\mathrm{ft}^{3} / \mathrm{s}\right.$, cubic feet per second; Conc, concentration; Q\%, percentile (percentage of data less than or equal to reported value or concentration) of drought-sample constituent; N, total number of historical and drought samples; $\mathrm{mg} / \mathrm{L}$, milligrams per liter; $\mu \mathrm{S} / \mathrm{cm}$, microsiemens per centimeter at $25^{\circ} \mathrm{Celsius;}{ }^{\circ} \mathrm{C}$, degrees Celsius; E. coli, Escherichia coli; $\mathrm{mL}$, milliliter; $\mu \mathrm{g} / \mathrm{L}$, micrograms per liter; $\mathrm{CaCO}_{3}$, calcium carbonate; $\mathrm{SiO}_{2}$, silica; $\mathrm{SO}_{4}$, sulfate; ID, insufficient number of samples (less than 20) to calculate percentile; <, less than; --, no drought-sample measurement; values or concentrations in bold type indicate 2002 Colorado water-quality exceedances; c, chronic water-quality exceedance]

\begin{tabular}{|c|c|c|c|c|c|c|c|c|}
\hline \multirow{2}{*}{$\begin{array}{l}\text { Site number } \\
\text { in table } 1 \\
\text { and fig. } 4\end{array}$} & \multirow{2}{*}{ Sampling date } & \multicolumn{3}{|c|}{$\begin{array}{l}\text { Manganese, total recoverable, in } \\
\mu \mathrm{g} / \mathrm{L}\end{array}$} & \multirow{2}{*}{$\begin{array}{c}\text { Mercury, } \\
\text { dissolved, in } \\
\mu \mathrm{g} / \mathrm{L}\end{array}$} & \multicolumn{3}{|c|}{$\begin{array}{l}\text { Mercury, total recoverable, } \\
\text { in } \mu \mathrm{g} / \mathrm{L}\end{array}$} \\
\hline & & Conc & $0 \%$ & $\mathrm{~N}$ & & Conc & $0 \%$ & $\mathrm{~N}$ \\
\hline 20 & $8-1-2002$ & -- & $\overline{--}$ & -- & -- & -- & -- & -- \\
\hline \multirow[t]{2}{*}{21} & $7-15-2002$ & -- & -- & -- & -- & -- & -- & -- \\
\hline & $9-4-2002$ & -- & -- & -- & -- & -- & -- & -- \\
\hline \multirow[t]{2}{*}{22} & $7-15-2002$ & -- & -- & -- & -- & -- & -- & -- \\
\hline & $9-4-2002$ & -- & -- & -- & -- & -- & -- & -- \\
\hline \multirow[t]{2}{*}{23} & $7-15-2002$ & -- & -- & -- & -- & -- & -- & -- \\
\hline & $9-4-2002$ & -- & -- & -- & -- & -- & -- & -- \\
\hline \multirow[t]{2}{*}{24} & $7-15-2002$ & -- & -- & -- & -- & -- & -- & -- \\
\hline & $9-4-2002$ & -- & -- & -- & -- & -- & -- & -- \\
\hline \multirow[t]{2}{*}{25} & $7-15-2002$ & -- & -- & -- & -- & -- & -- & -- \\
\hline & $9-4-2002$ & -- & -- & -- & -- & -- & -- & -- \\
\hline \multirow[t]{2}{*}{26} & $7-17-2002$ & -- & -- & -- & -- & -- & -- & -- \\
\hline & $9-6-2002$ & -- & -- & -- & -- & -- & -- & -- \\
\hline 27 & 8-1-2002 & -- & -- & -- & $<0.01$ & $<0.01$ & ID & 8 \\
\hline \multirow[t]{2}{*}{28} & 8-8-2002 & -- & -- & -- & -- & -- & -- & -- \\
\hline & $9-11-2002$ & -- & -- & -- & -- & -- & -- & -- \\
\hline \multirow[t]{2}{*}{29} & 8-8-2002 & -- & -- & -- & -- & -- & -- & -- \\
\hline & $9-11-2002$ & -- & -- & -- & -- & -- & -- & -- \\
\hline \multirow[t]{2}{*}{30} & $7-29-2002$ & 88 & 93.1 & 28 & $<0.01$ & $<0.01$ & ID & 14 \\
\hline & $9-10-2002$ & 141 & 96.6 & 28 & -- & -- & -- & 14 \\
\hline \multirow[t]{2}{*}{31} & $7-29-2002$ & 102 & 81.8 & 21 & $<0.01$ & $<0.01$ & ID & 14 \\
\hline & $9-10-2002$ & 296 & 90.9 & 21 & -- & -- & -- & -- \\
\hline \multirow[t]{2}{*}{32} & 8-8-2002 & -- & -- & -- & -- & -- & -- & -- \\
\hline & $9-11-2002$ & -- & -- & -- & -- & -- & -- & -- \\
\hline 33 & $7-30-2002$ & -- & -- & -- & -- & -- & -- & -- \\
\hline 34 & $7-30-2002$ & 12.4 & ID & 6 & $<0.01$ & -- & -- & -- \\
\hline \multirow[t]{2}{*}{35} & $7-30-2002$ & 1.9 & ID & 5 & $<0.01$ & -- & -- & -- \\
\hline & $9-3-2002$ & -- & -- & -- & -- & -- & -- & -- \\
\hline \multirow[t]{2}{*}{36} & $7-30-2002$ & 33 & ID & 9 & $<0.01$ & -- & -- & -- \\
\hline & $9-3-2002$ & -- & -- & -- & -- & -- & -- & -- \\
\hline \multirow[t]{2}{*}{37} & $7-31-2002$ & -- & -- & -- & $<0.01$ & $<0.01$ & ${ }^{2} 4.8-90.5$ & 20 \\
\hline & $9-4-2002$ & -- & -- & -- & -- & -- & -- & -- \\
\hline
\end{tabular}


Table 12. Water-quality data for the Upper Colorado River Basin, showing 2002 drought values, Colorado water-quality exceedances, and percentiles relative to historical data for selected properties and constituents from July through September, 1978-2002.—Continued

[ft $3 / \mathrm{s}$, cubic feet per second; Conc, concentration; $\mathrm{Q} \%$, percentile (percentage of data less than or equal to reported value or concentration) of drought-sample constituent; $\mathrm{N}$, total number of historical and drought samples; $\mathrm{mg} / \mathrm{L}$, milligrams per liter; $\mu \mathrm{S} / \mathrm{cm}$, microsiemens per centimeter at $25^{\circ} \mathrm{Celsius;}{ }^{\circ} \mathrm{C}$, degrees Celsius; E. coli, Escherichia coli; $\mathrm{mL}$, milliliter; $\mu \mathrm{g} / \mathrm{L}$, micrograms per liter; $\mathrm{CaCO}_{3}$, calcium carbonate; $\mathrm{SiO}_{2}$, silica; $\mathrm{SO}_{4}$, sulfate; ID, insufficient number of samples (less than 20) to calculate percentile; <, less than; --, no drought-sample measurement; values or concentrations in bold type indicate 2002 Colorado water-quality exceedances; $c$, chronic water-quality exceedance]

\begin{tabular}{|c|c|c|c|c|c|c|c|c|}
\hline \multirow{2}{*}{$\begin{array}{l}\text { Site number } \\
\text { in table } 1 \\
\text { and fig. } 4\end{array}$} & \multirow{2}{*}{ Sampling date } & \multicolumn{3}{|c|}{$\begin{array}{l}\text { Manganese, total recoverable, in } \\
\qquad \mu \mathrm{g} / \mathrm{L}\end{array}$} & \multirow{2}{*}{$\begin{array}{c}\text { Mercury, } \\
\text { dissolved, in } \\
\mu \mathrm{g} / \mathrm{L}\end{array}$} & \multicolumn{3}{|c|}{$\begin{array}{c}\text { Mercury, total recoverable, } \\
\text { in } \mu \mathrm{g} / \mathrm{L}\end{array}$} \\
\hline & & Conc & $0 \%$ & $\mathrm{~N}$ & & Conc & $0 \%$ & $\mathrm{~N}$ \\
\hline \multirow[t]{2}{*}{38} & $8-1-2002$ & -- & -- & -- & -- & -- & -- & -- \\
\hline & $9-5-2002$ & -- & -- & -- & -- & -- & -- & -- \\
\hline \multirow[t]{2}{*}{39} & $8-1-2002$ & -- & -- & -- & -- & -- & -- & -- \\
\hline & $9-6-2002$ & -- & -- & -- & -- & -- & -- & -- \\
\hline \multirow[t]{2}{*}{40} & $7-29-2002$ & 2.7 & ID & 6 & $<0.01$ & -- & -- & -- \\
\hline & $9-3-2002$ & -- & -- & -- & -- & -- & -- & -- \\
\hline \multirow[t]{2}{*}{41} & $7-29-2002$ & 10.6 & ID & 5 & $<0.01$ & -- & -- & -- \\
\hline & $9-4-2002$ & -- & -- & -- & -- & -- & -- & -- \\
\hline \multirow[t]{2}{*}{42} & $7-30-2002$ & 7.8 & ID & 6 & $<0.01$ & -- & -- & -- \\
\hline & 9-4-2002 & -- & -- & -- & -- & -- & -- & -- \\
\hline \multirow[t]{2}{*}{43} & $7-30-2002$ & 3.5 & ID & 3 & $<0.01$ & -- & -- & -- \\
\hline & 9-4-2002 & -- & -- & -- & -- & -- & -- & -- \\
\hline \multirow[t]{2}{*}{44} & $7-30-2002$ & 8.9 & ID & 9 & $<0.01$ & -- & -- & -- \\
\hline & $9-5-2002$ & -- & -- & -- & -- & -- & -- & -- \\
\hline \multirow[t]{2}{*}{45} & $7-31-2002$ & -- & -- & -- & -- & -- & -- & -- \\
\hline & $9-6-2002$ & -- & -- & -- & -- & -- & -- & -- \\
\hline 46 & $8-2-2002$ & -- & -- & -- & -- & -- & -- & -- \\
\hline \multirow[t]{2}{*}{47} & $8-1-2002$ & -- & -- & -- & -- & -- & -- & -- \\
\hline & $9-3-2002$ & -- & -- & -- & -- & -- & -- & -- \\
\hline \multirow[t]{2}{*}{48} & $8-1-2002$ & -- & -- & -- & -- & -- & -- & -- \\
\hline & $9-5-2002$ & -- & -- & -- & -- & -- & -- & -- \\
\hline 49 & $7-31-2002$ & -- & -- & -- & -- & -- & -- & -- \\
\hline 50 & $7-31-2002$ & -- & -- & -- & -- & -- & -- & -- \\
\hline 51 & 8-1-2002 & -- & -- & -- & -- & -- & -- & -- \\
\hline \multirow[t]{2}{*}{52} & $8-1-2002$ & -- & -- & -- & -- & -- & -- & -- \\
\hline & $9-17-2002$ & -- & -- & -- & -- & -- & -- & -- \\
\hline 53 & $7-31-2002$ & -- & -- & -- & -- & -- & -- & -- \\
\hline 54 & 8-1-2002 & -- & -- & -- & -- & -- & -- & -- \\
\hline 55 & $7-30-2002$ & 20 & ID & 5 & $<0.01$ & -- & -- & -- \\
\hline \multirow[t]{2}{*}{56} & $7-31-2002$ & -- & -- & -- & -- & -- & -- & -- \\
\hline & $9-5-2002$ & -- & -- & -- & -- & -- & -- & -- \\
\hline \multirow{2}{*}{57} & $7-31-2002$ & 39 & ID & 3 & $<0.01$ & -- & -- & -- \\
\hline & $9-4-2002$ & -- & -- & -- & -- & -- & -- & -- \\
\hline \multirow[t]{2}{*}{58} & 7-16-2002 & -- & -- & -- & -- & -- & -- & -- \\
\hline & $9-5-2002$ & -- & -- & -- & -- & -- & -- & -- \\
\hline
\end{tabular}


Table 12. Water-quality data for the Upper Colorado River Basin, showing 2002 drought values, Colorado water-quality exceedances, and percentiles relative to historical data for selected properties and constituents from July through September, 1978-2002.—Continued

[ft $3 t^{3}$, cubic feet per second; Conc, concentration; Q\%, percentile (percentage of data less than or equal to reported value or concentration) of drought-sample constituent; N, total number of historical and drought samples; $\mathrm{mg} / \mathrm{L}$, milligrams per liter; $\mu \mathrm{S} / \mathrm{cm}$, microsiemens per centimeter at $25^{\circ} \mathrm{Celsius;}{ }^{\circ} \mathrm{C}$, degrees Celsius; E. coli, Escherichia coli; $\mathrm{mL}$, milliliter; $\mu \mathrm{g} / \mathrm{L}$, micrograms per liter; $\mathrm{CaCO}_{3}$, calcium carbonate; $\mathrm{SiO}_{2}$, silica; $\mathrm{SO}_{4}$, sulfate; ID, insufficient number of samples (less than 20) to calculate percentile; <, less than; --, no drought-sample measurement; values or concentrations in bold type indicate 2002 Colorado water-quality exceedances; c, chronic water-quality exceedance]

\begin{tabular}{|c|c|c|c|c|c|c|c|}
\hline \multirow{2}{*}{$\begin{array}{l}\text { Site number } \\
\text { in table } 1 \\
\text { and fig. } 4\end{array}$} & \multirow{2}{*}{ Sampling date } & \multicolumn{3}{|c|}{$\begin{array}{l}\text { Molybdenum, dissolved, } \\
\text { in } \mu \mathrm{g} / \mathrm{L}\end{array}$} & \multicolumn{3}{|c|}{ Nickel, dissolved, in $\mu \mathrm{g} / \mathrm{L}$} \\
\hline & & Conc & $0 \%$ & $\mathrm{~N}$ & Conc & $0 \%$ & $\mathrm{~N}$ \\
\hline 20 & $8-1-2002$ & -- & -- & -- & -- & -- & $\overline{--}$ \\
\hline \multirow[t]{2}{*}{21} & $7-15-2002$ & -- & -- & -- & -- & -- & -- \\
\hline & $9-4-2002$ & -- & -- & -- & -- & -- & -- \\
\hline \multirow[t]{2}{*}{22} & $7-15-2002$ & -- & -- & -- & -- & -- & -- \\
\hline & $9-4-2002$ & -- & -- & -- & -- & -- & -- \\
\hline \multirow[t]{2}{*}{23} & $7-15-2002$ & -- & -- & -- & -- & -- & -- \\
\hline & $9-4-2002$ & -- & -- & -- & -- & -- & -- \\
\hline \multirow[t]{2}{*}{24} & $7-15-2002$ & -- & -- & -- & -- & -- & -- \\
\hline & $9-4-2002$ & -- & -- & -- & -- & -- & -- \\
\hline \multirow[t]{2}{*}{25} & $7-15-2002$ & -- & -- & -- & -- & -- & -- \\
\hline & $9-4-2002$ & -- & -- & -- & -- & -- & -- \\
\hline \multirow[t]{2}{*}{26} & $7-17-2002$ & -- & -- & -- & -- & -- & -- \\
\hline & $9-6-2002$ & -- & -- & -- & -- & -- & -- \\
\hline 27 & 8-1-2002 & -- & -- & -- & 0.23 & ID & 8 \\
\hline \multirow[t]{2}{*}{28} & 8-8-2002 & -- & -- & -- & -- & -- & -- \\
\hline & $9-11-2002$ & -- & -- & -- & -- & -- & -- \\
\hline \multirow[t]{2}{*}{29} & 8-8-2002 & -- & -- & -- & -- & -- & -- \\
\hline & $9-11-2002$ & -- & -- & -- & -- & -- & -- \\
\hline \multirow[t]{2}{*}{30} & $7-29-2002$ & 3.6 & ID & 14 & 3.6 & ID & 14 \\
\hline & $9-10-2002$ & -- & -- & -- & -- & -- & -- \\
\hline \multirow[t]{2}{*}{31} & $7-29-2002$ & 3.0 & ID & 14 & 2.8 & ID & 14 \\
\hline & $9-10-2002$ & -- & -- & -- & -- & -- & -- \\
\hline \multirow[t]{2}{*}{32} & $8-8-2002$ & -- & -- & -- & -- & -- & -- \\
\hline & $9-11-2002$ & -- & -- & -- & -- & -- & -- \\
\hline 33 & $7-30-2002$ & 24 & 63.6 & 21 & 0.25 & ${ }^{2} 4.5-90.9$ & 21 \\
\hline 34 & $7-30-2002$ & -- & -- & -- & -- & -- & -- \\
\hline \multirow[t]{2}{*}{35} & $7-30-2002$ & -- & -- & -- & $<0.06$ & ID & 5 \\
\hline & $9-3-2002$ & -- & -- & -- & -- & -- & -- \\
\hline \multirow[t]{2}{*}{36} & $7-30-2002$ & -- & -- & -- & -- & -- & -- \\
\hline & $9-3-2002$ & -- & -- & -- & -- & -- & -- \\
\hline \multirow[t]{2}{*}{37} & $7-31-2002$ & -- & -- & -- & $<2$ & ID & 18 \\
\hline & $9-4-2002$ & -- & -- & -- & -- & -- & -- \\
\hline
\end{tabular}


Table 12. Water-quality data for the Upper Colorado River Basin, showing 2002 drought values, Colorado water-quality exceedances, and percentiles relative to historical data for selected properties and constituents from July through September, 1978-2002.—Continued

[ft $3 / \mathrm{s}$, cubic feet per second; Conc, concentration; $\mathrm{Q} \%$, percentile (percentage of data less than or equal to reported value or concentration) of drought-sample constituent; $\mathrm{N}$, total number of historical and drought samples; $\mathrm{mg} / \mathrm{L}$, milligrams per liter; $\mu \mathrm{S} / \mathrm{cm}$, microsiemens per centimeter at $25^{\circ} \mathrm{Celsius;}{ }^{\circ} \mathrm{C}$, degrees Celsius; E. coli, Escherichia coli; $\mathrm{mL}$, milliliter; $\mu \mathrm{g} / \mathrm{L}$, micrograms per liter; $\mathrm{CaCO}_{3}$, calcium carbonate; $\mathrm{SiO}_{2}$, silica; $\mathrm{SO}_{4}$, sulfate; ID, insufficient number of samples (less than 20) to calculate percentile; <, less than; --, no drought-sample measurement; values or concentrations in bold type indicate 2002 Colorado water-quality exceedances; $c$, chronic water-quality exceedance]

\begin{tabular}{|c|c|c|c|c|c|c|c|}
\hline \multirow{2}{*}{$\begin{array}{l}\text { Site number } \\
\text { in table } 1 \\
\text { and fig. } 4\end{array}$} & \multirow{2}{*}{ Sampling date } & \multicolumn{3}{|c|}{$\begin{array}{l}\text { Molybdenum, dissolved, } \\
\text { in } \mu \mathrm{g} / \mathrm{L}\end{array}$} & \multicolumn{3}{|c|}{ Nickel, dissolved, in $\mu \mathrm{g} / \mathrm{L}$} \\
\hline & & Conc & $0 \%$ & $\mathrm{~N}$ & Conc & $0 \%$ & $\mathrm{~N}$ \\
\hline \multirow[t]{2}{*}{38} & $8-1-2002$ & -- & -- & -- & -- & -- & -- \\
\hline & $9-5-2002$ & -- & -- & -- & -- & -- & - \\
\hline \multirow[t]{2}{*}{39} & $8-1-2002$ & -- & -- & -- & -- & -- & -- \\
\hline & $9-6-2002$ & -- & -- & -- & -- & -- & -- \\
\hline \multirow[t]{2}{*}{40} & $7-29-2002$ & -- & -- & -- & -- & -- & -- \\
\hline & $9-3-2002$ & -- & -- & -- & -- & -- & -- \\
\hline \multirow[t]{2}{*}{41} & $7-29-2002$ & -- & -- & -- & -- & -- & -- \\
\hline & $9-4-2002$ & -- & -- & -- & -- & -- & -- \\
\hline \multirow[t]{2}{*}{42} & $7-30-2002$ & -- & -- & -- & -- & -- & -- \\
\hline & $9-4-2002$ & -- & -- & -- & -- & -- & -- \\
\hline \multirow[t]{2}{*}{43} & $7-30-2002$ & -- & -- & -- & -- & -- & -- \\
\hline & $9-4-2002$ & -- & -- & -- & -- & -- & -- \\
\hline \multirow[t]{2}{*}{44} & $7-30-2002$ & -- & -- & -- & -- & -- & -- \\
\hline & $9-5-2002$ & -- & -- & -- & -- & -- & -- \\
\hline \multirow[t]{2}{*}{45} & $7-31-2002$ & -- & -- & -- & -- & -- & -- \\
\hline & $9-6-2002$ & -- & -- & -- & -- & -- & -- \\
\hline 46 & $8-2-2002$ & -- & -- & -- & -- & -- & -- \\
\hline \multirow[t]{2}{*}{47} & $8-1-2002$ & -- & -- & -- & -- & -- & -- \\
\hline & $9-3-2002$ & -- & -- & -- & -- & -- & - \\
\hline \multirow[t]{2}{*}{48} & $8-1-2002$ & -- & -- & -- & -- & -- & -- \\
\hline & $9-5-2002$ & -- & -- & -- & -- & -- & - \\
\hline 49 & $7-31-2002$ & -- & -- & -- & -- & -- & - \\
\hline 50 & $7-31-2002$ & -- & -- & -- & -- & -- & -- \\
\hline 51 & 8-1-2002 & -- & -- & -- & -- & -- & -- \\
\hline \multirow[t]{2}{*}{52} & 8-1-2002 & -- & -- & -- & -- & -- & - \\
\hline & $9-17-2002$ & -- & -- & -- & -- & -- & - \\
\hline 53 & $7-31-2002$ & -- & -- & -- & -- & -- & - \\
\hline 54 & $8-1-2002$ & -- & -- & -- & -- & -- & - \\
\hline 55 & $7-30-2002$ & -- & -- & -- & -- & -- & - \\
\hline \multirow[t]{2}{*}{56} & $7-31-2002$ & -- & -- & -- & -- & -- & - \\
\hline & $9-5-2002$ & -- & -- & -- & -- & -- & - \\
\hline \multirow[t]{2}{*}{57} & $7-31-2002$ & -- & -- & -- & 0.33 & ID & 3 \\
\hline & $9-4-2002$ & -- & -- & -- & -- & -- & - \\
\hline \multirow[t]{2}{*}{58} & $7-16-2002$ & -- & -- & -- & -- & -- & - \\
\hline & $9-5-2002$ & -- & -- & -- & -- & -- & - \\
\hline
\end{tabular}


Table 12. Water-quality data for the Upper Colorado River Basin, showing 2002 drought values, Colorado water-quality exceedances, and percentiles relative to historical data for selected properties and constituents from July through September, 1978-2002.-Continued

[ft $3 / \mathrm{s}$, cubic feet per second; Conc, concentration; $\mathrm{Q} \%$, percentile (percentage of data less than or equal to reported value or concentration) of drought-sample constituent; $\mathrm{N}$, total number of historical and drought samples; $\mathrm{mg} / \mathrm{L}$, milligrams per liter; $\mu \mathrm{S} / \mathrm{cm}$, microsiemens per centimeter at $25^{\circ} \mathrm{Celsius;}{ }^{\circ} \mathrm{C}$, degrees Celsius; E. coli, Escherichia coli; $\mathrm{mL}$, milliliter; $\mu \mathrm{g} / \mathrm{L}$, micrograms per liter; $\mathrm{CaCO}_{3}$, calcium carbonate; $\mathrm{SiO}_{2}$, silica; $\mathrm{SO}_{4}$, sulfate; ID, insufficient number of samples (less than 20) to calculate percentile; <, less than; --, no drought-sample measurement; values or concentrations in bold type indicate 2002 Colorado water-quality exceedances; c, chronic water-quality exceedance]

\begin{tabular}{|c|c|c|c|c|c|c|c|}
\hline \multirow{2}{*}{$\begin{array}{l}\text { Site number } \\
\text { in table } 1 \\
\text { and fig. } 4\end{array}$} & \multirow{2}{*}{ Sampling date } & \multicolumn{3}{|c|}{$\begin{array}{l}\text { Nickel, total recoverable, } \\
\text { in } \mu \mathrm{g} / \mathrm{L}\end{array}$} & \multicolumn{3}{|c|}{ Selenium, dissolved, in $\mu \mathrm{g} / \mathrm{L}$} \\
\hline & & Conc & $0 \%$ & $\mathrm{~N}$ & Conc & $0 \%$ & $\mathrm{~N}$ \\
\hline 20 & $8-1-2002$ & -- & -- & -- & -- & -- & $\overline{--}$ \\
\hline \multirow[t]{2}{*}{21} & $7-15-2002$ & -- & -- & -- & -- & -- & -- \\
\hline & $9-4-2002$ & -- & -- & -- & -- & -- & -- \\
\hline \multirow[t]{2}{*}{22} & $7-15-2002$ & -- & -- & -- & -- & -- & -- \\
\hline & $9-4-2002$ & -- & -- & -- & -- & -- & -- \\
\hline \multirow[t]{2}{*}{23} & $7-15-2002$ & -- & -- & -- & -- & -- & -- \\
\hline & $9-4-2002$ & -- & -- & -- & -- & -- & -- \\
\hline \multirow[t]{2}{*}{24} & $7-15-2002$ & -- & -- & -- & -- & -- & -- \\
\hline & $9-4-2002$ & -- & -- & -- & -- & -- & -- \\
\hline \multirow[t]{2}{*}{25} & $7-15-2002$ & -- & -- & -- & -- & -- & -- \\
\hline & $9-4-2002$ & -- & -- & -- & -- & -- & -- \\
\hline \multirow[t]{2}{*}{26} & $7-17-2002$ & -- & -- & -- & -- & -- & -- \\
\hline & $9-6-2002$ & -- & -- & -- & -- & -- & -- \\
\hline 27 & $8-1-2002$ & $<1$ & ID & 8 & $<0.3$ & ID & 8 \\
\hline \multirow[t]{2}{*}{28} & 8-8-2002 & -- & -- & -- & -- & -- & -- \\
\hline & $9-11-2002$ & -- & -- & -- & -- & -- & -- \\
\hline \multirow[t]{2}{*}{29} & 8-8-2002 & -- & -- & -- & -- & -- & -- \\
\hline & $9-11-2002$ & -- & -- & -- & -- & -- & -- \\
\hline \multirow[t]{2}{*}{30} & $7-29-2002$ & 6 & ID & 14 & $<2$ & ID & 14 \\
\hline & $9-10-2002$ & -- & -- & -- & -- & -- & -- \\
\hline \multirow[t]{2}{*}{31} & $7-29-2002$ & 5 & ID & 14 & 2 & ID & 14 \\
\hline & $9-10-2002$ & -- & -- & -- & -- & -- & -- \\
\hline \multirow[t]{2}{*}{32} & 8-8-2002 & -- & -- & -- & -- & -- & -- \\
\hline & $9-11-2002$ & -- & -- & -- & -- & -- & -- \\
\hline 33 & $7-30-2002$ & -- & -- & -- & -- & -- & -- \\
\hline 34 & $7-30-2002$ & -- & -- & -- & $<2$ & ID & 6 \\
\hline \multirow[t]{2}{*}{35} & $7-30-2002$ & -- & -- & -- & $<0.3$ & ID & 5 \\
\hline & $9-3-2002$ & -- & -- & -- & -- & -- & -- \\
\hline \multirow[t]{2}{*}{36} & $7-30-2002$ & -- & -- & -- & $<2$ & ID & 9 \\
\hline & $9-3-2002$ & -- & -- & -- & -- & -- & -- \\
\hline \multirow[t]{2}{*}{37} & $7-31-2002$ & 1.1 & ID & 18 & $<2$ & ${ }^{2} 4.5-95.5$ & 21 \\
\hline & $9-4-2002$ & -- & -- & -- & -- & -- & -- \\
\hline
\end{tabular}


Table 12. Water-quality data for the Upper Colorado River Basin, showing 2002 drought values, Colorado water-quality exceedances, and percentiles relative to historical data for selected properties and constituents from July through September, 1978-2002.—Continued

[ft $\mathrm{t}^{3} / \mathrm{s}$, cubic feet per second; Conc, concentration; $\mathrm{Q} \%$, percentile (percentage of data less than or equal to reported value or concentration) of drought-sample constituent; N, total number of historical and drought samples; $\mathrm{mg} / \mathrm{L}$, milligrams per liter; $\mu \mathrm{S} / \mathrm{cm}$, microsiemens per centimeter at $25^{\circ} \mathrm{Celsius;}{ }^{\circ} \mathrm{C}$, degrees Celsius; E. coli, Escherichia coli; $\mathrm{mL}$, milliliter; $\mu \mathrm{g} / \mathrm{L}$, micrograms per liter; $\mathrm{CaCO}_{3}$, calcium carbonate; $\mathrm{SiO}_{2}$, silica; $\mathrm{SO}_{4}$, sulfate; $\mathrm{ID}$, insufficient number of samples (less than 20) to calculate percentile; <, less than; --, no drought-sample measurement; values or concentrations in bold type indicate 2002 Colorado water-quality exceedances; $\mathrm{c}$, chronic water-quality exceedance]

\begin{tabular}{|c|c|c|c|c|c|c|c|}
\hline \multirow{2}{*}{$\begin{array}{l}\text { Site number } \\
\text { in table } 1 \\
\text { and fig. } 4\end{array}$} & \multirow{2}{*}{ Sampling date } & \multicolumn{3}{|c|}{$\begin{array}{l}\text { Nickel, total recoverable, } \\
\text { in } \mu \mathrm{g} / \mathrm{L}\end{array}$} & \multicolumn{3}{|c|}{ Selenium, dissolved, in $\mu \mathrm{g} / \mathrm{L}$} \\
\hline & & Conc & $0 \%$ & $\mathrm{~N}$ & Conc & $0 \%$ & $\mathrm{~N}$ \\
\hline \multirow[t]{2}{*}{38} & $8-1-2002$ & -- & -- & -- & -- & -- & -- \\
\hline & $9-5-2002$ & -- & -- & -- & -- & -- & -- \\
\hline \multirow[t]{2}{*}{39} & $8-1-2002$ & -- & -- & -- & -- & -- & -- \\
\hline & $9-6-2002$ & -- & -- & -- & -- & -- & -- \\
\hline \multirow[t]{2}{*}{40} & $7-29-2002$ & -- & -- & -- & 2 & ID & 6 \\
\hline & $9-3-2002$ & -- & -- & -- & -- & -- & -- \\
\hline \multirow[t]{2}{*}{41} & $7-29-2002$ & -- & -- & -- & $<2$ & ID & 5 \\
\hline & $9-4-2002$ & -- & -- & -- & -- & -- & -- \\
\hline \multirow[t]{2}{*}{42} & $7-30-2002$ & -- & -- & -- & $<2$ & ID & 6 \\
\hline & $9-4-2002$ & -- & -- & -- & -- & -- & -- \\
\hline \multirow[t]{2}{*}{43} & $7-30-2002$ & -- & -- & -- & $<2$ & ID & 3 \\
\hline & $9-4-2002$ & -- & -- & -- & -- & -- & -- \\
\hline \multirow[t]{2}{*}{44} & $7-30-2002$ & -- & -- & -- & $<2$ & ID & 9 \\
\hline & $9-5-2002$ & -- & -- & -- & -- & -- & -- \\
\hline \multirow[t]{2}{*}{45} & $7-31-2002$ & -- & -- & -- & -- & -- & -- \\
\hline & $9-6-2002$ & -- & -- & -- & -- & -- & -- \\
\hline 46 & $8-2-2002$ & -- & -- & -- & -- & -- & -- \\
\hline \multirow[t]{2}{*}{47} & $8-1-2002$ & -- & -- & -- & 0.4 & ID & 19 \\
\hline & $9-3-2002$ & -- & -- & -- & 0.8 & ID & 19 \\
\hline \multirow[t]{2}{*}{48} & $8-1-2002$ & -- & -- & -- & 0.6 & ID & 7 \\
\hline & $9-5-2002$ & -- & -- & -- & -- & -- & -- \\
\hline 49 & $7-31-2002$ & -- & -- & -- & 0.6 & ID & 9 \\
\hline 50 & $7-31-2002$ & -- & -- & -- & 3.8 & ID & 7 \\
\hline 51 & 8-1-2002 & -- & -- & -- & $6.3 \mathrm{c}$ & ID & 7 \\
\hline \multirow[t]{2}{*}{52} & 8-1-2002 & -- & -- & -- & $8.8 \mathrm{c}$ & 83.3 & 47 \\
\hline & $9-17-2002$ & -- & -- & -- & $11 \mathrm{c}$ & 95.8 & 47 \\
\hline 53 & $7-31-2002$ & -- & -- & -- & 2.6 & ID & 4 \\
\hline 54 & $8-1-2002$ & -- & -- & -- & $5.8 \mathrm{c}$ & ID & 7 \\
\hline 55 & $7-30-2002$ & -- & -- & -- & $<2$ & ID & 5 \\
\hline \multirow[t]{2}{*}{56} & $7-31-2002$ & -- & -- & -- & -- & -- & -- \\
\hline & $9-5-2002$ & -- & -- & -- & -- & -- & -- \\
\hline \multirow[t]{2}{*}{57} & $7-31-2002$ & -- & -- & -- & 0.4 & ID & 3 \\
\hline & $9-4-2002$ & -- & -- & -- & -- & -- & -- \\
\hline \multirow[t]{2}{*}{58} & $7-16-2002$ & -- & -- & -- & -- & -- & -- \\
\hline & $9-5-2002$ & -- & -- & -- & -- & -- & -- \\
\hline
\end{tabular}


Table 12. Water-quality data for the Upper Colorado River Basin, showing 2002 drought values, Colorado water-quality exceedances, and percentiles relative to historical data for selected properties and constituents from July through September, 1978-2002.-Continued

[ft $3 / \mathrm{s}$, cubic feet per second; Conc, concentration; Q\%, percentile (percentage of data less than or equal to reported value or concentration) of drought-sample constituent; N, total number of historical and drought samples; mg/L, milligrams per liter; $\mu \mathrm{S} / \mathrm{cm}$, microsiemens per centimeter at $25^{\circ} \mathrm{Celsius;}{ }^{\circ} \mathrm{C}$, degrees Celsius; E. coli, Escherichia coli; $\mathrm{mL}$, milliliter; $\mu \mathrm{g} / \mathrm{L}$, micrograms per liter; $\mathrm{CaCO}_{3}$, calcium carbonate; $\mathrm{SiO}_{2}$, silica; $\mathrm{SO}_{4}$, sulfate; ID, insufficient number of samples (less than 20) to calculate percentile; <, less than; --, no drought-sample measurement; values or concentrations in bold type indicate 2002 Colorado water-quality exceedances; c, chronic water-quality exceedance]

\begin{tabular}{|c|c|c|c|c|c|c|c|c|}
\hline \multirow{2}{*}{$\begin{array}{l}\text { Site number } \\
\text { in table } 1 \\
\text { and fig. } 4\end{array}$} & \multirow{2}{*}{ Sampling date } & \multicolumn{3}{|c|}{$\begin{array}{l}\text { Selenium, total recoverable, in } \\
\qquad \mu \mathrm{g} / \mathrm{L}\end{array}$} & \multicolumn{3}{|c|}{ Silver, dissolved, in $\mu \mathrm{g} / \mathrm{L}$} & \multirow{2}{*}{$\begin{array}{c}\text { Silver, } \\
\text { total } \\
\text { recoverable, } \\
\text { in } \mu \mathrm{g} / \mathrm{L}\end{array}$} \\
\hline & & Conc & $0 \%$ & $\mathrm{~N}$ & Conc & $0 \%$ & $\mathrm{~N}$ & \\
\hline 20 & $8-1-2002$ & $\overline{--}$ & $\overline{--}$ & -- & -- & $\overline{--}$ & -- & -- \\
\hline \multirow[t]{2}{*}{21} & $7-15-2002$ & -- & -- & -- & -- & -- & -- & -- \\
\hline & $9-4-2002$ & -- & -- & -- & -- & -- & -- & -- \\
\hline \multirow[t]{2}{*}{22} & $7-15-2002$ & -- & -- & -- & -- & -- & -- & -- \\
\hline & $9-4-2002$ & -- & -- & -- & -- & -- & -- & -- \\
\hline \multirow[t]{2}{*}{23} & $7-15-2002$ & -- & -- & -- & -- & -- & -- & -- \\
\hline & $9-4-2002$ & -- & -- & -- & -- & -- & -- & -- \\
\hline \multirow[t]{2}{*}{24} & $7-15-2002$ & -- & -- & -- & -- & -- & -- & -- \\
\hline & $9-4-2002$ & -- & -- & -- & -- & -- & -- & -- \\
\hline \multirow[t]{2}{*}{25} & $7-15-2002$ & -- & -- & -- & -- & -- & -- & -- \\
\hline & $9-4-2002$ & -- & -- & -- & -- & -- & -- & -- \\
\hline \multirow[t]{2}{*}{26} & $7-17-2002$ & -- & -- & -- & -- & -- & -- & -- \\
\hline & 9-6-2002 & -- & -- & -- & -- & -- & -- & -- \\
\hline 27 & $8-1-2002$ & $<0.4$ & ID & 8 & $<1$ & ID & 8 & $<0.05$ \\
\hline \multirow[t]{2}{*}{28} & 8-8-2002 & -- & -- & -- & $<1$ & ID & 2 & -- \\
\hline & $9-11-2002$ & -- & -- & -- & $<1$ & ID & 2 & -- \\
\hline \multirow[t]{2}{*}{29} & $8-8-2002$ & -- & -- & -- & -- & -- & -- & -- \\
\hline & $9-11-2002$ & -- & -- & -- & -- & -- & -- & -- \\
\hline \multirow[t]{2}{*}{30} & 7-29-2002 & 0.7 & ID & 13 & $<0.1$ & ID & 14 & $<0.05$ \\
\hline & $9-10-2002$ & -- & -- & -- & -- & -- & -- & -- \\
\hline \multirow[t]{2}{*}{31} & $7-29-2002$ & 2.7 & ID & 12 & $<0.1$ & ID & 14 & $<0.05$ \\
\hline & $9-10-2002$ & -- & -- & -- & -- & -- & -- & -- \\
\hline \multirow[t]{2}{*}{32} & 8-8-2002 & -- & -- & -- & $<1$ & ID & 2 & -- \\
\hline & $9-11-2002$ & -- & -- & -- & $<1$ & ID & 2 & -- \\
\hline 33 & $7-30-2002$ & -- & -- & -- & $<1$ & ${ }^{2} 4.5-90.9$ & 21 & -- \\
\hline 34 & $7-30-2002$ & -- & -- & -- & $<0.1$ & ID & 6 & -- \\
\hline \multirow[t]{2}{*}{35} & $7-30-2002$ & -- & -- & -- & $<1$ & ID & 5 & -- \\
\hline & $9-3-2002$ & -- & -- & -- & -- & -- & -- & -- \\
\hline \multirow[t]{2}{*}{36} & $7-30-2002$ & -- & -- & -- & $<0.1$ & ID & 9 & -- \\
\hline & $9-3-2002$ & -- & -- & -- & -- & -- & -- & -- \\
\hline \multirow[t]{2}{*}{37} & $7-31-2002$ & 1.0 & ${ }^{2} 4.5-95.5$ & 21 & $<0.1$ & ID & 19 & $<0.3$ \\
\hline & $9-4-2002$ & -- & -- & -- & $<1$ & ID & 19 & -- \\
\hline
\end{tabular}


Table 12. Water-quality data for the Upper Colorado River Basin, showing 2002 drought values, Colorado water-quality exceedances, and percentiles relative to historical data for selected properties and constituents from July through September, 1978-2002.—Continued

$\left[\mathrm{ft}^{3} / \mathrm{s}\right.$, cubic feet per second; Conc, concentration; Q\%, percentile (percentage of data less than or equal to reported value or concentration) of drought-sample constituent; N, total number of historical and drought samples; $\mathrm{mg} / \mathrm{L}$, milligrams per liter; $\mu \mathrm{S} / \mathrm{cm}$, microsiemens per centimeter at $25^{\circ} \mathrm{Celsius;}{ }^{\circ} \mathrm{C}$, degrees Celsius; E. coli, Escherichia coli; $\mathrm{mL}$, milliliter; $\mu \mathrm{g} / \mathrm{L}$, micrograms per liter; $\mathrm{CaCO}_{3}$, calcium carbonate; $\mathrm{SiO}_{2}$, silica; $\mathrm{SO}_{4}$, sulfate; ID, insufficient number of samples (less than 20) to calculate percentile; <, less than; --, no drought-sample measurement; values or concentrations in bold type indicate 2002 Colorado water-quality exceedances; c, chronic water-quality exceedance]

\begin{tabular}{|c|c|c|c|c|c|c|c|c|}
\hline \multirow{2}{*}{$\begin{array}{l}\text { Site number } \\
\text { in table } 1 \\
\text { and fig. } 4\end{array}$} & \multirow{2}{*}{ Sampling date } & \multicolumn{3}{|c|}{$\begin{array}{l}\text { Selenium, total recoverable, in } \\
\qquad \mu \mathrm{g} / \mathrm{L}\end{array}$} & \multicolumn{3}{|c|}{ Silver, dissolved, in $\mu \mathrm{g} / \mathrm{L}$} & \multirow{2}{*}{$\begin{array}{c}\text { Silver, } \\
\text { total } \\
\text { recoverable, } \\
\text { in } \boldsymbol{\mu g} / \mathrm{L}\end{array}$} \\
\hline & & Conc & $0 \%$ & $\mathrm{~N}$ & Conc & $0 \%$ & $\mathrm{~N}$ & \\
\hline \multirow[t]{2}{*}{38} & $8-1-2002$ & -- & -- & -- & -- & -- & -- & -- \\
\hline & $9-5-2002$ & -- & -- & -- & -- & -- & -- & -- \\
\hline \multirow[t]{2}{*}{39} & $8-1-2002$ & -- & -- & -- & -- & -- & -- & -- \\
\hline & $9-6-2002$ & -- & -- & -- & -- & -- & -- & -- \\
\hline \multirow[t]{2}{*}{40} & $7-29-2002$ & -- & -- & -- & $<0.1$ & ID & 6 & -- \\
\hline & $9-3-2002$ & -- & -- & -- & -- & -- & -- & -- \\
\hline \multirow[t]{2}{*}{41} & $7-29-2002$ & -- & -- & -- & $<0.1$ & ID & 5 & -- \\
\hline & $9-4-2002$ & -- & -- & -- & -- & -- & -- & -- \\
\hline \multirow[t]{2}{*}{42} & $7-30-2002$ & -- & -- & -- & $<0.1$ & ID & 6 & -- \\
\hline & $9-4-2002$ & -- & -- & -- & -- & -- & -- & -- \\
\hline \multirow[t]{2}{*}{43} & $7-30-2002$ & -- & -- & -- & $<0.1$ & ID & 3 & -- \\
\hline & $9-4-2002$ & -- & -- & -- & -- & -- & -- & -- \\
\hline \multirow[t]{2}{*}{44} & $7-30-2002$ & -- & -- & -- & $<0.1$ & ID & 10 & -- \\
\hline & $9-5-2002$ & -- & -- & -- & $<1$ & ID & 10 & -- \\
\hline \multirow[t]{2}{*}{45} & $7-31-2002$ & -- & -- & -- & $<1$ & ID & 2 & -- \\
\hline & $9-6-2002$ & -- & -- & -- & $<1$ & ID & 2 & -- \\
\hline 46 & $8-2-2002$ & -- & -- & -- & -- & -- & -- & -- \\
\hline \multirow[t]{2}{*}{47} & $8-1-2002$ & -- & -- & -- & -- & -- & -- & -- \\
\hline & $9-3-2002$ & -- & -- & -- & -- & -- & -- & -- \\
\hline \multirow[t]{2}{*}{48} & $8-1-2002$ & -- & -- & -- & -- & -- & -- & -- \\
\hline & $9-5-2002$ & -- & -- & -- & -- & -- & -- & -- \\
\hline 49 & $7-31-2002$ & -- & -- & -- & -- & -- & -- & -- \\
\hline 50 & $7-31-2002$ & -- & -- & -- & -- & -- & -- & -- \\
\hline 51 & $8-1-2002$ & -- & -- & -- & -- & -- & -- & -- \\
\hline \multirow[t]{2}{*}{52} & $8-1-2002$ & -- & -- & -- & $<1$ & ${ }^{2} 4.3-87.0$ & 22 & -- \\
\hline & $9-17-2002$ & -- & -- & -- & $<1$ & ${ }^{2} 4.3-87.0$ & 22 & -- \\
\hline 53 & $7-31-2002$ & -- & -- & -- & -- & -- & -- & -- \\
\hline 54 & $8-1-2002$ & -- & -- & -- & -- & -- & -- & -- \\
\hline 55 & $7-30-2002$ & -- & -- & -- & $<0.1$ & ID & 5 & -- \\
\hline \multirow[t]{2}{*}{56} & $7-31-2002$ & -- & -- & -- & -- & -- & -- & -- \\
\hline & $9-5-2002$ & -- & -- & -- & -- & -- & -- & -- \\
\hline \multirow[t]{2}{*}{57} & $7-31-2002$ & -- & -- & -- & $<1$ & ID & 3 & -- \\
\hline & $9-4-2002$ & -- & -- & -- & -- & -- & -- & -- \\
\hline \multirow[t]{2}{*}{58} & $7-16-2002$ & -- & -- & -- & -- & -- & -- & -- \\
\hline & $9-5-2002$ & -- & -- & -- & -- & -- & -- & -- \\
\hline
\end{tabular}


Table 12. Water-quality data for the Upper Colorado River Basin, showing 2002 drought values, Colorado water-quality exceedances, and percentiles relative to historical data for selected properties and constituents from July through September, 1978-2002.-Continued

$\left[\mathrm{ft}^{3} / \mathrm{s}\right.$, cubic feet per second; Conc, concentration; Q\%, percentile (percentage of data less than or equal to reported value or concentration) of drought-sample constituent; $\mathrm{N}$, total number of historical and drought samples; $\mathrm{mg} / \mathrm{L}$, milligrams per liter; $\mu \mathrm{S} / \mathrm{cm}$, microsiemens per centimeter at $25^{\circ} \mathrm{Celsius;}{ }^{\circ} \mathrm{C}$, degrees Celsius; E. coli, Escherichia coli; $\mathrm{mL}$, milliliter; $\mu \mathrm{g} / \mathrm{L}$, micrograms per liter; $\mathrm{CaCO}_{3}$, calcium carbonate; $\mathrm{SiO}_{2}$, silica; $\mathrm{SO}_{4}$, sulfate; ID, insufficient number of samples (less than 20) to calculate percentile; <, less than; --, no drought-sample measurement; values or concentrations in bold type indicate 2002 Colorado water-quality exceedances; c, chronic water-quality exceedance]

\begin{tabular}{|c|c|c|c|c|c|c|c|}
\hline \multirow{2}{*}{$\begin{array}{l}\text { Site number } \\
\text { in table } 1 \\
\text { and fig. } 4\end{array}$} & \multirow{2}{*}{ Sampling date } & \multicolumn{3}{|c|}{ Zinc, dissolved, in $\mu \mathrm{g} / \mathrm{L}$} & \multicolumn{3}{|c|}{$\begin{array}{l}\text { Zinc, total recoverable, } \\
\text { in } \mu \mathrm{g} / \mathrm{L}\end{array}$} \\
\hline & & Conc & $0 \%$ & $\mathrm{~N}$ & Conc & $0 \%$ & $\mathrm{~N}$ \\
\hline 20 & $8-1-2002$ & -- & -- & -- & -- & -- & -- \\
\hline \multirow[t]{2}{*}{21} & $7-15-2002$ & -- & -- & -- & -- & -- & -- \\
\hline & $9-4-2002$ & -- & -- & -- & -- & -- & -- \\
\hline \multirow[t]{2}{*}{22} & $7-15-2002$ & -- & -- & -- & -- & -- & -- \\
\hline & $9-4-2002$ & -- & -- & -- & -- & -- & -- \\
\hline \multirow[t]{2}{*}{23} & $7-15-2002$ & -- & -- & -- & -- & -- & -- \\
\hline & $9-4-2002$ & -- & -- & -- & -- & -- & -- \\
\hline \multirow[t]{2}{*}{24} & $7-15-2002$ & -- & -- & -- & -- & -- & -- \\
\hline & $9-4-2002$ & -- & -- & -- & -- & -- & -- \\
\hline \multirow[t]{2}{*}{25} & $7-15-2002$ & -- & -- & -- & -- & -- & -- \\
\hline & $9-4-2002$ & -- & -- & -- & -- & -- & -- \\
\hline \multirow[t]{2}{*}{26} & $7-17-2002$ & -- & -- & -- & -- & -- & -- \\
\hline & $9-6-2002$ & -- & -- & -- & -- & -- & -- \\
\hline 27 & 8-1-2002 & 2 & ID & 8 & 4 & ID & 8 \\
\hline \multirow[t]{2}{*}{28} & $8-8-2002$ & 5 & ID & 2 & -- & -- & -- \\
\hline & $9-11-2002$ & 2 & ID & 2 & -- & -- & -- \\
\hline \multirow[t]{2}{*}{29} & 8-8-2002 & -- & -- & -- & -- & -- & -- \\
\hline & $9-11-2002$ & -- & -- & -- & -- & -- & -- \\
\hline \multirow[t]{2}{*}{30} & $7-29-2002$ & $<24$ & ID & 14 & 10 & ID & 14 \\
\hline & $9-10-2002$ & -- & -- & -- & -- & -- & -- \\
\hline \multirow[t]{2}{*}{31} & $7-29-2002$ & $<24$ & ID & 14 & 2 & ID & 14 \\
\hline & $9-10-2002$ & -- & -- & -- & -- & -- & -- \\
\hline \multirow[t]{2}{*}{32} & $8-8-2002$ & 3 & ID & 2 & -- & -- & -- \\
\hline & $9-11-2002$ & 2 & ID & 2 & -- & -- & -- \\
\hline 33 & $7-30-2002$ & 2 & ${ }^{2} 4.3-26.1$ & 22 & -- & -- & -- \\
\hline 34 & $7-30-2002$ & $<24$ & ID & 6 & -- & -- & -- \\
\hline \multirow[t]{2}{*}{35} & $7-30-2002$ & $<24$ & ID & 5 & -- & -- & -- \\
\hline & $9-3-2002$ & -- & -- & -- & -- & -- & -- \\
\hline \multirow[t]{2}{*}{36} & $7-30-2002$ & 16 & ID & 9 & -- & -- & -- \\
\hline & $9-3-2002$ & -- & -- & -- & -- & -- & -- \\
\hline \multirow[t]{2}{*}{37} & $7-31-2002$ & $<24$ & ${ }^{2} 4.3-87.0$ & 22 & $<20$ & 4.8 & 20 \\
\hline & $9-4-2002$ & 5 & ${ }^{2} 4.3-87.0$ & 22 & -- & -- & -- \\
\hline
\end{tabular}


Table 12. Water-quality data for the Upper Colorado River Basin, showing 2002 drought values, Colorado water-quality exceedances, and percentiles relative to historical data for selected properties and constituents from July through September, 1978-2002.—Continued

[ft $\mathrm{ft}^{3} / \mathrm{s}$, cubic feet per second; Conc, concentration; Q\%, percentile (percentage of data less than or equal to reported value or concentration) of drought-sample constituent; N, total number of historical and drought samples; $\mathrm{mg} / \mathrm{L}$, milligrams per liter; $\mu \mathrm{S} / \mathrm{cm}$, microsiemens per centimeter at $25^{\circ} \mathrm{Celsius;}{ }^{\circ} \mathrm{C}$, degrees Celsius; E. coli, Escherichia coli; $\mathrm{mL}$, milliliter; $\mu \mathrm{g} / \mathrm{L}$, micrograms per liter; $\mathrm{CaCO}_{3}$, calcium carbonate; $\mathrm{SiO}_{2}$, silica; $\mathrm{SO}_{4}$, sulfate; $\mathrm{ID}$, insufficient number of samples (less than 20) to calculate percentile; <, less than; --, no drought-sample measurement; values or concentrations in bold type indicate 2002 Colorado water-quality exceedances; c, chronic water-quality exceedance]

\begin{tabular}{|c|c|c|c|c|c|c|c|}
\hline \multirow{2}{*}{$\begin{array}{l}\text { Site number } \\
\text { in table } 1 \\
\text { and fig. } 4\end{array}$} & \multirow{2}{*}{ Sampling date } & \multicolumn{3}{|c|}{ Zinc, dissolved, in $\mu \mathrm{g} / \mathrm{L}$} & \multicolumn{3}{|c|}{$\begin{array}{l}\text { Zinc, total recoverable, } \\
\text { in } \mu \mathrm{g} / \mathrm{L}\end{array}$} \\
\hline & & Conc & $0 \%$ & $\mathrm{~N}$ & Conc & $0 \%$ & $\mathrm{~N}$ \\
\hline \multirow[t]{2}{*}{38} & $8-1-2002$ & -- & -- & -- & -- & -- & -- \\
\hline & $9-5-2002$ & -- & -- & -- & -- & -- & -- \\
\hline \multirow[t]{2}{*}{39} & $8-1-2002$ & -- & -- & -- & -- & -- & -- \\
\hline & $9-6-2002$ & -- & -- & -- & -- & -- & -- \\
\hline \multirow[t]{2}{*}{40} & $7-29-2002$ & $<24$ & ID & 6 & -- & -- & -- \\
\hline & $9-3-2002$ & -- & -- & -- & -- & -- & -- \\
\hline \multirow[t]{2}{*}{41} & $7-29-2002$ & $<24$ & ID & 5 & -- & -- & -- \\
\hline & $9-4-2002$ & -- & -- & -- & -- & -- & -- \\
\hline \multirow[t]{2}{*}{42} & $7-30-2002$ & $<24$ & ID & 6 & -- & -- & -- \\
\hline & $9-4-2002$ & -- & -- & -- & -- & -- & -- \\
\hline \multirow[t]{2}{*}{43} & $7-30-2002$ & $<24$ & ID & 3 & -- & -- & -- \\
\hline & $9-4-2002$ & -- & -- & -- & -- & -- & -- \\
\hline \multirow[t]{2}{*}{44} & $7-30-2002$ & $<24$ & ID & 10 & -- & -- & -- \\
\hline & $9-5-2002$ & 2 & ID & 10 & -- & -- & -- \\
\hline \multirow[t]{2}{*}{45} & $7-31-2002$ & 1 & ID & 2 & -- & -- & -- \\
\hline & $9-6-2002$ & 2 & ID & 2 & -- & -- & -- \\
\hline 46 & $8-2-2002$ & -- & -- & -- & -- & -- & -- \\
\hline \multirow[t]{2}{*}{47} & $8-1-2002$ & -- & -- & -- & -- & -- & -- \\
\hline & $9-3-2002$ & -- & -- & -- & -- & -- & -- \\
\hline \multirow[t]{2}{*}{48} & $8-1-2002$ & -- & -- & -- & -- & -- & -- \\
\hline & $9-5-2002$ & -- & -- & -- & -- & -- & - \\
\hline 49 & $7-31-2002$ & -- & -- & -- & -- & -- & -- \\
\hline 50 & $7-31-2002$ & -- & -- & -- & -- & -- & -- \\
\hline 51 & $8-1-2002$ & -- & -- & -- & -- & -- & - \\
\hline \multirow[t]{2}{*}{52} & $8-1-2002$ & 1 & ID & 19 & -- & -- & -- \\
\hline & $9-17-2002$ & 2 & ID & 19 & -- & -- & -- \\
\hline 53 & $7-31-2002$ & -- & -- & -- & -- & -- & - \\
\hline 54 & $8-1-2002$ & -- & -- & -- & -- & -- & - \\
\hline 55 & $7-30-2002$ & $<24$ & ID & 5 & -- & -- & - \\
\hline \multirow{2}{*}{56} & $7-31-2002$ & -- & -- & -- & -- & -- & - \\
\hline & $9-5-2002$ & -- & -- & -- & -- & -- & - \\
\hline \multirow[t]{2}{*}{57} & $7-31-2002$ & $<24$ & ID & 3 & -- & -- & - \\
\hline & $9-4-2002$ & -- & -- & -- & -- & -- & - \\
\hline \multirow[t]{2}{*}{58} & 7-16-2002 & -- & -- & -- & -- & -- & - \\
\hline & $9-5-2002$ & -- & -- & -- & -- & -- & - \\
\hline
\end{tabular}

${ }^{1}$ Possible percentile range because of uncensored ties in data.

${ }^{2}$ Possible percentile range because of censored or recensored ties in data. 
Table 13. Water-quality data for the Rio Grande Basin showing 2002 drought values, Colorado water-quality exceedances, and percentiles relative to historical data for selected properties and constituents from July through September, 1978-2002.

$\left[\mathrm{ft}^{3} / \mathrm{s}\right.$, cubic feet per second; Conc, concentration; Q\%, percentile (percentage of data less than or equal to reported value or concentration) of drought-sample constituent; N, total number of historical and drought samples; mg/L, milligrams per liter; $\mu \mathrm{S} / \mathrm{cm}$, microsiemens per centimeter at $25^{\circ} \mathrm{Celsius;}{ }^{\circ} \mathrm{C}$, degrees Celsius; E. coli, Escherichia coli; $\mathrm{mL}$, milliliter; $\mu \mathrm{g} / \mathrm{L}$, micrograms per liter; $\mathrm{CaCO}_{3}$, calcium carbonate; $\mathrm{SiO}_{2}$, silica; $\mathrm{SO}_{4}$, sulfate; ID, insufficient number of samples (less than 20) to calculate percentile; <, less than; --, no drought-sample measurement; values or concentrations in bold type indicate 2002 Colorado water-quality exceedances; ac, acute and chronic water-quality exceedance]

\begin{tabular}{|c|c|c|c|c|c|c|c|c|c|}
\hline \multirow{2}{*}{$\begin{array}{c}\text { Site number } \\
\text { in table } 1 \\
\text { and fig. } 5\end{array}$} & \multirow{2}{*}{$\begin{array}{l}\text { Sampling } \\
\text { date }\end{array}$} & \multirow{2}{*}{$\begin{array}{l}\text { Sampling } \\
\text { time }\end{array}$} & \multirow{2}{*}{$\begin{array}{c}\text { Discharge, } \\
\text { in } \mathrm{ft}^{3} / \mathrm{s}\end{array}$} & \multicolumn{3}{|c|}{ Oxygen, dissolved, in $\mathrm{mg} / \mathrm{L}$} & \multicolumn{3}{|c|}{$\mathrm{pH}$, in standard units } \\
\hline & & & & Conc & $0 \%$ & $\mathrm{~N}$ & Value & $0 \%$ & $\mathrm{~N}$ \\
\hline 59 & $9-18-2002$ & 1015 & 158 & 8.3 & ID & 2 & 7.9 & ID & 1 \\
\hline 60 & $9-18-2002$ & 1315 & 33 & 8.4 & ID & 1 & 8.3 & ID & 1 \\
\hline \multirow[t]{2}{*}{61} & $7-11-2002$ & 1130 & 12 & 9.5 & ${ }^{1} 88.2-91.2$ & 33 & 8.6 & ${ }^{1} 51.4-62.2$ & 36 \\
\hline & $8-15-2002$ & 1030 & 7 & 7.6 & ${ }^{1} 35.3-41.2$ & 33 & 9.0 & ${ }^{1} 91.9-97.3$ & 36 \\
\hline 62 & $9-18-2002$ & 0745 & 100 & 8.2 & ID & 1 & 7.6 & ID & 1 \\
\hline
\end{tabular}

\begin{tabular}{|c|c|c|c|c|c|c|c|c|}
\hline \multirow{2}{*}{$\begin{array}{l}\text { Site number } \\
\text { in table } 1 \\
\text { and fig. } 5\end{array}$} & \multirow{2}{*}{$\begin{array}{l}\text { Sampling } \\
\text { date }\end{array}$} & \multicolumn{3}{|c|}{ Specific conductance, in $\mu \mathrm{S} / \mathrm{cm}$} & \multicolumn{3}{|c|}{ Temperature, water, in ${ }^{\circ} \mathrm{C}$} & \multirow{2}{*}{$\begin{array}{l}\text { Hardness, } \\
\text { total, in } \mathrm{mg} / \mathrm{L} \\
\text { as } \mathrm{CaCO}_{3}\end{array}$} \\
\hline & & Value & $0 \%$ & $\mathrm{~N}$ & Value & $0 \%$ & $\mathrm{~N}$ & \\
\hline 59 & $9-18-2002$ & 111 & ID & 2 & 11.5 & ${ }^{1} 17.4-21.7$ & 45 & 36 \\
\hline 60 & $9-18-2002$ & 90 & ID & 1 & 11.5 & 24.4 & 44 & 37 \\
\hline \multirow[t]{2}{*}{61} & $7-11-2002$ & 461 & 68.6 & 34 & 21.5 & ${ }^{1} 83.9-87.5$ & 55 & 84 \\
\hline & $8-15-2002$ & 450 & 62.9 & 34 & 18.5 & ${ }^{1} 33.9-37.5$ & 55 & 58 \\
\hline 62 & $9-18-2002$ & 89 & ID & 1 & 9.5 & ID & 1 & 34 \\
\hline
\end{tabular}

\begin{tabular}{|c|c|c|c|c|c|c|c|c|}
\hline \multirow{2}{*}{$\begin{array}{l}\text { Site number } \\
\text { in table } 1 \\
\text { and fig. } 5\end{array}$} & \multirow{2}{*}{$\begin{array}{c}\text { Sampling } \\
\text { date }\end{array}$} & \multirow{2}{*}{$\begin{array}{c}\text { Calcium, } \\
\text { dissolved, in } \\
\mathrm{mg} / \mathrm{L}\end{array}$} & \multirow{2}{*}{$\begin{array}{c}\text { Magnesium, } \\
\text { dissolved, } \\
\text { in } \mathrm{mg} / \mathrm{L}\end{array}$} & \multirow{2}{*}{$\begin{array}{l}\text { Potassium, } \\
\text { dissolved, } \\
\text { in } \mathrm{mg} / \mathrm{L}\end{array}$} & \multirow{2}{*}{$\begin{array}{l}\text { Sodium, } \\
\text { dissolved, } \\
\text { in } \mathrm{mg} / \mathrm{L}\end{array}$} & \multicolumn{3}{|c|}{ Chloride, dissolved, in $\mathrm{mg} / \mathrm{L}$} \\
\hline & & & & & & Conc & $0 \%$ & $\mathrm{~N}$ \\
\hline 59 & $9-18-2002$ & 11.7 & 1.71 & 1.93 & 4.3 & 1.37 & ID & 2 \\
\hline 60 & $9-18-2002$ & 11.7 & 1.89 & 1.75 & 2.7 & 0.50 & ID & 1 \\
\hline \multirow[t]{2}{*}{61} & $7-11-2002$ & 21 & 7.8 & 7.8 & 64 & 15.4 & 85.7 & 34 \\
\hline & $8-15-2002$ & 12.1 & 6.7 & 8.3 & 70 & 17.6 & 97.1 & 34 \\
\hline 62 & $9-18-2002$ & 10.7 & 1.71 & 1.94 & 3.8 & 0.89 & ID & 1 \\
\hline
\end{tabular}

\begin{tabular}{|c|c|c|c|c|c|c|c|}
\hline \multirow{2}{*}{$\begin{array}{c}\text { Site number } \\
\text { in table } 1 \\
\text { and fig. } 5\end{array}$} & \multirow{2}{*}{$\begin{array}{c}\text { Sampling } \\
\text { date }\end{array}$} & \multicolumn{3}{|c|}{ Fluoride, dissolved, in $\mathrm{mg} / \mathrm{L}$} & \multicolumn{3}{|c|}{ Silica, dissolved, in $\mathrm{mg} / \mathrm{L}$ as $\mathrm{SiO}_{2}$} \\
\hline & & Conc & $0 \%$ & $\mathrm{~N}$ & Conc & $0 \%$ & $\mathrm{~N}$ \\
\hline 59 & 9-18-2002 & 0.1 & ID & 2 & 19.0 & ID & 2 \\
\hline 60 & $9-18-2002$ & 0.1 & ID & 1 & 20 & ID & 1 \\
\hline \multirow[t]{2}{*}{61} & $7-11-2002$ & 0.86 & 91.4 & 34 & 17.8 & 11.4 & 34 \\
\hline & $8-15-2002$ & 1.1 & 97.1 & 34 & 8.79 & 2.9 & 34 \\
\hline 62 & $9-18-2002$ & 0.1 & ID & 1 & 21 & ID & 1 \\
\hline
\end{tabular}


Table 13. Water-quality data for the Rio Grande Basin showing 2002 drought values, Colorado water-quality exceedances, and percentiles relative to historical data for selected properties and constituents from July through September, 1978-2002.—Continued

$\left[\mathrm{ft} \mathrm{t}^{3} / \mathrm{s}\right.$, cubic feet per second; Conc, concentration; Q\%, percentile (percentage of data less than or equal to reported value or concentration) of drought-sample constituent; $\mathrm{N}$, total number of historical and drought samples; $\mathrm{mg} / \mathrm{L}$, milligrams per liter; $\mu \mathrm{S} / \mathrm{cm}$, microsiemens per centimeter at $25^{\circ} \mathrm{Celsius} ;{ }^{\circ} \mathrm{C}$, degrees $\mathrm{Cel}-$ sius; E. coli, Escherichia coli; $\mathrm{mL}$, milliliter; $\mu \mathrm{g} / \mathrm{L}$, micrograms per liter; $\mathrm{CaCO}_{3}$, calcium carbonate; $\mathrm{SiO}_{2}$, silica; $\mathrm{SO}_{4}$, sulfate; ID, insufficient number of samples (less than 20) to calculate percentile; <, less than; --, no drought-sample measurement; values or concentrations in bold type indicate 2002 Colorado water-quality exceedances; ac, acute and chronic water-quality exceedance]

\begin{tabular}{|c|c|c|c|c|c|c|c|c|}
\hline \multirow{2}{*}{$\begin{array}{l}\text { Site number } \\
\text { in table } 1 \\
\text { and fig. } 5\end{array}$} & \multirow{2}{*}{$\begin{array}{l}\text { Sampling } \\
\text { date }\end{array}$} & \multicolumn{3}{|c|}{ Sulfate dissolved, in $\mathrm{mg} / \mathrm{L}$ as $\mathrm{SO}_{4}$} & \multirow{2}{*}{$\begin{array}{c}\text { Solids, dissolved, } \\
\text { residue on } \\
\text { evaporation at } \\
180^{\circ} \mathrm{C} \text {, in } \mathrm{mg} / \mathrm{L}\end{array}$} & \multicolumn{3}{|c|}{$\begin{array}{c}\text { Nitrogen, ammonia, dissolved } \\
\text { in } \mathrm{mg} / \mathrm{L} \text { as } \mathrm{N}\end{array}$} \\
\hline & & Conc & $0 \%$ & $\mathrm{~N}$ & & Conc & $0 \%$ & $\mathrm{~N}$ \\
\hline 59 & $9-18-2002$ & 9.6 & ID & 2 & 82 & $<0.015$ & ID & 2 \\
\hline 60 & $9-18-2002$ & 2.4 & ID & 1 & 74 & $<0.015$ & ID & 1 \\
\hline \multirow[t]{2}{*}{61} & $7-11-2002$ & 51 & 65.7 & 34 & 284 & $<0.04$ & ${ }^{2} 3.2-77.4$ & 30 \\
\hline & $8-15-2002$ & 53 & 68.6 & 34 & 263 & $<0.04$ & ${ }^{2} 3.2-77.4$ & 30 \\
\hline 62 & $9-18-2002$ & 6.3 & ID & 1 & 69 & $<0.015$ & ID & 1 \\
\hline
\end{tabular}

\begin{tabular}{|c|c|c|c|c|c|c|c|}
\hline \multirow{2}{*}{$\begin{array}{c}\text { Site number } \\
\text { in table } 1 \\
\text { and fig. } 5\end{array}$} & \multirow{2}{*}{$\begin{array}{l}\text { Sampling } \\
\text { date }\end{array}$} & \multicolumn{3}{|c|}{$\begin{array}{l}\text { Nitrogen, organic plus ammonia, } \\
\text { dissolved, in } \mathrm{mg} / \mathrm{L} \text { as } \mathrm{N}\end{array}$} & \multicolumn{3}{|c|}{$\begin{array}{l}\text { Nitrogen, organic plus ammonia, total } \\
\text { recoverable, in } \mathrm{mg} / \mathrm{L} \text { as } \mathrm{N}\end{array}$} \\
\hline & & Conc & $0 \%$ & $\mathrm{~N}$ & Conc & $0 \%$ & $\mathrm{~N}$ \\
\hline 59 & $9-18-2002$ & 0.12 & ID & 2 & 0.18 & ID & 2 \\
\hline 60 & $9-18-2002$ & 0.12 & ID & 1 & 0.12 & ID & 1 \\
\hline \multirow[t]{2}{*}{61} & $7-11-2002$ & 0.38 & 57.7 & 25 & 0.64 & 54.1 & 36 \\
\hline & $8-15-2002$ & 0.43 & 65.4 & 25 & 0.68 & 56.8 & 36 \\
\hline 62 & $9-18-2002$ & 0.10 & ID & 1 & 0.15 & ID & 1 \\
\hline
\end{tabular}

\begin{tabular}{|c|c|c|c|c|c|c|c|}
\hline \multirow{2}{*}{$\begin{array}{c}\text { Site number } \\
\text { in table } 1 \\
\text { and fig. } 5\end{array}$} & \multirow{2}{*}{$\begin{array}{l}\text { Sampling } \\
\text { date }\end{array}$} & \multicolumn{3}{|c|}{$\begin{array}{l}\text { Nitrogen, nitrite plus nitrate, dissolved, } \\
\text { in } \mathrm{mg} / \mathrm{L} \text { as } \mathrm{N}\end{array}$} & \multicolumn{3}{|c|}{$\begin{array}{l}\text { Nitrogen, nitrite, dissolved, } \\
\text { in } \mathrm{mg} / \mathrm{L} \text { as } \mathrm{N}\end{array}$} \\
\hline & & Conc & $0 \%$ & $\mathrm{~N}$ & Conc & $0 \%$ & $\mathrm{~N}$ \\
\hline 59 & $9-18-2002$ & $<0.013$ & ID & 2 & $<0.002$ & ID & 2 \\
\hline 60 & $9-18-2002$ & $<0.013$ & ID & 1 & $<0.002$ & ID & 1 \\
\hline \multirow[t]{2}{*}{61} & $7-11-2002$ & $<0.05$ & ${ }^{2} 3.0-87.9$ & 32 & $<0.008$ & ${ }^{2} 4.8-95.2$ & 20 \\
\hline & $8-15-2002$ & $<0.05$ & ${ }^{2} 3.0-87.9$ & 32 & $<0.008$ & ${ }^{2} 4.8-95.2$ & 20 \\
\hline 62 & $9-18-2002$ & $<0.013$ & ID & 1 & $<0.002$ & ID & 1 \\
\hline
\end{tabular}

\begin{tabular}{|c|c|c|c|c|c|c|c|}
\hline \multirow{2}{*}{$\begin{array}{c}\text { Site number } \\
\text { in table } 1 \\
\text { and fig. } 5\end{array}$} & \multirow{2}{*}{$\begin{array}{c}\text { Sampling } \\
\text { date }\end{array}$} & \multicolumn{3}{|c|}{ Phosphorus, dissolved, in $\mathrm{mg} / \mathrm{L}$ as $\mathrm{P}$} & \multicolumn{3}{|c|}{$\begin{array}{c}\text { Phosphorus, total recoverable, } \\
\text { in } \mathrm{mg} / \mathrm{L} \text { as } P\end{array}$} \\
\hline & & Conc & $0 \%$ & $\mathrm{~N}$ & Conc & $0 \%$ & $\mathrm{~N}$ \\
\hline 59 & $9-18-2002$ & 0.036 & ID & 2 & 0.051 & ID & 2 \\
\hline 60 & $9-18-2002$ & 0.015 & ID & 1 & 0.025 & ID & 1 \\
\hline \multirow[t]{2}{*}{61} & $7-11-2002$ & 0.011 & ${ }^{1} 5.4-8.1$ & 36 & 0.069 & 8.1 & 36 \\
\hline & $8-15-2002$ & 0.013 & 10.8 & 36 & 0.061 & ${ }^{1} 2.7-5.4$ & 36 \\
\hline 62 & $9-18-2002$ & 0.031 & ID & 1 & 0.043 & ID & 1 \\
\hline
\end{tabular}


Table 13. Water-quality data for the Rio Grande Basin showing 2002 drought values, Colorado water-quality exceedances, and percentiles relative to historical data for selected properties and constituents from July through September, 1978-2002.-Continued

$\left[\mathrm{ft}^{3} / \mathrm{s}\right.$, cubic feet per second; Conc, concentration; Q\%, percentile (percentage of data less than or equal to reported value or concentration) of drought-sample constituent; N, total number of historical and drought samples; mg/L, milligrams per liter; $\mu \mathrm{S} / \mathrm{cm}$, microsiemens per centimeter at $25^{\circ} \mathrm{Celsius;}{ }^{\circ} \mathrm{C}$, degrees $\mathrm{Cel}-$ sius; E. coli, Escherichia coli; $\mathrm{mL}$, milliliter; $\mu \mathrm{g} / \mathrm{L}$, micrograms per liter; $\mathrm{CaCO}_{3}$, calcium carbonate; $\mathrm{SiO}_{2}$, silica; $\mathrm{SO}_{4}$, sulfate; ID, insufficient number of samples (less than 20) to calculate percentile; <, less than; --, no drought-sample measurement; values or concentrations in bold type indicate 2002 Colorado water-quality exceedances; ac, acute and chronic water-quality exceedance]

\begin{tabular}{|c|c|c|c|c|c|c|c|}
\hline \multirow{2}{*}{$\begin{array}{l}\text { Site number } \\
\text { in table } 1 \\
\text { and fig. } 5\end{array}$} & \multirow{2}{*}{$\begin{array}{c}\text { Sampling } \\
\text { date }\end{array}$} & \multicolumn{3}{|c|}{$\begin{array}{l}\text { Phosphorus, orthophosphate, dissolved, in } \\
\qquad \mathrm{mg} / \mathrm{L} \text { as } P\end{array}$} & \multirow{2}{*}{$\begin{array}{l}\text { Carbon, } \\
\text { organic, } \\
\text { dissolved, } \\
\text { in } \mathrm{mg} / \mathrm{L}\end{array}$} & \multirow{2}{*}{$\begin{array}{l}\text { Carbon, } \\
\text { organic, total } \\
\text { recoverable, } \\
\text { in } \mathrm{mg} / \mathrm{L}\end{array}$} & \multirow{2}{*}{$\begin{array}{c}\text { Bacteria, } \\
\text { E. coli, } \\
\text { in colonies } \\
\text { per } 100 \mathrm{~mL}\end{array}$} \\
\hline & & Conc & $0 \%$ & $\mathrm{~N}$ & & & \\
\hline 59 & $9-18-2002$ & 0.028 & ID & 2 & 1.7 & 2.2 & 13 \\
\hline 60 & $9-18-2002$ & 0.012 & ID & 1 & 1.2 & 1.8 & 3 \\
\hline \multirow[t]{2}{*}{61} & $7-11-2002$ & $<0.02$ & ${ }^{2} 4.0-16.0$ & 24 & -- & -- & 20 \\
\hline & $8-15-2002$ & $<0.03$ & ${ }^{2} 4.0-16.0$ & 24 & -- & -- & 14 \\
\hline 62 & $9-18-2002$ & 0.025 & ID & 1 & 1.6 & 2.1 & 11 \\
\hline
\end{tabular}

\begin{tabular}{|c|c|c|c|c|c|c|c|}
\hline \multirow{2}{*}{$\begin{array}{c}\text { Site number } \\
\text { in table } 1 \\
\text { and fig. } 5\end{array}$} & \multirow{2}{*}{$\begin{array}{c}\text { Sampling } \\
\text { date }\end{array}$} & \multicolumn{3}{|c|}{$\begin{array}{l}\text { Bacteria, fecal coliform, in colonies per } \\
\qquad 100 \mathrm{~mL}\end{array}$} & \multirow{2}{*}{$\begin{array}{c}\text { Aluminum, } \\
\text { dissolved, } \\
\text { in } \mu \mathrm{g} / \mathrm{L}\end{array}$} & \multirow{2}{*}{$\begin{array}{c}\text { Arsenic, } \\
\text { dissolved, } \\
\text { in } \mu \mathrm{g} / \mathrm{L}\end{array}$} & \multirow{2}{*}{$\begin{array}{c}\text { Arsenic, } \\
\text { total } \\
\text { recoverable } \\
\text { in } \mu \mathrm{g} / \mathrm{L}\end{array}$} \\
\hline & & Conc & $0 \%$ & $\mathrm{~N}$ & & & \\
\hline 59 & $9-18-2002$ & 14 & ID & 1 & 5 & 1 & -- \\
\hline 60 & $9-18-2002$ & 5 & ID & 1 & 2 & 1 & -- \\
\hline \multirow[t]{2}{*}{61} & 7-11-2002 & -- & -- & -- & 6 & 5 & -- \\
\hline & $8-15-2002$ & 11 & 40.9 & 21 & -- & -- & -- \\
\hline 62 & $9-18-2002$ & 7 & ID & 1 & 4 & 1 & -- \\
\hline
\end{tabular}

\begin{tabular}{|c|c|c|c|c|c|c|c|c|}
\hline \multirow{2}{*}{$\begin{array}{c}\text { Site number } \\
\text { in table } 1 \\
\text { and fig. } 5\end{array}$} & \multirow{2}{*}{$\begin{array}{c}\text { Sampling } \\
\text { date }\end{array}$} & \multicolumn{3}{|c|}{ Barium, dissolved, in $\mathrm{mg} / \mathrm{L}$} & \multirow{2}{*}{$\begin{array}{c}\text { Beryllium, } \\
\text { dissolved, } \\
\text { in } \mu \mathrm{g} / \mathrm{L}\end{array}$} & \multirow{2}{*}{$\begin{array}{l}\text { Cadmium, } \\
\text { dissolved, } \\
\text { in } \mu \mathrm{g} / \mathrm{L}\end{array}$} & \multirow{2}{*}{$\begin{array}{l}\text { Cobalt, } \\
\text { dissolved, } \\
\text { in } \mu \mathrm{g} / \mathrm{L}\end{array}$} & \multirow{2}{*}{$\begin{array}{c}\text { Chromium, } \\
\text { dissolved, } \\
\text { in } \mu \mathrm{g} / \mathrm{L}\end{array}$} \\
\hline & & Conc & $0 \%$ & $\mathrm{~N}$ & & & & \\
\hline 59 & $9-18-2002$ & 22 & ID & 1 & $<0.06$ & 0.18 & 0.07 & $<0.8$ \\
\hline 60 & $9-18-2002$ & 17 & ID & 1 & $<0.06$ & $<0.04$ & 0.06 & $<0.8$ \\
\hline \multirow[t]{2}{*}{61} & $7-11-2002$ & 34 & 57.1 & 20 & $<0.06$ & 0.03 & $<3$ & $<0.8$ \\
\hline & $8-15-2002$ & -- & -- & -- & -- & -- & -- & -- \\
\hline 62 & $9-18-2002$ & 18 & ID & 1 & $<0.06$ & $<0.04$ & 0.07 & $<0.8$ \\
\hline
\end{tabular}


Table 13. Water-quality data for the Rio Grande Basin showing 2002 drought values, Colorado water-quality exceedances, and percentiles relative to historical data for selected properties and constituents from July through September, 1978-2002.—Continued

$\left[\mathrm{ft} \mathrm{t}^{3} / \mathrm{s}\right.$, cubic feet per second; Conc, concentration; Q\%, percentile (percentage of data less than or equal to reported value or concentration) of drought-sample constituent; $\mathrm{N}$, total number of historical and drought samples; $\mathrm{mg} / \mathrm{L}$, milligrams per liter; $\mu \mathrm{S} / \mathrm{cm}$, microsiemens per centimeter at $25^{\circ} \mathrm{Celsius} ;{ }^{\circ} \mathrm{C}$, degrees $\mathrm{Cel}-$ sius; E. coli, Escherichia coli; $\mathrm{mL}$, milliliter; $\mu \mathrm{g} / \mathrm{L}$, micrograms per liter; $\mathrm{CaCO}_{3}$, calcium carbonate; $\mathrm{SiO}_{2}$, silica; $\mathrm{SO}_{4}$, sulfate; ID, insufficient number of samples (less than 20) to calculate percentile; <, less than; --, no drought-sample measurement; values or concentrations in bold type indicate 2002 Colorado water-quality exceedances; ac, acute and chronic water-quality exceedance]

\begin{tabular}{|c|c|c|c|c|c|c|c|c|c|}
\hline \multirow{2}{*}{$\begin{array}{c}\text { Site number } \\
\text { in table } 1 \\
\text { and fig. } 5\end{array}$} & \multirow{2}{*}{$\begin{array}{l}\text { Sampling } \\
\text { date }\end{array}$} & \multirow{2}{*}{$\begin{array}{c}\text { Copper, } \\
\text { dissolved, } \\
\text { in } \mu \mathrm{g} / \mathrm{L}\end{array}$} & \multicolumn{3}{|c|}{ Iron, dissolved, in $\mu \mathrm{g} / \mathrm{L}$} & \multirow{2}{*}{$\begin{array}{l}\text { Lead, } \\
\text { solved, il } \\
\mu \mathrm{g} / \mathrm{L}\end{array}$} & \multicolumn{3}{|c|}{$\begin{array}{c}\text { Manganese, dissolved, } \\
\text { in } \mu \mathrm{g} / \mathrm{L}\end{array}$} \\
\hline & & & Conc & $0 \%$ & $\mathrm{~N}$ & & Conc & $0 \%$ & $\mathrm{~N}$ \\
\hline 59 & $9-18-2002$ & 1 & -- & -- & -- & 0.34 & 11.3 & ID & 2 \\
\hline 60 & $9-18-2002$ & 0.3 & -- & -- & -- & $<0.08$ & 4 & ID & 1 \\
\hline \multirow[t]{2}{*}{61} & $7-11-2002$ & -- & 7 & ${ }^{2} 3.7-22.2$ & 26 & 0.13 & 20.3 & ${ }^{1} 84.0-88.0$ & 24 \\
\hline & $8-15-2002$ & -- & 14 & 29.6 & 26 & -- & 20.3 & ${ }^{1} 84.0-88.0$ & 24 \\
\hline 62 & $9-18-2002$ & 0.6 & -- & -- & -- & $<0.08$ & 10.1 & ID & 1 \\
\hline
\end{tabular}

\begin{tabular}{|c|c|c|c|c|c|c|c|c|c|}
\hline \multirow{2}{*}{$\begin{array}{l}\text { Site number } \\
\text { in table } 1 \\
\text { and fig. } 5\end{array}$} & \multirow{2}{*}{$\begin{array}{l}\text { Sampling } \\
\text { date }\end{array}$} & \multirow{2}{*}{$\begin{array}{c}\text { Molybdenum, } \\
\text { dissolved, } \\
\text { in } \mu \mathrm{g} / \mathrm{L}\end{array}$} & \multirow{2}{*}{$\begin{array}{c}\text { Nickel, } \\
\text { dissolved, } \\
\text { in } \mu \mathrm{g} / \mathrm{L}\end{array}$} & \multicolumn{3}{|c|}{ Selenium, dissolved, in $\mu \mathrm{g} / \mathrm{L}$} & \multicolumn{3}{|c|}{ Silver, dissolved, in $\mu \mathrm{g} / \mathrm{L}$} \\
\hline & & & & Conc & $0 \%$ & $\mathrm{~N}$ & Conc & $0 \%$ & $\mathrm{~N}$ \\
\hline 59 & $9-18-2002$ & 0.5 & 0.66 & $<2$ & ID & 2 & $<1$ & ID & 1 \\
\hline 60 & $9-18-2002$ & 0.2 & 0.47 & $<2$ & ID & 1 & $<1$ & ID & 1 \\
\hline \multirow[t]{2}{*}{61} & $7-11-2002$ & 8.1 & 0.81 & $<2$ & ${ }^{2} 4.8-95.2$ & 20 & $<1$ & ${ }^{2} 4.8-95.2$ & 20 \\
\hline & $8-15-2002$ & -- & -- & -- & -- & -- & -- & -- & -- \\
\hline 62 & $9-18-2002$ & 0.4 & 0.47 & $<2$ & ID & 1 & $<1$ & ID & 1 \\
\hline
\end{tabular}

\begin{tabular}{cccc}
\hline $\begin{array}{c}\text { Site number } \\
\text { in table 1 } \\
\text { and fig. 5 }\end{array}$ & $\begin{array}{c}\text { Sampling } \\
\text { date }\end{array}$ & $\begin{array}{c}\text { Zinc, } \\
\text { dissolved, } \\
\text { in } \mu \mathrm{g} / \mathrm{L}\end{array}$ & $\begin{array}{c}\text { Uranium, } \\
\text { dissolved, } \\
\text { in } \mu \mathrm{g} / \mathrm{L}\end{array}$ \\
\hline 59 & $9-18-2002$ & $\mathbf{5 4 a c}$ & 0.21 \\
60 & $9-18-2002$ & $<1$ & 0.05 \\
61 & $7-11-2002$ & 1 & 1.96 \\
62 & $8-15-2002$ & -- & -- \\
& $9-18-2002$ & $<1$ & 0.12 \\
\hline
\end{tabular}

\footnotetext{
${ }^{1}$ Possible percentile range because of uncensored ties in data.

${ }^{2}$ Possible percentile range because of censored or recensored ties in data.
} 
Table 14. Water-quality data for the Gunnison River Basin showing 2002 drought values, Colorado water-quality exceedances, and percentiles relative to historical data for selected properties and constituents from July through September, 1978-2002.

[ft $\mathrm{ft}^{3} / \mathrm{s}$, cubic feet per second; Conc, concentration; Q\%, percentile (percentage of data less than or equal to reported value or concentration) of drought-sample constituent; $\mathrm{N}$, total number of historical and drought samples; $\mathrm{mg} / \mathrm{L}$, milligrams per liter; $\mu \mathrm{S} / \mathrm{cm}$, microsiemens per centimeter at $25^{\circ} \mathrm{Celsius;}{ }^{\circ} \mathrm{C}$, degrees Celsius; $E$. coli, Escherichia coli; $\mathrm{mL}$, milliliter; $\mu \mathrm{g} / \mathrm{L}$, micrograms per liter; $\mathrm{CaCO}_{3}$, calcium carbonate; $\mathrm{SiO}_{2}$, silica; $\mathrm{SO}_{4}$, sulfate; ID, insufficient number of samples (less than 20) to calculate percentile; <, less than; --, no drought-sample measurement; values or concentrations in bold type indicate 2002 Colorado water-quality exceedances; c, chronic water-quality exceedance]

\begin{tabular}{|c|c|c|c|c|c|c|c|c|c|c|c|c|}
\hline $\begin{array}{l}\text { Site } \\
\text { number in } \\
\text { table } 1 \\
\text { and fig. } 5\end{array}$ & $\begin{array}{l}\text { Sampling } \\
\text { date }\end{array}$ & $\begin{array}{l}\text { Sampling } \\
\text { time }\end{array}$ & $\begin{array}{c}\text { Discharge, } \\
\text { in } \mathrm{ft}^{3} / \mathrm{s}\end{array}$ & Conc & $0 \%$ & $\mathrm{~N}$ & Value & $0 \%$ & $\mathrm{~N}$ & Value & $0 \%$ & $\mathrm{~N}$ \\
\hline \multirow[t]{2}{*}{63} & $8-6-2002$ & 0905 & 79 & 7.6 & ${ }^{\mathrm{T}} 14.8-18.5$ & 26 & 8.2 & T22.2-33.3 & 26 & 323 & 86.8 & 37 \\
\hline & $9-3-2002$ & 1740 & 43 & 7.3 & 11.1 & 26 & 8.4 & ${ }^{1} 59.3-92.6$ & 26 & 335 & 94.7 & 37 \\
\hline 64 & $8-6-2002$ & 0900 & 119 & 8.1 & ${ }^{1} 40.9-50.0$ & 21 & 8.2 & ${ }^{1} 18.2-31.8$ & 21 & 343 & 92.6 & 67 \\
\hline 65 & 9-4-2002 & 1210 & 5.2 & 8.3 & ID & 8 & 8.6 & ID & 8 & 397 & ID & 9 \\
\hline 66 & $8-7-2002$ & 0905 & 357 & 8.4 & ID & 14 & 8.2 & ID & 14 & 232 & 87.0 & 76 \\
\hline \multirow[t]{2}{*}{67} & 8-7-2002 & 0910 & 39 & 7.7 & ID & 19 & 8.2 & ID & 19 & 377 & 94.2 & 68 \\
\hline & 9-4-2002 & 1350 & 18 & 9.9 & ID & 19 & 8.6 & ID & 19 & 330 & ${ }^{1} 71.0-72.5$ & 68 \\
\hline \multirow[t]{2}{*}{68} & $8-7-2002$ & 1239 & 482 & 8.4 & ${ }^{1} 12.5-16.7$ & 23 & 8.4 & 95.8 & 23 & 212 & 77.1 & 69 \\
\hline & $9-5-2002$ & 1440 & 444 & 8.6 & ${ }^{1} 25.0-33.3$ & 23 & 8.3 & 91.7 & 23 & 218 & 84.3 & 69 \\
\hline 69 & 8-8-2002 & 0930 & 48 & 7.5 & ID & 2 & 8.2 & ID & 2 & 1,590 & ID & 16 \\
\hline \multirow[t]{2}{*}{73} & 8-13-2002 & 1000 & 109 & 8.6 & ID & 9 & 8.2 & ${ }^{1} 21.4-75.0$ & 27 & 1,760 & 90.4 & 72 \\
\hline & 9-23-2002 & 0900 & 246 & 8.2 & ID & 9 & 8.2 & ${ }^{1} 21.4-75.0$ & 27 & 1,640 & 72.6 & 72 \\
\hline \multirow[t]{2}{*}{74} & 8-13-2002 & 1300 & 818 & 8.5 & ${ }^{1} 76.8-78.6$ & 55 & 8.4 & ${ }^{1} 62.7-86.4$ & 58 & 1,130 & 69.9 & 72 \\
\hline & $9-24-2002$ & 1000 & 1,010 & 7.6 & ${ }^{1} 33.9-37.5$ & 55 & 8.4 & ${ }^{1} 62.7-86.4$ & 58 & 1,250 & 82.2 & 72 \\
\hline \multirow[t]{2}{*}{75} & $8-7-2002$ & 1035 & 354 & 8.4 & ID & 17 & 8.3 & ID & 17 & 255 & ID & 17 \\
\hline & 9-5-2002 & 0830 & 116 & 8.1 & ID & 17 & 8.2 & ID & 17 & 288 & ID & 17 \\
\hline 76 & $8-6-2002$ & 1500 & 28 & 9.4 & ID & 3 & 8.8 & ID & 4 & 290 & ID & 4 \\
\hline 77 & $8-6-2002$ & 1140 & 8.3 & 7.6 & ID & 3 & 8.4 & ID & 3 & 198 & ID & 3 \\
\hline 78 & $8-8-2002$ & 1230 & 500 & 8.5 & ID & 15 & 8.2 & ID & 16 & 432 & ID & 15 \\
\hline 79 & $8-6-2002$ & 1010 & 43 & 7.9 & ID & 2 & 8.4 & ID & 2 & 331 & ID & 2 \\
\hline 80 & $8-5-2002$ & 1600 & 2.6 & 7.1 & ID & 2 & 8.2 & ID & 2 & 328 & ID & 2 \\
\hline 81 & $8-5-2002$ & 1410 & 9.2 & 6.1 & ID & 16 & 7.5 & ID & 16 & 136 & ID & 16 \\
\hline 82 & $8-5-2002$ & 1240 & 1.8 & 6.1 & ID & 2 & 8.3 & ID & 2 & 280 & ID & 2 \\
\hline 83 & $8-5-2002$ & 1630 & 14 & 6.6 & ID & 12 & 8.3 & ID & 12 & 385 & ID & 12 \\
\hline
\end{tabular}


Table 14. Water-quality data for the Gunnison River Basin showing 2002 drought values, Colorado water-quality exceedances, and percentiles relative to historical data for selected properties and constituents from July through September, 1978-2002. - Continued

[ft $3 / \mathrm{s}$, cubic feet per second; Conc, concentration; Q\%, percentile (percentage of data less than or equal to reported value or concentration) of drought-sample constituent; $\mathrm{N}$, total number of historical and drought samples; $\mathrm{mg} / \mathrm{L}$, milligrams per liter; $\mu \mathrm{S} / \mathrm{cm}$, microsiemens per centimeter at $25^{\circ}$ Celsius; ${ }^{\circ} \mathrm{C}$, degrees Celsius; E. coli, Escherichia coli; mL, milliliter; $\mu \mathrm{g} / \mathrm{L}$, micrograms per liter; $\mathrm{CaCO}_{3}$, calcium carbonate; $\mathrm{SiO}_{2}$, silica; $\mathrm{SO}_{4}$, sulfate; ID, insufficient number of samples (less than 20) to calculate percentile; <, less than; --, no drought-sample measurement; values or concentrations in bold type indicate 2002 Colorado water-quality exceedances; c, chronic water-quality exceedance]

\begin{tabular}{|c|c|c|c|c|c|c|c|c|c|c|c|c|c|}
\hline \multirow{2}{*}{$\begin{array}{c}\text { Site } \\
\text { number in } \\
\text { table } 1 \\
\text { and fig. } 5\end{array}$} & \multirow{2}{*}{$\begin{array}{l}\text { Sampling } \\
\text { date }\end{array}$} & \multicolumn{3}{|c|}{ Temperature, water, in ${ }^{\circ} \mathrm{C}$} & \multirow{2}{*}{$\begin{array}{l}\text { Hardness, } \\
\text { total, in } \mathrm{mg} / \mathrm{L} \\
\text { as } \mathrm{CaCO}_{3}\end{array}$} & \multirow{2}{*}{$\begin{array}{c}\text { Calcium, } \\
\text { dissolved, } \\
\text { in } \mathrm{mg} / \mathrm{L}\end{array}$} & \multirow{2}{*}{$\begin{array}{l}\text { Magnesium, } \\
\text { dissolved, } \\
\text { in } \mathrm{mg} / \mathrm{L}\end{array}$} & \multirow{2}{*}{$\begin{array}{l}\text { Potassium, } \\
\text { dissolved, } \\
\text { in } \mathrm{mg} / \mathrm{L}\end{array}$} & \multirow{2}{*}{$\begin{array}{c}\text { Sodium, } \\
\text { dissolved, } \\
\text { in } \mathrm{mg} / \mathrm{L}\end{array}$} & \multirow{2}{*}{$\begin{array}{c}\text { Acid- } \\
\text { neutralizing } \\
\text { capacity, } \\
\text { in } \mathrm{mg} / \mathrm{L} \text { as } \\
\mathrm{CaCO}_{3}\end{array}$} & \multicolumn{3}{|c|}{ Chloride, dissolved, in $\mathrm{mg} / \mathrm{L}$} \\
\hline & & Value & Q\% & N & & & & & & & Conc & $0 \%$ & $\mathrm{~N}$ \\
\hline \multirow[t]{2}{*}{63} & $8-6-2002$ & 12.0 & $55.3-57.9$ & 37 & 150 & 49 & 7.8 & 1.02 & 3.8 & 135 & 2.0 & 96.2 & 25 \\
\hline & 9-3-2002 & 14.0 & ${ }^{1} 78.9-81.6$ & 37 & 160 & 51 & 9.0 & 1.18 & 4.8 & 135 & 1.98 & 92.3 & 25 \\
\hline \multirow[t]{2}{*}{64} & 8-6-2002 & 12.7 & 48.5 & 67 & 170 & 54 & 8.5 & 1.04 & 3.8 & 150 & 1.34 & ID & 8 \\
\hline & 9-4-2002 & 12.0 & ${ }^{1} 32.4-42.6$ & 67 & 180 & 56 & 9.3 & 1.24 & 4.2 & 158 & 1.44 & ID & 8 \\
\hline \multirow[t]{2}{*}{65} & $8-6-2002$ & 21.5 & ID & 9 & 190 & 54 & 13.7 & 1.91 & 6.4 & 199 & 1.69 & ID & 2 \\
\hline & $9-4-2002$ & 15.5 & ID & 9 & 210 & 58 & 15.3 & 2.0 & 6.8 & 198 & 2.3 & ID & 2 \\
\hline 66 & 8-7-2002 & 13.0 & ${ }^{1} 34.2-42.1$ & 75 & 110 & 33 & 6.7 & 0.91 & 3.0 & 104 & 1.34 & ID & 6 \\
\hline \multirow[t]{2}{*}{67} & $8-7-2002$ & 14.5 & ${ }^{1} 25.0-26.5$ & 67 & 190 & 53 & 13.3 & 2.7 & 8.5 & 181 & 4.0 & ID & 8 \\
\hline & 9-4-2002 & 20.0 & ${ }^{1} 69.1-75.0$ & 67 & 170 & 47 & 12.6 & 2.3 & 5.7 & 158 & 3.8 & ID & 8 \\
\hline \multirow[t]{2}{*}{68} & 8-7-2002 & 12.5 & ${ }^{1} 65.7-72.9$ & 69 & 93 & 28 & 5.7 & 1.53 & 4.6 & 90 & 1.22 & ID & 17 \\
\hline & 9-5-2002 & 14.0 & ${ }^{1} 92.9-98.6$ & 69 & 100 & 31 & 6.2 & 1.49 & 4.8 & 91 & 1.65 & ID & 17 \\
\hline \multirow[t]{2}{*}{69} & 8-8-2002 & 17.0 & ID & 16 & 830 & 210 & 74 & 4.6 & 73 & 272 & 7.2 & ID & 2 \\
\hline & $9-10-2002$ & 22.0 & ID & 16 & 760 & 194 & 67 & 5.3 & 75 & 271 & 9.2 & ID & 2 \\
\hline \multirow[t]{2}{*}{70} & 8-8-2002 & 22.0 & ${ }^{1} 93.0-95.8$ & 70 & 330 & 90 & 26 & 3.4 & 46 & 143 & 7.2 & ID & 11 \\
\hline & $9-11-2002$ & 18.0 & ${ }^{1} 53.5-62.0$ & 70 & -- & -- & -- & -- & -- & -- & -- & -- & -- \\
\hline 71 & $8-7-2002$ & 13.5 & ID & 3 & 250 & 92 & 4.3 & 1.37 & 11.2 & 32 & 4.2 & ID & 3 \\
\hline 72 & 8-7-2002 & 13.0 & ${ }^{1} 29.5-34.4$ & 60 & 330 & 111 & 13.7 & 2.6 & 25 & 138 & 5.7 & ID & 13 \\
\hline \multirow[t]{2}{*}{73} & 8-13-2002 & 16.0 & ${ }^{1} 23.3-30.1$ & 72 & 800 & 224 & 58 & 3.5 & 111 & 269 & 10.8 & 82.8 & 28 \\
\hline & 9-23-2002 & 12.0 & 4.1 & 72 & 750 & 208 & 55 & 3.9 & 106 & 155 & 10.0 & 62.1 & 28 \\
\hline \multirow[t]{2}{*}{74} & 8-13-2002 & 20.0 & ${ }^{1} 72.7-75.3$ & 76 & 490 & 135 & 37 & 3.8 & 64 & 169 & 8.9 & 72.4 & 57 \\
\hline & $9-24-2002$ & 15.0 & ${ }^{1} 9.1-10.4$ & 76 & 550 & 155 & 41 & 3.8 & 74 & 127 & 10 & 82.8 & 57 \\
\hline \multirow[t]{2}{*}{75} & $8-7-2002$ & 14.7 & ID & 17 & 120 & 36 & 7.7 & 1.14 & 3.9 & 114 & 1.51 & ID & 11 \\
\hline & 9-5-2002 & 11.0 & ID & 17 & 150 & 44 & 9.1 & 1.3 & 4.2 & 131 & 1.9 & ID & 11 \\
\hline 76 & 8-6-2002 & 21.4 & ID & 3 & 140 & 39 & 9.6 & 2.4 & 8.6 & 149 & 2.2 & ID & 2 \\
\hline 77 & 8-6-2002 & 14.8 & ID & 3 & 96 & 28 & 6.2 & 0.84 & 2.0 & 94 & 0.34 & ID & 1 \\
\hline 78 & 8-8-2002 & 17.0 & ID & 4 & 180 & 47 & 14.7 & 2.5 & 19.2 & 114 & 6 & ID & 7 \\
\hline 79 & 8-6-2002 & 14.0 & ID & 15 & 160 & 53 & 7.7 & 0.88 & 2.1 & 137 & 0.41 & ID & 0 \\
\hline 80 & $8-5-2002$ & 16.3 & ID & 2 & 130 & 44 & 4.8 & -- & -- & -- & -- & -- & -- \\
\hline 81 & $8-5-2002$ & 14.9 & ID & 16 & 58 & 19.6 & 2.2 & -- & -- & -- & -- & -- & -- \\
\hline 82 & 8-5-2002 & 18.0 & ID & 2 & -- & -- & -- & -- & -- & -- & -- & -- & - \\
\hline 83 & 8-5-2002 & 18.5 & ID & 12 & 160 & 51.1 & 7.2 & 0.73 & 2.2 & 129 & 0.55 & ID & 4 \\
\hline
\end{tabular}


Table 14. Water-quality data for the Gunnison River Basin showing 2002 drought values, Colorado water-quality exceedances, and percentiles relative to historical data for selected properties and constituents from July through September, 1978-2002. - Continued

$\left[\mathrm{ft}^{3} / \mathrm{s}\right.$, cubic feet per second; Conc, concentration; $\mathrm{Q} \%$, percentile (percentage of data less than or equal to reported value or concentration) of drought-sample constituent; $\mathrm{N}$, total number of historical and drought samples; $\mathrm{mg} / \mathrm{L}$, milligrams per liter; $\mu \mathrm{S} / \mathrm{cm}$, microsiemens per centimeter at $25^{\circ}$ Celsius; ${ }^{\circ} \mathrm{C}$, degrees Celsius; E. coli,

Escherichia coli; $\mathrm{mL}$, milliliter; $\mu \mathrm{g} / \mathrm{L}$, micrograms per liter; $\mathrm{CaCO}_{3}$, calcium carbonate; $\mathrm{SiO}_{2}$, silica; $\mathrm{SO}_{4}$, sulfate; ID, insufficient number of samples (less than 20) to calculate percentile; <, less than; --, no drought-sample measurement; values or concentrations in bold type indicate 2002 Colorado water-quality exceedances; c, chronic water-quality exceedance]

\begin{tabular}{|c|c|c|c|c|c|c|c|c|c|c|c|c|c|}
\hline \multirow{2}{*}{$\begin{array}{c}\text { Site } \\
\text { number in } \\
\text { table } 1 \\
\text { and fig. } 5\end{array}$} & \multirow{2}{*}{$\begin{array}{l}\text { Sampling } \\
\text { date }\end{array}$} & \multicolumn{3}{|c|}{ Fluoride, dissolved, in $\mathrm{mg} / \mathrm{L}$} & \multicolumn{3}{|c|}{ Silica, dissolved, in $\mathrm{mg} / \mathrm{L}$ as $\mathrm{SiO}_{2}$} & \multicolumn{3}{|c|}{ Sulfate dissolved, in $\mathrm{mg} / \mathrm{L}$ as $\mathrm{SO}_{4}$} & \multicolumn{3}{|c|}{$\begin{array}{l}\text { Nitrogen, ammonia, dissolved, } \\
\text { in } \mathrm{mg} / \mathrm{L} \text { as } \mathrm{N}\end{array}$} \\
\hline & & Conc & $0 \%$ & $\mathrm{~N}$ & Conc & $0 \%$ & $\mathrm{~N}$ & Conc & $0 \%$ & $\mathrm{~N}$ & Conc & $0 \%$ & $\mathrm{~N}$ \\
\hline \multirow[t]{2}{*}{63} & $8-6-2002$ & $\overline{--}$ & $\overline{--}$ & $\overline{--}$ & 7.1 & 65.4 & 25 & 32 & 80.8 & 25 & $<0.015$ & ${ }^{2} 3.7-66.7$ & 26 \\
\hline & 9-3-2002 & -- & -- & -- & 8.2 & 96.2 & 25 & 34 & 96.2 & 25 & $<0.015$ & ${ }^{2} 3.7-66.7$ & 26 \\
\hline 64 & 8-6-2002 & -- & -- & -- & 8.0 & ID & 8 & 28 & ID & 8 & $<0.015$ & ID & 18 \\
\hline 65 & 9-4-2002 & -- & -- & -- & 19.3 & ID & 2 & 18.2 & ID & 2 & $<0.015$ & ID & 8 \\
\hline 66 & $8-7-2002$ & -- & -- & -- & 8.6 & ID & 6 & 14.6 & ID & 6 & $<0.015$ & ID & 14 \\
\hline \multirow{2}{*}{67} & $8-7-2002$ & -- & -- & -- & 20.4 & ID & 8 & 17.5 & ID & 8 & 0.009 & ID & 16 \\
\hline & 9-4-2002 & -- & -- & -- & 14.4 & ID & 8 & 16.1 & ID & 8 & $<0.015$ & ID & 16 \\
\hline \multirow[t]{2}{*}{68} & $8-7-2002$ & -- & -- & -- & 10.4 & ID & 16 & 17.3 & ID & 17 & $<0.015$ & ${ }^{2} 4.3-69.6$ & 22 \\
\hline & 9-5-2002 & -- & -- & -- & 10.5 & ID & 16 & 18.0 & ID & 17 & $<0.015$ & ${ }^{2} 4.3-69.6$ & 22 \\
\hline 69 & $8-8-2002$ & -- & -- & -- & 17.7 & ID & 2 & 646 & ID & 2 & $<0.04$ & ID & 2 \\
\hline \multirow[t]{2}{*}{73} & $8-13-2002$ & -- & -- & -- & 17.5 & ${ }^{1} 64.3-67.9$ & 27 & 743 & 89.7 & 28 & $<0.04$ & ID & 3 \\
\hline & $9-23-2002$ & 0.8 & ${ }^{1} 70.4-85.2$ & 26 & 17.5 & ${ }^{1} 64.3-67.9$ & 27 & 702 & 75.9 & 28 & -- & -- & -- \\
\hline \multirow[t]{2}{*}{74} & $8-13-2002$ & -- & -- & -- & 11.0 & ${ }^{1} 6.9-10.3$ & 57 & 433 & 72.4 & 57 & $<0.04$ & ${ }^{2} 2.3-68.2$ & 43 \\
\hline & $9-24-2002$ & -- & -- & -- & 14.7 & 65.5 & 57 & 503 & 81.0 & 57 & $<0.04$ & ${ }^{2} 2.3-68.2$ & 43 \\
\hline \multirow[t]{2}{*}{75} & 8-7-2002 & -- & -- & -- & 10.1 & ID & 11 & 15.6 & ID & 11 & $<0.015$ & ID & 17 \\
\hline & 9-5-2002 & -- & -- & -- & 11.2 & ID & 11 & 16.4 & ID & 11 & $<0.015$ & ID & 17 \\
\hline 76 & $8-6-2002$ & -- & -- & -- & 17.4 & ID & 2 & 8.0 & ID & 2 & $<0.015$ & ID & 4 \\
\hline 77 & $8-6-2002$ & -- & -- & -- & 9.6 & ID & 1 & 8.0 & ID & 1 & $<0.015$ & ID & 3 \\
\hline 78 & $8-8-2002$ & 0.3 & ID & 6 & 10.6 & ID & 7 & 94 & ID & 7 & -- & -- & -- \\
\hline 79 & $8-6-2002$ & -- & -- & -- & 6.4 & ID & 0 & 37 & ID & 0 & $<0.015$ & ID & 2 \\
\hline 80 & $8-5-2002$ & -- & -- & -- & -- & -- & -- & -- & -- & -- & $<0.015$ & ID & 2 \\
\hline 81 & $8-5-2002$ & -- & -- & -- & -- & -- & -- & -- & -- & -- & $<0.015$ & ID & 16 \\
\hline 82 & $8-5-2002$ & -- & -- & -- & -- & --. & -- & -- & -- & -- & $<0.015$ & ID & 2 \\
\hline 83 & $8-5-2002$ & -- & -- & -- & 5.8 & ID & 4 & 34 & ID & 4 & $<0.015$ & ID & 12 \\
\hline
\end{tabular}


Table 14. Water-quality data for the Gunnison River Basin showing 2002 drought values, Colorado water-quality exceedances, and percentiles relative to historical data for selected properties and constituents from July through September, 1978-2002.-Continued

$\left[\mathrm{ft}^{3} / \mathrm{s}\right.$, cubic feet per second; Conc, concentration; Q\%, percentile (percentage of data less than or equal to reported value or concentration) of drought-sample constituent; $\mathrm{N}$, total number of historical and drought samples; $\mathrm{mg} / \mathrm{L}$, milligrams per liter; $\mu \mathrm{S} / \mathrm{cm}$, microsiemens per centimeter at $25^{\circ}$ Celsius; ${ }^{\circ} \mathrm{C}$, degrees Celsius; E. coli, Escherichia coli; mL, milliliter; $\mathrm{ug} / \mathrm{L}$, micrograms per liter; $\mathrm{CaCO}_{3}$, calcium carbonate; $\mathrm{SiO}_{2}$, silica; $\mathrm{SO}_{4}$, sulfate; ID, insufficient number of samples (less than 20) to calculate percentile; <, less than; --, no drought-sample measurement; values or concentrations in bold type indicate 2002 Colorado water-quality exceedances; c, chronic water-quality exceedance]

\begin{tabular}{|c|c|c|c|c|c|c|c|c|c|c|c|c|c|}
\hline \multirow{2}{*}{$\begin{array}{l}\text { Site } \\
\text { number in } \\
\text { table } 1 \\
\text { and fig. } 5\end{array}$} & \multirow{2}{*}{$\begin{array}{l}\text { Sampling } \\
\text { date }\end{array}$} & \multicolumn{3}{|c|}{$\begin{array}{l}\text { Nitrogen, organic plus ammonia, } \\
\text { dissolved, in } \mathrm{mg} / \mathrm{L} \text { as } \mathrm{N}\end{array}$} & \multicolumn{3}{|c|}{$\begin{array}{l}\text { Nitrogen, organic plus ammonia, total } \\
\text { recoverable, in } \mathrm{mg} / \mathrm{L} \text { as } \mathrm{N}\end{array}$} & \multicolumn{3}{|c|}{$\begin{array}{l}\text { Nitrogen, nitrite plus nitrate, dissolved, } \\
\text { in } \mathrm{mg} / \mathrm{L} \text { as } \mathrm{N}\end{array}$} & \multicolumn{3}{|c|}{$\begin{array}{l}\text { Nitrogen, nitrite, dissolved, } \\
\text { in } \mathrm{mg} / \mathrm{L} \text { as } \mathrm{N}\end{array}$} \\
\hline & & Conc & $0 \%$ & $N$ & Conc & $0 \%$ & $\mathrm{~N}$ & Conc & $0 \%$ & $\mathrm{~N}$ & Conc & $0 \%$ & $\mathrm{~N}$ \\
\hline \multirow[t]{2}{*}{63} & 8-6-2002 & 0.07 & ${ }^{2} 3.7-96.3$ & 26 & 0.1 & ${ }^{2} 3.8-96.2$ & 25 & 0.049 & ${ }^{2} 3.7-40.7$ & 26 & 0.002 & ${ }^{2} 3.7-88.9$ & 26 \\
\hline & $9-3-2002$ & 0.06 & ${ }^{2} 3.7-96.3$ & 26 & 0.08 & ${ }^{2} 3.8-96.2$ & 25 & 0.027 & ${ }^{2} 3.7-40.7$ & 26 & $<0.002$ & ${ }^{2} 3.7-88.9$ & 26 \\
\hline \multirow[t]{2}{*}{64} & 8-6-2002 & ID & ID & 18 & 0.11 & ID & 17 & 0.016 & ID & 18 & $<0.002$ & ID & 18 \\
\hline & 9-4-2002 & ID & ID & 18 & 0.08 & ID & 17 & 0.011 & ID & 18 & $<0.002$ & ID & 18 \\
\hline \multirow[t]{2}{*}{65} & 8-6-2002 & ID & ID & 8 & 0.23 & ID & 8 & $<0.013$ & ID & 8 & $<0.002$ & ID & 8 \\
\hline & 9-4-2002 & ID & ID & 8 & 0.17 & ID & 8 & $<0.013$ & ID & 8 & $<0.002$ & ID & 8 \\
\hline 66 & $8-7-2002$ & ID & ID & 14 & 0.13 & ID & 14 & 0.012 & ID & 14 & $<0.002$ & ID & 14 \\
\hline \multirow[t]{2}{*}{67} & $8-7-2002$ & ID & ID & 16 & 0.41 & ID & 16 & 0.020 & ID & 16 & 0.002 & ID & 16 \\
\hline & 9-4-2002 & ID & ID & 16 & 0.22 & ID & 16 & $<0.013$ & ID & 16 & $<0.002$ & ID & 16 \\
\hline \multirow[t]{2}{*}{68} & $8-7-2002$ & 0.11 & ${ }^{2} 4.8-95.2$ & 20 & -- & -- & -- & $<0.013$ & ${ }^{2} 4.3-65.2$ & 22 & $<0.002$ & ${ }^{2} 4.3-95.7$ & 22 \\
\hline & 9-5-2002 & 0.12 & ${ }^{2} 4.8-95.2$ & 20 & -- & -. & -. & $<0.013$ & ${ }^{2} 4.3-65.2$ & 22 & $<0.002$ & ${ }^{2} 4.3-95.7$ & 22 \\
\hline \multirow[t]{2}{*}{69} & $8-8-2002$ & ID & ID & 2 & -- & -- & -- & $<0.05$ & ID & 2 & $<0.008$ & ID & 2 \\
\hline & $9-10-2002$ & ID & ID & 2 & -- & -- & -- & 0.083 & ID & 2 & 0.017 & ID & 2 \\
\hline \multirow[t]{2}{*}{70} & 8-8-2002 & -- & -- & -- & -- & -- & -- & -- & -- & -- & -- & -- & -- \\
\hline & $9-11-2002$ & -- & -- & -- & -- & -- & -- & -- & -- & -- & -- & -- & -- \\
\hline 71 & $8-7-2002$ & ID & ID & 3 & 0.08 & ID & 3 & 0.265 & ID & 3 & $<0.002$ & ID & 3 \\
\hline 72 & $8-7-2002$ & ID & ID & 13 & 0.29 & ID & 13 & 0.219 & ID & 13 & 0.004 & ID & 13 \\
\hline \multirow[t]{2}{*}{73} & 8-13-2002 & ID & ID & 2 & -- & -- & -- & 4.0 & ID & 10 & 0.013 & ID & 3 \\
\hline & $9-23-2002$ & -- & -- & -- & -- & -- & -- & -- & -- & -- & -- & -- & -- \\
\hline \multirow[t]{2}{*}{74} & $8-13-2002$ & 0.28 & 50.0 & 23 & -- & -- & -- & 1.46 & 68.2 & 43 & 0.006 & ${ }^{2} 2.9-55.9$ & 33 \\
\hline & $9-24-2002$ & 0.27 & 45.8 & 23 & -- & -- & -- & 1.58 & 75.0 & 43 & 0.005 & ${ }^{2} 2.9-55.9$ & 33 \\
\hline \multirow[t]{2}{*}{75} & $8-7-2002$ & ID & ID & 17 & 0.17 & ID & 17 & 0.037 & ID & 17 & $<0.002$ & ID & 17 \\
\hline & 9-5-2002 & ID & ID & 17 & 0.12 & ID & 17 & 0.078 & ID & 17 & 0.002 & ID & 17 \\
\hline 76 & 8-6-2002 & ID & ID & 4 & 0.27 & ID & 4 & $<0.013$ & ID & 4 & $<0.002$ & ID & 4 \\
\hline 77 & 8-6-2002 & ID & ID & 3 & 0.1 & ID & 3 & 0.021 & ID & 3 & $<0.002$ & ID & 3 \\
\hline 78 & 8-8-2002 & -- & -- & -- & -- & -- & -- & -- & -- & -- & -- & -- & -- \\
\hline 79 & 8-6-2002 & ID & ID & 2 & 0.11 & ID & 2 & 0.023 & ID & 2 & $<0.002$ & ID & 15 \\
\hline 80 & $8-5-2002$ & ID & ID & 2 & 0.08 & ID & 2 & 0.026 & ID & 2 & $<0.002$ & ID & 2 \\
\hline 81 & $8-5-2002$ & ID & ID & 16 & $<0.1$ & ID & 16 & 0.045 & ID & 16 & $<0.002$ & ID & 16 \\
\hline 82 & $8-5-2002$ & ID & ID & 2 & 0.48 & ID & 2 & 2.7 & ID & 2 & 0.021 & ID & 2 \\
\hline 83 & $8-5-2002$ & ID & ID & 12 & 0.11 & ID & 12 & $<0.013$ & ID & 12 & $<0.002$ & ID & 12 \\
\hline
\end{tabular}


Table 14. Water-quality data for the Gunnison River Basin showing 2002 drought values, Colorado water-quality exceedances, and percentiles relative to historical data for selected properties and constituents from July through September, 1978-2002.-Continued

$\left[\mathrm{ft}^{3} / \mathrm{s}\right.$, cubic feet per second; Conc, concentration; $\mathrm{Q} \%$, percentile (percentage of data less than or equal to reported value or concentration) of drought-sample constituent; N, total number of historical and drought samples; $\mathrm{mg} / \mathrm{L}$, milligrams per liter; $\mu \mathrm{S} / \mathrm{cm}$, microsiemens per centimeter at $25^{\circ}$ Celsius; ${ }^{\circ} \mathrm{C}$, degrees Celsius; E. coli, Escherichia coli; $\mathrm{mL}$, milliliter; $\mu \mathrm{g} / \mathrm{L}$, micrograms per liter; $\mathrm{CaCO}{ }_{3}$, calcium carbonate; $\mathrm{SiO}_{2}$, silica; $\mathrm{SO}_{4}$, sulfate; ID, insufficient number of samples (less than 20) to calculate percentile; <, less than; --, no drought-sample measurement; values or concentrations in bold type indicate 2002 Colorado water-quality exceedances; c, chronic water-quality exceedance]

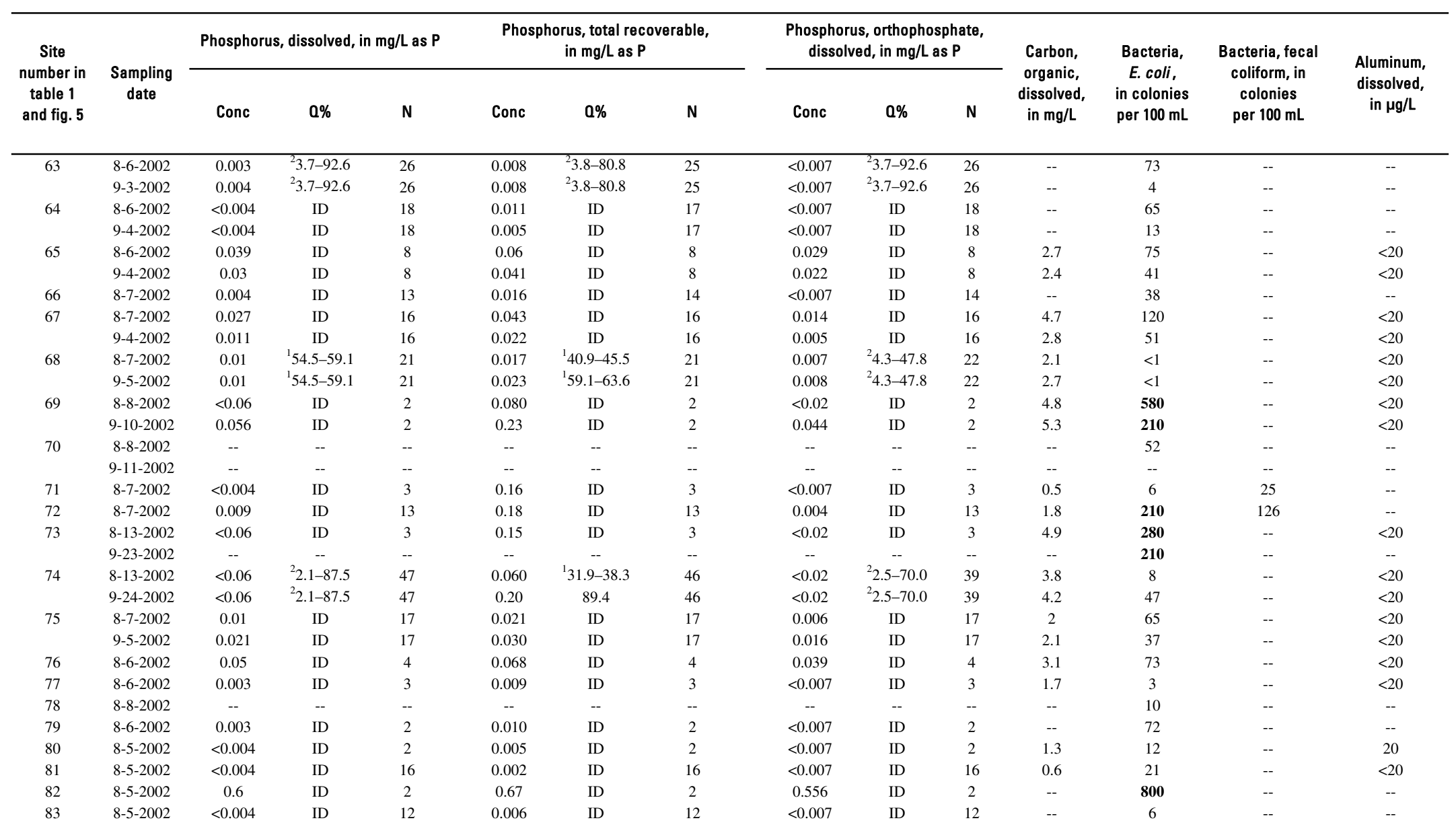


Table 14. Water-quality data for the Gunnison River Basin showing 2002 drought values, Colorado water-quality exceedances, and percentiles relative to historical data for selected properties and constituents from July through September, 1978-2002. - Continued

[ft $3 / \mathrm{s}$, cubic feet per second; Conc, concentration; Q\%, percentile (percentage of data less than or equal to reported value or concentration) of drought-sample constituent; N, total number of historical and drought samples; $\mathrm{mg} / \mathrm{L}$, milligrams per liter; $\mu \mathrm{S} / \mathrm{cm}$, microsiemens per centimeter at $25^{\circ}$ Celsius; ${ }^{\circ} \mathrm{C}$, degrees Celsius; E. coli, Escherichia coli $\mathrm{mL}$, milliliter; $\mu \mathrm{g} / \mathrm{L}$, micrograms per liter; $\mathrm{CaCO}_{3}$, calcium carbonate; $\mathrm{SiO}_{2}$, silica; $\mathrm{SO}_{4}$, sulfate; ID, insufficient number of samples (less than 20) to calculate percentile; <, less than; --, no drought-sample measurement; values or concentrations in bold type indicate 2002 Colorado water-quality exceedances; c, chronic water-quality exceedance]

\begin{tabular}{|c|c|c|c|c|c|c|c|c|c|c|c|c|c|c|}
\hline \multirow{2}{*}{$\begin{array}{c}\text { Site } \\
\text { number in } \\
\text { table } 1 \\
\text { and fig. } 5\end{array}$} & \multirow{2}{*}{$\begin{array}{l}\text { Sampling } \\
\text { date }\end{array}$} & \multicolumn{3}{|c|}{ Cadmium, dissolved, in $\mathrm{mg} / \mathrm{L}$} & \multicolumn{3}{|c|}{ Copper, dissolved, in $\mu \mathrm{g} / \mathrm{L}$} & \multicolumn{3}{|c|}{ Iron, dissolved, in $\mu \mathrm{g} / \mathrm{L}$} & \multirow{2}{*}{$\begin{array}{l}\text { Iron, total } \\
\text { recoverable, } \\
\text { in } \mu \mathrm{g} / \mathrm{L}\end{array}$} & \multicolumn{3}{|c|}{ Lead, dissolved, in $\mu \mathrm{g} / \mathrm{L}$} \\
\hline & & Conc & $0 \%$ & $\mathrm{~N}$ & Conc & $0 \%$ & $\mathrm{~N}$ & Conc & $0 \%$ & $\mathrm{~N}$ & & Conc & $0 \%$ & $\mathrm{~N}$ \\
\hline \multirow[t]{2}{*}{63} & 8-6-2002 & -- & -- & -- & -- & -- & -- & 6 & ${ }^{2} 4.0-40.0$ & 24 & -- & -- & -- & -- \\
\hline & 9-3-2002 & -- & -- & -- & -- & -- & -- & 6 & ${ }^{2} 4.0-40.0$ & 24 & -- & -- & -- & -- \\
\hline \multirow[t]{2}{*}{64} & 8-6-2002 & -- & -- & -- & -- & -- & -- & 8 & ID & 9 & -- & -- & -- & -- \\
\hline & $9-4-2002$ & -- & -- & -- & -- & -- & -- & 10 & ID & 9 & -- & -- & -- & -- \\
\hline \multirow[t]{2}{*}{65} & 8-6-2002 & $<0.04$ & ID & 3 & 0.5 & ID & 3 & 33 & ID & 3 & -- & $<0.08$ & ID & 3 \\
\hline & $9-4-2002$ & $<0.04$ & ID & 3 & 0.6 & ID & 3 & 15 & ID & 3 & -- & $<0.08$ & ID & 3 \\
\hline 66 & 8-7-2002 & -- & -- & -- & -- & -- & -- & 9 & ID & 5 & -- & -- & -- & -- \\
\hline \multirow[t]{2}{*}{67} & 8-7-2002 & $<0.1$ & ID & 12 & 0.8 & ID & 12 & 48 & ID & 8 & -- & $<1$ & ID & 12 \\
\hline & $9-4-2002$ & $<0.04$ & ID & 12 & 0.6 & ID & 12 & 26 & ID & 8 & -- & 0.09 & ID & 12 \\
\hline \multirow[t]{2}{*}{68} & 8-7-2002 & $<0.04$ & ID & 6 & 0.7 & ID & 6 & $<10$ & ID & 15 & -- & 0.04 & ID & 6 \\
\hline & 9-5-2002 & $<0.04$ & ID & 6 & 0.7 & ID & 6 & $<10$ & ID & 15 & -- & 0.11 & ID & 6 \\
\hline \multirow[t]{2}{*}{69} & 8-8-2002 & 0.02 & ID & 2 & 2.7 & ID & 2 & 7 & ID & 2 & -- & $<0.08$ & ID & 2 \\
\hline & $9-10-2002$ & $<0.04$ & ID & 2 & 2.8 & ID & 2 & $<10$ & ID & 2 & -- & $<0.08$ & ID & 2 \\
\hline \multirow[t]{2}{*}{70} & 8-8-2002 & -- & -- & -- & -- & -- & -- & -- & -- & -- & -- & -- & -- & -- \\
\hline & $9-11-2002$ & -- & -- & -- & -- & -- & -- & -- & -- & -- & -- & -- & -- & -- \\
\hline 71 & 8-7-2002 & 0.3 & ID & 3 & 2.9 & ID & 3 & -- & -- & -- & $4,050 \mathrm{c}$ & $<1$ & ID & 3 \\
\hline 72 & 8-7-2002 & 0.1 & ID & 13 & 2.3 & ID & 13 & -- & -- & -- & $2,640 \mathrm{c}$ & $<1$ & ID & 13 \\
\hline \multirow[t]{2}{*}{73} & 8-13-2002 & 0.03 & ID & 7 & 4.2 & ID & 7 & $<10$ & ID & 3 & -- & 0.06 & ID & 7 \\
\hline & $9-23-2002$ & -- & -- & -- & -- & -- & -- & -- & -- & -- & -- & -- & -- & -- \\
\hline \multirow[t]{2}{*}{74} & 8-13-2002 & $<0.04$ & ${ }^{2} 3.8-92.3$ & 25 & 2.7 & 61.5 & 25 & $<10$ & ${ }^{2} 2.3-65.1$ & 42 & -- & $<0.08$ & ${ }^{2} 3.8-88.5$ & 25 \\
\hline & 9-24-2002 & 0.02 & ${ }^{2} 3.8-92.3$ & 25 & 4 & ${ }^{1} 73.1-80.8$ & 25 & $<10$ & ${ }^{2} 2.3-65.1$ & 42 & -- & $<0.08$ & ${ }^{2} 3.8-88.5$ & 25 \\
\hline \multirow[t]{2}{*}{75} & 8-7-2002 & $<0.2$ & ID & 9 & $<3$ & ID & 9 & 13 & ID & 15 & -- & 3 & ID & 9 \\
\hline & 9-5-2002 & $<0.04$ & ID & 9 & 0.5 & ID & 9 & 19 & ID & 15 & -- & $<0.08$ & ID & 9 \\
\hline 76 & 8-6-2002 & $<0.1$ & ID & 2 & 0.7 & ID & 2 & 45 & ID & 3 & -- & $<1$ & ID & 2 \\
\hline 77 & 8-6-2002 & $<0.1$ & ID & 2 & 1 & ID & 2 & 76 & ID & 2 & -- & 1 & ID & 2 \\
\hline 78 & 8-8-2002 & -- & -- & -- & -- & -- & -- & -- & -- & -- & -- & -- & -- & -- \\
\hline 79 & 8-6-2002 & -- & -- & -- & -- & -- & -- & 8 & ID & 2 & -- & -- & -- & -- \\
\hline 80 & $8-5-2002$ & 0.3 & ID & 2 & $<1$ & ID & 2 & 7 & ID & 2 & -- & $<1$ & ID & 2 \\
\hline 81 & 8-5-2002 & 0.1 & ID & 8 & 0.6 & ID & 8 & 17 & ID & 9 & -- & 1 & ID & 8 \\
\hline 82 & 8-5-2002 & -- & -- & -- & -- & -- & -- & -- & -- & -- & -- & -- & -- & -- \\
\hline 83 & 8-5-2002 & -- & -- & -- & -- & -- & -- & 38 & ID & 2 & -- & -- & -- & -- \\
\hline
\end{tabular}


Table 14. Water-quality data for the Gunnison River Basin showing 2002 drought values, Colorado water-quality exceedances, and percentiles relative to historical data for selected properties and constituents from July through September, 1978-2002.-Continued

$\left[\mathrm{ft}^{3} / \mathrm{s}\right.$, cubic feet per second; Conc, concentration; Q\%, percentile (percentage of data less than or equal to reported value or concentration) of drought-sample constituent; $\mathrm{N}$, total number of historical and drought samples; $\mathrm{mg} / \mathrm{L}$, milligrams per liter; $\mu \mathrm{S} / \mathrm{cm}$, microsiemens per centimeter at $25^{\circ} \mathrm{Celsius} ;{ }^{\circ} \mathrm{C}$, degrees Celsius; E. coli, Escherichia coli; $\mathrm{mL}$, milliliter; $\mu \mathrm{g} / \mathrm{L}$, micrograms per liter; CaCO ${ }_{3}$, calcium carbonate; $\mathrm{SiO}_{2}$, silica; $\mathrm{SO}_{4}$, sulfate; ID, insufficient number of samples (less than 20) to calculate percentile; <, less than; --, no drought-sample measurement; values or concentrations in bold type indicate 2002 Colorado water-quality exceedances; c, chronic water-quality exceedance]

\begin{tabular}{|c|c|c|c|c|c|c|c|c|c|c|c|c|c|c|c|}
\hline \multirow{2}{*}{$\begin{array}{c}\text { Site } \\
\text { number in } \\
\text { table } 1 \\
\text { and fig. } 5\end{array}$} & \multirow{2}{*}{$\begin{array}{l}\text { Sampling } \\
\text { date }\end{array}$} & \multicolumn{3}{|c|}{ Manganese, dissolved, in $\mu \mathrm{g} / \mathrm{L}$} & \multirow{2}{*}{$\begin{array}{c}\text { Manganese, } \\
\text { total } \\
\text { recoverable, } \\
\text { in } \mu \mathrm{g} / \mathrm{L}\end{array}$} & \multirow{2}{*}{$\begin{array}{l}\text { Mercury, } \\
\text { dissolved, } \\
\text { in } \mu \mathrm{g} / \mathrm{L}\end{array}$} & \multicolumn{3}{|c|}{ Selenium, dissolved, in $\boldsymbol{\mu g} / \mathrm{L}$} & \multicolumn{3}{|c|}{ Silver, dissolved, in $\boldsymbol{\mu g} / \mathbf{L}$} & \multicolumn{3}{|c|}{ Zinc, dissolved, in $\mu \mathrm{g} / \mathrm{L}$} \\
\hline & & Conc & $0 \%$ & $\mathrm{~N}$ & & & Conc & $0 \%$ & $\mathrm{~N}$ & Conc & $0 \%$ & $\mathrm{~N}$ & Conc & $0 \%$ & $\mathrm{~N}$ \\
\hline \multirow[t]{2}{*}{63} & $8-6-2002$ & -- & -- & -- & -- & -- & -- & -- & -- & -- & -- & -- & -- & -- & - \\
\hline & $9-3-2002$ & -- & -- & -- & -- & -- & -- & -- & -- & -- & -- & -- & -- & -- & -- \\
\hline \multirow[t]{2}{*}{64} & 8-6-2002 & -- & -- & -- & -- & -- & -- & -- & -- & -- & -- & -- & -- & -- & -- \\
\hline & 9-4-2002 & -- & -- & -- & -- & -- & -- & -- & -- & -- & -- & -- & -- & -- & -- \\
\hline \multirow[t]{2}{*}{65} & 8-6-2002 & 27 & ID & 3 & -- & -- & -- & -- & -- & $<1$ & ID & 3 & $<1$ & ID & 3 \\
\hline & 9-4-2002 & 26 & ID & 3 & -- & -- & -- & -- & -- & $<1$ & ID & 3 & $<1$ & ID & 3 \\
\hline 66 & $8-7-2002$ & -- & -- & -- & -- & -- & -- & -- & -- & -- & -- & -- & -- & -- & -- \\
\hline \multirow[t]{2}{*}{67} & $8-7-2002$ & $57 \mathrm{c}$ & ID & 11 & -- & -- & -- & -- & -- & $<0.1$ & ID & 7 & $<24$ & ID & 12 \\
\hline & 9-4-2002 & 10.6 & ID & 11 & .- & -. & -- & -- & -. & $<1$ & ID & 7 & $<1$ & ID & 12 \\
\hline \multirow[t]{2}{*}{68} & $8-7-2002$ & 1.5 & ID & 16 & -- & -- & $<0.3$ & ID & 9 & $<1$ & ID & 4 & $<1$ & ID & 6 \\
\hline & 9-5-2002 & $<2$ & ID & 16 & -- & -- & $<0.3$ & ID & 9 & $<1$ & ID & 4 & 1 & ID & 6 \\
\hline \multirow[t]{2}{*}{69} & $8-8-2002$ & 88 & ID & 2 & -- & -- & 4 & ID & 2 & $<1$ & ID & 2 & 3 & ID & 2 \\
\hline & $9-10-2002$ & 94 & ID & 2 & -- & -- & 3 & ID & 2 & $<1$ & ID & 2 & 2 & ID & 2 \\
\hline \multirow[t]{2}{*}{70} & $8-8-2002$ & -- & -- & -- & -- & -- & $5 c$ & ID & 14 & -- & -- & -- & -- & -- & -. \\
\hline & $9-11-2002$ & -- & -- & -- & -- & -- & $7 \mathrm{c}$ & ID & 14 & -. & -- & -- & -- & -- & -. \\
\hline 71 & $8-7-2002$ & 350 & ID & 3 & 422 & $<0.01$ & $<2$ & ID & 3 & $<0.1$ & ID & 3 & 65 & ID & 3 \\
\hline 72 & $8-7-2002$ & 36 & ID & 13 & 198 & $<0.01$ & $<2$ & ID & 12 & $<0.1$ & ID & 13 & $<24$ & ID & 13 \\
\hline \multirow[t]{2}{*}{73} & 8-13-2002 & 48 & ID & 6 & -- & -- & 10 & ${ }^{1} 21.4-28.6$ & 27 & $<1$ & ID & 4 & 4 & ID & 7 \\
\hline & 9-23-2002 & -- & -- & -- & -- & -. & 12 & ${ }^{1} 50.0-71.4$ & 27 & -- & -- & -- & -. & -- & -- \\
\hline \multirow[t]{2}{*}{74} & $8-13-2002$ & 16.9 & 79.1 & 42 & -- & -- & 6 & ${ }^{1} 40.4-46.2$ & 51 & $<1$ & ${ }^{2} 3.3-93.3$ & 29 & 2 & ${ }^{2} 3.8-80.8$ & 25 \\
\hline & $9-24-2002$ & 8.4 & ${ }^{2} 2.3-48.8$ & 42 & -- & -. & $8 \mathrm{c}$ & ${ }^{1} 71.2-80.8$ & 51 & $<1$ & ${ }^{2} 3.3-93.3$ & 29 & 2 & ${ }^{2} 3.8-80.8$ & 25 \\
\hline \multirow[t]{2}{*}{75} & $8-7-2002$ & 14.4 & ID & 15 & -- & -- & $<0.3$ & ID & 4 & $<0.2$ & ID & 7 & $<24$ & ID & 9 \\
\hline & 9-5-2002 & 25 & ID & 15 & -- & -- & -- & -- & -- & $<1$ & ID & 7 & 5 & ID & 9 \\
\hline 76 & 8-6-2002 & 29 & ID & 3 & -- & -- & -- & -- & -- & $<0.1$ & ID & 2 & $<24$ & ID & 2 \\
\hline 77 & 8-6-2002 & 1.8 & ID & 2 & -- & -- & -- & -- & -- & $<0.1$ & ID & 2 & $<24$ & ID & 2 \\
\hline 78 & $8-8-2002$ & -- & -- & -- & -- & -- & 0.5 & ID & 5 & -- & -- & -- & -- & -- & . \\
\hline 79 & 8-6-2002 & -- & -- & -- & -- & -- & -- & -- & -- & -- & -- & -- & -- & -- & .. \\
\hline 80 & $8-5-2002$ & 13.8 & ID & 2 & -- & -- & -- & -- & -- & $<0.1$ & ID & 2 & 45 & ID & 2 \\
\hline 81 & $8-5-2002$ & 7.3 & ID & 9 & -- & -- & -- & -- & -- & $<0.1$ & ID & 5 & $<24$ & ID & 8 \\
\hline 82 & $8-5-2002$ & -- & -- & -- & -- & -- & -- & -- & -- & -- & -- & -- & -- & -- & . \\
\hline 83 & $8-5-2002$ & -- & -- & -- & -- & -- & -- & -- & -- & -- & -- & -- & -- & -- & - \\
\hline
\end{tabular}

${ }^{1}$ Possible percentile range because of uncensored ties in data.

${ }^{2}$ Possible percentile range because of censored or recensored ties in data. 
Table 15. Water-quality data for the Dolores River Basin showing 2002 drought values, Colorado water-quality exceedances, and percentiles relative to historical data for selected properties and constituents from July through September 1978-2002.

$\left[\mathrm{ft} \mathrm{t}^{3} / \mathrm{s}\right.$, cubic feet per second; Conc, concentration; Q\%, percentile (percentage of data less than or equal to reported value or concentration) of drought-sample constituent; N, total number of historical and drought samples; $\mathrm{mg} / \mathrm{L}$, milligrams per liter; $\mu \mathrm{S} / \mathrm{cm}$, microsiemens per centimeter at $25^{\circ} \mathrm{Celsius;}{ }^{\circ} \mathrm{C}$, degrees Celsius; E. coli, Escherichia coli; $\mathrm{mL}$, milliliter; $\mu \mathrm{g} / \mathrm{L}$, micrograms per liter; $\mathrm{CaCO}_{3}$, calcium carbonate; $\mathrm{SiO}_{2}$, silica; $\mathrm{SO}_{4}$, sulfate; ID, insufficient number of samples (less than 20) to calculate percentile; <, less than; --, no drought-sample measurement; values or concentrations in bold type indicate 2002 Colorado water-quality exceedances]

\begin{tabular}{|c|c|c|c|c|c|c|c|}
\hline \multirow{2}{*}{$\begin{array}{l}\text { Site number } \\
\text { in table } 1 \\
\text { and fig. } 5\end{array}$} & \multirow{2}{*}{$\begin{array}{l}\text { Sampling } \\
\text { date }\end{array}$} & \multirow{2}{*}{$\begin{array}{l}\text { Sampling } \\
\text { time }\end{array}$} & \multirow{2}{*}{$\begin{array}{c}\text { Discharge, } \\
\text { in } \mathrm{ft}^{3} / \mathrm{s}\end{array}$} & \multirow{2}{*}{$\begin{array}{l}\text { Oxygen, } \\
\text { dissolved, } \\
\text { in } \mathrm{mg} / \mathrm{L}\end{array}$} & \multicolumn{3}{|c|}{$\mathrm{pH}$, in standard units } \\
\hline & & & & & Value & $0 \%$ & $\mathrm{~N}$ \\
\hline 84 & $8-8-2002$ & 1000 & 58 & 7.5 & 8.3 & ID & 1 \\
\hline \multirow[t]{2}{*}{85} & $8-5-2002$ & 1215 & 2.5 & 7.4 & 8.5 & ${ }^{1} 80.9-95.7$ & 46 \\
\hline & $9-26-2002$ & 1130 & 9.5 & 8.7 & 8.5 & ${ }^{1} 80.9-95.7$ & 46 \\
\hline \multirow[t]{2}{*}{86} & $8-5-2002$ & 1415 & 2.1 & 8.6 & 8.6 & ${ }^{1} 95.7-97.9$ & 46 \\
\hline & $9-26-2002$ & 1445 & 9.8 & 7.8 & 8.5 & ${ }^{1} 85.1-93.6$ & 46 \\
\hline \multirow[t]{2}{*}{87} & $8-6-2002$ & 850 & 47 & 7.9 & 8.3 & ID & 2 \\
\hline & $9-25-2002$ & 1230 & 88 & 9.2 & 8.3 & ID & 2 \\
\hline \multirow[t]{2}{*}{88} & $8-6-2002$ & 1130 & 9.4 & 7.8 & 8.3 & ID & 7 \\
\hline & $9-25-2002$ & 1645 & 49 & 8.1 & 8.6 & ID & 7 \\
\hline
\end{tabular}

\begin{tabular}{|c|c|c|c|c|c|c|c|}
\hline \multirow{2}{*}{$\begin{array}{l}\text { Site number } \\
\text { in table } 1 \\
\text { and fig. } 5\end{array}$} & \multirow{2}{*}{$\begin{array}{c}\text { Sampling } \\
\text { date }\end{array}$} & \multicolumn{3}{|c|}{ Specific conductance, in $\mu \mathrm{S} / \mathrm{cm}$} & \multicolumn{3}{|c|}{ Temperature, water, in ${ }^{\circ} \mathrm{C}$} \\
\hline & & Value & $0 \%$ & $\mathrm{~N}$ & Value & $0 \%$ & $\mathrm{~N}$ \\
\hline 84 & $8-8-2002$ & 385 & 93.6 & 46 & 17.6 & ${ }^{1} 75.5-77.6$ & 48 \\
\hline \multirow[t]{2}{*}{85} & $8-5-2002$ & 545 & 28.6 & 76 & 24.0 & ${ }^{1} 76.6-81.8$ & 76 \\
\hline & $9-26-2002$ & 1,790 & 90.9 & 76 & 18.5 & 15.6 & 76 \\
\hline \multirow[t]{2}{*}{86} & $8-5-2002$ & 9,960 & 87.5 & 71 & 24.5 & ${ }^{1} 79.2-80.6$ & 71 \\
\hline & $9-26-2002$ & 3,990 & 81.9 & 71 & 23.0 & ${ }^{1} 61.1-68.1$ & 71 \\
\hline \multirow[t]{2}{*}{87} & $8-6-2002$ & 398 & 93.0 & 56 & 13.0 & ${ }^{1} 43.9-52.6$ & 56 \\
\hline & $9-25-2002$ & 367 & 80.7 & 56 & 10.0 & ${ }^{\mathrm{l}} 14.0-19.3$ & 56 \\
\hline \multirow[t]{2}{*}{88} & $8-6-2002$ & 1,700 & ${ }^{1} 92.9-94.6$ & 55 & 22.0 & ${ }^{1} 53.6-71.4$ & 55 \\
\hline & $9-25-2002$ & 1,130 & ${ }^{1} 80.4-82.1$ & 55 & 17.5 & 10.7 & 55 \\
\hline
\end{tabular}

\begin{tabular}{|c|c|c|c|c|c|c|c|}
\hline $\begin{array}{l}\text { Site number } \\
\text { in table } 1 \\
\text { and fig. } 5\end{array}$ & $\begin{array}{c}\text { Sampling } \\
\text { date }\end{array}$ & $\begin{array}{l}\text { Hardness, } \\
\text { total, in } \mathrm{mg} / \mathrm{L} \\
\text { as } \mathrm{CaCO}_{3}\end{array}$ & $\begin{array}{l}\text { Calcium, } \\
\text { dissolved, } \\
\text { in } \mathrm{mg} / \mathrm{L}\end{array}$ & $\begin{array}{l}\text { Magnesium, } \\
\text { dissolved, } \\
\text { in } \mathrm{mg} / \mathrm{L}\end{array}$ & $\begin{array}{l}\text { Potassium, } \\
\text { dissolved, } \\
\text { in } \mathrm{mg} / \mathrm{L}\end{array}$ & $\begin{array}{l}\text { Sodium, } \\
\text { dissolved, } \\
\text { in } \mathrm{mg} / \mathrm{L}\end{array}$ & $\begin{array}{c}\text { Acid-neutralizing } \\
\text { capacity, } \\
\text { in } \mathrm{mg} / \mathrm{L} \text { as } \mathrm{CaCO}_{3}\end{array}$ \\
\hline 84 & $8-8-2002$ & 160 & 49 & 8.7 & 3.1 & 15.8 & 113 \\
\hline \multirow[t]{2}{*}{85} & $8-5-2002$ & 180 & 38 & 22 & 4.6 & 43 & 108 \\
\hline & $9-26-2002$ & 330 & 94 & 22 & 9.7 & 217 & 158 \\
\hline \multirow[t]{2}{*}{86} & $8-5-2002$ & 430 & 65 & 64 & 100 & 1,890 & 100 \\
\hline & $9-26-2002$ & 440 & 117 & 35 & 31.6 & 641 & 159 \\
\hline \multirow[t]{2}{*}{87} & $8-6-2002$ & 200 & 67 & 6.9 & 1.1 & 4.9 & 115 \\
\hline & $9-25-2002$ & 180 & 61 & 6.4 & 0.88 & 4.7 & 134 \\
\hline \multirow[t]{2}{*}{88} & $8-6-2002$ & 900 & 214 & 89 & 5.0 & 54 & 110 \\
\hline & $9-25-2002$ & 600 & 155 & 51 & 3.2 & 31 & 156 \\
\hline
\end{tabular}


Table 15. Water-quality data for the Dolores River Basin showing 2002 drought values, Colorado water-quality exceedances, and percentiles relative to historical data for selected properties and constituents from July through September 1978-2002.—Continued

[ft $3 / \mathrm{s}$, cubic feet per second; Conc, concentration; Q\%, percentile (percentage of data less than or equal to reported value or concentration) of drought-sample constituent; $\mathrm{N}$, total number of historical and drought samples; $\mathrm{mg} / \mathrm{L}$, milligrams per liter; $\mu \mathrm{S} / \mathrm{cm}$, microsiemens per centimeter at $25^{\circ} \mathrm{Celsius;}{ }^{\circ} \mathrm{C}$, degrees Celsius; E. coli, Escherichia coli; $\mathrm{mL}$, milliliter; $\mu \mathrm{g} / \mathrm{L}$, micrograms per liter; $\mathrm{CaCO}_{3}$, calcium carbonate; $\mathrm{SiO}_{2}$, silica; $\mathrm{SO}_{4}$, sulfate; $\mathrm{ID}$, insufficient number of samples (less than 20) to calculate percentile; <, less than; --, no drought-sample measurement; values or concentrations in bold type indicate 2002 Colorado water-quality exceedances]

\begin{tabular}{|c|c|c|c|c|c|c|c|}
\hline \multirow{2}{*}{$\begin{array}{c}\text { Site number } \\
\text { in table } 1 \\
\text { and fig. } 5\end{array}$} & \multirow{2}{*}{$\begin{array}{c}\text { Sampling } \\
\text { date }\end{array}$} & \multicolumn{3}{|c|}{ Chloride, dissolved, in $\mathrm{mg} / \mathrm{L}$} & \multicolumn{3}{|c|}{ Fluoride, dissolved, in $\mathrm{mg} / \mathrm{L}$} \\
\hline & & Conc & $0 \%$ & $\mathrm{~N}$ & Conc & $0 \%$ & $\mathrm{~N}$ \\
\hline 84 & $8-8-2002$ & 19.9 & ID & 1 & -- & -- & -- \\
\hline \multirow[t]{2}{*}{85} & $8-5-2002$ & 56 & 24.0 & 49 & 0.20 & ${ }^{1} 48.0-82.0$ & 49 \\
\hline & $9-26-2002$ & 371 & 98.0 & 49 & 0.24 & 84.0 & 49 \\
\hline \multirow[t]{2}{*}{86} & $8-5-2002$ & 3,130 & 98.0 & 49 & 0.23 & 78.0 & 49 \\
\hline & $9-26-2002$ & 1,050 & 92.0 & 49 & 0.26 & 80.0 & 49 \\
\hline \multirow[t]{2}{*}{87} & $8-6-2002$ & 3.5 & ID & 2 & -- & -- & -- \\
\hline & $9-25-2002$ & 2.8 & ID & 2 & -- & -- & -- \\
\hline \multirow[t]{2}{*}{88} & $8-6-2002$ & 9.1 & ID & 3 & -- & -- & -- \\
\hline & $9-25-2002$ & 6.9 & ID & 3 & -- & -- & -- \\
\hline
\end{tabular}

\begin{tabular}{|c|c|c|c|c|c|c|c|}
\hline \multirow{2}{*}{$\begin{array}{c}\text { Site number } \\
\text { in table } 1 \\
\text { and fig. } 5\end{array}$} & \multirow{2}{*}{$\begin{array}{c}\text { Sampling } \\
\text { date }\end{array}$} & \multicolumn{3}{|c|}{ Silica, dissolved, in $\mathrm{mg} / \mathrm{L}$ as $\mathrm{SiO}_{2}$} & \multicolumn{3}{|c|}{ Sulfate dissolved, in $\mathrm{mg} / \mathrm{L}_{\text {as }} \mathrm{SO}_{4}$} \\
\hline & & Conc & $0 \%$ & $\mathrm{~N}$ & Conc & $0 \%$ & $\mathrm{~N}$ \\
\hline 84 & $8-8-2002$ & 7.6 & ID & 1 & 41 & ID & 1 \\
\hline \multirow[t]{2}{*}{85} & $8-5-2002$ & 4.8 & 74.0 & 49 & 21 & 2.0 & 49 \\
\hline & $9-26-2002$ & 7.2 & 94.0 & 49 & 180 & 82.0 & 49 \\
\hline \multirow[t]{2}{*}{86} & $8-5-2002$ & 1.05 & 2.0 & 49 & 187 & 80.0 & 49 \\
\hline & $9-26-2002$ & 7.0 & 96.0 & 49 & 264 & 92.0 & 49 \\
\hline \multirow[t]{2}{*}{87} & $8-6-2002$ & 8.5 & ID & 2 & 108 & ID & 2 \\
\hline & $9-25-2002$ & 7.7 & ID & 2 & 100 & ID & 2 \\
\hline \multirow[t]{2}{*}{88} & $8-6-2002$ & 5.8 & ID & 3 & 823 & ID & 3 \\
\hline & $9-25-2002$ & 7.9 & ID & 3 & 480 & ID & 3 \\
\hline
\end{tabular}

\begin{tabular}{|c|c|c|c|c|c|c|c|}
\hline $\begin{array}{l}\text { Site number } \\
\text { in table } 1 \\
\text { and fig. } 5\end{array}$ & $\begin{array}{c}\text { Sampling } \\
\text { date }\end{array}$ & $\begin{array}{l}\text { Nitrogen, } \\
\text { ammonia, } \\
\text { dissolved, } \\
\text { in } \mathrm{mg} / \mathrm{L} \text { as } \mathrm{N}\end{array}$ & $\begin{array}{c}\text { Nitrogen, nitrite } \\
\text { plus nitrate, } \\
\text { dissolved, } \\
\text { in } \mathrm{mg} / \mathrm{L} \text { as } \mathrm{N}\end{array}$ & $\begin{array}{c}\text { Nitrogen, nitrite, } \\
\text { dissolved, } \\
\text { in } \mathrm{mg} / \mathrm{L} \text { as } \mathrm{N}\end{array}$ & $\begin{array}{l}\text { Bacteria, } \\
\text { E. coli, } \\
\text { in colonies } \\
\text { per } 100 \mathrm{~mL}\end{array}$ & $\begin{array}{l}\text { Cadmium, } \\
\text { dissolved, } \\
\text { in } \mu \mathrm{g} / \mathrm{L}\end{array}$ & $\begin{array}{l}\text { Copper, } \\
\text { dissolved, } \\
\text { in } \mu \mathrm{g} / \mathrm{L}\end{array}$ \\
\hline 84 & $8-8-2002$ & 0.008 & $<0.013$ & $<0.002$ & 67 & $<0.04$ & 0.7 \\
\hline \multirow[t]{2}{*}{85} & $8-5-2002$ & $<0.04$ & $<0.05$ & $<0.008$ & 60 & -- & -- \\
\hline & $9-26-2002$ & -- & -- & -- & 32 & -- & -- \\
\hline \multirow[t]{2}{*}{86} & $8-5-2002$ & 0.11 & 0.05 & 0.011 & 17 & $<0.1$ & 2 \\
\hline & $9-26-2002$ & $<0.04$ & 0.23 & 0.009 & 43 & $<0.07$ & 3.9 \\
\hline \multirow[t]{2}{*}{87} & $8-6-2002$ & $<0.04$ & 0.43 & $<0.008$ & 210 & -- & -- \\
\hline & $9-25-2002$ & 0.009 & 0.25 & 0.002 & 5 & -- & -- \\
\hline \multirow[t]{2}{*}{88} & $8-6-2002$ & $<0.04$ & $<0.05$ & $<0.008$ & 30 & 0.03 & 3.4 \\
\hline & $9-25-2002$ & 0.014 & $<0.013$ & $<0.002$ & 13 & $<0.04$ & 4.3 \\
\hline
\end{tabular}


Table 15. Water-quality data for the Dolores River Basin showing 2002 drought values, Colorado water-quality exceedances, and percentiles relative to historical data for selected properties and constituents from July through September 1978-2002.—Continued

[ft $t^{3} / \mathrm{s}$, cubic feet per second; Conc, concentration; $\mathrm{Q} \%$, percentile (percentage of data less than or equal to reported value or concentration) of drought-sample constituent; $\mathrm{N}$, total number of historical and drought samples; $\mathrm{mg} / \mathrm{L}$, milligrams per liter; $\mu \mathrm{S} / \mathrm{cm}$, microsiemens per centimeter at $25^{\circ} \mathrm{Celsius;}{ }^{\circ} \mathrm{C}$, degrees Celsius; E. coli, Escherichia coli; $\mathrm{mL}$, milliliter; $\mu \mathrm{g} / \mathrm{L}$, micrograms per liter; $\mathrm{CaCO}_{3}$, calcium carbonate; $\mathrm{SiO}_{2}$, silica; $\mathrm{SO}_{4}$, sulfate; ID, insufficient number of samples (less than 20) to calculate percentile; <, less than; --, no drought-sample measurement; values or concentrations in bold type indicate 2002 Colorado water-quality exceedances]

\begin{tabular}{ccccccc}
\hline $\begin{array}{c}\text { Site number } \\
\text { in table 1 } \\
\text { and fig. } 5\end{array}$ & $\begin{array}{c}\text { Sampling } \\
\text { date }\end{array}$ & $\begin{array}{c}\text { Iron, } \\
\text { dissolved, } \\
\text { in } \mu \text { g/L }\end{array}$ & $\begin{array}{c}\text { Lead, } \\
\text { dissolved, } \\
\text { in } \mu \mathrm{g} / \mathrm{L}\end{array}$ & $\begin{array}{c}\text { Manganese, } \\
\text { dissolved, } \\
\text { in } \mu \mathrm{g} / \mathrm{L}\end{array}$ & $\begin{array}{c}\text { Silver, } \\
\text { dissolved, } \\
\text { in } \mu \mathrm{g} / \mathrm{L}\end{array}$ & $\begin{array}{c}\text { Zinc, } \\
\text { dissolved, } \\
\text { in } \mu \mathrm{g} / \mathrm{L}\end{array}$ \\
\hline 84 & $8-8-2002$ & 18 & $<0.08$ & 8.9 & $<1$ & $<1$ \\
85 & $8-5-2002$ & $<10$ & -- & -- & -- & -- \\
& $9-26-2002$ & -- & -- & -- & -- & - \\
86 & $8-5-2002$ & $<100$ & $<0.3$ & 21 & $<4$ & 6 \\
87 & $9-26-2002$ & $<30$ & $<0.2$ & 26 & $<2$ & $<2$ \\
8 & $8-6-2002$ & $<10$ & -- & -- & -- & -- \\
& $9-25-2002$ & 7 & -- & -- & -- & - \\
& $8-6-2002$ & 12 & 0.08 & 56 & $<1$ & 7 \\
& $9-25-2002$ & 5 & 0.05 & 42 & $<1$ & 2 \\
\hline
\end{tabular}

${ }^{1}$ Possible percentile range because of uncensored ties in data. 
Table 16. Water-quality data for the San Juan River Basin showing 2002 drought values, Colorado water-quality exceedances, and percentiles relative to historical data for selected properties and constituents from July through September,1978-2002.

$\left[\mathrm{ft}^{3} / \mathrm{s}\right.$, cubic feet per second; Conc, concentration; Q\%, percentile (percentage of data less than or equal to reported value or concentration) of drought-sample constituent; $\mathrm{N}$, total number of historical and drought samples; $\mathrm{mg} / \mathrm{L}$, milligrams per liter; $\mu \mathrm{S} / \mathrm{cm}$, microsiemens per centimeter at $25^{\circ} \mathrm{Celsius} ;{ }^{\circ} \mathrm{C}$, degrees Celsius; $E$. coli, Escherichia coli; $\mathrm{mL}$, milliliter; $\mu \mathrm{g} / \mathrm{L}$, micrograms per liter; $\mathrm{CaCO}_{3}$, calcium carbonate; $\mathrm{SiO}_{2}$, silica; $\mathrm{SO}_{4}$, sulfate; ID, insufficient number of samples (less than 20) to calculate percentile; <, less than; --, no drought-sample measurement; values or concentrations in bold type indicate 2002 Colorado water-quality exceedances; c, chronic water-quality exceedance]

\begin{tabular}{|c|c|c|c|c|c|c|c|c|c|c|}
\hline \multirow{2}{*}{$\begin{array}{c}\text { Site number } \\
\text { in table } 1 \\
\text { and fig. } 5\end{array}$} & \multirow{2}{*}{$\begin{array}{l}\text { Sampling } \\
\text { date }\end{array}$} & \multirow{2}{*}{$\begin{array}{l}\text { Sampling } \\
\text { time }\end{array}$} & \multirow{2}{*}{$\begin{array}{c}\text { Discharge, } \\
\text { in } \mathrm{ft}^{3} / \mathrm{s}\end{array}$} & \multirow{2}{*}{$\begin{array}{c}\text { Oxygen, } \\
\text { dissolved, } \\
\text { in } \mathrm{mg} / \mathrm{L}\end{array}$} & \multicolumn{3}{|c|}{$\mathrm{pH}$, in standard units } & \multicolumn{3}{|c|}{ Specific conductance, in $\mu \mathrm{S} / \mathrm{cm}$} \\
\hline & & & & & Value & $0 \%$ & $\mathrm{~N}$ & Value & $0 \%$ & $\mathrm{~N}$ \\
\hline \multirow[t]{2}{*}{89} & $8-5-2002$ & 1400 & 24 & 7.2 & 8.4 & ID & 2 & 585 & 96.0 & 49 \\
\hline & $9-6-2002$ & 1230 & 6.8 & 8.8 & 8.4 & ID & 2 & 626 & 98.0 & 49 \\
\hline 90 & $7-30-2002$ & 1000 & 27 & 8.5 & 7.6 & ${ }^{1} 69.7-78.8$ & 32 & 74 & 90.0 & 39 \\
\hline \multirow[t]{2}{*}{91} & $8-2-2002$ & 1000 & 11 & 8.9 & 8.4 & ID & 6 & 337 & 97.6 & 41 \\
\hline & $9-19-2002$ & 1430 & 48 & 8.2 & 8.7 & ID & 6 & 304 & 95.2 & 41 \\
\hline 92 & $7-31-2002$ & 1030 & 74 & 8.1 & 6.3 & ID & 2 & 496 & ID & 9 \\
\hline \multirow[t]{2}{*}{93} & $8-1-2002$ & 0945 & 132 & 7.8 & 8.1 & ID & 2 & 881 & ID & 8 \\
\hline & $9-3-2002$ & 1015 & 119 & 8.6 & 8.2 & ID & 2 & 898 & ID & 8 \\
\hline \multirow[t]{2}{*}{94} & $8-2-2002$ & 1230 & 4.8 & 7.3 & 8.6 & ID & 11 & 568 & ID & 11 \\
\hline & $9-4-2002$ & 1600 & 1.9 & 6.7 & 8.6 & ID & 11 & 478 & ID & 11 \\
\hline \multirow[t]{2}{*}{95} & $8-8-2002$ & 1430 & 1.8 & 7.8 & 8.5 & ID & 14 & 911 & ID & 14 \\
\hline & $9-4-2002$ & 1030 & 0.93 & 8.8 & 8.4 & ID & 14 & 889 & ID & 14 \\
\hline \multirow[t]{2}{*}{96} & $8-8-2002$ & 1215 & 7.9 & 7.0 & 8.3 & ID & 2 & 239 & ID & 2 \\
\hline & $9-4-2002$ & 1320 & 1.1 & 7.4 & 8.3 & ID & 2 & 302 & ID & 2 \\
\hline \multirow[t]{2}{*}{97} & $8-7-2002$ & 0945 & 5.4 & 6.9 & 8.3 & ${ }^{1} 69.6-91.3$ & 22 & 2,360 & 79.4 & 33 \\
\hline & $9-18-2002$ & 1045 & 0.81 & 8.6 & 8.2 & ${ }^{1} 17.4-65.2$ & 22 & 4,870 & 97.1 & 33 \\
\hline \multirow[t]{2}{*}{98} & $8-7-2002$ & 1130 & 16 & 10.0 & 8.4 & ${ }^{1} 77.1-94.3$ & 34 & 1,890 & 94.7 & 37 \\
\hline & $9-10-2002$ & 1115 & 9.5 & 7.2 & 8.2 & ${ }^{1} 5.7-22.9$ & 34 & 2,680 & 97.4 & 37 \\
\hline \multirow[t]{2}{*}{99} & $8-7-2002$ & 1400 & 4.5 & 8.1 & 8.3 & ${ }^{1} 60.3-77.8$ & 62 & 2,660 & 92.5 & 79 \\
\hline & $9-10-2002$ & 1430 & 13 & 6.7 & 8.2 & ${ }^{1} 31.7-58.7$ & 62 & 2,760 & 93.8 & 79 \\
\hline \multirow[t]{2}{*}{100} & $8-1-2002$ & 1445 & 106 & 8.0 & 8.3 & ID & 3 & 797 & ID & 3 \\
\hline & $9-3-2002$ & 1545 & 86 & 10.3 & 8.6 & ID & 3 & 783 & ID & 3 \\
\hline \multirow[t]{2}{*}{101} & $8-1-2002$ & 1215 & 128 & 7.8 & 8.6 & ID & 2 & 834 & ID & 2 \\
\hline & $9-3-2002$ & 1245 & 118 & 10.9 & 8.5 & ID & 2 & 858 & ID & 2 \\
\hline \multirow[t]{2}{*}{102} & $8-5-2002$ & 1115 & 16 & 7.2 & 8.5 & ID & 2 & 943 & ID & 2 \\
\hline & $9-6-2002$ & 0945 & 6.6 & 10.7 & 8.5 & ID & 2 & 1,480 & ID & 2 \\
\hline
\end{tabular}


Table 16. Water-quality data for the San Juan River Basin showing 2002 drought values, Colorado water-quality exceedances, and percentiles relative to historical data for selected properties and constituents from July through September,1978-2002. - Continued

$\left[\mathrm{ft}^{3} / \mathrm{s}\right.$, cubic feet per second; Conc, concentration; Q\%, percentile (percentage of data less than or equal to reported value or concentration) of drought-sample constituent; $\mathrm{N}$, total number of historical and drought samples; $\mathrm{mg} / \mathrm{L}$, milligrams per liter; $\mu \mathrm{S} / \mathrm{cm}$, microsiemens per centimeter at $25^{\circ}$ Celsius; ${ }^{\circ} \mathrm{C}$, degrees Celsius; E. coli, Escherichia coli; $\mathrm{mL}$, milliliter; $\mu \mathrm{g} / \mathrm{L}$, micrograms per liter; $\mathrm{CaCO}_{3}$, calcium carbonate; $\mathrm{SiO}_{2}$, silica; $\mathrm{SO}_{4}$, sulfate; ID, insufficient number of samples (less than 20) to calculate percentile; <, less than; --, no droughtsample measurement; values or concentrations in bold type indicate 2002 Colorado water-quality exceedances; c, chronic water-quality exceedance]

\begin{tabular}{|c|c|c|c|c|c|c|c|c|c|c|c|}
\hline \multirow{2}{*}{$\begin{array}{c}\text { Site number } \\
\text { in table } 1 \\
\text { and fig. } 5\end{array}$} & \multirow{2}{*}{$\begin{array}{c}\text { Sampling } \\
\text { date }\end{array}$} & \multicolumn{3}{|c|}{ Temperature, water, in ${ }^{\circ} \mathrm{C}$} & \multirow{2}{*}{$\begin{array}{l}\text { Hardness, } \\
\text { total, in } \mathrm{mg} / \mathrm{L} \\
\text { as } \mathrm{CaCO}_{3}\end{array}$} & \multirow{2}{*}{$\begin{array}{l}\text { Calcium, } \\
\text { dissolved, } \\
\text { in } \mathrm{mg} / \mathrm{L}\end{array}$} & \multirow{2}{*}{$\begin{array}{l}\text { Magnesium, } \\
\text { dissolved, } \\
\text { in } \mathrm{mg} / \mathrm{L}\end{array}$} & \multirow{2}{*}{$\begin{array}{c}\text { Potassium, } \\
\text { dissolved, } \\
\text { in } \mathrm{mg} / \mathrm{L}\end{array}$} & \multirow{2}{*}{$\begin{array}{l}\text { Sodium, } \\
\text { dissolved, } \\
\text { in } \mathrm{mg} / \mathrm{L}\end{array}$} & \multirow{2}{*}{$\begin{array}{c}\text { Alkalinity, } \\
\text { laboratory, } \\
\text { in } \mathrm{mg} / \mathrm{L} \text { as } \\
\mathrm{CaCO}_{3}\end{array}$} & \multirow{2}{*}{$\begin{array}{c}\text { Alkalinity, } \\
\text { onsite, } \\
\text { in } \mathrm{mg} / \mathrm{L} \text { as } \\
\mathrm{CaCO}_{3}\end{array}$} \\
\hline & & Value & $0 \%$ & $\mathrm{~N}$ & & & & & & & \\
\hline \multirow[t]{2}{*}{89} & $8-5-2002$ & 26.2 & 96.1 & 50 & 220 & 71 & 10.5 & 3.6 & 33 & 129 & -- \\
\hline & $9-6-2002$ & 20.2 & 62.7 & 50 & 250 & 81 & 12.3 & 3.7 & 36 & 126 & -- \\
\hline 90 & $7-30-2002$ & 12.0 & ${ }^{1} 81.1-90.6$ & 52 & 30 & 8.8 & 2.0 & 0.60 & 0.97 & -- & 22 \\
\hline \multirow{2}{*}{91} & $8-2-2002$ & 20.2 & 67.4 & 42 & 140 & 42 & 7.8 & 3.2 & 19.1 & 164 & -- \\
\hline & $9-19-2002$ & 18.1 & 48.8 & 42 & 130 & 42 & 6.1 & 2.6 & 13.3 & 143 & -- \\
\hline 92 & $7-31-2002$ & 11.5 & ID & 9 & 240 & 88 & 5.3 & 0.88 & 3.3 & -- & 6 \\
\hline \multirow{2}{*}{93} & $8-1-2002$ & 17.2 & ID & 8 & 350 & 113 & 17.4 & 6.4 & 46 & 197 & -- \\
\hline & $9-3-2002$ & 16.0 & ID & 8 & 360 & 115 & 17.6 & 6.5 & 47 & 188 & -- \\
\hline \multirow[t]{2}{*}{94} & $8-2-2002$ & 23.2 & ID & 11 & 230 & 70 & 12.8 & 5.7 & 37 & 274 & -- \\
\hline & $9-4-2002$ & 23.4 & ID & 11 & 170 & 46 & 13.1 & 2.7 & 40 & 199 & -- \\
\hline \multirow[t]{2}{*}{95} & $8-8-2002$ & 27.7 & 97.1 & 22 & 400 & 77 & 49 & 1.55 & 44 & 180 & -- \\
\hline & $9-4-2002$ & 17.7 & 47.1 & 22 & 390 & 81 & 46 & 1.31 & 45 & 177 & -- \\
\hline \multirow[t]{2}{*}{96} & $8-8-2002$ & 18.4 & ID & 2 & 110 & 34 & 5.7 & 0.91 & 4.2 & 68 & -- \\
\hline & $9-4-2002$ & 20.7 & ID & 2 & 140 & 41 & 8.6 & 1.15 & 6.9 & 78 & -- \\
\hline \multirow[t]{2}{*}{97} & $8-7-2002$ & 18.5 & ${ }^{1} 22.9-28.6$ & 34 & 1,200 & 293 & 125 & 5.1 & 119 & 242 & -- \\
\hline & $9-18-2002$ & 12.7 & 8.6 & 34 & 2,100 & 376 & 292 & 8.6 & 486 & 215 & -- \\
\hline \multirow[t]{2}{*}{98} & 8-7-2002 & 22.0 & 86.8 & 37 & 950 & 210 & 102 & 5.0 & 94 & 203 & -- \\
\hline & $9-10-2002$ & 19.0 & 34.2 & 37 & 1,400 & 305 & 144 & 7.1 & 169 & 207 & -- \\
\hline \multirow[t]{2}{*}{99} & $8-7-2002$ & 27.0 & 98.8 & 80 & 1,300 & 269 & 163 & 7.2 & 174 & 298 & -- \\
\hline & $9-10-2002$ & 22.3 & ${ }^{1} 88.9-90.1$ & 80 & 1,300 & 297 & 142 & 7.8 & 184 & 235 & -- \\
\hline \multirow[t]{2}{*}{100} & 8-1-2002 & 22.0 & ID & 3 & -- & -- & -- & -- & -- & -- & -- \\
\hline & $9-3-2002$ & 19.8 & ID & 3 & -- & -- & -- & -- & -- & -- & -- \\
\hline \multirow[t]{2}{*}{101} & $8-1-2002$ & 19.0 & ID & 2 & -- & -- & -- & -- & -- & -- & -- \\
\hline & $9-3-2002$ & 17.3 & ID & 2 & -- & -- & -- & -- & -- & -- & -- \\
\hline \multirow[t]{2}{*}{102} & $8-5-2002$ & 24.6 & ID & 2 & 200 & 60 & 12.3 & 12.7 & 113 & 197 & -- \\
\hline & $9-6-2002$ & 17.3 & ID & 2 & 280 & 86 & 14.9 & 21 & 206 & 265 & -- \\
\hline
\end{tabular}


Table 16. Water-quality data for the San Juan River Basin showing 2002 drought values, Colorado water-quality exceedances, and percentiles relative to historical data for selected properties and constituents from July through September,1978-2002.-Continued

[ $\mathrm{ft}^{3} / \mathrm{s}$, cubic feet per second; Conc, concentration; $\mathrm{Q} \%$, percentile (percentage of data less than or equal to reported value or concentration) of drought-sample constituent; $\mathrm{N}$, total number of historical and drought samples; $\mathrm{mg} / \mathrm{L}$, milligrams per liter; $\mu \mathrm{S} / \mathrm{cm}$, microsiemens per centimeter at $25^{\circ}$ Celsius; ${ }^{\circ} \mathrm{C}$, degrees Celsius; E. coli, Escherichia coli; $\mathrm{mL}, \mathrm{milliliter} ; \mu \mathrm{g} / \mathrm{L}$, micrograms per liter; $\mathrm{CaCO}{ }_{3}$, calcium carbonate; $\mathrm{SiO}_{2}$, silica; $\mathrm{SO}_{4}$, sulfate; ID, insufficient number of samples (less than 20) to calculate percentile; <, less than; --, no drought-sample measurement; values or concentrations in bold type indicate 2002 Colorado water-quality exceedances; c, chronic water-quality exceedance]

\begin{tabular}{|c|c|c|c|c|c|c|c|c|c|c|c|c|c|}
\hline \multirow{2}{*}{$\begin{array}{c}\text { Site number } \\
\text { in table } 1 \\
\text { and fig. } 5\end{array}$} & \multirow{2}{*}{$\begin{array}{l}\text { Sampling } \\
\text { date }\end{array}$} & \multicolumn{3}{|c|}{ Chloride, dissolved, in $\mathrm{mg} / \mathrm{L}$} & \multicolumn{3}{|c|}{ Fluoride, dissolved, in $\mathrm{mg} / \mathrm{L}$} & \multicolumn{3}{|c|}{ Silica, dissolved, in $\mathrm{mg} / \mathrm{L}$ as $\mathrm{SiO}_{2}$} & \multicolumn{3}{|c|}{ Sulfate, dissolved, in $\mathrm{mg} / \mathrm{L}$ as $\mathrm{SO}_{4}$} \\
\hline & & Conc & $0 \%$ & $\mathrm{~N}$ & Conc & $0 \%$ & $\mathrm{~N}$ & Conc & $0 \%$ & $\mathrm{~N}$ & Conc & $0 \%$ & $\mathrm{~N}$ \\
\hline \multirow[t]{2}{*}{89} & $8-5-2002$ & 8.2 & ID & 2 & -- & -- & -- & 16.8 & ID & 2 & 155 & 33.3 & 2 \\
\hline & $9-6-2002$ & 9.0 & ID & 2 & -- & -- & -- & 16.5 & ID & 2 & 167 & 66.7 & 2 \\
\hline 90 & $7-30-2002$ & 0.25 & 64.4 & 44 & -- & -- & -- & 3.7 & 88.9 & 44 & 10.7 & 91.1 & 44 \\
\hline \multirow[t]{2}{*}{91} & $8-2-2002$ & 3.3 & ID & 6 & -- & -- & -- & 4.6 & ID & 5 & 14.6 & 71.4 & 6 \\
\hline & $9-19-2002$ & 3.2 & ID & 6 & -- & -- & -- & 7.3 & ID & 5 & 16.1 & 85.7 & 6 \\
\hline 92 & $7-31-2002$ & 0.69 & ID & 2 & -- & -- & -- & 15.2 & ID & 2 & 235 & ID & 2 \\
\hline \multirow[t]{2}{*}{93} & $8-1-2002$ & 51 & ID & 2 & -- & -- & -- & 9.5 & ID & 2 & 167 & 60.0 & 2 \\
\hline & $9-3-2002$ & 52 & ID & 2 & -- & -- & -- & 11.4 & ID & 2 & 171 & ID & 2 \\
\hline \multirow[t]{2}{*}{94} & $8-2-2002$ & 11 & ID & 1 & -- & -- & -- & 10.8 & ID & 1 & 28 & ID & 1 \\
\hline & $9-4-2002$ & 12.4 & ID & 1 & -- & -- & -- & 6.1 & ID & 1 & 25 & ID & 1 \\
\hline \multirow[t]{2}{*}{95} & 8-8-2002 & 22 & ID & 14 & -- & -- & -- & 14.7 & ID & 14 & 276 & ID & 14 \\
\hline & $9-4-2002$ & 21 & ID & 14 & -- & -- & -- & 13.8 & ID & 14 & 263 & ID & 14 \\
\hline \multirow[t]{2}{*}{96} & $8-8-2002$ & 0.5 & ID & 2 & -- & -- & -- & 8.7 & ID & 2 & 50 & ID & 2 \\
\hline & $9-4-2002$ & 0.9 & ID & 2 & -- & -- & -- & 9.2 & ID & 2 & 71 & ID & 2 \\
\hline \multirow[t]{2}{*}{97} & $8-7-2002$ & 27 & 92.0 & 24 & 0.31 & 60.0 & 24 & 10.3 & ${ }^{1} 29.2-33.3$ & 23 & 1,190 & 88.0 & 24 \\
\hline & $9-18-2002$ & 71 & 96.0 & 24 & 0.43 & 92.0 & 24 & 7.1 & 4.2 & 23 & 2,800 & 96.0 & 24 \\
\hline \multirow[t]{2}{*}{98} & $8-7-2002$ & 25 & 92.1 & 37 & 0.33 & 68.4 & 37 & 7.8 & 5.3 & 37 & 901 & 94.7 & 37 \\
\hline & $9-10-2002$ & 34 & 97.4 & 37 & 0.45 & 97.4 & 37 & 10.7 & 21.1 & 37 & 1,410 & 97.4 & 37 \\
\hline \multirow[t]{2}{*}{99} & $8-7-2002$ & 35 & 84.8 & 65 & 0.36 & 30.8 & 64 & 12.5 & 59.1 & 65 & 1,370 & 90.9 & 65 \\
\hline & $9-10-2002$ & 35 & 86.4 & 65 & 0.45 & 89.2 & 64 & 12.1 & 53.0 & 65 & 1,440 & 92.4 & 65 \\
\hline \multirow[t]{2}{*}{100} & $8-1-2002$ & -- & -- & -- & -- & -- & -- & -- & -- & -- & -- & -- & -- \\
\hline & $9-3-2002$ & -- & -- & -- & -- & -- & -- & -- & -- & -- & -- & -- & -- \\
\hline \multirow[t]{2}{*}{101} & $8-1-2002$ & -- & -- & -- & -- & -- & -- & -- & -- & -- & -- & -- & -- \\
\hline & $9-3-2002$ & -- & -- & -- & -- & -- & -- & -- & -- & -- & -- & -- & -- \\
\hline \multirow[t]{2}{*}{102} & $8-5-2002$ & 25 & ID & 2 & -- & -- & -- & 18.6 & ID & 2 & 236 & ID & 2 \\
\hline & $9-6-2002$ & 45 & ID & 2 & -- & -- & -- & 20 & ID & 2 & 419 & ID & 2 \\
\hline
\end{tabular}


Table 16. Water-quality data for the San Juan River Basin showing 2002 drought values, Colorado water-quality exceedances, and percentiles relative to historical data for selected properties and constituents from July through September,1978-2002.—Continued

[ft $\mathrm{f}^{3} \mathrm{~s}$, cubic feet per second; Conc, concentration; Q\%, percentile (percentage of data less than or equal to reported value or concentration) of drought-sample constituent; $\mathrm{N}$, total number of historical and drought samples; $\mathrm{mg} / \mathrm{L}$, milligrams per liter; $\mu \mathrm{S} / \mathrm{cm}$, microsiemens per centimeter at $25^{\circ}$ Celsius; ${ }^{\circ} \mathrm{C}$, degrees Celsius; E. coli, Escherichia coli; $\mathrm{mL}$, milliliter; $\mu \mathrm{g} / \mathrm{L}$, micrograms per liter; $\mathrm{CaCO}_{3}$, calcium carbonate; $\mathrm{SiO}_{2}$, silica; $\mathrm{SO}_{4}$, sulfate; ID, insufficient number of samples (less than 20) to calculate percentile; <, less than; --, no drought-sample measurement; values or concentrations in bold type indicate 2002 Colorado water-quality exceedances; c, chronic water-quality exceedance]

\begin{tabular}{|c|c|c|c|c|c|c|c|c|c|c|c|c|}
\hline \multirow{2}{*}{$\begin{array}{l}\text { Site number } \\
\text { in table } 1 \\
\text { and fig. } 5\end{array}$} & \multirow{2}{*}{$\begin{array}{l}\text { Sampling } \\
\text { date }\end{array}$} & \multicolumn{3}{|c|}{$\begin{array}{l}\text { Nitrogen, ammonia, dissolved, } \\
\text { in } \mathrm{mg} / \mathrm{L} \text { as } \mathrm{N}\end{array}$} & \multirow{2}{*}{$\begin{array}{l}\text { Nitrogen, } \\
\text { organic plus } \\
\text { ammonia, } \\
\text { dissolved, } \\
\text { in } \mathrm{mg} / \mathrm{L} \text { as } \mathrm{N}\end{array}$} & \multicolumn{3}{|c|}{$\begin{array}{l}\text { Nitrogen, organic plus ammonia, total } \\
\text { recoverable, in } \mathrm{mg} / \mathrm{L} \text { as } \mathrm{N}\end{array}$} & \multicolumn{3}{|c|}{$\begin{array}{l}\text { Nitrogen, nitrite plus nitrate, } \\
\text { dissolved, in } \mathrm{mg} / \mathrm{L} \text { as } \mathrm{N}\end{array}$} & \multirow{2}{*}{$\begin{array}{c}\text { Nitrogen, } \\
\text { nitrite, } \\
\text { in } \mathrm{mg} / \mathrm{L} \\
\text { as } \mathrm{N}\end{array}$} \\
\hline & & Conc & $0 \%$ & $N$ & & Conc & $0 \%$ & $\mathrm{~N}$ & Conc & $0 \%$ & $\mathrm{~N}$ & \\
\hline \multirow[t]{2}{*}{89} & $8-5-2002$ & $<0.015$ & ID & 2 & 0.12 & -- & -- & -- & $<0.013$ & ID & 2 & $<0.002$ \\
\hline & $9-6-2002$ & $<0.04$ & ID & 2 & 0.17 & -- & -- & -- & $<0.05$ & ID & 2 & $<0.008$ \\
\hline 90 & $7-30-2002$ & $<0.015$ & ${ }^{2} 2.8-58.3$ & 35 & 0.06 & $<0.1$ & ${ }^{2} 4.3-47.8$ & 22 & 0.143 & 94.7 & 37 & $<0.002$ \\
\hline \multirow[t]{2}{*}{91} & $8-2-2002$ & $<0.04$ & ID & 2 & 0.39 & -- & -- & -- & 0.040 & ID & 5 & $<0.008$ \\
\hline & $9-19-2002$ & $<0.015$ & ID & 2 & 0.30 & -- & -- & -- & $<0.013$ & ID & 5 & 0.002 \\
\hline 92 & $7-31-2002$ & 0.043 & ID & 1 & -- & 0.13 & ID & 1 & 0.058 & ID & 1 & 0.003 \\
\hline \multirow[t]{2}{*}{93} & $8-1-2002$ & 0.036 & ID & 2 & 0.07 & -- & -- & -- & 0.086 & ID & 2 & 0.005 \\
\hline & $9-3-2002$ & 0.028 & ID & 2 & 0.08 & -- & -- & -- & 0.172 & ID & 2 & 0.013 \\
\hline \multirow[t]{2}{*}{94} & $8-2-2002$ & $<0.04$ & ID & 1 & 0.44 & -- & -- & -- & 0.070 & ID & 1 & 0.009 \\
\hline & $9-4-2002$ & $<0.04$ & ID & 1 & 0.24 & -- & -- & -- & $<0.05$ & ID & 1 & $<0.008$ \\
\hline \multirow[t]{2}{*}{95} & $8-8-2002$ & $<0.04$ & ID & 2 & 0.33 & -- & -- & -- & 0.060 & ID & 2 & $<0.008$ \\
\hline & $9-4-2002$ & $<0.04$ & ID & 2 & 0.18 & -- & -- & -- & 0.140 & ID & 2 & $<0.008$ \\
\hline \multirow[t]{2}{*}{96} & $8-8-2002$ & $<0.015$ & ID & 2 & 0.09 & -- & -- & -- & $<0.013$ & ID & 2 & $<0.002$ \\
\hline & $9-4-2002$ & $<0.015$ & ID & 2 & 0.08 & -- & -- & -- & $<0.013$ & ID & 2 & $<0.002$ \\
\hline \multirow[t]{2}{*}{97} & $8-7-2002$ & $<0.04$ & ID & 1 & 0.53 & -- & -- & -- & 2.9 & ID & 2 & 0.010 \\
\hline & $9-18-2002$ & -- & -- & -- & -- & -- & -- & -- & -- & -- & -- & -- \\
\hline \multirow[t]{2}{*}{98} & $8-7-2002$ & $<0.04$ & ID & 1 & 0.44 & -- & -- & -- & 0.36 & ID & 2 & 0.004 \\
\hline & $9-10-2002$ & -- & -- & -- & -- & -- & -- & -- & -- & -- & -- & -- \\
\hline \multirow[t]{2}{*}{99} & $8-7-2002$ & $<0.04$ & ID & 2 & 0.45 & -- & -- & -- & 0.020 & ID & 14 & $<0.008$ \\
\hline & $9-10-2002$ & 0.16 & ID & 2 & 0.65 & -- & -- & -- & 1.49 & ID & 14 & 0.045 \\
\hline \multirow[t]{2}{*}{100} & $8-1-2002$ & $<0.04$ & ID & 3 & -- & 0.10 & ID & 3 & $<0.05$ & ID & 3 & $<0.008$ \\
\hline & $9-3-2002$ & $<0.04$ & ID & 3 & -- & 0.20 & ID & 3 & $<0.05$ & ID & 3 & $<0.008$ \\
\hline \multirow[t]{2}{*}{101} & $8-1-2002$ & $<0.04$ & ID & 2 & -- & 0.14 & ID & 2 & 0.060 & ID & 2 & 0.010 \\
\hline & $9-3-2002$ & $<0.04$ & ID & 2 & -- & 0.19 & ID & 2 & 0.200 & ID & 2 & 0.011 \\
\hline \multirow[t]{2}{*}{102} & $8-5-2002$ & 0.094 & ID & 2 & 0.37 & -- & -- & -- & 0.064 & ID & 2 & 0.024 \\
\hline & $9-6-2002$ & $<0.015$ & ID & 2 & 0.32 & -- & -- & -- & 0.258 & ID & 2 & 0.007 \\
\hline
\end{tabular}


Table 16. Water-quality data for the San Juan River Basin showing 2002 drought values, Colorado water-quality exceedances, and percentiles relative to historical data for selected properties and constituents from July through September,1978-2002.-Continued

[fts $/ \mathrm{s}$, cubic feet per second; Conc, concentration; $\mathrm{Q} \%$, percentile (percentage of data less than or equal to reported value or concentration) of drought-sample constituent; $\mathrm{N}$, total number of historical and drought samples; mg/L, milligrams per liter; $\mu \mathrm{S} / \mathrm{cm}$, microsiemens per centimeter at $25^{\circ}$ Celsius; ${ }^{\circ} \mathrm{C}$, degrees Celsius; E. coli, Escherichia coli; $\mathrm{mL}$, milliliter; $\mu \mathrm{g} / \mathrm{L}$, micrograms per liter; $\mathrm{CaCO}{ }_{3}$, calcium carbonate; $\mathrm{SiO}_{2}$, silica; $\mathrm{SO}$, sulfate; ID, insufficient number of samples (less than 20) to calculate percentile; <, less than; --, no drought-sample measurement; values or concentrations in bold type indicate 2002 Colorado water-quality exceedances; c, chronic water-quality exceedance]

\begin{tabular}{|c|c|c|c|c|c|c|c|c|c|c|c|c|c|}
\hline \multirow{2}{*}{$\begin{array}{l}\text { Site number } \\
\text { in table } 1 \\
\text { and fig. } 5\end{array}$} & \multirow{2}{*}{$\begin{array}{l}\text { Sampling } \\
\text { date }\end{array}$} & \multicolumn{3}{|c|}{$\begin{array}{l}\text { Phosphorus, dissolved, } \\
\text { in } \mathrm{mg} / \mathrm{L} \text { as } \mathrm{P}\end{array}$} & \multicolumn{3}{|c|}{$\begin{array}{c}\text { Phosphorus, total recoverable, in } \\
\mathrm{mg} / \mathrm{L} \text { as } P\end{array}$} & \multirow{2}{*}{$\begin{array}{l}\text { Phosphorus, } \\
\text { orthophosphate, } \\
\text { dissolved, } \\
\text { in } \mathrm{mg} / \mathrm{L} \text { as } \mathrm{P}\end{array}$} & \multirow{2}{*}{$\begin{array}{c}\text { Carbon, } \\
\text { organic, } \\
\text { dissolved, } \\
\text { in } \mathrm{mg} / \mathrm{L}\end{array}$} & \multirow{2}{*}{$\begin{array}{c}\text { Bacteria, } \\
\text { E. Coli, } \\
\text { in colonies per } \\
100 \mathrm{~mL}\end{array}$} & \multirow{2}{*}{$\begin{array}{c}\text { Aluminum, } \\
\text { dissolved, } \\
\text { in } \mu \mathrm{g} / \mathrm{L}\end{array}$} & \multirow{2}{*}{$\begin{array}{l}\text { Aluminum, } \\
\text { total } \\
\text { recoverable, } \\
\text { in } \mu \mathrm{g} / \mathrm{L}\end{array}$} & \multirow{2}{*}{$\begin{array}{c}\text { Cadmium, } \\
\text { dissolved, } \\
\text { in } \mu \mathrm{g} / \mathrm{L}\end{array}$} \\
\hline & & Conc & $0 \%$ & $\mathrm{~N}$ & Conc & $0 \%$ & $\mathrm{~N}$ & & & & & & \\
\hline \multirow[t]{2}{*}{89} & $8-5-2002$ & 0.003 & ID & 2 & 0.024 & ID & 2 & $<0.007$ & -- & 16 & -- & -- & -- \\
\hline & $9-6-2002$ & $<0.06$ & ID & 2 & $<0.06$ & ID & 2 & $<0.02$ & -- & 5 & -- & -- & -- \\
\hline 90 & $7-30-2002$ & $<0.004$ & ${ }^{2} 4.3-65.2$ & 22 & $<0.004$ & ${ }^{2} 3.2-51.6$ & 30 & $<0.007$ & 0.5 & -- & -- & -- & -- \\
\hline \multirow[t]{2}{*}{91} & $8-2-2002$ & 0.030 & ID & 2 & 0.060 & ID & 5 & 0.030 & 5.3 & 17 & $<20$ & -- & $<0.04$ \\
\hline & $9-19-2002$ & 0.059 & ID & 2 & 0.171 & ID & 5 & 0.046 & 4.5 & 83 & $<20$ & -- & $<0.04$ \\
\hline 92 & $7-31-2002$ & -- & -- & -- & 0.021 & ID & 1 & $<0.007$ & 0.4 & 8 & 50 & 2,060 & 1.4 \\
\hline \multirow[t]{2}{*}{93} & $8-1-2002$ & 0.003 & ID & 2 & 0.015 & ID & 2 & $<0.007$ & 0.7 & 38 & 20 & -- & 0.05 \\
\hline & $9-3-2002$ & 0.006 & ID & 2 & 0.023 & ID & 2 & $<0.007$ & 1.2 & 36 & $<20$ & -- & 0.05 \\
\hline \multirow[t]{2}{*}{94} & $8-2-2002$ & 0.070 & ID & 1 & 0.27 & ID & 1 & 0.070 & 5.8 & 140 & 30 & -- & $<0.04$ \\
\hline & $9-4-2002$ & $<0.06$ & ID & 1 & 0.100 & ID & 1 & 0.010 & 3.3 & 90 & 20 & -- & $<0.04$ \\
\hline \multirow[t]{2}{*}{95} & $8-8-2002$ & $<0.06$ & ID & 14 & $<0.06$ & ID & 14 & $<0.02$ & 3.1 & 21 & $<20$ & -- & -- \\
\hline & $9-4-2002$ & $<0.06$ & ID & 14 & $<0.06$ & ID & 14 & $<0.02$ & 2.7 & 380 & $<20$ & -- & -- \\
\hline \multirow[t]{2}{*}{96} & $8-8-2002$ & 0.002 & ID & 2 & 0.006 & ID & 2 & $<0.007$ & -- & 71 & -- & -- & -- \\
\hline & $9-4-2002$ & 0.003 & ID & 2 & 0.006 & ID & 2 & $<0.007$ & -- & 27 & -- & -- & -- \\
\hline \multirow[t]{2}{*}{97} & $8-7-2002$ & 0.050 & ID & 1 & 0.22 & ID & 1 & 0.050 & -- & 220 & -- & -- & -- \\
\hline & $9-18-2002$ & -- & -- & -- & -- & -- & -- & -- & -- & 260 & -- & -- & -- \\
\hline \multirow[t]{2}{*}{98} & $8-7-2002$ & $<0.06$ & ID & 1 & 0.030 & ID & 1 & $<0.02$ & -- & 61 & -- & -- & -- \\
\hline & $9-10-2002$ & -- & -- & -- & -- & -- & -- & -- & -- & 540 & -- & -- & -- \\
\hline \multirow[t]{2}{*}{99} & $8-7-2002$ & $<0.06$ & ID & 12 & 0.060 & ID & 12 & $<0.02$ & 7.5 & 220 & $<20$ & -- & $<0.07$ \\
\hline & $9-10-2002$ & 0.030 & ID & 12 & 1.66 & ID & 12 & 0.020 & 10 & 1,100 & $<20$ & -- & $<0.07$ \\
\hline \multirow[t]{2}{*}{100} & $8-1-2002$ & -- & -- & -- & 0.008 & ID & 3 & $<0.02$ & -- & 3 & -- & -- & -- \\
\hline & $9-3-2002$ & -- & -- & -- & 0.017 & ID & 3 & 0.010 & 1.5 & 4 & -- & -- & -- \\
\hline \multirow[t]{2}{*}{101} & $8-1-2002$ & -- & -- & -- & 0.039 & ID & 2 & 0.010 & -- & 9 & -- & -- & -- \\
\hline & $9-3-2002$ & -- & -- & -- & 0.061 & ID & 2 & 0.040 & 1.5 & 25 & -- & -- & -- \\
\hline \multirow[t]{2}{*}{102} & $8-5-2002$ & 0.107 & ID & 2 & 0.149 & ID & 2 & 0.086 & -- & 28 & -- & -- & -- \\
\hline & $9-6-2002$ & 0.141 & ID & 2 & 0.191 & ID & 2 & 0.121 & -- & 100 & -- & -- & -- \\
\hline
\end{tabular}


Table 16. Water-quality data for the San Juan River Basin showing 2002 drought values, Colorado water-quality exceedances, and percentiles relative to historical data for selected properties and constituents from July through September,1978-2002.-Continued

[ft $3 t^{3} / \mathrm{s}$, cubic feet per second; Conc, concentration; Q\%, percentile (percentage of data less than or equal to reported value or concentration) of drought-sample constituent; N, total number of historical and drought samples; $\mathrm{mg} / \mathrm{L}$, milligrams per liter; $\mu \mathrm{S} / \mathrm{cm}$, microsiemens per centimeter at $25^{\circ}$ Celsius; ${ }^{\circ} \mathrm{C}$, degrees Celsius; E. coli, Escherichia coli; $\mathrm{mL}$, milliliter; $\mu \mathrm{g} / \mathrm{L}$, micrograms per liter; $\mathrm{CaCO}{ }_{3}$, calcium carbonate; $\mathrm{SiO}_{2}$, silica; $\mathrm{SO}_{4}$, sulfate; ID, insufficient number of samples (less than 20) to calculate percentile; <, less than; --, no drought-sample measurement; values or concentrations in bold type indicate 2002 Colorado water-quality exceedances; c, chronic water-quality exceedance]

\begin{tabular}{|c|c|c|c|c|c|c|c|c|c|c|c|}
\hline $\begin{array}{c}\text { Site number } \\
\text { in table } 1 \\
\text { and fig. } 5\end{array}$ & $\begin{array}{l}\text { Sampling } \\
\text { date }\end{array}$ & $\begin{array}{l}\text { Copper, } \\
\text { dissolved, } \\
\text { in } \mu \mathrm{g} / \mathrm{L}\end{array}$ & $\begin{array}{c}\text { Copper, total } \\
\text { recoverable, } \\
\text { in } \mu \mathrm{g} / \mathrm{L}\end{array}$ & $\begin{array}{c}\text { Iron, } \\
\text { dissolved, } \\
\text { in } \mu \mathrm{g} / \mathrm{L}\end{array}$ & $\begin{array}{l}\text { Iron, total } \\
\text { recoverable, } \\
\text { in } \mu \mathrm{g} / \mathrm{L}\end{array}$ & $\begin{array}{l}\text { Lead, } \\
\text { dissolved, } \\
\text { in } \mu \mathrm{g} / \mathrm{L}\end{array}$ & $\begin{array}{c}\text { Manganese, } \\
\text { total } \\
\text { recoverable, } \\
\text { in } \mu \mathrm{g} / \mathrm{L}\end{array}$ & $\begin{array}{l}\text { Mercury, } \\
\text { dissolved, } \\
\text { in } \mu \mathrm{g} / \mathrm{L}\end{array}$ & $\begin{array}{c}\text { Selenium, } \\
\text { dissolved, } \\
\text { in } \mu \mathrm{g} / \mathrm{L}\end{array}$ & $\begin{array}{c}\text { Silver, } \\
\text { dissolved, } \\
\text { in } \mu \mathrm{g} / \mathrm{L}\end{array}$ & $\begin{array}{l}\text { Zinc, dissolved, } \\
\text { in } \mu \mathrm{g} / \mathrm{L}\end{array}$ \\
\hline \multirow[t]{2}{*}{89} & $8-5-2002$ & -- & -- & 7 & -- & -- & -- & -- & -- & -- & - \\
\hline & $9-6-2002$ & -- & -- & $<10$ & -- & -- & -- & -- & -- & -- & -- \\
\hline 90 & $7-30-2002$ & -- & -- & $<10$ & -- & -- & -- & -- & -- & -- & -- \\
\hline \multirow[t]{2}{*}{91} & $8-2-2002$ & 1.1 & -- & 28 & -- & $<0.08$ & -- & -- & -- & $<1$ & $<1$ \\
\hline & $9-19-2002$ & 0.8 & -- & 8 & -- & $<0.08$ & -- & -- & -- & $<1$ & 1 \\
\hline 92 & $7-31-2002$ & 5.1 & 10 & $1,900 \mathrm{c}$ & 3,170 & $<1$ & 953 & $<0.01$ & $<2$ & $<0.1$ & 406 \\
\hline \multirow[t]{2}{*}{93} & $8-1-2002$ & 1.6 & -- & 30 & -- & 0.09 & -- & -- & -- & $<1$ & 7 \\
\hline & $9-3-2002$ & 1.5 & -- & 17 & -- & 0.07 & -- & -- & -- & $<1$ & 7 \\
\hline \multirow[t]{2}{*}{94} & $8-2-2002$ & 1.2 & -- & $<10$ & -- & 0.07 & -- & -- & -- & $<1$ & $<1$ \\
\hline & $9-4-2002$ & 0.9 & -- & $<10$ & -- & 0.06 & -- & -- & -- & $<1$ & $<1$ \\
\hline \multirow[t]{2}{*}{95} & $8-8-2002$ & 2.0 & -- & $<10$ & -- & $<0.08$ & -- & -- & -- & $<1$ & 1 \\
\hline & $9-4-2002$ & 1.8 & -- & $<10$ & -- & $<0.08$ & -- & -- & -- & $<1$ & 2 \\
\hline \multirow[t]{2}{*}{96} & $8-8-2002$ & -- & -- & 23 & -- & -- & -- & -- & -- & -- & -- \\
\hline & $9-4-2002$ & -- & -- & 37 & -- & -- & -- & -- & -- & -- & -- \\
\hline \multirow[t]{2}{*}{97} & $8-7-2002$ & -- & -- & 30 & -- & -- & -- & -- & -- & -- & -- \\
\hline & $9-18-2002$ & -- & -- & -- & -- & -- & -- & -- & -- & -- & -- \\
\hline \multirow[t]{2}{*}{98} & $8-7-2002$ & -- & -- & $<10$ & -- & -- & -- & -- & -- & -- & -- \\
\hline & $9-10-2002$ & -- & -- & -- & -- & -- & -- & -- & -- & -- & -- \\
\hline \multirow[t]{2}{*}{99} & $8-7-2002$ & 5.0 & -- & 19 & -- & $<0.2$ & -- & -- & -- & $<2$ & 4 \\
\hline & $9-10-2002$ & 5.9 & -- & $<10$ & -- & $<0.2$ & -- & -- & -- & $<2$ & 4 \\
\hline \multirow[t]{2}{*}{100} & $8-1-2002$ & -- & -- & -- & -- & -- & -- & -- & -- & -- & -- \\
\hline & $9-3-2002$ & -- & -- & -- & -- & -- & -- & -- & -- & -- & -- \\
\hline \multirow[t]{2}{*}{101} & $8-1-2002$ & -- & -- & -- & -- & -- & -- & -- & -- & -- & -- \\
\hline & $9-3-2002$ & -- & -- & -- & -- & -- & -- & -- & -- & -- & -- \\
\hline \multirow[t]{2}{*}{102} & $8-5-2002$ & -- & -- & 56 & -- & -- & -- & -- & -- & -- & -- \\
\hline & $9-6-2002$ & -- & -- & 43 & -- & -- & -- & -- & -- & -- & -- \\
\hline
\end{tabular}

${ }^{1}$ Possible percentile range because of uncensored ties in data.

${ }^{2}$ Possible percentile range because of censored or recensored ties in data. 
Table 17. Water-quality data for the South Platte River Basin showing 2002 drought values, Colorado water-quality exceedances, and percentiles relative to historical data for selected properties and constituents from July through September, 1978-2002.

$\left[\mathrm{ft}^{3} / \mathrm{s}\right.$, cubic feet per second; Conc, concentration; $\mathrm{Q} \%$, percentile (percentage of data less than or equal to reported value or concentration) of drought-sample constituent; N, total number of historical and drought samples; $\mathrm{mg} / \mathrm{L}$, milligrams per liter; $\mu \mathrm{S} / \mathrm{cm}$, microsiemens per centimeter at $25^{\circ} \mathrm{Celsius;}{ }^{\circ} \mathrm{C}$, degrees Celsius; E. coli, Escherichia coli; $\mathrm{mL}$, milliliter; $\mu \mathrm{g} / \mathrm{L}$, micrograms per liter; $\mathrm{CaCO}_{3}$, calcium carbonate; $\mathrm{SiO}_{2}$, silica; $\mathrm{SO}_{4}$, sulfate; ID, insufficient number of samples (less than 20) to calculate percentile; <, less than; --, no drought-sample measurement; values or concentrations in bold type indicate 2002 Colorado water-quality exceedances; c, chronic water-quality exceedance; ac, acute and chronic water-quality exceedance]

\begin{tabular}{|c|c|c|c|c|c|c|c|c|c|}
\hline \multirow{2}{*}{$\begin{array}{c}\text { Site number } \\
\text { in table } 1 \\
\text { and fig. } 6\end{array}$} & \multirow{2}{*}{$\begin{array}{l}\text { Sampling } \\
\text { date }\end{array}$} & \multirow{2}{*}{$\begin{array}{l}\text { Sampling } \\
\text { time }\end{array}$} & \multirow{2}{*}{$\begin{array}{c}\text { Discharge, } \\
\text { in } \mathrm{ft}^{3} / \mathrm{s}\end{array}$} & \multicolumn{3}{|c|}{$\begin{array}{l}\text { Oxygen, dissolved, } \\
\text { in } \mathrm{mg} / \mathrm{L}\end{array}$} & \multicolumn{3}{|c|}{$\mathrm{pH}$, in standard units } \\
\hline & & & & Conc & $0 \%$ & $\mathrm{~N}$ & Value & $0 \%$ & $\mathrm{~N}$ \\
\hline \multirow[t]{2}{*}{103} & $8-5-2002$ & 1400 & 12 & 8.5 & 61.9 & 20 & 8.6 & ${ }^{1} 81.0-85.7$ & 20 \\
\hline & $9-5-2002$ & 0940 & 9.0 & 10.4 & 90.5 & 20 & 8.5 & ${ }^{1} 71.4-76.2$ & 20 \\
\hline \multirow[t]{2}{*}{104} & $8-13-2002$ & 1200 & 32 & 16.8 & 97.6 & 41 & 8.4 & ${ }^{1} 87.8-92.7$ & 40 \\
\hline & $9-10-2002$ & 0940 & 211 & 6.9 & ${ }^{1} 7.1-9.5$ & 41 & 7.9 & ${ }^{1} 7.3-19.5$ & 40 \\
\hline \multirow[t]{2}{*}{105} & $8-15-2002$ & 1020 & 58 & 8.4 & ID & 19 & 8.3 & ID & 19 \\
\hline & $9-12-2002$ & 1430 & 72 & 8 & ID & 19 & 8.1 & ID & 19 \\
\hline \multirow[t]{2}{*}{106} & $8-13-2002$ & 0900 & 86 & 6.8 & ${ }^{1} 44.0-48.0$ & 24 & 7.9 & ${ }^{1} 72.0-76.0$ & 24 \\
\hline & $9-10-2002$ & 1340 & 458 & 6.4 & ${ }^{1} 28.0-40.0$ & 24 & 7.0 & 4.0 & 24 \\
\hline \multirow[t]{2}{*}{107} & $8-5-2002$ & 1005 & 47 & 7.6 & ID & 8 & 7.8 & ID & 8 \\
\hline & $9-9-2002$ & 0850 & 22 & 7.9 & ID & 8 & 7.6 & ID & 8 \\
\hline \multirow[t]{2}{*}{108} & $8-5-2002$ & 1245 & 553 & 6.9 & 10.0 & 49 & 7.9 & ${ }^{1} 87.8-89.8$ & 48 \\
\hline & $9-9-2002$ & 1220 & 256 & 7 & ${ }^{1} 12.0-14.0$ & 49 & 9.1 & 98.0 & 48 \\
\hline \multirow[t]{2}{*}{109} & $8-5-2002$ & 1202 & 71 & 7.4 & ID & 8 & 8.0 & ID & 8 \\
\hline & $9-9-2002$ & 1102 & 49 & 7.5 & ID & 8 & 9.1 & ID & 8 \\
\hline \multirow[t]{2}{*}{110} & $8-6-2002$ & 1317 & 16 & 7.2 & ID & 8 & 7.9 & ID & 8 \\
\hline & $9-10-2002$ & 1322 & 11 & 7.9 & ID & 8 & 8.0 & ID & 8 \\
\hline \multirow[t]{2}{*}{111} & $8-8-2002$ & 1000 & 86 & 7.5 & 5.3 & 74 & 8.0 & ${ }^{1} 13.5-20.3$ & 73 \\
\hline & $9-11-2002$ & 1325 & 38 & 9.5 & 65.3 & 74 & 8.5 & ${ }^{1} 77.0-87.8$ & 73 \\
\hline \multirow[t]{2}{*}{112} & 8-8-2002 & 1150 & 30 & 7.1 & 29.2 & 23 & 8.4 & ${ }^{1} 69.6-73.9$ & 22 \\
\hline & $9-12-2002$ & 0900 & 3.4 & 6.4 & 12.5 & 23 & 8.1 & ${ }^{1} 26.1-47.8$ & 22 \\
\hline \multirow[t]{2}{*}{113} & $8-23-2002$ & 1545 & 36 & 7.6 & ${ }^{1} 8.6-15.7$ & 69 & 8.2 & ${ }^{1} 51.4-65.3$ & 71 \\
\hline & $9-12-2002$ & 1525 & 9.5 & 8.2 & ${ }^{1} 22.9-25.7$ & 69 & 8.1 & ${ }^{1} 36.1-50.0$ & 71 \\
\hline \multirow[t]{2}{*}{114} & $8-23-2002$ & 1440 & 7.4 & 12.1 & ${ }^{1} 91.3-92.8$ & 68 & 8.8 & ${ }^{1} 88.6-90.0$ & 69 \\
\hline & $9-12-2002$ & 1250 & 3.8 & 7.6 & ${ }^{1} 21.7-23.2$ & 68 & 7.9 & ${ }^{1} 7.1-11.4$ & 69 \\
\hline 115 & $8-6-2002$ & 0940 & 0.11 & 13.2 & ID & 18 & 8.4 & ID & 17 \\
\hline \multirow[t]{2}{*}{116} & $8-8-2002$ & 1030 & 113 & 7.6 & 36.0 & 36 & 7.9 & ${ }^{1} 7.5-12.5$ & 39 \\
\hline & $9-3-2002$ & 1050 & 73 & 10.8 & 36.0 & 36 & 8.1 & ${ }^{1} 50.0-65.0$ & 39 \\
\hline \multirow[t]{2}{*}{117} & $8-2-2002$ & 1145 & 86 & 11 & 90.2 & 50 & 8.4 & ${ }^{1} 61.5-82.7$ & 51 \\
\hline & $9-17-2002$ & 1125 & 192 & 8.9 & 58.8 & 50 & 8.3 & ${ }^{1} 36.5-59.6$ & 51 \\
\hline 118 & $9-19-2002$ & 1325 & 18 & 11 & ${ }^{1} 78.1-81.3$ & 31 & 8.4 & ${ }^{1} 63.3-80.0$ & 29 \\
\hline \multirow[t]{2}{*}{119} & $8-6-2002$ & 0932 & 551 & 7 & ${ }^{1} 9.7-11.3$ & 61 & 8.4 & ${ }^{1} 85.2-91.8$ & 60 \\
\hline & $9-10-2002$ & 0852 & 249 & 7.3 & ${ }^{1} 16.1-22.6$ & 61 & 9.3 & 98.4 & 60 \\
\hline \multirow[t]{2}{*}{120} & $8-22-2002$ & 1200 & 1.1 & 9.1 & ID & 2 & 8.1 & ID & 2 \\
\hline & $9-16-2002$ & 1100 & 1.5 & 9.1 & ID & 2 & 8.0 & ID & 2 \\
\hline 121 & $8-7-2002$ & 1040 & 29 & 9.5 & ID & 15 & 7.2 & ID & 16 \\
\hline & $9-4-2002$ & 1030 & 10 & 8.7 & ID & 15 & 7.4 & ID & 16 \\
\hline 122 & $8-7-2002$ & 1255 & 132 & 7.3 & ID & 8 & 8.1 & ID & 8 \\
\hline & $9-11-2002$ & 1150 & 82 & 8.6 & ID & 8 & 8.5 & ID & 8 \\
\hline 123 & $9-30-2002$ & 1030 & 0.74 & 10.1 & ID & 1 & 8.3 & ID & 1 \\
\hline 124 & 8-6-2002 & 1147 & 82 & 7.4 & ID & 8 & 8.1 & ID & 8 \\
\hline & $9-10-2002$ & 1152 & 49 & 8.1 & ID & 8 & 8.3 & ID & 8 \\
\hline 125 & $8-14-2002$ & 1040 & $<0.01$ & 7.1 & ID & 2 & 8.1 & ID & 2 \\
\hline & $9-11-2002$ & 1120 & 0.09 & 8 & ID & 2 & 7.8 & ID & 2 \\
\hline
\end{tabular}


Table 17. Water-quality data for the South Platte River Basin showing 2002 drought values, Colorado water-quality exceedances, and percentiles relative to historical data for selected properties and constituents from July through September, 1978-2002.-Continued

$\left[\mathrm{ft}^{3} / \mathrm{s}\right.$, cubic feet per second; Conc, concentration; Q\%, percentile (percentage of data less than or equal to reported value or concentration) of drought-sample constituent; $\mathrm{N}$, total number of historical and drought samples; $\mathrm{mg} / \mathrm{L}$, milligrams per liter; $\mu \mathrm{S} / \mathrm{cm}$, microsiemens per centimeter at $25^{\circ} \mathrm{Celsius;}{ }^{\circ} \mathrm{C}$, degrees Celsius; E. coli, Escherichia coli; $\mathrm{mL}$, milliliter; $\mu \mathrm{g} / \mathrm{L}$, micrograms per liter; $\mathrm{CaCO}_{3}$, calcium carbonate; $\mathrm{SiO}_{2}$, silica; $\mathrm{SO}_{4}$, sulfate; ID, insufficient number of samples (less than 20) to calculate percentile; <, less than; --, no drought-sample measurement; values or concentrations in bold type indicate 2002 Colorado water-quality exceedances; c, chronic water-quality exceedance; ac, acute and chronic water-quality exceedance]

\begin{tabular}{|c|c|c|c|c|c|c|c|c|c|}
\hline \multirow{2}{*}{$\begin{array}{c}\text { Site number } \\
\text { in table } 1 \\
\text { and fig. } 6\end{array}$} & \multirow{2}{*}{$\begin{array}{l}\text { Sampling } \\
\text { date }\end{array}$} & \multicolumn{3}{|c|}{$\begin{array}{l}\text { Specific conductance, } \\
\text { in } \mu \mathrm{S} / \mathrm{cm}\end{array}$} & \multicolumn{3}{|c|}{ Temperature, water, in ${ }^{\circ} \mathrm{C}$} & \multirow{2}{*}{$\begin{array}{l}\text { Hardness, } \\
\text { total, } \\
\text { in } \mathrm{mg} / \mathrm{L} \\
\text { as } \mathrm{CaCO}_{3}\end{array}$} & \multirow{2}{*}{$\begin{array}{l}\text { Calcium, } \\
\text { dissolved, in } \\
\mathrm{mg} / \mathrm{L}\end{array}$} \\
\hline & & Value & $0 \%$ & $\mathrm{~N}$ & Value & $0 \%$ & $\mathrm{~N}$ & & \\
\hline \multirow[t]{2}{*}{103} & $8-5-2002$ & 1,040 & 75.7 & 69 & 27.5 & 98.6 & 68 & 330 & 104 \\
\hline & $9-5-2002$ & 1,030 & 71.4 & 69 & 18.0 & ${ }^{1} 15.9-20.3$ & 68 & 340 & 106 \\
\hline \multirow[t]{2}{*}{104} & $8-13-2002$ & 992 & 95.2 & 41 & 21.5 & ${ }^{1} 67.4-68.5$ & 91 & 270 & 81 \\
\hline & $9-10-2002$ & 704 & 66.7 & 41 & 18.0 & ${ }^{1} 21.7-23.9$ & 91 & 180 & 54 \\
\hline \multirow[t]{2}{*}{105} & $8-15-2002$ & 250 & 95.7 & 45 & 15.0 & ${ }^{1} 65.7-77.6$ & 66 & 79 & 23 \\
\hline & $9-12-2002$ & 277 & 97.8 & 45 & 17.0 & ${ }^{1} 89.6-92.5$ & 66 & 91 & 25 \\
\hline \multirow[t]{2}{*}{106} & $8-13-2002$ & 944 & 88.0 & 24 & 18.5 & ${ }^{1} 17.1-18.6$ & 69 & 220 & 64 \\
\hline & $9-10-2002$ & 881 & 56.0 & 24 & 20.5 & ${ }^{1} 45.7-52.9$ & 69 & 210 & 62 \\
\hline \multirow[t]{2}{*}{107} & $8-5-2002$ & 23 & ID & 8 & 16.0 & ${ }^{1} 90.3-93.5$ & 30 & 8 & 2.3 \\
\hline & $9-9-2002$ & 36 & ID & 8 & 14.0 & ${ }^{1} 80.6-87.1$ & 30 & 12 & 3.2 \\
\hline \multirow[t]{2}{*}{108} & $8-5-2002$ & 47 & 94.0 & 49 & 18.5 & 96.0 & 49 & 19 & 5.7 \\
\hline & $9-9-2002$ & 57 & 98.0 & 49 & 17.0 & ${ }^{1} 86.0-90.0$ & 49 & 23 & 6.9 \\
\hline \multirow[t]{2}{*}{109} & $8-5-2002$ & 48 & ID & 8 & 18.5 & ${ }^{1} 93.8-96.9$ & 31 & 19 & 5.9 \\
\hline & $9-9-2002$ & 57 & ID & 8 & 17.0 & ${ }^{1} 71.9-78.1$ & 31 & 23 & 7.0 \\
\hline \multirow[t]{2}{*}{110} & $8-6-2002$ & 30 & ID & 8 & 20.0 & ID & 8 & 10 & 2.9 \\
\hline & $9-10-2002$ & 36 & ID & 8 & 15.5 & ID & 8 & 12 & 3.3 \\
\hline \multirow[t]{2}{*}{111} & $8-8-2002$ & 281 & 15.4 & 77 & 19.5 & ${ }^{1} 64.6-69.6$ & 78 & 120 & 34 \\
\hline & $9-11-2002$ & 424 & 38.5 & 77 & 18.5 & ${ }^{1} 54.4-59.5$ & 78 & 170 & 45 \\
\hline \multirow[t]{2}{*}{112} & 8-8-2002 & 376 & 4.3 & 22 & 22.0 & 83.3 & 23 & 150 & 42 \\
\hline & $9-12-2002$ & 774 & 52.2 & 22 & 17.0 & ${ }^{1} 25.0-29.2$ & 23 & 310 & 73 \\
\hline \multirow[t]{2}{*}{113} & $8-23-2002$ & 191 & 54.2 & 71 & 20.5 & ${ }^{1} 92.0-93.3$ & 74 & 82 & 24 \\
\hline & $9-12-2002$ & 394 & 90.3 & 71 & 20.5 & ${ }^{1} 92.0-93.3$ & 74 & 180 & 51 \\
\hline \multirow[t]{2}{*}{114} & $8-23-2002$ & 650 & 37.9 & 65 & 25.0 & ${ }^{1} 91.4-92.9$ & 69 & 280 & 75 \\
\hline & $9-12-2002$ & 853 & 40.9 & 65 & 21.0 & ${ }^{1} 55.7-60.0$ & 69 & 400 & 106 \\
\hline 115 & 8-6-2002 & 1,830 & ID & 18 & 22.0 & ID & 18 & 770 & 204 \\
\hline \multirow[t]{2}{*}{116} & $8-8-2002$ & 1,510 & 70.7 & 40 & 22.3 & 75.0 & 47 & 540 & 126 \\
\hline & $9-3-2002$ & 1,660 & 95.1 & 40 & 19.0 & ${ }^{1} 31.3-37.5$ & 47 & 590 & 143 \\
\hline \multirow[t]{2}{*}{117} & $8-2-2002$ & 1,710 & 84.6 & 51 & 20.5 & ${ }^{1} 35.7-38.6$ & 69 & 600 & 143 \\
\hline & $9-17-2002$ & 1,570 & 55.8 & 51 & 18.5 & ${ }^{1} 21.4-25.7$ & 69 & 520 & 127 \\
\hline 118 & $9-19-2002$ & 2,080 & 63.3 & 29 & 21.0 & ${ }^{1} 57.1-62.9$ & 34 & 720 & 195 \\
\hline \multirow[t]{2}{*}{119} & $8-6-2002$ & 52 & 91.9 & 61 & 18.5 & 95.2 & 62 & 22 & 6.6 \\
\hline & $9-10-2002$ & 60 & 98.4 & 61 & 16.0 & ${ }^{1} 60.3-68.3$ & 62 & 23 & 7.2 \\
\hline \multirow[t]{2}{*}{120} & $8-22-2002$ & 897 & ID & 2 & 19.0 & ID & 2 & 340 & 102 \\
\hline & $9-16-2002$ & 783 & ID & 2 & 16.0 & ID & 2 & 290 & 84 \\
\hline 121 & $8-7-2002$ & 17 & ID & 16 & 15.3 & ID & 16 & 5 & 1.48 \\
\hline & $9-4-2002$ & 19 & ID & 16 & 13.0 & ID & 16 & 6 & 1.71 \\
\hline 122 & $8-7-2002$ & 51 & ID & 8 & 20.5 & ID & 8 & 20 & 6.0 \\
\hline & $9-11-2002$ & 61 & ID & 8 & 17.5 & ID & 8 & 21 & 6.5 \\
\hline 123 & $9-30-2002$ & 595 & ID & 1 & 13.0 & ID & 1 & 280 & 80 \\
\hline 124 & $8-6-2002$ & 57 & ID & 8 & 19.0 & ID & 8 & 21 & 6.1 \\
\hline & $9-10-2002$ & 67 & ID & 8 & 15.5 & ID & 8 & 24 & 7.2 \\
\hline 125 & $8-14-2002$ & 1,450 & ID & 2 & 17.5 & ID & 2 & 620 & 135 \\
\hline & $9-11-2002$ & 2,150 & ID & 2 & 17.0 & ID & 2 & 940 & 192 \\
\hline
\end{tabular}


Table 17. Water-quality data for the South Platte River Basin showing 2002 drought values, Colorado water-quality exceedances, and percentiles relative to historical data for selected properties and constituents from July through September, 1978-2002.-Continued

$\left[\mathrm{ft}^{3} / \mathrm{s}\right.$, cubic feet per second; Conc, concentration; $\mathrm{Q} \%$, percentile (percentage of data less than or equal to reported value or concentration) of drought-sample constituent; N, total number of historical and drought samples; $\mathrm{mg} / \mathrm{L}$, milligrams per liter; $\mu \mathrm{S} / \mathrm{cm}$, microsiemens per centimeter at $25^{\circ} \mathrm{Celsius;}{ }^{\circ} \mathrm{C}$, degrees Celsius; E. coli, Escherichia coli; $\mathrm{mL}$, milliliter; $\mu \mathrm{g} / \mathrm{L}$, micrograms per liter; $\mathrm{CaCO}_{3}$, calcium carbonate; $\mathrm{SiO}_{2}$, silica; $\mathrm{SO}_{4}$, sulfate; ID, insufficient number of samples (less than 20) to calculate percentile; <, less than; --, no drought-sample measurement; values or concentrations in bold type indicate 2002 Colorado water-quality exceedances; c, chronic water-quality exceedance; ac, acute and chronic water-quality exceedance]

\begin{tabular}{|c|c|c|c|c|c|c|}
\hline $\begin{array}{l}\text { Site number } \\
\text { in table } 1 \\
\text { and fig. } 6\end{array}$ & $\begin{array}{c}\text { Sampling } \\
\text { date }\end{array}$ & $\begin{array}{l}\text { Magnesium, } \\
\text { dissolved, } \\
\text { in } \mathrm{mg} / \mathrm{L}\end{array}$ & $\begin{array}{l}\text { Potassium, } \\
\text { dissolved, in } \\
\mathrm{mg} / \mathrm{L}\end{array}$ & $\begin{array}{l}\text { Sodium, } \\
\text { dissolved, in } \\
\mathrm{mg} / \mathrm{L}\end{array}$ & $\begin{array}{c}\text { Acid- } \\
\text { neutralizing } \\
\text { capacity, } \\
\text { in } \mathrm{mg} / \mathrm{L} \\
\text { as } \mathrm{CaCO}_{3}\end{array}$ & $\begin{array}{c}\text { Alkalinity, onsite, } \\
\text { in } \mathrm{mg} / \mathrm{L} \\
\text { as } \mathrm{CaCO}_{3}\end{array}$ \\
\hline \multirow[t]{2}{*}{103} & $8-5-2002$ & 17.3 & -- & 85 & -- & 208 \\
\hline & $9-5-2002$ & 17.8 & -- & 85 & -- & 214 \\
\hline \multirow[t]{2}{*}{104} & $8-13-2002$ & 16.2 & 10.3 & 97 & -- & 176 \\
\hline & $9-10-2002$ & 11.6 & 6.9 & 69 & -- & 128 \\
\hline \multirow[t]{2}{*}{105} & $8-15-2002$ & 5.6 & 2.6 & 16.1 & -- & 40 \\
\hline & $9-12-2002$ & 6.6 & 2.8 & 16.8 & -- & 44 \\
\hline \multirow[t]{2}{*}{106} & $8-13-2002$ & 13.5 & 12.2 & 98 & -- & 165 \\
\hline & $9-10-2002$ & 13.8 & 9.4 & 90 & -- & 152 \\
\hline \multirow[t]{2}{*}{107} & $8-5-2002$ & 0.63 & 0.30 & 1.45 & -- & 10 \\
\hline & $9-9-2002$ & 0.92 & 0.46 & 2.0 & -- & 14 \\
\hline \multirow[t]{2}{*}{108} & $8-5-2002$ & 1.12 & 0.49 & 2.1 & -- & 21 \\
\hline & $9-9-2002$ & 1.29 & 0.57 & 2.2 & -- & 27 \\
\hline \multirow[t]{2}{*}{109} & $8-5-2002$ & 1.11 & 0.51 & 2.1 & -- & 21 \\
\hline & $9-9-2002$ & 1.3 & 0.58 & 2.2 & -- & 27 \\
\hline \multirow[t]{2}{*}{110} & $8-6-2002$ & 0.69 & 0.51 & 2.2 & -- & 13 \\
\hline & $9-10-2002$ & 0.81 & 0.63 & 2.3 & -- & 15 \\
\hline \multirow[t]{2}{*}{111} & 8-8-2002 & 8.6 & 1.13 & 9.2 & -- & 63 \\
\hline & $9-11-2002$ & 15.1 & 1.39 & 15.9 & -- & 69 \\
\hline \multirow[t]{2}{*}{112} & $8-8-2002$ & 12.3 & 1.73 & 15.7 & -- & 72 \\
\hline & $9-12-2002$ & 31 & 3.3 & 40 & -- & 110 \\
\hline \multirow[t]{2}{*}{113} & $8-23-2002$ & 5.6 & -- & -- & 75 & -- \\
\hline & $9-12-2002$ & 12.6 & -- & 10.6 & 137 & -- \\
\hline \multirow[t]{2}{*}{114} & $8-23-2002$ & 22 & -- & -- & 139 & -- \\
\hline & $9-12-2002$ & 33 & -- & 39 & 165 & 167 \\
\hline 115 & $8-6-2002$ & 62 & -- & 125 & -- & 280 \\
\hline \multirow[t]{2}{*}{116} & $8-8-2002$ & 55 & -- & 116 & -- & 210 \\
\hline & $9-3-2002$ & 57 & -- & 138 & -- & 241 \\
\hline \multirow[t]{2}{*}{117} & $8-2-2002$ & 60 & 9.5 & 170 & 224 & -- \\
\hline & $9-17-2002$ & 50 & 8.4 & 132 & 244 & -- \\
\hline 118 & $9-19-2002$ & 57 & -- & 196 & -- & -- \\
\hline \multirow[t]{2}{*}{119} & $8-6-2002$ & 1.2 & 0.47 & 2.1 & -- & 25 \\
\hline & $9-10-2002$ & 1.33 & 0.59 & 2.1 & -- & 28 \\
\hline \multirow[t]{2}{*}{120} & $8-22-2002$ & 22 & 2.8 & 56 & -- & 247 \\
\hline & $9-16-2002$ & 19.3 & 3.0 & 51 & -- & 192 \\
\hline \multirow[t]{2}{*}{121} & $8-7-2002$ & 0.36 & -- & 0.82 & -- & 6 \\
\hline & $9-4-2002$ & 0.45 & -- & 1.04 & -- & 7 \\
\hline \multirow[t]{2}{*}{122} & 8-7-2002 & 1.16 & 0.65 & 2.4 & -- & 22 \\
\hline & $9-11-2002$ & 1.26 & 0.84 & 3.1 & -- & 25 \\
\hline 123 & $9-30-2002$ & 21 & -- & 21 & -- & -- \\
\hline \multirow[t]{2}{*}{124} & 8-6-2002 & 1.26 & 0.88 & 3.4 & -- & 23 \\
\hline & $9-10-2002$ & 1.4 & 0.89 & 3.6 & -- & 27 \\
\hline \multirow[t]{2}{*}{125} & 8-14-2002 & 69 & 2.3 & 87 & -- & 314 \\
\hline & $9-11-2002$ & 112 & 3.4 & 157 & -- & 282 \\
\hline
\end{tabular}


Table 17. Water-quality data for the South Platte River Basin showing 2002 drought values, Colorado water-quality exceedances, and percentiles relative to historical data for selected properties and constituents from July through September, 1978-2002.-Continued

$\left[\mathrm{ft}^{3} / \mathrm{s}\right.$, cubic feet per second; Conc, concentration; Q\%, percentile (percentage of data less than or equal to reported value or concentration) of drought-sample constituent; $\mathrm{N}$, total number of historical and drought samples; $\mathrm{mg} / \mathrm{L}$, milligrams per liter; $\mu \mathrm{S} / \mathrm{cm}$, microsiemens per centimeter at $25^{\circ} \mathrm{Celsius;}{ }^{\circ} \mathrm{C}$, degrees Celsius; E. coli, Escherichia coli; $\mathrm{mL}$, milliliter; $\mu \mathrm{g} / \mathrm{L}$, micrograms per liter; $\mathrm{CaCO}_{3}$, calcium carbonate; $\mathrm{SiO}_{2}$, silica; $\mathrm{SO}_{4}$, sulfate; ID, insufficient number of samples (less than 20) to calculate percentile; <, less than; --, no drought-sample measurement; values or concentrations in bold type indicate 2002 Colorado water-quality exceedances; c, chronic water-quality exceedance; ac, acute and chronic water-quality exceedance]

\begin{tabular}{|c|c|c|c|c|c|c|c|}
\hline \multirow{2}{*}{$\begin{array}{c}\text { Site number } \\
\text { in table } 1 \\
\text { and fig. } 6\end{array}$} & \multirow{2}{*}{$\begin{array}{c}\text { Sampling } \\
\text { date }\end{array}$} & \multicolumn{3}{|c|}{ Chloride, dissolved, in $\mathrm{mg} / \mathrm{L}$} & \multicolumn{3}{|c|}{$\begin{array}{l}\text { Silica, dissolved, } \\
\text { in } \mathrm{mg} / \mathrm{L} \text { as } \mathrm{SiO}_{2}\end{array}$} \\
\hline & & Conc & $0 \%$ & $\mathrm{~N}$ & Conc & $0 \%$ & $\mathrm{~N}$ \\
\hline \multirow[t]{2}{*}{103} & $8-5-2002$ & 83 & ID & 19 & 21 & ID & 19 \\
\hline & $9-5-2002$ & 83 & ID & 19 & 20 & ID & 19 \\
\hline \multirow[t]{2}{*}{104} & $8-13-2002$ & 92 & 96.2 & 25 & 10.8 & 80.8 & 25 \\
\hline & $9-10-2002$ & 58 & 80.8 & 25 & 8.8 & 34.6 & 25 \\
\hline \multirow[t]{2}{*}{105} & $8-15-2002$ & 11.0 & ID & 17 & 7.1 & ID & 17 \\
\hline & $9-12-2002$ & 16.6 & ID & 17 & 7.2 & ID & 17 \\
\hline \multirow[t]{2}{*}{106} & $8-13-2002$ & 93 & 95.7 & 22 & 11.1 & 69.6 & 22 \\
\hline & $9-10-2002$ & 82 & 87.0 & 22 & 9.1 & 13.0 & 22 \\
\hline \multirow[t]{2}{*}{107} & $8-5-2002$ & 0.69 & ID & 8 & -- & -- & -- \\
\hline & $9-9-2002$ & 1.40 & ID & 8 & -- & -- & -- \\
\hline \multirow[t]{2}{*}{108} & $8-5-2002$ & 0.63 & 83.3 & 47 & -- & -- & -- \\
\hline & $9-9-2002$ & 0.58 & 64.6 & 47 & -- & -- & -- \\
\hline \multirow[t]{2}{*}{109} & $8-5-2002$ & 0.60 & ID & 8 & -- & -- & -- \\
\hline & $9-9-2002$ & 0.56 & ID & 8 & -- & -- & -- \\
\hline \multirow[t]{2}{*}{110} & $8-6-2002$ & 0.77 & ID & 8 & -- & -- & -- \\
\hline & $9-10-2002$ & 1.41 & ID & 8 & -- & -- & -- \\
\hline \multirow[t]{2}{*}{111} & $8-8-2002$ & 3.9 & 53.1 & 31 & -- & -- & -- \\
\hline & $9-11-2002$ & 4.6 & 65.6 & 31 & -- & -- & -- \\
\hline \multirow[t]{2}{*}{112} & $8-8-2002$ & 5.4 & ID & 11 & -- & -- & -- \\
\hline & $9-12-2002$ & 13.8 & ID & 11 & -- & -- & -- \\
\hline \multirow[t]{2}{*}{113} & 8-23-2002 & -- & -- & 31 & -- & -- & -- \\
\hline & $9-12-2002$ & 7.2 & 84.4 & 31 & 9.7 & 97.6 & 41 \\
\hline \multirow[t]{2}{*}{114} & $8-23-2002$ & -- & -- & 26 & -- & -- & -- \\
\hline & $9-12-2002$ & 15.8 & 59.3 & 26 & 9.6 & 83.3 & 35 \\
\hline 115 & $8-6-2002$ & 46 & ID & 16 & 26 & ID & 16 \\
\hline \multirow[t]{2}{*}{116} & $8-8-2002$ & 80 & 95.0 & 39 & 14.2 & 95.0 & 39 \\
\hline & $9-3-2002$ & 86 & 97.5 & 39 & 14.0 & ${ }^{1} 87.5-92.5$ & 39 \\
\hline \multirow[t]{2}{*}{117} & $8-2-2002$ & 98 & 98.0 & 49 & 13.9 & 76.0 & 49 \\
\hline & $9-17-2002$ & 84 & 92.0 & 49 & 13.4 & 74.0 & 49 \\
\hline 118 & $9-19-2002$ & -- & -- & 25 & 25 & 74.1 & 26 \\
\hline \multirow{2}{*}{119} & $8-6-2002$ & 0.38 & ${ }^{2} 1.7-84.7$ & 58 & -- & -- & -- \\
\hline & $9-10-2002$ & 0.38 & ${ }^{2} 1.7-84.7$ & 58 & -- & -- & -- \\
\hline \multirow[t]{2}{*}{120} & $8-22-2002$ & 67 & ID & 2 & 18.1 & ID & 2 \\
\hline & $9-16-2002$ & 58 & ID & 2 & 11.6 & ID & 2 \\
\hline \multirow[t]{2}{*}{121} & 8-7-2002 & $<0.3$ & ID & 10 & 3.1 & ID & 10 \\
\hline & $9-4-2002$ & 0.33 & ID & 10 & 3.8 & ID & 10 \\
\hline \multirow[t]{2}{*}{122} & 8-7-2002 & 1.00 & ID & 8 & -- & -- & -- \\
\hline & $9-11-2002$ & 1.57 & ID & 8 & -- & -- & -- \\
\hline 123 & $9-30-2002$ & -- & -- & -- & 15 & ID & 1 \\
\hline \multirow[t]{2}{*}{124} & $8-6-2002$ & 1.89 & ID & 8 & -- & -- & -- \\
\hline & $9-10-2002$ & 2.1 & ID & 8 & -- & -- & -- \\
\hline 125 & 8-14-2002 & 23 & ID & 2 & 12.4 & ID & 2 \\
\hline & $9-11-2002$ & 24 & ID & 2 & 10.2 & ID & 2 \\
\hline
\end{tabular}


Table 17. Water-quality data for the South Platte River Basin showing 2002 drought values, Colorado water-quality exceedances, and percentiles relative to historical data for selected properties and constituents from July through September, 1978-2002.-Continued

$\left[\mathrm{ft}^{3} / \mathrm{s}\right.$, cubic feet per second; Conc, concentration; $\mathrm{Q} \%$, percentile (percentage of data less than or equal to reported value or concentration) of drought-sample constituent; N, total number of historical and drought samples; $\mathrm{mg} / \mathrm{L}$, milligrams per liter; $\mu \mathrm{S} / \mathrm{cm}$, microsiemens per centimeter at $25^{\circ} \mathrm{Celsius;}{ }^{\circ} \mathrm{C}$, degrees Celsius; E. coli, Escherichia coli; $\mathrm{mL}$, milliliter; $\mu \mathrm{g} / \mathrm{L}$, micrograms per liter; $\mathrm{CaCO}_{3}$, calcium carbonate; $\mathrm{SiO}_{2}$, silica; $\mathrm{SO}_{4}$, sulfate; ID, insufficient number of samples (less than 20) to calculate percentile; <, less than; --, no drought-sample measurement; values or concentrations in bold type indicate 2002 Colorado water-quality exceedances; c, chronic water-quality exceedance; ac, acute and chronic water-quality exceedance]

\begin{tabular}{|c|c|c|c|c|c|c|c|c|}
\hline \multirow{2}{*}{$\begin{array}{l}\text { Site number } \\
\text { in table } 1 \\
\text { and fig. } 6\end{array}$} & \multirow{2}{*}{$\begin{array}{l}\text { Sampling } \\
\text { date }\end{array}$} & \multicolumn{3}{|c|}{$\begin{array}{l}\text { Sulfate, dissolved, } \\
\text { in } \mathrm{mg} / \mathrm{L} \text { as } \mathrm{SO}_{4}\end{array}$} & \multirow{2}{*}{$\begin{array}{l}\text { Solids, } \\
\text { dissolved, } \\
\text { residue on } \\
\text { evaporation } \\
\text { at } 180^{\circ} \mathrm{C} \text {, } \\
\text { in } \mathrm{mg} / \mathrm{L}\end{array}$} & \multicolumn{3}{|c|}{$\begin{array}{c}\text { Nitrogen, ammonia, dissolved, in } \\
\mathrm{mg} / \mathrm{L} \text { as } \mathrm{N}\end{array}$} \\
\hline & & Conc & $0 \%$ & $\mathrm{~N}$ & & Conc & $0 \%$ & $\mathrm{~N}$ \\
\hline \multirow[t]{2}{*}{103} & $8-5-2002$ & 185 & ID & 19 & -- & 0.020 & ${ }^{2} 4.8-57.1$ & 20 \\
\hline & $9-5-2002$ & 176 & ID & 19 & -- & $<0.04$ & ${ }^{2} 4.8-57.1$ & 20 \\
\hline \multirow[t]{2}{*}{104} & $8-13-2002$ & 166 & 96.2 & 25 & -- & $0.89 \mathrm{c}$ & 90.3 & 30 \\
\hline & $9-10-2002$ & 115 & 57.7 & 25 & -- & $0.51 c$ & 77.4 & 30 \\
\hline \multirow[t]{2}{*}{105} & $8-15-2002$ & 56 & ID & 17 & -- & $<0.04$ & ID & 10 \\
\hline & $9-12-2002$ & 62 & ID & 17 & -- & $<0.04$ & ID & 10 \\
\hline \multirow[t]{2}{*}{106} & 8-13-2002 & 145 & 56.5 & 22 & -- & $4.65 c$ & 80.0 & 24 \\
\hline & $9-10-2002$ & 139 & 34.8 & 22 & -- & $2.71 \mathrm{c}$ & 60.0 & 24 \\
\hline \multirow[t]{2}{*}{107} & $8-5-2002$ & 1.6 & ID & 8 & 28 & $<0.015$ & ID & 8 \\
\hline & $9-9-2002$ & 1.9 & ID & 8 & $<10$ & $<0.015$ & ID & 8 \\
\hline \multirow[t]{2}{*}{108} & $8-5-2002$ & 2.3 & 85.4 & 47 & 38 & $0.047 \mathrm{c}$ & ID & 18 \\
\hline & $9-9-2002$ & 2.4 & 85.4 & 47 & 39 & 0.012 & ID & 18 \\
\hline \multirow[t]{2}{*}{109} & $8-5-2002$ & 2.4 & ID & 8 & 42 & $0.030 \mathrm{c}$ & ID & 8 \\
\hline & $9-9-2002$ & 2.4 & ID & 8 & 43 & 0.009 & ID & 8 \\
\hline \multirow[t]{2}{*}{110} & $8-6-2002$ & 1.6 & ID & 8 & 34 & $<0.015$ & ID & 8 \\
\hline & $9-10-2002$ & 1.4 & ID & 8 & 29 & $<0.015$ & ID & 8 \\
\hline \multirow[t]{2}{*}{111} & $8-8-2002$ & 69 & 18.8 & 31 & 186 & 0.010 & ${ }^{2} 1.5-29.4$ & 67 \\
\hline & $9-11-2002$ & 136 & 53.1 & 31 & 283 & $<0.015$ & ${ }^{2} 1.5-29.4$ & 67 \\
\hline \multirow[t]{2}{*}{112} & $8-8-2002$ & 99 & ID & 11 & 241 & 0.035 & ${ }^{1} 68.2-72.7$ & 21 \\
\hline & $9-12-2002$ & 255 & ID & 11 & 553 & 0.022 & 54.5 & 21 \\
\hline \multirow[t]{2}{*}{113} & $8-23-2002$ & -- & -- & -- & -- & $<0.04$ & ${ }^{2} 1.4-75.0$ & 71 \\
\hline & $9-12-2002$ & 55 & 93.8 & 31 & 242 & $<0.04$ & ${ }^{2} 1.4-75.0$ & 71 \\
\hline \multirow[t]{2}{*}{114} & $8-23-2002$ & -- & -- & -- & -- & $<0.04$ & ${ }^{2} 1.5-27.9$ & 67 \\
\hline & $9-12-2002$ & 299 & 44.4 & 26 & 659 & $<0.04$ & ${ }^{2} 1.5-27.9$ & 67 \\
\hline 115 & $8-6-2002$ & 642 & ID & 16 & -- & 0.020 & ID & 17 \\
\hline \multirow[t]{2}{*}{116} & $8-8-2002$ & 440 & 52.5 & 39 & -- & $1.81 \mathrm{c}$ & 96.6 & 28 \\
\hline & $9-3-2002$ & 496 & 72.5 & 39 & -- & $0.90 \mathrm{c}$ & 89.7 & 28 \\
\hline \multirow[t]{2}{*}{117} & $8-2-2002$ & 562 & 62.0 & 49 & 1290 & 0.011 & ID & 17 \\
\hline & $9-17-2002$ & 475 & 44.0 & 49 & 1130 & $<0.015$ & 11.1 & 17 \\
\hline 118 & $9-19-2002$ & -- & -- & -- & -- & 0.070 & 60.9 & 22 \\
\hline \multirow[t]{2}{*}{119} & $8-6-2002$ & 2.5 & 48.2 & 55 & 47 & $<0.015$ & ${ }^{2} 3.3-53.3$ & 29 \\
\hline & $9-10-2002$ & 2.5 & 48.2 & 55 & 46 & $<0.015$ & ${ }^{2} 3.3-53.3$ & 29 \\
\hline \multirow[t]{2}{*}{120} & $8-22-2002$ & 137 & ID & 2 & -- & $<0.04$ & ID & 2 \\
\hline & $9-16-2002$ & 126 & ID & 2 & -- & $<0.04$ & ID & 2 \\
\hline \multirow[t]{2}{*}{121} & $8-7-2002$ & 1.4 & ID & 10 & -- & $<0.015$ & ID & 16 \\
\hline & $9-4-2002$ & 1.9 & ID & 10 & -- & $<0.015$ & ID & 16 \\
\hline 122 & 8-7-2002 & 2.5 & ID & 8 & 39 & $0.024 c$ & ID & 8 \\
\hline & $9-11-2002$ & 2.7 & ID & 8 & 18 & $0.022 \mathrm{c}$ & ID & 8 \\
\hline 123 & $9-30-2002$ & -- & -- & -- & -- & $<0.04$ & ID & 1 \\
\hline 124 & $8-6-2002$ & 3.1 & ID & 8 & 41 & 0.016 & ID & 8 \\
\hline & $9-10-2002$ & 3.0 & ID & 8 & 48 & $<0.015$ & ID & 8 \\
\hline 125 & $8-14-2002$ & 491 & ID & 2 & -- & $<0.04$ & ID & 2 \\
\hline & $9-11-2002$ & 964 & ID & 2 & -- & $<0.04$ & ID & 2 \\
\hline
\end{tabular}


Table 17. Water-quality data for the South Platte River Basin showing 2002 drought values, Colorado water-quality exceedances, and percentiles relative to historical data for selected properties and constituents from July through September, 1978-2002.-Continued

$\left[\mathrm{ft}^{3} / \mathrm{s}\right.$, cubic feet per second; Conc, concentration; Q\%, percentile (percentage of data less than or equal to reported value or concentration) of drought-sample constituent; N, total number of historical and drought samples; $\mathrm{mg} / \mathrm{L}$, milligrams per liter; $\mu \mathrm{S} / \mathrm{cm}$, microsiemens per centimeter at $25^{\circ} \mathrm{Celsius;}{ }^{\circ} \mathrm{C}$, degrees Celsius; E. coli, Escherichia coli; $\mathrm{mL}$, milliliter; $\mu \mathrm{g} / \mathrm{L}$, micrograms per liter; $\mathrm{CaCO}_{3}$, calcium carbonate; $\mathrm{SiO}_{2}$, silica; $\mathrm{SO}_{4}$, sulfate; ID, insufficient number of samples (less than 20) to calculate percentile; <, less than; --, no drought-sample measurement; values or concentrations in bold type indicate 2002 Colorado water-quality exceedances; c, chronic water-quality exceedance; ac, acute and chronic water-quality exceedance]

\begin{tabular}{|c|c|c|c|c|c|c|c|}
\hline \multirow{2}{*}{$\begin{array}{c}\text { Site number } \\
\text { in table } 1 \\
\text { and fig. } 6\end{array}$} & \multirow{2}{*}{$\begin{array}{l}\text { Sampling } \\
\text { date }\end{array}$} & \multicolumn{3}{|c|}{$\begin{array}{l}\text { Nitrogen, organic plus ammonia, } \\
\text { dissolved, in } \mathrm{mg} / \mathrm{L} \text { as } \mathrm{N}\end{array}$} & \multicolumn{3}{|c|}{$\begin{array}{l}\text { Nitrogen, organic plus ammonia, tota } \\
\text { recoverable, in } \mathrm{mg} / \mathrm{L} \text { as } \mathrm{N}\end{array}$} \\
\hline & & Conc & $0 \%$ & $\mathrm{~N}$ & Conc & $0 \%$ & $\mathrm{~N}$ \\
\hline \multirow[t]{2}{*}{103} & $8-5-2002$ & -- & -- & -- & 0.51 & 54.5 & 21 \\
\hline & $9-5-2002$ & -- & -- & -- & 0.29 & 4.5 & 21 \\
\hline \multirow[t]{2}{*}{104} & $8-13-2002$ & 1.9 & 92.9 & 27 & -- & -- & -- \\
\hline & $9-10-2002$ & 1.5 & ${ }^{1} 82.1--85.7$ & 27 & -- & -- & -- \\
\hline \multirow[t]{2}{*}{105} & $8-15-2002$ & 0.10 & ID & 17 & -- & -- & -- \\
\hline & $9-12-2002$ & 0.10 & ID & 17 & -- & -- & -- \\
\hline \multirow[t]{2}{*}{106} & $8-13-2002$ & 6.0 & ID & 14 & -- & -- & -- \\
\hline & $9-10-2002$ & 3.9 & ID & 14 & -- & -- & -- \\
\hline \multirow[t]{2}{*}{107} & $8-5-2002$ & -- & -- & -- & 0.13 & ID & 8 \\
\hline & $9-9-2002$ & -- & -- & -- & 0.14 & ID & 8 \\
\hline \multirow[t]{2}{*}{108} & $8-5-2002$ & -- & -- & -- & 0.29 & ID & 19 \\
\hline & $9-9-2002$ & -- & -- & -- & 0.34 & ID & 19 \\
\hline \multirow[t]{2}{*}{109} & $8-5-2002$ & -- & -- & -- & 0.29 & ID & 8 \\
\hline & $9-9-2002$ & -- & -- & -- & 0.32 & ID & 8 \\
\hline \multirow[t]{2}{*}{110} & $8-6-2002$ & -- & -- & -- & 0.21 & ID & 8 \\
\hline & $9-10-2002$ & -- & -- & -- & 0.17 & ID & 8 \\
\hline \multirow[t]{2}{*}{111} & $8-8-2002$ & -- & -- & -- & 0.44 & 39.4 & 32 \\
\hline & $9-11-2002$ & -- & -- & -- & 0.29 & 9.1 & 32 \\
\hline \multirow[t]{2}{*}{112} & $8-8-2002$ & -- & -- & -- & 1.2 & ID & 6 \\
\hline & $9-12-2002$ & -- & -- & -- & 0.57 & ID & 6 \\
\hline \multirow[t]{2}{*}{113} & $8-23-2002$ & -- & -- & -- & -- & -- & -- \\
\hline & $9-12-2002$ & -- & -- & -- & -- & -- & -- \\
\hline \multirow[t]{2}{*}{114} & $8-23-2002$ & -- & -- & -- & -- & -- & -- \\
\hline & $9-12-2002$ & -- & -- & -- & -- & -- & -- \\
\hline 115 & $8-6-2002$ & -- & -- & -- & 0.77 & ID & 17 \\
\hline \multirow[t]{2}{*}{116} & $8-8-2002$ & -- & -- & -- & 3.3 & 96.4 & 27 \\
\hline & $9-3-2002$ & -- & -- & -- & 2.7 & 89.3 & 27 \\
\hline \multirow[t]{2}{*}{117} & $8-2-2002$ & -- & -- & -- & 0.88 & ID & 18 \\
\hline & $9-17-2002$ & -- & -- & -- & 0.73 & 42.1 & 18 \\
\hline 118 & $9-19-2002$ & -- & -- & -- & 0.47 & 10.3 & 28 \\
\hline \multirow[t]{2}{*}{119} & $8-6-2002$ & -- & -- & -- & 0.21 & 24.2 & 61 \\
\hline & $9-10-2002$ & -- & -- & -- & 0.38 & ${ }^{1} 54.8-56.5$ & 61 \\
\hline \multirow[t]{2}{*}{120} & $8-22-2002$ & 0.21 & ID & 2 & -- & -- & -- \\
\hline & $9-16-2002$ & 0.26 & ID & 2 & -- & -- & -- \\
\hline \multirow[t]{2}{*}{121} & $8-7-2002$ & 0.10 & ID & 16 & 0.10 & ID & 16 \\
\hline & $9-4-2002$ & 0.06 & ID & 16 & 0.10 & ID & 16 \\
\hline \multirow[t]{2}{*}{122} & 8-7-2002 & -- & -- & -- & 0.26 & ID & 8 \\
\hline & $9-11-2002$ & -- & -- & -- & 0.32 & ID & 8 \\
\hline 123 & $9-30-2002$ & -- & -- & -- & 0.18 & ID & 1 \\
\hline \multirow[t]{2}{*}{124} & 8-6-2002 & -- & -- & -- & 0.31 & ID & 8 \\
\hline & $9-10-2002$ & -- & -- & -- & 0.35 & ID & 8 \\
\hline 125 & $8-14-2002$ & 0.15 & ID & 2 & -- & -- & -- \\
\hline & $9-11-2002$ & 0.30 & ID & 2 & -- & -- & -- \\
\hline
\end{tabular}


Table 17. Water-quality data for the South Platte River Basin showing 2002 drought values, Colorado water-quality exceedances, and percentiles relative to historical data for selected properties and constituents from July through September, 1978-2002.-Continued

$\left[\mathrm{ft}^{3} / \mathrm{s}\right.$, cubic feet per second; Conc, concentration; $\mathrm{Q} \%$, percentile (percentage of data less than or equal to reported value or concentration) of drought-sample constituent; N, total number of historical and drought samples; $\mathrm{mg} / \mathrm{L}$, milligrams per liter; $\mu \mathrm{S} / \mathrm{cm}$, microsiemens per centimeter at $25^{\circ} \mathrm{Celsius;}{ }^{\circ} \mathrm{C}$, degrees Celsius; E. coli, Escherichia coli; $\mathrm{mL}$, milliliter; $\mu \mathrm{g} / \mathrm{L}$, micrograms per liter; $\mathrm{CaCO}_{3}$, calcium carbonate; $\mathrm{SiO}_{2}$, silica; $\mathrm{SO}_{4}$, sulfate; ID, insufficient number of samples (less than 20) to calculate percentile; <, less than; --, no drought-sample measurement; values or concentrations in bold type indicate 2002 Colorado water-quality exceedances; c, chronic water-quality exceedance; ac, acute and chronic water-quality exceedance]

\begin{tabular}{|c|c|c|c|c|c|c|c|}
\hline \multirow{2}{*}{$\begin{array}{l}\text { Site number } \\
\text { in table } 1 \\
\text { and fig. } 6\end{array}$} & \multirow{2}{*}{$\begin{array}{l}\text { Sampling } \\
\text { date }\end{array}$} & \multicolumn{3}{|c|}{$\begin{array}{l}\text { Nitrogen, nitrite plus nitrate, dissolved, in } \\
\mathrm{mg} / \mathrm{L} \text { as } \mathrm{N}\end{array}$} & \multicolumn{3}{|c|}{$\begin{array}{c}\text { Nitrogen, nitrite, dissolved, in } \mathrm{mg} / \mathrm{L} \\
\text { as } \mathrm{N}\end{array}$} \\
\hline & & Conc & $0 \%$ & $\mathrm{~N}$ & Conc & $0 \%$ & $\mathrm{~N}$ \\
\hline \multirow[t]{2}{*}{103} & $8-5-2002$ & 3.0 & 71.4 & 20 & 0.046 & 71.4 & 20 \\
\hline & $9-5-2002$ & 3.7 & 95.2 & 20 & 0.018 & 14.3 & 20 \\
\hline \multirow[t]{2}{*}{104} & $8-13-2002$ & 9.7 & 96.8 & 30 & 0.344 & 85.7 & 27 \\
\hline & $9-10-2002$ & 5.4 & 90.3 & 30 & 0.219 & 82.1 & 27 \\
\hline \multirow[t]{2}{*}{105} & $8-15-2002$ & 0.150 & ID & 17 & $<0.008$ & ID & 10 \\
\hline & $9-12-2002$ & 0.140 & ID & 17 & $<0.008$ & ID & 10 \\
\hline \multirow[t]{2}{*}{106} & $8-13-2002$ & 5.3 & 76.0 & 24 & 1.21 & 96.0 & 24 \\
\hline & $9-10-2002$ & 4.8 & 64.0 & 24 & 0.638 & 72.0 & 24 \\
\hline \multirow[t]{2}{*}{107} & $8-5-2002$ & 0.127 & ID & 8 & -- & -- & -- \\
\hline & $9-9-2002$ & 0.098 & ID & 8 & -- & -- & -- \\
\hline \multirow[t]{2}{*}{108} & $8-5-2002$ & 0.034 & ${ }^{2} 2.0-90.0$ & 49 & -- & -- & -- \\
\hline & $9-9-2002$ & 0.014 & ${ }^{2} 2.0-90.0$ & 49 & -- & -- & -- \\
\hline \multirow[t]{2}{*}{109} & $8-5-2002$ & 0.029 & ID & 8 & -- & -- & -- \\
\hline & $9-9-2002$ & 0.014 & ID & 8 & -- & -- & -- \\
\hline \multirow[t]{2}{*}{110} & $8-6-2002$ & 0.094 & ID & 8 & -- & -- & -- \\
\hline & $9-10-2002$ & 0.072 & ID & 8 & -- & -- & -- \\
\hline \multirow[t]{2}{*}{111} & $8-8-2002$ & 0.085 & 35.6 & 58 & -- & -- & -- \\
\hline & $9-11-2002$ & 0.128 & 55.9 & 58 & -- & -- & -- \\
\hline \multirow[t]{2}{*}{112} & 8-8-2002 & 1.11 & 52.4 & 20 & -- & -- & -- \\
\hline & $9-12-2002$ & 3.7 & 95.2 & 20 & -- & -- & -- \\
\hline \multirow[t]{2}{*}{113} & $8-23-2002$ & 0.016 & 3.2 & 61 & $<0.008$ & ${ }^{2} 1.5-95.5$ & 65 \\
\hline & $9-12-2002$ & 0.107 & 32.3 & 61 & 0.007 & ${ }^{2} 1.5-95.5$ & 65 \\
\hline \multirow[t]{2}{*}{114} & $8-23-2002$ & 0.392 & 29.3 & 57 & 0.010 & ${ }^{1} 11.9-19.4$ & 66 \\
\hline & $9-12-2002$ & 0.636 & 48.3 & 57 & 0.032 & 52.2 & 66 \\
\hline 115 & $8-6-2002$ & 12.4 & ID & 17 & 0.146 & ID & 17 \\
\hline \multirow[t]{2}{*}{116} & $8-8-2002$ & 6.0 & 82.9 & 40 & 0.212 & 93.1 & 28 \\
\hline & $9-3-2002$ & 7.4 & 95.1 & 40 & 0.288 & 96.6 & 28 \\
\hline \multirow[t]{2}{*}{117} & $8-2-2002$ & 3.4 & 51.0 & 50 & 0.046 & ID & 17 \\
\hline & $9-17-2002$ & 3.8 & 56.9 & 50 & 0.024 & 55.6 & 17 \\
\hline 118 & $9-19-2002$ & 3.6 & 91.7 & 23 & 0.043 & ID & 12 \\
\hline \multirow[t]{2}{*}{$\begin{array}{l}110 \\
119\end{array}$} & $8-6-2002$ & $<0.013$ & ${ }^{2} 1.7-93.2$ & 58 & -- & - & -- \\
\hline & $9-10-2002$ & $<0.013$ & ${ }^{2} 1.7-93.2$ & 58 & -- & -- & -- \\
\hline \multirow[t]{2}{*}{120} & $8-22-2002$ & 1.03 & ID & 2 & 0.015 & ID & 2 \\
\hline & $9-16-2002$ & 0.650 & ID & 2 & 0.005 & ID & 2 \\
\hline \multirow[t]{2}{*}{121} & $8-7-2002$ & 0.190 & ID & 16 & $<0.002$ & ID & 16 \\
\hline & $9-4-2002$ & 0.133 & ID & 16 & $<0.002$ & ID & 16 \\
\hline \multirow[t]{2}{*}{122} & $8-7-2002$ & 0.091 & ID & 8 & -- & -- & -- \\
\hline & $9-11-2002$ & 0.149 & ID & 8 & -- & -- & -- \\
\hline 123 & $9-30-2002$ & 0.060 & ID & 1 & $<0.008$ & ID & 1 \\
\hline \multirow[t]{2}{*}{124} & $8-6-2002$ & 0.363 & ID & 8 & -- & -- & -- \\
\hline & $9-10-2002$ & 0.546 & ID & 8 & -- & -- & -- \\
\hline 125 & $8-14-2002$ & 0.880 & ID & 2 & 0.004 & ID & 2 \\
\hline & $9-11-2002$ & 0.990 & ID & 2 & 0.020 & ID & 2 \\
\hline
\end{tabular}


Table 17. Water-quality data for the South Platte River Basin showing 2002 drought values, Colorado water-quality exceedances, and percentiles relative to historical data for selected properties and constituents from July through September, 1978-2002.-Continued

$\left[\mathrm{ft}^{3} / \mathrm{s}\right.$, cubic feet per second; Conc, concentration; Q\%, percentile (percentage of data less than or equal to reported value or concentration) of drought-sample constituent; N, total number of historical and drought samples; $\mathrm{mg} / \mathrm{L}$, milligrams per liter; $\mu \mathrm{S} / \mathrm{cm}$, microsiemens per centimeter at $25^{\circ} \mathrm{Celsius;}{ }^{\circ} \mathrm{C}$, degrees Celsius; E. coli, Escherichia coli; $\mathrm{mL}$, milliliter; $\mu \mathrm{g} / \mathrm{L}$, micrograms per liter; $\mathrm{CaCO}_{3}$, calcium carbonate; $\mathrm{SiO}_{2}$, silica; $\mathrm{SO}_{4}$, sulfate; ID, insufficient number of samples (less than 20) to calculate percentile; <, less than; --, no drought-sample measurement; values or concentrations in bold type indicate 2002 Colorado water-quality exceedances; c, chronic water-quality exceedance; ac, acute and chronic water-quality exceedance]

\begin{tabular}{|c|c|c|c|c|c|c|c|}
\hline \multirow{2}{*}{$\begin{array}{l}\text { Site number } \\
\text { in table } 1 \\
\text { and fig. } 6\end{array}$} & \multirow{2}{*}{$\begin{array}{l}\text { Sampling } \\
\text { date }\end{array}$} & \multicolumn{3}{|c|}{$\begin{array}{c}\text { Phosphorus, dissolved, in } \mathrm{mg} / \mathrm{L} \\
\text { as } \mathrm{P}\end{array}$} & \multicolumn{3}{|c|}{$\begin{array}{c}\text { Phosphorus, total recoverable, in } \mathrm{mg} / \mathrm{L} \\
\text { as } \mathrm{P}\end{array}$} \\
\hline & & Conc & $0 \%$ & $\mathrm{~N}$ & Conc & $0 \%$ & $\mathrm{~N}$ \\
\hline \multirow[t]{2}{*}{103} & $8-5-2002$ & -- & -- & -- & 0.28 & ${ }^{1} 57.1-71.4$ & 20 \\
\hline & $9-5-2002$ & -- & -- & -- & 0.22 & ${ }^{1} 14.3-19.0$ & 20 \\
\hline \multirow[t]{2}{*}{104} & 8-13-2002 & 1.56 & 96.4 & 27 & 1.6 & 90.3 & 30 \\
\hline & $9-10-2002$ & 0.86 & 85.7 & 27 & 1.12 & 87.1 & 30 \\
\hline \multirow[t]{2}{*}{105} & $8-15-2002$ & $<0.06$ & ID & 17 & $<0.06$ & ID & 17 \\
\hline & $9-12-2002$ & $<0.06$ & ID & 17 & $<0.06$ & ID & 17 \\
\hline \multirow[t]{2}{*}{106} & $8-13-2002$ & 2.2 & 76.0 & 24 & 2.2 & 73.9 & 22 \\
\hline & $9-10-2002$ & 1.11 & 36.0 & 24 & 1.47 & 34.8 & 22 \\
\hline \multirow[t]{2}{*}{107} & $8-5-2002$ & 0.005 & ID & 8 & 0.008 & ID & 8 \\
\hline & $9-9-2002$ & 0.004 & ID & 8 & 0.011 & ID & 8 \\
\hline \multirow[t]{2}{*}{108} & $8-5-2002$ & 0.007 & ${ }^{2} 2.1-42.6$ & 46 & 0.023 & 56.0 & 49 \\
\hline & $9-9-2002$ & 0.005 & ${ }^{2} 2.1-42.6$ & 46 & 0.024 & 58.0 & 49 \\
\hline \multirow[t]{2}{*}{109} & $8-5-2002$ & 0.009 & ID & 8 & 0.021 & ID & 8 \\
\hline & $9-9-2002$ & 0.004 & ID & 8 & 0.020 & ID & 8 \\
\hline \multirow[t]{2}{*}{110} & $8-6-2002$ & 0.009 & ID & 8 & 0.023 & ID & 8 \\
\hline & $9-10-2002$ & 0.008 & ID & 8 & 0.021 & ID & 8 \\
\hline \multirow[t]{2}{*}{111} & $8-8-2002$ & 0.009 & ${ }^{2} 1.6-46.0$ & 62 & 0.077 & ID & 8 \\
\hline & $9-11-2002$ & 0.017 & ${ }^{2} 1.6-46.0$ & 62 & 0.037 & ID & 8 \\
\hline \multirow[t]{2}{*}{112} & 8-8-2002 & 0.184 & ID & 13 & 0.48 & ID & 6 \\
\hline & $9-12-2002$ & 0.46 & ID & 13 & 0.47 & ID & 6 \\
\hline \multirow[t]{2}{*}{113} & $8-23-2002$ & $<0.06$ & ${ }^{2} 1.7-95.0$ & 59 & -- & -- & -- \\
\hline & $9-12-2002$ & $<0.06$ & ${ }^{2} 1.7-95.0$ & 59 & -- & -- & -- \\
\hline \multirow[t]{2}{*}{114} & $8-23-2002$ & 0.11 & ${ }^{1} 80.7-82.5$ & 56 & -- & -- & -- \\
\hline & $9-12-2002$ & 0.10 & ${ }^{1} 77.2-78.9$ & 56 & -- & -- & -- \\
\hline 115 & $8-6-2002$ & -- & -- & -- & 0.071 & ID & 17 \\
\hline \multirow[t]{2}{*}{116} & $8-8-2002$ & -- & -- & -- & 0.79 & 85.7 & 27 \\
\hline & $9-3-2002$ & -- & -- & -- & 0.81 & 89.3 & 27 \\
\hline \multirow[t]{2}{*}{117} & $8-2-2002$ & 0.049 & 6.7 & 44 & 0.130 & ID & 18 \\
\hline & $9-17-2002$ & 0.19 & ${ }^{1} 42.2-46.7$ & 44 & 0.28 & ID & 18 \\
\hline 118 & $9-19-2002$ & -- & -- & -- & 0.024 & 17.9 & 27 \\
\hline \multirow[t]{2}{*}{119} & $8-6-2002$ & 0.004 & ${ }^{2} 1.9-57.4$ & 53 & 0.015 & ${ }^{1} 50.8-52.5$ & 58 \\
\hline & $9-10-2002$ & 0.003 & ${ }^{2} 1.9-57.4$ & 53 & 0.015 & ${ }^{1} 50.8-52.5$ & 58 \\
\hline \multirow[t]{2}{*}{120} & $8-22-2002$ & 0.04 & ID & 2 & 0.040 & ID & 2 \\
\hline & $9-16-2002$ & $<0.06$ & ID & 2 & 0.040 & ID & 2 \\
\hline \multirow[t]{2}{*}{121} & $8-7-2002$ & $<0.004$ & ID & 16 & 0.007 & ID & 16 \\
\hline & $9-4-2002$ & 0.002 & ID & 16 & 0.006 & ID & 16 \\
\hline \multirow[t]{2}{*}{122} & $8-7-2002$ & 0.03 & ID & 8 & 0.047 & ID & 8 \\
\hline & $9-11-2002$ & 0.061 & ID & 8 & 0.084 & ID & 8 \\
\hline 123 & $9-30-2002$ & -- & -- & -- & 0.012 & ID & 1 \\
\hline \multirow[t]{2}{*}{124} & $8-6-2002$ & 0.138 & ID & 8 & 0.167 & ID & 8 \\
\hline & $9-10-2002$ & 0.098 & ID & 8 & 0.130 & ID & 8 \\
\hline 125 & $8-14-2002$ & $<0.06$ & ID & 2 & $<0.06$ & ID & 2 \\
\hline & $9-11-2002$ & $<0.06$ & ID & 2 & 0.030 & ID & 2 \\
\hline
\end{tabular}


Table 17. Water-quality data for the South Platte River Basin showing 2002 drought values, Colorado water-quality exceedances, and percentiles relative to historical data for selected properties and constituents from July through September, 1978-2002.-Continued

$\left[\mathrm{ft}^{3} / \mathrm{s}\right.$, cubic feet per second; Conc, concentration; $\mathrm{Q} \%$, percentile (percentage of data less than or equal to reported value or concentration) of drought-sample constituent; N, total number of historical and drought samples; $\mathrm{mg} / \mathrm{L}$, milligrams per liter; $\mu \mathrm{S} / \mathrm{cm}$, microsiemens per centimeter at $25^{\circ} \mathrm{Celsius;}{ }^{\circ} \mathrm{C}$, degrees Celsius; E. coli, Escherichia coli; $\mathrm{mL}$, milliliter; $\mu \mathrm{g} / \mathrm{L}$, micrograms per liter; $\mathrm{CaCO}_{3}$, calcium carbonate; $\mathrm{SiO}_{2}$, silica; $\mathrm{SO}_{4}$, sulfate; ID, insufficient number of samples (less than 20) to calculate percentile; <, less than; --, no drought-sample measurement; values or concentrations in bold type indicate 2002 Colorado water-quality exceedances; c, chronic water-quality exceedance; ac, acute and chronic water-quality exceedance]

\begin{tabular}{|c|c|c|c|c|c|c|c|}
\hline \multirow{2}{*}{$\begin{array}{c}\text { Site number } \\
\text { in table } 1 \\
\text { and fig. } 6\end{array}$} & \multirow{2}{*}{$\begin{array}{l}\text { Sampling } \\
\text { date }\end{array}$} & \multicolumn{3}{|c|}{$\begin{array}{l}\text { Phosphorus, orthophosphate, dissolved, in } \\
\mathrm{mg} / \mathrm{L} \text { as } \mathrm{P}\end{array}$} & \multicolumn{3}{|c|}{ Carbon, organic, dissolved, in $\mathrm{mg} / \mathrm{L}$} \\
\hline & & Conc & $0 \%$ & $\mathrm{~N}$ & Conc & $0 \%$ & $\mathrm{~N}$ \\
\hline \multirow[t]{2}{*}{103} & $8-5-2002$ & 0.220 & ${ }^{1} 57.1-61.9$ & 20 & 4.6 & ID & 17 \\
\hline & $9-5-2002$ & 0.190 & 28.6 & 20 & 2.9 & ID & 17 \\
\hline \multirow[t]{2}{*}{104} & $8-13-2002$ & 1.5 & 96.8 & 30 & -- & -- & -- \\
\hline & $9-10-2002$ & 0.800 & 83.9 & 30 & 25 & 92.6 & 26 \\
\hline \multirow[t]{2}{*}{105} & $8-15-2002$ & $<0.02$ & ID & 10 & -- & -- & -- \\
\hline & $9-12-2002$ & $<0.02$ & ID & 10 & 1.4 & ID & 9 \\
\hline \multirow[t]{2}{*}{106} & $8-13-2002$ & 2.0 & 80.0 & 24 & -- & -- & -- \\
\hline & $9-10-2002$ & 1.0 & ${ }^{1} 28.0-32.0$ & 24 & 11.7 & ID & 10 \\
\hline \multirow[t]{2}{*}{107} & $8-5-2002$ & $<0.007$ & ID & 8 & -- & -- & -- \\
\hline & $9-9-2002$ & $<0.007$ & ID & 8 & -- & -- & -- \\
\hline \multirow[t]{2}{*}{108} & $8-5-2002$ & $<0.007$ & ${ }^{2} 4.8-81.0$ & 20 & -- & -- & -- \\
\hline & $9-9-2002$ & $<0.007$ & ${ }^{2} 4.8-81.0$ & 20 & -- & -- & -- \\
\hline \multirow[t]{2}{*}{109} & $8-5-2002$ & $<0.007$ & ID & 8 & -- & -- & -- \\
\hline & $9-9-2002$ & $<0.007$ & ID & 8 & -- & -- & -- \\
\hline \multirow[t]{2}{*}{110} & $8-6-2002$ & $<0.007$ & ID & 8 & -- & -- & -- \\
\hline & $9-10-2002$ & $<0.007$ & ID & 8 & -- & -- & -- \\
\hline \multirow[t]{2}{*}{111} & $8-8-2002$ & $<0.007$ & ${ }^{2} 2.4-63.4$ & 40 & -- & -- & -- \\
\hline & $9-11-2002$ & 0.007 & ${ }^{2} 2.4-63.4$ & 40 & -- & -- & -- \\
\hline \multirow[t]{2}{*}{112} & $8-8-2002$ & 0.162 & ID & 11 & -- & -- & -- \\
\hline & $9-12-2002$ & 0.414 & ID & 11 & -- & -- & -- \\
\hline \multirow[t]{2}{*}{113} & $8-23-2002$ & $<0.02$ & ${ }^{2} 2.6-87.2$ & 38 & -- & -- & -- \\
\hline & $9-12-2002$ & $<0.02$ & ${ }^{2} 2.6-87.2$ & 38 & -- & -- & -- \\
\hline \multirow[t]{2}{*}{114} & $8-23-2002$ & 0.100 & ${ }^{1} 88.9-91.7$ & 35 & -- & -- & -- \\
\hline & $9-12-2002$ & 0.080 & 83.3 & 35 & -- & -- & -- \\
\hline 115 & $8-6-2002$ & 0.040 & ID & 17 & 5.3 & ID & 16 \\
\hline \multirow[t]{2}{*}{116} & $8-8-2002$ & 0.540 & 88.6 & 34 & 6.9 & 96.4 & 27 \\
\hline & $9-3-2002$ & 0.550 & 91.4 & 34 & 4.3 & ${ }^{1} 32.1-35.7$ & 27 \\
\hline \multirow[t]{2}{*}{117} & $8-2-2002$ & 0.037 & 8.3 & 23 & -- & -- & -- \\
\hline & $9-17-2002$ & 0.189 & 58.3 & 23 & 4.3 & ID & 1 \\
\hline 118 & $9-19-2002$ & $<0.02$ & ID & 16 & 3.9 & ID & 6 \\
\hline \multirow[t]{2}{*}{119} & $8-6-2002$ & $<0.007$ & ${ }^{2} 3.1-81.3$ & 31 & -- & -- & -- \\
\hline & $9-10-2002$ & $<0.007$ & ${ }^{2} 3.1-81.3$ & 31 & -- & -- & -- \\
\hline \multirow[t]{2}{*}{120} & $8-22-2002$ & 0.020 & ID & 2 & 2.7 & ID & 2 \\
\hline & $9-16-2002$ & $<0.02$ & ID & 2 & 3.3 & ID & 2 \\
\hline \multirow[t]{2}{*}{121} & $8-7-2002$ & $<0.007$ & ID & 16 & 1.5 & ID & 10 \\
\hline & $9-4-2002$ & $<0.007$ & ID & 16 & 1.5 & ID & 10 \\
\hline \multirow[t]{2}{*}{122} & 8-7-2002 & 0.020 & ID & 8 & -- & -- & -- \\
\hline & $9-11-2002$ & 0.048 & ID & 8 & -- & -- & -- \\
\hline & $9-30-2002$ & 0.010 & ID & 1 & 2.7 & ID & 1 \\
\hline \multirow[t]{2}{*}{124} & $8-6-2002$ & 0.125 & ID & 8 & -- & -- & -- \\
\hline & $9-10-2002$ & 0.085 & ID & 8 & -- & -- & -- \\
\hline 125 & $8-14-2002$ & $<0.02$ & ID & 2 & -- & -- & -- \\
\hline & $9-11-2002$ & $<0.02$ & ID & 2 & -- & -- & -- \\
\hline
\end{tabular}


Table 17. Water-quality data for the South Platte River Basin showing 2002 drought values, Colorado water-quality exceedances, and percentiles relative to historical data for selected properties and constituents from July through September, 1978-2002.-Continued

$\left[\mathrm{ft}^{3} / \mathrm{s}\right.$, cubic feet per second; Conc, concentration; Q\%, percentile (percentage of data less than or equal to reported value or concentration) of drought-sample constituent; N, total number of historical and drought samples; $\mathrm{mg} / \mathrm{L}$, milligrams per liter; $\mu \mathrm{S} / \mathrm{cm}$, microsiemens per centimeter at $25^{\circ} \mathrm{Celsius;}{ }^{\circ} \mathrm{C}$, degrees Celsius; E. coli, Escherichia coli; $\mathrm{mL}$, milliliter; $\mu \mathrm{g} / \mathrm{L}$, micrograms per liter; $\mathrm{CaCO}_{3}$, calcium carbonate; $\mathrm{SiO}_{2}$, silica; $\mathrm{SO}_{4}$, sulfate; ID, insufficient number of samples (less than 20) to calculate percentile; <, less than; --, no drought-sample measurement; values or concentrations in bold type indicate 2002 Colorado water-quality exceedances; c, chronic water-quality exceedance; ac, acute and chronic water-quality exceedance]

\begin{tabular}{|c|c|c|c|c|c|c|c|}
\hline \multirow{2}{*}{$\begin{array}{c}\text { Site number } \\
\text { in table } 1 \\
\text { and fig. } 6\end{array}$} & \multirow{2}{*}{$\begin{array}{l}\text { Sampling } \\
\text { date }\end{array}$} & \multicolumn{3}{|c|}{$\begin{array}{l}\text { Carbon, organic, total recoverable, in } \\
\qquad \mathrm{mg} / \mathrm{L}\end{array}$} & \multirow{2}{*}{$\begin{array}{c}\text { Aluminum, } \\
\text { dissolved, } \\
\text { in } \mu \mathrm{g} / \mathrm{L}\end{array}$} & \multirow{2}{*}{$\begin{array}{l}\text { Arsenic, } \\
\text { dissolved, } \\
\text { in } \mu \mathrm{g} / \mathrm{L}\end{array}$} & \multirow{2}{*}{$\begin{array}{c}\text { Cadmium, } \\
\text { dissolved, } \\
\text { in } \mu \mathrm{g} / \mathrm{L}\end{array}$} \\
\hline & & Conc & $0 \%$ & $\mathrm{~N}$ & & & \\
\hline \multirow[t]{2}{*}{103} & $8-5-2002$ & 1.2 & ID & 16 & -- & -- & -- \\
\hline & $9-5-2002$ & 0.3 & ID & 16 & -- & -- & -- \\
\hline \multirow[t]{2}{*}{104} & $8-13-2002$ & -- & -- & -- & -- & -- & -- \\
\hline & $9-10-2002$ & -- & -- & -- & 10 & -- & 0.08 \\
\hline \multirow[t]{2}{*}{105} & $8-15-2002$ & -- & -- & -- & 20 & -- & 0.21 \\
\hline & $9-12-2002$ & -- & -- & -- & 20 & -- & 0.28 \\
\hline \multirow[t]{2}{*}{106} & $8-13-2002$ & -- & -- & -- & -- & -- & -- \\
\hline & $9-10-2002$ & -- & -- & -- & -- & -- & -- \\
\hline \multirow[t]{2}{*}{107} & $8-5-2002$ & -- & -- & -- & -- & $<0.2$ & -- \\
\hline & $9-9-2002$ & -- & -- & -- & -- & 0.1 & -- \\
\hline \multirow[t]{2}{*}{108} & $8-5-2002$ & -- & -- & -- & -- & 0.2 & -- \\
\hline & $9-9-2002$ & -- & -- & -- & -- & 0.3 & -- \\
\hline \multirow[t]{2}{*}{109} & $8-5-2002$ & -- & -- & -- & -- & 0.3 & -- \\
\hline & $9-9-2002$ & -- & -- & -- & -- & 0.3 & -- \\
\hline \multirow[t]{2}{*}{110} & 8-6-2002 & -- & -- & -- & -- & 0.1 & -- \\
\hline & $9-10-2002$ & -- & -- & -- & -- & 0.1 & -- \\
\hline \multirow[t]{2}{*}{111} & $8-8-2002$ & -- & -- & -- & -- & 0.6 & -- \\
\hline & $9-11-2002$ & -- & -- & -- & -- & 0.6 & -- \\
\hline \multirow[t]{2}{*}{112} & $8-8-2002$ & -- & -- & -- & -- & 0.7 & -- \\
\hline & $9-12-2002$ & -- & -- & -- & -- & 1.0 & -- \\
\hline \multirow[t]{2}{*}{113} & $8-23-2002$ & -- & -- & -- & -- & -- & -- \\
\hline & $9-12-2002$ & -- & -- & -- & 3 & 1.0 & $<0.1$ \\
\hline \multirow[t]{2}{*}{114} & $8-23-2002$ & -- & -- & -- & -- & -- & -- \\
\hline & $9-12-2002$ & -- & -- & -- & 1 & $<2$ & 0.10 \\
\hline 115 & 8-6-2002 & 0.4 & ID & 16 & -- & -- & -- \\
\hline \multirow[t]{2}{*}{116} & $8-8-2002$ & 4.0 & 88.9 & 26 & -- & -- & -- \\
\hline & $9-3-2002$ & 1.2 & 25.9 & 26 & -- & -- & -- \\
\hline \multirow[t]{2}{*}{117} & 8-2-2002 & -- & -- & -- & -- & -- & 0.10 \\
\hline & $9-17-2002$ & -- & -- & -- & -- & -- & $<0.1$ \\
\hline 118 & $9-19-2002$ & -- & -- & -- & $<20$ & -- & 0.03 \\
\hline \multirow[t]{2}{*}{119} & $8-6-2002$ & -- & -- & -- & -- & 0.3 & -- \\
\hline & $9-10-2002$ & -- & -- & -- & -- & 0.4 & -- \\
\hline \multirow[t]{2}{*}{120} & $8-22-2002$ & -- & -- & -- & $<20$ & -- & $<0.04$ \\
\hline & $9-16-2002$ & -- & -- & -- & $<20$ & -- & $<0.04$ \\
\hline \multirow[t]{2}{*}{121} & $8-7-2002$ & 0.3 & ID & 9 & -- & -- & -- \\
\hline & $9-4-2002$ & 0.2 & ID & 9 & -- & -- & -- \\
\hline 122 & 8-7-2002 & -- & -- & -- & -- & 0.2 & -- \\
\hline & $9-11-2002$ & -- & -- & -- & -- & 0.3 & -- \\
\hline 123 & $9-30-2002$ & -- & -- & -- & $<20$ & -- & $<0.04$ \\
\hline 124 & 8-6-2002 & -- & -- & -- & -- & 0.2 & -- \\
\hline & $9-10-2002$ & -- & -- & -- & -- & 0.3 & -- \\
\hline 125 & 8-14-2002 & -- & -- & -- & -- & -- & -- \\
\hline & $9-11-2002$ & -- & -- & -- & -- & -- & -- \\
\hline
\end{tabular}


Table 17. Water-quality data for the South Platte River Basin showing 2002 drought values, Colorado water-quality exceedances, and percentiles relative to historical data for selected properties and constituents from July through September, 1978-2002.-Continued

$\left[\mathrm{ft}^{3} / \mathrm{s}\right.$, cubic feet per second; Conc, concentration; $\mathrm{Q} \%$, percentile (percentage of data less than or equal to reported value or concentration) of drought-sample constituent; N, total number of historical and drought samples; $\mathrm{mg} / \mathrm{L}$, milligrams per liter; $\mu \mathrm{S} / \mathrm{cm}$, microsiemens per centimeter at $25^{\circ} \mathrm{Celsius;}{ }^{\circ} \mathrm{C}$, degrees Celsius; E. coli, Escherichia coli; $\mathrm{mL}$, milliliter; $\mu \mathrm{g} / \mathrm{L}$, micrograms per liter; $\mathrm{CaCO}_{3}$, calcium carbonate; $\mathrm{SiO}_{2}$, silica; $\mathrm{SO}_{4}$, sulfate; ID, insufficient number of samples (less than 20) to calculate percentile; <, less than; --, no drought-sample measurement; values or concentrations in bold type indicate 2002 Colorado water-quality exceedances; c, chronic water-quality exceedance; ac, acute and chronic water-quality exceedance]

\begin{tabular}{|c|c|c|c|c|c|c|c|}
\hline \multirow{2}{*}{$\begin{array}{c}\text { Site number } \\
\text { in table } 1 \\
\text { and fig. } 6\end{array}$} & \multirow{2}{*}{$\begin{array}{c}\text { Sampling } \\
\text { date }\end{array}$} & \multicolumn{3}{|c|}{ Copper, dissolved, in $\mu \mathrm{g} / \mathrm{L}$} & \multicolumn{3}{|c|}{ Iron, dissolved, in $\mu \mathrm{g} / \mathrm{L}$} \\
\hline & & Conc & $0 \%$ & $\mathrm{~N}$ & Conc & $0 \%$ & $\mathrm{~N}$ \\
\hline \multirow[t]{2}{*}{103} & $8-5-2002$ & -- & -- & -- & $<10$ & ID & 19 \\
\hline & $9-5-2002$ & -- & -- & -- & 8 & ID & 19 \\
\hline \multirow[t]{2}{*}{104} & $8-13-2002$ & -- & -- & -- & 35 & 70.4 & 26 \\
\hline & $9-10-2002$ & 4.4 & ID & 8 & 44 & 81.5 & 26 \\
\hline \multirow[t]{2}{*}{105} & $8-15-2002$ & 4.3 & ID & 8 & 19 & ID & 18 \\
\hline & $9-12-2002$ & 4.1 & ID & 8 & 25 & ID & 18 \\
\hline \multirow[t]{2}{*}{106} & $8-13-2002$ & -- & -- & -- & 65 & ID & 18 \\
\hline & $9-10-2002$ & -- & -- & -- & 41 & ID & 18 \\
\hline \multirow[t]{2}{*}{107} & $8-5-2002$ & 0.6 & ID & 8 & 129 & ID & 8 \\
\hline & 9-9-2002 & 0.6 & ID & 8 & 140 & ID & 8 \\
\hline \multirow[t]{2}{*}{108} & $8-5-2002$ & 0.6 & ID & 18 & 86 & 67.4 & 45 \\
\hline & $9-9-2002$ & 0.5 & ID & 18 & 36 & 6.5 & 45 \\
\hline \multirow[t]{2}{*}{109} & $8-5-2002$ & 0.6 & ID & 8 & 65 & ID & 8 \\
\hline & $9-9-2002$ & 0.5 & ID & 8 & 45 & ID & 8 \\
\hline \multirow[t]{2}{*}{110} & $8-6-2002$ & 1.9ac & ID & 8 & 64 & ID & 8 \\
\hline & $9-10-2002$ & 0.5 & ID & 8 & 83 & ID & 8 \\
\hline \multirow[t]{2}{*}{111} & $8-8-2002$ & 1.1 & ${ }^{1} 35.7-40.5$ & 41 & 8 & ${ }^{2} 3.6-14.3$ & 27 \\
\hline & $9-11-2002$ & 2.2 & 83.3 & 41 & 26 & 53.6 & 27 \\
\hline \multirow[t]{2}{*}{112} & $8-8-2002$ & 1.6 & ${ }^{2} 4.8-47.6$ & 20 & 6 & ID & 6 \\
\hline & $9-12-2002$ & 2.5 & 71.4 & 20 & 18 & ID & 6 \\
\hline \multirow[t]{2}{*}{113} & $8-23-2002$ & 1.3 & 31.8 & 43 & 18 & 8.6 & 34 \\
\hline & $9-12-2002$ & 1.1 & 27.3 & 43 & 32 & 25.7 & 34 \\
\hline \multirow[t]{2}{*}{114} & $8-23-2002$ & 1.8 & ${ }^{1} 59.6-63.8$ & 46 & 24 & 27.6 & 28 \\
\hline & $9-12-2002$ & 2.2 & 80.9 & 46 & 12 & 13.8 & 28 \\
\hline 115 & 8-6-2002 & -- & -- & -- & $<10$ & ID & 16 \\
\hline \multirow[t]{2}{*}{116} & $8-8-2002$ & -- & -- & -- & $<10$ & ${ }^{2} 2.5-75.0$ & 39 \\
\hline & $9-3-2002$ & -- & -- & -- & 20 & ${ }^{1} 77.5-80.0$ & 39 \\
\hline \multirow[t]{2}{*}{117} & $8-2-2002$ & 3.3 & ID & 16 & $<10$ & ${ }^{2} 2.0-64.0$ & 49 \\
\hline & $9-17-2002$ & 3.5 & ID & 16 & 5 & ${ }^{2} 2.0-64.0$ & 49 \\
\hline 118 & $9-19-2002$ & 3.7 & ID & 15 & $<10$ & ID & 18 \\
\hline \multirow[t]{2}{*}{119} & 8-6-2002 & 0.6 & ${ }^{2} 2.4-95.2$ & 41 & 36 & 65.5 & 57 \\
\hline & $9-10-2002$ & 0.5 & ${ }^{2} 2.4-95.2$ & 41 & 13 & ${ }^{1} 1.7-3.4$ & 57 \\
\hline \multirow[t]{2}{*}{120} & $8-22-2002$ & 1.8 & ID & 2 & 28 & ID & 2 \\
\hline & $9-16-2002$ & 1.2 & ID & 2 & 21 & ID & 2 \\
\hline \multirow[t]{2}{*}{121} & $8-7-2002$ & -- & -- & -- & 68 & ID & 13 \\
\hline & $9-4-2002$ & -- & -- & -- & 111 & ID & 13 \\
\hline \multirow[t]{2}{*}{122} & $8-7-2002$ & 1.3 & ID & 7 & 53 & ID & 8 \\
\hline & $9-11-2002$ & 1.7 & ID & 7 & 40 & ID & 8 \\
\hline & $9-30-2002$ & 1.3 & ID & 1 & 20 & ID & 1 \\
\hline \multirow[t]{2}{*}{124} & $8-6-2002$ & 0.8 & ID & 8 & 44 & ID & 8 \\
\hline & $9-10-2002$ & 0.7 & ID & 8 & 26 & ID & 8 \\
\hline 125 & $8-14-2002$ & -- & -- & -- & $<10$ & ID & 2 \\
\hline & $9-11-2002$ & -- & -- & -- & $<10$ & ID & 2 \\
\hline
\end{tabular}


Table 17. Water-quality data for the South Platte River Basin showing 2002 drought values, Colorado water-quality exceedances, and percentiles relative to historical data for selected properties and constituents from July through September, 1978-2002.-Continued

$\left[\mathrm{ft}^{3} / \mathrm{s}\right.$, cubic feet per second; Conc, concentration; Q\%, percentile (percentage of data less than or equal to reported value or concentration) of drought-sample constituent; $\mathrm{N}$, total number of historical and drought samples; $\mathrm{mg} / \mathrm{L}$, milligrams per liter; $\mu \mathrm{S} / \mathrm{cm}$, microsiemens per centimeter at $25^{\circ} \mathrm{Celsius;}{ }^{\circ} \mathrm{C}$, degrees Celsius; E. coli, Escherichia coli; $\mathrm{mL}$, milliliter; $\mu \mathrm{g} / \mathrm{L}$, micrograms per liter; $\mathrm{CaCO}_{3}$, calcium carbonate; $\mathrm{SiO}_{2}$, silica; $\mathrm{SO}_{4}$, sulfate; ID, insufficient number of samples (less than 20) to calculate percentile; <, less than; --, no drought-sample measurement; values or concentrations in bold type indicate 2002 Colorado water-quality exceedances; c, chronic water-quality exceedance; ac, acute and chronic water-quality exceedance]

\begin{tabular}{|c|c|c|c|c|c|c|c|}
\hline \multirow{2}{*}{$\begin{array}{l}\text { Site number } \\
\text { in table } 1 \\
\text { and fig. } 6\end{array}$} & \multirow{2}{*}{$\begin{array}{c}\text { Sampling } \\
\text { date }\end{array}$} & \multicolumn{3}{|c|}{ Iron, total recoverable, in $\mu \mathrm{g} / \mathrm{L}$} & \multicolumn{3}{|c|}{ Lead, dissolved, in $\mu \mathrm{g} / \mathrm{L}$} \\
\hline & & Conc & $0 \%$ & $\mathrm{~N}$ & Conc & $0 \%$ & $\mathrm{~N}$ \\
\hline \multirow[t]{2}{*}{103} & $8-5-2002$ & -- & -- & -- & -- & -- & -- \\
\hline & $9-5-2002$ & -- & -- & -- & -- & -- & -- \\
\hline \multirow[t]{2}{*}{104} & $8-13-2002$ & -- & -- & -- & -- & -- & -- \\
\hline & $9-10-2002$ & -- & -- & -- & 0.25 & ID & 8 \\
\hline \multirow[t]{2}{*}{105} & $8-15-2002$ & -- & -- & -- & 0.23 & ID & 8 \\
\hline & $9-12-2002$ & -- & -- & -- & 0.23 & ID & 8 \\
\hline \multirow[t]{2}{*}{106} & $8-13-2002$ & -- & -- & -- & -- & -- & -- \\
\hline & $9-10-2002$ & -- & -- & -- & -- & -- & -- \\
\hline \multirow[t]{2}{*}{107} & $8-5-2002$ & -- & -- & -- & $<0.08$ & ID & 8 \\
\hline & $9-9-2002$ & -- & -- & -- & 0.10 & ID & 8 \\
\hline \multirow[t]{2}{*}{108} & $8-5-2002$ & -- & -- & -- & $<0.08$ & ID & 18 \\
\hline & $9-9-2002$ & -- & -- & -- & $<0.08$ & ID & 18 \\
\hline \multirow[t]{2}{*}{109} & $8-5-2002$ & -- & -- & -- & $<0.08$ & ID & 8 \\
\hline & $9-9-2002$ & -- & -- & -- & $<0.08$ & ID & 8 \\
\hline \multirow[t]{2}{*}{110} & $8-6-2002$ & -- & -- & -- & 0.14 & ID & 8 \\
\hline & $9-10-2002$ & -- & -- & -- & 0.07 & ID & 8 \\
\hline \multirow[t]{2}{*}{111} & 8-8-2002 & -- & -- & -- & 0.10 & ID & 19 \\
\hline & $9-11-2002$ & -- & -- & -- & 0.11 & ID & 19 \\
\hline \multirow[t]{2}{*}{112} & $8-8-2002$ & -- & -- & -- & 0.06 & ID & 11 \\
\hline & $9-12-2002$ & -- & -- & -- & 0.11 & ID & 11 \\
\hline \multirow[t]{2}{*}{113} & $8-23-2002$ & 200 & ${ }^{1} 52.0-56.0$ & 49 & -- & -- & -- \\
\hline & $9-12-2002$ & 210 & ${ }^{1} 58.0-62.0$ & 49 & $<1$ & ID & 14 \\
\hline \multirow[t]{2}{*}{114} & $8-23-2002$ & 240 & ${ }^{1} 15.4-21.2$ & 51 & -- & -- & -- \\
\hline & $9-12-2002$ & 250 & ${ }^{1} 23.1-25.0$ & 51 & $<1$ & ID & 15 \\
\hline 115 & $8-6-2002$ & -- & -- & -- & -- & -- & -- \\
\hline \multirow[t]{2}{*}{116} & $8-8-2002$ & -- & -- & -- & -- & -- & -- \\
\hline & $9-3-2002$ & -- & -- & -- & -- & -- & -- \\
\hline \multirow[t]{2}{*}{117} & $8-2-2002$ & -- & -- & -- & 0.06 & ID & 16 \\
\hline & $9-17-2002$ & -- & -- & -- & 0.17 & ID & 16 \\
\hline 118 & $9-19-2002$ & -- & -- & -- & $<0.08$ & ID & 15 \\
\hline \multirow[t]{2}{*}{119} & $8-6-2002$ & -- & -- & -- & 0.04 & ${ }^{2} 2.9-94.1$ & 33 \\
\hline & $9-10-2002$ & -- & -- & -- & 0.05 & ${ }^{2} 2.9-94.1$ & 33 \\
\hline \multirow[t]{2}{*}{120} & $8-22-2002$ & -- & -- & -- & 0.04 & ID & 2 \\
\hline & $9-16-2002$ & -- & -- & -- & 0.05 & ID & 2 \\
\hline \multirow[t]{2}{*}{121} & $8-7-2002$ & -- & -- & -- & -- & -- & -- \\
\hline & $9-4-2002$ & -- & -- & -- & -- & -- & -- \\
\hline \multirow[t]{2}{*}{122} & $8-7-2002$ & -- & -- & -- & 0.07 & ID & 8 \\
\hline & $9-11-2002$ & -- & -- & -- & 0.08 & ID & 8 \\
\hline 123 & $9-30-2002$ & -- & -- & -- & 0.04 & ID & 1 \\
\hline \multirow[t]{2}{*}{124} & $8-6-2002$ & -- & -- & -- & $<0.08$ & ID & 8 \\
\hline & $9-10-2002$ & -- & -- & -- & 0.05 & ID & 8 \\
\hline 125 & $8-14-2002$ & -- & -- & -- & -- & -- & -- \\
\hline & $9-11-2002$ & -- & -- & -- & -- & -- & -- \\
\hline
\end{tabular}


Table 17. Water-quality data for the South Platte River Basin showing 2002 drought values, Colorado water-quality exceedances, and percentiles relative to historical data for selected properties and constituents from July through September, 1978-2002.-Continued

$\left[\mathrm{ft}^{3} / \mathrm{s}\right.$, cubic feet per second; Conc, concentration; $\mathrm{Q} \%$, percentile (percentage of data less than or equal to reported value or concentration) of drought-sample constituent; N, total number of historical and drought samples; $\mathrm{mg} / \mathrm{L}$, milligrams per liter; $\mu \mathrm{S} / \mathrm{cm}$, microsiemens per centimeter at $25^{\circ} \mathrm{Celsius;}{ }^{\circ} \mathrm{C}$, degrees Celsius; E. coli, Escherichia coli; $\mathrm{mL}$, milliliter; $\mu \mathrm{g} / \mathrm{L}$, micrograms per liter; $\mathrm{CaCO}_{3}$, calcium carbonate; $\mathrm{SiO}_{2}$, silica; $\mathrm{SO}_{4}$, sulfate; ID, insufficient number of samples (less than 20) to calculate percentile; <, less than; --, no drought-sample measurement; values or concentrations in bold type indicate 2002 Colorado water-quality exceedances; c, chronic water-quality exceedance; ac, acute and chronic water-quality exceedance]

\begin{tabular}{|c|c|c|c|c|c|c|c|c|}
\hline \multirow{2}{*}{$\begin{array}{c}\text { Site number } \\
\text { in table } 1 \\
\text { and fig. } 6\end{array}$} & \multirow{2}{*}{$\begin{array}{c}\text { Sampling } \\
\text { date }\end{array}$} & \multicolumn{3}{|c|}{ Manganese, dissolved, in $\mu \mathrm{g} / \mathrm{L}$} & \multirow{2}{*}{$\begin{array}{l}\text { Mercury, } \\
\text { dissolved, } \\
\text { in } \mu \mathrm{g} / \mathrm{L}\end{array}$} & \multicolumn{3}{|c|}{ Nickel, dissolved, in $\mu \mathrm{g} / \mathrm{L}$} \\
\hline & & Conc & $0 \%$ & $\mathrm{~N}$ & & Conc & $0 \%$ & $\mathrm{~N}$ \\
\hline \multirow[t]{2}{*}{103} & $8-5-2002$ & 18.9 & ID & 19 & -- & -- & -- & -- \\
\hline & $9-5-2002$ & 13.7 & ID & 19 & -- & -- & -- & -- \\
\hline \multirow[t]{2}{*}{104} & $8-13-2002$ & -- & -- & -- & -- & -- & -- & -- \\
\hline & $9-10-2002$ & 46 & 50.0 & 25 & -- & -- & -- & -- \\
\hline \multirow[t]{2}{*}{105} & $8-15-2002$ & 21 & ID & 18 & -- & -- & -- & -- \\
\hline & $9-12-2002$ & 36 & ID & 18 & -- & -- & -- & -- \\
\hline \multirow[t]{2}{*}{106} & $8-13-2002$ & -- & -- & -- & -- & -- & -- & -- \\
\hline & $9-10-2002$ & -- & -- & -- & -- & -- & -- & -- \\
\hline \multirow[t]{2}{*}{107} & $8-5-2002$ & 3.4 & ID & 8 & $<0.01$ & 0.10 & ID & 8 \\
\hline & $9-9-2002$ & 6.8 & ID & 8 & $<0.01$ & 0.21 & ID & 8 \\
\hline \multirow[t]{2}{*}{108} & $8-5-2002$ & 2.0 & ${ }^{2} 2.0-88.0$ & 49 & $<0.01$ & 0.16 & ID & 18 \\
\hline & $9-9-2002$ & 0.7 & ${ }^{2} 2.0-88.0$ & 49 & $<0.01$ & 0.32 & ID & 18 \\
\hline \multirow[t]{2}{*}{109} & $8-5-2002$ & 1.5 & ID & 8 & $<0.01$ & 0.15 & ID & 8 \\
\hline & $9-9-2002$ & 1.7 & ID & 8 & $<0.01$ & 0.31 & ID & 8 \\
\hline \multirow[t]{2}{*}{110} & 8-6-2002 & 3.4 & ID & 8 & $<0.01$ & 0.11 & ID & 8 \\
\hline & $9-10-2002$ & 4.7 & ID & 8 & $<0.01$ & 0.19 & ID & 8 \\
\hline \multirow[t]{2}{*}{111} & 8-8-2002 & 9.1 & 25.0 & 31 & $<0.01$ & 0.77 & ID & 19 \\
\hline & $9-11-2002$ & 16.5 & 53.1 & 31 & $<0.01$ & 1.68 & ID & 19 \\
\hline \multirow[t]{2}{*}{112} & 8-8-2002 & 19.6 & ID & 6 & $<0.01$ & 1.11 & ID & 11 \\
\hline & $9-12-2002$ & 47 & ID & 6 & $<0.01$ & 2.7 & ID & 11 \\
\hline \multirow[t]{2}{*}{113} & $8-23-2002$ & -- & -- & -- & -- & -- & -- & -- \\
\hline & $9-12-2002$ & -- & -- & -- & $<0.01$ & 1.2 & ID & 14 \\
\hline \multirow[t]{2}{*}{114} & $8-23-2002$ & -- & -- & -- & -- & -- & -- & 15 \\
\hline & $9-12-2002$ & -- & -- & -- & $<0.01$ & 1.5 & ID & 15 \\
\hline 115 & $8-6-2002$ & 10.2 & ID & 16 & -- & -- & -- & -- \\
\hline \multirow[t]{2}{*}{116} & $8-8-2002$ & 177 & 97.5 & 39 & -- & -- & -- & -- \\
\hline & $9-3-2002$ & 167 & 95.0 & 39 & -- & -- & -- & -- \\
\hline \multirow[t]{2}{*}{117} & 8-2-2002 & 40 & 98.0 & 49 & -- & 4.2 & ID & 16 \\
\hline & $9-17-2002$ & 23 & 88.0 & 49 & -- & 6.3 & ID & 16 \\
\hline 118 & $9-19-2002$ & 7.9 & ID & 18 & -- & -- & -- & -- \\
\hline \multirow[t]{2}{*}{119} & $8-6-2002$ & 1.5 & ${ }^{2} 1.6-98.4$ & 61 & $<0.01$ & 0.16 & ${ }^{2} 2.9-97.1$ & 34 \\
\hline & $9-10-2002$ & 0.8 & ${ }^{2} 1.6-98.4$ & 61 & $<0.01$ & 0.31 & ${ }^{2} 2.9-97.1$ & 34 \\
\hline \multirow[t]{2}{*}{120} & $8-22-2002$ & 69 & ID & 2 & -- & -- & -- & -- \\
\hline & $9-16-2002$ & 63 & ID & 2 & -- & -- & -- & -- \\
\hline \multirow[t]{2}{*}{121} & $8-7-2002$ & 2.9 & ID & 13 & -- & -- & -- & -- \\
\hline & $9-4-2002$ & 4.0 & ID & 13 & -- & -- & -- & -- \\
\hline 122 & 8-7-2002 & 3.0 & ID & 8 & $<0.01$ & 0.16 & ID & 8 \\
\hline & $9-11-2002$ & 5.1 & ID & 8 & $<0.01$ & 0.36 & ID & 8 \\
\hline 123 & $9-30-2002$ & 14.3 & ID & 1 & -- & -- & -- & -- \\
\hline 124 & $8-6-2002$ & 2.9 & ID & 8 & $<0.01$ & 0.22 & ID & 8 \\
\hline & $9-10-2002$ & 2.9 & ID & 8 & 0.01 & 0.40 & ID & 8 \\
\hline 125 & $8-14-2002$ & -- & -- & -- & -- & -- & -- & -- \\
\hline & $9-11-2002$ & -- & -- & -- & -- & -- & -- & -- \\
\hline
\end{tabular}


Table 17. Water-quality data for the South Platte River Basin showing 2002 drought values, Colorado water-quality exceedances, and percentiles relative to historical data for selected properties and constituents from July through September, 1978-2002.-Continued

$\left[\mathrm{ft}^{3} / \mathrm{s}\right.$, cubic feet per second; Conc, concentration; Q\%, percentile (percentage of data less than or equal to reported value or concentration) of drought-sample constituent; N, total number of historical and drought samples; $\mathrm{mg} / \mathrm{L}$, milligrams per liter; $\mu \mathrm{S} / \mathrm{cm}$, microsiemens per centimeter at $25^{\circ} \mathrm{Celsius;}{ }^{\circ} \mathrm{C}$, degrees Celsius; E. coli, Escherichia coli; $\mathrm{mL}$, milliliter; $\mu \mathrm{g} / \mathrm{L}$, micrograms per liter; $\mathrm{CaCO}_{3}$, calcium carbonate; $\mathrm{SiO}_{2}$, silica; $\mathrm{SO}_{4}$, sulfate; ID, insufficient number of samples (less than 20) to calculate percentile; <, less than; --, no drought-sample measurement; values or concentrations in bold type indicate 2002 Colorado water-quality exceedances; c, chronic water-quality exceedance; ac, acute and chronic water-quality exceedance]

\begin{tabular}{|c|c|c|c|c|c|}
\hline \multirow{2}{*}{$\begin{array}{c}\text { Site number } \\
\text { in table } 1 \\
\text { and fig. } 6\end{array}$} & \multirow{2}{*}{$\begin{array}{c}\text { Sampling } \\
\text { date }\end{array}$} & \multicolumn{3}{|c|}{ Silver, dissolved, in $\mu \mathrm{g} / \mathrm{L}$} & \multirow{2}{*}{$\begin{array}{l}\text { Zinc, dissolved, } \\
\text { in } \mu \mathrm{g} / \mathrm{L}\end{array}$} \\
\hline & & Conc & $0 \%$ & $\mathrm{~N}$ & \\
\hline \multirow[t]{2}{*}{103} & $8-5-2002$ & -- & -- & -- & -- \\
\hline & $9-5-2002$ & -- & -- & -- & -- \\
\hline \multirow[t]{2}{*}{104} & $8-13-2002$ & -- & -- & -- & -- \\
\hline & $9-10-2002$ & $<1$ & ID & 5 & 18 \\
\hline \multirow[t]{2}{*}{105} & $8-15-2002$ & $<1$ & ID & 6 & 33 \\
\hline & $9-12-2002$ & $<1$ & ID & 6 & 41 \\
\hline \multirow[t]{2}{*}{106} & $8-13-2002$ & -- & -- & -- & -- \\
\hline & $9-10-2002$ & -- & -- & -- & -- \\
\hline \multirow[t]{2}{*}{107} & $8-5-2002$ & $<1$ & ID & 8 & -- \\
\hline & $9-9-2002$ & $<1$ & ID & 8 & -- \\
\hline \multirow[t]{2}{*}{108} & $8-5-2002$ & $<1$ & ID & 18 & -- \\
\hline & $9-9-2002$ & $<1$ & ID & 18 & -- \\
\hline \multirow[t]{2}{*}{109} & $8-5-2002$ & $<1$ & ID & 8 & -- \\
\hline & 9-9-2002 & $<1$ & ID & 8 & -- \\
\hline \multirow[t]{2}{*}{110} & $8-6-2002$ & $<1$ & ID & 8 & -- \\
\hline & $9-10-2002$ & $<1$ & ID & 8 & -- \\
\hline \multirow[t]{2}{*}{111} & $8-8-2002$ & $<1$ & ${ }^{2} 2.4-97.6$ & 40 & -- \\
\hline & $9-11-2002$ & $<1$ & ${ }^{2} 2.4-97.6$ & 40 & -- \\
\hline \multirow[t]{2}{*}{112} & 8-8-2002 & $<1$ & ID & 16 & -- \\
\hline & $9-12-2002$ & $<1$ & ID & 16 & -- \\
\hline \multirow[t]{2}{*}{113} & $8-23-2002$ & $<0.1$ & ${ }^{2} 2.7-86.5$ & 36 & -- \\
\hline & $9-12-2002$ & $<0.1$ & ${ }^{2} 2.7-86.5$ & 36 & 2 \\
\hline \multirow[t]{2}{*}{114} & $8-23-2002$ & $<0.1$ & ${ }^{2} 2.5-95.0$ & 39 & -- \\
\hline & $9-12-2002$ & $<0.1$ & ${ }^{2} 2.5-95.0$ & 39 & 3 \\
\hline 115 & 8-6-2002 & -- & -- & -- & -- \\
\hline \multirow[t]{2}{*}{116} & $8-8-2002$ & -- & -- & -- & -- \\
\hline & $9-3-2002$ & -- & -- & -- & -- \\
\hline \multirow[t]{2}{*}{117} & $8-2-2002$ & $<1$ & ID & 16 & 3 \\
\hline & $9-17-2002$ & $<1$ & ID & 16 & 24 \\
\hline 118 & $9-19-2002$ & $<1$ & ID & 18 & 4 \\
\hline \multirow[t]{2}{*}{119} & $8-6-2002$ & $<1$ & ${ }^{2} 3.8-76.9$ & 25 & -- \\
\hline & $9-10-2002$ & $<1$ & ${ }^{2} 3.8-76.9$ & 25 & -- \\
\hline \multirow[t]{2}{*}{120} & $8-22-2002$ & $<1$ & ID & 2 & 15 \\
\hline & $9-16-2002$ & $<1$ & ID & 2 & 4 \\
\hline \multirow[t]{2}{*}{121} & 8-7-2002 & -- & -- & -- & -- \\
\hline & $9-4-2002$ & -- & -- & -- & -- \\
\hline \multirow[t]{2}{*}{122} & $8-7-2002$ & $<1$ & ID & 8 & -- \\
\hline & $9-11-2002$ & $<1$ & ID & 8 & -- \\
\hline 123 & $9-30-2002$ & $<1$ & ID & 1 & $<1$ \\
\hline \multirow[t]{2}{*}{124} & 8-6-2002 & $<1$ & ID & 8 & -- \\
\hline & $9-10-2002$ & $<1$ & ID & 8 & -- \\
\hline \multirow[t]{2}{*}{125} & $8-14-2002$ & -- & -- & -- & -- \\
\hline & $9-11-2002$ & -- & -- & -- & -- \\
\hline
\end{tabular}

${ }^{1}$ Possible percentile range because of uncensored ties in data.

${ }^{2}$ Possible percentile range because of censored or recensored ties in data. 
Table 18. Water-quality data for the Arkansas River Basin showing 2002 drought values, Colorado water-quality exceedances, and percentiles relative to historical data for selected properties and constituents from July through September, 1978-2002.

$\left[\mathrm{ft}^{3} / \mathrm{s}\right.$, cubic feet per second; Conc, concentration; Q\%, percentile (percentage of data less than or equal to reported value or concentration) of drought-sample constituent; $\mathrm{N}$, total number of historical and drought samples; $\mathrm{mg} / \mathrm{L}$, milligrams per liter; $\mu \mathrm{S} / \mathrm{cm}$, microsiemens per centimeter at $25^{\circ} \mathrm{Celsius;}{ }^{\circ} \mathrm{C}$, degrees Celsius; E. coli, Escherichia coli; $\mathrm{mL}$, milliliter; $\mu \mathrm{g} / \mathrm{L}$, micrograms per liter; $\mathrm{CaCO}_{3}$, calcium carbonate; $\mathrm{SiO}_{2}$, silica; $\mathrm{SO}_{4}$, sulfate; ID, insufficient number of samples (less than 20) to calculate percentile; <, less than; --, no drought-sample measurement; values or concentrations in bold type indicate 2002 Colorado water-quality exceedances; c, chronic water-quality exceedance; ac, acute and chronic water-quality exceedance]

\begin{tabular}{|c|c|c|c|c|c|c|}
\hline \multirow{2}{*}{$\begin{array}{c}\text { Site } \\
\text { number in } \\
\text { table } 1 \\
\text { and fig. } 7\end{array}$} & \multirow{2}{*}{$\begin{array}{l}\text { Sampling } \\
\text { date }\end{array}$} & \multirow{2}{*}{$\begin{array}{l}\text { Sampling } \\
\text { time }\end{array}$} & \multirow{2}{*}{$\begin{array}{c}\text { Discharge, } \\
\text { in } \mathrm{ft}^{3} / \mathrm{s}\end{array}$} & \multicolumn{3}{|c|}{ Oxygen, dissolved, in $\mathrm{mg} / \mathrm{L}$} \\
\hline & & & & Conc & $0 \%$ & $\mathrm{~N}$ \\
\hline \multirow[t]{2}{*}{126} & $7-19-2002$ & 1640 & 126 & 6.8 & ID & 8 \\
\hline & 8-26-2002 & 1340 & 139 & 7.5 & ID & 8 \\
\hline \multirow[t]{2}{*}{127} & $7-31-2002$ & 1215 & 171 & 8.4 & ID & 8 \\
\hline & $8-26-2002$ & 1000 & 175 & 8.3 & ID & 8 \\
\hline \multirow[t]{2}{*}{128} & $7-31-2002$ & 1345 & 139 & 12.9 & 96.1 & 50 \\
\hline & $8-26-2002$ & 1115 & 150 & 9.4 & 70.6 & 50 \\
\hline \multirow[t]{2}{*}{129} & $7-31-2002$ & 915 & 165 & 7.6 & ID & 16 \\
\hline & 8-26-2002 & 1300 & 125 & 11.0 & ID & 16 \\
\hline \multirow[t]{2}{*}{130} & $7-22-2002$ & 1505 & 298 & 10.2 & 93.3 & 29 \\
\hline & $8-27-2002$ & 1110 & 9.5 & 6.4 & 3.3 & 29 \\
\hline \multirow[t]{2}{*}{131} & $7-22-2002$ & 1315 & 185 & 9.8 & ID & 15 \\
\hline & $8-27-2002$ & 1225 & 4.4 & 8.7 & ID & 15 \\
\hline 132 & $8-21-2002$ & 915 & 2.9 & 7.9 & ${ }^{1} 29.7-47.3$ & 73 \\
\hline 133 & $8-21-2002$ & 1100 & 2.8 & 8.0 & ${ }^{1} 41.5-47.2$ & 52 \\
\hline 134 & $8-21-2002$ & 1230 & 4.7 & 8.3 & ID & 11 \\
\hline 135 & $8-20-2002$ & 1230 & 6.6 & 7.6 & ${ }^{1} 71.2-76.3$ & 58 \\
\hline 136 & $8-20-2002$ & 1035 & 7.9 & 6.9 & ${ }^{1} 23.9-26.1$ & 87 \\
\hline 137 & $8-20-2002$ & 1630 & 66 & 7.1 & ${ }^{1} 56.8-59.3$ & 80 \\
\hline 138 & $8-20-2002$ & 1500 & 84 & 6.6 & ID & 19 \\
\hline 139 & 8-19-2002 & 1020 & 73 & 7.1 & ID & 12 \\
\hline 140 & $8-19-2002$ & 1300 & 45 & 7.1 & ${ }^{1} 61.3-64.5$ & 30 \\
\hline \multirow[t]{2}{*}{141} & $7-24-2002$ & 1130 & 276 & 7.5 & 75.8 & 32 \\
\hline & $8-27-2002$ & 1430 & 103 & 8.4 & ${ }^{1} 87.9-90.9$ & 32 \\
\hline \multirow[t]{2}{*}{142} & $7-23-2002$ & 1100 & 23 & 8.4 & ID & 13 \\
\hline & $8-28-2002$ & 1415 & 5.7 & 8.9 & ID & 13 \\
\hline \multirow[t]{2}{*}{143} & $7-23-2002$ & 1600 & 20 & 8.2 & ID & 2 \\
\hline & $8-28-2002$ & 1250 & 6.4 & 9.0 & ID & 2 \\
\hline \multirow[t]{2}{*}{144} & $7-23-2002$ & 1245 & 28 & 8.2 & ID & 14 \\
\hline & $9-4-2002$ & 1515 & 12 & 7.3 & ID & 14 \\
\hline \multirow[t]{2}{*}{145} & $7-23-2002$ & 1115 & 10 & 8.4 & ${ }^{1} 72.4-75.9$ & 28 \\
\hline & $9-4-2002$ & 1045 & 13 & 9.0 & 89.7 & 28 \\
\hline 146 & $8-28-2002$ & 1400 & 1.9 & 6.9 & ID & 3 \\
\hline 147 & $9-4-2002$ & 1225 & 0.21 & 5.8 & ID & 1 \\
\hline 148 & $9-4-2002$ & 0915 & 5.8 & 7.8 & ID & 4 \\
\hline
\end{tabular}


Table 18. Water-quality data for the Arkansas River Basin showing 2002 drought values, Colorado water-quality exceedances, and percentiles relative to historical data for selected properties and constituents from July through September, 1978-2002.—Continued

$\left[\mathrm{ft}^{3} / \mathrm{s}\right.$, cubic feet per second; Conc, concentration; Q\%, percentile (percentage of data less than or equal to reported value or concentration) of drought-sample constituent; $\mathrm{N}$, total number of historical and drought samples; $\mathrm{mg} / \mathrm{L}$, milligrams per liter; $\mu \mathrm{S} / \mathrm{cm}$, microsiemens per centimeter at $25^{\circ} \mathrm{Celsius;}{ }^{\circ} \mathrm{C}$, degrees Celsius; E. coli, Escherichia coli; $\mathrm{mL}$, milliliter; $\mu \mathrm{g} / \mathrm{L}$, micrograms per liter; $\mathrm{CaCO}_{3}$, calcium carbonate; $\mathrm{SiO}_{2}$, silica; $\mathrm{SO}_{4}$, sulfate; ID, insufficient number of samples (less than 20) to calculate percentile; <, less than; --, no drought-sample measurement; values or concentrations in bold type indicate 2002 Colorado water-quality exceedances; c, chronic water-quality exceedance; ac, acute and chronic water-quality exceedance]

\begin{tabular}{|c|c|c|c|c|c|c|c|}
\hline \multirow{2}{*}{$\begin{array}{c}\text { Site } \\
\text { number in } \\
\text { table } 1 \\
\text { and fig. } 7\end{array}$} & \multirow{2}{*}{$\begin{array}{c}\text { Sampling } \\
\text { date }\end{array}$} & \multicolumn{3}{|c|}{$\mathrm{pH}$, in standard units } & \multicolumn{3}{|c|}{ Specific conductance, in $\mu S / \mathrm{cm}$} \\
\hline & & Value & $0 \%$ & $\mathrm{~N}$ & Value & $0 \%$ & $\mathrm{~N}$ \\
\hline \multirow[t]{2}{*}{126} & $7-19-2002$ & 8.3 & ID & 9 & 129 & 65.2 & 45 \\
\hline & $8-26-2002$ & 7.4 & ID & 9 & 105 & ${ }^{1} 39.1-41.3$ & 45 \\
\hline \multirow[t]{2}{*}{127} & $7-31-2002$ & 8.5 & ID & 9 & 244 & 48.0 & 24 \\
\hline & $8-26-2002$ & 8.2 & ID & 9 & 239 & 44.0 & 24 \\
\hline \multirow[t]{2}{*}{128} & $7-31-2002$ & 8.7 & ${ }^{1} 79.3-82.8$ & 57 & 472 & ${ }^{1} 81.1-82.4$ & 73 \\
\hline & $8-26-2002$ & 8.5 & ${ }^{1} 58.6-63.8$ & 57 & 520 & 91.9 & 73 \\
\hline \multirow[t]{2}{*}{129} & $7-31-2002$ & 8.2 & ID & 16 & 559 & ID & 16 \\
\hline & $8-26-2002$ & 8.6 & ID & 16 & 593 & ID & 16 \\
\hline \multirow[t]{2}{*}{130} & $7-22-2002$ & 8.1 & ${ }^{1} 36.4-51.5$ & 32 & 606 & 95.1 & 40 \\
\hline & $8-27-2002$ & 8.1 & ${ }^{1} 36.4-51.5$ & 32 & 657 & 97.6 & 40 \\
\hline \multirow[t]{2}{*}{131} & $7-22-2002$ & 8.7 & ID & 17 & 651 & 90.5 & 20 \\
\hline & $8-27-2002$ & 8.0 & ID & 17 & 1,780 & 95.2 & 20 \\
\hline 132 & $8-21-2002$ & 8.4 & ${ }^{1} 84.6--94.9$ & 77 & 558 & 99.3 & 146 \\
\hline 133 & $8-21-2002$ & 8.3 & ${ }^{1} 37.7-45.3$ & 52 & 467 & 98.9 & 94 \\
\hline 134 & $8-21-2002$ & 8.6 & ID & 12 & 445 & 95.7 & 46 \\
\hline 135 & $8-20-2002$ & 8.4 & ${ }^{1} 52.5-76.3$ & 58 & 902 & 98.3 & 59 \\
\hline 136 & $8-20-2002$ & 8.2 & ${ }^{1} 51.1-68.5$ & 91 & 1,040 & 99.0 & 195 \\
\hline 137 & $8-20-2002$ & 7.9 & ${ }^{1} 57.1-75.0$ & 83 & 751 & 53.4 & 102 \\
\hline 138 & $8-20-2002$ & 8.4 & ${ }^{1} 90.9-95.5$ & 21 & 788 & 65.1 & 108 \\
\hline 139 & 8-19-2002 & 8.2 & ID & 12 & 995 & 38.5 & 25 \\
\hline 140 & $8-19-2002$ & 8.4 & ${ }^{1} 84.4-96.9$ & 31 & 1,100 & ${ }^{1} 56.7-57.9$ & 170 \\
\hline \multirow[t]{2}{*}{141} & $7-24-2002$ & 8.1 & ${ }^{1} 34.3-48.6$ & 34 & 912 & 89.1 & 63 \\
\hline & $8-27-2002$ & 8.2 & ${ }^{1} 51.4-82.9$ & 34 & 1,220 & 96.9 & 63 \\
\hline \multirow[t]{2}{*}{142} & $7-23-2002$ & 8.4 & ID & 14 & 1,080 & 81.8 & 32 \\
\hline & $8-28-2002$ & 8.2 & ID & 14 & 1,900 & 97.0 & 32 \\
\hline \multirow[t]{2}{*}{143} & $7-23-2002$ & 8.5 & ID & 2 & 2,170 & ID & 2 \\
\hline & $8-28-2002$ & 8.2 & ID & 2 & 2,360 & ID & 2 \\
\hline \multirow[t]{2}{*}{144} & $7-23-2002$ & 8.2 & ID & 16 & 2,310 & 77.3 & 21 \\
\hline & $9-4-2002$ & 8.2 & ID & 16 & 2,460 & 86.4 & 21 \\
\hline \multirow[t]{2}{*}{145} & $7-23-2002$ & 8.1 & ${ }^{1} 23.3-50.0$ & 29 & 3,320 & ${ }^{1} 82.4-83.0$ & 181 \\
\hline & $9-4-2002$ & 8.1 & ${ }^{1} 23.3-50.0$ & 29 & 3,300 & ${ }^{1} 80.2-81.3$ & 181 \\
\hline 146 & $8-28-2002$ & 8.7 & ID & 6 & 1,070 & 99.0 & 95 \\
\hline 147 & $9-4-2002$ & 8.0 & ID & 1 & 409 & ID & 1 \\
\hline 148 & $9-4-2002$ & 8.0 & ID & 5 & 3,680 & 88.9 & 80 \\
\hline
\end{tabular}


Table 18. Water-quality data for the Arkansas River Basin showing 2002 drought values, Colorado water-quality exceedances, and percentiles relative to historical data for selected properties and constituents from July through September, 1978-2002.—Continued

$\left[\mathrm{ft}^{3} / \mathrm{s}\right.$, cubic feet per second; Conc, concentration; $\mathrm{Q} \%$, percentile (percentage of data less than or equal to reported value or concentration) of drought-sample constituent; $\mathrm{N}$, total number of historical and drought samples; $\mathrm{mg} / \mathrm{L}$, milligrams per liter; $\mu \mathrm{S} / \mathrm{cm}$, microsiemens per centimeter at $25^{\circ} \mathrm{Celsius;}{ }^{\circ} \mathrm{C}$, degrees Celsius; E. coli, Escherichia coli; $\mathrm{mL}$, milliliter; $\mu \mathrm{g} / \mathrm{L}$, micrograms per liter; $\mathrm{CaCO}_{3}$, calcium carbonate; $\mathrm{SiO}_{2}$, silica; $\mathrm{SO}_{4}$, sulfate; ID, insufficient number of samples (less than 20) to calculate percentile; <, less than; --, no drought-sample measurement; values or concentrations in bold type indicate 2002 Colorado water-quality exceedances; c, chronic water-quality exceedance; ac, acute and chronic water-quality exceedance]

\begin{tabular}{|c|c|c|c|c|c|c|c|}
\hline \multirow{2}{*}{$\begin{array}{c}\text { Site } \\
\text { number in } \\
\text { table } 1 \\
\text { and fig. } 7\end{array}$} & \multirow{2}{*}{$\begin{array}{l}\text { Sampling } \\
\text { date }\end{array}$} & \multicolumn{3}{|c|}{ Temperature, water, in ${ }^{\circ} \mathrm{C}$} & \multirow{2}{*}{$\begin{array}{l}\text { Hardness, total, } \\
\text { in } \mathrm{mg} / \mathrm{L} \text { as } \mathrm{CaCO}_{3}\end{array}$} & \multirow{2}{*}{$\begin{array}{c}\text { Calcium, } \\
\text { dissolved, in } \\
\mathrm{mg} / \mathrm{L}\end{array}$} & \multirow{2}{*}{$\begin{array}{c}\text { Magnesium, } \\
\text { dissolved, in } \mathrm{mg} / \mathrm{L}\end{array}$} \\
\hline & & Value & $0 \%$ & $\mathrm{~N}$ & & & \\
\hline \multirow[t]{2}{*}{126} & $7-19-2002$ & 21.5 & 96.3 & 26 & 55 & 14.5 & 4.58 \\
\hline & $8-26-2002$ & 17.0 & 92.6 & 26 & 46 & 12.6 & 3.47 \\
\hline \multirow[t]{2}{*}{127} & $7-31-2002$ & 22.5 & ID & 15 & 100 & 29.2 & 6.72 \\
\hline & $8-26-2002$ & 19.5 & ID & 15 & 95 & 27.6 & 6.33 \\
\hline \multirow[t]{2}{*}{128} & $7-31-2002$ & 27.0 & 98.7 & 74 & 190 & 52.6 & 14.8 \\
\hline & $8-26-2002$ & 21.0 & ${ }^{1} 57.3-60.0$ & 74 & 200 & 55.7 & 15.5 \\
\hline \multirow[t]{2}{*}{129} & $7-31-2002$ & 21.0 & ID & 16 & -- & -- & -- \\
\hline & $8-26-2002$ & 24.0 & ID & 16 & -- & -- & -- \\
\hline \multirow[t]{2}{*}{130} & $7-22-2002$ & 19.0 & ${ }^{1} 21.7-34.8$ & 45 & 260 & 70.7 & 19.6 \\
\hline & $8-27-2002$ & 20.5 & ${ }^{1} 63-71.7$ & 45 & -- & -- & -- \\
\hline \multirow[t]{2}{*}{131} & $7-22-2002$ & 23.0 & 85.7 & 20 & 270 & 72.4 & 21.6 \\
\hline & $8-27-2002$ & 21.0 & ${ }^{1} 38.1-47.6$ & 20 & -- & -- & -- \\
\hline 132 & $8-21-2002$ & 15.5 & ${ }^{1} 69.7-73.5$ & 154 & 200 & 59.1 & 12.7 \\
\hline 133 & $8-21-2002$ & 20.5 & ${ }^{1} 58.8-61.9$ & 96 & 110 & 29.4 & 9.47 \\
\hline 134 & $8-21-2002$ & 21.0 & ${ }^{1} 53.1-59.2$ & 48 & 150 & 47.8 & 8.23 \\
\hline 135 & $8-20-2002$ & 21.5 & ${ }^{1} 36.1-39.3$ & 60 & 330 & 95.1 & 21.4 \\
\hline 136 & $8-20-2002$ & 23.0 & ${ }^{1} 72.2-77.1$ & 204 & 370 & 104 & 27.7 \\
\hline 137 & $8-20-2002$ & 23.5 & ${ }^{1} 86.9-90.7$ & 106 & 190 & 45.7 & 18.9 \\
\hline 138 & $8-20-2002$ & 27.5 & ${ }^{1} 90.8-95.4$ & 108 & 190 & 48.3 & 17.9 \\
\hline 139 & $8-19-2002$ & 20.5 & ${ }^{1} 34.6-38.5$ & 25 & 280 & 72 & 24.6 \\
\hline 140 & $8-19-2002$ & 26.5 & ${ }^{1} 71.7-76.1$ & 158 & 330 & 85.3 & 27.5 \\
\hline \multirow[t]{2}{*}{141} & $7-24-2002$ & 25.0 & ${ }^{1} 91.3-92.8$ & 68 & 330 & 80.5 & 31.9 \\
\hline & $8-27-2002$ & 24.5 & ${ }^{1} 87.0-89.9$ & 68 & -- & -- & -- \\
\hline \multirow[t]{2}{*}{142} & $7-23-2002$ & 24.5 & ID & 18 & 430 & 108 & 38.7 \\
\hline & $8-28-2002$ & 31.5 & ID & 18 & -- & -- & -- \\
\hline \multirow[t]{2}{*}{143} & $7-23-2002$ & 30.5 & ID & 2 & 810 & 192 & 79.2 \\
\hline & $8-28-2002$ & 26.0 & ID & 2 & -- & -- & -- \\
\hline \multirow[t]{2}{*}{144} & $7-23-2002$ & 29.5 & 89.7 & 77 & 960 & 250 & 82.8 \\
\hline & $9-4-2002$ & 32.0 & 94.9 & 77 & -- & -- & -- \\
\hline \multirow[t]{2}{*}{145} & $7-23-2002$ & 26.5 & ${ }^{1} 73.4-73.9$ & 183 & 1,300 & 325 & 125 \\
\hline & $9-4-2002$ & 22.5 & ${ }^{1} 38.0-39.1$ & 183 & -- & -- & -- \\
\hline 146 & $8-28-2002$ & 23.5 & ${ }^{1} 89.0-90.1$ & 90 & 140 & 29.4 & 17.4 \\
\hline 147 & $9-4-2002$ & 22.5 & 27.3 & 32 & 170 & 51.9 & 9.93 \\
\hline 148 & $9-4-2002$ & 19.0 & ${ }^{1} 16.5-20.3$ & 78 & 1,400 & 320 & 156 \\
\hline
\end{tabular}


Table 18. Water-quality data for the Arkansas River Basin showing 2002 drought values, Colorado water-quality exceedances, and percentiles relative to historical data for selected properties and constituents from July through September, 1978-2002.—Continued

$\left[\mathrm{ft}^{3} / \mathrm{s}\right.$, cubic feet per second; Conc, concentration; $\mathrm{Q} \%$, percentile (percentage of data less than or equal to reported value or concentration) of drought-sample constituent; N, total number of historical and drought samples; $\mathrm{mg} / \mathrm{L}$, milligrams per liter; $\mu \mathrm{S} / \mathrm{cm}$, microsiemens per centimeter at $25^{\circ} \mathrm{Celsius;}{ }^{\circ} \mathrm{C}$, degrees Celsius; E. coli, Escherichia coli; $\mathrm{mL}$, milliliter; $\mu \mathrm{g} / \mathrm{L}$, micrograms per liter; $\mathrm{CaCO}_{3}$, calcium carbonate; $\mathrm{SiO}_{2}$, silica; $\mathrm{SO}_{4}$, sulfate; ID, insufficient number of samples (less than 20) to calculate percentile; <, less than; --, no drought-sample measurement; values or concentrations in bold type indicate 2002 Colorado water-quality exceedances; c, chronic water-quality exceedance; ac, acute and chronic water-quality exceedance]

\begin{tabular}{|c|c|c|c|c|c|c|c|}
\hline \multirow{3}{*}{$\begin{array}{c}\text { Site } \\
\text { number in } \\
\text { table } 1 \\
\text { and fig. } 7 \\
126\end{array}$} & \multirow{3}{*}{$\begin{array}{c}\begin{array}{c}\text { Sampling } \\
\text { date }\end{array} \\
7-19-2002\end{array}$} & \multirow{3}{*}{ 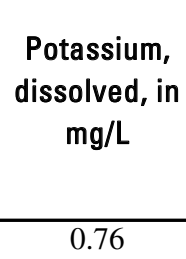 } & \multirow{3}{*}{$\begin{array}{c}\begin{array}{c}\text { Sodium, } \\
\text { dissolved, in } \\
\mathrm{mg} / \mathrm{L}\end{array} \\
3.2\end{array}$} & \multirow{3}{*}{$\begin{array}{c}\text { Acid- } \\
\text { neutralizing } \\
\text { capacity, in } \\
\mathrm{mg} / \mathrm{L} \text { as } \mathrm{CaCO}_{3}\end{array}$} & \multicolumn{3}{|c|}{ Chloride, dissolved, in $\mathrm{mg} / \mathrm{L}$} \\
\hline & & & & & \multirow{2}{*}{$\begin{array}{c}\text { Conc } \\
--\end{array}$} & \multirow{2}{*}{$\begin{array}{c}0 \% \\
--\end{array}$} & \multirow{2}{*}{$\begin{array}{l}\mathrm{N} \\
--\end{array}$} \\
\hline & & & & & & & \\
\hline & $8-26-2002$ & 0.68 & 2.5 & 34 & -- & -- & -- \\
\hline \multirow[t]{2}{*}{127} & $7-31-2002$ & 1.6 & 10.2 & 88 & 7.2 & ID & 1 \\
\hline & $8-26-2002$ & 1.6 & 9.4 & 84 & -- & -- & -- \\
\hline \multirow[t]{2}{*}{128} & $7-31-2002$ & 2.8 & 27 & 127 & 9.8 & 95.0 & 39 \\
\hline & $8-26-2002$ & 2.8 & 30 & 131 & -- & -- & -- \\
\hline \multirow[t]{2}{*}{129} & $7-31-2002$ & -- & -- & -- & -- & -- & -- \\
\hline & $8-26-2002$ & -- & -- & -- & -- & -- & -- \\
\hline \multirow[t]{2}{*}{130} & $7-22-2002$ & 3.0 & 30 & -- & 10.2 & ID & 16 \\
\hline & $8-27-2002$ & -- & -- & -- & -- & -- & -- \\
\hline \multirow[t]{2}{*}{131} & $7-22-2002$ & 3.0 & 35 & -- & 11.8 & ID & 9 \\
\hline & $8-27-2002$ & -- & -- & -- & -- & -- & -- \\
\hline 132 & $8-21-2002$ & -- & -- & -- & -- & -- & -- \\
\hline 133 & $8-21-2002$ & -- & -- & -- & -- & -- & -- \\
\hline 134 & $8-21-2002$ & -- & -- & -- & -- & -- & -- \\
\hline 135 & $8-20-2002$ & -- & -- & -- & -- & -- & -- \\
\hline 136 & $8-20-2002$ & -- & -- & -- & -- & -- & -- \\
\hline 137 & $8-20-2002$ & -- & -- & -- & -- & -- & -- \\
\hline 138 & $8-20-2002$ & -- & -- & -- & -- & -- & -- \\
\hline 139 & 8-19-2002 & -- & -- & -- & -- & -- & -- \\
\hline 140 & 8-19-2002 & -- & -- & -- & -- & -- & -- \\
\hline \multirow[t]{2}{*}{141} & $7-24-2002$ & 4.3 & 59 & -- & 24 & ID & 10 \\
\hline & $8-27-2002$ & -- & -- & -- & -- & -- & -- \\
\hline \multirow[t]{2}{*}{142} & $7-23-2002$ & 4.0 & 73 & -- & 25 & ID & 7 \\
\hline & $8-28-2002$ & -- & -- & -- & -- & -- & -- \\
\hline \multirow[t]{2}{*}{143} & $7-23-2002$ & 6.6 & 210 & -- & 79 & ID & 1 \\
\hline & $8-28-2002$ & -- & -- & -- & -- & -- & -- \\
\hline \multirow[t]{2}{*}{144} & $7-23-2002$ & 5.0 & 174 & -- & 53 & ID & 2 \\
\hline & $9-4-2002$ & -- & -- & -- & -- & -- & -- \\
\hline \multirow[t]{2}{*}{145} & $7-23-2002$ & 4.8 & 344 & -- & 90 & ID & 7 \\
\hline & $9-4-2002$ & -- & -- & -- & -- & -- & -- \\
\hline 146 & 8-28-2002 & 3.3 & 174 & -- & 110 & ID & 4 \\
\hline 147 & $9-4-2002$ & 4.5 & 12.8 & -- & 2.89 & ID & 1 \\
\hline 148 & $9-4-2002$ & 7.0 & 381 & -- & 95 & ID & 2 \\
\hline
\end{tabular}


Table 18. Water-quality data for the Arkansas River Basin showing 2002 drought values, Colorado water-quality exceedances, and percentiles relative to historical data for selected properties and constituents from July through September, 1978-2002.—Continued

$\left[\mathrm{ft}^{3} / \mathrm{s}\right.$, cubic feet per second; Conc, concentration; $\mathrm{Q} \%$, percentile (percentage of data less than or equal to reported value or concentration) of drought-sample constituent; N, total number of historical and drought samples; $\mathrm{mg} / \mathrm{L}$, milligrams per liter; $\mu \mathrm{S} / \mathrm{cm}$, microsiemens per centimeter at $25^{\circ} \mathrm{Celsius;}{ }^{\circ} \mathrm{C}$, degrees Celsius; E. coli, Escherichia coli; $\mathrm{mL}$, milliliter; $\mu \mathrm{g} / \mathrm{L}$, micrograms per liter; $\mathrm{CaCO}_{3}$, calcium carbonate; $\mathrm{SiO}_{2}$, silica; $\mathrm{SO}_{4}$, sulfate; ID, insufficient number of samples (less than 20) to calculate percentile; <, less than; --, no drought-sample measurement; values or concentrations in bold type indicate 2002 Colorado water-quality exceedances; c, chronic water-quality exceedance; ac, acute and chronic water-quality exceedance]

\begin{tabular}{|c|c|c|c|c|c|c|c|c|}
\hline \multirow{2}{*}{$\begin{array}{c}\text { Site } \\
\text { number in } \\
\text { table } 1 \\
\text { and fig. } 7\end{array}$} & \multirow{2}{*}{$\begin{array}{c}\text { Sampling } \\
\text { date }\end{array}$} & \multicolumn{3}{|c|}{ Fluoride, dissolved, in $\mathrm{mg} / \mathrm{L}$} & \multirow{2}{*}{$\begin{array}{c}\text { Silica, } \\
\text { dissolved, in } \\
\mathrm{mg} / \mathrm{L} \text { as } \mathrm{SiO}_{2}\end{array}$} & \multicolumn{3}{|c|}{ Sulfate dissolved, in $\mathrm{mg} / \mathrm{L}$ as $\mathrm{SO}_{4}$} \\
\hline & & Conc & $0 \%$ & $\mathrm{~N}$ & & Conc & $0 \%$ & $\mathrm{~N}$ \\
\hline \multirow[t]{2}{*}{126} & $7-19-2002$ & 0.10 & ID & 4 & -- & 19.2 & ID & 9 \\
\hline & $8-26-2002$ & 0.16 & ID & 4 & -- & 15.9 & ID & 9 \\
\hline \multirow[t]{2}{*}{127} & $7-31-2002$ & 0.45 & ID & 2 & -- & 24.4 & ID & 2 \\
\hline & $8-26-2002$ & 0.43 & ID & 2 & -- & 25.5 & ID & 2 \\
\hline \multirow[t]{2}{*}{128} & $7-31-2002$ & 0.57 & 66.7 & 35 & -- & 106 & 78.0 & 40 \\
\hline & $8-26-2002$ & 0.59 & 69.4 & 35 & -- & 119 & 90.2 & 40 \\
\hline \multirow[t]{2}{*}{129} & $7-31-2002$ & -- & -- & -- & -- & -- & -- & -- \\
\hline & 8-26-2002 & -- & -- & -- & -- & -- & -- & -- \\
\hline \multirow[t]{2}{*}{130} & $7-22-2002$ & 0.58 & ID & 8 & 7.2 & 158 & 95.5 & 21 \\
\hline & $8-27-2002$ & -- & -- & -- & -- & -- & -- & -- \\
\hline \multirow[t]{2}{*}{131} & $7-22-2002$ & 0.60 & ID & 4 & 6.0 & 186 & ID & 13 \\
\hline & $8-27-2002$ & -- & -- & -- & -- & -- & -- & -- \\
\hline 132 & $8-21-2002$ & 2.7 & ${ }^{1} 58.8-76.5$ & 33 & -- & 22.2 & 98.2 & 54 \\
\hline 133 & $8-21-2002$ & 1.5 & 78.6 & 27 & -- & 31.5 & 89.4 & 46 \\
\hline 134 & $8-21-2002$ & 0.94 & ID & 13 & -- & 50 & ID & 10 \\
\hline 135 & $8-20-2002$ & 1.2 & ${ }^{1} 25.0-50.0$ & 27 & -- & 240 & 98.0 & 50 \\
\hline 136 & $8-20-2002$ & 1.3 & ${ }^{1} 25.0-35.0$ & 39 & -- & 300 & 96.9 & 64 \\
\hline 137 & $8-20-2002$ & 1.4 & ${ }^{1} 10.5-18.4$ & 37 & -- & 160 & ${ }^{1} 45.0-50.0$ & 39 \\
\hline 138 & $8-20-2002$ & 1.3 & ID & 13 & -- & 170 & ID & 12 \\
\hline 139 & 8-19-2002 & 1.4 & ID & 8 & -- & 270 & ID & 8 \\
\hline 140 & 8-19-2002 & 1.7 & ID & 17 & -- & 290 & ID & 17 \\
\hline \multirow[t]{2}{*}{141} & $7-24-2002$ & 0.80 & ID & 6 & 8.1 & 293 & ID & 14 \\
\hline & $8-27-2002$ & -- & -- & -- & -- & -- & -- & -- \\
\hline \multirow[t]{2}{*}{142} & $7-23-2002$ & 1.0 & ID & 3 & 8.3 & 369 & ID & 11 \\
\hline & $8-28-2002$ & -- & -- & -- & -- & -- & -- & -- \\
\hline \multirow[t]{2}{*}{143} & $7-23-2002$ & 1.6 & ID & 1 & 9.5 & 929 & ID & 1 \\
\hline & $8-28-2002$ & -- & -- & -- & -- & -- & -- & -- \\
\hline \multirow[t]{2}{*}{144} & $7-23-2002$ & 1.2 & ID & 1 & 13 & 1,080 & ID & 6 \\
\hline & $9-4-2002$ & -- & -- & -- & -- & -- & -- & -- \\
\hline \multirow[t]{2}{*}{145} & $7-23-2002$ & 1.2 & ID & 3 & 17.9 & 1,590 & ID & 11 \\
\hline & $9-4-2002$ & -- & -- & -- & -- & -- & -- & -- \\
\hline 146 & $8-28-2002$ & 0.70 & ID & 4 & 3.2 & 76.2 & ID & 4 \\
\hline 147 & $9-4-2002$ & 0.20 & ID & 1 & 8.0 & 105 & ID & 1 \\
\hline 148 & $9-4-2002$ & 1.1 & ID & 1 & 17.6 & 1,830 & ID & 2 \\
\hline
\end{tabular}


Table 18. Water-quality data for the Arkansas River Basin showing 2002 drought values, Colorado water-quality exceedances, and percentiles relative to historical data for selected properties and constituents from July through September, 1978-2002.—Continued

$\left[\mathrm{ft}^{3} / \mathrm{s}\right.$, cubic feet per second; Conc, concentration; $\mathrm{Q} \%$, percentile (percentage of data less than or equal to reported value or concentration) of drought-sample constituent; $\mathrm{N}$, total number of historical and drought samples; $\mathrm{mg} / \mathrm{L}$, milligrams per liter; $\mu \mathrm{S} / \mathrm{cm}$, microsiemens per centimeter at $25^{\circ} \mathrm{Celsius;}{ }^{\circ} \mathrm{C}$, degrees Celsius; E. coli, Escherichia coli; $\mathrm{mL}$, milliliter; $\mu \mathrm{g} / \mathrm{L}$, micrograms per liter; $\mathrm{CaCO}_{3}$, calcium carbonate; $\mathrm{SiO}_{2}$, silica; $\mathrm{SO}_{4}$, sulfate; ID, insufficient number of samples (less than 20) to calculate percentile; <, less than; --, no drought-sample measurement; values or concentrations in bold type indicate 2002 Colorado water-quality exceedances; c, chronic water-quality exceedance; ac, acute and chronic water-quality exceedance]

\begin{tabular}{|c|c|c|c|c|c|c|}
\hline \multirow{2}{*}{$\begin{array}{c}\text { Site } \\
\text { number in } \\
\text { table } 1 \\
\text { and fig. } 7\end{array}$} & \multirow{2}{*}{$\begin{array}{l}\text { Sampling } \\
\text { date }\end{array}$} & \multirow{2}{*}{$\begin{array}{l}\text { Solids, } \\
\text { dissolved, } \\
\text { residue on } \\
\text { evaporation at } \\
180^{\circ} \mathrm{C} \text {, in } \mathrm{mg} / \mathrm{L}\end{array}$} & \multicolumn{3}{|c|}{$\begin{array}{c}\text { Nitrogen, ammonia, dissolved, in } \mathrm{mg} / \mathrm{L} \text { as } \\
\mathrm{N}\end{array}$} & \multirow{2}{*}{$\begin{array}{c}\text { Nitrogen, } \\
\text { organic plus } \\
\text { ammonia, } \\
\text { dissolved, in } \\
\mathrm{mg} / \mathrm{L} \text { as } \mathrm{N}\end{array}$} \\
\hline & & & Conc & $0 \%$ & $\mathrm{~N}$ & \\
\hline \multirow[t]{2}{*}{126} & $7-19-2002$ & $\overline{--}$ & $<0.04$ & ID & 2 & -- \\
\hline & $8-26-2002$ & -- & $<0.04$ & ID & 2 & -- \\
\hline \multirow[t]{2}{*}{127} & $7-31-2002$ & -- & $<0.04$ & ID & 2 & -- \\
\hline & $8-26-2002$ & -- & $<0.04$ & ID & 2 & -- \\
\hline \multirow[t]{2}{*}{128} & $7-31-2002$ & -- & $<0.04$ & ${ }^{2} 3.7-59.3$ & 26 & -- \\
\hline & $8-26-2002$ & -- & $<0.04$ & ${ }^{2} 3.7-59.3$ & 26 & -- \\
\hline \multirow[t]{2}{*}{129} & $7-31-2002$ & -- & 0.012 & ID & 7 & 0.22 \\
\hline & $8-26-2002$ & -- & 0.011 & ID & 7 & -- \\
\hline \multirow[t]{2}{*}{130} & $7-22-2002$ & 395 & 0.05 & ID & 9 & -- \\
\hline & $8-27-2002$ & -- & 0.04 & ID & 9 & 0.21 \\
\hline \multirow[t]{2}{*}{131} & $7-22-2002$ & 435 & $<0.04$ & ID & 6 & -- \\
\hline & $8-27-2002$ & -- & 1.65 & ID & 6 & -- \\
\hline 132 & $8-21-2002$ & -- & $<0.015$ & ${ }^{2} 3.8-61.5$ & 25 & -- \\
\hline 133 & $8-21-2002$ & -- & 0.009 & ${ }^{2} 4.5-45.5$ & 21 & -- \\
\hline 134 & $8-21-2002$ & -- & $<0.015$ & ID & 12 & -- \\
\hline 135 & $8-20-2002$ & -- & 0.008 & ID & 19 & -- \\
\hline 136 & $8-20-2002$ & -- & 0.009 & 28.6 & 41 & -- \\
\hline 137 & $8-20-2002$ & -- & 0.039 & 7.7 & 38 & -- \\
\hline 138 & $8-20-2002$ & -- & 0.20 & ID & 17 & -- \\
\hline 139 & $8-19-2002$ & -- & 0.20 & ID & 7 & -- \\
\hline 140 & $8-19-2002$ & -- & $<0.015$ & ID & 15 & -- \\
\hline \multirow[t]{2}{*}{141} & $7-24-2002$ & 639 & $<0.04$ & ID & 6 & -- \\
\hline & $8-27-2002$ & -- & 0.07 & ID & 6 & -- \\
\hline \multirow[t]{2}{*}{142} & $7-23-2002$ & 775 & $<0.04$ & ID & 6 & -- \\
\hline & $8-28-2002$ & -- & $<0.04$ & ID & 6 & -- \\
\hline \multirow[t]{2}{*}{143} & $7-23-2002$ & 1,780 & $<0.04$ & ID & 2 & -- \\
\hline & $8-28-2002$ & -- & 0.12 & ID & 2 & -- \\
\hline \multirow[t]{2}{*}{144} & $7-23-2002$ & 1,990 & 0.04 & ID & 6 & -- \\
\hline & $9-4-2002$ & -- & 0.04 & ID & 6 & -- \\
\hline \multirow[t]{2}{*}{145} & $7-23-2002$ & 2,970 & 0.05 & ID & 6 & -- \\
\hline & $9-4-2002$ & -- & 0.04 & ID & 6 & -- \\
\hline 146 & $8-28-2002$ & 555 & $<0.015$ & ID & 1 & 0.24 \\
\hline 147 & $9-4-2002$ & 261 & 0.17 & ID & 1 & 0.55 \\
\hline 148 & $9-4-2002$ & 3,210 & 0.06 & ID & 1 & -- \\
\hline
\end{tabular}


Table 18. Water-quality data for the Arkansas River Basin showing 2002 drought values, Colorado water-quality exceedances, and percentiles relative to historical data for selected properties and constituents from July through September, 1978-2002.—Continued

$\left[\mathrm{ft}^{3} / \mathrm{s}\right.$, cubic feet per second; Conc, concentration; $\mathrm{Q} \%$, percentile (percentage of data less than or equal to reported value or concentration) of drought-sample constituent; N, total number of historical and drought samples; $\mathrm{mg} / \mathrm{L}$, milligrams per liter; $\mu \mathrm{S} / \mathrm{cm}$, microsiemens per centimeter at $25^{\circ} \mathrm{Celsius;}{ }^{\circ} \mathrm{C}$, degrees Celsius; E. coli, Escherichia coli; $\mathrm{mL}$, milliliter; $\mu \mathrm{g} / \mathrm{L}$, micrograms per liter; $\mathrm{CaCO}_{3}$, calcium carbonate; $\mathrm{SiO}_{2}$, silica; $\mathrm{SO}_{4}$, sulfate; ID, insufficient number of samples (less than 20) to calculate percentile; <, less than; --, no drought-sample measurement; values or concentrations in bold type indicate 2002 Colorado water-quality exceedances; c, chronic water-quality exceedance; ac, acute and chronic water-quality exceedance]

\begin{tabular}{|c|c|c|c|c|c|c|c|c|}
\hline \multirow{2}{*}{$\begin{array}{c}\text { Site } \\
\text { number in } \\
\text { table } 1 \\
\text { and fig. } 7\end{array}$} & \multirow{2}{*}{$\begin{array}{l}\text { Sampling } \\
\text { date }\end{array}$} & \multicolumn{3}{|c|}{$\begin{array}{l}\text { Nitrogen, organic plus ammonia, } \\
\text { total recoverable, in } \mathrm{mg} / \mathrm{L} \text { as } \mathrm{N}\end{array}$} & \multicolumn{3}{|c|}{$\begin{array}{l}\text { Nitrogen, nitrite plus nitrate, in } \mathrm{mg} / \mathrm{L} \\
\text { as } \mathrm{N}\end{array}$} & \multirow{2}{*}{$\begin{array}{c}\text { Nitrogen, } \\
\text { nitrite, } \\
\text { dissolved, in } \\
\text { mg/L as } \mathrm{N}\end{array}$} \\
\hline & & Conc & $0 \%$ & $\mathrm{~N}$ & Conc & $0 \%$ & $\mathrm{~N}$ & \\
\hline \multirow[t]{2}{*}{126} & $7-19-2002$ & 0.13 & ID & 2 & $<0.05$ & ID & 2 & $<0.008$ \\
\hline & $8-26-2002$ & 0.13 & ID & 2 & $<0.05$ & ID & 2 & $<0.008$ \\
\hline \multirow[t]{2}{*}{127} & $7-31-2002$ & 0.27 & ID & 2 & $<0.05$ & ID & 2 & $<0.008$ \\
\hline & $8-26-2002$ & 0.21 & ID & 2 & $<0.05$ & ID & 2 & $<0.008$ \\
\hline \multirow[t]{2}{*}{128} & $7-31-2002$ & 0.49 & 56.3 & 31 & 0.05 & ${ }^{2} 3.2-16.1$ & 30 & 0.004 \\
\hline & $8-26-2002$ & 0.39 & 46.9 & 31 & 0.16 & ${ }^{1} 41.9-51.6$ & 30 & 0.005 \\
\hline \multirow[t]{2}{*}{129} & $7-31-2002$ & -- & -- & -- & 0.11 & ID & 12 & 0.004 \\
\hline & $8-26-2002$ & -- & -- & -- & 0.30 & ID & 12 & 0.009 \\
\hline \multirow[t]{2}{*}{130} & $7-22-2002$ & 0.23 & ID & 8 & 0.40 & ID & 10 & 0.007 \\
\hline & $8-27-2002$ & 0.28 & ID & 8 & 0.24 & ID & 10 & 0.003 \\
\hline \multirow[t]{2}{*}{131} & $7-22-2002$ & 0.24 & ID & 2 & 0.49 & ID & 6 & 0.008 \\
\hline & $8-27-2002$ & 2.7 & ID & 2 & 1.06 & ID & 6 & 0.333 \\
\hline 132 & $8-21-2002$ & -- & -- & -- & 0.51 & ${ }^{1} 29.6-33.3$ & 26 & -- \\
\hline 133 & $8-21-2002$ & -- & -- & -- & $<0.05$ & ${ }^{2} 4.8-23.8$ & 20 & -- \\
\hline 134 & $8-21-2002$ & -- & -- & -- & 0.80 & ID & 13 & -- \\
\hline 135 & $8-20-2002$ & -- & -- & -- & 3.2 & 42.9 & 20 & -- \\
\hline 136 & $8-20-2002$ & -- & -- & -- & 3.2 & 95.3 & 42 & -- \\
\hline 137 & $8-20-2002$ & -- & -- & -- & 5.41 & 97.6 & 40 & -- \\
\hline 138 & $8-20-2002$ & -- & -- & -- & 3.13 & ID & 18 & -- \\
\hline 139 & 8-19-2002 & -- & -- & -- & 4.16 & ID & 8 & -- \\
\hline 140 & 8-19-2002 & -- & -- & -- & 2.28 & ID & 15 & -- \\
\hline \multirow[t]{2}{*}{141} & $7-24-2002$ & 0.71 & ID & 8 & 1.48 & ID & 6 & 0.024 \\
\hline & $8-27-2002$ & 0.79 & ID & 8 & 2.77 & ID & 6 & 0.095 \\
\hline \multirow[t]{2}{*}{142} & $7-23-2002$ & 0.44 & ID & 2 & 1.20 & ID & 6 & 0.009 \\
\hline & $8-28-2002$ & 0.38 & ID & 2 & 2.11 & ID & 6 & 0.036 \\
\hline \multirow[t]{2}{*}{143} & $7-23-2002$ & 2.0 & ID & 2 & 1.22 & ID & 2 & 0.014 \\
\hline & $8-28-2002$ & 1.2 & ID & 2 & 3.8 & ID & 2 & 0.041 \\
\hline \multirow[t]{2}{*}{144} & $7-23-2002$ & 0.85 & ID & 2 & 2.6 & ID & 7 & 0.007 \\
\hline & $9-4-2002$ & 0.81 & ID & 2 & 3.1 & ID & 7 & 0.027 \\
\hline \multirow[t]{2}{*}{145} & $7-23-2002$ & 0.39 & ID & 2 & 1.37 & ID & 6 & 0.017 \\
\hline & $9-4-2002$ & 0.39 & ID & 2 & 1.11 & ID & 6 & 0.019 \\
\hline 146 & $8-28-2002$ & 0.31 & ID & 2 & $<0.013$ & ID & 4 & $<0.002$ \\
\hline 147 & $9-4-2002$ & 0.95 & ID & 1 & 0.53 & ID & 1 & 0.054 \\
\hline 148 & $9-4-2002$ & 0.43 & ID & 1 & 1.24 & ID & 2 & 0.022 \\
\hline
\end{tabular}


Table 18. Water-quality data for the Arkansas River Basin showing 2002 drought values, Colorado water-quality exceedances, and percentiles relative to historical data for selected properties and constituents from July through September, 1978-2002.—Continued

$\left[\mathrm{ft}^{3} / \mathrm{s}\right.$, cubic feet per second; Conc, concentration; Q\%, percentile (percentage of data less than or equal to reported value or concentration) of drought-sample constituent; N, total number of historical and drought samples; $\mathrm{mg} / \mathrm{L}$, milligrams per liter; $\mu \mathrm{S} / \mathrm{cm}$, microsiemens per centimeter at $25^{\circ} \mathrm{Celsius;}{ }^{\circ} \mathrm{C}$, degrees Celsius; E. coli, Escherichia coli; $\mathrm{mL}$, milliliter; $\mu \mathrm{g} / \mathrm{L}$, micrograms per liter; $\mathrm{CaCO}_{3}$, calcium carbonate; $\mathrm{SiO}_{2}$, silica; $\mathrm{SO}_{4}$, sulfate; ID, insufficient number of samples (less than 20) to calculate percentile; <, less than; --, no drought-sample measurement; values or concentrations in bold type indicate 2002 Colorado water-quality exceedances; c, chronic water-quality exceedance; ac, acute and chronic water-quality exceedance]

\begin{tabular}{|c|c|c|c|c|c|c|c|c|}
\hline \multirow{2}{*}{$\begin{array}{c}\text { Site } \\
\text { number in } \\
\text { table } 1 \\
\text { and fig. } 7\end{array}$} & \multirow{2}{*}{$\begin{array}{l}\text { Sampling } \\
\text { date }\end{array}$} & \multirow{2}{*}{$\begin{array}{c}\text { Phosphorus, } \\
\text { dissolved, in } \mathrm{mg} / \mathrm{L}\end{array}$} & \multicolumn{3}{|c|}{$\begin{array}{c}\text { Phosphorus, total recoverable, in } \\
\mathrm{mg} / \mathrm{L} \text { as } \mathrm{P}\end{array}$} & \multicolumn{3}{|c|}{$\begin{array}{l}\text { Phosphorus, orthophosphate, dissolved, } \\
\text { in } \mathrm{mg} / \mathrm{L} \text { as } \mathrm{P}\end{array}$} \\
\hline & & & Conc & $0 \%$ & $\mathrm{~N}$ & Conc & $0 \%$ & $\mathrm{~N}$ \\
\hline \multirow[t]{2}{*}{126} & $7-19-2002$ & -- & 0.009 & ID & 2 & $<0.02$ & ID & 2 \\
\hline & $8-26-2002$ & -- & 0.007 & ID & 2 & $<0.02$ & ID & 2 \\
\hline \multirow[t]{2}{*}{127} & $7-31-2002$ & -- & 0.033 & ID & 2 & $<0.02$ & ID & 2 \\
\hline & $8-26-2002$ & -- & 0.023 & ID & 2 & $<0.02$ & ID & 2 \\
\hline \multirow[t]{2}{*}{128} & $7-31-2002$ & -- & 0.087 & 71.1 & 37 & 0.01 & ${ }^{1} 12.5-25.0$ & 23 \\
\hline & $8-26-2002$ & -- & 0.142 & 84.2 & 37 & 0.07 & 95.8 & 23 \\
\hline \multirow[t]{2}{*}{129} & $7-31-2002$ & 0.025 & 0.085 & ID & 4 & 0.015 & ID & 8 \\
\hline & 8-26-2002 & 0.131 & 0.200 & ID & 4 & 0.12 & ID & 8 \\
\hline \multirow[t]{2}{*}{130} & $7-22-2002$ & -- & 0.022 & ID & 16 & $<0.02$ & ID & 10 \\
\hline & $8-27-2002$ & 0.011 & 0.022 & ID & 16 & $<0.007$ & ID & 10 \\
\hline \multirow[t]{2}{*}{131} & $7-22-2002$ & -- & 0.020 & ID & 10 & $<0.02$ & ID & 6 \\
\hline & $8-27-2002$ & -- & 0.23 & ID & 10 & 0.12 & ID & 6 \\
\hline 132 & $8-21-2002$ & -- & $<0.06$ & ${ }^{2} 4.0-64.0$ & 24 & $<0.02$ & ${ }^{2} 4.0-76.0$ & 24 \\
\hline 133 & $8-21-2002$ & -- & 2.2 & ID & 4 & 1.7 & ID & 19 \\
\hline 134 & $8-21-2002$ & -- & 0.210 & ID & 11 & 0.16 & ID & 11 \\
\hline 135 & $8-20-2002$ & -- & 0.070 & ID & 11 & 0.04 & ID & 19 \\
\hline 136 & $8-20-2002$ & -- & 0.060 & ${ }^{1} 12.1-18.2$ & 32 & 0.03 & ${ }^{1} 17.1-37.1$ & 34 \\
\hline 137 & $8-20-2002$ & -- & 1.3 & 58.3 & 23 & 1.2 & 78.8 & 32 \\
\hline 138 & $8-20-2002$ & -- & 0.76 & ID & 15 & 0.66 & ID & 15 \\
\hline 139 & 8-19-2002 & -- & 0.80 & ID & 4 & 0.73 & ID & 4 \\
\hline 140 & 8-19-2002 & -- & 0.47 & ID & 10 & 0.22 & ID & 11 \\
\hline \multirow[t]{2}{*}{141} & 7-24-2002 & -- & 0.33 & ID & 8 & 0.10 & ID & 6 \\
\hline & $8-27-2002$ & -- & 0.31 & ID & 8 & 0.19 & ID & 6 \\
\hline \multirow[t]{2}{*}{142} & $7-23-2002$ & -- & 0.153 & ID & 8 & 0.03 & ID & 6 \\
\hline & $8-28-2002$ & -- & 0.037 & ID & 8 & $<0.02$ & ID & 6 \\
\hline \multirow[t]{2}{*}{143} & $7-23-2002$ & -- & 0.199 & ID & 2 & $<0.02$ & ID & 2 \\
\hline & $8-28-2002$ & -- & 0.076 & ID & 2 & $<0.02$ & ID & 2 \\
\hline \multirow[t]{2}{*}{144} & $7-23-2002$ & -- & 0.119 & ID & 2 & $<0.02$ & ID & 6 \\
\hline & $9-4-2002$ & -- & 0.103 & ID & 2 & $<0.02$ & ID & 6 \\
\hline \multirow[t]{2}{*}{145} & $7-23-2002$ & -- & 0.034 & ID & 8 & $<0.02$ & ID & 6 \\
\hline & $9-4-2002$ & -- & 0.029 & ID & 8 & $<0.02$ & ID & 6 \\
\hline 146 & $8-28-2002$ & 0.012 & 0.035 & ID & 2 & 0.006 & ID & 4 \\
\hline 147 & $9-4-2002$ & 0.030 & 0.172 & ID & 1 & 0.017 & ID & 1 \\
\hline 148 & $9-4-2002$ & -- & 0.024 & ID & 1 & $<0.02$ & ID & 1 \\
\hline
\end{tabular}


Table 18. Water-quality data for the Arkansas River Basin showing 2002 drought values, Colorado water-quality exceedances, and percentiles relative to historical data for selected properties and constituents from July through September, 1978-2002.—Continued

$\left[\mathrm{ft}^{3} / \mathrm{s}\right.$, cubic feet per second; Conc, concentration; $\mathrm{Q} \%$, percentile (percentage of data less than or equal to reported value or concentration) of drought-sample constituent; N, total number of historical and drought samples; $\mathrm{mg} / \mathrm{L}$, milligrams per liter; $\mu \mathrm{S} / \mathrm{cm}$, microsiemens per centimeter at $25^{\circ} \mathrm{Celsius} ;{ }^{\circ} \mathrm{C}$, degrees Celsius; E. coli, Escherichia coli; $\mathrm{mL}$, milliliter; $\mu \mathrm{g} / \mathrm{L}$, micrograms per liter; $\mathrm{CaCO}_{3}$, calcium carbonate; $\mathrm{SiO}_{2}$, silica; $\mathrm{SO}_{4}$, sulfate; ID, insufficient number of samples (less than 20) to calculate percentile; <, less than; --, no drought-sample measurement; values or concentrations in bold type indicate 2002 Colorado water-quality exceedances; c, chronic water-quality exceedance; ac, acute and chronic water-quality exceedance]

\begin{tabular}{|c|c|c|c|c|c|c|c|}
\hline \multirow{2}{*}{$\begin{array}{c}\text { Site } \\
\text { number in } \\
\text { table } 1 \\
\text { and fig. } 7\end{array}$} & \multirow{2}{*}{$\begin{array}{l}\text { Sampling } \\
\text { date }\end{array}$} & \multirow{2}{*}{$\begin{array}{l}\text { Carbon, } \\
\text { organic, } \\
\text { dissolved, } \\
\text { in } \mathrm{mg} / \mathrm{L}\end{array}$} & \multirow{2}{*}{$\begin{array}{c}\text { Carbon, organic, } \\
\text { total } \\
\text { recoverable, } \\
\text { in } \mathrm{mg} / \mathrm{L}\end{array}$} & \multirow{2}{*}{$\begin{array}{c}\text { Bacteria, } \\
\text { E. coli, } \\
\text { in colonies per } \\
100 \mathrm{~mL}\end{array}$} & \multicolumn{3}{|c|}{$\begin{array}{l}\text { Bacteria, fecal coliform, in colonies } \\
\text { per } 100 \mathrm{~mL}\end{array}$} \\
\hline & & & & & Conc & $0 \%$ & $\mathrm{~N}$ \\
\hline \multirow[t]{2}{*}{126} & $7-19-2002$ & -- & -- & 4 & $<3$ & ID & 2 \\
\hline & $8-26-2002$ & -- & -- & 2 & 2 & ID & 2 \\
\hline \multirow[t]{2}{*}{127} & $7-31-2002$ & -- & -- & 3 & 2 & ID & 2 \\
\hline & $8-26-2002$ & -- & -- & 9 & 16 & ID & 2 \\
\hline \multirow[t]{2}{*}{128} & $7-31-2002$ & 2.6 & 4.4 & 290 & 230 & ${ }^{1} 51.9-55.6$ & 26 \\
\hline & $8-26-2002$ & 2.5 & 3.3 & 54 & 100 & ${ }^{1} 25.9-29.6$ & 26 \\
\hline \multirow[t]{2}{*}{129} & $7-31-2002$ & 2.9 & -- & -- & -- & -- & -- \\
\hline & $8-26-2002$ & 2.7 & -- & -- & -- & -- & -- \\
\hline \multirow[t]{2}{*}{130} & $7-22-2002$ & 2.3 & -- & 10 & 5 & ID & 12 \\
\hline & $8-27-2002$ & 2.5 & -- & 4 & 11 & ID & 12 \\
\hline \multirow[t]{2}{*}{131} & $7-22-2002$ & -- & -- & 730 & 700 & ID & 8 \\
\hline & $8-27-2002$ & -- & -- & 1,800 & $\mathbf{5 , 5 0 0}$ & ID & 8 \\
\hline 132 & $8-21-2002$ & -- & -- & 930 & 600 & 60.6 & 65 \\
\hline 133 & $8-21-2002$ & -- & -- & 300 & 260 & 91.3 & 45 \\
\hline 134 & $8-21-2002$ & -- & -- & 40 & 60 & ID & 12 \\
\hline 135 & $8-20-2002$ & -- & -- & 200 & 420 & 44.0 & 49 \\
\hline 136 & $8-20-2002$ & -- & -- & 440 & 440 & ${ }^{1} 28.1-31.3$ & 63 \\
\hline 137 & $8-20-2002$ & -- & -- & 460 & 500 & 38.9 & 53 \\
\hline 138 & $8-20-2002$ & -- & -- & 3,900 & 4,200 & ID & 9 \\
\hline 139 & $8-19-2002$ & -- & -- & 430 & 660 & ID & 4 \\
\hline 140 & $8-19-2002$ & -- & -- & 520 & 940 & ID & 14 \\
\hline \multirow[t]{2}{*}{141} & $7-24-2002$ & -- & -- & 270 & 380 & ID & 14 \\
\hline & $8-27-2002$ & -- & -- & 160 & 270 & ID & 14 \\
\hline \multirow[t]{2}{*}{142} & $7-23-2002$ & -- & -- & 240 & 210 & ID & 7 \\
\hline & $8-28-2002$ & -- & -- & 86 & 280 & ID & 7 \\
\hline \multirow[t]{2}{*}{143} & $7-23-2002$ & -- & -- & 2,500 & 3,300 & ID & 2 \\
\hline & $8-28-2002$ & -- & -- & 540 & 940 & ID & 2 \\
\hline \multirow[t]{2}{*}{144} & $7-23-2002$ & -- & -- & 200 & 120 & ID & 5 \\
\hline & $9-4-2002$ & -- & -- & 68 & 150 & ID & 5 \\
\hline \multirow[t]{2}{*}{145} & $7-23-2002$ & -- & -- & 140 & 120 & ID & 11 \\
\hline & $9-4-2002$ & -- & -- & 140 & 290 & ID & 11 \\
\hline 146 & $8-28-2002$ & -- & 3.8 & 75 & 77 & ID & 1 \\
\hline 147 & $9-4-2002$ & 6.4 & 8.6 & 200 & 110 & ID & 1 \\
\hline 148 & $9-4-2002$ & -- & -- & 100 & 110 & ID & 4 \\
\hline
\end{tabular}


Table 18. Water-quality data for the Arkansas River Basin showing 2002 drought values, Colorado water-quality exceedances, and percentiles relative to historical data for selected properties and constituents from July through September, 1978-2002.—Continued

$\left[\mathrm{ft}^{3} / \mathrm{s}\right.$, cubic feet per second; Conc, concentration; Q\%, percentile (percentage of data less than or equal to reported value or concentration) of drought-sample constituent; N, total number of historical and drought samples; $\mathrm{mg} / \mathrm{L}$, milligrams per liter; $\mu \mathrm{S} / \mathrm{cm}$, microsiemens per centimeter at $25^{\circ} \mathrm{Celsius;}{ }^{\circ} \mathrm{C}$, degrees Celsius; E. coli, Escherichia coli; $\mathrm{mL}$, milliliter; $\mu \mathrm{g} / \mathrm{L}$, micrograms per liter; $\mathrm{CaCO}_{3}$, calcium carbonate; $\mathrm{SiO}_{2}$, silica; $\mathrm{SO}_{4}$, sulfate; ID, insufficient number of samples (less than 20) to calculate percentile; <, less than; --, no drought-sample measurement; values or concentrations in bold type indicate 2002 Colorado water-quality exceedances; c, chronic water-quality exceedance; ac, acute and chronic water-quality exceedance]

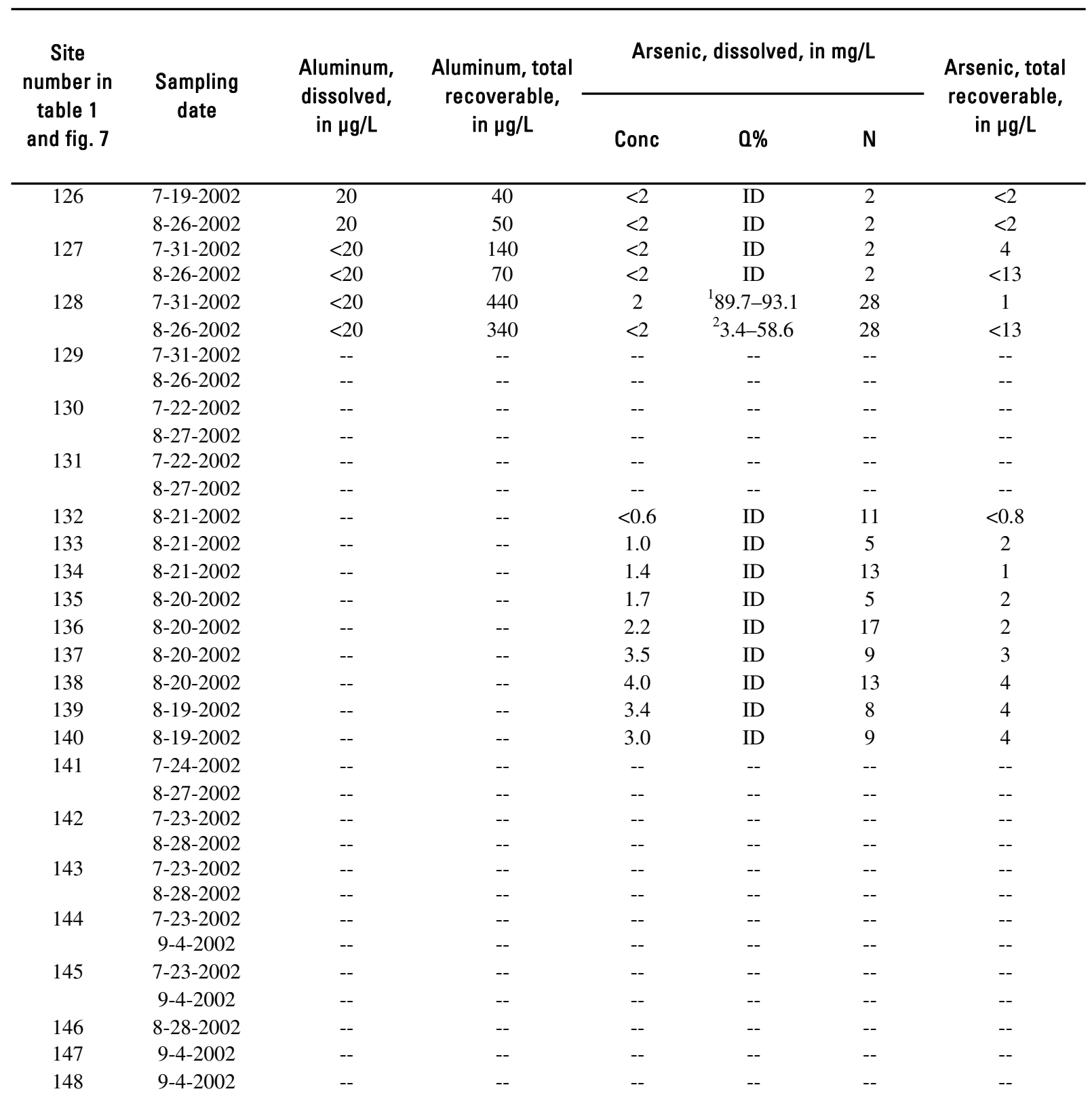


Table 18. Water-quality data for the Arkansas River Basin showing 2002 drought values, Colorado water-quality exceedances, and percentiles relative to historical data for selected properties and constituents from July through September, 1978-2002.—Continued

$\left[\mathrm{ft}^{3} / \mathrm{s}\right.$, cubic feet per second; Conc, concentration; $\mathrm{Q} \%$, percentile (percentage of data less than or equal to reported value or concentration) of drought-sample constituent; N, total number of historical and drought samples; $\mathrm{mg} / \mathrm{L}$, milligrams per liter; $\mu \mathrm{S} / \mathrm{cm}$, microsiemens per centimeter at $25^{\circ} \mathrm{Celsius} ;{ }^{\circ} \mathrm{C}$, degrees Celsius; E. coli, Escherichia coli; $\mathrm{mL}$, milliliter; $\mu \mathrm{g} / \mathrm{L}$, micrograms per liter; $\mathrm{CaCO}_{3}$, calcium carbonate; $\mathrm{SiO}_{2}$, silica; $\mathrm{SO}_{4}$, sulfate; ID, insufficient number of samples (less than 20) to calculate percentile; <, less than; --, no drought-sample measurement; values or concentrations in bold type indicate 2002 Colorado water-quality exceedances; c, chronic water-quality exceedance; ac, acute and chronic water-quality exceedance]

\begin{tabular}{|c|c|c|c|c|c|c|}
\hline \multirow{2}{*}{$\begin{array}{c}\text { Site } \\
\text { number in } \\
\text { table } 1 \\
\text { and fig. } 7\end{array}$} & \multirow{2}{*}{$\begin{array}{c}\text { Sampling } \\
\text { date }\end{array}$} & \multirow{2}{*}{$\begin{array}{l}\text { Boron, } \\
\text { dissolved, } \\
\text { in } \mu \mathrm{g} / \mathrm{L}\end{array}$} & \multirow{2}{*}{$\begin{array}{l}\text { Boron, total } \\
\text { recoverable, } \\
\text { in } \mu \mathrm{g} / \mathrm{L}\end{array}$} & \multicolumn{3}{|c|}{ Cadmium, dissolved, in $\mathrm{mg} / \mathrm{L}$} \\
\hline & & & & Conc & $0 \%$ & $\mathrm{~N}$ \\
\hline \multirow[t]{2}{*}{126} & $7-19-2002$ & -- & -- & 0.1 & ID & 9 \\
\hline & $8-26-2002$ & $<10$ & -- & 0.1 & ID & 9 \\
\hline \multirow[t]{2}{*}{127} & $7-31-2002$ & 20 & -- & $<0.1$ & ID & 2 \\
\hline & $8-26-2002$ & 10 & -- & $<0.1$ & ID & 2 \\
\hline \multirow[t]{2}{*}{128} & $7-31-2002$ & 50 & -- & $<0.1$ & ${ }^{2} 3.1-90.6$ & 31 \\
\hline & $8-26-2002$ & 50 & -- & $<0.1$ & ${ }^{2} 3.1-90.6$ & 31 \\
\hline \multirow[t]{2}{*}{129} & $7-31-2002$ & -- & -- & -- & -- & -- \\
\hline & $8-26-2002$ & -- & -- & -- & -- & -- \\
\hline \multirow[t]{2}{*}{130} & $7-22-2002$ & -- & -- & -- & -- & -- \\
\hline & $8-27-2002$ & -- & -- & -- & -- & -- \\
\hline \multirow[t]{2}{*}{131} & $7-22-2002$ & -- & -- & -- & -- & -- \\
\hline & $8-27-2002$ & -- & -- & -- & -- & -- \\
\hline 132 & $8-21-2002$ & 80 & 70 & -- & -- & -- \\
\hline 133 & $8-21-2002$ & 270 & 260 & 0.03 & ${ }^{2} 3.3-93.3$ & 29 \\
\hline 134 & $8-21-2002$ & 80 & 80 & $<0.1$ & ID & 12 \\
\hline 135 & $8-20-2002$ & 90 & 80 & $<0.1$ & ${ }^{2} 3.3-96.7$ & 29 \\
\hline 136 & $8-20-2002$ & 120 & 120 & $<0.1$ & ${ }^{2} 2.6-92.3$ & 38 \\
\hline 137 & $8-20-2002$ & 230 & 220 & -- & -- & -- \\
\hline 138 & $8-20-2002$ & 250 & 240 & -- & -- & -- \\
\hline 139 & 8-19-2002 & 240 & 240 & -- & -- & -- \\
\hline 140 & 8-19-2002 & 250 & 250 & -- & -- & -- \\
\hline \multirow[t]{2}{*}{141} & $7-24-2002$ & -- & -- & -- & -- & -- \\
\hline & $8-27-2002$ & -- & -- & -- & -- & -- \\
\hline \multirow[t]{2}{*}{142} & $7-23-2002$ & -- & -- & -- & -- & -- \\
\hline & $8-28-2002$ & -- & -- & -- & -- & -- \\
\hline \multirow[t]{2}{*}{143} & $7-23-2002$ & -- & -- & -- & -- & -- \\
\hline & $8-28-2002$ & -- & -- & -- & -- & -- \\
\hline \multirow[t]{2}{*}{144} & $7-23-2002$ & -- & -- & -- & -- & -- \\
\hline & $9-4-2002$ & -- & -- & -- & -- & -- \\
\hline \multirow[t]{2}{*}{145} & $7-23-2002$ & -- & -- & -- & -- & -- \\
\hline & $9-4-2002$ & -- & -- & -- & -- & -- \\
\hline 146 & $8-28-2002$ & -- & -- & -- & -- & -- \\
\hline 147 & $9-4-2002$ & -- & -- & -- & -- & -- \\
\hline 148 & $9-4-2002$ & -- & -- & -- & -- & -- \\
\hline
\end{tabular}


Table 18. Water-quality data for the Arkansas River Basin showing 2002 drought values, Colorado water-quality exceedances, and percentiles relative to historical data for selected properties and constituents from July through September, 1978-2002.—Continued

$\left[\mathrm{ft}^{3} / \mathrm{s}\right.$, cubic feet per second; Conc, concentration; Q\%, percentile (percentage of data less than or equal to reported value or concentration) of drought-sample constituent; N, total number of historical and drought samples; $\mathrm{mg} / \mathrm{L}$, milligrams per liter; $\mu \mathrm{S} / \mathrm{cm}$, microsiemens per centimeter at $25^{\circ} \mathrm{Celsius;}{ }^{\circ} \mathrm{C}$, degrees Celsius; E. coli, Escherichia coli; $\mathrm{mL}$, milliliter; $\mu \mathrm{g} / \mathrm{L}$, micrograms per liter; $\mathrm{CaCO}_{3}$, calcium carbonate; $\mathrm{SiO}_{2}$, silica; $\mathrm{SO}_{4}$, sulfate; ID, insufficient number of samples (less than 20) to calculate percentile; <, less than; --, no drought-sample measurement; values or concentrations in bold type indicate 2002 Colorado water-quality exceedances; c, chronic water-quality exceedance; ac, acute and chronic water-quality exceedance]

\begin{tabular}{|c|c|c|c|c|c|c|c|c|c|c|}
\hline \multirow{2}{*}{$\begin{array}{c}\text { Site } \\
\text { number in } \\
\text { table } 1 \\
\text { and fig. } 7\end{array}$} & \multirow{2}{*}{$\begin{array}{l}\text { Sampling } \\
\text { date }\end{array}$} & \multicolumn{3}{|c|}{$\begin{array}{l}\text { Cadmium, total recoverable, in } \\
\qquad \mu \mathrm{g} / \mathrm{L}\end{array}$} & \multicolumn{3}{|c|}{ Chromium, dissolved, in $\mu \mathrm{g} / \mathrm{L}$} & \multicolumn{3}{|c|}{$\begin{array}{c}\text { Chromium, total recoverable, } \\
\text { in } \mu \mathrm{g} / \mathrm{L}\end{array}$} \\
\hline & & Conc & $0 \%$ & $\mathrm{~N}$ & Conc & $0 \%$ & $\mathrm{~N}$ & Conc & $0 \%$ & $\mathrm{~N}$ \\
\hline \multirow[t]{2}{*}{126} & $7-19-2002$ & 0.1 & ID & 9 & -- & -- & -- & -- & -- & -- \\
\hline & $8-26-2002$ & 0.1 & ID & 9 & -- & -- & -- & -- & -- & -- \\
\hline \multirow[t]{2}{*}{127} & $7-31-2002$ & 0.1 & ID & 2 & -- & -- & -- & -- & -- & -- \\
\hline & $8-26-2002$ & $<0.1$ & ID & 2 & -- & -- & -- & -- & -- & -- \\
\hline \multirow[t]{2}{*}{128} & $7-31-2002$ & 0.1 & ID & 19 & -- & -- & -- & -- & -- & -- \\
\hline & 8-26-2002 & 0.1 & ID & 19 & -- & -- & -- & -- & -- & -- \\
\hline \multirow[t]{2}{*}{129} & $7-31-2002$ & -- & -- & -- & -- & -- & -- & -- & -- & -- \\
\hline & $8-26-2002$ & -- & -- & -- & -- & -- & -- & -- & -- & -- \\
\hline \multirow[t]{2}{*}{130} & $7-22-2002$ & -- & -- & -- & -- & -- & -- & -- & -- & -- \\
\hline & $8-27-2002$ & -- & -- & -- & -- & -- & -- & -- & -- & -- \\
\hline \multirow[t]{2}{*}{131} & $7-22-2002$ & -- & -- & -- & -- & -- & -- & -- & -- & -- \\
\hline & $8-27-2002$ & -- & -- & -- & -- & -- & -- & -- & -- & -- \\
\hline 132 & $8-21-2002$ & $<0.1$ & ${ }^{2} 1.8-91.1$ & 55 & -- & -- & -- & 1 & ${ }^{1} 60.0-74.3$ & 34 \\
\hline 133 & $8-21-2002$ & $<0.1$ & ${ }^{2} 2.1-89.6$ & 47 & $<0.8$ & ${ }^{2} 2.1-95.7$ & 46 & $<0.8$ & ${ }^{2} 3.4-86.2$ & 28 \\
\hline 134 & $8-21-2002$ & $<0.1$ & ID & 3 & -- & -- & -- & $<1$ & ID & 13 \\
\hline 135 & $8-20-2002$ & $<0.1$ & ${ }^{2} 2.0-93.9$ & 48 & -- & -- & -- & 2 & ${ }^{1} 58.6-72.4$ & 28 \\
\hline 136 & 8-20-2002 & -- & -- & -- & -- & -- & -- & 2 & ${ }^{1} 48.7-56.4$ & 38 \\
\hline 137 & $8-20-2002$ & $<0.1$ & ${ }^{2} 2.6-97.4$ & 37 & -- & -- & -- & 1 & ${ }^{1} 22.2-36.1$ & 35 \\
\hline 138 & $8-20-2002$ & $<0.1$ & ID & 1 & -- & -- & -- & 2 & ID & 19 \\
\hline 139 & 8-19-2002 & -- & -- & -- & -- & -- & -- & 3 & ID & 5 \\
\hline 140 & 8-19-2002 & -- & -- & -- & -- & -- & -- & 4 & ID & 13 \\
\hline \multirow[t]{2}{*}{141} & $7-24-2002$ & -- & -- & -- & -- & -- & -- & -- & -- & -- \\
\hline & $8-27-2002$ & -- & -- & -- & -- & -- & -- & -- & -- & -- \\
\hline \multirow[t]{2}{*}{142} & $7-23-2002$ & -- & -- & -- & -- & -- & -- & -- & -- & -- \\
\hline & $8-28-2002$ & -- & -- & -- & -- & -- & -- & -- & -- & -- \\
\hline \multirow[t]{2}{*}{143} & 7-23-2002 & -- & -- & -- & -- & -- & -- & -- & -- & -- \\
\hline & $8-28-2002$ & -- & -- & -- & -- & -- & -- & -- & -- & -- \\
\hline \multirow[t]{2}{*}{144} & $7-23-2002$ & -- & -- & -- & -- & -- & -- & -- & -- & -- \\
\hline & $9-4-2002$ & -- & -- & -- & -- & -- & -- & -- & -- & -- \\
\hline \multirow[t]{2}{*}{145} & $7-23-2002$ & -- & -- & -- & -- & -- & -- & -- & -- & -- \\
\hline & $9-4-2002$ & -- & -- & -- & -- & -- & -- & -- & -- & -- \\
\hline 146 & $8-28-2002$ & -- & -- & -- & -- & -- & -- & -- & -- & -- \\
\hline 147 & $9-4-2002$ & -- & -- & -- & -- & -- & -- & -- & -- & -- \\
\hline 148 & $9-4-2002$ & -- & -- & -- & -- & -- & -- & -- & -- & -- \\
\hline
\end{tabular}


Table 18. Water-quality data for the Arkansas River Basin showing 2002 drought values, Colorado water-quality exceedances, and percentiles relative to historical data for selected properties and constituents from July through September, 1978-2002.—Continued

$\left[\mathrm{ft}^{3} / \mathrm{s}\right.$, cubic feet per second; Conc, concentration; $\mathrm{Q} \%$, percentile (percentage of data less than or equal to reported value or concentration) of drought-sample constituent; N, total number of historical and drought samples; $\mathrm{mg} / \mathrm{L}$, milligrams per liter; $\mu \mathrm{S} / \mathrm{cm}$, microsiemens per centimeter at $25^{\circ} \mathrm{Celsius;}{ }^{\circ} \mathrm{C}$, degrees Celsius; E. coli, Escherichia coli; $\mathrm{mL}$, milliliter; $\mu \mathrm{g} / \mathrm{L}$, micrograms per liter; $\mathrm{CaCO}_{3}$, calcium carbonate; $\mathrm{SiO}_{2}$, silica; $\mathrm{SO}_{4}$, sulfate; ID, insufficient number of samples (less than 20) to calculate percentile; <, less than; --, no drought-sample measurement; values or concentrations in bold type indicate 2002 Colorado water-quality exceedances; c, chronic water-quality exceedance; ac, acute and chronic water-quality exceedance]

\begin{tabular}{|c|c|c|c|c|c|c|c|c|c|c|}
\hline \multirow{2}{*}{$\begin{array}{c}\text { Site } \\
\text { number in } \\
\text { table } 1 \\
\text { and fig. } 7\end{array}$} & \multirow{2}{*}{$\begin{array}{l}\text { Sampling } \\
\text { date }\end{array}$} & \multicolumn{3}{|c|}{ Copper, dissolved, in $\mu \mathrm{g} / \mathrm{L}$} & \multicolumn{3}{|c|}{$\begin{array}{c}\text { Copper, total recoverable, } \\
\text { in } \mu \mathrm{g} / \mathrm{L}\end{array}$} & \multicolumn{3}{|c|}{ Iron, dissolved, in $\mu \mathrm{g} / \mathrm{L}$} \\
\hline & & Conc & $0 \%$ & $\mathrm{~N}$ & Conc & $0 \%$ & $\mathrm{~N}$ & Conc & $0 \%$ & $\mathrm{~N}$ \\
\hline \multirow[t]{2}{*}{126} & $7-19-2002$ & 1.1 & ID & 8 & 1.8 & ID & 8 & 51 & ID & 9 \\
\hline & $8-26-2002$ & -- & -- & -- & -- & -- & -- & 28 & ID & 9 \\
\hline \multirow[t]{2}{*}{127} & $7-31-2002$ & 0.8 & ID & 1 & 1.7 & ID & 1 & 15 & ID & 2 \\
\hline & $8-26-2002$ & -- & -- & -- & -- & -- & -- & 13 & ID & 2 \\
\hline \multirow[t]{2}{*}{128} & $7-31-2002$ & 1.1 & 26.1 & 22 & 2.7 & ID & 17 & 11 & ${ }^{1} 4.9-7.3$ & 40 \\
\hline & $8-26-2002$ & -- & -- & -- & -- & -- & -- & 9 & 2.4 & 40 \\
\hline \multirow[t]{2}{*}{129} & $7-31-2002$ & -- & -- & -- & -- & -- & -- & -- & -- & -- \\
\hline & 8-26-2002 & -- & -- & -- & -- & -- & -- & -- & -- & -- \\
\hline \multirow[t]{2}{*}{130} & $7-22-2002$ & -- & -- & -- & -- & -- & -- & $<10$ & ID & 17 \\
\hline & $8-27-2002$ & -- & -- & -- & -- & -- & -- & $<10$ & ID & 17 \\
\hline \multirow[t]{2}{*}{131} & $7-22-2002$ & -- & -- & -- & -- & -- & -- & $<10$ & ID & 13 \\
\hline & $8-27-2002$ & -- & -- & -- & -- & -- & -- & 25 & ID & 13 \\
\hline 132 & $8-21-2002$ & -- & -- & -- & -- & -- & -- & 10 & ${ }^{1} 18.6-20.3$ & 58 \\
\hline 133 & $8-21-2002$ & 1.4 & 46.4 & 27 & 2.4 & 62.8 & 42 & 44 & 23.9 & 45 \\
\hline 134 & $8-21-2002$ & -- & -- & -- & -- & -- & -- & 40 & ID & 13 \\
\hline 135 & $8-20-2002$ & -- & -- & -- & -- & -- & -- & $<10$ & ${ }^{2} 1.9-56.6$ & 52 \\
\hline 136 & $8-20-2002$ & -- & -- & -- & -- & -- & -- & $<10$ & ${ }^{2} 1.5-45.5$ & 65 \\
\hline 137 & $8-20-2002$ & -- & -- & -- & -- & -- & -- & 30 & ${ }^{1} 38.1-47.6$ & 41 \\
\hline 138 & $8-20-2002$ & -- & -- & -- & -- & -- & -- & $<10$ & ID & 12 \\
\hline 139 & $8-19-2002$ & -- & -- & -- & -- & -- & -- & $<10$ & ID & 8 \\
\hline 140 & $8-19-2002$ & -- & -- & -- & 10 & ID & 16 & $<10$ & ${ }^{2} 4.8-71.4$ & 20 \\
\hline \multirow[t]{2}{*}{141} & $7-24-2002$ & -- & -- & -- & -- & -- & -- & $<10$ & ID & 11 \\
\hline & $8-27-2002$ & -- & -- & -- & -- & -- & -- & $<10$ & ID & 11 \\
\hline \multirow[t]{2}{*}{142} & $7-23-2002$ & -- & -- & -- & -- & -- & -- & $<10$ & ID & 11 \\
\hline & $8-28-2002$ & -- & -- & -- & -- & -- & -- & $<10$ & ID & 11 \\
\hline \multirow[t]{2}{*}{143} & $7-23-2002$ & -- & -- & -- & -- & -- & -- & $<30$ & ID & 2 \\
\hline & $8-28-2002$ & -- & -- & -- & -- & -- & -- & $<30$ & ID & 2 \\
\hline \multirow[t]{2}{*}{144} & $7-23-2002$ & -- & -- & -- & -- & -- & -- & $<30$ & ID & 5 \\
\hline & $9-4-2002$ & -- & -- & -- & -- & -- & -- & $<30$ & ID & 5 \\
\hline \multirow[t]{2}{*}{145} & $7-23-2002$ & -- & -- & -- & -- & -- & -- & $<30$ & ID & 11 \\
\hline & $9-4-2002$ & -- & -- & -- & -- & -- & -- & $<30$ & ID & 11 \\
\hline 146 & $8-28-2002$ & -- & -- & -- & -- & -- & -- & $<10$ & ID & 4 \\
\hline 147 & $9-4-2002$ & -- & -- & -- & -- & -- & -- & $<10$ & ID & 1 \\
\hline 148 & $9-4-2002$ & -- & -- & -- & -- & -- & -- & $<30$ & ID & 1 \\
\hline
\end{tabular}


Table 18. Water-quality data for the Arkansas River Basin showing 2002 drought values, Colorado water-quality exceedances, and percentiles relative to historical data for selected properties and constituents from July through September, 1978-2002.—Continued

$\left[\mathrm{ft}^{3} / \mathrm{s}\right.$, cubic feet per second; Conc, concentration; Q\%, percentile (percentage of data less than or equal to reported value or concentration) of drought-sample constituent; N, total number of historical and drought samples; $\mathrm{mg} / \mathrm{L}$, milligrams per liter; $\mu \mathrm{S} / \mathrm{cm}$, microsiemens per centimeter at $25^{\circ} \mathrm{Celsius;}{ }^{\circ} \mathrm{C}$, degrees Celsius; E. coli, Escherichia coli; $\mathrm{mL}$, milliliter; $\mu \mathrm{g} / \mathrm{L}$, micrograms per liter; $\mathrm{CaCO}_{3}$, calcium carbonate; $\mathrm{SiO}_{2}$, silica; $\mathrm{SO}_{4}$, sulfate; ID, insufficient number of samples (less than 20) to calculate percentile; <, less than; --, no drought-sample measurement; values or concentrations in bold type indicate 2002 Colorado water-quality exceedances; c, chronic water-quality exceedance; ac, acute and chronic water-quality exceedance]

\begin{tabular}{|c|c|c|c|c|c|c|c|c|}
\hline \multirow{2}{*}{$\begin{array}{c}\text { Site } \\
\text { number in } \\
\text { table } 1 \\
\text { and fig. } 7\end{array}$} & \multirow{2}{*}{$\begin{array}{c}\text { Sampling } \\
\text { date }\end{array}$} & \multicolumn{3}{|c|}{ Iron, total recoverable, in $\mu \mathrm{g} / \mathrm{L}$} & \multicolumn{3}{|c|}{ Lead, dissolved, in $\mu \mathrm{g} / \mathrm{L}$} & \multirow{2}{*}{$\begin{array}{l}\text { Lead, total } \\
\text { recoverable, } \\
\text { in } \mu \mathrm{g} / \mathrm{L}\end{array}$} \\
\hline & & Conc & $0 \%$ & $\mathrm{~N}$ & Conc & $0 \%$ & $\mathrm{~N}$ & \\
\hline \multirow[t]{2}{*}{126} & $7-19-2002$ & 120 & ID & 9 & $<1$ & ID & 9 & 2 \\
\hline & $8-26-2002$ & 110 & ID & 9 & $<1$ & ID & 9 & 1 \\
\hline \multirow[t]{2}{*}{127} & $7-31-2002$ & 190 & ID & 2 & $<1$ & ID & 2 & 1 \\
\hline & $8-26-2002$ & 110 & ID & 2 & $<1$ & ID & 2 & -- \\
\hline \multirow[t]{2}{*}{128} & $7-31-2002$ & 650 & 36.4 & 21 & $<1$ & ${ }^{2} 4.3-87$ & 22 & 2 \\
\hline & 8-26-2002 & 500 & 22.7 & 21 & $<1$ & ${ }^{2} 4.3-87$ & 22 & 1 \\
\hline \multirow[t]{2}{*}{129} & $7-31-2002$ & -- & -- & -- & -- & -- & -- & -- \\
\hline & $8-26-2002$ & -- & -- & -- & -- & -- & -- & -- \\
\hline \multirow[t]{2}{*}{130} & $7-22-2002$ & -- & -- & -- & -- & -- & -- & -- \\
\hline & $8-27-2002$ & -- & -- & -- & -- & -- & -- & -- \\
\hline \multirow[t]{2}{*}{131} & $7-22-2002$ & -- & -- & -- & -- & -- & -- & -- \\
\hline & $8-27-2002$ & -- & -- & -- & -- & -- & -- & -- \\
\hline 132 & $8-21-2002$ & 90 & 1.5 & 64 & $<0.2$ & ${ }^{2} 2.9-91.2$ & 33 & -- \\
\hline 133 & $8-21-2002$ & 1,130 & 83.3 & 47 & 0.2 & ${ }^{2} 3.4-82.8$ & 28 & -- \\
\hline 134 & $8-21-2002$ & 330 & ID & 13 & $<0.2$ & ID & 8 & -- \\
\hline 135 & $8-20-2002$ & 640 & ${ }^{1} 8.8-10.5$ & 56 & $<0.2$ & ${ }^{2} 3.4-93.1$ & 28 & -- \\
\hline 136 & $8-20-2002$ & 340 & 3.0 & 66 & $<0.2$ & ${ }^{2} 2.5-97.5$ & 39 & -- \\
\hline 137 & $8-20-2002$ & 150 & 2.3 & 42 & -- & -- & -- & -- \\
\hline 138 & $8-20-2002$ & 1,000 & ID & 11 & -- & -- & -- & -- \\
\hline 139 & 8-19-2002 & 1,170 & ID & 5 & -- & -- & -- & -- \\
\hline 140 & 8-19-2002 & $3,840 \mathrm{c}$ & ID & 19 & $<0.2$ & ID & 16 & -- \\
\hline \multirow[t]{2}{*}{141} & $7-24-2002$ & -- & -- & -- & -- & -- & -- & -- \\
\hline & $8-27-2002$ & -- & -- & -- & -- & -- & -- & -- \\
\hline \multirow[t]{2}{*}{142} & $7-23-2002$ & -- & -- & -- & -- & -- & -- & -- \\
\hline & 8-28-2002 & -- & -- & -- & -- & -- & -- & -- \\
\hline \multirow[t]{2}{*}{143} & $7-23-2002$ & -- & -- & -- & -- & -- & -- & -- \\
\hline & $8-28-2002$ & -- & -- & -- & -- & -- & -- & -- \\
\hline \multirow[t]{2}{*}{144} & $7-23-2002$ & -- & -- & -- & -- & -- & -- & -- \\
\hline & $9-4-2002$ & -- & -- & -- & -- & -- & -- & -- \\
\hline \multirow[t]{2}{*}{145} & $7-23-2002$ & -- & -- & -- & -- & -- & -- & -- \\
\hline & $9-4-2002$ & -- & -- & -- & -- & -- & -- & -- \\
\hline 146 & $8-28-2002$ & -- & -- & -- & -- & -- & -- & -- \\
\hline 147 & $9-4-2002$ & -- & -- & -- & -- & -- & -- & -- \\
\hline 148 & $9-4-2002$ & -- & -- & -- & -- & -- & -- & -- \\
\hline
\end{tabular}


Table 18. Water-quality data for the Arkansas River Basin showing 2002 drought values, Colorado water-quality exceedances, and percentiles relative to historical data for selected properties and constituents from July through September, 1978-2002.—Continued

$\left[\mathrm{ft}^{3} / \mathrm{s}\right.$, cubic feet per second; Conc, concentration; $\mathrm{Q} \%$, percentile (percentage of data less than or equal to reported value or concentration) of drought-sample constituent; N, total number of historical and drought samples; $\mathrm{mg} / \mathrm{L}$, milligrams per liter; $\mu \mathrm{S} / \mathrm{cm}$, microsiemens per centimeter at $25^{\circ} \mathrm{Celsius;}{ }^{\circ} \mathrm{C}$, degrees Celsius; E. coli, Escherichia coli; $\mathrm{mL}$, milliliter; $\mu \mathrm{g} / \mathrm{L}$, micrograms per liter; $\mathrm{CaCO}_{3}$, calcium carbonate; $\mathrm{SiO}_{2}$, silica; $\mathrm{SO}_{4}$, sulfate; ID, insufficient number of samples (less than 20) to calculate percentile; <, less than; --, no drought-sample measurement; values or concentrations in bold type indicate 2002 Colorado water-quality exceedances; c, chronic water-quality exceedance; ac, acute and chronic water-quality exceedance]

\begin{tabular}{|c|c|c|c|c|c|c|c|c|}
\hline \multirow{2}{*}{$\begin{array}{c}\text { Site } \\
\text { number in } \\
\text { table } 1 \\
\text { and fig. } 7\end{array}$} & \multirow{2}{*}{$\begin{array}{l}\text { Sampling } \\
\text { date }\end{array}$} & \multicolumn{3}{|c|}{ Manganese, dissolved, in $\mu \mathrm{g} / \mathrm{L}$} & \multicolumn{3}{|c|}{$\begin{array}{l}\text { Manganese, total recoverable, } \\
\text { in } \mu \mathrm{g} / \mathrm{L}\end{array}$} & \multirow{2}{*}{$\begin{array}{c}\text { Mercury, } \\
\text { dissolved, } \\
\text { in } \mu \mathrm{g} / \mathrm{L}\end{array}$} \\
\hline & & Conc & $0 \%$ & $\mathrm{~N}$ & Conc & $0 \%$ & $\mathrm{~N}$ & \\
\hline \multirow[t]{2}{*}{126} & $7-19-2002$ & 9.5 & ID & 9 & 20.4 & ID & 9 & -- \\
\hline & $8-26-2002$ & 10.7 & ID & 9 & 22.6 & ID & 9 & -- \\
\hline \multirow[t]{2}{*}{127} & $7-31-2002$ & 7.7 & ID & 2 & 28.5 & ID & 2 & -- \\
\hline & $8-26-2002$ & 7.7 & ID & 2 & 25.4 & ID & 2 & -- \\
\hline \multirow[t]{2}{*}{128} & $7-31-2002$ & 18.9 & 47.5 & 39 & 77 & ${ }^{1} 40.9-45.5$ & 21 & -- \\
\hline & $8-26-2002$ & 29 & 80.0 & 39 & 77 & ${ }^{1} 40.9-45.5$ & 21 & -- \\
\hline \multirow[t]{2}{*}{129} & $7-31-2002$ & 33 & ID & 6 & -- & -- & -- & -- \\
\hline & $8-26-2002$ & -- & -- & -- & -- & -- & -- & -- \\
\hline \multirow[t]{2}{*}{130} & $7-22-2002$ & 50 & ID & 16 & -- & -- & -- & -- \\
\hline & $8-27-2002$ & $162 \mathrm{c}$ & ID & 16 & -- & -- & -- & -- \\
\hline \multirow[t]{2}{*}{131} & $7-22-2002$ & 13.2 & ID & 14 & -- & -- & -- & -- \\
\hline & $8-27-2002$ & $141 \mathrm{c}$ & ID & 14 & -- & -- & -- & -- \\
\hline 132 & $8-21-2002$ & 10 & ${ }^{1} 5.3-10.5$ & 56 & 10.0 & 1.5 & 64 & $<0.01$ \\
\hline 133 & $8-21-2002$ & $52 \mathrm{c}$ & 50.0 & 47 & 158 & 81.3 & 47 & $<0.01$ \\
\hline 134 & $8-21-2002$ & 40 & ID & 10 & 60 & ID & 13 & $<0.01$ \\
\hline 135 & $8-20-2002$ & -- & -- & -- & 20.0 & ${ }^{1} 7.0-12.3$ & 56 & $<0.01$ \\
\hline 136 & $8-20-2002$ & 20 & ${ }^{1} 46.6-53.4$ & 57 & 50 & ${ }^{1} 6.0-7.5$ & 66 & $<0.01$ \\
\hline 137 & $8-20-2002$ & 40 & $19.0-31.0$ & 41 & 40 & ${ }^{1} 2.7-5.4$ & 36 & $<0.01$ \\
\hline 138 & $8-20-2002$ & -- & -- & -- & 60 & ID & 12 & $<0.01$ \\
\hline 139 & 8-19-2002 & -- & -- & -- & 60 & ID & 5 & $<0.01$ \\
\hline 140 & 8-19-2002 & -- & -- & -- & 170 & ID & 19 & $<0.01$ \\
\hline \multirow[t]{2}{*}{141} & $7-24-2002$ & 7.4 & ID & 11 & -- & -- & -- & -- \\
\hline & $8-27-2002$ & $71 c$ & ID & 11 & -- & -- & -- & -- \\
\hline \multirow[t]{2}{*}{142} & $7-23-2002$ & 12.8 & ID & 10 & -- & -- & -- & -- \\
\hline & $8-28-2002$ & 24 & ID & 10 & -- & -- & -- & -- \\
\hline \multirow[t]{2}{*}{143} & $7-23-2002$ & 36 & ID & 2 & -- & -- & -- & -- \\
\hline & $8-28-2002$ & $115 c$ & ID & 2 & -- & -- & -- & -- \\
\hline \multirow[t]{2}{*}{144} & $7-23-2002$ & 8.1 & ID & 6 & -- & -- & -- & -- \\
\hline & $9-4-2002$ & 15.4 & ID & 6 & -- & -- & -- & -- \\
\hline \multirow[t]{2}{*}{145} & $7-23-2002$ & 43 & ID & 12 & -- & -- & -- & -- \\
\hline & $9-4-2002$ & $133 c$ & ID & 12 & -- & -- & -- & -- \\
\hline 146 & $8-28-2002$ & 8.2 & ID & 3 & -- & -- & -- & -- \\
\hline 147 & $9-4-2002$ & 66 & ID & 1 & -- & -- & -- & -- \\
\hline 148 & $9-4-2002$ & 643 & ID & 1 & -- & -- & -- & -- \\
\hline
\end{tabular}


Table 18. Water-quality data for the Arkansas River Basin showing 2002 drought values, Colorado water-quality exceedances, and percentiles relative to historical data for selected properties and constituents from July through September, 1978-2002.—Continued

$\left[\mathrm{ft}^{3} / \mathrm{s}\right.$, cubic feet per second; Conc, concentration; Q\%, percentile (percentage of data less than or equal to reported value or concentration) of drought-sample constituent; N, total number of historical and drought samples; $\mathrm{mg} / \mathrm{L}$, milligrams per liter; $\mu \mathrm{S} / \mathrm{cm}$, microsiemens per centimeter at $25^{\circ} \mathrm{Celsius;}{ }^{\circ} \mathrm{C}$, degrees Celsius; E. coli, Escherichia coli; $\mathrm{mL}$, milliliter; $\mu \mathrm{g} / \mathrm{L}$, micrograms per liter; $\mathrm{CaCO}_{3}$, calcium carbonate; $\mathrm{SiO}_{2}$, silica; $\mathrm{SO}_{4}$, sulfate; ID, insufficient number of samples (less than 20) to calculate percentile; <, less than; --, no drought-sample measurement; values or concentrations in bold type indicate 2002 Colorado water-quality exceedances; c, chronic water-quality exceedance; ac, acute and chronic water-quality exceedance]

\begin{tabular}{|c|c|c|c|c|c|c|c|c|c|c|}
\hline \multirow{2}{*}{$\begin{array}{c}\text { Site } \\
\text { number in } \\
\text { table } 1 \\
\text { and fig. } 7\end{array}$} & \multirow{2}{*}{$\begin{array}{l}\text { Sampling } \\
\text { date }\end{array}$} & \multicolumn{3}{|c|}{$\begin{array}{l}\text { Mercury, total recoverable, } \\
\text { in } \mu \mathrm{g} / \mathrm{L}\end{array}$} & \multicolumn{3}{|c|}{ Nickel, dissolved, in $\mu \mathrm{g} / \mathrm{L}$} & \multicolumn{3}{|c|}{$\begin{array}{c}\text { Nickel, total recoverable, } \\
\text { in } \mu \mathrm{g} / \mathrm{L}\end{array}$} \\
\hline & & Conc & $0 \%$ & $\mathrm{~N}$ & Conc & $0 \%$ & $\mathrm{~N}$ & Conc & $0 \%$ & $\mathrm{~N}$ \\
\hline \multirow[t]{2}{*}{126} & $7-19-2002$ & -- & -- & -- & -- & -- & -- & -- & -- & -- \\
\hline & $8-26-2002$ & -- & -- & -- & -- & -- & -- & -- & -- & -- \\
\hline \multirow[t]{2}{*}{127} & $7-31-2002$ & -- & -- & -- & -- & -- & -- & -- & -- & -- \\
\hline & $8-26-2002$ & -- & -- & -- & -- & -- & -- & -- & -- & -- \\
\hline \multirow[t]{2}{*}{128} & $7-31-2002$ & -- & -- & -- & -- & -- & -- & -- & -- & -- \\
\hline & $8-26-2002$ & -- & -- & -- & -- & -- & -- & -- & -- & -- \\
\hline \multirow[t]{2}{*}{129} & $7-31-2002$ & -- & -- & -- & -- & -- & -- & -- & -- & -- \\
\hline & $8-26-2002$ & -- & -- & -- & -- & -- & -- & -- & -- & -- \\
\hline \multirow[t]{2}{*}{130} & $7-22-2002$ & -- & -- & -- & -- & -- & -- & -- & -- & -- \\
\hline & $8-27-2002$ & -- & -- & -- & -- & -- & -- & -- & -- & -- \\
\hline \multirow[t]{2}{*}{131} & $7-22-2002$ & -- & -- & -- & -- & -- & -- & -- & -- & -- \\
\hline & $8-27-2002$ & -- & -- & -- & -- & -- & -- & -- & -- & -- \\
\hline 132 & 8-21-2002 & $<0.01$ & ID & 16 & -- & -- & -- & -- & -- & -- \\
\hline 133 & 8-21-2002 & 0.01 & ID & 4 & 2.7 & 79.3 & 28 & 3.9 & 89.3 & 27 \\
\hline 134 & $8-21-2002$ & 0.01 & ID & 12 & -- & -- & -- & -- & -- & -- \\
\hline 135 & 8-20-2002 & $<0.01$ & ID & 10 & -- & -- & -- & -- & -- & -- \\
\hline 136 & $8-20-2002$ & $<0.01$ & ${ }^{2} 4.8-90.5$ & 20 & -- & -- & -- & -- & -- & -- \\
\hline 137 & $8-20-2002$ & $<0.01$ & ID & 12 & -- & -- & -- & -- & -- & -- \\
\hline 138 & $8-20-2002$ & 0.01 & ID & 9 & -- & -- & -- & -- & -- & -- \\
\hline 139 & $8-19-2002$ & 0.01 & ID & 3 & -- & -- & -- & -- & -- & -- \\
\hline 140 & $8-19-2002$ & 0.01 & ID & 9 & -- & -- & -- & -- & -- & -- \\
\hline \multirow[t]{2}{*}{141} & $7-24-2002$ & -- & -- & -- & -- & -- & -- & -- & -- & -- \\
\hline & $8-27-2002$ & -- & -- & -- & -- & -- & -- & -- & -- & -- \\
\hline \multirow[t]{2}{*}{142} & $7-23-2002$ & -- & -- & -- & -- & -- & -- & -- & -- & -- \\
\hline & $8-28-2002$ & -- & -- & -- & -- & -- & -- & -- & -- & -- \\
\hline \multirow[t]{2}{*}{143} & $7-23-2002$ & -- & -- & -- & -- & -- & -- & -- & -- & -- \\
\hline & $8-28-2002$ & -- & -- & -- & -- & -- & -- & -- & -- & -- \\
\hline \multirow[t]{2}{*}{144} & $7-23-2002$ & -- & -- & -- & -- & -- & -- & -- & -- & -- \\
\hline & $9-4-2002$ & -- & -- & -- & -- & -- & -- & -- & -- & -- \\
\hline \multirow[t]{2}{*}{145} & $7-23-2002$ & -- & -- & -- & -- & -- & -- & -- & -- & -- \\
\hline & $9-4-2002$ & -- & -- & -- & -- & -- & -- & -- & -- & -- \\
\hline 146 & $8-28-2002$ & -- & -- & -- & -- & -- & -- & -- & -- & -- \\
\hline 147 & $9-4-2002$ & -- & -- & -- & -- & -- & -- & -- & -- & -- \\
\hline 148 & $9-4-2002$ & -- & -- & -- & -- & -- & -- & -- & -- & -- \\
\hline
\end{tabular}


Table 18. Water-quality data for the Arkansas River Basin showing 2002 drought values, Colorado water-quality exceedances, and percentiles relative to historical data for selected properties and constituents from July through September, 1978-2002.—Continued

$\left[\mathrm{ft}^{3} / \mathrm{s}\right.$, cubic feet per second; Conc, concentration; $\mathrm{Q} \%$, percentile (percentage of data less than or equal to reported value or concentration) of drought-sample constituent; N, total number of historical and drought samples; $\mathrm{mg} / \mathrm{L}$, milligrams per liter; $\mu \mathrm{S} / \mathrm{cm}$, microsiemens per centimeter at $25^{\circ} \mathrm{Celsius;}{ }^{\circ} \mathrm{C}$, degrees Celsius; E. coli, Escherichia coli; $\mathrm{mL}$, milliliter; $\mu \mathrm{g} / \mathrm{L}$, micrograms per liter; $\mathrm{CaCO}_{3}$, calcium carbonate; $\mathrm{SiO}_{2}$, silica; $\mathrm{SO}_{4}$, sulfate; ID, insufficient number of samples (less than 20) to calculate percentile; <, less than; --, no drought-sample measurement; values or concentrations in bold type indicate 2002 Colorado water-quality exceedances; c, chronic water-quality exceedance; ac, acute and chronic water-quality exceedance]

\begin{tabular}{|c|c|c|c|c|c|c|c|c|}
\hline \multirow{2}{*}{$\begin{array}{c}\text { Site } \\
\text { number in } \\
\text { table } 1 \\
\text { and fig. } 7\end{array}$} & \multirow{2}{*}{$\begin{array}{l}\text { Sampling } \\
\text { date }\end{array}$} & \multicolumn{3}{|c|}{ Selenium, dissolved, in $\mu \mathrm{g} / \mathrm{L}$} & \multirow{2}{*}{$\begin{array}{c}\text { Selenium, } \\
\text { total } \\
\text { recoverable, } \\
\text { in } \mu \mathrm{g} / \mathrm{L}\end{array}$} & \multicolumn{3}{|c|}{ Silver, dissolved, in $\mu \mathrm{g} / \mathrm{L}$} \\
\hline & & Conc & $0 \%$ & $\mathrm{~N}$ & & Conc & $0 \%$ & $\mathrm{~N}$ \\
\hline \multirow[t]{2}{*}{126} & $7-19-2002$ & -- & -- & $\overline{--}$ & -- & 0.1c & ID & 2 \\
\hline & $8-26-2002$ & -- & -- & -- & -- & $<0.1$ & ID & 2 \\
\hline \multirow[t]{2}{*}{127} & $7-31-2002$ & -- & -- & -- & -- & $<0.1$ & ID & 2 \\
\hline & $8-26-2002$ & -- & -- & -- & -- & $<0.1$ & ID & 2 \\
\hline \multirow[t]{2}{*}{128} & $7-31-2002$ & -- & -- & -- & -- & $<0.2$ & ${ }^{2} 3.4-93.1$ & 28 \\
\hline & $8-26-2002$ & -- & -- & -- & -- & $<0.1$ & ${ }^{2} 3.4-93.1$ & 28 \\
\hline \multirow[t]{2}{*}{129} & $7-31-2002$ & -- & -- & -- & -- & -- & -- & -- \\
\hline & 8-26-2002 & -- & -- & -- & -- & -- & -- & -- \\
\hline \multirow[t]{2}{*}{130} & $7-22-2002$ & 4 & ID & 13 & 5 & -- & -- & -- \\
\hline & $8-27-2002$ & 4 & ID & 13 & 5 & -- & -- & -- \\
\hline \multirow[t]{2}{*}{131} & $7-22-2002$ & 8 & ID & 13 & 9 & -- & -- & -- \\
\hline & $8-27-2002$ & 28ac & ID & 13 & 32 & -- & -- & -- \\
\hline 132 & $8-21-2002$ & 2 & ID & 8 & 1 & $<0.04$ & ID & 11 \\
\hline 133 & $8-21-2002$ & $<2$ & ID & 4 & $<2$ & $<1$ & ID & 5 \\
\hline 134 & $8-21-2002$ & 2 & ID & 8 & 2 & $<0.04$ & ID & 13 \\
\hline 135 & $8-20-2002$ & $15 \mathrm{c}$ & ID & 5 & 13 & $<0.04$ & ID & 5 \\
\hline 136 & $8-20-2002$ & $14 c$ & 96.3 & 26 & 13 & $<0.04$ & ID & 17 \\
\hline 137 & $8-20-2002$ & 6 & ${ }^{1} 66.7-79.2$ & 23 & 5 & $<0.04$ & ID & 9 \\
\hline 138 & $8-20-2002$ & -- & -- & -- & -- & $<0.04$ & ID & 13 \\
\hline 139 & 8-19-2002 & 6 & ID & 8 & 6 & $<0.04$ & ID & 8 \\
\hline 140 & 8-19-2002 & 7 & ID & 12 & 7 & $<0.04$ & ID & 9 \\
\hline \multirow[t]{2}{*}{141} & $7-24-2002$ & 11 & ID & 10 & 10 & -- & -- & -- \\
\hline & $8-27-2002$ & 15 & ID & 10 & 18 & -- & -- & -- \\
\hline \multirow[t]{2}{*}{142} & $7-23-2002$ & 9 & ID & 12 & 10 & -- & -- & -- \\
\hline & 8-28-2002 & 13 & ID & 12 & 14 & -- & -- & -- \\
\hline \multirow[t]{2}{*}{143} & $7-23-2002$ & 7 & ID & 2 & 7 & -- & -- & -- \\
\hline & $8-28-2002$ & 9 & ID & 2 & 9 & -- & -- & -- \\
\hline \multirow[t]{2}{*}{144} & $7-23-2002$ & 13 & ID & 7 & 13 & -- & -- & -- \\
\hline & $9-4-2002$ & 9 & ID & 7 & 11 & -- & -- & -- \\
\hline \multirow[t]{2}{*}{145} & $7-23-2002$ & 12 & ID & 12 & 11 & -- & -- & -- \\
\hline & $9-4-2002$ & 10 & ID & 12 & 7 & -- & -- & -- \\
\hline 146 & $8-28-2002$ & $<2$ & ID & 2 & $<2$ & -- & -- & -- \\
\hline 147 & $9-4-2002$ & 1 & ID & 1 & $<2$ & -- & -- & -- \\
\hline 148 & $9-4-2002$ & 5 & ID & 2 & 7 & -- & -- & -- \\
\hline
\end{tabular}


Table 18. Water-quality data for the Arkansas River Basin showing 2002 drought values, Colorado water-quality exceedances, and percentiles relative to historical data for selected properties and constituents from July through September, 1978-2002.—Continued

$\left[\mathrm{ft}^{3} / \mathrm{s}\right.$, cubic feet per second; Conc, concentration; Q\%, percentile (percentage of data less than or equal to reported value or concentration) of drought-sample constituent; N, total number of historical and drought samples; $\mathrm{mg} / \mathrm{L}$, milligrams per liter; $\mu \mathrm{S} / \mathrm{cm}$, microsiemens per centimeter at $25^{\circ} \mathrm{Celsius;}{ }^{\circ} \mathrm{C}$, degrees Celsius; E. coli, Escherichia coli; $\mathrm{mL}$, milliliter; $\mu \mathrm{g} / \mathrm{L}$, micrograms per liter; $\mathrm{CaCO}_{3}$, calcium carbonate; $\mathrm{SiO}_{2}$, silica; $\mathrm{SO}_{4}$, sulfate; ID, insufficient number of samples (less than 20) to calculate percentile; <, less than; --, no drought-sample measurement; values or concentrations in bold type indicate 2002 Colorado water-quality exceedances; c, chronic water-quality exceedance; ac, acute and chronic water-quality exceedance]

\begin{tabular}{|c|c|c|c|c|c|c|c|c|}
\hline \multirow{2}{*}{$\begin{array}{c}\text { Site } \\
\text { number in } \\
\text { table } 1 \\
\text { and fig. } 7\end{array}$} & \multirow{2}{*}{$\begin{array}{l}\text { Sampling } \\
\text { date }\end{array}$} & \multirow{2}{*}{$\begin{array}{c}\text { Silver, } \\
\text { total } \\
\text { recoverable, } \\
\text { in } \mu \mathrm{g} / \mathrm{L}\end{array}$} & \multicolumn{3}{|c|}{ Zinc, dissolved, in $\mu \mathrm{g} / \mathrm{L}$} & \multicolumn{3}{|c|}{ Zinc, total recoverable, in $\mu \mathrm{g} / \mathrm{L}$} \\
\hline & & & Conc & $0 \%$ & $\mathrm{~N}$ & Conc & $0 \%$ & $\mathrm{~N}$ \\
\hline \multirow[t]{2}{*}{126} & $7-19-2002$ & $<0.3$ & $<24$ & ID & 9 & 40 & ID & 8 \\
\hline & $8-26-2002$ & $<0.3$ & $<24$ & ID & 9 & 20 & ID & 8 \\
\hline \multirow[t]{2}{*}{127} & $7-31-2002$ & $<0.3$ & $<24$ & ID & 2 & -- & -- & -- \\
\hline & $8-26-2002$ & $<0.3$ & $<24$ & ID & 2 & $<20$ & ID & 1 \\
\hline \multirow[t]{2}{*}{128} & $7-31-2002$ & $<0.3$ & $<24$ & ${ }^{2} 3.1-78.1$ & 31 & -- & -- & -- \\
\hline & $8-26-2002$ & $<0.3$ & $<24$ & ${ }^{2} 3.1-78.1$ & 31 & $<20$ & ${ }^{2} 4.8-14.3$ & 20 \\
\hline \multirow[t]{2}{*}{129} & $7-31-2002$ & -- & -- & -- & -- & -- & -- & -- \\
\hline & $8-26-2002$ & -- & -- & -- & -- & -- & -- & -- \\
\hline \multirow[t]{2}{*}{130} & $7-22-2002$ & -- & -- & -- & -- & -- & -- & -- \\
\hline & $8-27-2002$ & -- & -- & -- & -- & -- & -- & -- \\
\hline \multirow[t]{2}{*}{131} & $7-22-2002$ & -- & -- & -- & -- & -- & -- & -- \\
\hline & $8-27-2002$ & -- & -- & -- & -- & -- & -- & -- \\
\hline 132 & $8-21-2002$ & $<0.04$ & $<6$ & ${ }^{2} 2.9-55.9$ & 33 & $<9$ & ${ }^{2} 1.6-22.6$ & 61 \\
\hline 133 & $8-21-2002$ & $<0.3$ & 12 & 71.4 & 27 & 20 & ${ }^{2} 70.2-83$ & 46 \\
\hline 134 & $8-21-2002$ & $<0.04$ & $<6$ & ID & 8 & $<9$ & ID & 11 \\
\hline 135 & $8-20-2002$ & $<0.04$ & $<6$ & ${ }^{2} 3.4-96.6$ & 28 & $<9$ & ${ }^{2} 1.8-21.4$ & 55 \\
\hline 136 & $8-20-2002$ & $<0.04$ & 10 & ${ }^{1} 81.1-86.5$ & 36 & 20 & ${ }^{1} 18.2-31.8$ & 65 \\
\hline 137 & $8-20-2002$ & 0.04 & 40 & 82.5 & 39 & 40 & ${ }^{1} 27.9-41.9$ & 42 \\
\hline 138 & $8-20-2002$ & 0.09 & 30 & ID & 10 & 40 & ID & 13 \\
\hline 139 & 8-19-2002 & 0.06 & 20 & ID & 6 & 30 & ID & 5 \\
\hline 140 & $8-19-2002$ & 0.07 & -- & -- & -- & 30 & ID & 19 \\
\hline \multirow[t]{2}{*}{141} & $7-24-2002$ & -- & -- & -- & -- & -- & -- & -- \\
\hline & $8-27-2002$ & -- & -- & -- & -- & -- & -- & -- \\
\hline \multirow[t]{2}{*}{142} & $7-23-2002$ & -- & -- & -- & -- & -- & -- & -- \\
\hline & $8-28-2002$ & -- & -- & -- & -- & -- & -- & -- \\
\hline \multirow[t]{2}{*}{143} & $7-23-2002$ & -- & -- & -- & -- & -- & -- & -- \\
\hline & $8-28-2002$ & -- & -- & -- & -- & -- & -- & -- \\
\hline \multirow[t]{2}{*}{144} & $7-23-2002$ & -- & -- & -- & -- & -- & -- & -- \\
\hline & $9-4-2002$ & -- & -- & -- & -- & -- & -- & -- \\
\hline \multirow[t]{2}{*}{145} & $7-23-2002$ & -- & -- & -- & -- & -- & -- & -- \\
\hline & $9-4-2002$ & -- & -- & -- & -- & -- & -- & -- \\
\hline 146 & $8-28-2002$ & -- & -- & -- & -- & -- & -- & -- \\
\hline 147 & $9-4-2002$ & -- & -- & -- & -- & -- & -- & -- \\
\hline 148 & $9-4-2002$ & -- & -- & -- & -- & -- & -- & -- \\
\hline
\end{tabular}

${ }^{1}$ Possible percentile range because of uncensored ties in data.

${ }^{2}$ Possible percentile range because of censored or recensored ties in data. 


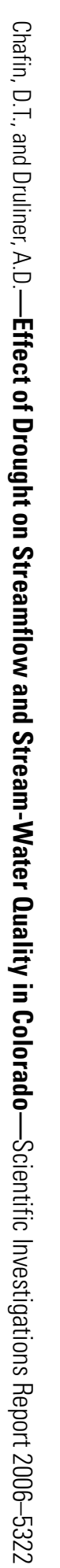

